\title{
Estrategias de diseño y ensamblaje de Objetos de Aprendizaje
}

\author{
GustaVo Javier Astudillo \\ Cecilia Sanz (Directora) \\ Liliana Patricia Santacruz-Valencia (Co-Directora)
}

Tesis presentada para obtener el grado de Magister en Tecnología Informática Aplicada en Educación

Febrero, 2016

Facultad de Informática
UNIVERSIDAD NACIONAL DE LA PLATA 



\section{DEDiCATORIA}

A Paula, Elsa y Humberto 


\section{AGRADECIMIETNTOS}

A Paula por ser mi sostén

A Cecilia por estar siempre

A Liliana por la guía y el apoyo, pese a la distancia

A Carlos Becerra, a Jorge Madonado y a la Biblioteca de la UNLP por el apoyo para conseguir bibliografía A la Facultad de Ciencias Exactas y Naturales, $y$ a la Universidad Nacional de la Pampa 


\section{ÍNDICE DE CONTENIDOS}

Dedicatoria. i

Agradecimientos...........................................................................................................iii

Índice de contenidos.............................................................................................v

Índice de figuras..................................................................................................ix

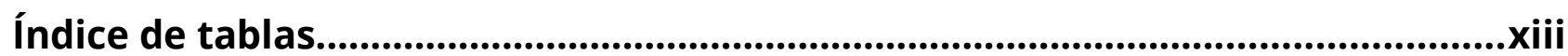

Resumen....................................................................................................................xV

Capítulo 1. Introducción ................................................................................1

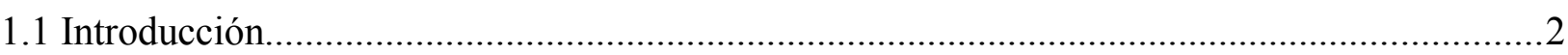

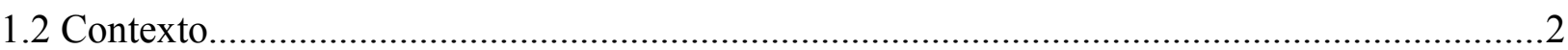

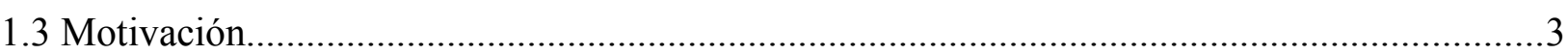

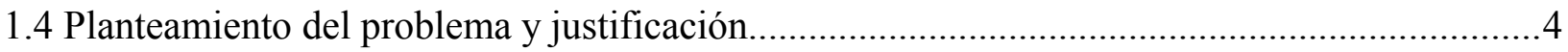

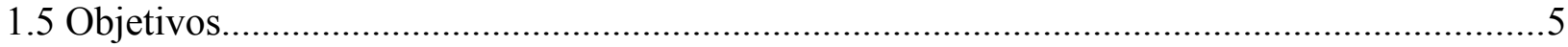

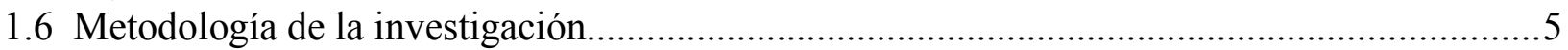

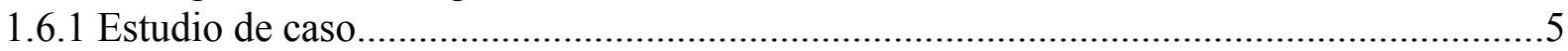

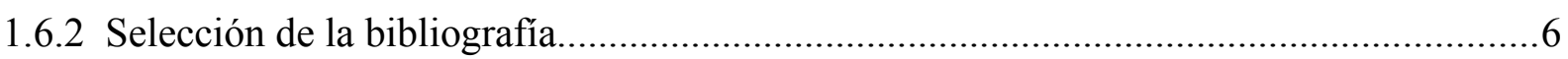

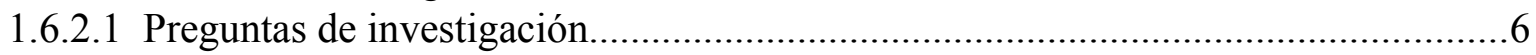

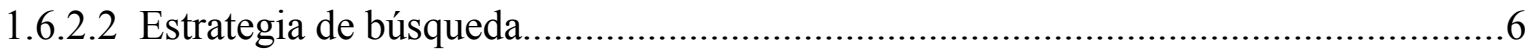

1.6.2.3 Criterios de inclusión/exclusión......................................................................... 7

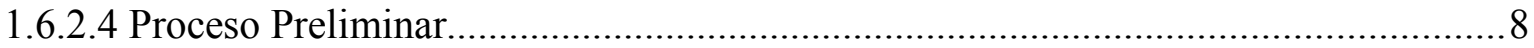

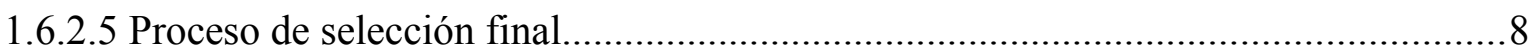

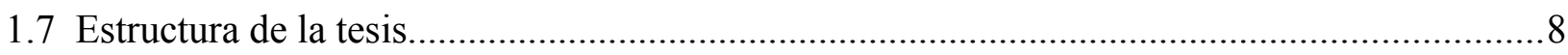

Capítulo 2. Estado del Arte (primera parte) ............................................................11

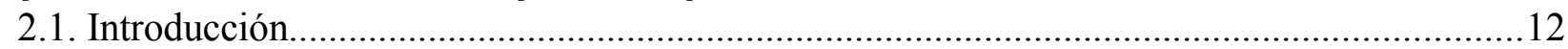

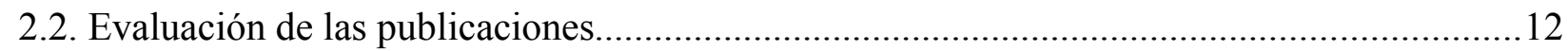

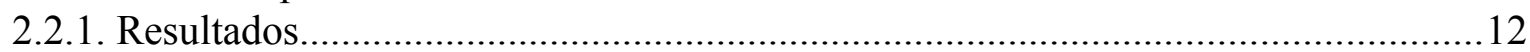

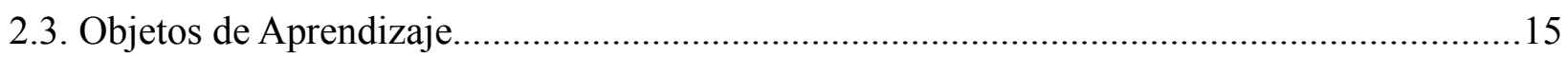

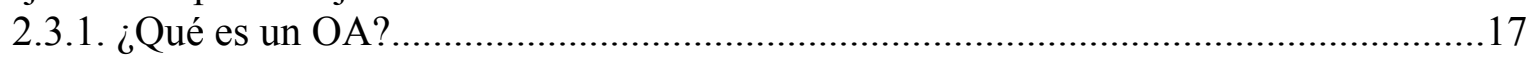

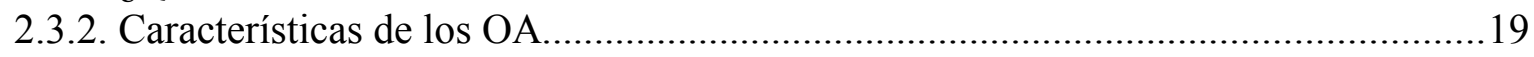

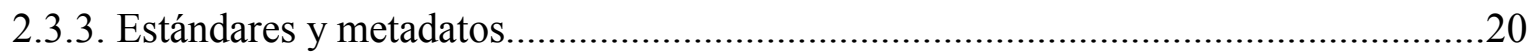

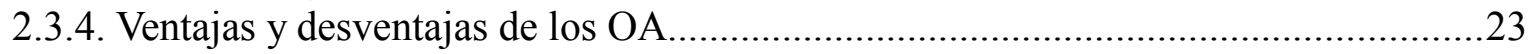

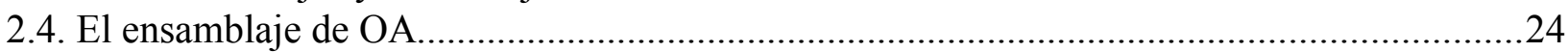

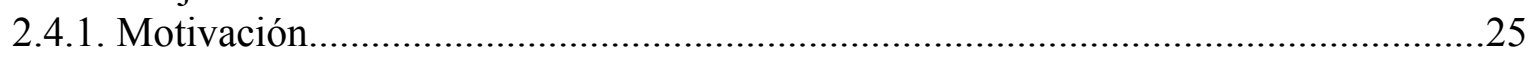

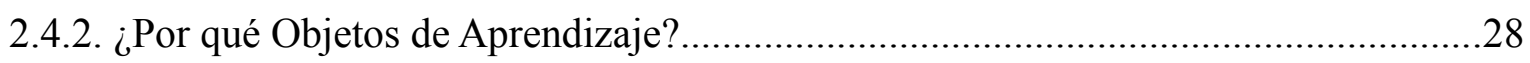

2.4.2.1. Definiciones de OA utilizadas por las metodologías de ensamblaje.....................29

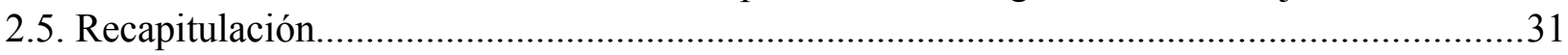


Capítulo 3. Estado del Arte (segunda parte) .33

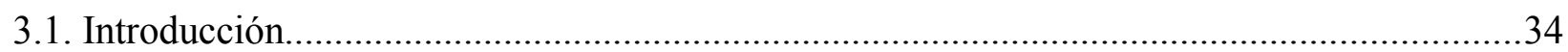

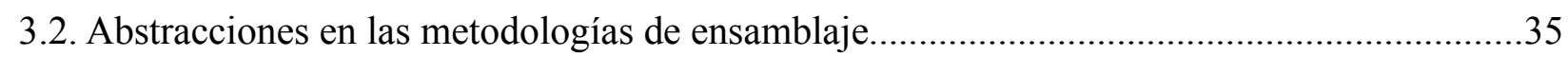

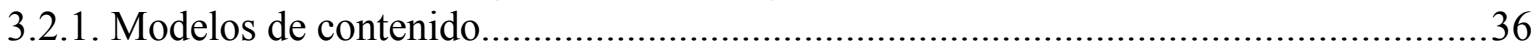

3.2.2. El modelo de estudiante.............................................................................. 43

3.2.3. Modelo de profesor/Modelo de enseñanza o instrucción...........................................44

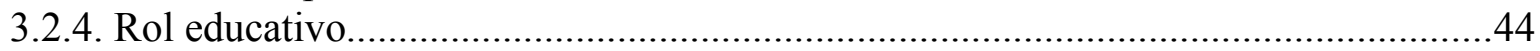

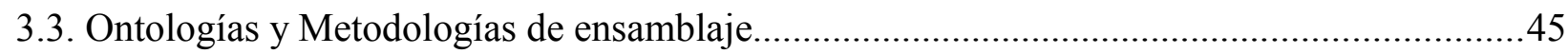

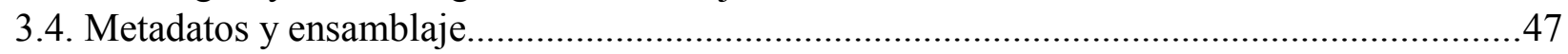

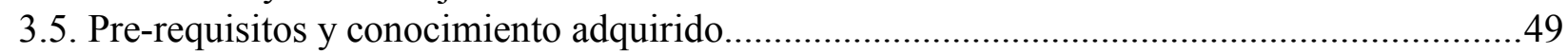

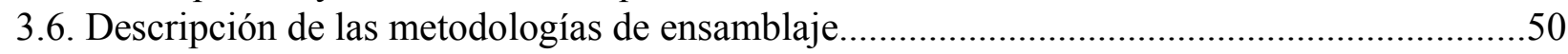

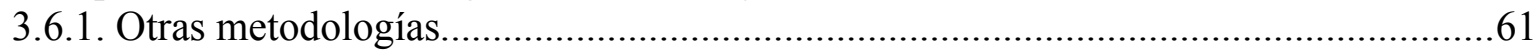

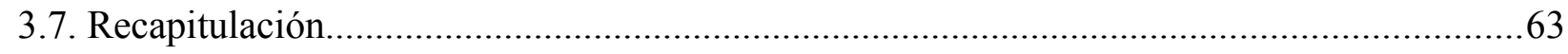

Capítulo 4. Criterios de Evaluación ..........................................................................65

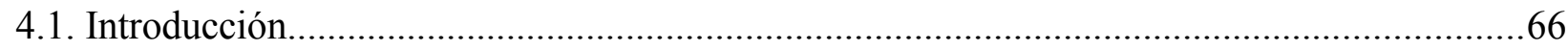

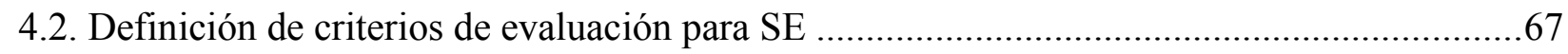

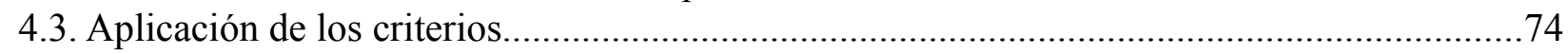

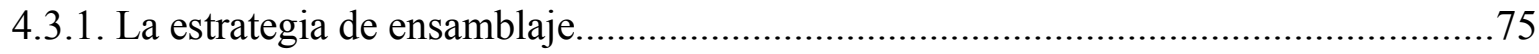

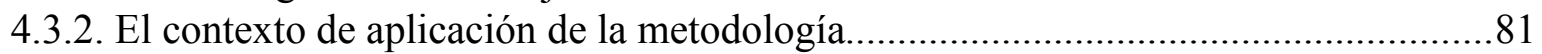

4.3.3. El software que implementa la metodología........................................................ 83

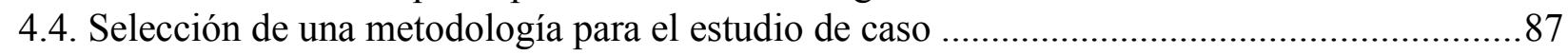

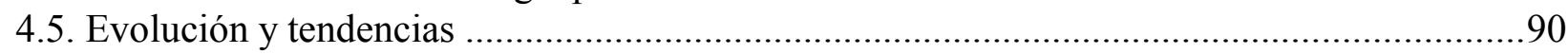

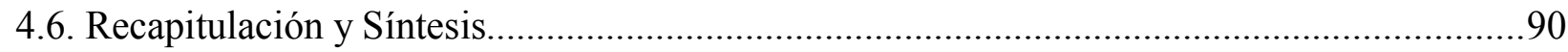

Capítulo 5. Estudio de Caso ....................................................................................93

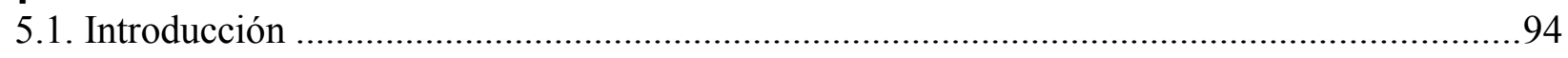

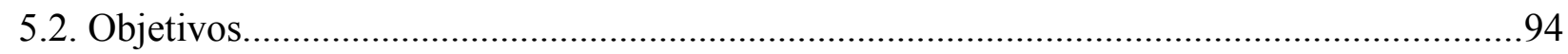

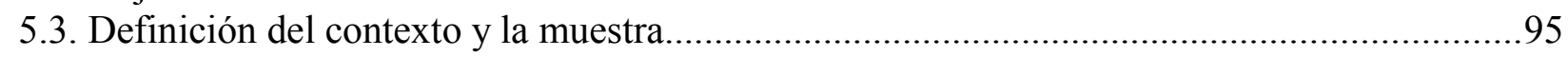

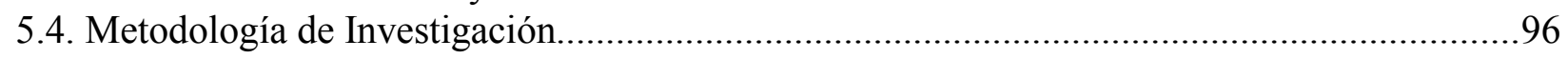

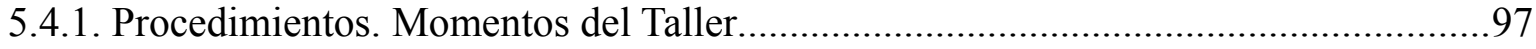

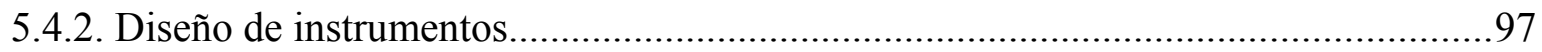

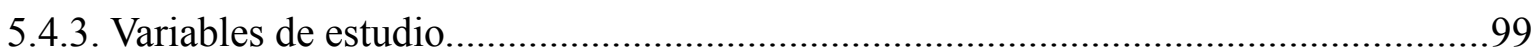

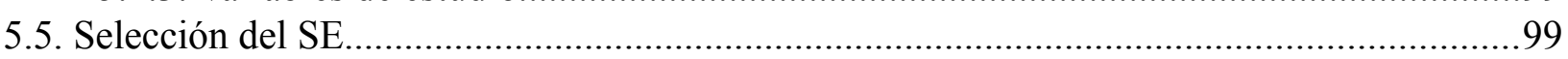

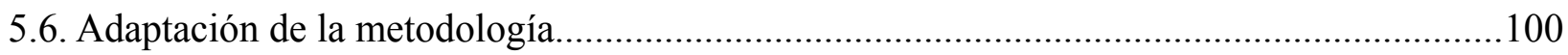

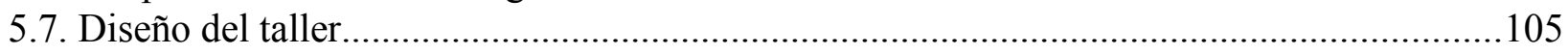

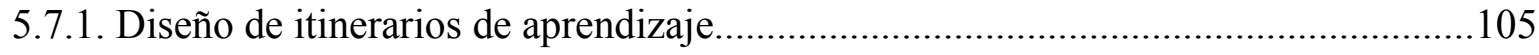

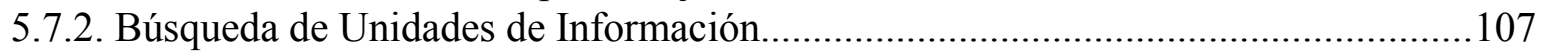

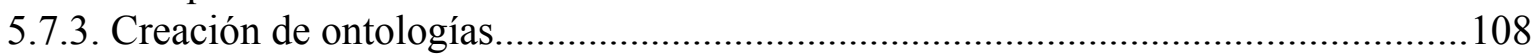

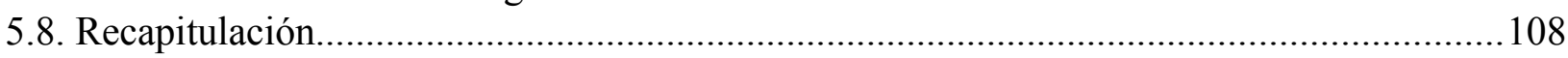

Capítulo 6. Resultados y Discusión .....................................................................111

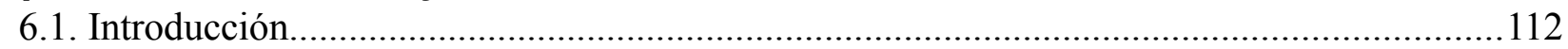

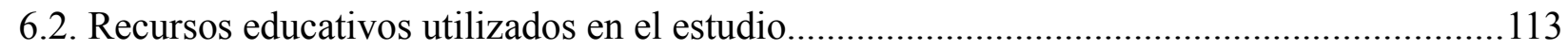

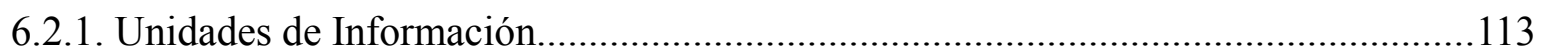

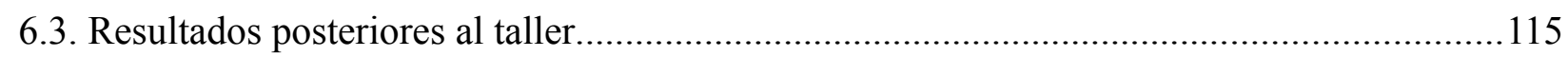

6.3.1. Nivel de dificultad al completar la tarea........................................................... 115

6.3.2. Nivel de comprensión de la metodología de ensamblaje.......................................118

6.3.3. Nivel de comprensión de las adaptaciones realizadas............................................120

6.3.4. Fortalezas y debilidades de la metodología de ensamblaje......................................122

6.3.5. Utilidad de la metodología para crear itinerarios de aprendizaje............................ 124

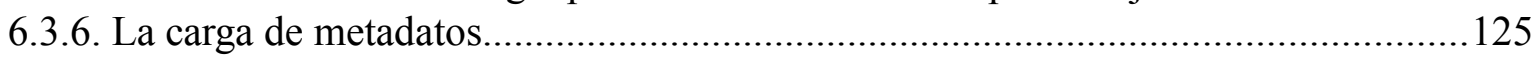


6.3.7. Nivel de satisfacción de los usuarios con ELO-Tool 127

6.3.8. Nivel de satisfacción de los usuarios con ELOs-Viewer...........................................130

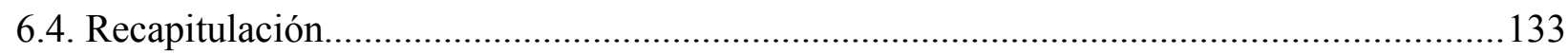

Capítulo 7. Conclusiones y Trabajos Futuros ..................................................135

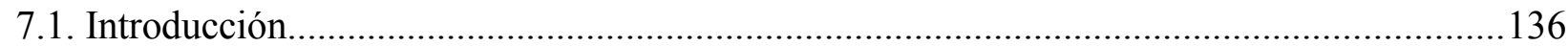

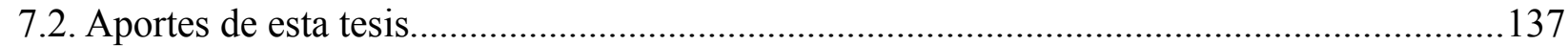

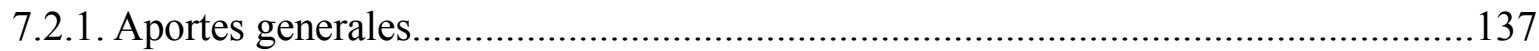

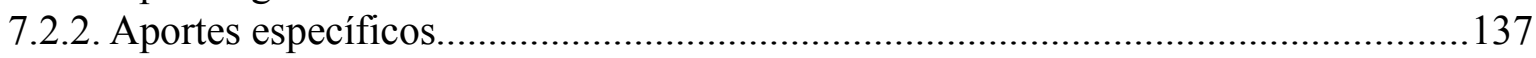

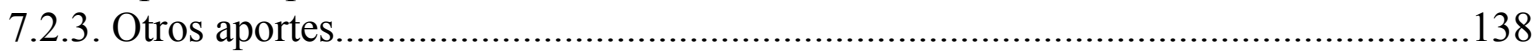

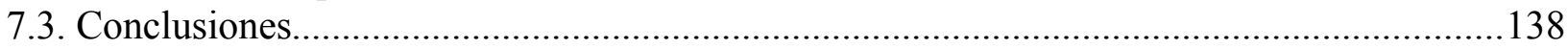

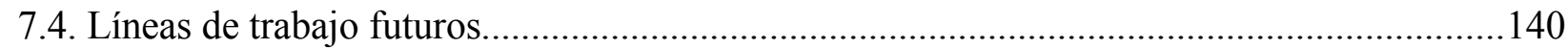

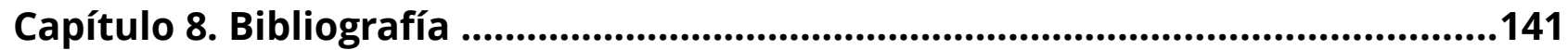

Acrónimos......................................................................................................151

Anexo I. Lista de publicaciones seleccionadas ....................................................155

Anexo II. Aplicación de los criterios de evaluación ..........................................159

Anexo III. Instrumentos de recolección de datos .................................................177

Anexo IV. Caracterización de ELO-Tool y la Metodología de Ensamblaje que Implementa ..............................................................................................187

Anexo V. Diagrama de los itinerarios de aprendizaje ............................................195

Anexo VI. Ontologías ..................................................................................199

Anexo VII. Opiniones de los informantes ......................................................209 


\section{ÍNDICE DE FIGURAS}

\section{Capítulo 2}

Figura 2.1: Jerarquía completa de DCMES (imagen de producción propia)

Figura 2.2: Jerarquía completa de IEEE LOM (imagen producción propia). 22

Figura 2.3. Vinculación entre los OA y las metodologías de ensamblaje.

\section{Capítulo 3}

Figura 3.1. Esquema que representa el modelo de contenidos de SCORM

(ADL, 2009, fig. 2.1.5a). .36

Figura 3.2. Esquema que representa el modelo de contenidos para un ELO

(Santacruz-Valencia et al., 2005, fig. 3.1).

Figura 3.3. Esquema que representa el modelo de contenidos del recurso educativo

(Li \& Huang, 2006, fig. 1) o knowledge unit (Anh et al., 2008, fig. 1).

Figura 3.4: Diferentes tipos de contenido definidos en ALOCoM Content Type (Jovanović, Gasevic, Verbert, \& Duval, 2010, fig. 1)...

Figura 3.5. Caracterización de las metodologías de ensamblaje de OA.

Figura 3.6. Función de metadatos y ontologías en el contexto de las metodología de ensamblaje de OA.

\section{Capítulo 4}

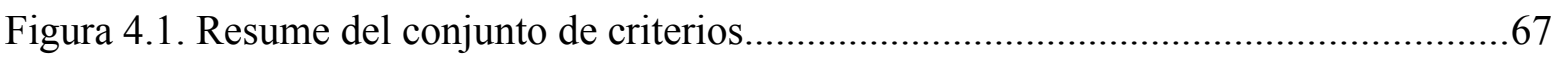

Figura 4.2. Criterio a.i: ensambladores vs recomendadores................................................ 76

Figura 4.3. Criterio a.ii: motivación que llevó a los investigadores a desarrollar la metodología.

Figura 4.4. Criterio a.iii: cómo relaciona la metodología un OA con el siguiente.................77

Figura 4.5. Criterio a.iv: niveles de automatización del proceso de ensamblaje....................78

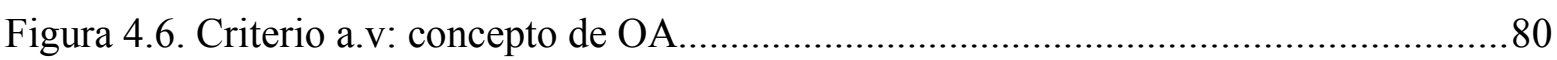

Figura 4.7. Las metodologías recurren a distintas disciplinas para su diseño.......................80

Figura 4.8. Presencia de las tres disciplinas analizadas en la construcción de la metodología.

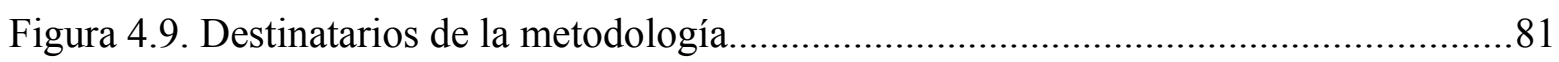

Figura 4.10. Nivel de actividad para metodologías destinadas a estudiantes y docentes.......82

Figura 4.11. De qué forma las metodologías abordan el aspecto pedagógico. .82 
Figura 4.12. Las distintas estrategias de evaluación de las metodologías...........................83

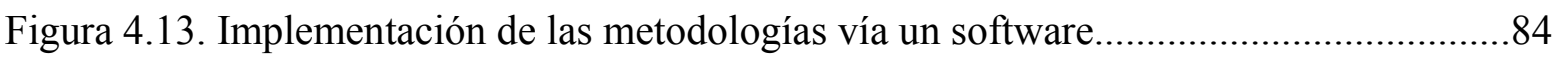

Figura 4.14. Disquisición entre aplicaciones web y de escritorio........................................84

Figura 4.15. Necesidad de credenciales, criterio aplicado sobre las metodología que

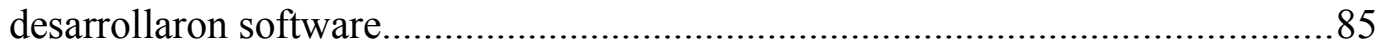

Figura 4.16. Necesidad de credenciales, criterio aplicado sobre el software accesible..........85

Figura 4.17. Fuente de datos de las 25 metodologías que desarrollaron software.................86

Figura 4.18. Estándar de metadatos utilizados por las metodologías en sus

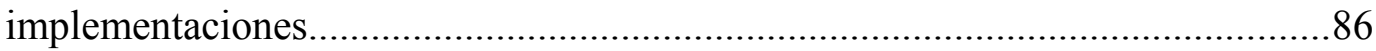

Figura 4.19. Tipo de material utilizado por las metodologías........................................... 87

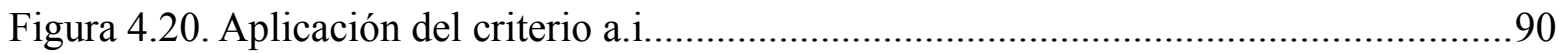

Figura 4.21: Agrupación de las metodologías por Destinatarios.........................................91

Figura 4.22. Accesibilidad del software desarrollado.......................................................92

\section{Capítulo 5}

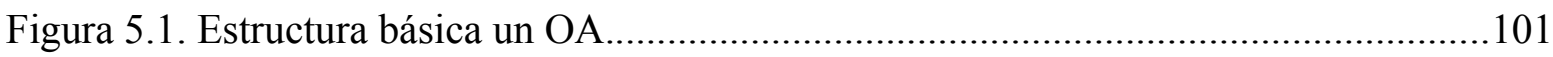

Figura 5.2. Esquema del OA: ESTRUCTURA REPETITIVA FOR ...................................102

Figura 5.3. Esquema del itinerario de aprendizaje INTRODUCCIÓN A ARREGLOS

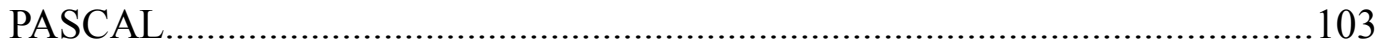

Figura 5.4. Visualizando un ELO en ELO-Tool.............................................................. 103

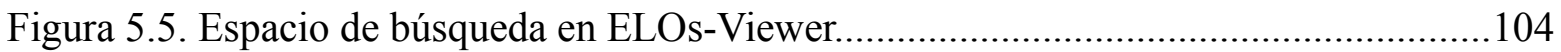

Figura 5.6. Estructura del OA “UD01. ESTRUCTURAS REPETITIVAS PASCAL.

EL FOR" en ELOs-Viewer.

Figura 5.7. Estructura de Itinerario "IA01. INTRODUCCIÓN A ARREGLOS PASCAL"

en ELOs-Viewer..

Figura 5.8. Diagrama que muestra la estructura del Itinerario de Aprendizaje

"IA01. BURBUJA".

Figura 5.9. Diagrama que muestra la estructura del Itinerario de Aprendizaje "IA02.

SELECCION"

Figura 5.10. Diagrama del Itinerario de Aprendizaje "IA03. COMPARAR

REPETITIVAS"....

Figura 5.11. Estructura del Itinerario de Aprendizaje

"IA04. ARCHIVO SECUENCIAL".

Figura 5.12. Estructura del Itinerario de Aprendizaje

"IA05. POLYA VS DESARROLLO".

Figura 5.13. Resumen del proceso realizado para el estudio de caso...

\section{Capítulo 6}

Figura 6.1. Distribución de UIs localizadas en la web por tema. 113

Figura 6.2. Esquema visual que muestra las variables de estudio......................................115

Figura 6.3. Encuesta 2. ¿Qué tan complejo le resultó el proceso de ensamblaje?................115

Figura 6.4. Encuesta de satisfacción sobre ELO-Tool.

"El ensamblaje de los OA le resultó:"

Figura 6.5. La imagen muestra una captura de pantalla donde al ensamblar los OA correspondientes al IA03b los requerimientos aparecen vacíos 
Figura 6.6. Encuesta 1. "Según lo expuesto hasta aquí en el taller, considera Ud. que el ensamblaje de OA es".....

Figura 6.7. Encuesta 1. "En la metodología de ensamblaje, el CA es".

Figura 6.8. Encuesta 1. "Según la ADAPTACIÓN propuesta, qué tipo de ELO son considerados recursos educativos"...

Figura 6.9. Encuesta 1. "Según la ADAPTACIÓN propuesta, un Objeto de Aprendizaje equivale a"

Figura 6.10: Encuesta 1. "Según la ADAPTACIÓN propuesta, un OA debe contener".......121

Figura 6.11. Encuesta 1. "Según la metodología de ensamblaje, qué se obtiene al ensamblar".

Figura 6.12. Encuesta 2. "El nivel de automatización del proceso de ensamblaje que le propone la metodología le pareció"....

Figura 6.13. Encuesta 2. "La cantidad de metadatos que tuvo que cargar para concretar el ensamblaje, le pareció"..

Figura 6.14: Encuesta 2. "La cantidad de información que aporta el Conocimiento Asociado (CA) para el ensamblaje, en todos los casos, le parece".

Figura 6.15: Encuesta 2. "Cuando tuvo que utilizar las ontologías para completar los requerimientos/competencias, la elección del Conocimiento Asociado (CA) más apropiado le resultó".

Figura 6.16. Encuesta 2. "La metodología depende de las ontologías para el ensamblaje, esto la vuelve una metodología".

Figura 6.17: Encuesta 2. "Utilizaría, en el futuro, el ensamblaje (con esta herramienta u otra) como una técnica para la creación de material educativo".

Figura 6.18. Encuesta de satisfacción de ELO-Tool . "La carga de metadatos le resultó"...126

Figura 6.19. Encuesta de satisfacción de ELO-Tool. "La carga de CA de los OA le resultó"...

Figura 6.20. Encuesta de satisfacción de ELO-Tool. "Su percepción en relación a la claridad de cómo el sistema le permite realizar las tareas solicitadas es"..

Figura 6.21. Encuesta de satisfacción de ELO-Tool. "La navegación a través del sistema le resulta".

Figura 6.22. Encuesta de satisfacción de ELO-Tool. "La apariencia general del sistema le resulta".

Figura 6.23. Encuesta de satisfacción de ELO-Tool. "La representatividad del texto de las opciones respecto a la función de las mismas le resultaron".

Figura 6.24. Encuesta de satisfacción de ELO-Tool. "La estructura y organización del sistema le resultó".

Figura 6.25. Encuesta de satisfacción de ELO-Tool. "El sistema para los distintos tipos de usuario que hay en la Facultad resulta"...

Figura 6.26. Parte de los metadatos del itinerario "IA05 - POLYA VS DESARROLLO". El círculo muestra la evaluación vacía. Disponible en: http://solaris.fdi.ucm.es:40000/elo-toolv1/elos/elo793.xml.

Figura 6.27: Encuesta de satisfacción de ELO-Viewer. "Su percepción en relación a la claridad de cómo el sistema le permite realizar las tareas solicitadas es".....

Figura 6.28: Encuesta de satisfacción de ELO-Viewer. "La navegación a través del sistema le resulta"

Figura 6.29: Encuesta de satisfacción de ELO-Viewer. "La apariencia general del sistema le resulta". 
Figura 6.30: Encuesta de satisfacción de ELO-Viewer. "La representatividad del texto de las opciones respecto a la función de las mismas le resultaron".

Figura 6.31: Encuesta de satisfacción de ELO-Viewer. "La estructura y organización del sistema le resultó".

Figura 6.32: Encuesta de satisfacción de ELO-Viewer. "La forma en la que se presentan los ELOs le resultó".

Figura 6.33: Encuesta de satisfacción de ELO-Viewer. "La forma en la que se presentan los metadatos le resultó"....

Figura 6.34: Encuesta de satisfacción de ELO-Viewer. "La forma en la que se presenta el CA de los OA le resultó".

Figura 6.35: Encuesta de satisfacción de ELO-Viewer. "La información que le muestra la herramienta le fue de utilidad en el proceso de ensamblaje"

Figura 6.36: Encuesta de satisfacción de ELO-Viewer. "El sistema para los distintos tipos de usuario que hay en la Facultad resulta".

Figura 6.37. El diagrama ilustra el proceso de creación de IA en este estudio de caso

Figura 6.38. Distribución de las UIs alojadas en ELO-Tool de acuerdo a la fuente de obtención......

Figura 6.39. El diagrama muestra las variables de estudio y los valores obtenidos en base a los registros

Figura 6.40. Restricciones impuestas a las UIs para ser publicadas con vistas al taller. 


\section{ÍNDICE DE TABLAS}

\section{Capítulo 1}

Tabla 1.1. Etapas del estudio de caso.

\section{Capítulo 2}

Tabla 2.1: Lista de lecturas completas. Se incluyen publicaciones seleccionadas en el

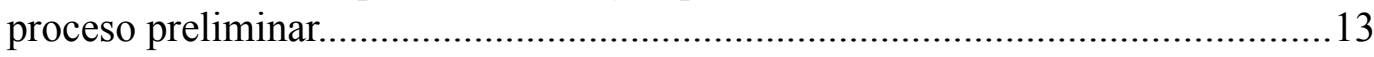

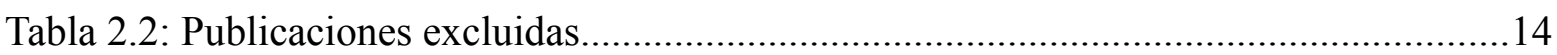

Tabla 2.3: Publicaciones correspondientes al período 2013-2015 .......................................14

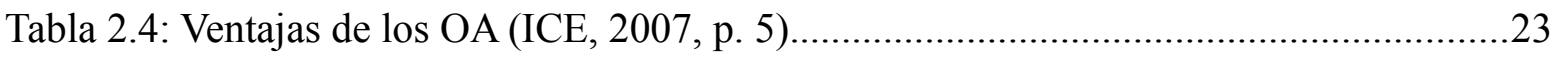

Tabla 2.5: Principales motivaciones para la investigación en la temática.............................28

Tabla 2.6: Caracterización de las definiciones de OA propuestas en las publicaciones analizadas.

\section{Capítulo 3}

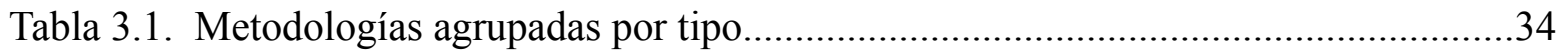

Tabla 3.2: Modelos de contenido...............................................................................42-43

Tabla 3.3: Lista de metodología que utilizan el estándar LOM............................................48

Tabla 3.4: Pre-requisito y conocimiento adquirido...........................................................50

\section{Capítulo 4}

Tabla 4.1: Asignación de una codificación a cada metodología.

Tabla 4.2. Criterio a.ii: motivación que llevó a los investigadores a desarrollar la

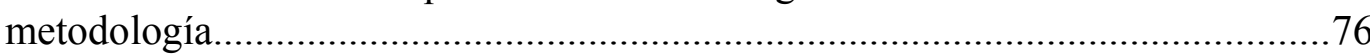

Tabla 4.3. Caracterización de OA. Comparativa con la definición de Sanz (2015)..............79

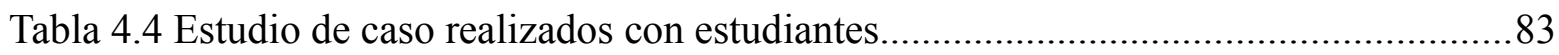

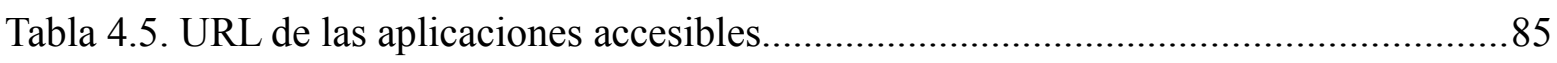

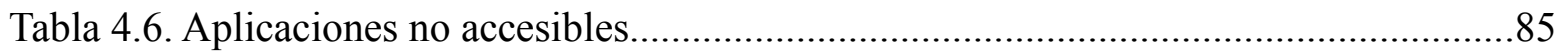

Tabla 4.7. Comparativa para la selección de la metodología para el estudio de caso............89

\section{Capítulo 5}

Tabla 5.1. Posibles resultados de ensamblar dos ELOs

(Santacruz-Valencia et al., 2005, p. 81 Tabla 3.5). 


\section{Capítulo 6}

Tabla 6.1. Codificación e información sobre el registro de audio de las entrevistas. 113

Tabla 6.2. Cantidad de UIs en función de la fuente de obtención de las mismas. 114

Tabla 6.3: Lista de itinerarios de aprendizaje (finalizado el taller). 114

Tabla 6.4. Tiempo de creación del itinerario. Comparativa entre la percepción del informante y el registro audiovisual. 116

Tabla 6.5. Contenido de la Planilla estructura de observación. 118

Tabla 6.6. Encuesta 1. Comparativa de respuestas de los participantes acerca de qué describen las ontologías.

Tabla 6.7. Encuesta 1. Comparativa de respuestas de los participantes acerca la función de las ontologías 
El aprendizaje es un proceso que necesita de la secuenciación del contenido -y de las estrategias que la vehiculizan-, y es precisamente, en este aspecto del proceso educativo en el que se enfoca el ensamblaje. Para lograr una apropiada secuenciación del itinerario de aprendizaje es necesario la selección de los materiales educativos digitales apropiados, pero para ello el conocimiento debe estar organizado de forma que sea posible su localización. Asimismo, sería deseable que para la construcción de un itinerario de aprendizaje se tengan en cuenta distintos aspectos del perfil del estudiante, apuntando a una personalización del contenido. Lograr, además, que la secuenciación de contenidos sea automática (o semi-automática) redundaría en un mejoramiento de los sistemas e-learning, lo que impactaría positivamente en los procesos educativos mediados por TIC y en una reutilización genuina de los materiales educativos. Todas estas son motivaciones que sustentan la investigación en la temática de ensamblaje.

Las metodologías de ensamblaje de OA tienen como objetivo principal la definición de un itinerario de aprendizaje con base en un conjunto de objetos alojados en repositorios. Se trata de un proceso que involucra tres etapas: búsqueda o definición de la temática, definición de la secuencia de aprendizaje y conexión con los OA apropiados.

En este trabajo de tesis se llevó adelante, inicialmente, un proceso de búsqueda y selección publicaciones sobre la temática. Luego de aplicar criterios de inclusión y exclusión se eligieron 35 publicaciones para su revisión en detalle. A partir de allí, se obtienen las 27 metodologías que se analizan en esta tesis.

Luego del análisis realizado, pudo observarse, que de los distintos tipos de materiales educativos, las metodologías de ensamblaje adoptan, en general, los OA. Aunque hay coincidencia en la adopción de éstos, no ocurre lo mismo al definir el concepto. Esto dificultó el proceso de comparación de las metodologías.

Con el objetivo de profundizar el análisis de las metodologías de ensamblaje se definieron 17 de criterios de evaluación. Los mismos permiten caracterizar la estrategia de ensamblaje, el contexto de aplicación y el software que implementa la metodología. La aplicación de cada criterio, permitió tanto la evaluación de las metodologías, como la caracterización y clasificación de las mismas en relación a las categorías. 
Así se pudo establecer que las metodologías de ensamblaje de OA podrían ser agrupadas en tres categorías: las que crean un OA vía ensamblaje, aquellas que crean un itinerario de aprendizaje de OA, y las que recomiendan un itinerario de aprendizaje basado en el perfil del usuario.

Dependiendo del enfoque, las metodologías, pueden trabajar sólo con OA o con estos y los perfiles de los usuarios (docentes/estudiantes), así como con las relaciones entre ellos. Estas entidades -y las relaciones- son representadas, usualmente, con ontologías y metadatos. Un itinerario puede ser generado a través de distintos niveles de automatización y puede (o no) tener en cuenta el perfil del usuario.

Asimismo, fue posible identificar, tomando en cuenta los criterios, las metodologías candidatas para llevar adelante un estudio de caso. Finalmente se seleccionó la metodología con la que se llevó adelante el taller foco de dicho estudio.

El estudio de caso permitió: $(i)$ indagar sobre las opiniones de docentes en relación a la generación de itinerarios de aprendizaje, (ii) identificar fortalezas y debilidades de la metodología de ensamblaje seleccionada, (iii) evaluar el nivel de usabilidad de las herramientas de software utilizadas y (iv) generar un proceso metodológico de aplicación de ELO-Tool para ser utilizado por docentes del área de ciencias de la computación.

Para la implementación del taller, en el contexto de la definición de OA utilizada en esta tesis, fue necesario realizar una serie de adaptaciones. Esto derivó en la creación de un proceso metodológico de aplicación de una metodología de ensamblaje para la creación de itinerarios de aprendizaje.

Finalmente, el aporte resultado del proceso de investigación y de la implementación del estudio de caso, se compone de un estado de la cuestión, un proceso metodológico para la implementación de actividades con ensamblaje de $\mathrm{OA}$, y un conjunto de criterios para la evaluación/caraterización de metodologías de ensamblaje de OA. Así como conjunto de conclusiones derivadas de la investigación. 


\section{Capítulo}

\section{INTRODUCCIÓN}

\section{RESUMEN}

Esta tesis de maestría pone el foco en la investigación de las metodologías y los sistemas de ensamblaje de objetos de aprendizaje.

Motiva esta investigación la necesidad de profundizar el conocimiento de las metodologías de ensamblaje, sus formas de relacionar el material educativo y cómo esto se implementa sobre una aplicación de software. Asimismo, indagar sobre la utilización que estas metodologías hacen de los Objetos de Aprendizaje y el impacto que tienen sobre el diseño de materiales. En este primer capítulo se presenta una introducción a los fundamentos de este trabajo, el contexto y los alcances de esta investigación. Finalmente, se presenta una síntesis de la temática que abordará cada capítulo. 


\subsection{Introducción}

Uno de los principales obstáculos que tienen los docentes y estudiantes al momento de seleccionar un material educativo, de la web, es la ingente cantidad del mismo disponible en ella. Además, es necesario evaluarlos de manera que sea posible reconocer su pertinencia frente al tema y a los destinatarios del mismo.

Si bien mucho de dicho material cuenta con información que facilita su localización y recuperación, la decisión sobre cuál es el más apropiado continúa siendo compleja. Es por esto que se están desarrollando mecanismos que apoyen y faciliten este proceso de selección. Los mismos permiten desde la recomendación de los materiales hasta una propuesta sobre cómo podrían ser ensamblados o secuenciados para conseguir los aprendizajes o competencias que se desean alcanzar o que se pretende que logren los estudiantes.

En la Sección 1.1 se presenta la motivación y el contexto de este trabajo de investigación, así como el planteamiento del problema y su justificación. Asimismo, se enuncian los objetivos y se describe la metodología de la investigación. En la Sección 1.2 se presenta la metodología utilizada para la selección de las publicaciones que llevaron a la elección del conjunto de metodologías analizadas en este trabajo de tesis. Finalmente, en la Sección 1.3, se describe la estructura de la tesis.

\subsection{Contexto}

La web ofrece un amplio abanico de recursos y Materiales Educativos Digitales ${ }^{1}$ (MED) que pueden ser utilizados y reutilizados tanto por diseñadores instruccionales, como docentes $o$ estudiantes. Pero al momento de hallar el que mejor se ajusta a las necesidades u objetivos de aprendizaje, los motores de búsqueda se basan únicamente en un conjunto de palabras claves y proporcionan una variedad de enlaces a contenidos que, por lo general, no reflejan objetivos ni estilos de aprendizaje, competencias o ideas previas necesarias para su abordaje, o para los resultados esperados o destinatarios; por nombrar algunos de los aspectos que ayudarían a caracterizar a un material educativo.

Una alternativa para la búsqueda de este tipo de materiales lo constituyen los repositorios específicos (Astudillo, Willging, \& García, 2011). Estos almacenes, en general de acceso libre, cuentan con herramientas de búsqueda que cubren aspectos pedagógico-didácticos. Los materiales alojados en estos almacenes cuentan con información que describe el material, el ámbito en el que puede ser utilizado y el formato -entre otros datos-, además, de evaluación de pares y/o expertos. Asimismo, se encuentran respaldados por instituciones u organizaciones ligadas a la educación. Todo esto hace que los MED - $\mathrm{o}$ recursos- disponibles en los repositorios sean apropiados cuando, un docente o diseñador instruccional, diseña un curso o actividad educativa, o bien, para un estudiante que necesita avanzar con su auto-aprendizaje.

Sin embargo, la creación de este tipo de almacenes se incrementa año tras año -el proyecto OpenDOAR ${ }^{2}$ puede dar cuenta de esta situación- y con ellos la cantidad de materiales disponibles. Por tanto, el problema de la selección del material más apropiado, paso necesario para la reutilización, continúa siendo una tarea compleja y que demanda mucho tiempo y esfuerzo, aún utilizando repositorios.

1 Pere Marquès (2011) define recursos educativos como "cualquier material que, en un contexto educativo determinado, sea utilizado con una finalidad didáctica o para facilitar el desarrollo de las actividades formativas" y lo distingue de los medios didácticos, "cualquier material elaborado con la intención de facilitar los procesos de enseñanza y aprendizaje". En este trabajo denominaremos a estos últimos materiales educativos, englobando en esta denominación a todo medio didáctico digital.

2 OpenDOAR, un sitio web de la Universidad de Nottingham (Reino Unido) que se encarga de recopilar información sobre repositorios de acceso abierto. Gráficas disponibles en: http://www.opendoar.org/find.php?format=charts (ver Growth of the OpenDOAR Database) 


\subsection{Motivación}

La popularización de la educación virtual y, por ende, la creciente demanda de cursos, talleres, seminarios y postgrados -tanto en modalidades híbridas o a distancia- puso el foco en el diseño y distribución de MED reutilizables. "Debido a las características del eLearning, la creación de contenido reutilizable que pueda ser compartido por varias personas o aplicaciones es una idea que cobra fuerza" (Santacruz-Valencia, Delgado Kloos, \& Cuevas Aedo, 2005, p. 3). Esto impulsó la búsqueda de metodologías flexibles que permitieran; (i) generar MED reutilizables, (ii) minimizar las tareas de diseño de cursos e (iii) implementar formas eficientes para su ensamblaje y reutilización.

La idea de material educativo reutilizable $-\mathrm{y}$ con posibilidad de ensamblaje- puede rastrearse hasta finales de los 60, donde Gerard (1969, pp. 29-30), hablaba de "unidades curriculares que pueden hacerse más pequeñas y combinarse, como piezas de un Meccano". Sin embargo, es tres décadas más tarde donde esa idea comienza a materializarse a través del concepto de Objetos de Aprendizaje (OA) convirtiéndose así en una de las innovaciones tecno-educativas más influyentes en el campo del diseño de materiales educativos (Chan Núñez, 2003; Santacruz-Valencia et al., 2005). Tanto el concepto de reutilizable, como el de ensamblaje, pueden observarse en las metáforas que en los inicios del paradigma intentaban explicarlo: los objetos como piezas de LEGO $^{3}$ o la metáfora del átomo.

Una de las tareas centrales en el diseño de cursos es la selección del material de estudio. El mismo debe estar ajustado a los destinatarios y apoyar la consecución de los objetivos planteados. Una alternativa es desarrollar materiales ad-hoc, pero demanda mucho tiempo y esfuerzo. También es posible adaptar un material ya existente (aunque este es un tema controversial) o bien optar por la reutilización de un MED, tal y como lo diseñó su autor. Cualquiera de estas opciones es muy válida, pero como ya se mencionó antes en el capítulo, el abanico de materiales y recursos que ofrece la web actualmente es inmenso, y por tanto, hallar el material apropiado es una tarea compleja y que demanda mucho tiempo. En un intento por minimizar las tareas que le son propias al diseño de un curso, se han comenzado a integrar, al ámbito educativo, herramientas que originalmente fueron de uso comercial: los Sistemas Recomendadores (SR). Las mismas ofrecen una solución al problema de recuperar materiales educativos ajustados al perfil educativo del destinatario. En el escenario educativo, los SR permiten buscar recursos o materiales educativos en uno o varios Repositorios, $\mathrm{y}$ sugiere aquellos que mejor se adaptan no sólo a la búsqueda, sino también al perfil o necesidades educativas de la persona (Manouselis, Drachsler, Verbert, \& Duval, 2012). Para ello tienen en cuenta: palabras claves, objetivos y estilos de enseñanza (docentes) y de aprendizaje (estudiantes) y/o competencias, entre otros.

El reutilizar material educativo no es novedoso, aunque sí lo es diseñar el material con la intención de que se pueda reutilizar. Así, esta característica se ha trasformado en la marca distintiva de los OA. Como la reutilización, el ensamblaje, es una característica que distingue a los OA de otros tipos de MED. Se trata de "combinar varios [OA] en una unidad instruccional de alto nivel y que cumple con objetivos específicos de aprendizaje bajo circunstancias contextuales concretas" (Lopez Cobo, Arroyo, Sicilia, \& Sánchez, 2007, p. 7). Así pues, se están desarrollando metodologías que permitan crear sistemas que, además de la selección de MED, ofrezcan una secuencia de aprendizaje en la que los mismos se podrían abordar con un fin educativo determinado. A estos sistemas se los denominará en este trabajo como Sistemas Ensambladores (SE).

El ensamblaje de un OA es una tarea compleja y requiere de un conjunto de pre-condiciones que se deben dar para lograrlo (Santacruz-Valencia et al., 2005, p. 69): la localización y descripción del material, la expresión formal de los objetivos y un conjunto de reglas de composición. El hecho de que un MED permita su ensamblaje se constituye en una ventaja sustancial respeto a otros tipos que no lo hagan, ya que: (i) logra reducir costos y esfuerzos de diseño, (ii) favorece la calidad del

3 LEGO es una empresa, fundada en 1932 por la familia Kirk Kristiansen, que cuenta con un popular juego infantil en que se pueden construir diferentes objetos a través del encastre de piezas denominadas "ladrillitos" (http://www.lego.com). 
material, (iii) en función de cómo se ensamble, permite la personalización y adaptación para diferentes objetivos y estilos de aprendizaje y, además, $(i v)$ propicia la reutilización.

Los SR/SE hacen uso, por lo general, de OA. Esto se debe, principalmente, a que los mismos cumplen con dos aspectos que son esenciales para estos sistemas: $(i)$ son diseñados para ser reutilizados y (ii) cuentan con información que los describe o metadatos.

La primera acción para generar un itinerario de aprendizaje, previa al ensamblaje, es la localización de los MED. La forma tradicional de búsqueda en la web, que incluye a los OA, sólo ofrece un listado de estos, incluso dentro de los propios repositorios. Aunque los OA se diseñan con la posibilidad de ser ensamblados -algo implícito en el paradigma, asociación con LEGO o Meccano, o la metáfora de los átomos-, no se incluye en los resultados una posible ruta o itinerario que los vincule (Becerra, Calvo, Muñoz, \& Noël, 2013). Es pues un desafío, no sólo la selección de los objetos de aprendizaje apropiados (ajustados al tema y a las preferencias del usuario), sino también la definición de las relación entre estos (Garrido \& Onaindia, 2013) y que las mismas sean tenidas en cuenta en la generación de los resultados de la búsqueda. Como afirman Casali, Cechinel \& Ochoa (2015) es necesario "enfocar esfuerzos de investigación a un tema que se daba por terminado como es el de proveer acceso al material adecuado, a la persona indicada, en el mejor momento y en el formato que sea el más apto para la situación." (p. 77).

\subsection{Planteamiento del problema y justificación}

La reutilización es uno de los aspectos centrales, tanto en el proceso de diseño de MED como en la consecución de una secuencia de aprendizaje apropiada. Docentes y diseñadores instruccionales retoman y resignifican materiales propios y ajenos. Si bien los OA, por sus características, propician dicha forma de trabajo, también plantean un desafío al momento de su creación, ya que su diseño está orientado a la reutilización y deberían poder ensamblarse de manera que permitan crear unidades de aprendizaje de mayor granularidad, acorde al contexto educativo en el que necesiten aplicarse. Son estas características propias y distintivas de los OA las que siguen poniendo al paradigma como tema de la agenda de la investigación educativa. Se ha avanzado mucho sobre el diseño de OA desarrollando metodologías que facilitan la creación de este tipo de MED, sin embargo, las estrategias y mecanismos de ensamblaje de OA aún se debaten y es necesario profundizar las investigaciones en este sentido. No se cuenta con metodologías validadas que guíen el proceso de ensamblaje y que faciliten la construcción de unidades de aprendizaje más complejas (clases, módulos, cursos).

La creciente oferta en la web de MED, junto con la proliferación de repositorios a nivel mundial hace que la cantidad de materiales que docentes y estudiantes tienen a su alcance se torne inmanejable. Si bien, varios grupos de investigación están trabajando en la implementación de SR que ofrezcan a los usuarios un conjunto de alternativas (MED) que se ajusten a su perfil educativo, las mismas se encuentran, en general, en fase de desarrollo. El contar con SR facilitaría la selección del material, pero no su ensamblaje.

Sumado a lo anterior, tanto las metodologías, como las aplicaciones que las implementan y que permiten la automatización de parte o todo el proceso de composición de OA están en una etapa de prueba. Esto es, la mayoría de las metodologías están en estado experimental y sus implementaciones se basan en prototipos. Si bien, muchas afirman trabajar con OA, las aplicaciones trabajan con recursos que no verifican los requerimientos mínimos del paradigma. Además, las pruebas realizadas, en general, se han llevado a cabo en laboratorio, para pequeños grupos de estudiantes y/o docentes. Todo esto ofrece indicios de que la investigación sobre el ensamblaje de OA no está cerrada y es posible el análisis y evaluación de las metodologías existentes y su prueba sobre grupos concretos de docentes.

Esta tesis toma como punto de partida la experiencia adquirida en la realización del trabajo de Especialización en Tecnología Informática Aplicada en Educación (Astudillo, Sanz, \& Willging, 2011) en la que se realizó una revisión bibliográfica que permitió dar cuenta del estado del arte del paradigma OA y la tesis doctoral de Liliana Santacruz-Valencia (2005) donde se abordó el 
ensamblaje de OA, desarrollando una metodología y una herramienta web que permite la composición de los mismos. Con esta base, se analizaron las diferentes metodologías y herramientas de recomendación y ensamblaje de OA disponibles con el fin de poder caracterizarlas, definir criterios para su evaluación, y finalmente, seleccionar una implementación que permitia llevar adelante un estudio de caso.

\subsection{Objetivos}

El presente trabajo de investigación tiene como objetivo principal realizar un estudio y análisis de las metodologías de ensamblaje de OA que favorezcan la reutilización. En particular, se ha descompuesto este objetivo en los siguientes sub-objetivos:

Estudiar y analizar metodologías de ensamblaje de OA.

Definir criterios que permitan la comparación entre las diferentes metodologías de ensamblaje de OA.

Estudiar y analizar herramientas actuales que permitan el ensamblaje de OA. Partiendo de este estudio realizar una comparativa de las mismas.

Seleccionar una metodología de ensamblaje de OA y una herramienta de ensamblaje y aplicarlos a un estudio de caso.

Realizar el análisis de los resultados obtenidos.

\subsection{Metodología de la investigación}

Se propone realizar un trabajo experimental en el que se evaluarán distintas metodologías y herramientas para el ensamblaje de OA.

En primer lugar, se realiza una revisión, búsqueda, registro y evaluación documental y bibliográfica vinculada a los temas de investigación planteados. Esto permite definir el estado de la cuestión, identificar las metodologías y herramientas que permiten el ensamblaje de OA; asimismo permite la definición de un conjunto de criterios de análisis con vistas a evaluar: $(i)$ cuál es su base pedagógica/didáctica, (ii) cuál es el rol del docente, (iii) cuál es el rol del estudiante, (iv) cuál es la definición de OA que se utiliza, ( $v$ ) cómo y desde dónde se extraen los $\mathrm{OA}$, ( $v i)$ qué criterios se utilizan para la selección y secuenciación de contenidos, (vii) cuáles son las características de las interfaces de las herramientas de ensamblaje (si es que las hubiera), (viii) qué estrategias hay disponibles para su integración a prácticas educativas.

Se desarrolla, luego, un estudio de caso que $(i)$ permite poner en juego la metodología y la herramienta de ensamblaje de OA seleccionada - a partir del análisis previo basado en criterios de evaluación-, y (ii) posibilita su evaluación en profundidad.

\subsubsection{Estudio de caso}

El estudio de caso se lleva adelante en la Facultad de Ciencias Exactas y Naturales (UNLPam), en el marco de un curso de formación docente del que participan docentes de las cátedras Introducción a la Computación y Programación I. Ambas asignaturas se desarrollan durante el primer año de la carrera Profesorado en Computación (primer y segundo cuatrimestre, respectivamente). Se aborda el trabajo en dos etapas: $(a)$ creación de OA, $(b)$ ensamblaje de los mismos utilizando la metodología y herramienta seleccionada. La primera etapa, es previa a la implementación del taller y consta de dos sub-etapas: (a.i) búsqueda y selección de los recursos a utilizar en proceso de ensamblaje, (a.ii) creación y publicación de los OA para que sean accesibles desde la herramienta seleccionada. La segunda etapa se desarrolla durante el taller y se divide en dos sub-etapas: (b.i) la familiarización con la metodología y la herramienta por parte de los participantes y (b.ii) la creación de itinerarios de aprendizaje, a partir del ensamblaje de los OA diseñados en la etapa anterior. Para focalizar el trabajo de los participantes en el ensamblaje de OA, la creación de los mismos estará a 
cargo del autor de esta tesis (ver Tabla 1.1).

Para la recolección de datos, se utiliza la observación participante, encuestas de opinión y satisfacción y entrevistas. El registro de las observaciones fue estructurado. El haber estado a cargo de la coordinación y desarrollo del taller, facilitó su realización y la observación cercana del caso.

Una vez obtenidos todos los registros se analizaron los datos obtenidos y se elaboraron los resultados.

Tabla 1.1. Etapas del estudio de caso

\begin{tabular}{|c|c|c|c|c|c|c|c|c|c|c|c|}
\hline & \multirow[b]{2}{*}{ Actividad/Semanas } & \multicolumn{8}{|c|}{ Creación de OA } & \multicolumn{2}{|c|}{ Taller } \\
\hline & & 1 & 2 & 3 & 4 & 5 & 6 & 7 & 8 & 9 & 10 \\
\hline \multicolumn{12}{|l|}{ Búsqueda y selección } \\
\hline \multicolumn{12}{|l|}{ Creación y publicación } \\
\hline $\begin{array}{l}\text { Familiarización con la } \\
\text { herramienta }\end{array}$ & metodología y la & & & & & & & & & & \\
\hline Ensamblaje de OA & & & & & & & & & & & \\
\hline
\end{tabular}

\subsubsection{Selección de la bibliografía}

La selección del material bibliográfico con el que se trabaja en esta tesis se basa en la propuesta realizada por Barbara Kitchenham (2004). A continuación se describe, en detalle, el proceso de selección de publicaciones.

\subsubsection{Preguntas de investigación}

Teniendo en cuenta los objetivos (1.1.3) se definieron diez preguntas que contienen los criterios apropiados para la inclusión y/o exclusión de publicaciones, a saber:

[CEx1]: ¿Para qué se realiza el ensamblaje de OA? (motivación)

[CEx2]: ¿Quién o quiénes son sus destinatarios?

[CEx3]: ¿Qué metodologías son utilizadas para ensamblar de OA?¿Cuál es la definición de OA que involucran?

[CEx4]: ¿Existen metodologías de ensamblaje que utilicen otros tipos de MED?

[CEx5]: ¿Cómo se realiza el ensamblaje?¿Qué tipo de técnicas son utilizadas?

[CEx6]: Las secuencias de OA ¿son lineales, forman árboles o constituyen una red de OA?

[CEx7]: ¿Las metodologías han desarrollado alguna herramienta de software que la implemente?

[CEx8]: La herramienta de software ¿esta accesible? (vía la web o como aplicación de escritorio)

[CEx9]: ¿Cuáles son las funcionalidades que ofrece la herramienta?

[CEx10]: ¿Qué tipo de material se obtiene luego de ensamblaje?

\subsubsection{Estrategia de búsqueda}

\section{- Fuentes bibiográficas}

Se utilizaron, principalmente, tres fuentes documentales digitales/electrónicas para la localización de los documentos bibliográficos: 
- IEEE Xplore digital library ${ }^{4}$

- Springer $^{5}$

- ACM Digital Library 6

Las bases de datos antes mencionadas fueron seleccionadas por ser librerías digitales que brindan acceso a artículos publicados en revistas y actas de congresos de reconocidos por la comunidad científica internacional.

También se consultó la Comunidad Latinoamericana de Objetos de Aprendizaje (LACLO) ${ }^{7}$, por ser un ámbito especializado en la temática. Asimismo, se realizaron búsquedas utilizando el motor de búsqueda Google Académico ${ }^{8}$.

Los idiomas elegidos fueron español e inglés y los tipos de documentos: actas de congresos, artículos en revistas, libros/capítulos de libros, tesis, capítulos de tesis o reportes de investigación publicados entre 2004 y 2012 inclusive. Se eligió la década próxima pasada debido a que en la misma se popularizó la web como espacio de publicación de materiales educativos, particularmente a través de la utilización OA. A fines de 2015 se realizó una nueva revisión para analizar las últimas investigaciones en relación a metodologías de ensamblaje de OA. Se trabajó con siete publicaciones 2013-2015 que permitieron confirmar algunas tendencias en la temática.

- Palabras clave

Las palabras clave fueron: ensamblaje/assembly, objetos de aprendizaje/learning object, composición/composition, automático/automatic, dinámico/dynamic, metodología/methodology, secuencia de aprendizaje/itinerario de aprendizaje/learning path/learning route.

Si bien todas las palabras se tuvieron en cuenta, no se utilizaron todas en las cadenas de búsqueda.

\section{- Cadenas de búsqueda}

Se realizaron las siguientes combinaciones de palabras clase sobre los buscadores utilizados:

- ensamblaje objetos de aprendizaje / assembly learning object

- ensamblaje "objetos de aprendizaje" / assembly "learning object"

- "ensamblaje de objetos de aprendizaje" / "assembly learning object"

- composición objetos de aprendizaje / learning object composition

- composición "objetos de aprendizaje" / "learning object" composition

- "composición de objetos de aprendizaje" / "learning object composition"

\subsubsection{Criterios de inclusión/exclusión}

Se utilizaron los siguientes criterios para la inclusión de artículos:

[CEx1]: Artículos que propusieran una metodología de ensamblaje de MED de forma automática, semi-automática o manual.

[CEx2]: Artículos que propusieran una metodología de ensamblaje de OA de forma automática, semi-automática o manual.

[CEx3]: Artículos que usen sistemas recomendadores para lograr el ensamblaje de OA que

4 http://ieeexplore.ieee.org/Xplore/home.jsp

5 http://rd.springer.com/

6 http://dl.acm.org/

7 http://laclo.org/papers/index.php

8 http://scholar.google.com 
formarán parte de un itinerario de aprendizaje o learning path.

[CEx4]: Descripción de una técnica para el ensamblaje.

[CEx5]: Descripción de una herramienta de software que implementa una metodología de ensamblaje de MED/OA.

Se utilizaron los siguientes criterios para la excluir artículos:

[CEx1]: El idioma del trabajo.

[CEx2]: Artículos a los que no se pudo acceder al texto completo.

[CEx3]: Artículos que sólo realizan el trabajo de recomendación de MED.

[CEx4]: Literatura informal.

\subsubsection{Proceso Preliminar}

Se realizó un proceso preliminar de selección aplicando las cadenas de búsqueda en las fuentes seleccionadas. Para cada una de las publicaciones que arrojó el proceso de búsqueda se leyó: el título, resumen y palabras clave; y se aplicaron los criterios de inclusión y exclusión. Este proceso generó el listado de artículos considerados para su lectura completa.

Cabe destacar que la lectura de los artículos, a su vez, propició la selección de otros trabajos presenten en las referencias o bibliografía que ofrecía el trabajo.

Asimismo, se realizó una búsqueda basada en los autores para identificar trabajos relacionados/derivados sobre la temática desarrollados por el mismo autor.

\subsubsection{Proceso de selección final}

Los artículos aprobados en la fase preliminar fueron sometidos a una lectura completa. En esta etapa se volvieron a aplicar los criterios de inclusión/exclusión, de manera que si no estaba relacionada con objetivo de investigación, el artículo se descartó.

Para finalizar, aquellos artículos seleccionados fueron registrados en una base bibliográfica y se respondieron las preguntas de investigación.

\subsection{Estructura de la tesis}

Esta memoria está organizada en ocho capítulos y siete anexos: I. Introducción, II. y III. Estado de arte, IV. Criterios de evaluación, V. Caso de Estudio, VI. Resultados y discusión, VII. Conclusiones $\mathrm{y}$ trabajos futuros, y VIII. Bibliografía.

Los anexos por su parte contienen: Anexo I. Lista de publicaciones seleccionadas, las tablas con los resultados de aplicar las preguntas de evaluación a la lista de publicaciones; Anexo II. Aplicación de criterios de evaluación, las tablas correspondientes a la aplicación de criterios de evaluación, el Anexo III. Instrumentos de recolección de datos, las encuestas y las preguntas de las entrevistas aplicadas a los informantes; el Anexo IV. ELO-Tool, una descripción de la herramienta de software utilizada en el estudio de caso; el Anexo V. Diagrama de los Itinerarios de Aprendizaje, contiene los esquemas de los itinerarios de aprendizaje proporcionados a los participantes del taller para realizar la actividad de ensamblaje; el Anexo VI. Ontologías de dominio, presenta las ontologías creadas para el estudio de caso en lenguaje OWL; y finalmente, el Anexo VII. Opiniones de los informantes, ofrece parte de la transcripción de las opiniones de los informantes.

El Capítulo I, dividido en siete secciones, presenta una introducción a la temática de la Tesis y al proceso de investigación. En la primera sección, se presenta el capítulo. Luego en las secciones dos y tres se establece la motivación y el contexto del trabajo. En la cuarta se hace el planteamiento del problema y de la hipótesis de partida. En la quinta sección se enuncian los objetivos de investigación. La sección seis describe la metodología de investigación, y finalmente, en esta séptima y última sección se describe la estructura de la tesis. 
El Capítulo II, dividido en cinco secciones, expone la primera parte del estado de la cuestión. La primera sección presenta el capítulo, y seguidamente, en la sección dos, se describe la metodología de selección de las publicaciones y se listan los resultados de este proceso. En la sección tres, se aborda el paradigma de OA a partir de una breve historia del desarrollo del mismo, se define el concepto y se enumeran sus características. También, en la misma sección, se define el concepto de metadatos y se establece su relación con los OA, así como la función de los repositorios y su importancia en el paradigma; para terminar la sección se enuncian ventajas y desventajas del uso de OA. La sección cuatro, desarrolla el tema de ensambladores de OA, introduce el concepto, lista las motivaciones que llevan a los investigadores a enfocarse en la temática y discute el por qué se eligen los OA como insumo del proceso de ensamblaje. Para finalizar, en la sección cinco, se hace una recapitulación.

El Capítulo III, dividido en siete secciones, aborda la segunda, y última parte, del estado de la cuestión. La primera sección presenta el capítulo, se define el concepto de ensamblaje y se propone una tipificación de las metodologías. En la segunda sección se describen las abstracciones con las que trabajan las metodologías de ensamblaje (modelo de contenido, de estudiante, de profesor y de enseñanza). En la tercera sección, se discute sobre el papel que juegan las ontologías en las metodologías de ensamblaje analizadas en esta tesis. En la sección cuatro, se explica la importancia de los metadatos y, seguidamente, en la sección cinco, se hace lo propio para los pre-requisitos y el conocimiento adquirido. En la ante-última sección se hace una descripción de las 27 metodologías de ensamblaje de OA analizadas en este trabajo y una revisión de siete metodologías correspondientes al período 2013-2015 con vistas a la actualización del estado del arte. Finalmente, en la sección siete, se presenta una recapitulación.

En el Capítulo IV, dividido en cinco secciones, se aborda la definición de los criterios que permitirán la clasificación y evaluación de las metodologías de ensamblaje. La primera sección presenta el capítulo, en ella se describen las categorías en las que estarán agrupados los criterios de evaluación. En la sección dos se define cada uno de los criterios y se describen los posibles valores para estos, para seguidamente, en la sección tres, mostrar los resultados de su aplicación. La cuarta sección, con base en información obtenida de la aplicación de los criterios, se discute la elección de una metodología de ensamblaje para el estudio de caso. Finalmente, en la quita sección se expone una recapitulación y una síntesis, donde se presenta un re-agrupamiento y caracterización de las metodologías a partir de la aplicación de algunos de los criterios.

En el Capítulo V, dividido en ocho secciones, se describe el Estudio de Caso y el diseño del taller sobre el que el mismo se implementa. La primera sección introduce el capítulo. Los objetivos del Estudio de Caso, así como la definición del contexto y la muestra se definen en las secciones dos y tres, respectivamente. En la sección cuatro, se presenta la metodología de investigación y se describe: el diseño de instrumentos y procedimientos, el proceso de recolección de datos y las variables de estudio. En la sección cinco se discute la elección de la metodología de ensamblaje. La sexta sección presenta el conjunto de adaptaciones realizadas para llevar adelante el taller. Seguidamente, en la sección siete, se describe el diseño de dicho taller. Finalmente, en la octava, y última sección, se hace una recapitulación.

En el Capítulo VI, dividido en cuatro secciones, se presenta los resultados obtenidos del caso de estudio y la discusión de los mismos. La primera sección introduce el capítulo. La sección dos resume los resultados obtenidos en el proceso de localización y publicación de los recursos educativos que permitieron la creación de OA y posteriormente de los itinerarios de aprendizaje. La sección tres presenta los resultados obtenidos en el estudio de caso organizados a través de las variables de estudio. En la misma sección se discuten los resultados a medida que se presentan los mismos. Finalmente, en la octava, y última sección, se hace una recapitulación.

El Capítulo VII, dividido en cuatro secciones, expone las conclusiones y las futuras líneas de investigación. En la primera sección se retoman los objetivos y la motivación a modo de introducción. Seguidamente, en la sección dos, se presentan los aportes derivados de las actividades realizadas en esta tesis. En la tercera sección se exhibe conclusiones, donde se identifican las principales contribuciones, clasificadas en tres tipos de aportaciones: generales, específicas y las 
publicaciones realizadas. Para finalizar el capítulo, en la sección cuatro se presentan las futuras líneas de trabajo.

Para concluir esta Tesis, en el Capítulo VIII, se detalla la bibliografía referenciada. 


\section{Capítulo}

\section{ESTADO DE ARTE (PRIMERA PARTE)}

\section{RESUMEN}

La elección del conjunto de publicaciones que se analizan en esta tesis tuvo un proceso de selección basado en la definición de criterios de inclusión y exclusión. Se obtuvieron así, 35 publicaciones que describen 27 metodologías de ensamblaje de $O A$.

Este segundo capítulo presenta la primera parte del estado del arte. Inicialmente, se describe el proceso de selección de las publicaciones y los resultados obtenidos del mismo. Luego, se aborda el paradigma de Objetos de Aprendizaje (las metáforas, su definición, sus características, estándares y metadatos) para, seguidamente, ponerlos en el contexto del ensamblaje. Se presentan las motivaciones de los investigadores para enfocarse en la temática y se analiza por qué los $O A$ son el material elegido por las metodologías de ensamblaje y cómo éstos son definidos en las mismas. 


\subsection{Introducción}

Como afirman Cobo Romaní y Pardo Kuklinski (2007) “en esta nueva Web la red digital deja de ser una simple vidriera de contenidos multimedia para convertirse en una plataforma abierta, construida sobre una arquitectura basada en la participación de los usuarios" (p. 15). Esto ha provocado el ingente crecimiento en la publicación de contenidos en la web, y de MED en particular. La cantidad de materiales que se obtienen al realizar una búsqueda es tal, que insume mucho tiempo la selección del más apropiado. Sumado a lo anterior, una vez hecha la selección, se debe crear una secuencia de aprendizaje a partir de los MED localizados. La secuenciación de contenidos es una tarea compleja, para un docente que diseña actividades y, más aún, para un estudiante que desea gestionar su propio aprendizaje.

La problemática expuesta, ha motivado a parte de la comunidad científica a desarrollar metodologías que apoyen y faciliten este proceso de selección y ensamblaje de MED. Las diferentes propuestas van desde la recomendación de materiales basados en el perfil del usuario (docente o estudiante), pasando por el ensamblaje de los MED con distintos grados de automatización del proceso, hasta aquellos que recomiendan un itinerario de aprendizaje ${ }^{1}$ (learning path) basado en el perfil del usuario.

A continuación (Sección 2.2) se presentan los resultados obtenidos a partir del proceso de selección de publicaciones descrito en el Capítulo 1. Luego, en la Sección 2.3, se realiza una introducción al paradigma de OA y al conceptos metadatos (asociado a OA) para, seguidamente (Sección 2.4), enunciar las motivaciones que impulsan a los investigadores a enfocarse en la temática de esta tesis. En la misma Sección se exponen, además, los motivos por los cuales se eligen los OA como material para el ensamblaje y se discute la elección de la definición de OA en las publicaciones seleccionadas. Finalmente, se ofrece una recapitulación (Sección 2.5).

\subsection{Evaluación de las publicaciones}

La evaluación de cada una de las publicaciones seleccionadas (lectura completa) se realizó basada en las preguntas de investigación planteadas. Se las denomina preguntas de evaluación (PE) y se utilizó una calificación binaria: Sí/No (S/N) para identificar si en la publicación se responde (o no) a cada pregunta. Las preguntas de evaluación son las siguientes:

[PE1]: ¿Se expresa/n la/s motivación/es que lleva/n a desarrollar una metodología de ensamblaje?

[PE2]: ¿Propone una metodología de ensamblaje?

[PE3]: ¿Describe cómo se realiza el ensamblaje?

[PE4]: ¿Se enuncian los destinatarios y sus funciones?

[PE5]: ¿Utiliza OA?

[PE6]: ¿Define el concepto de OA?

[PE7]: ¿Describe alguna herramienta de software que implemente la metodología?

\subsubsection{Resultados}

Durante el proceso de búsqueda y selección se localizaron 61 publicaciones. Luego de aplicar los criterios de inclusión y exclusión se eligieron 40 publicaciones para su lectura completa (ver Tabla 2.1), de las que se excluyeron cinco (Tabla 2.2) por no proponer una metodología de ensamblaje.

1 Un itinerario de aprendizaje puede asemejarse a "un mapa conceptual que nos guía en el aprendizaje sobre un tema presentando una serie de competencias que deben comprenderse, dominarse y demostrarse para entenderlo, y que [...] se ocupa del cómo aprenderlo. Supone, por tanto, una forma de organizar la secuencia de aprendizaje y responde a la necesidad de guía de los alumnos por los contenidos, procesos y actividades, proporcionando, al mismo tiempo, suficiente flexibilidad para que ejerza cierta autonomía en el proceso de aprendizaje" (de Benito, Darder, \& Salinas, 2012, p. 3). 
Las 35 publicaciones seleccionadas se agrupan, finalmente, en función de las metodologías de ensamblaje propuestas, así se obtienen las 27 metodologías que se analizan en esta tesis.

Tabla 2.1: Lista de lecturas completas. Se incluyen publicaciones seleccionadas en el proceso preliminar.

\begin{tabular}{|c|c|c|c|}
\hline Autor & Fecha & Fuente & País \\
\hline S01 Kellar et al. & 2004 & IEEE & Canada \\
\hline S02 Farrell, Liburd \& Thomas & 2004 & $\mathrm{ACM}$ & EEUU \\
\hline S03 Pahl et al. & 2004 & otro & Irlanda \\
\hline S04 Roig Vila & 2005 & otro & España \\
\hline S05 Colucci et al. & 2005 & Springer & Italia \\
\hline S06 Santacruz Valencia, Delgado Kloos \& Aedo Cuevas & 2005 & otro & España \\
\hline S07 Farrell \& White & 2006 & otro & EEUU \\
\hline S08 Li \& Huang & 2006 & otro & China \\
\hline S09 Anh \& Dam & 2006 & IEEE & Korea \\
\hline S10 Jovanovic, Gašević \& Devedžić & 2006 & Springer & Rusia/Candá \\
\hline S11 Tsai, Chiu, Lee \& Wang & 2006 & IEEE & Taiwan \\
\hline S12 Doan, Bourda \& Dumitrascu & 2006 & IEEE & Francia \\
\hline S13 Lopes Gançarski et al. & 2007 & otro & Francia \\
\hline S14 Rigaux \& Spyratos & 2007 & otro & Francia \\
\hline S15 Sánchez Alonso et al. & 2007 & Springer & España \\
\hline S16 Thyagharajan \& Nayak & 2007 & otro & India \\
\hline S17 Karam, Linckels \& Meinel & 2007 & Springer & Alemania \\
\hline S18 Anh, Ha \& Dam & 2008 & otro & Korea \\
\hline S19 Santacruz Valencia, et al. & 2008 & otro & España \\
\hline S20 Shahin et al. & 2008 & IEEE & Siria \\
\hline S21 Schreurs, Vanhove \& Al-Zoubi & 2008 & otro & Belgica y Jordania \\
\hline S22 Sarasa et al. & 2008 & otro & España \\
\hline S23 Wetzlinger, Auinger \& Stary & 2008 & otro & Australia \\
\hline S24 López, Miguel \& Montaño & 2008 & otro & Venezuela \\
\hline S25 Lalithsena, Hewagamage \& Jayaratne & 2008 & otro & Sri Lanka \\
\hline S26 Bouzeghoub et al. & 2009 & Springer & Francia \\
\hline S27 Bouzeghoub \& Selmi & 2009 & IEEE & Francia \\
\hline S28 Garrido, Onaindia \& Sapena & 2009 & IEEE & España \\
\hline S29 Verbert, Wiley \& Duval & 2009 & Springer & Belgica y EEUU \\
\hline S30 Jovanovic, Gašević \& Devedžić & 2009 & Springer & Rusia y Canadá \\
\hline S31 Huang et al. & 2009 & otro & Taiwan \\
\hline S32 Ullrich \& Melis & 2009 & otro & China y Alemania \\
\hline S33 Menéndez, Prieto \& Zapata & 2010 & IEEE & México \\
\hline S34 Ruiz-Iniesta, Jiménez-Díaz \& Gómez-Albarrán & 2010 & IEEE & España \\
\hline S35 Menéndez, et al. & 2011 & otro & México \\
\hline S36 Stănică \& Crișan & 2011 & otro & Rumania \\
\hline S37 Caro Piñares, Hernández \& Jiménez Builes & 2011 & otro & Colombia \\
\hline S38 Stănică \& Crișan & 2012 & otro & Rumania \\
\hline S39 Verbert et al. & 2012 & otro & Belgica, Ecuador, Alemania y España \\
\hline S40 Chellatamilan \& Suresh & 2012 & IEEE & India \\
\hline
\end{tabular}

Del total de publicaciones (40), diez se localizaron en IEEE, siete en Springer, una en ACM y 22 pertenecen a actas de congresos, artículos en revistas y tesis. De acuerdo a la distribución por año tres pertenecen a 2004 , tres a 2005 , seis a 2006 , cinco a 2007 , ocho a 2008 , siete a 2009 , dos a 2010 , 
tres a 2011 y tres a 2012. Los trabajos analizados incluyen a investigadores de Universidades de más de 20 países que representan a cuatro de los cinco continentes, con preeminencia de europeos.

Se analizaron, además, siete publicaciones correspondientes al período 2013-2015 a fin de aportar, al presente estado de la cuestión, las últimas tendencias en la temática (ver Tabla 2.3).

Tabla 2.2: Publicaciones excluidas.

\begin{tabular}{|c|c|c|c|}
\hline Autor & Fecha & PE2 & PE3 \\
\hline S11 Tsai, Chiu, Lee \& Wang & 2006 & $\mathrm{~N}$ & $\mathrm{~N}$ \\
\hline S12 Doan, Bourda \& Dumitrascu & 2006 & $\mathrm{~N}$ & $\mathrm{~N}$ \\
\hline S34 Ruiz-Iniesta, Jiménez-Díaz \& Gómez-Albarrán & 2010 & $\mathrm{~N}$ & $\mathrm{~N}$ \\
\hline S37 Caro Piñares, Hernández \& Jiménez Builes & 2011 & $\mathrm{~N}$ & $\mathrm{~N}$ \\
\hline S40 Chellatamilan \& Suresh & 2012 & $\mathrm{~N}$ & $\mathrm{~N}$ \\
\hline
\end{tabular}

Tabla 2.3: Publicaciones correspondientes al periodo 2013-2015.

\begin{tabular}{lccc}
\hline \multicolumn{1}{c}{ Autor } & Fecha & Fuente & País \\
\hline Becerra et al. & 2013 & LACLO & Chile \\
Garrido \& Onaindia & 2013 & IEEE & España \\
Colace et al. & 2014 & iJET & Italia \\
Torres \& Guzmán-Luna & 2015 & Springer & Colombia \\
Shmelev et al. & 2015 & Elsevier & Rusia \\
Štuikys et al. & 2015 & Springer & Lituania \\
Ben Mahmoud et al. & 2015 & IEEE & Túnez/Francia \\
\hline
\end{tabular}

Respecto a las preguntas de evaluación (ver Anexo I), las metodologías presentan una variedad de motivaciones que impulsan la investigación. Si bien todas proponen una metodología de ensamblaje, sólo 18 de ellas describen en detalle el proceso. La mayoría (25) enuncian claramente la función de los usuarios al utilizar el sistema que implementa la metodología de ensamblaje. Aunque el total elige los OA para la creación de los itinerarios de aprendizaje, sólo 17 enuncia una definición, el resto usa el concepto sin definirlo. Además, no hay una definición acordada o común entre las distintas propuestas (ver 2.4.2.1 en este capítulo). De todas las metodologías analizadas, 17 presentan un software que la implementa.

Las 35 publicaciones analizadas permitieron la localización de 27 metodologías de ensamblaje de OA, las cuales conforman el insumo para el análisis de las metodologías realizado en el presente trabajo tesis. Cabe aclarar que la siguiente lista se realiza agrupando las publicaciones que describen la misma metodología:

1. Kellar et al.

2. Pahl et al.

3. Roig Vila

4. Colucci et al.

5. Santacruz Valencia, Delgado Kloos \& Aedo Cuevas

6. Farrell et al.

7. Li \& Huang

8. Lopes Gançarski et al.

9. Rigaux \& Spyratos

10. Sánchez Alonso et al.

11. Thyagharajan \& Nayak

12. Karam, Linckels \& Meinel

13. Anh et al. 

14. Shahin et al.
15. Schreurs, Vanhove \& Al-Zoubi
16. Sarasa et al.
17. Wetzlinger, Auinger \& Stary
18. López, Miguel \& Montaño
19. Lalithsena, Hewagamage \& Jayaratne
20. Bouzeghoub et al.
21. Garrido, Onaindia \& Sapena
22. Jovanovic et al.
23. Huang et al.
24. Ullrich \& Melis
25. Menéndez et al.
26. Stănică \& Crișan
27. Verbert et al.

\subsection{Objetos de Aprendizaje}

Las metodologías de ensamblaje aquí analizadas hacen uso de un tipo particular de MED: los Objetos de Aprendizaje (OA). Esto se debe, principalmente, a que cumplen con dos aspectos que son esenciales para la implementación de estas metodologías: $(i)$ son diseñados para ser reutilizados, (ii) cuentan con metadatos (Astudillo, Sanz, \& Santacruz Valencia, 2013). Además, es el propio paradigma de diseño de $\mathrm{OA}$ el que propone, desde sus inicios, la composición de distintos OA para alcanzar MED de mayor granularidad² (Barritt, Lewis, \& Wieseler, 1999; Gerard, 1969; L'Allier, 1998; Wiley, 1999a).

Los docentes han encontrado en la web un lugar donde compartir sus materiales y recursos educativos tanto con sus estudiantes, como con sus colegas. Esto ha provocado que se diseñen o adapten una gran cantidad de recursos y materiales educativos y sean distribuidas a través de la red. La web se constituye, entonces, como el espacio obligado donde buscar. Sin embargo, el volumen de la oferta, así como, en muchos casos, la falta de información sobre el propósito educativo del recurso/material, provocan que utilizar (o reutilizar) el mismo no sea una tarea sencilla.

No solo se trata de evaluar la granularidad de los recursos/materiales -la cual varía desde simples archivos hasta cursos completos-, o si es posible reutilizarlos, o si son apropiados a los destinatarios, o si se adaptan al estilo pedagógico del docente, sino que además, es necesario ubicarlos dentro de una secuencia de aprendizaje que permita la consecución de los objetivos de aprendizaje planteados.

Estas problemáticas son abordadas desde el paradigma de OA. La idea de combinar MED para adaptarlos a los destinatarios y reutilizarlos en varios contexto es un viejo anhelo que se remonta los años 60. En 1969, Gerard planteaba el diseño de "pequeñas unidades curriculares que pueden ser combinadas, como las piezas estandarizadas de un juego de Meccano, en una gran variedad de programas específicos hechos a la medida de cada estudiante." (Gerard, 1969, pp. 29-30).

En los inicios de los noventa, Merrill, Li, \& Jones (1990) utilizan el término marcos (frames) para indicar objetos que podían representar el conocimiento, los mismos que podían unirse con otros objetos para formar un curso. Dicen los autores:

"Proponemos para representar el conocimiento en términos de objetos que llamamos marcos; cada marco tiene una estructura interna [...], y enlaces a otros marcos. [...] Al conjunto de todos los marcos elaborados en conjunto, que contiene todos los conocimientos necesarios para ser impartidos en un curso, se denominan red de marcos" (p.12).

2 La granularidad refiere a qué tan grande es un MED en relación a los objetivos que se propone. Cuanto menor sea la cantidad de tópicos que se abordan en un MED, más baja es su granularidad. Es importante destacar que la granularidad no hace referencia a la cantidad de archivos y/o recursos que se utilicen para la consecución del objetivo de aprendizaje. 
Pero tuvieron que pasar veinticinco años, desde la propuesta de Gerard, para que el término "objeto de aprendizaje" viera la luz. Fue Wayne Hodgins quien lo utiliza por primera vez, en 1994, inspirado, esta vez, en las piezas de $\mathrm{LEGO}^{3}$. Los bloques del juego le sirvieron como metáfora para explicar la formación de materiales educativos utilizando pequeñas unidades que son interconectadas entre sí para apoyar el aprendizaje.

Wiley (1999b) critica la metáfora del LEGO (similar a la del Mecano sugerida por Gerard) que se utiliza para explicar el concepto de OA. En ella los OA son "pequeñas piezas instruccionales (LEGOs) que pueden ser ensambladas (apiladas juntas) en una estructura instruccional más grande (castillo o nave espacial)" (p. 1). El autor la considera demasiado simplista e identifica tres problemas (Wiley, 1999b, p. 1): ( $i$ ) un bloque se puede combinar con cualquier otro, (ii) de cualquier manera y (iii) al ser tan sencillos cualquiera puede ensamblarlos. En su lugar propone la metáfora de la molécula, en ella cada OA es un átomo. Esta propuesta es superadora respecto al enfoque de un OA como material educativo ya que (Wiley, 1999b, p. 2): ( $i$ ) no todo átomo es combinable con cualquier otro, (ii) los átomos sólo pueden ser ensamblados en ciertas formas prescritas por su estructura y (iii) es necesaria algún tipo de formación o saber para poder aplicar el proceso de ensamblaje.

Una tercera metáfora, que permite explicar el concepto de OA y que, también, los asocia con el ensamblaje, es la del ladrillo y cemento (the brick-and-mortar metaphor). También propuesta por Wiley (2007), asocia a los OA con ladrillos y propone que la teoría de diseño instruccional, debería ser la forma apropiada de unirlos (el cemento entre los OA). Los OA "por ser de una variedad de formas y tamaños, son difíciles de ensamblar de manera significativa, sin algún tipo de pegamento contextual que los mantenga juntos." (p.348).

Las metáforas muestran las ideas que subyacen en el paradigma de OA: la reutilización, la personalización del contenido y el ensamblaje. Sin embargo, este último, se plantea más en término de diseño, que de proceso. Esto es, los OA deben ser diseñados con vistas al ensamblaje, pero no se hace explícito cómo este proceso debiera hacerse o se deja en manos de especialistas -como en la metáfora de la molécula.

Los sistemas de e-learning debieran evolucionar para dejar ser administradores de contenido y herramientas de autor que permiten la creación de actividades/cursos. Sería deseable que comenzaran a proporcionar funcionalidades que faciliten tanto la recomendación de contenidos como la construcción de itinerarios de aprendizaje de forma automática o semi-automática. Los OA se proponen desde sus inicios como un tipo de MED que facilitarían la incorporación de estas funcionalidades a los sistemas.

Sin embargo, la falta de acuerdo sobre una definición de OA hace que el abanico de recursos y materiales digitales que se identifican como OA limite la evolución de las metodologías de ensamblaje. En la práctica, las metodologías utilizan, en muchos casos, definiciones genéricas del concepto $-\mathrm{o}$ no lo definen-. Proponen, entonces, implementaciones con materiales que no se corresponden con el concepto de OA abordado por varios autores y tomado en esta tesis. Sí podrían ser considerados recursos educativos - como es el caso de las imágenes o las diapositivas-. En otros casos la definición termina adaptándose a las necesidades de la metodología, lo que hace que se limite la cantidad de OA con los que se pueda trabajar al implementar la misma en la práctica.

Como se puede observar, es central presentar una definición de OA que sea lo suficientemente genérica para que incluya a los OA existentes, pero que además caracterice apropiadamente a este tipo particular de MED y sea, a su vez, aplicable en la práctica. A continuación, se presentan algunas de las definiciones más relevantes y se explicita la que se utilizará en el presente trabajo de tesis.

3 LEGO es una empresa, fundada en 1932 por la familia Kirk Kristiansen, que cuenta con un popular juego infantil en que se pueden construir diferentes objetos a través del encastre de piezas denominadas "ladrillitos" (http://www.lego.com). 


\subsection{1 ¿Qué es un OA?}

Aunque hay acuerdo en los beneficios del diseño de MED bajo el paradigma de OA, ya ha pasado más de una década y media desde las primeras definiciones del concepto y, aún, no hay una única respuesta a la pregunta ¿Qué es un Objeto de Aprendizaje? Varios autores y organizaciones propusieron definiciones del concepto, la mayoría de las cuales resultan muy genéricas y de difícil aplicación en la práctica (Astudillo, Sanz, \& Willging, 2011).

A finales de los '90, L'Allier (1998), define OA como "la experiencia de formación independiente más pequeña que contiene un objetivo, actividades de aprendizaje, y una evaluación". Este autor, además, fue uno de los primeros en ofrecer una metodología, basada en prácticas de diseño instruccional, para el ensamblaje utilizando lo que él denomina tópicos y un conjunto de reglas que ayudan al diseñador instruccional a crear estructuras de aprendizaje más complejas: lecciones, unidades y cursos. Wiley (1999a), como ya se mencionó, adhiere a esta metodología al utilizar la metáfora del cemento y los ladrillos, y afirma que debe ser el diseño instruccional el cemento que permita pegar (ensamblar) los OA entre sí, como si fueran ladrillos.

También a finales de los '90, Barritt, Lewis, \& Wieseler (1999), de Cisco System, presentan Reusable Learning Objects (RLO). Un RLO se crea combinando una vista, un resumen, una evaluación, y entre 5 y 7 Objetos Informativos Reutilizables (RIO, por sus siglas en inglés). Un RIO "es una porción de información reutilizable y con estructura granular, que es independiente del medio que se utiliza para distribuirlo" (Barritt et al., 1999, p. 2). Cada RIO, a su vez, puede estar compuestos por contenidos, actividades y evaluaciones que son combinados para la consecución de un objetivo de aprendizaje simple.

En el año 2000 el Learning Technology Standards Committee de la IEEE presenta, dentro de LOM Working draft v4.1, una de las definiciones con mayor acuerdo en la comunidad científica, pero también de gran controversia (Polsani, 2003; Wiley, 2000). Para la IEEE un OA es: "cualquier entidad -digital o no- que puede ser usada para aprender, enseñar o utilizar en estrategias de formación" (IEEE LTSC, 2002). Entre las observaciones que se le hicieron, Wiley (2000) advierte que la "definición es extremadamente amplia [y...] falla al no excluir personas, lugares, cosas o ideas que han existido en cualquier momento en la historia del universo" (p. 5). En este sentido dice Polsani (2003), "la definición de OA [de la IEEE] es no solo general sino también impracticable, en parte porque objetos no digitales como equipamiento informático u objetos digitales como imágenes gozan del mismo estatus conceptual, así se hace imposible usar el término Objeto de Aprendizaje de forma significativa." (p. 3).

David Wiley (2000), por su parte, apoyado en la definición del IEEE propone una versión superadora de la misma en la que un OA es "cualquier recurso digital que puede ser reutilizado como soporte para el aprendizaje” (p.5). Así, restringe el concepto sólo a OA digitales.

Las definiciones de, L'Allier, IEEE, Wiley y Cisco han sentado las bases y dado origen a un importante número de trabajos que han adaptado/mejorado estas definiciones, así como también los que han propuesto nuevas definiciones del concepto. A continuación se presentan, algunas de las definiciones que, a criterio de los autores, definen apropiadamente el concepto, incluyendo las características básicas de los OA y/o una noción de su forma o estructura interna y son de aplicación práctica.

En 2003, Polsani (2003) en su trabajo "Use and Abuse of Reusable Learning Objects" después de analizar varias definiciones (Wiley, IEEE, L'Allier, entre otras) y características de los OA los define como "una unidad de aprendizaje de contenidos autónoma e independiente que está predispuesta al reuso en múltiples contextos educativos" (p. 3).

El repositorio chileno APROA (APROA, 2004), basados en la definición de L'Allier, proponen que "un Objeto de Aprendizaje (OA) corresponde cualquier material educativo digital, que presenta una estructura independiente, que contiene un objetivo, una actividad de aprendizaje, una metadata (descripción del contenido) y un mecanismo de evaluación, el cual puede ser desarrollado con tecnologías de infocomunicación (TIC), con el fin de posibilitar su reutilización, interoperabilidad, 
accesibilidad y duración en el tiempo".

Santacruz-Valencia et al. (2005), en su tesis de doctorado "Automatización de los procesos para la generación ensamblaje y reutilización de Objetos de Aprendizaje" donde realizan una propuesta integral para la generación, ensamblaje y reutilización de OA, definen un OA como "aquel recurso educativo descrito por meta-datos y organizado en una estructura multicapa, de tal forma que los elementos más avanzados de esta estructura incluyen conocimiento asociado (competencias y requisitos), con vistas al ensamblaje y la reutilización" (p. 74).

Desde el año 2006, el Ministerio de Educación Colombiano (MENC) junto con Instituciones de Educación Superior de ese país, llevan adelante un proyecto denominado Banco Nacional de Objetos de Aprendizaje e Informativos ${ }^{4}$, destinado a almacenar OA en repositorios (Zapata \& Zapata, 2009). En este contexto trabajaron para llevar adelante un marco conceptual que incluyó una definición para OA. La misma está basada en la definición de Cisco y propone que "un objeto de aprendizaje es un conjunto de recursos digitales, autocontenido y reutilizable, con un propósito educativo y constituido por al menos tres componentes internos: contenidos, actividades de aprendizaje y elementos de contextualización. El objeto de aprendizaje debe tener una estructura de información externa (metadatos) que facilite su almacenamiento, identificación y recuperación" (Leal Fonseca, 2008, p. 80).

Para Chiappe et. al. (2007) se definen como "una entidad digital, auto-contenible y reutilizable, con un claro propósito educativo constituido por al menos tres componentes internos editables: contenidos, actividades de aprendizaje y elementos de contextualización. A manera de complemento, los objetos de aprendizaje han de tener una estructura (externa) de información que facilite su identificación, almacenamiento y recuperación: los metadatos" (p. 675).

En 2009, en el marco del curso "Diseño y evaluación contenidos y actividades educativas reutilizables" impartido desde la Universidad de Alcalá ${ }^{5}$, Sicilia Urbán y Sánchez Alonso proponen la siguiente definición: "un objeto de aprendizaje es una unidad didáctica en formato digital, independiente, autocontenida, perdurable y predispuesta para su reutilización en varios contextos educativos por la inclusión de información autodescriptiva en forma de metadatos" (M.-A. Sicilia Urbán \& Sánchez Alonso, 2009b, p. 9).

También en 2009, Advanced Distributed Learning ${ }^{6}$ (ADL) en su 4ta edición de los libros de SCORM caracteriza este tipo de MED. En el libro de información general (ADL, 2009b) se afirma que "SCORM se apoya en la noción de contenidos hechos de objetos de contenido reutilizable agrupados bajo la forma de unidades de instrucción" (p. 3). Los objetos de contenido son representados en SCORM por los Sharable Content Object (SCO) y asset (ADL, 2009a). En ambos casos se trata de una colección de uno o más recursos (imágenes, textos, videos, etc.), la diferencia radica en que los SCO permiten la comunicación con un LMS (que respete este estándar) y los asset son contenidos estáticos que se le presentan al estudiante. Desde ADL se sugiere que los SCO sean libres de contexto para propiciar su reutilización. Además, se propone la combinación de varios SCOs para formar unidades de aprendizaje. Los "SCO [son] pequeñas unidades, [con] el potencial de ser reutilizadas en múltiples contextos de aprendizaje” (ADL, 2009a, p. 4).

Astudillo et al. (2011) en su trabajo "Análisis del estado del arte de los objetos de aprendizaje. Revisión de su definición y sus posibilidades" analizada un conjunto de definiciones y características de los OA para luego proponer una definición del concepto. Los autores definen los OA como "una unidad didáctica digital diseñada para alcanzar un objetivo de aprendizaje simple, y para ser reutilizada en diferentes Entornos Virtuales de Enseñanza y Aprendizaje, y en distintos contextos de aprendizaje. Debe contar, además, con metadatos que propicien su localización, y permitan abordar su contextualización" (p. 34).

Este trabajo retoma la definición de Astudillo et al. (2011) y adaptada por Sanz (2015) para definir un OA como:

4 Disponible en: http://www.colombiaaprende.edu.co/html/directivos/1598/article-99543.html

5 Disponible en: http://www.cc.uah.es/ie/cursos/learningobjects09/

6 Disponible en: http://www.adlnet.org/ 
"Un tipo de material educativo digital, que se caracteriza, desde el punto de vista pedagógico, por orientarse a un objetivo específico de aprendizaje, y presentar mínimamente: una serie de contenidos con el fin de abordar la temática relacionada con el objetivo, una actividad que permita al alumno poner en práctica o problematizar el contenido presentado, y una autoevaluación que posibilite conocer al alumno, si ha podido comprender esos contenidos vinculados al objetivo. Desde el punto de vista tecnológico, se caracteriza por contener un conjunto de metadatos estandarizados para su búsqueda, y recuperación, y estar integrado, utilizando un modelo de empaquetamiento que respete estándares, y de esta manera, permita su diálogo con diferentes entornos tecnológicos." (Sanz, 2015, p. 34).

\subsubsection{Características de los OA}

Entre las características más importantes del paradigma de OA, están: reutilización, accesibilidad, e interoperabilidad (Polsani, 2003), además de la contextualizacion y el ensamblaje (Longmire, 2000; Santacruz-Valencia et al., 2005; M.-A. Sicilia Urbán \& Sánchez Alonso, 2009b).

Reutilización. Esta es la característica central y distintiva de los OA. La reutilización "es la idea fundamental detrás de los objetos de aprendizaje" (Wiley, 2000, p. 3). Se trata de la capacidad de un OA de utilizarse en distintos contextos educativos (Chan Núñez, 2004; García Aretio, 2005; Wiley, Gibbons, \& Recker, 2000). Se trata de una característica central para el paradigma, tal es así, que el resto de las características soportan o apoyan la reutilización.

Cabe aclarar que algunos autores proponen que la reutilización debe ser en diferentes contextos tecnológicos, en este trabajo a esta característica se la denominará interoperabilidad.

Accesibilidad. Es la capacidad de los OA de ser localizados y accedidos (ADL, 2009b; García Aretio, 2005; McGreal, 2004).

Esta característica depende fuertemente de que los OA sean publicados en repositorios y etiquetados con metadatos (ver 2.3.3), de manera que puedan ser catalogados apropiadamente y, por ende, fácilmente localizables.

Interoperabilidad. Esta característica permite que un OA pueda ser utilizado en distintas plataformas tecnológicas y busca la independencia de los objetos de una herramienta/aplicación particular. Así la interoperabilidad es "la habilidad de tomar un componente instruccional de un lugar o localización, desarrollado con un conjunto de herramientas particulares, y usarlo en otra localización y con un conjunto diferente de herramientas o plataformas" (ADL, 2009b; McGreal, 2004, p. 1). García Aretio, también asocia la interoperabilidad con las plataformas y define esta característica como "la capacidad para poder integrarse en estructuras y sistemas (plataformas) diferentes" (2005, p. 3).

Para que esta característica se refleje en la práctica es importante la utilización de estándares reconocidos (ver 2.3.3). En este sentido Zapata Ros (2005), define interoperabilidad como una capacidad del OA que le permita "intercambiar información a través de todas las plataformas [...] que adopten los estándares” (p. 12).

Contextualización. Esta característica, aún controversial en la ámbito de los OA, enuncia que estos pueden ser adaptados al de estudiantes o necesidades didácticas de la actividad planteada. La contextualización se puede abordar desde dos enfoques: ( $i$ ) desde el contenido del OA o (ii) desde sus metadatos. En el primer caso, o bien el objeto debe ser diseñado para ser utilizado en varios contextos, o debe permitir su adaptación. Dicen Sicilia Urbán \& Sánchez Alonso (2009b) "si el material se diseña desde un principio para ser reutilizado en diversos contextos, será más fácilmente reutilizable que otros materiales diseñados 'a la manera tradicional', pues estos últimos deberán ser adaptados a cada nuevo contexto" (p. 6). En el segundo caso, se involucra a los metadatos, los cuales brindan información sobre el OA o sus posibles usos o contextos de uso. Una buena descripción de los OA propicia su reutilización (Santacruz-Valencia et al., 2005). 
"La contextualización es un punto clave para la personalización del contenido. Sin embargo, los conceptos de reutilización y contextualización originan la llamada paradoja de la reusabilidad" (Santacruz-Valencia et al., 2005, p. 50). Esto es, por una parte los OA debieran ser descontextualizados para propiciar su reutilización, y por otra, es necesario darles contexto que permita la adaptación del contenido a la situación de aprendizaje (Friesen, Caws, \& Beaudoin, 2006; Wiley et al., 2004). Por tanto, como el resto de las características, la contextualización impacta sobre la reutilización. En este sentido advierte Zapata Ros (2005) "un objeto no es absolutamente reutilizable en distintos contextos tecnológicos o curriculares. Puesto que la reusabilidad se refiere a situaciones anticipadas y usos futuros, [...] exige la especificación de los contextos posibles de uso [lo que determinará] el grado de reusabilidad del objeto de aprendizaje." (p. 14).

Es importante destacar que la contextualización de un OA también está definida en función de la propuesta pedagógica. En este sentido, es el docente, quien a través de la selección apropiada de los OA y su posterior ensamblaje, conforma una propuesta didáctica y propone el contexto en el que utilizarán. Para que esto sea posible, los OA deben contar con información (metadatos) que facilite su ensamblaje y su integración a diferentes contextos educativos.

Ensamblaje. Esta característica permite que los OA puedan agruparse de manera de crear unidades de aprendizaje coherentes y de mayor granularidad. Esto es, no se trata aquí que únicamente de conseguir una metodología que permita acoplar un objeto con otro, sino que además, debe haber una correspondencia semántica entre ellos - que puedan ser combinados para alcanzar los objetivos pedagógicos propuestos.

El ensamblaje requiere de $(i)$ la accesibilidad del OA, por ende necesaria su (ii) descripción con metadatos y (iii) un conjunto de reglas de composición (Santacruz-Valencia et al., 2005).

\subsubsection{Estándares y metadatos}

Los metadatos conforman "información sobre un objeto, sea este físico o digital" (IEEE LTSC, 2002, p. ii). Dentro del paradigma de OA, García Aretio (2005), lo define metadatos como "una estructura detallada del texto, que describe atributos, propiedades y características, distribuidos en diferentes campos que identifican claramente al objeto, con el fin de que pueda encontrarse, ensamblarse, [y] utilizarse" (p.2).

Como afirman Sicilia Urbán \& Sánchez Alonso (2009b), los metadatos "son esencia y no accidente en el paradigma de los OA. Si eliminamos este elemento, podríamos perfectamente hablar de diseño de recursos digitales educativos, de manera genérica", por tanto, los metadatos debieran ser parte constitutiva del propio OA.

La importancia del etiquetado de los OA está dada por los alcances que tiene el mismo: $(i)$ hace posible la identificación del objeto, (ii) facilita la búsqueda, (iii) permite el almacenamiento, (iv) favorece la reutilización y (v) propicia la interoperabilidad (Astudillo et al., 2011), así como también, dan lugar al ensamblaje (Santacruz-Valencia et al., 2005).

El principal objetivo de los metadatos es describir, almacenar, indexar los OA, en un repositorio, con el fin de recuperarlos o reutilizarlos en diferentes contextos educativos (Bouzeghoub \& Selmi, 2009). Los metadatos juegan un papel central, ya que "permiten a estudiantes y diseñadores instruccionales elegir OA que puedan ser adaptados para un propósito educativo específico" (Parrish, 2004, p. 14). En el mismo sentido, Sicilia Urbán (2005) afirma que: "desde el punto de vista del tutor o educador que busca [OA] para una situación concreta, la disponibilidad de herramientas de búsqueda y composición automatizadas, además de ahorrar tiempo en el diseño, amplían las posibilidades de encontrar lo adecuado a las necesidades concretas [...y] abren un escenario completamente diferente a la construcción de herramientas de búsqueda, más allá de los buscadores convencionales" (p.10).

Sicilia Urbán y Sánchez Alonso (2009a) proponen una estructura mínima de contenidos para los metadatos: debe contener información relacionada al contenido del propio recurso (por ejemplo 
matemática), requisitos técnicos (por ejemplo flash) y posibles contextos de uso (por ejemplo educación primaria). McGreal (2004), llama a este tipo de metadatos "metadatos objetivos", y agrega lo que denomina "metadatos subjetivos". Esto es, aquella información provista por los usuarios, como por ejemplo, comentarios a los OA o la puntuación de los mismos. Habría que agregar para el contexto de ensambladores los "metadatos semánticos" (Bouzeghoub \& Selmi, 2009; Garrido, Onaindia, \& Sapena, 2009; Sánchez-Alonso, Sicilia, López-Cobo, \& Arroyo, 2007), que hacen referencia a significados y relaciones entre el contenido y, al igual que los anteriores, deben estructurarse de manera formal para dar lugar al procedimiento automático.

"En el marco de las comunidades de usuarios basadas en las tecnologías, surge la necesidad de colaborar y compartir recursos e información. Para ello es necesario contar con [...] estándares aceptados a nivel internacional" (López, Miguel, \& Montaño, 2008, p. 4).

En el contexto educativo, para el etiquetado de MED, y de OA en particular, se utilizan tanto Learning Object Metadata ${ }^{7}$ (LOM) como Dublin Core Metadata Element Set ${ }^{8}$ (DCMES). Ambos estándares son ampliamente aceptados y utilizados por la comunidad e-learning (Hilera González \& Hoya Marón, 2010).

DCMES pertenece a Dublin Core Metadata Initiative, es un proyecto de la Association for Information Science and Technology, cuyo estándar cuenta 15 definiciones semánticas que permiten la descripción de recursos web. El conjunto de elementos de la iniciativa de Dublin Core es el siguiente: título, creador, tema, descripción, casa editorial, colaborador, fecha, tipo, formato, identificador, fuente, idioma, relación, cobertura y derechos (ver Figura 2.1).

LOM fue desarrollado por el IEEE y especifica cómo deben describirse los OA. Cuenta con nueve categorías: general, ciclo de vida, meta-metadatos, técnica, uso educativo, derechos, relación, anotación y clasificación. Al extender cada categoría, la cantidad de campos del estándar hace un total de 76 elementos, además el estándar es extensible (ver Figura 2.2).

La organización ISO/IEC está desarrollando un nuevo estándar para el etiquetado de MED, basado en LOM y DCMI, denominado ISO/IEC 19788 Metadata Learning Resource (MLR) (Betrián, Hilera, \& Pagés-Arévalo, 2011). El conjunto de elementos de MLR son: identificador, nombre del establecimiento, definición, indicador lingüístico, dominio, alcance, contenido, refina, ejemplo (s) y nota (s).

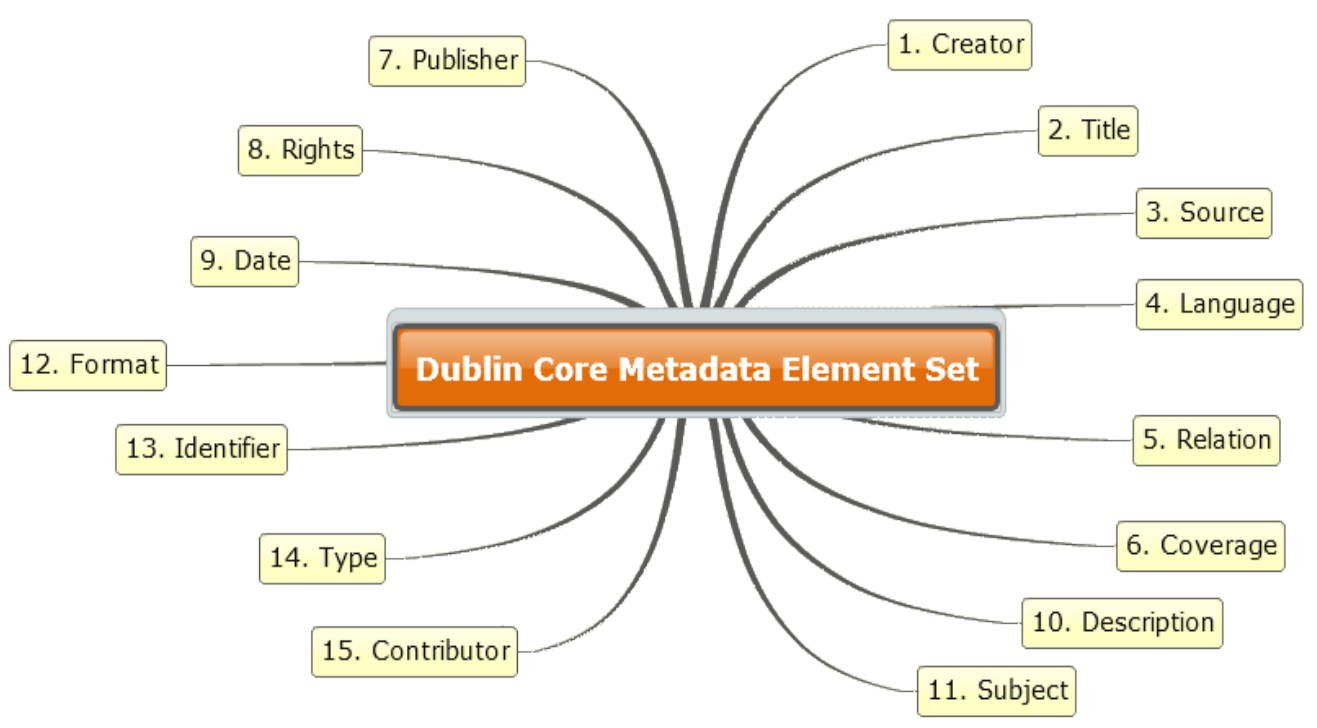

Figura 2.1: Jerarquía completa de DCMES (imagen de producción propia).

7 Disponible en http://www.ieeeltsc.org

8 Disponible en http://dublincore.org

9 Disponible en: https://www.iso.org/obp/ui/\#iso:std:iso-iec:19788:-1:ed-1:v1:en 


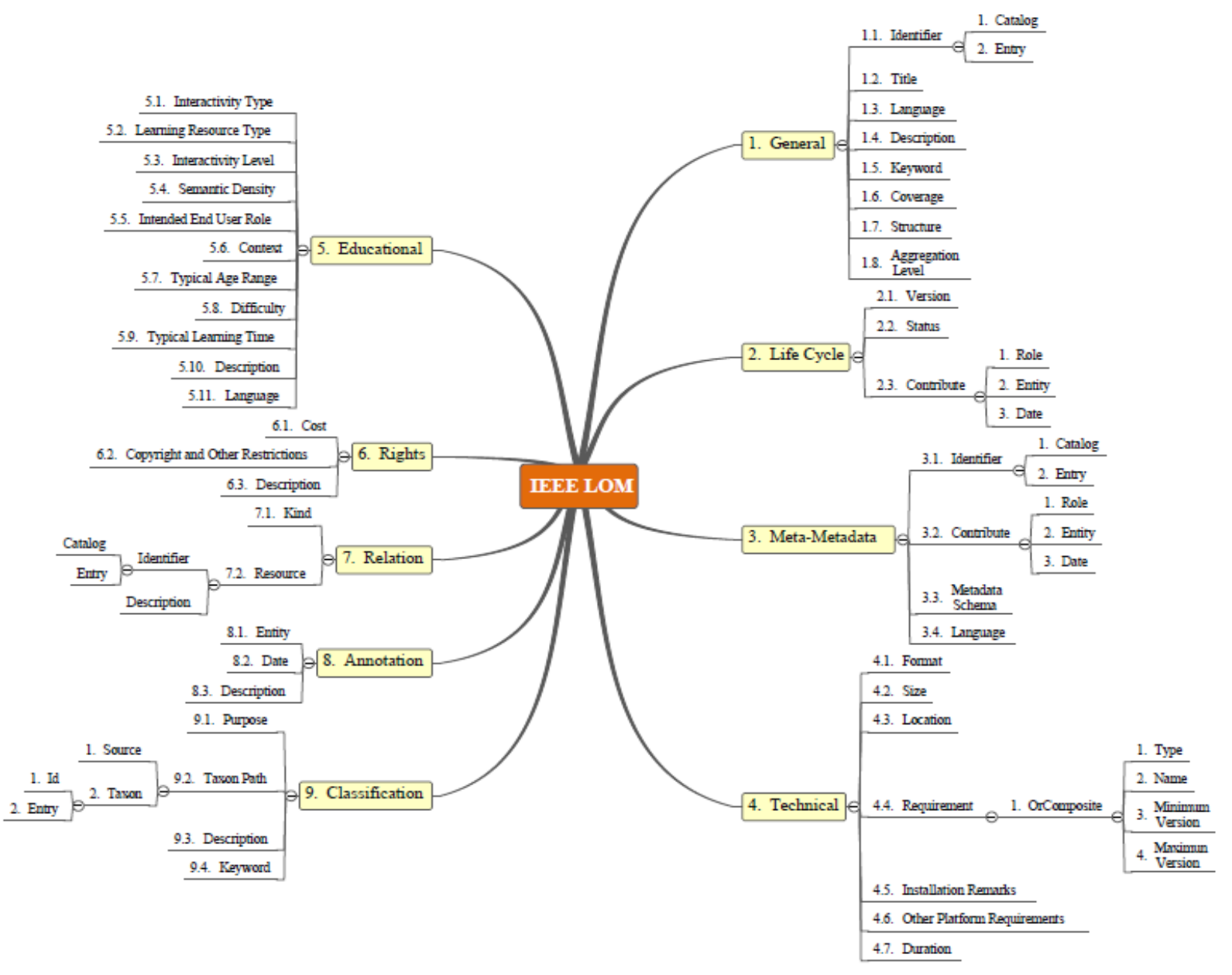

Figura 2.2: Jerarquía completa de IEEE LOM (imagen producción propia).

Otro de los aspectos que aborda la estandarización en el contexto educativo es el empaquetamiento de contenidos. En este caso el estándar más difundido y utilizado es Sharable Content Object Referente Model $^{10}$ (SCORM). Esta iniciativa, perteneciente a ADL compendia un conjunto de varias iniciativas a nivel global (IEEE, IMS y AICC). "SCORM es un 'modelo de referencia' que integra un conjunto de estándares, especificaciones y guías interrelacionadas diseñadas para satisfacer requerimientos de alto nivel para sistemas y contenido educativo" (ADL, 2009b, p. 1). SCORM propone un modelo de agregación de contenidos (Content Aggregation Model, CAM), un entorno de tiempo de ejecución (Run-Time Environment, RTE) y la secuenciación y navegación (Sequencing and Navigation, SN) de los contenidos (ADL, 2009b). SCORM propicia la reutilización y la interoperabilidad, ya que el material empaquetado bajo este estándar puede ser utilizado y reutilizado sobre los distintos sistemas de e-learning que adopten el mismo. Para el etiquetado del material se puede utilizar tanto LOM como DCMES.

Existe actualmente una evolución del estándar SCORM denominada Tin Can. Esta nueva generación del estándar permite la comunicación con otros entornos y dispositivos. Al igual que SCORM, Tin Can registran actividades del usuario. Pero este último, permite tomar registro de una mayor variedad de actividades realizadas por los usuarios e incluso la interacción entre ellos (aprendizaje social). Mientras estos realizan sus actividades de aprendizaje (aprendizaje móvil, simulaciones, mundos virtuales, juegos serios, entre otros) Tin Can puede guardarlas con el formato sujeto - verbo - objeto en el Learning Record Store (LRS). La información de este almacén de actividades puede ser compartida y recuperada por las plataformas apegadas al estándar (Ledda, 2013; Rustici Software, s. f.). 


\subsubsection{Ventajas y desventajas de los OA}

Dadas las características planteadas es posible proponer un conjunto de ventajas y desventajas con las que se pudieran encontrar quienes utilicen el paradigma de OA para llevar adelante el diseño de actividades de enseñanza y de aprendizaje.

Como afirman Llamas Nistal et al. (2007) en "términos generales, los objetos de aprendizaje suponen, por un lado, un ahorro de tiempo para el profesor en la preparación de recursos de aprendizaje de calidad, y por otro lado, una disponibilidad constante de dichos recursos para el alumno" (p. 70).

Desde la Universidad Politécnica de Valencia (ICE, 2007), en el "Plan de acciones para la convergencia europea" proponen la siguiente tabla que permite resumir el conjunto de ventajas del paradigma de OA (Tabla 2.4).

Si bien son varias las ventajas (Tabla 2.4) que ofrece la creación de material educativo bajo el paradigma de OA, existen algunos aspectos que pueden limitar o complejizar su adopción.

Una de las características distintivas de los OA es la reutilización. Así como es un aspecto central y distintivo de este tipo de MED, y lo pone en ventaja frente a otros que no la posen, también aporta complejidad al diseño de los mismos.

Son varios los aspectos a tener en cuenta para conseguir un OA reutilizable: $(i)$ granularidad, $(i i)$ contextualización, (iii) interoperabilidad, (iv) accesibilidad y (v) el uso de estándares. Cada uno de los aspecto listados encierra, en sí mismo, su propia complejidad.

Tabla 2.4: Ventajas de los OA (ICE, 2007, p. 5).

\begin{tabular}{|c|c|c|}
\hline Ventajas & Estudiantes & Profesores \\
\hline Personalización & $\begin{array}{l}\text { Permiten la individualización del } \\
\text { aprendizaje en función de sus intereses, } \\
\text { necesidades y estilos de aprendizaje. }\end{array}$ & $\begin{array}{l}\text { Ofrecen caminos de } \\
\text { alternativos. Adaptan los prondizaje } \\
\text { formativos a necesidades de los estudiantes. }\end{array}$ \\
\hline Interoperabilidad & $\begin{array}{l}\text { Acceden a los objetos independientemente } \\
\text { de la plataforma y hardware. }\end{array}$ & $\begin{array}{l}\text { Utilizan materiales desarrollados en otros } \\
\text { contextos y sistemas de aprendizaje. }\end{array}$ \\
\hline Inmediatez/accesibilidad & $\begin{array}{l}\text { Tienen acceso, en cualquier momento, a los } \\
\text { objetos de aprendizaje que se desee. }\end{array}$ & $\begin{array}{l}\text { Obtienen, al momento, los objetos que } \\
\text { necesitan para construir sus lecciones. }\end{array}$ \\
\hline Reutilización & $\begin{array}{l}\text { Provee materiales que ya han sido utilizados } \\
\text { con criterios de calidad. }\end{array}$ & $\begin{array}{l}\text { Disminuyen el tiempo invertido en el } \\
\text { desarrollo del material didáctico. }\end{array}$ \\
\hline Flexibilidad & $\begin{array}{l}\text { Se integran en el proceso de aprendizaje. Se } \\
\text { adaptan al ritmo de aprendizaje del alumno. }\end{array}$ & $\begin{array}{l}\text { Es de fácil adaptación a: } \\
\text { - los distintos contextos de aprendizaje. } \\
\text { - las diferentes metodologías de enseñanza- } \\
\text { aprendizaje. }\end{array}$ \\
\hline Durabilidad/Actualización & $\begin{array}{l}\text { Acceden a contenidos que se adaptan } \\
\text { fácilmente a los cambios tecnológicos. }\end{array}$ & $\begin{array}{l}\text { Crean contenidos que pueden ser re- } \\
\text { diseñados y adaptados a nuevas tecnologías. }\end{array}$ \\
\hline
\end{tabular}

Un aspecto a que permite echar luz sobre la complejidad de trabajo con OA es el ciclo de vida de los OA. Como afirman el Menéndez et al. (2010) "el ciclo de vida de un OA está fuertemente influenciado por la reusabilidad" (p. 56). Si observamos la propuesta de Collis \& Strijker (2004) la misma consta de seis fases: (i) obtener, (ii) etiquetar, (iii) ofrecer, (iv) seleccionar, (v) utilizar y (vi) mantener. Al analizar este ciclo de vida, es posible observar la complejidad en el proceso de diseño y utilización de un OA.

La creación del OA es sólo la primera fase (obtener) del ciclo de vida del objeto, y como todo proceso de creación de MED es una tarea compleja y que requiere de un esfuerzo considerable -si se desea generar material de calidad. Esta tarea conlleva, por una parte, el análisis de los contenidos en busca de su división en pequeñas partes reutilizables, y por otra, contar con una metodología de diseño, -basada en general, en el diseño instruccional-. Suele plantearse la necesidad de un equipo 
multidisciplinario, o en su defecto, tener experiencia en el diseño de MED. Al tratarse de material digital, se debe tener en cuenta el manejo de alguna/s herramienta/s de autor que permita crear el OA y también completar sus metadatos. Por tanto, para poder seguir los lineamientos de este paradigma de diseño de material educativo se deben tener tanto conocimientos de pedagogía, como de informática.

En la segunda fase (etiquetar) se encuentra la asociación de metadatos al OA, esta tarea requiere del uso de estándares, y por tanto, de cierto nivel de conocimiento de los mismos para su desarrollo. Poder identificar qué tipo de información y en qué formato (vocabulario) debe ser ingresada, para cada uno de los campos del formulario, es central. Contar con metadatos de calidad es muy importante para la toma de decisiones - de usuarios y de los sistemas-. Sumado a lo anterior, cada organización/institución puede definir un vocabulario específico, lo que agrega al proceso de carga aún más complejidad.

La tercera fase (ofrecer) propone la publicación del OA. Si bien no es una tarea compleja, se debe seleccionar el repositorio y pasar por los proceso de validación o evaluación del objeto para que el mismo esté disponible. Además, en algunos casos es necesario completar -nuevamente- los metadatos. También, es posible, publicar el OA directamente sobre un entorno de e-learning (LMS, por ejemplo). En este caso, debe analizarse cuidadosamente la compatibilidad y el funcionamiento adecuado del OA en el entorno. Tanto la elección de la herramienta de autor -primera fase- como el entorno sobre el que se va a publicar el OA deben ser cuidadosamente estudiados y probados. Esto contribuirá a minimizar los inconvenientes con los que se puedan encontrar los estudiantes (y los propios docentes) al utilizar los OA (Astudillo et al., 2011).

La cuarta fase (seleccionar), también está asociada a los repositorios, en ella se tienen en cuenta las herramientas con las que cuenta estos almacenes para la recuperación de los OA. Aquí la complejidad puede estar dada por localizar el material más apropiado. Esto dependerá no sólo de la forma en la que busca el usuario, sino además de las posibilidades del repositorio. Las cuales pueden ir desde una búsqueda por palabra clave y búsqueda avanzada, hasta incluir funcionalidades de recomendación.

La quinta fase es utilizar y aquí es donde se hace necesario hacer uso de la información con la que cuenta el OA para saber cómo y dónde podría ser integrado, además de la experticia del docente para incorporarlo en la secuencia de aprendizaje. En esta fase es posible contar con la ayuda de los Sistemas Ensambladores (SE).

La última fase (mantener) es una tarea de la que se debería encargar el/los autores, sin embargo, los repositorios pueden ayudar no sólo recordando a estos que debiera actualizarse, sino también, facilitando esta operación. La tarea de actualización del material, al igual que su creación, insume tiempo y esfuerzo, sin embargo, si se tratara de un objeto abierto puede ser mantenido por la comunidad de forma colaborativa.

Finalmente, se debe destacar el hecho de que los estándares y especificaciones, así como los repositorios, cuentan en forma implícita o explícita con una definición de OA, y sumado a esto, la falta de consenso por parte de los especialistas en el tema sobre una definición, hace que no se logre una correlación entre la teoría y la adopción del paradigma en la práctica (Astudillo et al., 2011). La proliferación de OA heterogéneos hace que las diferentes propuestas sobre diseño, recomendación (Maldonado \& Astudillo, 2014) o ensamblaje de OA puedan ser, en general, aplicadas sólo a un grupo reducido de OA, justamente, aquellos que verifican la definición adoptada.

\subsection{El ensamblaje de $O A$}

Como afirma Zapara Ros (2010):

"La organización, selección y secuenciación de los contenidos de aprendizaje ha sido un tema de interés central para el diseño curricular de los programas instruccionales a los distintos niveles, desde la unidad didáctica hasta los estudios de ciclo formativo o carrera" (p. 20). 
El aprendizaje es un proceso que necesita de la secuenciación del contenido $-\mathrm{y}$ del material apropiado-. La "secuenciación de contenidos, de tareas y de actividades ha de ir propiciando un acercamiento progresivo desde la situación inicial de aprendizaje de los alumnos hasta los objetivos propuestos para el programa formativo en que estemos inmersos" (Zapata Ros, 2010, p. 18). El concepto de secuenciación de contenidos sustenta a la "noción de ensamblaje, la cual se puede describir en términos de la correspondencia sintáctica, estructural y semántica entre los recursos educativos" (Santacruz-Valencia et al., 2005, p. 80).

La secuenciación de contenidos apoyada por tecnología facilitaría el proceso de auto-aprendizaje a los estudiantes y el diseño de actividades/cursos a los docentes. Pero, ¿qué motivaciones subyacen a la búsqueda de metodologías para ensamblar MED y más en particular, OA? ¿Se vinculan con la idea de secuenciación de contenidos antes mencionadas? Algunas respuestas a estas preguntas se presentan en la siguiente sub-sección. En forma posterior, se presentan una serie de metodologías que permiten el ensamblaje de OA, de manera de comenzar a hacer foco el tema central de esta tesis.

\subsubsection{Motivación}

La motivación para desarrollar metodologías de ensamblaje no es única pero la sobre-abundancia de recursos en la web hace necesario contar con un procedimiento para la curación de contenidos ${ }^{11}$.

Sin duda, una de las principales motivaciones está dada, justamente, por el rápido crecimiento de la cantidad de información en la web (Anh, Ha, \& Dam, 2008; Karam, Linckels, \& Meinel, 2007; Li \& Huang, 2006; Lopes Gançarski, Bouzeghoub, Defude, \& Lecocq, 2007; Shahin, Barakat, Mahmoud, \& Alkassar, 2008; Wetzlinger, Auinger, \& Stary, 2008). Como afirman Li \& Huang (2006), en su búsqueda por conocimiento la gente tiene que luchar contra la sobrecarga de información. Si bien muchos MED están alojados en repositorios (Shahin et al., 2008), las funcionalidades de estas herramientas, centradas en la búsqueda, están limitadas frente a la creciente cantidad de OA u otro tipo de recursos (Karam et al., 2007; Lopes Gançarski et al., 2007).

Como afirman Anh et al. (2008) Internet trajo consigo el desarrollo de sistemas basados en la web que permiten a los estudiantes aprender en cualquier momento y lugar, pero, es complejo para ellos obtener el conocimiento que necesitan debido la enorme cantidad de material disponible.

Sumado a lo anterior, se presenta otro aspecto donde pueden aportar los SE y que motiva a investigarlos: el conocimiento no está organizado eficientemente (Farrell, Liburd, \& Thomas, 2004; Huang, Huang, Wang, \& Hwang, 2009; Li \& Huang, 2006; Thyagharajan \& Nayak, 2007). Esto hace que se invierta demasiado tiempo en la obtención del mismo, sumado a que en muchos casos, los estudiantes no cuentan con las habilidades o competencias necesarias para elegir o seleccionar el material apropiado (Huang et al., 2009). Afirma Farrell (2004, p. 162), "el conocimiento que se adquiere de forma desconectada, a menudo se olvida o no puede integrarse efectivamente a la práctica".

La ingente cantidad de información así como su falta de organización deriva en otro de los motivos que esgrimen los autores para llevar adelante sus investigaciones sobre ensamblaje: la dificultad para buscar/localizar MED (Doan, Bourda, \& Dumitrascu, 2006; Farrell et al., 2004; Karam et al., 2007). Como afirma Farrell (2004, p. 167), es necesario que "los estudiantes dediquen más tiempo a aprender y menos a buscar y navegar". Tanto estudiantes como docentes, invierten demasiado tiempo y esfuerzo en localizar los materiales más apropiados (Karam et al., 2007).

Otra de las motivaciones que mayoritariamente plantean los investigadores es la necesidad de personalización del contenido (Colucci, Di Noia, Di Sciascio, Donini, \& Ragone, 2005; Garrido et al., 2009; Jovanović, Gašević, \& Devedžić, 2009; Kellar, Stern, Watters, \& Shepherd, 2004; Lalithsena, Hewagamage, \& Jayaratne, 2008; Li \& Huang, 2006; Roig Vila, 2005; Schreurs, Vanhove, \& Al-Zoubi, 2008; Thyagharajan \& Nayak, 2007; Verbert et al., 2012). La misma,

11 "La curación de contenidos consiste en buscar, agrupar, organizar y compartir el contenido más relevante de un determinado tema, transformándolo en conocimiento" (Bhargava, 2009). 
también está asociada con la creciente cantidad de MED en la red. Cómo localizar o seleccionar un contenido que se adapte al perfil del estudiante. Este trabajo es realizado, habitualmente, por el docente y se propone en varias metodologías de ensamblaje que este proceso de selección sea apoyado por el SE (Colucci et al., 2005).

El informe Horizon 2013, plantea la personalización del contenido con una implantación a dos o tres años. "El aprendizaje personalizado ha evolucionado desde hace algún tiempo, e incluye una amplia variedad de aproximaciones para apoyar el autoaprendizaje y el aprendizaje colectivo, que puede ser diseñado conforme a los objetivos de cada uno de los usuarios, incluyendo entornos de aprendizaje personalizados y redes, herramientas de aprendizaje adaptativas y algunas otras" (Johnson et al., 2013, p. 11).

Si lo que se busca es llevar adelante un diseño didáctico centrado en el estudiantes, es deseable que el aprendizaje sea personalizado (Roig Vila, 2005). El aprendizaje personalizado involucra, no sólo la adaptación de contenido, sino además una evaluación continua del estudiante que permita detectar el nivel alcanzado por el mismo para la adaptación del resto del itinerario de aprendizaje (Thyagharajan \& Nayak, 2007). Cada estudiante tiene necesidades, intereses diferentes, así como diferentes niveles de competencia o experiencia sobre un tema, por lo que no deberían ser tratados de manera uniforme (Lalithsena et al., 2008).

Habitualmente, los docentes planifican sus clases y diseñan los materiales para las mismas desde cero o bien reutilizando alguno ya existentes -o partes de éstos-. Sin embargo, en muchos casos, es complejo localizar MED que se adapten al estilo, la experiencia y/o las preferencias del docente (Verbert et al., 2012).

Los estudiantes, por su parte, al intentar llevar adelante un proceso de auto-aprendizaje se encuentran a menudo recursos y materiales que son poco relevantes, ya sea porque "no cuentan con el conocimiento necesario o porque tienen parte o todo el conocimiento, lo que puede producirles experiencias desconectadas, sobrecarga cognitiva y/o un ambiente de aprendizaje menos productivo." (Kellar et al., 2004, p. 2).

Afirma Verbert et al. (2012) "la adaptación a un aprendizaje individual o a los estilos docentes, los conocimientos, las experiencias, los intereses o preferencias, generalmente, no son posibles, a menos que el contenido sea específicamente diseñado con el propósito de ser personalizado" (p. 1258).

Los MED disponibles en la red, en muchos casos, no pueden adaptarse a las necesidades de cada estudiante -conocimientos previos, habilidades, competencias, objetivos o estilos de aprendizaje(Li \& Huang, 2006; Thyagharajan \& Nayak, 2007) o la información con la que cuentan (metadatos) no es suficientes para identificar los más apropiados (Thyagharajan \& Nayak, 2007). Dicen Jovanović et al. (2009) "estándares y especificaciones no captan la suficiente información que se requiere para niveles avanzados del proceso personalización del aprendizaje.” (p. 6).

Otro aspecto a tener en cuenta en la personalización del contenido es el abanico de tecnologías que los estudiantes utilizan para acceder al material. Éstos deben estar preparados/adaptados a todos o a la mayoría de los dispositivos (Schreurs et al., 2008).

Muchos sistemas de e-learning, aún proponen un modelo donde hay un único conjunto de recursos educativos para todos los estudiantes. En contraposición con esto, se afirma que el aprendizaje debe ser dinámico y altamente personalizado (Lalithsena et al., 2008). En este sentido, afirma Garrido et al. (2009), los sistemas inteligentes orientados a la educación deben asegurar que el contenido pueda ser adaptado al perfil del usuario. En este sentido el informe Horizon (Johnson et al., 2013) afirma que "a medida que las nuevas pedagogías hacen más énfasis en el aprendizaje personalizado, crece la demanda de alternativas en línea centradas en el estudiante. Los entornos de aprendizaje online, diseñados correctamente, poseen potencial a escala global" (p. 5).

El problema de los sistemas de e-learning y cómo mejorarlos y adaptarlos a la actual forma de administrar los contenidos es otra de las motivaciones que impulsan las implementación de las metodologías de ensamblaje (Bouzeghoub et al., 2009; Garrido et al., 2009; Kellar et al., 2004; 
Lalithsena et al., 2008; López et al., 2008; Sánchez-Alonso et al., 2007; Santacruz-Valencia, et al., 2008; Sarasa, Piquer, Arriola, \& Iglesia, 2008; Ullrich \& Melis, 2009). La mejora de este tipo de aplicaciones, para que brinden más soporte a los docentes/diseñadores instruccionales/estudiantes es una necesidad (Bouzeghoub et al., 2009).

Los sistemas que gestionan material educativo permiten administrar $-o$ deberían hacerlo- un importante abanico de MED. Pero, además, deberían tener la capacidad para ensamblar los mismos en una secuencia de aprendizaje apropiada (Santacruz-Valencia et al., 2008; Ullrich \& Melis, 2009). Para lograr esto, es necesario que los sistemas cuenten con información. La selección de OA requiere de registros de metadatos suficientes y completos que apoyen la toma decisiones $\mathrm{y}$ permitan a los sistemas de e-learning incluir un objeto dado en un itinerario de aprendizaje específico (Sánchez-Alonso et al., 2007, p. 179; Santacruz-Valencia et al., 2008).

Otro aspecto, relacionado con las aplicaciones de e-learning, que también aparece en la motivación es la preponderancia del trabajo manual que, aún, persiste en el diseño de cursos y MED (Li \& Huang, 2006; Rigaux \& Spyratos, 2007; Santacruz-Valencia et al., 2008; Shahin et al., 2008; Verbert et al., 2012). Los docentes y diseñadores que deciden diseñar un OA (o cursos) desde cero invierten mucho tiempo y esfuerzo en esta tarea (Santacruz-Valencia et al., 2008; Verbert et al., 2012), cuando la tecnología informática podría hacerse cargo de parte de la carga de trabajo.

Esto lleva, también, a otra de los las motivaciones: las TIC pueden mejorar el proceso educativo (Huang et al., 2009; Menéndez et al., 2010; Pahl \& Barrett, 2004; Thyagharajan \& Nayak, 2007; Verbert et al., 2012). Dicen Thyagharajan et al. (2007, p. 828) "los rápidos progresos de las [TIC] crean nuevas y numerosas oportunidades para la mejora de la calidad de la educación". Los avances en las investigaciones han permitido que se sumen una variedad de técnicas tanto al proceso de ensamblaje como al de recuperación de los materiales. Esto coincide con los momentos en los que, según Verbert (2012, p. 1258), los docentes requieren a las TIC: (a) durante el proceso de diseño y (b) en la selección de recursos relevantes para el diseño de actividades.

Es necesario contar con aplicaciones de e-learning que sean más flexibles a la hora de diseñar las lecciones (Kellar et al., 2004) para poder adaptar el contenido al perfil del usuario (Garrido et al., 2009; Lalithsena et al., 2008). Lo cual también redundaría en un aumento de la reutilización.

Precisamente, la reutilización, es otro de los motivos esgrimidos por varios autores (Bouzeghoub et al., 2009; Garrido et al., 2009; Jovanović et al., 2009; Li \& Huang, 2006; Lopes Gançarski et al., 2007; Santacruz-Valencia et al., 2008; Stănică \& Crișan, 2012). Es claro que la reutilización es central frente a la abundancia de MED ¿por qué crear un material que ya está disponible? Tarea, esta última, demandante y que requiere de un esfuerzo considerable; y en ocasiones onerosa (Li \& Huang, 2006; Stănică \& Crișan, 2012). Sin embargo, si la intención es crear un itinerario de aprendizaje, reutilizando material, el trabajo de secuenciar el mismo de forma manual, también implica un actividad que insume tiempo y esfuerzo (Jovanović et al., 2009; Lopes Gançarski et al., 2007).

La reutilización, en los procesos de ensamblaje, está directamente relacionada con una correcta y completa descripción de los OA a través de metadatos. Los cuales no sólo deben describir el material sino además contar con la semántica suficiente para permitir una apropiada secuenciación de los mismos (Bouzeghoub et al., 2009; Garrido et al., 2009; Santacruz-Valencia et al., 2008). Así como también la posibilidad de contar con operadores que permitan relacionar dos recursos o materiales heterogéneos (Bouzeghoub et al., 2009; Santacruz-Valencia et al., 2008).

La tabla 2.5 resume las principales motivaciones que impulsaron la investigación sobre ensamblaje de OA. 
Tabla 2.5: Principales motivaciones para la investigación en la temática.

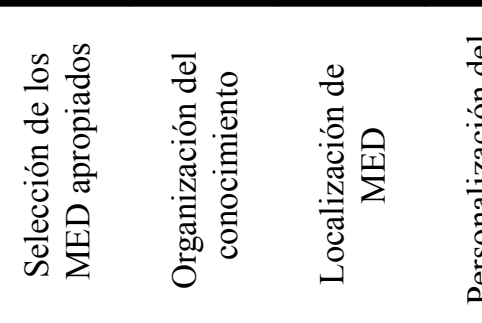

Kellar et al., 2004

Pahl \& Barrett, 2004

Colucci et al., 2005

Roig Vila, 2006

Farrell et al., 2004/2006

Li \& Huang, 2006

Karam et al., 2007

Lopes Gançarski et al., 2007

Rigaux \& Spyratos, 2007

Sánchez Alonso et al., 2007

Thyagharajan \& Nayak, 2007

Anh \& Dam, 2008

Lalithsena et al., 2008

Santacruz Valencia et al., 2008

Sarasa et al., 2008

Schreurs et al., 2008

Shahin et al., 2008

Wetzlinger et al., 2008

Bouzeghoub , 2009

Garrido et al., 2009

Huang et al., 2009

Jovanovic et al., 2009

Ullrich \& Melis, 2009

Menéndez et al., 2010

López et al., 2011

Stănică \& Crișan, 2012

Verbert et al., 2009/2012

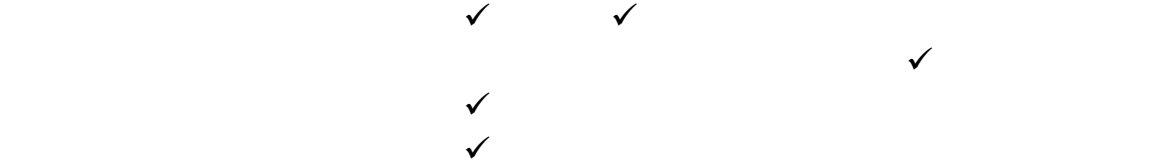

\subsection{2 ¿Por qué Objetos de Aprendizaje?}

Los SE, de forma genérica, buscan la generación de itinerario de aprendizaje personalizados a partir de recursos o materiales educativos ya existentes. Es por esto que el material debe cumplir con un conjunto de requisitos que permita utilizarlo en el contexto de estos sistemas. Básicamente estos requerimientos se podrían resumir como sigue:

- El material debe ser digital.

- El material debe contar con información que permita relacionarse con otro.

- El material debe contar con información que lo describa tanto técnicamente como pedagógicamente.

- El material debe estar alojado en repositorios.

Como afirman Stănică \& Crișan (2012) "los objetos de aprendizaje son vistos como la tecnología del futuro para el diseño, desarrollo y distribución de la próxima generación de software educativo, debido a la capacidad de adaptación, reutilización y escalabilidad potencial que ofrecen" (p. 29). Estas ventajas que ofrece el paradigma de OA están sustentadas en un tipo de MED que desde sus inicios propone el ensamblaje, así como el etiquetado por medio de metadatos y el diseño con vistas a la reutilización. 
En pos de maximizar la reutilización del material (característica distintiva de los OA): los metadatos deben apegarse a un estándar y los OA deben ser alojarlos en repositorios. Los OA cumplen, al menos en teoría, con los requerimientos impuestos por los SE al material educativo con el que se crean los itinerarios de aprendizaje.

Esta inclinación por la elección de los OA, que hacen los SE, se puede observar al analizar las publicaciones abordadas en este trabajo. Si bien eligen los OA para la creación de los itinerarios de aprendizaje, sólo 17 enuncian una definición, el resto usa el concepto sin definirlo. A continuación se presenta un análisis de las definiciones OA propuestas por las metodologías de ensamblaje.

\subsubsection{Definiciones de OA utilizadas por las metodologías de ensamblaje}

En este trabajo se han analizado un conjunto de publicaciones que pueden dar cuenta de la variedad $\mathrm{y}$ disparidad entre las diferentes definiciones de OA adoptadas por los distintos autores en el contexto de las metodologías de ensamblaje.

Del total de publicaciones analizadas que proponen una definición de OA, algunas de ellas (7) optan por una conceptualización genérica en consonancia con la propuesta por David Wiley (2000). Así un OA es definido como:

- "cualquier documento digital que se puede utilizar para el aprendizaje [...] descrito por metadatos" (Bouzeghoub \& Selmi, 2009, p. 175)

- "cualquier pedazo de información (texto, imagen, sonido, etc) que se puede identificar unívocamente" (Rigaux \& Spyratos, 2007, p. 2).

- "contenido crudo (raw content) u objetos de aprendizaje atómicos (texto, figuras, resumen de texto, palabras clave de texto)" (Schreurs et al., 2008, p. 15).

- "un elemento con documentos digitales reciclables de contenido multimedia, que tienen un propósito y algún uso en la enseñanza y el aprendizaje, a la vez que cumplen con cierta especificación tecnológica". Un OA está constituido por un recurso educativo multimedia y un conjunto de metadatos (Menéndez et al., 2010, p. 142).

- "pequeños componentes curriculares que puedan ser reutilizados varias veces en diferentes contextos de aprendizaje" (Roig Vila, 2005, p. 3). ${ }^{12}$

- "un recurso educativo atómico y auto-contenido, que está identificado unívocamente y es direccionable por un URI (identificador uniforme de recursos)" (Ullrich \& Melis, 2009, p. 9320).

- "es un nuevo tipo de elemento instruccional basado en el paradigma de la Orientación a Objetos del área Ingeniería de Software, orientado al soporte del aprendizaje en línea, que se crea una sola vez y se puede utilizar muchas veces más y en distintos contextos" (López et al., 2008, p. 3).

Las siguientes definiciones se caracterizan por atribuir a los OA la capacidad de poder combinarse o ensamblarse. Para los autores que proponen este tipo de definiciones (7), los OA son:

- "una unidad de contenido de aprendizaje con un objetivo específico, a menudo utilizada como bloque de construcción en diseño y ensamblaje de cursos. Los OA forman colecciones, son etiquetados con metadatos y almacenados en repositorios" (Farrell, 2006, p. $1)$.

- "la unidad de ensamblaje en entornos de enseñanza y aprendizaje más grandes construidos a partir de unidades más pequeñas" (Pahl \& Barrett, 2004, p. 446).

- "recursos educativos descritos por meta-datos y organizados en una estructura multicapa, de tal forma que los elementos más avanzados de esta estructura incluyen conocimiento asociado (CA) con vistas al ensamblaje y la reutilizacion" (Santacruz-Valencia et al., 2005, p. 74).

12 Estos autores parten explícitamente de la definición de David Wiley. 
- "fragmentos o partes de un curso que puede variar en tamaño y complejidad desde un gráfico sencillo de todo el curso" (Stănică \& Crișan, 2012, p. 2). Además debe cumplir las siguientes características: auto-contenido, independiente, reutilizable, de baja granularidad, que pueda ser combinado, personalizado y etiquetado con metadatos .

- "recursos educativos pequeños y fácilmente reutilizables que pueden ser compuestos para permitir una instrucción personalizada y la creación de cursos" (Colucci et al., 2005, p. 633).

- "materiales de aprendizaje auto-contenido que una vez desarrollados, puede ser intercambiados, compuestos, y pueden volver a utilizarse". Un OA es de granularidad pequeña, por lo que en la mayoría de los casos es necesario ensamblar varios para alcanzar un determinado objetivo de aprendizaje (Li \& Huang, 2006, p. 1).

- "unidades reutilizables de contenido educativo que pueden ser secuenciados en unidades más grandes para permitir el aprendizaje personalizado" (Karam et al., 2007, p. 716). Si bien retoman esta definición, completan el concepto diciendo que un OA es "cada subparte es una entidad educativa que se trata de un tema concreto del campo de la conferencia/clase (lecture)" (Karam et al., 2007, p. 720).

En las siguientes tres definiciones, se puede destacar que los autores retoman algunas de las características básicas del concepto de OA al definirlo.

- Para es Sarasa et al. (2008) los OA son reutilizables y contienen metadatos: "unidades mínima de información reutilizables [...que combinan] tres elementos: contenidos, descripciones del comportamiento del objeto, y un conjunto de metadatos que se refieren a los objetos" (p. 242).

- Para Wetzlinger et al. (2008) los OA son de granularidad fina, auto-contenidos y reutilizables: "elementos de granularidad fina para la transferencia de conocimiento [... que] permiten a los ingenieros de contenido diseñar unidades didácticas modulares autocontenidas, re-compuestas en nuevos cursos" (p. 33).

- Kellar et al., por su parte, (2004) afirma que los OA son reutilizables: "material pedagógico, accesibles desde la web, que pueden ser diseñado para su uso y reutilización en una variedad de contextos, desde la remediación hasta la preparación de una lección".

En el resto de las publicaciones analizadas (9), si bien se utiliza el concepto de OA, el mismo no se define explícitamente.

La Tabla 2.6 propone un resumen sobre cuál/es de las características más relevantes de OA se reflejan las definiciones propuestas en las publicaciones analizadas. 


\begin{tabular}{|c|c|c|c|c|c|c|c|}
\hline Autor & 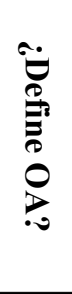 & $\begin{array}{l}\stackrel{0}{0} \\
\frac{0}{0} \\
\frac{0}{0} \\
\frac{0}{0}\end{array}$ & 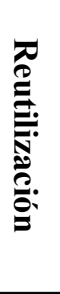 & 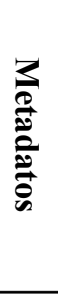 & 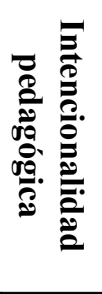 & 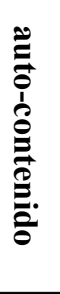 & 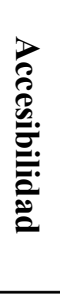 \\
\hline Kellar et al. (2004) & Sí & & $\checkmark$ & & $\checkmark$ & & \\
\hline Pahl et al. (2004) & Sí & $\checkmark$ & & & $\checkmark$ & & \\
\hline Colucci et al. (2005) & Sí & $\checkmark$ & $\checkmark$ & & $\checkmark$ & & \\
\hline Roig Vila (2005) & Sí & & & $\checkmark$ & $\checkmark$ & & \\
\hline Santacruz Valencia, Delgado Kloos \& Aedo Cuevas (2005) & Sí & $\checkmark$ & $\checkmark$ & $\checkmark$ & $\checkmark$ & & \\
\hline Farrell et al. (2006) & Sí & $\checkmark$ & & $\checkmark$ & $\checkmark$ & & \\
\hline Li \& Huang (2006) & Sí & $\checkmark$ & $\checkmark$ & & $\checkmark$ & $\checkmark$ & \\
\hline Karam, Linckels \& Meinel (2007) & Sí & $\checkmark$ & $\checkmark$ & & $\checkmark$ & & \\
\hline Lopes Gançarski et al. (2007) & No & $\checkmark$ & & & & & \\
\hline Rigaux \& Spyratos (2007) & Sí & $\checkmark$ & & & & & \\
\hline Sánchez Alonso et al. (2007) & No & & & & & & \\
\hline Thyagharajan \& Nayak (2007) & No & & & & & & \\
\hline Anh et al. (2008) & No & & & & & & \\
\hline Lalithsena, Hewagamage \& Jayaratne (2008) & No & & & & & & \\
\hline López, Miguel \& Montaño (2008) & Sí & & $\checkmark$ & & $\checkmark$ & & \\
\hline Sarasa et al. (2008) & Sí & & $\checkmark$ & $\checkmark$ & & & \\
\hline Schreurs, Vanhove \& Al-Zoubi (2008) & Sí & & & & & & \\
\hline Shahin et al. (2008) & No & & & & & & \\
\hline Wetzlinger, Auinger \& Stary (2008) & Sí & & $\checkmark$ & & $\checkmark$ & $\checkmark$ & \\
\hline Bouzeghoub et al. (2009) & Sí & & & $\checkmark$ & $\checkmark$ & & \\
\hline Garrido, Onaindia \& Sapena (2009) & No & & & & & & \\
\hline Huang et al. (2009) & No & & & & & & \\
\hline Jovanovic et al. (2009) & No & & & & & & \\
\hline Ullrich \& Melis (2009) & Sí & & & & $\checkmark$ & $\checkmark$ & \\
\hline Menéndez et al. (2010) & Sí & & $\checkmark$ & $\checkmark$ & $\checkmark$ & & \\
\hline Stănică \& Crișan (2012) & Sí & $\checkmark$ & $\checkmark$ & $\checkmark$ & & $\checkmark$ & \\
\hline Verbert et al. (2012) & No & & & & & & \\
\hline
\end{tabular}

\subsection{Recapitulación}

La ingente cantidad de MED disponibles a través de la web se ha convertido en uno de los factores que propician la adopción del paradigma de OA, así como también para el desarrollo de SE. Estos últimos han adoptado a los OA para la creación de los itinerarios de aprendizaje que generan. Esto se debe, en principio, a que es el propio paradigma de OA el que propone desde sus inicios el ensamblaje de OA para alcanzar MED de mayor granularidad. A lo que se le agrega estar diseñados para la reutilización, contar con metadatos que los describen y estar alojados en repositorios, características deseables en el proceso de ensamblaje (ver Figura 2.3).

Los OA y los SE comparten, entonces, la idea de combinar MED para adaptarlos a los destinatarios y reutilizarlos en varios contexto. Metáforas como la de LEGO (similar a la del Mecano sugerida por Gerard), de la molécula o la del ladrillo y cemento (the brick-and-mortar metaphor) se han utilizado para explicar el concepto de OA y en ellas la idea de ensamblaje está siempre presente. 


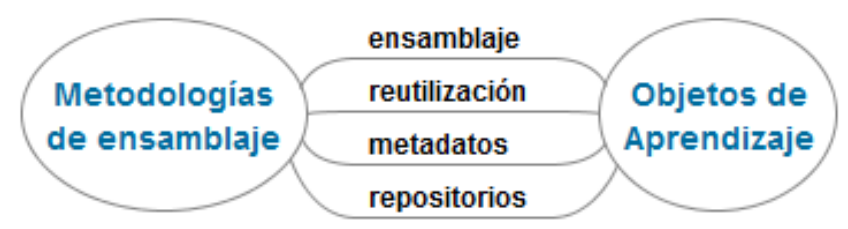

Figura 2.3. Vinculación entre los OA y las metodologías de ensamblaje

El proceso de selección de las metodologías de ensamblaje, para esta investigación, inició con 61 publicaciones, de las cuales se eligieron, a través de los criterios de inclusión y exclusión, 40 para su lectura completa. Dicha lectura, permitió descartar otras cinco publicaciones. Las 35 restantes se agruparon de acuerdo a la metodología de ensamblaje abordada y, así, se definieron las 27 metodologías de ensamblaje de OA analizadas en esta tesis. Cabe aclarar que se agregaron siete publicaciones, por fuera de las seleccionadas para el análisis, con el objetivo de mostrar avances y/o tendencias en la temática.

A partir de la revisión de las 27 metodologías se pudo realizar un análisis preliminar. Se observó que, aunque hay coincidencia en la adopción de los OA por parte de los SE, no ocurre lo mismo al definir el concepto. Esto se debe, principalmente, a que el propio paradigma de OA no cuenta, aún, con una única respuesta a la pregunta ¿Qué es un objeto de aprendizaje? De esta manera, 18 de las 27 metodologías optan por definir el concepto y el resto lo utilizan sin definirlo. De las que lo hacen, siete optan por una definición genérica y en otras siete se enuncia la capacidad del OA como unidad de ensamblaje. Sólo en tres de las definiciones propuestas se incluyen, al menos, algunas de las características acordadas por los especialistas en la temática para paradigma.

El aprendizaje es un proceso que necesita de una secuenciación del contenido, y es precisamente, en este concepto en el que se sustenta la noción de ensamblaje. Para lograr una apropiada secuenciación o itinerario de aprendizaje es necesario la selección de los MED apropiados, pero para ello el conocimiento debe estar organizado de forma que sea posible su localización. También, es deseable que un itinerario de aprendizaje se ajuste al perfil del estudiante (personalización del contenido). Lograr, además, que la secuencia sea generada de forma automática (o semi-automática) redundaría en un mejoramiento de los sistemas e-learning, lo que impactaría positivamente en los procesos educativos mediados por TIC y en una reutilización genuina de MED. Estas son motivaciones que sustentan las investigaciones en la temática de ensamblaje dentro de las publicaciones analizadas en esta tesis.

En el Capítulo siguiente se continúa con el desarrollo de estado de la cuestión. Se retoma la revisión de las metodologías de ensamblaje y se profundiza en su caracterización, así como también se describen el conjunto de metodologías analizadas en el presente trabajo de tesis. 


\section{ESTADO DE ARTE (SEGUNDA PARTE)}

\section{RESUMEN}

Las metodologías de ensamblaje de OA tienen como objetivo principal la definición de un itinerario de aprendizaje utilizando objetos alojados en repositorios. Para lograrlo deben definir cómo conectar un OA con otro, pero además cómo representar las distintas entidades involucradas en el proceso (OA, estudiantes $y / o$ docentes).

Este tercer capitulo presenta la segunda parte del estado de arte. Primeramente se define el concepto de ensamblaje y se tipifican las metodologías. Luego, se describen las abstracciones con las que éstas trabajan (modelo de contenido, de estudiante, de profesor y de enseñanza). Seguidamente, se establece el papel de los metadatos y, de los pre-requisitos y el conocimiento adquirido en los procesos de ensamblaje. Finalmente, se bace un descripción de las 27 metodologías de ensamblaje de OA estudiadas. 


\subsection{Introducción}

Las metodologías de ensamblaje de OA tienen como objetivo principal la definición de un itinerario de aprendizaje en base a un conjunto de este tipo MED alojados en repositorios. El mismo puede estar generado por distintos niveles de automatización y tener (o no) en cuenta el perfil de usuario.

El proceso de ensamblaje consta de tres etapas (Farrell, Liburd, \& Thomas, 2004; Thyagharajan \& Nayak, 2007):

(i) buscar las conexiones del material relevante para una secuencia de aprendizaje,

(ii) secuenciar el material y

(iii) conectarlo dentro de una estructura organizada.

Es posible distinguir entre tres tipos de metodologías de ensamblaje (ver Tabla 3.1):

(i) aquellas que crean un itinerario de aprendizaje de OA (en este trabajo se denominarán como de Tipo 1),

(ii) las que crean un $\mathrm{OA}$-el itinerario está implícito, por ser una unidad didáctica- (en este trabajo se denominarán como de Tipo 2) y

(iii) las que recomiendan un itinerario de aprendizaje o el objeto siguiente en la secuencia, con base en el perfil del usuario (en este trabajo se denominarán como de Tipo 3).

Tabla 3.1. Metodologías agrupadas por tipo.

\begin{tabular}{|c|c|c|}
\hline Tipo & Autor & Fecha \\
\hline \multirow{10}{*}{ T1 } & Pahl et al. & 2004 \\
\hline & Roig Vila & 2005 \\
\hline & Santacruz Valencia, Delgado Kloos \& Aedo Cuevas & 2005 \\
\hline & Farrell et al. & 2006 \\
\hline & Sánchez Alonso et al. & 2007 \\
\hline & López, Miguel \& Montaño & 2008 \\
\hline & Sarasa et al. & 2008 \\
\hline & Wetzlinger, Auinger \& Stary & 2008 \\
\hline & Menéndez et al. & 2010 \\
\hline & Verbert et al. & 2012 \\
\hline \multirow{5}{*}{$\mathbf{T} 2$} & Lopes Gançarski et al. & 2007 \\
\hline & Schreurs, Vanhove \& Al-Zoubi & 2008 \\
\hline & Bouzeghoub et al. & 2009 \\
\hline & Jovanovic et al. & 2009 \\
\hline & Stănică \& Crișan & 2012 \\
\hline \multirow{12}{*}{$\mathbf{T 3}$} & Kellar et al. & 2004 \\
\hline & Colucci et al. & 2005 \\
\hline & Li \& Huang & 2006 \\
\hline & Karam, Linckels \& Meinel & 2007 \\
\hline & Rigaux \& Spyratos & 2007 \\
\hline & Thyagharajan \& Nayak & 2007 \\
\hline & Anh et al. & 2008 \\
\hline & Lalithsena, Hewagamage \& Jayaratne & 2008 \\
\hline & Shahin et al. & 2008 \\
\hline & Garrido, Onaindia \& Sapena & 2009 \\
\hline & Huang et al. & 2009 \\
\hline & Ullrich \& Melis & 2009 \\
\hline
\end{tabular}


El proceso de ensamblaje de OA se puede realizar de forma manual, semi-automática o automática. En el primer caso, son los expertos y/o los propios docentes los que proponen la forma en que los contenidos serán secuenciados y qué $\mathrm{OA}$ se incluirán en el itinerario (incluso la adaptación de al perfil del estudiante). En este caso las metodologías se basan, en general, en el diseño intruccional para proponer un conjunto de buenas prácticas para el ensamblaje de OA.

En el segundo caso, proceso semi-automático, proponen la participación de expertos/docentes que:

(i) diseñan una secuencia o red de OA, la que luego, el sistema completará de forma automática con los OA más apropiados en función de los contenidos abordados $\mathrm{y}$, podría tomar en cuenta el perfil del usuario. Cada OA agregado respeta el esquema definido por el autor para la composición y al que el sistema asigna OA (Bouzeghoub, Buffat, Lopes Gançarski, et al., 2009).

(ii) proponen la secuenciación de los OA y el sistema se encarga de ensamblarlos (esto incluye la validación de que el ensamblaje propuesto es posible). Es necesario contar con metadatos de calidad y reglas que permitan el acoplamiento de los OA (Santacruz-Valencia, Delgado Kloos, \& Cuevas Aedo, 2005).

Cuando se hace de forma automatizada o dinámica, el estudiante o el docente proponen un tema y/o los objetivos, y el sistema ofrece uno o varios itinerarios de aprendizaje. Los mismos puede generarse completamente -requiere, por lo general, de la validación del docente- o puede crearse paso a paso a medida que el estudiante finaliza el abordaje de cada OA. El "ensamblaje dinámico de [OA] se basa en la coincidencia respecto del contenido del [OA] y los metadatos con las necesidades del estudiante, las preferencias, el contexto y las limitaciones de aprendizaje" (Santacruz-Valencia, Navarro, Delgado Kloos, \& Aedo, 2008, p. 104). El ensamblaje dinámico pone el foco en cómo construir automáticamente una secuencia ad-hoc o personalizada, en base a una búsqueda y las preferencia del usuario, que permita la consecución de ciertos objetivos de aprendizaje (Bouzeghoub, Buffat, Lopes Gançarski, et al., 2009; Thyagharajan \& Nayak, 2007; Ullrich \& Melis, 2009; Wetzlinger, Auinger, \& Stary, 2008). Para lograr el armado del itinerario de aprendizaje, de forma automática, se recurre en general a la utilización de patrones, los cuales se pueden conseguir a través de un enfoque top-down, basados en el diseño instruccional, o bottom-up, basados en la experiencia de los usuarios (cómo aprenden) (Verbert et al., 2012).

A continuación (sección 3.2) se presentan un conjunto de abstracciones que son utilizadas por las diferentes metodologías de ensamblaje para gestionar las distintas entidades que deben administrar. Seguidamente (Sección 3.3) se aborda el concepto de ontologías y su relación con el proceso de ensamblaje. La Sección 3.4 se enfoca en la relación de los metadatos con el ensamblaje de OA. En la Sección 3.5 se describen las metodologías de ensamblaje que se analizaron en esta tesis. Para finalizar, se presenta una recapitulación.

\subsection{Abstracciones en las metodologías de ensamblaje}

Las metodologías de ensamblaje deben representar o abstraer varios aspectos de la realidad, dando prioridad a lo educativo. De manera general, cada metodología puede contar con -parte o todas- de las siguientes abstracciones: ( $i$ ) un modelo de contenido (content model), (ii) un modelo de estudiante (learner model), (iii) un modelo profesor (teacher model/teaching/instructional model) y/o (iv) un rol educativo (Ullrich \& Melis, 2009; Verbert et al., 2012).

Uno de los aspectos que comparten la mayoría de las metodologías es la definición del Modelo de Contenido (MC). Dado que el mismo no es único, en la siguiente sección, se presentan las distintas representaciones utilizadas por las metodologías analizadas en el presente trabajo de investigación. Del mismo modo, se hace una descripción del modelo de estudiante, del modelo de profesor y del modelo de contenido. 


\subsubsection{Modelos de contenido}

"El modelo de contenido [...] describe los componentes utilizados en una experiencia de aprendizaje, cuál es la relación entre dichos componentes, cómo se describen para facilitar su búsqueda y descubrimiento y las reglas que hacen posible el ensamblaje de dichos componentes para su posterior reutilización." (Santacruz-Valencia et al., 2005, p. 73). En este sentido afirman Menéndez et al. (2010) que un OA "está constituido por dos elementos básicos: una colección de recursos o contenidos y un conjunto de descriptores de las características del objeto, denominados metadatos. Adicionalmente, puede incluir un conjunto de descriptores del comportamiento o de los requerimientos para su utilización y ensamble.” (p. 57).

El MC SCORM, definido por ADL, es adoptado (o adaptado) por varias de las metodologías (Anh, Ha, \& Dam, 2008; Huang, Huang, Wang, \& Hwang, 2009; Jovanović, Gašević, \& Devedžić, 2009; Li \& Huang, 2006; Menéndez et al., 2010; Santacruz-Valencia et al., 2005; Schreurs, Vanhove, \& Al-Zoubi, 2008; Stănică \& Crișan, 2012; Verbert et al., 2012; Wetzlinger et al., 2008).

El MC de SCORM (ver Figura 3.1) está compuesto por (ADL, 2009, p. 3):

- Asset

- Shareable Content Objects (SCO)

- Activity

- Content organization

- Content aggregation

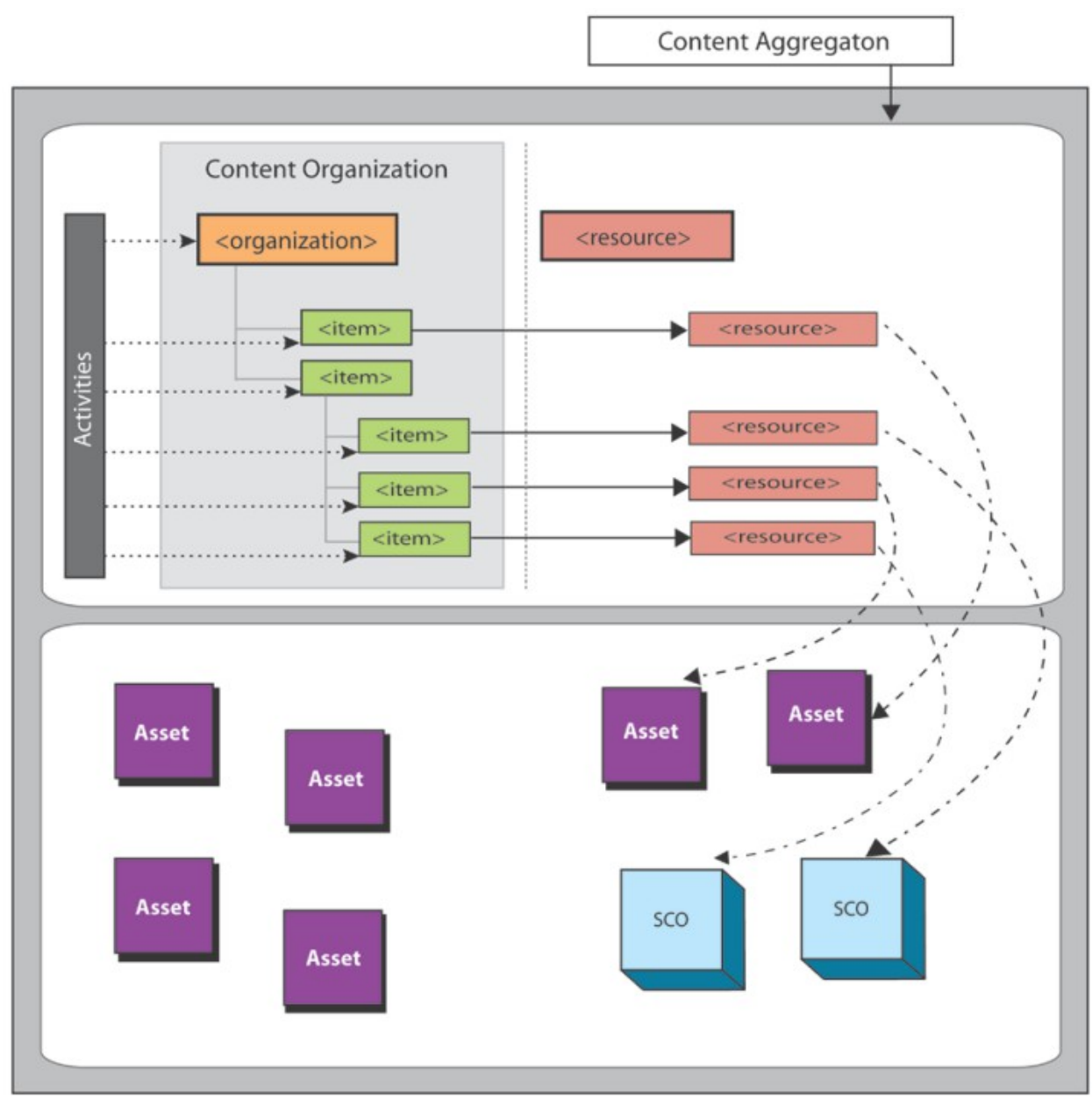

Figura 3.1. Esquema que representa el modelo de contenidos de SCORM (ADL, 2009, fig. 2.1.5a).

Los asset o recursos corresponden a recursos digitales, tales como textos, imágenes, sonidos, videos o animaciones, páginas web (o cualquier otro recurso que pueda ser interpretado por un cliente 
web). Un asset se constituye como el "bloque de construcción básico del recurso de aprendizaje" (ADL, 2009, p. 3). Los asset pueden ser etiquetados con metadatos.

Los SCO, por su parte, son una colección de uno o más asset, que se diferencian de éstos por tener comunicación con el LMS. En ambos casos, los recursos pueden estar diseñados o no con una intencionalidad pedagógica. Los SCO deben estar etiquetados con metadatos.

Una activity o actividad "es algo que el estudiante hace mientras avanza a través de la instrucción" (ADL, 2009, p. 5), pueden estar asociadas tanto a un asset como un SCO. Las activity que se agrupan en otras actividades se llaman clusters y estos pueden ser etiquetados con metadatos.

La content organization u organización de contenidos permite definir la secuencia en la que las actividades deberían ser presentadas al estudiante. Los content organization pueden ser etiquetados con metadatos.

La content aggregation o agregación de contenidos, por su parte, permite describir la granularidad de objetos de contenido.

Los niveles de granularidad de contenidos propuesto por SCORM (asset, SCO, activity) son retomados por varias de las metodología y/o adaptados en función de la definición de OA y la manera en que proponen que se realice el ensamblaje de los mismos.

Santacruz-Valencia et al. (2005) proponen un MC (ver Figura 3.2) con tres niveles de agregación para los objetos que denominan Electronic Learning Object (ELO):

- Unidades de información

- Unidades de contenido

- Unidades didácticas

Las unidades de información representan "elementos atómicos y son auto-contenidos y altamente reutilizables" (Santacruz-Valencia et al., 2008, p. 104). Al igual que los asset, hacen referencia a archivos multimedia los cuales sólo toman un sentido educativo en el contexto del OA. Los metadatos asociados a las unidades de información son descriptivos y bajo el estándar LOM.

En un segundo nivel de agregación se encuentran las unidades de contenido, las mismas se forman agrupando unidades de información o éstas con otras unidades de contenidos. En virtud de que las unidades de contenido deben constituir una experiencia educativa para el estudiante "necesita de unos requisitos para su comprensión y proporciona unas competencias tras su comprensión" (Santacruz-Valencia et al., 2005, p. 77), lo cual se representa a través de la incorporación de metadatos, denominado por los autores "conocimiento asociado". Los mismos se implementan extendiendo la categoría educational del estándar LOM.

Las unidades didácticas son el tercer nivel de agregación, se forman combinando varias unidades de contenido y/o éstas con otras unidades didácticas. Representa "el conocimiento referente a un área tras la acumulación de diversas experiencias educativas relacionadas" (Santacruz-Valencia et al., 2005 , p. 78). Al igual que las unidades de contenido cuentan con conocimiento asociado que permite describirla, incluyendo: un resumen, los objetivos de aprendizaje (competencias) de la misma y los mecanismos de evaluación. El conocimiento asociado a la unidad didáctica, también se implementan extendiendo la categoría educational del estándar LOM.

Cabe destacar que son los propios autores los que establecen una equivalencia entre su propuesta y el estándar SCORM (Santacruz-Valencia et al., 2005):

- Las unidades de información "son asimilables a los assets" (p.76).

- Las unidades de contenido "son asimilables a los SCOs [...] con la diferencia fundamental de que las [unidades de contenido] tienen conocimiento asociado" (p.77).

- Una unidad didáctica “es asimilable a un Content Organization” (p.78). 


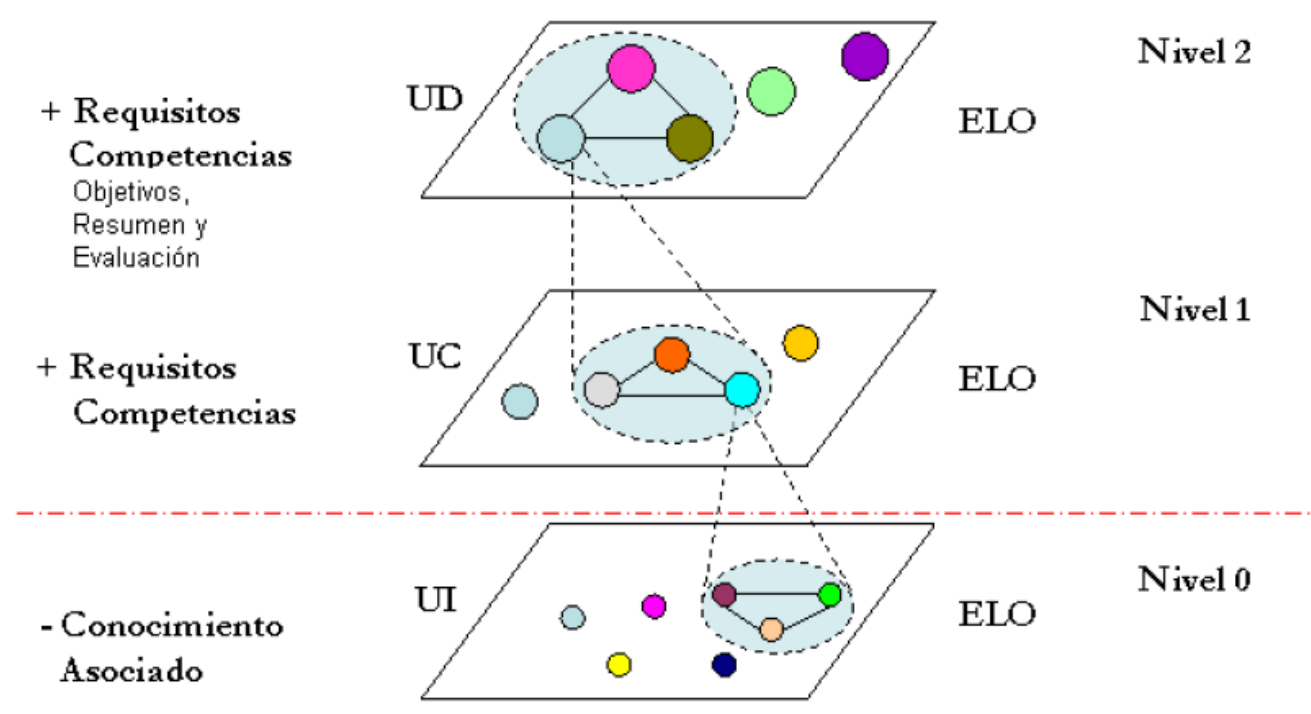

Figura 3.2. Esquema que representa el modelo de contenidos para un ELO (Santacruz-Valencia et al., 2005, fig. 3.1).

Li \& Huang (2006) y Anh, Ha \& Dam (2008) utilizan un MC (ver Figura 3.3) en sus metodologías de ensamblaje para representar los OA y este está formado por:

- Asset

- Learning object

- Knowledge map

Un asset, como en los casos anteriores, hacen referencia a recursos digitales multimedia (páginas web, documentos PDF, animaciones y audios, según los autores). Estos recursos no cuentan con metadatos asociados, pero contarán con una descripción semántica una vez incluidos en un OA.

Un learning object es un conjunto de asset que constituyen un material educativo, el cual debe ser "auto-contenido" e "independiente de la forma, granularidad y funcionalidad" (Li \& Huang, 2006, p. 570). Deben estar etiquetados con metadatos que describen: el objeto (content metadata), la relación del objetos con otro/s objeto/s (structural metadata) y en qué contexto pedagógico utilizar el objeto (context metadata).

El knowledge map describe el dominio específico de conocimiento donde se explicitan relaciones semánticas entre los objetos (learning object). Esto permite establecer pre-requisitos para el acceso a los objetos.

Anh, Ha \& Dam (2008), por su parte, agregan las knowledge unit. La misma está constituida por un conjunto de OA y cuentan con los siguientes atributos o metadatos (Anh et al., 2008, p. 2):

- Pre-requisitos: requerimientos para acceder al objeto.

- Nivel de experiencia (master level): principiante, intermedio, avanzado y experto.

- Nivel de dificultad: fácil o no.

- Tiempo requerido: tiempo mínimo, estimado, para completar la knowledge unit.

- Relación de la unidad con otras.

- Estilo de interactividad: de arriba hacia abajo, de abajo hacia arriba, en consecuencia, en paralelo.

- Habilidades: enseñanza de habilidades de aprendizaje (la comprensión, la deducción, etc.). 


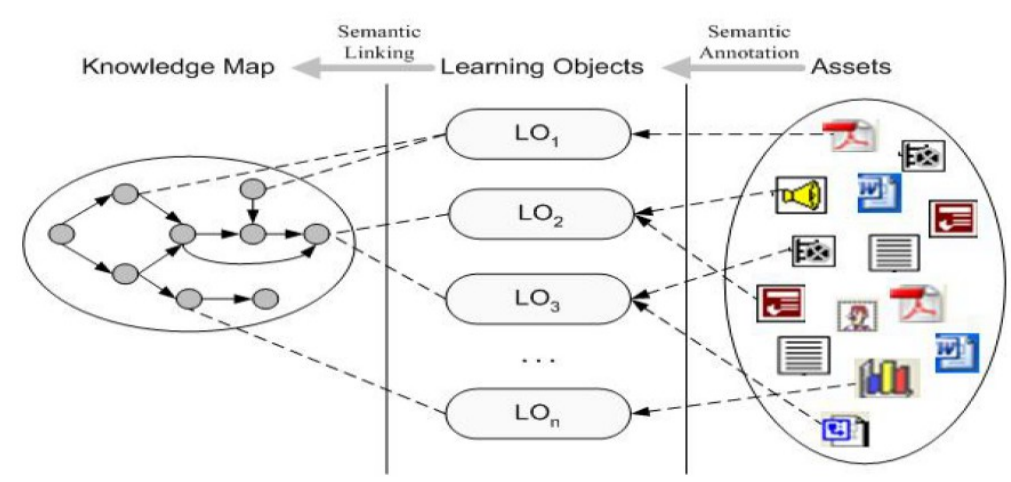

Figura 3.3. Esquema que representa el modelo de contenidos del recurso educativo (Li \& Huang, 2006, fig. 1) o knowledge unit (Anh et al., 2008, fig. 1).

Otro de los autores que retoman un modelo basado en SCORM son Schreurs et al. (2008) asociando el mismo con un libro (capítulos, unidades y bloques). El MC, cuenta así, con los siguientes componentes:

- Atamic Learning Object (ALO)

- Block

- Unit

- Chapter

Cada ALO es asimilable a un asset y refiere al contenido crudo (raw content). Al realizar la equivalencia del modelo de contenido con un libro, cada ALO puede ser: el resumen, palabras claves, tablas, textos, figuras, y se agregan animaciones, videos, Q\&A ${ }^{1}$, la URL para información adicional, entre otros. Los metadatos para cada ALO son: título, la descripción y los derechos de autor.

Cada block es asimilable a un SCO y se compone de un conjunto de ALO que permiten aprender el tópico de bloque. Los bloques pueden estar divididos en sub-bloques. Además de los metadatos requeridos para los ALO, un block debe contar con: idioma y el tiempo transcurrido.

Una unit está compuesta por un conjunto de blocks y representan una parte del contenido por aprender. Una unit es un OA organizado como un content aggregation model (de SCORM). Además de los metadatos que se agregan a un block, la unidad debe contar con: título, el nivel de aprendizaje de los contenidos y el conocimiento previo necesario.

Cada chapter o capítulo es un conjunto de OA o unit.

Stănică \& Crișan (2012) definen, en su MC, tres niveles de agregación²:

- Fundamental objects

- Instructional components

- Compound objects

Un fundamental object es el objeto más simple, tiene una baja granularidad y representa: un texto, una función o una fórmula matemática.

Un instructional component se constituye de uno o más fundamental objects y está asociado al concepto de función matemática. Puede ser combinado o ensamblado con otros componentes (reutilizado) y el comportamiento del mismo se determina por su capacidad de acoplamiento.

Un compound object, es una combinación de instructional components y se constituyen en una lección.

1 Questions and answers o actividades de preguntas y respuestas.

2 El trabajo de Stănică \& Crișan se centra en desarrollar un ambiente de aprendizaje integrado para el aprendizaje de matemática, es por eso que los objetos se centran en este campo de conocimiento. 
Verbert \& Duval (2004) desarrollaron un modelo de objetos de contenido (object content model) denominado ALOCoM (Abstract Learning Object Content Model), el mismo es retomado por la autora (Verbert et al., 2012) y adaptado por Jovanović et al. (2006; 2009) para desarrollar metodologías de ensamblaje de OA.

En el MC de ALOCoM se definen tres niveles de agregación (Verbert \& Duval, 2004, 2008):

- Content Fragment

- Content Object

- Learning Object

Un content fragment es la forma más básica de contenido, la cual no puede dividirse. Se trata de cualquier recurso digital multimedia.

Un content object es un conjunto de content fragment y puede incluir otros content object. Puede utilizarse tanto para explicar un concepto, ilustrar un principio, como para describir un proceso.

Un learning object es una agrupación de content fragment y/o content object. Puede haber tres tipos de learning objects: (i) los de objetivo educativo simple (single-objective LO), que explican conceptos, hechos, principios, procesos o procedimientos; (ii) los de objetivo mayor (larger objective $L O$ ) que agrupan objetos de objetivo simple para formar lecciones o capítulos; o (iii) las agregaciones (LO aggregations) que representan unidades, cursos o planes de estudios.

En virtud de que ALOCoM está basado, en parte, en SCORM los content fragment puede asemejarse a los asset y los content object a los SCO (Zouaq, Nkambou, \& Frasson, 2007). En este sentido afirman Verbert \& Duval (2008, p. 60) "en el modelo ALOCoM, una agregación de contenidos combina actividades, y una actividad combina dos tipos de recursos de aprendizaje, SCO y asset".

Algunos autores (Farrell et al., 2004; Garrido, Onaindia, \& Sapena, 2009; Kellar, Stern, Watters, \& Shepherd, 2004; Sarasa, Piquer, Arriola, \& Iglesia, 2008) elijen para su MC las especificaciones de IMS GLOBAL: IMS Content Packaging Specification ${ }^{3}$. Esta especificación, a la que SCORM "adhiere estrictamente" (ADL, 2009, p. 3), "describe las estructuras de datos que pueden ser utilizadas para el intercambio de datos entre sistemas que desean importar, exportar, agregar y desagregar los paquetes de contenido.” (IMS Global Learning Consortium, 2007, p. 5).

En el MC de la especificación se definen los siguientes niveles agregación (IMS Global Learning Consortium, 2007):

- Content file

- Content/Unit of content

- Logical package

Content file son archivos informáticos individuales que pueden ser referenciados en el paquete lógico.

Content es un término genérico para las unidades lógicas de información utilizables (y reutilizables). Un contenido es descrito en un paquete lógico.

Logical package o paquete lógico, se constituye de una o más unidades contenido.

La especificación cuenta también con una “organización" que representa las relaciones lógicas entre unidades de contenido. Asimismo, tanto los archivos, los contenidos como los paquetes son descritos a través de metadatos.

Farrel et al. (2004) utilizan el estándar de metadatos LOM y extienden la categoría Educational e incorporan dentro del tipo de recursos de aprendizaje "rol educativo" (instructional role, ver 3.2.4). Tanto Kellar et al. (2004), como Garrido et al. (2009) utilizan IMS-LRMS como estándar de

3 Disponible en: http://www.imsglobal.org/content/packaging/ 
metadatos, aunque el mismo es compatible con LOM (Barker, 2005).

Cabe aclarar que, si bien Kellar et al. (2004) utilizan IMS Content Packaging Specification para empaquetar el contenido, definen los siguientes niveles de agregación:

- Atom

- Component

- $\quad$ Page

- Lesson

Un atom es la unidad más pequeña de contenido de aprendizaje. Puede ser representado por uno o más archivos multimedia.

Los component son contenedores reutilizables para los atom.

Una page representa un concepto a aprender.

Cada lesson está compuesta por una lista ordenada de page.

"Un módulo de aprendizaje es una lección en línea, compuesta de una lista ordenada de páginas. [...] Las lecciones que se producen en este sistema se empaquetan y se cumple con IMS Content Packaging Specification" (Kellar et al., 2004, p. 3).

López et al. (2008), por su parte, proponen que los OA sean compatibles con los estándares IMS$\mathrm{LD}^{4}$ y SCORM. Sin embargo, utilizan los siguientes niveles de agregación:

- OA Fundamentales

- OA Generativo-Instruccionales

- Estrategias

Los OA Fundamentales representan "contenido independiente del contexto" y "pueden incluir gráficos, videos, definiciones, explicaciones descontextualizadas o lecturas, casos particulares y definición de problemas, entre otros" (López et al., 2008, p. 8).

OA Generativo-Instruccionales puede representar "contenido con un contexto mínimo añadido (por ejemplo, enlaces dentro de un caso de estudio a perspectivas y temas, pudiendo incluir metas de aprendizaje) hasta actividades de aprendizaje más completas o estrategias instruccionales como tutoriales, micromundos, simulaciones, etc." (López et al., 2008, p. 8).

Las estrategias son “objetos que están contextualizados por la implementación de enfoques instruccionales específicos (hipertextos cognitivos flexibles, aprendizaje basado en problemas, etc.) y pueden incorporar otros OA y varias clases de enlaces.” (López et al., 2008, p. 8).

Bouzeghoub et al. (2004a) desarrollaron un esquema llamado SIMBAD ${ }^{5}$ que incluye tres modelos semánticos: el dominio, el alumno y OA compuestos. Este modelo es retomado por Bouzeghoub \& Selmi (2009) y por Lopes Gançarski et al. (2007) para proponer metodologías de ensamblaje de OA. El MC propone dos niveles de agregación:

- Atomic

- Complex

"Un OA complejo se construye mediante la aplicación de operadores (si es necesario, de forma recursiva) de composición de OA (atómico o complejo). Un OA complejo [está representado por] un grafo" (Bouzeghoub \& Selmi, 2009, p. 764). En el grafo "los nodos representan conceptos y

4 IMS Learning Design o IMS-LD es una especificación que permite a los sistemas de aprendizaje compatibles soportar una amplia gama de pedagogías de aprendizaje en línea. Proporcionando un lenguaje genérico y flexible que permite modelar procesos de enseñanza y aprendizaje desde diferentes enfoques pedagógicos (IMS Global Learning Consortium, 2003).

5 SIMBAD define: una ontología de dominio, un modelo de usuario y un modelo de composición (Bouzeghoub, Defude, Ammour, Duitama, \& Lecocq, 2004b; Duitama, Defude, Bouzeghoub, \& Lecocq, 2005). 
aristas representan las relaciones entre dos conceptos" (Lopes Gançarski et al., 2007, p. 9). Cada OA cuenta con metadatos que describen el contenido, los requisitos previos para acceder al objeto y el conocimiento obtenido al utilizarlo. En ambas propuestas se utiliza el estándar de metadatos IEEE LOM.

De forma similar Rigaux \& Spyratos (2007) proponen un MC donde hay sólo dos niveles de agregación:

- atomic LOs

- composite LOs

Un atomic $L O$, es definido como "el bloque básico de construcción". Una pieza de información, que como el asset, puede ser cualquier archivo multimedia.

Un composite $L O$, es un conjunto de atomic LO o composite LO. Éstos forman un grafo con forma de árbol denominado grafo de composición (composition graph). Cada nodo del árbol es un OA y es pre-requisito (salvo las hojas) para acceder al próximo OA.

Cada OA atómico cuenta con un conjunto de metadatos y de éstos se derivan o heredan al OA compuesto.

Thyagharajan \& Nayak (2007) proponen un MC basado en:

- pagelet

- page/module

Una pagelet, que representa "una unidad discreta de información sobre un concepto" (p.833). Éstas se agrupan para forma páginas o módulos.

La tabla 3.2 resume los modelos de contenido utilizado por las distintas metodologías de ensamblaje. Cada columna refiere a distintos niveles de granularidad (creciente de izquierda a derecha): los componentes de granularidad más baja, el contenido u OA con una granularidad media que agrupan a los componentes y la composición con una granularidad mayor que agrupan a los OA.

También en la Tabla 3.2, se puede observar que la mayoría de las propuestas de ensamblaje utilizan tres niveles de agregación de contenidos (de granularidad creciente): (i) archivos multimedia, asociado con recursos que pueden tener o no una intencionalidad pedagógica, (ii) las unidades de contenido, que vinculan o agrupan los recursos, contextualizan las actividades y se orientan a la consecución de un OA y (iii) lo que se podría denominar un itinerario de aprendizaje que el estudiante debe seguir para apropiarse de los contenidos o alcanzar las competencias. Dependiendo del autor y de la definición de OA que utilice, éste último, puede ser considerado como un OA o un conjunto de éstos.

Tabla 3.2: Modelos de contenido.

\begin{tabular}{|c|c|c|c|c|}
\hline Autor/es & año & Componentes & Contenido/OA & Composición \\
\hline ADL SCORM & & Asset & $\begin{array}{c}\text { SCO, Activity, } \\
\text { Content organization }\end{array}$ & Content aggregation \\
\hline Kellar et al. & 2004 & Atom & Component / Page & Lesson \\
\hline Roig Vila & 2005 & & & WebQuest \\
\hline Farrell et al. & 2004 & Content file & Content/Unit of content & Logical package \\
\hline Li \& Huang & 2006 & Asset & Learning object & Knowledge map \\
\hline Lopes Gançarski et al. & 2007 & & Atomic & Complex \\
\hline Rigaux \& Spyratos & 2007 & & atomic LOs & composite LOs \\
\hline Thyagharajan \& Nayak & 2007 & pagelet & & module \\
\hline Anh \& Dam & 2008 & Asset & Learning object & knowledge unit \\
\hline Santacruz Valencia et al. & 2008 & Unidades de información & Unidades de contenido & Unidades didácticas \\
\hline Sarasa et al. & 2008 & Content file & Content/Unit of content & Logical package \\
\hline
\end{tabular}


Tabla 3.2: Modelos de contenido (continuación).

\begin{tabular}{|c|c|c|c|c|}
\hline Autor/es & año & Componentes & Contenido/OA & Composición \\
\hline$\overline{\text { Schreurs et al. }}$ & 2008 & $A L O$ & Block/Unit & Chapter \\
\hline Bouzeghoub & 2009 & & Atomic & Complex \\
\hline Garrido et al. & 2009 & Content file & Content/Unit of content & Logical package \\
\hline Jovanovic et al. & 2009 & Content Fragment & Content Object & Learning Object \\
\hline Menéndez et al. & 2010 & Asset & $\begin{array}{c}\text { SCO, Activity, } \\
\text { Content organization }\end{array}$ & Content aggregation \\
\hline López et al. & 2011 & & OA Fundamentales & $\begin{array}{l}\text { OA Generativo- } \\
\text { Instruccionales }\end{array}$ \\
\hline Stănică \& Crișan & 2012 & Fundamental objects & Instructional components & Compound objects \\
\hline Verbert et al. & 2012 & Content Fragment & Content Object & Learning Object \\
\hline
\end{tabular}

\subsubsection{El modelo de estudiante}

Varias metodologías de ensamblaje proponen la creación de itinerarios de aprendizaje que se ajusten al perfil del estudiante. Para ello deben contar con un modelo de estudiante (learner model) el cual les permita registrar y mantener actualizada las interacciones de los estudiantes con el material (Ullrich \& Melis, 2009). "Un modelo estudiante contiene supuestos modelados explícitamente que representan las características del estudiante que son pertinentes para el sistema [y] permite a [éste] personalizar la interacción entre el estudiante y el contenido" (Thyagharajan \& Nayak, 2007, p. 832).

El modelo debe poder representar características del estudiante como: su nivel de conocimiento, las tareas realizadas y sus objetivos (Verbert et al., 2012). Otros autores (Anh \& Dam, 2006; Chellatamilan \& Suresh, 2012; Garrido et al., 2009; Huang et al., 2009; Jovanović et al., 2009; Kellar et al., 2004; Schreurs et al., 2008; Thyagharajan \& Nayak, 2007) agregan a la lista de características el estilo de aprendizaje del estudiante.

Los estudiantes hacen propia la información, de forma más eficiente, de acuerdo a su estilo de aprendizaje, necesidades e intereses. También, aprenden con diferente ritmo, distintos enfoques del tema, dependiendo del ambiente y de las rutas de aprendizaje propuestas (Thyagharajan \& Nayak, 2007). En éste sentido afirman Chellatamilan \& Suresh (2012), en la fase de modelado de usuario se identifica y clasifica al estudiante en el sistema con el objetivo de ajustar los contenidos al perfil del mismo. La forma en la que se enlazan los contenidos debe tener su base en el estilo de aprendizaje de los estudiantes. Para construir cursos adaptado al perfil de usuario se debe evaluar, en primer lugar, al estudiante con el fin de indagar sobre los requerimientos, habilidades, conocimientos y estilos de aprendizaje (Anh \& Dam, 2006; Anh et al., 2008). Basado en esta información es que es posible seleccionar el contenido que mejor se adapta al estudiante. Esta evaluación se puede hacer, inicialmente, a través de una test o diagnóstico y luego puede mantenerse a través de la interacción del estudiante con el SE. Por ejemplo, Jovanović et al. (2009) proponen que el estudiante se registre y en este proceso se le requiere la información que permite crear la primera versión del modelo de estudiante. Para esto utilizan un cuestionario cuyas preguntas son adaptadas del modelo de estilos de aprendizaje propuesto por Felder \& Silverman ${ }^{6}$.

Para Lalithsena et al. (2008), modelar al estudiante es un "aspecto crítico" de cualquier ambiente que persiga la personalización. Se debe contar, además de la información general, con objetivos y preferencias (como el idioma, tipo de archivo y el tipo de dispositivo). El conocimiento de cada estudiante se actualiza después de generar el itinerario de aprendizaje y que se haya completado exitosamente un test asociado con sus objetivos.

De forma similar, Thyagharajan \& Nayak (2007) crean el modelo de estudiante basado, inicialmente, en información provista por este, y posteriormente que la misma se actualice a partir de los diferentes test que se le presentan, al usuario, por cada tema o tópico. Esto permite un refinamiento del modelo de estudiante.

6 Este modelo reconoce cinco categorías de estilos de aprendizaje: (i) visual-verbal, (ii) percepción-intuitivo, (iii) secuencial-global, (iv) inductivo-deductivo y (v) activo-reflexivo (Felder \& Silverman, 1988). 
Para Bouzeghoub et al. (2009; 2004a) y Lopes Gançarski et al. (2007) el modelo de estudiante se define como una tupla compuesta por la identificación del estudiantes, sus preferencias y el dominio de la temática. Este último se define a través de los conceptos visitados y de qué tipo son éstos (una introducción, una definición, una descripción, o una aplicación del concepto), así como la complejidad (muy bajo, bajo, medio, alto, muy alto).

\subsubsection{Modelo de profesor}

Los SE deben contar con la impronta del docente, por tanto, es necesario reflejar o modelar de algún modo su participación en el proceso de ensamblaje. Sea creando un modelo de profesor o incluyendo dentro de las fases de la construcción del itinerario de aprendizaje su participación.

El modelo de profesor refleja las preferencias de éste (Verbert et al., 2012) y el modelo de enseñanza contiene el conocimiento pedagógico (las estrategias) sobre cómo ensamblar el material en una secuencia para cada estudiante (Ullrich \& Melis, 2009; Verbert et al., 2012).

La incorporación de un modelo de profesor en una metodología está sujeta a los destinatarios del SE. En este estudio sólo Verbert et al. (2012) incorporan a su metodología de ensamblaje este modelo. Según Verbert, Wiley, \& Duval (2009) "el modelo de profesor define atributos de éste que permiten la agregación personalizada de recursos de aprendizaje. El modelo incluye atributos para representar el nivel de experiencia de los docentes, sus intereses y actividades, las estrategias enseñanza que prefieren, sus antecedentes y estilos de presentación.” (p. 2).

En el modelo de enseñanza, los SE toman en cuenta las estrategias docentes o lo estilos de enseñanza como parte de las metodologías de ensamblaje. Para Chellatamilan \& Suresh (2012) es necesario contar con sistemas proactivos basados en las actividades que realiza el docente al preparar sus clases. Shahin et al. (2008), por su parte, optan por una intervención docente, en la cual sea éste quien defina el método educativo que mejor se adapta al estudiante.

\subsubsection{Rol educativo}

Otra de las representaciones que utilizan, algunos autores, tiene que ver con la función pedagógica o didáctica que tendrán los OA en el contexto del itinerario de aprendizaje. Dice Verbert et al. (2012) "algunos sistemas también distinguen un modelo de contenido a partir de un modelo de dominio que define los tipos de contenido, tales como definiciones, ejemplos, ilustraciones, etc." (p. 1258). A esta clasificación o taxonomía de los OA otros autores la denominan como rol educativo (instructional role), dice Ullrich \& Melis (2009) "cada recurso tiene un tipo que especifica su función educativa, por ejemplo, si se trata de una definición, un ejemplo, un ejercicio, etc." (p. 9320). "Estos tipos representan posibles funciones para el objeto de aprendizaje en futuros ensamblajes." (Farrell et al., 2004, p. 4).

Definir la función educativa que tendrán los OA favorece el ensamblaje. Si esta información está presente sería posible ubicar al OA dentro de distintos itinerarios en distintos momentos del proceso de aprendizaje. Así pues, un ejemplo podría colocarse antes de una definición (de lo particular a lo general) o después de la misma (de lo general a lo particular). Farrel et al. (2004) utiliza el rol educativo asociado a los OA para definir cómo se ordenarán estos al presentar un tema o tópico. Algunos autores dividen el rol educativo en tres categorías: (i) retórico (por ejemplo, introducción, motivación y conclusión), (ii) cognitivo ${ }^{7}$ (hechos, principios, conceptos, procesos, procedimientos) y (iii) específico del dominio de conocimiento. De forma simular Jovanović et al. (2009), quienes se basan en ALOCoM Content Type (ver Figura 3.4), identifican tres tipos de roles educativos: (i) cognitivo (por ejemplo, hecho, definición, procedimiento), (ii) retórico (por ejemplo, introducción, conclusión) y (iii) de apoyo (por ejemplo, descripción, ejemplo, ejercicio).

7 Basados en el artículo “Component display theory” de David Merrill (1983).

8 Disponible en: http://jelenajovanovic.net/ontologies/loco/alocom-content-type/spec/\#overview 


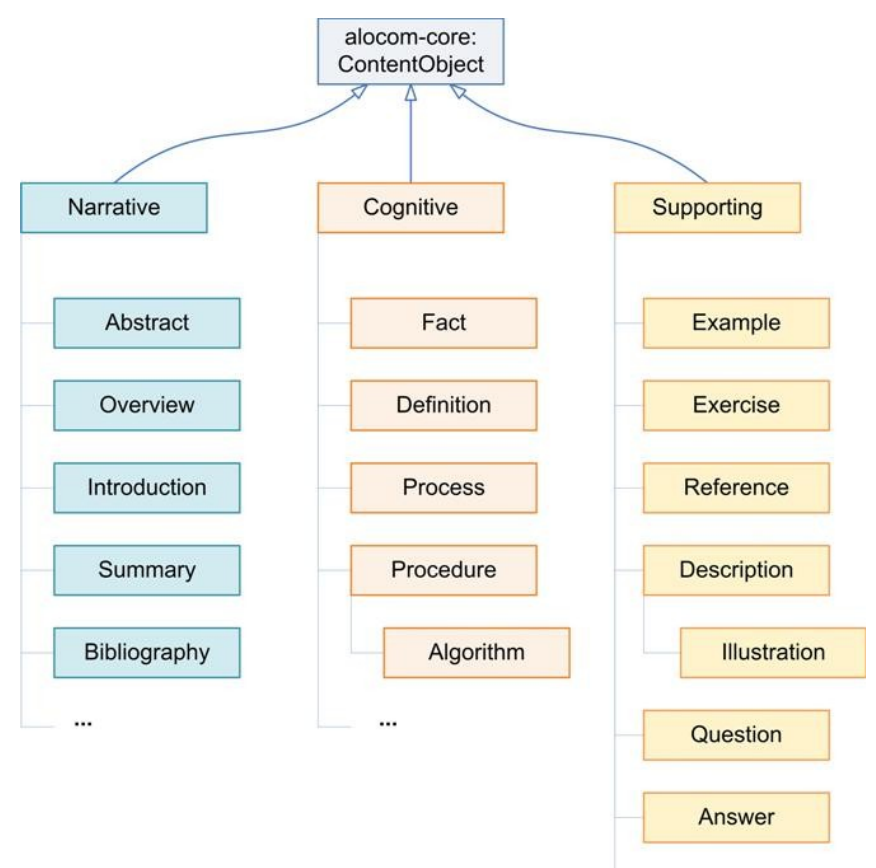

Figura 3.4: Diferentes tipos de contenido definidos en ALOCoM Content Type (Jovanović, Gasevic, Verbert, \& Duval, 2010, fig. 1).

Shahin et al. (2008), lo denominan rol pedagógico (pedagogical role), y afirman que al identificar a los OA como constituidos por una introducción, un ejemplo, una evaluación, etc. se incluye la dimensión pedagógica, lo que permitiría la adopción de teorías pedagógicas en el proceso de generación de secuencias de aprendizaje.

Esta información asociada a los OA, también ayuda al usuario (estudiante/docente) en la navegación (Li \& Huang, 2006) y búsqueda de materiales, así como propicia la reutilización.

\subsection{Ontologías y Metodologías de ensamblaje}

En el marco de las metodologías de ensamblaje de OA analizadas en este trabajo, se destaca la adopción de las ontologías como parte constitutiva del proceso de ensamblaje, ya que las mismas permiten representar: $(i)$ conceptos, $(i i)$ relaciones entre ellos y (iii) sus atributos.

Una ontología es "una vista abstracta y simplificada del mundo que queremos representar para un propósito específico" (Santacruz-Valencia et al., 2008, p. 106). Según Tom Gruber "en el contexto de las ciencias de la informática y de información, una ontología define un conjunto de primitivas de representación con la que modelar un dominio de conocimiento o discurso. Las primitivas de representación son típicamente las clases (o conjuntos), atributos (o propiedades), y las relaciones (o las relaciones entre los miembros de la clase). [...] Debido a su independencia de los modelos de datos del nivel inferior, las ontologías son utilizadas para la integración de bases de datos heterogéneas, lo que permite la interoperabilidad entre sistemas dispares.” (Gruber, 2009, p. 1963).

Una ontología no solo permite la descripción de conceptos y las relaciones entre ellos, sino que también organiza el conocimiento de forma jerárquica y estructurada, lo que favorece el razonamiento automático (López et al., 2008). Es decir, se puede utilizarse como un método de estructuración de los conocimientos de una manera procesable por una máquina (Thyagharajan \& Nayak, 2007).

El uso de ontologías permite: $(i)$ estructurar un dominio de conocimiento y ( $i$ i) hacer comparaciones entre términos sintácticamente distintos, aunque semánticamente iguales (Lalithsena et al., 2008; Santacruz-Valencia et al., 2005).

En el ámbito educativo "la definición de ontologías relacionadas con estrategias de enseñanzaaprendizaje es útil porque permite especificar dentro del objeto de aprendizaje información relevante para el procesamiento de dicho objeto de aprendizaje desde el punto de vista pedagógico. 
Esto favorece la personalización de la enseñanza basada en las preferencias, el estilo de aprendizaje del estudiante y el diseño particular del [OA]" (Santacruz-Valencia et al., 2005, p. 64).

Como se pudo observar en las secciones anteriores, las metodologías de ensamblaje utilizan distintos modelos para representar, entre otros, usuarios y contenidos, y son las ontologías (y los metadatos) las que permiten modelados y establecer relaciones entre ellos (Lalithsena et al., 2008; Manouselis, Drachsler, Verbert, \& Duval, 2012). Como afirma Doan et al. (2006) un sistema de $e-$ learning necesita administrar, al menos, dos ontologías: una de dominio ${ }^{9}$ y otra que describa los OA en sí mismos y las relaciones entre ellos. Por su parte, Colucci et al. (2005) afirma que "el descubrimiento y la composición de [...OA] de una forma automática requiere la asociación de metadatos sin ambigüedades y semánticamente ricos, que se definen de conformidad con las ontologías" (p.633).

Así por ejemplo, Lalithsena et al. (2008) utilizan: una ontología para representar el conocimiento (ontología de dominio), otra para almacenar y mantener actualizado el perfil del estudiante (user profile ontology) -incluye en este último, conocimientos previos, competencia y preferencias- y una tercera de mapeo (ontology mapping) que permite analizar los objetivos de los estudiantes con los anotados en la ontología de dominio. Karam et al. (2007) por su parte proponen utilizar una ontología de dominio que, en combinación con una consulta, le permita recuperar el contenido de mayor relevancia para el estudiante. Bouzeghoub et al. (2009), así como Lopes Gançarski et al. (2007) implementan el esquema SIMBAD, el cual utiliza una ontología de dominio para representar todos los conceptos del dominio de conocimientos. Para Jovanović et al., (2006), que basa su propuesta en ALOCoM Ontology (Jovanovic, Gasevic, Verbert, \& Duval, 2005), utiliza: (i) una ontología de dominio que le permite la descripción semántica del contenido de cada OA, (ii) una ontología estructural que describe la estructura de los OA, (iii) una ontologías de contexto que especifican el rol didáctico del contenido, (iv) una ontología de itinerario de aprendizaje (learning paths ontology) que define trayectorias de aprendizaje a través de los temas definidos en la ontología de dominio y $(v)$ una ontología de modelo de usuario (user model ontology) para describir los perfiles tanto de autores como de estudiantes - para esto últimos se incluye en la ontología, entre otros, los estilos de aprendizaje-. Sánchez-Alonso et al. (2007), en su enfoque basado en los contratos, expresan estos últimos en términos de una ontología, lo cual les permite recuperar conjunto de OA ajustados al los objetivos del usuario. Por su parte, Santacruz-Valencia et al. (2005, 2008) utilizan una ontología, denominada OntoGlue, para representar el conocimiento asociado a cada OA (requisitos y competencias), lo cual, les permite realizar búsquedas semánticas, comparar OA heterogéneos y chequear si las competencias de un OA cubren los requisitos de otro y facilita el cálculo de los metadatos de OA resultante del ensamblaje. En el mismo sentido, López et al. (2008) utilizan una ontología de dominio para representar el conocimiento y hacer consultas semánticas. Thyagharajan \& Nayak (2007) utilizan ontologías para representar los conceptos y las relaciones entre ellos (ontología de dominio), así como aspectos pedagógicos, lo que permite al sistema recuperar el conjunto de OA que deben ser incluidos en el itinerario de aprendizaje para estudiar un tema específico.

Cabe mencionar que las ontologías deben estar representadas en un lenguaje de ontologías. Varios autores (Colucci et al., 2005; Jovanović et al., 2006; Karam et al., 2007; Lalithsena et al., 2008; López et al., 2008; Santacruz-Valencia et al., 2008) utilizan Ontology Web Language (OWL ${ }^{10}$ ) para escribir las ontologías y representar las relaciones entre las entidades.

Para finalizar, se debe tener en cuenta que aquellas metodologías que utilizan ontologías dependen fuertemente de éstas, las cuales son costosas de construir (Farrell et al., 2004) y deben generarse para cada dominio.

9 Una ontología de dominio es una formalización del conocimiento de un área o dominio en particular. Representa cosas que existen dentro de un dominio particular de la realidad.

10 Ontology Web Language es un lenguaje de marcado semántico, desarrollado por la W3C, para publicar y compartir ontologías en la World Wide Web (http://www.w3.org/TR/owl-ref/). 


\subsection{Metadatos y ensamblaje}

Los metadatos son esenciales tanto para los OA, como para el ensamblaje de éstos. La "estructura de metadatos supone contar con una detallada disposición textual, que describe atributos, propiedades y características distribuidos en diferentes campos que identifican claramente al objeto, con el fin de que pueda encontrarse [y] ensamblarse" (García Aretio, 2013, p. 4). La mayoría de los SE necesitan la información contenida en los metadatos para llevar a adelante el proceso de ensamblaje.

Como afirma García Aretio (2013) “si los objetos cumplen con determinados estándares sus posibilidades aumentan al permitirse combinarlos, ensamblarlos, agruparlos, catalogarlos, secuenciarlos, permutarlos, etc." (p.4). Por lo que el uso de metadatos que estén estandarizados es central para el ensamblaje.

Sin embargo, la información necesaria para llevar adelante el proceso de ensamblaje va más allá de un conjunto de datos técnicos sobre el OA, las etiquetas deben tener semántica. La descripción de los OA con metadatos semánticos apropiados es necesaria para permitir una verdadera reutilización y ensamblaje de OA (Gançarski et al., 2007; Santacruz-Valencia et al., 2008). "Describir los OA simplemente mediante el uso de un conjunto de metadatos es insuficiente; la semántica tienen que ser añadida a esta descripción con el fin de enriquecer búsqueda, reutilizar y componer." (Bouzeghoub et al., 2009, p. 763).

Si bien varios autores adoptan LOM (ver tabla 3.3) en la implementación de las metodología de ensamblaje, hacen notar el problema de la falta de semántica. Este estándar, por defecto, no proporciona suficiente descripción semántica a los OA (Gançarski et al., 2007; Jovanović et al., 2009; Sánchez-Alonso et al., 2007; Santacruz-Valencia et al., 2008). Para Sánchez-Alonso et al. (2007) "los estándares [como LOM y SCORM] no cuenta con el suficiente nivel de especificación semántica para trabajar de forma consistente la semántica en tiempo de ejecución" (p. 179). Como afirman Colucci et al. (2005) "para ser ensamblado de forma automatizada, los metadatos tienen que ser explotados, asociados a descripciones no ambiguas y semánticamente ricos" (p. 633). Por su parte, Santacruz-Valencia et al. (2008) aseveran que los metadatos semánticos son el mecanismo básico para el ensamblaje, ya que no solo apoyan el proceso de composición, sino también la búsqueda y comparación de OA heterogéneos.

Para Colucci (2005) y Santacruz-Valencia et al. (2005) el estándar LOM permite describir libremente los recursos educativos. Pahl \& Barrett (2004), Lopes Gançarski et al. (2007) y Jovanović et al. (2009) lo utilizan para definir las características educativas de los OA. Por su parte, López et al. (2008) y Wetzlinger et al. (2008) lo destacan como una herramienta de catalogación sobre la que basan sus procesos de localización de los OA. Sánchez-Alonso et al. (2007) basan su propuesta de Learning object Design by Contract en el estándar LOM, así como Garrido et al. (2009) propone un planificador automático compatible con LOM y Sarasa et al. (2008) una herramienta, denominada LOMEditor, para la composición y clasificación de OA.

Algunos autores (Anh \& Dam, 2006; Farrell et al., 2004; Garrido et al., 2009; Santacruz-Valencia et al., 2008) proponen la extensión del conjunto de metadatos de forma de mejorar la descripción y aportar semántica a los OA. "Hemos ampliado los metadatos de los objetos de aprendizaje para incluir un tipo especial de recursos de aprendizaje llamado 'rol educativo' [...el cual] representa los potenciales roles del objeto de aprendizaje en futuras ensambles [y permite] ordenar los recursos en la secuencia de aprendizaje." (Farrell et al., 2004, p. 5). "En particular se utiliza LOM como esquema de meta-datos para la descripción de los [OA], aunque incluyendo una extensión, con lo que se consigue una descripción más adecuada [... en particular] LOM no era suficiente para describir [OA] de la forma adecuada en vistas al proceso de ensamblaje, se ha realizado una extensión [...] en la categoría 5. Educational añadiendo nuevos elementos a la misma." (SantacruzValencia et al., 2005, p. 78).

Además de la falta de semántica, otros problemas que plantean los autores en relación a los metadatos y su impacto sobre el proceso de ensamblaje son: 
- La carga de metadatos es una tarea que requiere un importante esfuerzo. "El etiquetado con meta-datos es una tarea ardua y que a menudo no consigue su objetivo, esto a llevado a repositorios donde el contenido educativo no reúne los requerimientos necesarios para servir de base para automatizar actividades de aprendizaje" (Garrido et al., 2009, p. 178).

- Los registros de metadatos deben ser de alta calidad. "La selección y la composición de objetos de aprendizaje son dos actividades esenciales en los enfoques automatizados para el aprendizaje basado en la web. Tales actividades requieren registros de metadatos de alta calidad las especificaciones y estándares actuales no cumplen aún." (Sánchez-Alonso et al., 2007, p. 179).

- Los registros de metadatos deben estar completos. "La selección de objetos de aprendizaje requiere de registros de metadatos suficientemente completos para permitir a un LMS decidir la inclusión de un objeto dado en una composición..." (Sánchez-Alonso et al., 2007, p. 180).

- El estándar LOM es complejo y extenso. "LOM maneja un conjunto de metadatos a menudo complejo y muy largo para ser descrito (nueve categorías y varias decenas de atributos) y es difícil motivar a los autores para que describan sus producciones con un modelo de este tipo." (Bouzeghoub \& Selmi, 2009, p. 176). "El llenado en forma manual de largas listas de metadatos para describir un recurso (para el caso del estándar IEEE-LOM son más de 60) ocasiona errores de captura, selección de valores incorrectos, malas interpretaciones o desconocimiento pleno del significado del metadato, entre otros." (Menéndez et al., 2010, p. 144).

- Los estándares no capturan la suficiente información asociada a lo educativo. Para Garrido et al. (2009) los estándares de metadatos deberían permitir asociar a un OA información asociada a lo educativo: ( i) información acerca de los estilos de aprendizaje, (ii) requerimientos para el uso de los OA, (iii) tiempo necesarios para el abordaje del material y (iv) la relación entre un OA y los otros. Para Jovanović et al. (2009) "estándares y especificaciones no capturan la información suficiente requerida para niveles avanzados de personalización del proceso de aprendizaje, como [...] las preferencias de los estudiantes, los estilos de aprendizaje, y/o los objetivos." (p. 6).

Tabla 3.3: Lista de metodología que utilizan el estándar LOM.

\begin{tabular}{l} 
Autor \\
\hline Pahl et al. (2004) \\
Colucci et al. (2005) \\
Santacruz Valencia, Delgado Kloos \& Aedo Cuevas (2005) \\
Farrell et al. (2006) \\
Lopes Gançarski, et al. (2007) \\
Sánchez Alonso, et al. (2007) \\
Anh et al. (2008) \\
López, Miguel \& Montaño (2008) \\
Sarasa et al. (2008) \\
Shahin et al. (2008) \\
Wetzlinger, Auinger \& Stary (2008) \\
Bouzeghoub et al. (2009) \\
Garrido, Onaindia \& Sapena (2009) \\
Jovanovic et al. (2009) \\
Menéndez et al. (2010)
\end{tabular}




\subsection{Pre-requisitos y conocimiento adquirido}

Las distintas propuestas de ensamblaje de OA utilizan diferentes estrategias para relacionar un objeto con el siguiente. Sin embargo, existe una coincidencia en varias de las metodologías en este aspecto: trabajar con pre-requisitos y conocimiento adquirido.

Los primeros definen aquel conocimiento con el que debería contar un estudiante para acceder a un $\mathrm{OA}$, y el segundo, es el conocimiento y/o competencia que se espera adquiera éste al haber completado el OA.

En aquellas metodologías en las que toma en cuenta el perfil del estudiante, los pre-requisitos no solo permiten relacionar los OA, sino que además seleccionar aquellos que se ajustan mejor a los conocimientos previos del estudiante. Como afirman Karam et al. (2007) "la composición tiene en cuenta el conocimiento del usuario sobre el tema a fin de proponer la mejor secuencia para los OA recuperados." (p. 716).

Colucci et al. (2005), por ejemplo, define un OA como una dupla donde a cada objeto se le asocia pre-requisitos o conocimientos previos (background) y conocimiento adquirido.

donde

$$
\text { Objeto de aprendizaje: } \lambda=<\lambda D, \lambda B K>
$$

$D$ representa conocimiento adquirido al usar el OA $\lambda$

$B K$ representa conocimientos previos necesarios para usar el OA $\lambda$

Sánchez-Alonso et al. (2007) en su diseño basado en contratos definen para cada OA un conjunto de pre y post-condiciones. Las primeras son las circunstancias bajos las cuales un OA puede ser utilizado y las post-condiciones los resultados de aprendizaje esperados.

Un contrato de un OA se expresa como sigue (Sánchez-Alonso et al., 2007, p. 180):

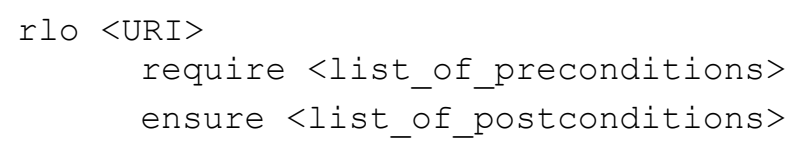

Santacruz-Valencia et al. (2005) utiliza el concepto de "conocimiento asociado" para referirse tanto a los pre-requisitos como al conocimiento adquirido. Según los autores, el conocimiento asociado "representa la característica esencial que hace posible el ensamblaje [...]. Se refiere a los requisitos necesarios para la comprensión del [OA] y las competencias adquiridas después de su comprensión." (p. 74).

Garrido et al. (2009) utiliza el concepto de tarea, la cual encapsula todos los OA para aprender un concepto. Al utilizar una tarea el estudiante aprende los conceptos (conocimiento adquirido) y estos mismos conceptos actúan como pre-requisitos. Entonces los conceptos funcionan, al mismo tiempo, como pre-requisitos o conocimiento adquirido.

Karam et al. (2007) proponen incluir en la secuencia aquellos OA que se definen como pre-requisito para el objeto que el estudiante desea abordar. "En nuestra solución, los OA se organizan de manera que el usuario puede adquirir tal conocimiento requerido [(pre-requisito)] con la lectura de otros OA. [Los mismos] son detectados por el sistema y se añade a la secuencia resultante." (p.720). Para Huang et al. (2009), mostrar aquellos OA que forman parte de los pre-requisitos pone en evidencia, ante el estudiante, la relación entre los distintos tópicos. Thyagharajan \& Nayak (2007) proponen que aquellos estudiantes novatos comiencen directamente por la introducción al tema o tópico, y aquellos con más experiencia completen un examen que les permita acreditar los pre-requisitos del material al que desean acceder.

Para Bouzeghoub et al. (2004a; 2009) y Lopes Gançarski et al. (2007) los pre-requisitos van más allá del contenido. Para estos autores son triplas de la forma (concepto, rol, nivel). Donde el concepto refiere al OA, el rol representa la función educativa del objeto (introducción, definición, descripción, aplicación, evaluación, demostración, sumarias) y el nivel refiere al "nivel de conocimientos" sobre ese tópico con el que cuenta el estudiante. 
Varios autores (Anh et al., 2008; Jovanović et al., 2009; Thyagharajan \& Nayak, 2007) afirman que la utilización de pre-requisitos permite una personalización de la propuesta de aprendizaje, debido a que se le ofrece al estudiante una guía que indica qué contenidos debe seguir para alcanzar sus objetivos de aprendizaje. Esta guía - por ende los pre-requisitos- debería ser definida por el docente o el autor del curso o actividad (Bouzeghoub \& Selmi, 2009; Lalithsena et al., 2008; Rigaux \& Spyratos, 2007).

La Tabla 3.4 resume el conjunto de autores que utilizan pre-requisitos y/o conocimiento adquirido en sus metodologías de ensamblaje:

Tabla 3.4: Pre-requisito y conocimiento adquirido

\begin{tabular}{lcc}
\hline \multicolumn{1}{c}{ Autor/es } & pre-requisito & $\begin{array}{c}\text { conocimiento } \\
\text { adquirido }\end{array}$ \\
\hline Colucci et al. (2005) & $\checkmark$ & $\checkmark$ \\
Santacruz Valencia et al. (2005) & $\checkmark$ & $\checkmark$ \\
Karam et al. (2007) & $\checkmark$ & \\
Lopes Gançarski et al. (2007) & $\checkmark$ & \\
Rigaux \& Spyratos (2007) & $\checkmark$ & \\
Sánchez Alonso et al. (2007) & $\checkmark$ & \\
Thyagharajan \& Nayak (2007) & $\checkmark$ & \\
Anh \& Dam (2008) & $\checkmark$ & \\
Lalithsena et al. (2008) & $\checkmark$ & \\
Shahin et al. (2008) & $\checkmark$ & \\
Bouzeghoub et al. (2009) & $\checkmark$ & \\
Garrido et al. (2009) & $\checkmark$ & \\
Huang et al. (2009) & $\checkmark$ & \\
Jovanovic et al. (2009) & $\checkmark$ & \\
Ullrich \& Melis (2009) & $\checkmark$ &
\end{tabular}

\subsection{Descripción de las metodologías de ensamblaje}

En esta sección se realiza una breve descripción de las metodologías de ensamblaje de OA que se han abordado en este trabajo de tesis:

En Kellar et al. (2004) describen una arquitectura para la web que permite, dinámicamente, seleccionar y ensamblar OA basados en el perfil del estudiante. "La arquitectura se basa en una base de datos de $[\mathrm{OA}]$ reutilizables meta-etiquetados que se pueden combinar dinámicamente para crear lecciones personalizadas" (p.1). El itinerario de aprendizaje es diseñado por diseñadores de contenido y el estudiante, una vez completada la auto-evalaución, recibe una secuencia en base a su perfil. La misma es generada dinámicamente a partir del contenido alojado en la base de datos. La metodología se evaluó a través de dos casos de estudio: se desarrolló un prototipo que implementa la arquitectura para una escuela media (con material remedial para matemática) y otro para una cátedra de educación informática (health informatics education) para la salud. Utiliza fuente de datos local con materiales desarrollados ad-hoc.

Pahl \& Barrett (2004) proponen una arquitectura, basada en servicios, en la que introducen una serie de patrones que favorecen la localización y ensamblaje de OA. El ensamblaje se basa en "dos dimensiones" de metadatos (basados en LOM): los descriptores de contenido que "abordan aspectos educativos relacionados con la utilización de objetos." (Pahl \& Barrett, 2004, p. 446), y los descriptores de infraestructura que "abordan aspectos técnicos relacionados con el ensamblaje de los objetos de aprendizaje." (Pahl \& Barrett, 2004, p. 446). Los patrones permiten relacionar OA independientemente de su estructura (son servicios), representar entidades (como los estudiantes, objetos y docentes) y mantener el estado de avance del estudiante. No se encontró evidencia del desarrollo una aplicación. Proponen un marco de trabajo (o framework) arquitectónico genérico para el diseño de software de apoyo a cursos (Courseware system). 
Roig Vila (2005) plantea un ensamblaje manual de OA para el diseño de una WebQuest" . “Cómo pueden aplicarse los criterios de diseño pedagógico a los objetos de aprendizaje?. Pues bien, a través de este texto, pretendemos describir una propuesta de diseño de materiales docentes electrónicos llamados WebQuests que aportan la visión de ensamblaje de los objetos de aprendizaje." (Roig Vila, 2005, p. 1). La autora plantea la utilización de la aplicación web EDUTIC $^{12}$ para ensamblar los OA que compondrán la WebQuest. Si bien manifiesta que la propuesta está siendo experimentada en dos proyectos, en las publicaciones analizadas, no se encontraron datos sobre la implementación.

Colucci et al. (2005) proponen la composición personalizada y dinámica de cursos basado en OA, los cuales son secuenciados a partir de los conocimientos previos requeridos para el OA. Los OA son obtenidos desde repositorios de OA y para la implementación hace uso metadatos. La propuesta esta basada en "la idea de que cursos de e-learning personalizados puede ser construido por la composición dinámica de los objetos de aprendizaje distribuidos, disponibles como servicios web." (Colucci et al., 2005, p. 633). Cada OA cuenta con información sobre el conocimiento requerido para utilizarlo (o background) y el que el estudiante adquiere una vez usado el OA. El estudiante hace una consulta y el SE busca en el repositorio un conjunto de OA que satisfacen "tanto como sea posible" la consulta. De este conjunto de OA se arma la secuencia, donde el conocimiento que permite alcanzar un objeto sea suficiente para abordar el próximo. La secuencia comienza con un objeto para el que el conocimiento del estudiante sea al menos el conocimiento requerido por el objeto. Para la evaluación de esta propuesta, se presenta un ejemplo teórico y se plantea la confección de un prototipo en los trabajos futuros.

Santacruz-Valencia et al. (2005) proponen un mecanismo de ensamblaje de OA basado en metadatos y ontologías. La metodología plantea la utilización de requerimientos y las competencias (conocimiento asociado) de manera que, dos ELOs (Electronic Learning Objects) pueden ensamblarse si las competencias de uno cubren los requerimientos del otro -puede que se necesite más de un OA para completar los requerimientos de otro-. El conocimiento asociado es representado a través de la extensión del estándar LOM y el ensamblaje se hace utilizando ontologías. "En particular, se aborda la correspondencia semántica, representada mediante ontologías. Esto permite la construcción del mecanismo para el ensamblaje de objetos de aprendizaje heterogéneos, denominado OntoGlue. El elemento fundamental del mecanismo propuesto es el concepto de conocimiento asociado." (Santacruz-Valencia et al., 2005, p. 80). Esta metodología utiliza unidades de información (UI), unidades de contenido (UC) y unidades didácticas (UD). Así "es posible obtener UCs a partir del ensamblaje de UIs o del ensamblaje entre UIs y UCs. [...] El ensamblaje de UCs da como resultado una UD. El ensamblaje entre UCs y UDs genera una nueva UD. Finalmente, el ensamblaje UDs da como resultado una nueva UD" (Santacruz-Valencia et al., 2005, p. 81). La metodología está plasmada en una aplicación web denominada ELO-Tools ${ }^{13}$, la cual permite: almacenar los metadatos de los OA y las ontologías en repositorios locales y ensamblar ELOs para generar nuevos a partir de otros ya existentes. Para la evaluación de la metodología se han hecho pruebas de laboratorio con ELO-Tools. La aplicación es totalmente funcional y, si bien, cuenta con un repositorio local donde almacena metadatos es posible obtener esta información desde otros repositorios o la web.

Farrell et al. (2006; 2004), proponen el ensamblaje dinámico de OA basado en la coincidencia de estos y sus metadatos con las necesidades de aprendizaje, el contexto, las preferencias y las restricciones impuestas por los estudiantes. La propuesta "se centra en el problema de cómo ensamblar automáticamente los objetos de aprendizaje dentro de 'cursos personalizados' que sean simples, cortos, enfocados y basados en Web. Este proceso, al que llamamos 'Ensamblaje Dinámico', incluye el proceso de conexión de resultados relevantes en un itinerario de aprendizaje, la secuenciación de los objetos de aprendizaje seleccionados en el itinerario, y la vinculación de los objetos de aprendizaje seleccionados en una estructura organizada." (Farrell et al., 2004, p. 162).

11 Una WebQuest es una actividad orientada a la indagación en la que algunos o la totalidad de la información con la que los alumnos interactúan proviene de recursos en Internet (Dodge, 1997).

12 Disponible en: http://www.edutic.ua.es/edutic-webquest/

13 Disponible en: http://solaris.fdi.ucm.es:8080/elo-toolv1/ 
Los autores desarrollaron una aplicación denominada Custom Course System, la cual permite al estudiante buscar un tema, a partir del cual se genera el itinerario de aprendizaje (o instructional role sequence) y luego utilizar el mismo (course player). Dicho itinerario, puede ser editado por el estudiante. El ordenamiento de los OA se hace de manera de colocar aquellos más básicos primero y utilizando lo que denominan "rol instruccional" (introducción, conceptos, procedimientos y conclusión) -implementado a través de la extensión de LOM-. Este rol permite asociar a cada OA la posición en el que debiera estar dentro de una secuencia de aprendizaje. La aplicación trabaja con repositorios externos. En 2004, la aplicación fue probada en un estudio piloto en una capacitación para 114 empleados de IBM.

Li \& Huang (2006) proponen un itinerario de aprendizaje generado por mapa de conocimiento (knowledge map) que relaciona los OA, el cual es creado por el docente. El enfoque pretende crear el itinerario a partir de OA "para hacer frente a las necesidades individuales de aprendizaje de los estudiantes" (Li \& Huang, 2006, p. 569). La metodología toma en cuenta las características de los OA y perfil del estudiantes para seleccionar, secuenciar y enlazar dinámicamente OA de manera de generar actividades coherentes y centradas en el estudiante (Li \& Huang, 2006). La composición del itinerario de aprendizaje se basa en la utilización de metadatos: de contenido, de estructura y de contexto. Los primeros describen al OA, los segundos establecen la relación con otros OA y los de contexto definen cuándo se debe presentar el OA al estudiante. La relación entre los OA se realiza a través de mapa de conocimiento. El estudiante propone una búsqueda y el sistema revisa el mapa para determinar, basado en el perfil del estudiante, dónde comenzar y qué nodos visitar. Para la evaluación de la metodología los autores desarrollaron una herramienta de autor (de escritorio) e integraron a $W e b C L^{14}$ un módulo para la creación itinerarios de aprendizaje personalizados. La prueba del módulo de ensamblaje se plantea como trabajo futuro.

Lopes Gançarski et al. (2007), proponen una búsqueda interactiva que permite al usuario componer OA. El usuario navega sobre la estructura de los OA y elige las partes que son de su interés apoyado por el sistema en la toma de decisiones-. Se trata de "un enfoque interactivo para buscar y componer OA definidos por el modelo SIMBAD” (Lopes Gançarski et al., 2007, p. 12). La metodología utiliza dos tipos de OA, atómico y compuestos, en ambos casos, etiquetados con metadatos LOM. Los docentes/autores podrían agregar OA atómicos o componer en base a estos últimos. Así generan un grafo de composición (composition graphs) que representa el itinerario de aprendizaje. Los estudiantes, por su parte, podrían seleccionar un OA, realizar una búsqueda o definir el tema, el tipo de material (o rol educativo del mismo) y el nivel de éste para que el sistema proponga el material de estudio. La búsqueda iterativa se hace escribiendo la consulta en un lenguaje de consulta iterativa denominado ILO-QL. Se plantea como trabajo futuro la implementación de un prototipo.

Rigaux \& Spyratos (2007), proponen una arquitectura y las funciones para implementar una Red de Auto-aprendizaje ( $\mathrm{SeLeNe}$, por sus siglas en inglés). SeLeNe es visto como un repositorio, donde "una comunidad de autores coopera a través de un [...] mediador en la creación de [OA] que serán usados por una comunidad de estudiantes." (Rigaux \& Spyratos, 2007, p. 1). La metodología asume los OA como bloques de construcción (atomic LO) de otros objetos compuestos por ellos (composite LO). El mediador es un software que actúa como servidor: registra, localiza y recomienda un itinerario de aprendizaje, en base a la consulta del estudiante. Para este último tiene en cuenta sus conocimientos previos. Los docentes son los que publican los OA, para hacerlo deberán indicar si el objeto es pre-requisito para acceder a otro objeto (creando así el el grafo de dependencia o dependency graph) y si es parte de un objeto compuesto. Una vez que el mediador identifica un OA, basado en el perfil del estudiante, utiliza el grafo de dependencia para proponer el itinerario de aprendizaje. Se implementó un prototipo y se hicieron pruebas de laboratorio para validar la metodología.

Sánchez Alonso et al. (2007), proponen la utilización del paradigma Diseño por contrato ${ }^{15}$ como

$14 \mathrm{Si}$ bien en el trabajo se presenta la URL http://www.webcl.net.cn, la misma no esta disponible a la fecha.

15 Es una notación basada en LOM y enriquecida con semántica, permite especificar un conjunto de pre- y postcondiciones para cada OA, y las relaciones entre ellos (Sánchez-Alonso et al. 2007). 
una metodología para especificar y manejar la selección OA y la composición de una secuencia con ellos. "Los contratos permiten esencialmente la especificación de un conjunto de condiciones previas (circunstancias bajo las cuales un objeto se puede utilizar) y post-condiciones (resultados de aprendizaje esperados) para cada objeto de aprendizaje, las cuales se puede utilizar para especificar claramente las relaciones entre los objetos de aprendizaje" (Sánchez-Alonso et al., 2007, p. 180). La metodología se basa en ontologías para definir los contratos y metadatos que describen a los OA. El primer paso de la metodología es crear el arquetipo (archetype), en el cual el usuario especifica las necesidades y restricciones -incluyendo el contexto de aprendizaje y el tecnológico-. Basado en éste el sistema busca los OA compatibles sobre un repositorio. La información del arquetipo también permite definir una secuencia para el abordaje de los OA. Los autores proponen una arquitectura basada en web service ${ }^{16}$, que provee: localización de OA en función de sus contratos, su descarga y la recuperación de sus metadatos. También plantean utilizar servicios para acceder a los repositorios. Si bien los servicios son descritos, no se indica su localización o una evaluación de la metodología.

Thyagharajan \& Nayak (2007) abordan la selección y composición automática de cursos basados en OA y en los objetivos de aprendizaje de los estudiantes. Éstos son guiados a través de una secuencia en función de la consecución de sus objetivos. La metodología apunta principalmente a la personalización del contenido, "al seleccionar y combinar los asset apropiados en un objeto de aprendizaje pueden ser tenidas en cuenta las necesidades y las preferencias del estudiante" (Thyagharajan \& Nayak, 2007, p. 828). La metodología cuenta con un modelo de estudiante que se actualiza en función de las actividades que éste realiza (esta información se obtiene, inicialmente, con el formulario de registro). Los estudiantes se dividen en principiantes y experimentados. Utilizan una "ontología de dominio que modela los conceptos y las relaciones básicas en el dominio, y una ontología pedagógica que se utiliza para la generación de procesos de aprendizaje flexibles y personalizados" (Thyagharajan \& Nayak, 2007, p. 830). El contenido esta organizado en fragmentos denominados pagelets, los cuales son ensamblados. Para acceder a un pagelets el estudiante debe cumplir los pre-requisitos del mismo. Si se trata de un principiante debe iniciar por la introducción, sino puede elegir el módulo de su interés -al cual accede si pasa el test de prerequisitos- . La metodología se implementa a través de una aplicación web basada en web service. La evaluación se hace sobre un dominio específico y el contenido es dividido en capítulos y subcapítulos, aunque no se ofrecen datos sobre actividades realizadas con estudiantes.

Karam et al. (2007) crean una secuencia de OA que cubra tanto como sea posible la consulta del estudiante, basado en la información de los OA y conocimiento de usuario. La secuencia está basada en pre-requisitos para el abordaje de los OA y los conocimientos adquiridos al usarlos. Los autores proponen un algoritmo para aprendizaje personalizado denominado "LectureComposer que toma como entrada una consulta del usuario y un repositorio de OA [...]. El algoritmo [...] recupera OA que cubran tanto como sea posible la consulta del usuario [... y] compone una secuencia de OA acuerdo a los requisitos previos. La composición tiene en cuenta el conocimiento del usuario sobre el tema a fin de proponer la mejor secuencia entre los OA recuperados" (Karam et al., 2007, p. 716). En la metodología, a partir del conjunto de OA recuperados desde el repositorio, luego de la búsqueda, se establece el conocimiento ofrecido (knowledge offered) y los pre-requisitos (prerequisites needed) de este grupo de objetos; en ambos casos, se define como la conjunción del conocimiento/pre-requisitos de cada OA. El conocimiento del estudiante (background knowledge) debe cubrir los pre-requisitos de, al menos, uno de los OA del conjunto recuperado. El itinerario de aprendizaje (o composition flow) se crea en función de que cada OA cubre con su conocimiento los pre-requisitos del OA siguiente. Los autores proponen un ejemplo teórico donde aplican la metodología (basado en las notas de clase sobre el tema redes de computadoras que están alojadas en tele-TASK ${ }^{17}$ ). Afirman que se encuentran desarrollando una ontología y un prototipo que se integrará a tele-TASK con el objetivo de llevar adelante un estudio de caso para la temática "Internetworking".

16 Un servicio web es un sistema de software diseñado para mantener interacciones interoperables de máquina-amáquina sobre una red (Haas \& Brown, 2004).

17 Disponible en: http://www.tele-task.de/ 
Anh et al. $(2006 ; 2008)$ proponen la generación de itinerario de aprendizaje basados en OA y adaptados al perfil del estudiante. Dicho itinerario se construye a partir de calcular el camino más corto sobre un mapa de conocimiento (knowledge map) -basado en el perfil del estudiante-. "Para cada estudiante que participa en el curso, nuestro sistema genera automáticamente el mejor itinerario de aprendizaje [...] basado en su perfil, así como en el mapa de conocimiento que había sido diseñado por el docente" (Anh et al., 2008). La metodología tiene en cuenta para el ensamblaje los OA necesarios para abordar un objeto (pre-requisitos) y la relación del OA con otros en la secuencia. Así como también, su nivel de dificultad, el tiempo necesario para recorrerlo, su estilo de interacción, el nivel de experticia necesario para su abordaje y las habilidades que permite aprender. Para generar el itinerario de aprendizaje, se utilizan cuatro pasos: $(i)$ se evalúa el estudiante para generar su perfil e identificar sus demandas u objetivos, (ii) el docente o diseñador instruccional genera el mapa de conocimiento basado en el perfil del estudiante, (iii) se generan los posibles itinerarios de aprendizaje (candidate learning path) en función de las demandas del estudiante y (iv) se genera el itinerario de aprendizaje que mejor se adapte a las demandas del estudiantes basado en cálculos de probabilidad. Para la implementación, los autores, desarrollaron lo que denominan Sistema Generado de Cursos Adapatativo (ACG por sus siglas en inglés). El mismo está desarrollado como una aplicación web y cuenta con tres módulos: ( $i$ ) un módulo para que administrar el perfil de los estudiantes, $(\mathrm{ii})$ otra para generar los itinerarios de aprendizaje adaptados al perfil del estudiante y (iii) el tercero permite visualizar el itinerario. Además, proveen para el docente una herramienta de autor que permite generar los mapas de conocimiento. La aplicación sigue el estándar SCORM. En 2006 (Anh \& Dam, 2006), crearon dos cursos (Programación en $\mathrm{C}++\mathrm{y}$ estructuras de datos y algoritmos) que fueron tomados por, aproximadamente, setenta estudiantes a quienes le hicieron una encuesta para evaluar estructura, interfaz, la capacidad de adaptación y satisfacción de las demandas al utilizar ACG. El sistema fue evaluado positivamente en todas sus características. En la publicación de 2008, no se encontró evidencia de otras actividades con estudiantes.

Shahin et al. (2008) parten de un plan de contenidos, creado por el docente, para armar el curso con los OA apropiados. El plan define pre-requisitos para armar la secuencia. Esta metodología esta enfocada en resolver el problema del ensamblaje manual, para ello presenta la "generación dinámica de cursos adaptados, cuyo objetivo es ensamblar dinámicamente cursos completos desde [OA] ya existentes. El objetivo es generar dinámicamente un curso adaptado a las necesidades de un determinado grupo de estudiantes, teniendo en cuenta sus objetivos de aprendizaje, los conocimientos previos y preferencias" (Shahin et al., 2008, p. 1). En esta metodología, el conocimiento se representa a través de un grafo dirigido, donde los nodos son conceptos y los arcos relaciones entre ellos. Las relaciones que definen el grafo son dos: has-part, es un sub-concepto de... y has-prerequisite, ser pre-requisitos de.... El grafo es administrado por expertos (los docentes, por ejemplo). Cada concepto está asociado con un conjunto de OA. Los objetos están alojados en repositorios y deben tener asociado su rol pedagógico (una introducción, un ejemplo, una evaluación, etc.). Esta información representa a través de metadatos compatibles con LOM. El proceso de ensamblaje involucra cuatro pasos: $(i)$ se genera automáticamente un plan de contenido basado en los objetivos de aprendizaje y los conocimientos del estudiante -que puede ser ajustado por el docente-, (ii) con este plan inicial, el docente debe decidir cómo se presenta el material al estudiante dividiéndolo en tarea y sub-tareas -utilizando un método de diseño instruccional-, (iii) luego se seleccionan automáticamente los OA que se utilizarán en el plan de contenidos para presentar los conceptos, finalmente, (iv) se determina la estructura final del itinerario de aprendizaje, esto se hace en base al orden establecido por el plan de contenidos y el de OA que se asociaron a él. El curso generado es empaquetado como SCORM. Si bien se menciona la utilización de una herramienta de software (como un editor para administrar las tareas del plan de contenidos) no se ofrecen detalles, ni capturas de pantalla de la misma. La metodología fue probada en "el dominio estructuras de datos y algoritmos y mostró buenos resultados" (Shahin et al., 2008, p. 4) pero no se presentan detalles de la prueba y/o los resultados.

Schreurs et al. (2008) proponen una metodología manual para en ensamblaje de OA a cargo de especialistas: el autor descompone el contenido en fragmentos para construir contenido reutilizable 
y un diseñador lo ensambla y almacena. Para este enfoque, "cada lección es un conjunto de OA los cuales están compuestos de un conjunto de bloques de información que se componen de un conjunto de elementos básicos, texto completo, resumen, palabras clave, figuras, etc., generalmente denominados OA atómicos o simplemente ALOs" (Schreurs et al., 2008, p. 15). La producción de un curso se realiza en seis fases: ( $i$ ) descomponer el material, (ii) definir su nivel de interacción, aspecto y dónde será incluido, (iii) diseñar el contenido, (iv) crear el contenido (ALO), (v) almacenarlo, y (vi) ensamblarlo y empaquetarlo. Las primeras tres fases son responsabilidad del docente o diseñador instruccional y las otras tres de un diseñado de cursos de e-learning. La descomposición es un proceso manual que divide el contenido en capítulos, unidades y bloques (un curso es asimilado a un libro). El proceso de descomposición continua hasta obtener los ALO y etiquetarlos con metadatos. Para la descomposición, los docentes, cuentan con una plantilla desarrollada en Microsoft Excel. Esta metodología fue probada para el diseño de cursos de capacitación en Agfa HealthCare. Como herramienta se utilizó Microsoft PowerPoint con el complemento Articulate Studio ${ }^{18}$. En el trabajo se evalúa la pertinencia de la elección de las herramientas, pero no la metodología.

Sarasa et al. (2008) diseñan una herramienta de autor (LOMEditor ${ }^{19}$ ) que permite crear paquetes a través del ensamblaje manual de OA. La herramienta permite componer, de forma manual, un itinerario de aprendizaje a partir de OA ya existentes. "En este sentido uno de los objetivos es simplificar la creación de recursos a través del ensamblaje de recursos existentes que pueden estar situados de forma local o remotamente" (Sarasa et al., 2008, p. 241). Los OA siguen las especificaciones de IMS Content Packaging (compatibles con SCORM), por lo que los contenidos se estructuran en forma de árbol. Asimismo, los objetos son etiquetados en base a IMS-LRMS (compatible con LOM). La composición, permite que que se ensamblen dos OA de manera de obtener un único objeto. Primero, el docente, selecciona $(i)$ los dos OA que desea componer y, luego, (ii) puede optar por dos posibilidades de composición: paralela y en profundidad. En la primera, crea dos rutas o secuencias de contenidos (otro hijo de la raíz del árbol de contenidos), y la otra añade un camino al interior del OA (un hijo del otro hijo en el árbol). Después de este proceso, el nuevo objeto se valida. En el trabajo no se aclara si la validación es sólo sintáctica (estructura) o también semántica (aspecto educativo). La metodología se implementa a través de LOMEditor, una herramienta de autor de escritorio, que permite la edición, creación, composición y evaluación de OA. Las funciones básicas que ofrece la herramienta son: (i) abrir/visualizar el OA, (ii) editar los metadatos del OA, (iii) empaquetar el objeto, $(i v)$ creación de un objeto desde cero, (v) evaluar de la calidad del objeto - de forma manual o automática-, (vi) consultas a una base de datos que contiene información sobre los $\mathrm{OA}$, (vii) clasificación automática de $\mathrm{OA}$-de acuerdo con los mismos atributos utilizados en su evaluación- y (viii) composición de los objetos de aprendizaje. No se encontró evidencia acerca de posibles evaluaciones de la aplicación y/o la metodología.

Wetzlinger et al. (2008) presentan un sistema que permite la conexión con diferentes repositorios distribuidos para buscar y recuperar OA e integrarlos en un nuevo material de aprendizaje. La metodología "propone un sistema ad-hoc de composición de objetos de aprendizaje. Permite la conexión de repositorios distribuidos con sistemas de gestión de contenidos (LMCS), buscar, solicitar y recuperar los objetos de aprendizaje, adaptándolos al LCMS y, por último, la integración de los objetos recuperados en un nuevo material de aprendizaje" (Wetzlinger et al., 2008, p. 33). En este enfoque se introduce una forma de realizar la composición con OA través de un sistema que utiliza XML para representar los OA y los metadatos asociados los mismos. Se propone la utilización de web service para la recuperación de los OA, así como para la composición. Como resultado de una búsqueda, en el repositorio local, quedan disponibles todos OA encontrados en la red de pares -que satisfacen los criterios de búsqueda-. Se tiene acceso, entonces, al material para integrarlo y adaptarlo en un nuevo material de aprendizaje. Para habilitar la búsqueda y distribución de los OA, usando esta arquitectura, cada par en la red tiene que mantener el almacenamiento de los OA como documentos XML y proporcionar los servicios de búsqueda. La metodología propone el uso de SCORM que cuenta con información en XML. La misma se ha implementado a través de un

18 Disponible en: https://www.articulate.com/products/presenter.php

19 La herramienta actualmente no esta disponible. 
prototipo con el que han realizado pruebas de laboratorio con dos repositorios -creados $a d-h o c-$ en la red de pares. Según los autores la propuesta "sólo se centra en los aspectos técnicos para permitir la búsqueda y recuperación de objetos de aprendizaje $[\ldots y]$ en un siguiente paso, el enfoque tiene que contar con el uso de esta arquitectura para componer resultados semánticamente correctas y consistentes" (Wetzlinger et al., 2008, p. 38).

López et al. (2008) presentan un sistema generador de ambientes de enseñanza-aprendizaje que denominan AMBAR. El mismo permite combinar OA existentes. Esto se hace a través de un conjunto de reglas de producción que definen las combinaciones posibles. AMBAR está "basado en una visión interdisciplinaria que incluye un enfoque Cognitivo-Constructivista de aprendizaje, el concepto de Objetos de Aprendizaje (OA) y repositorios de OA, los estándares para el desarrollo de ambientes de aprendizaje en línea, como SCORM y Learning Design, y la visión de Web Semántica" (López et al., 2008, p. 2). La metodología propone tres tipos de OA y dos reglas de producción para combinarlos. Se dispone de OA fundamentales, OA generativo-instruccionales y OA generativo-estrategias. Los dos últimos se generan a través de las reglas de producción a partir de los OA fundamentales -los cuales pueden ser importados de otros repositorios-. Cuenta, además, con una ontología para representar el dominio de conocimiento que permite las consultas semánticas al repositorio. AMBAR es una aplicación web que cuenta con tres ambientes: $(i)$ uno para el diseño -para el docente-, (ii) uno para la colaboración entre estudiantes y docentes, (iii) y el tercero para el mantenimiento. "De los tres ambientes que forman parte de AMBAR, actualmente ya se cuenta con el primer prototipo funcional del repositorio de OA" (López et al., 2008, p. 9). Los OA utilizados están etiquetados con LOM y se ajustan al estándar SCORM.

Lalithsena et al. (2008) proponen la creación de un itinerario de aprendizaje en base a la información representada a través de ontologías. Los autores plantean que el "modelo propuesto genera el itinerario de aprendizaje que más coincide con los objetivos de aprendizaje del estudiante" (Lalithsena et al., 2008, p. 30). Las ontologías son utilizadas para representar tanto el conocimiento, como el perfil del estudiante. En la metodología se mapean los objetivos con estas ontologías, lo que permite generar un itinerario de aprendizaje. Cabe destacar que al crear la ontología de dominio, los expertos, definen los pre-requisitos de cada concepto. Antes de que el estudiante acceda a la secuencia se le realiza un pre-test. Entonces el "itinerario de aprendizaje generado [...] puede ser considerado como el camino óptimo con respecto al objetivo del usuario y que es compatible con sus intereses, conocimientos previos, nivel de competencia y preferencias" (Lalithsena et al., 2008, p. 33). Basado en el itinerario de aprendizaje se asocian los recursos o materiales de aprendizaje que alojan en una librería de recursos o repositorio. Una vez que el estudiante recorre el itinerario se le propone un post-test que permite actualizar el perfil del usuario. Los autores desarrollaron un prototipo para evaluar el modelo. La ontología de dominio se centró en aprendizaje del lenguaje de programación JAVA. La evaluación se hizo sobre 10 estudiantes del primer año de la University of Colombo School of Computing, que permitió a los autores evaluar los itinerarios generados por el prototipo y el rendimiento de los estudiantes que los utilizaron frente a un grupo testigo.

Bouzeghoub et al. $(2009 ; 2009)$, proponen un ambiente virtual que soporta tanto la búsqueda como la composición de OA. Los autores proponen "un enfoque iterativo para buscar OA: el usuario final navega por un conjunto de OA y dentro de la estructura interna de cada uno y elige los que él/ella está interesado/a. El usuario final compone su propio OA" (Bouzeghoub et al., 2009, p. 764). La metodología está basada en SIMBAD por lo que cuenta con tres modelos: $(i)$ dominio de conocimiento -representado a través de una ontología de dominio-, (ii) de estudiantes -que refleja el conocimiento y las preferencias de los mismos-y (iii) de OA -que modela el contenido, los prerequisitos y el conocimiento adquirido al utilizarlo-. Existen dos tipos de OA: atómico o complejo. Este último se construye mediante la aplicación operadores de composición ${ }^{20}$ sobre objetos atómicos o complejos. La composición (OA complejos) se representa, entonces, con un grafo (composition graph) cuyos nodos tiene OA y sus arcos son relaciones entre estos. Para la aplicación de la metodología se desarrolló una aplicación de escritorio que asiste al docente en el proceso

20 Se proponen cinco operadores: SEQ, PAR, ALT, AGG y PROJ . Se sugiere acceder a la bibliografía para más detalles (Bouzeghoub et al., 2004b; Duitama et al., 2005). 
composición y creación de cursos. Basado un criterio de búsqueda propuesto por el docente, el sistema, busca sobre el servidor de conocimiento y regresa la recomendación. A través de un editor gráfico el autor ensambla un OA complejo utilizando (re-utilizando) todo o parte de los objetos recuperados. Previo al almacenamiento del nuevo OA se deben cargar y validar los metadatos. La validación evalúa la estructura del composition graph y la coherencia de la secuencia de aprendizaje (por ejemplo, que sea incremental y que el estudiante tiene los pre-requisitos para accederla). Al cargar los metadatos (basados en LOM) el sistema genera parte automáticamente. Los estudiantes pueden buscar OA o seguir lecciones. Si bien la metodología se implementó, sólo se utilizó el sistema en pruebas de laboratorio. Afirman los autores "nuestro sistema es una herramienta completa para la gestión OA: creación, búsqueda, validación, anotación y de inserción en la base de conocimientos. Como siguiente paso tenemos la intención de probar el sistema con usuarios reales en un contexto real." (Bouzeghoub et al., 2009, p. 768).

Garrido et al. (2009), presentan un planificador automático para crear cursos personalizados. Los autores presentan "un enfoque [...] compatible LOM que utiliza un planificador automático para crear cursos personalizados que son portables a cualquier entorno compatible con IMS-LD, mientras que también se enfoca en el modelado de OA reutilizables." (Garrido et al., 2009, p. 178). La metodología define tres elementos: tareas, conceptos y perfiles de los estudiantes. Las tareas encapsulan los OA necesarios para aprender los conceptos. Los OA se vinculan a través de tres tipos de relación: requiere (requires), basado en (IsBasedOn) y referencia a (references), las cuales permiten ensamblar los OA. El docente puede diseñar la estructura del cursos desde cero o reutilizando OA alojados en repositorios. La estructura del curso y la información del estudiante forman lo que los autores denominan un problema de planificación que es tomado por el planificador inteligente, el cual genera el itinerario de aprendizaje (o learning route) para cada estudiante. Los estudiantes, por su parte, pueden seguir un curso o sólo una parte -en base a sus características personales (perfil), conocimientos y preferencias-- Los OA son etiquetados con el estándar de metadatos IMS-LRMS ${ }^{21}$ (compatible con LOM). Los autores desarrollaron una aplicación de escritorio de modelado visual que permite al diseñador instruccional crear cursos por medio de arrastrar y soltar. Asimismo, "la herramienta permite al docente modelar explícitamente y etiquetar las relaciones estructurales entre las tareas, los conceptos y la adaptación de las tareas a tipología y particularidades del estudiante" (Garrido et al., 2009, p. 179). Además, los docentes pueden especificar el tipo de material, el formato y los recursos necesarios para la consecución de las tareas. La herramienta se probó con ocho estudiantes de doctorado, que la utilizaron para obtener sus itinerarios de aprendizaje para un curso de inteligencia artificial.

Jovanović et al. (2006, 2009), presentan "un enfoque basado en ontologías para la descomposición automática de $\mathrm{OA}$ en fragmentos reutilizables, y un re-ensamblaje dinámico de estos fragmentos dentro contenido de aprendizaje personalizado" (Jovanović et al., 2009, p. 6). La propuesta se basa ALOCoM, por tanto, el contenido es dividido en: content fragment, content object (CO) y learning object. Usan ontologías para describir la estructura de los OA y agregan semántica a su descripción. Así definen una $(i)$ ontología de dominio para describir el contenido, una (ii) ontología estructural para describir la estructura del contenido y (iii) una ontología de contexto para definir el rol educativo del contenido. "En nuestro enfoque, un OA está representado por la ontología de estructura, mientras que la ontología de dominio se utilizan para describir semánticamente el contenido de los OA. Además, la ontología de contextos se utiliza para marcar semánticamente los OA con un rol educativo" (Jovanović et al., 2009, p. 7). También utilizan ontologías para modelar los estudiantes, el modelo instruccional y para la definición del itinerario de aprendizaje (learning paths ontology). Esta última permite establecer relaciones para el ensamblaje de los OA, tales como: pre-requisitos entre los $\mathrm{OA}$, asignarles un nivel de dificultad, definir niveles de agregación (hasPart o isPartOf) y relaciones de navegación (preceeds o follows). El proceso de esamblaje se lleva adelante en base a una consulta de estudiantes, a partir de la cual se localizan en el repositorio el conjunto de $\mathrm{CO}$ que permiten cubrir la consulta, luego, se agrupan en función del perfil del estudiante (permitiendo la recomendación) y se ordenan con la información de learning paths ontology. La evaluación de la propuesta se hizo a través del desarrollo de una aplicación web

21 Disponible en: $\underline{\text { http://www.imsglobal.org/metadata/ }}$ 
denominada TANGRAM. Un ambiente de aprendizaje inteligente que se diseñó para un curso sistemas de información inteligentes. Cuenta con cuatro módulos: $(i)$ un administrador de contenidos que permite publicar y etiquetar los OA -utiliza un perfil de LOM-, (ii) un administrador de usuarios, (iii) el módulo de ensamblaje y (iv) el módulo de interacción con los usuarios. Al acceder por primera vez, el estudiante, debe completar un cuestionario que permite al sistema construir su perfil. En la sesiones siguientes sólo debe elegir el tópico sobre el que desea aprender y el sistema le propone un conjunto itinerarios de aprendizaje entre los que puede optar. El software se probó con estudiantes del curso Sistema de Información Inteligentes (alrededor de 30 por curso) del Departamento de Ingeniería de Software de la Universidad de Belgrado durante más de dos años. Los autores afirman haber "recibido reacciones principalmente positivas". Cabe aclarar que el sistema trabaja únicamente con presentaciones multimedia (Microsoft PowerPoint o OpenOffice Impress), los "nuevos OA se generan dinámicamente como páginas HTML, a partir de un repositorio que almacena OA [...en formato de presentaciones multimedia...] preparadas por los profesores y autores de contenido." (Jovanović et al., 2009, p. 7).

Huang et al. (2009), proponen el uso de un sistema de recomendación de secuencias de aprendizaje. Las recomendaciones se basan en las trayectorias de aprendizaje realizadas por los usuarios para aprender un tema. "En este estudio, se argumenta que la transferencia del aprendizaje en línea se puede lograr de manera efectiva partiendo de un grupo secuencias de aprendizaje. [...El] Sistema de Recomendación de Secuencias de Aprendizaje (LSRS), analiza las experiencias de aprendizaje de grupos para predecir y proporcionar una lista personal de aprendizaje a cada estudiante mediante el seguimiento de los patrones de aprendizaje de los demás con respecto a ciertos temas." (Huang et al., 2009, p. 145). Este grupo de experiencias de aprendizaje (group-learning experience) permiten derivar patrones de aprendizaje. Los cuales permiten calcular posibles secuencias de aprendizaje personalizadas para cada estudiante. Así como los OA se encuentran alojados en repositorios, los perfiles de los estudiantes se almacenan en una base de datos. Esto permite conectar los OA a los que accede cada estudiante para aprender un tema y así obtener la secuencia de OA que utilizó. El seguimiento de cada estudiante, permite en esta propuesta, generar patrones de secuencia aprendizaje. Este enfoque utiliza procesos estocásticos para poder predecir el próximo OA, en la secuencia, al que debe acceder el estudiante. Además, para cada secuencia el estudiante debe proporcionar una retroalimentación (feedback) mejorando así el proceso de recomendación. La propuesta fue incorporada a una aplicación web que utiliza paquetes SCORM para implementar los cursos que toman los estudiantes. Estos acceden a la aplicación con su usuario y contraseña. En el área de recomendación, cada estudiante, selecciona un tema y el sistema le ofrece una lista de posibles secuencias. La metodología se evaluó en tres cursos tomados en un semestre (otoño de 2005) por un grupo de 113 estudiantes y 23 profesores de una universidad en Taiwan. El estudio se enfocó, principalmente, en evaluar la utilidad del sistema para mejorar la transferencia de aprendizaje de los estudiantes. Según los autores, "a partir de los resultados de la evaluación, se encontró que el uso de la secuencia de aprendizaje por los estudiantes fue capaz de promover significativamente su transferencia de aprendizaje. [...] Para los profesores, el sistema ofrece soporte a la secuenciación de aprendizaje proporcionando buenas recomendaciones. [...] Para los estudiantes [...] las recomendaciones, en particular, pueden reducir la carga de trabajo en la búsqueda y acceso a la información.” (Huang et al., 2009, p. 161).

Ullrich \& Melis (2009), presentan un framework para generar cursos de matemática a partir del ensamblaje de OA utilizando criterios pedagógicos como: contexto, nivel de competencias, conocimientos previos, entre otros. La propuesta "utiliza la información acerca de los objetos de aprendizaje, el estudiante y sus objetivos de aprendizaje para generar automáticamente una secuencia adaptada de recursos que ayudan al estudiante a lograr sus metas. La generación de cursos ofrece un camino que se encuentra a la mitad entre los cursos pre-diseñados donde hay 'una sola talla para todos' y la búsqueda individual de objetos de aprendizaje" (Ullrich \& Melis, 2009, p. 9319). La metodología propone que el curso (o secuencia de OA) sea generado dinámicamente, seleccionando los OA que se presentarán al estudiante paso a paso. Utilizan un planificador HTN ${ }^{22}$

22 Un HTM (Hierarchical Task Network) o red jerárquica de tareas es un algoritmo de planificación automática, perteneciente al campo de la Inteligencia Artificial, que crea un plan por descomposición de tareas en sub-tareas 
para la descomposición de tareas en sub-tareas. El resultado del proceso de planificación es un plan que consiste en una secuencia de operadores (pedagogically neutral operators) que, cuando se aplican, generan una secuencia de tareas en las cuales se referencian los OA alojados en el repositorio. La generación del curso se realiza para un estudiante en base a sus objetivos de aprendizaje, identificados como tareas objetivo o goal task. Estas últimas son las que están asociadas al conjunto de recursos que permiten alcanzar el objetivo. El curso incluye, además, el conjunto de conceptos que el estudiante necesita para comprender un tema (pre-requisitos). Comienza con una descripción de sus objetivos y su estructura. Para cada concepto asociado a la goal task, se presenta: una introducción, desarrollo, demostraciones, práctica, conexiones y reflexión. La implementación de esta metodología se denomina PAIGOS, un generador de cursos basado en web service-, que se incluyó dentro del ambiente de aprendizaje ActiveMath ${ }^{23}$. El conocimiento se representa a través de ontologías y es posible registrar repositorios en la aplicación para que el material alojado en éstos este disponible (Ullrich, 2008). La evaluación de la aplicación -y por ende la metodología que implementa- se hizo poniendo foco en el rendimiento de ésta en la construcción de cursos. Según los autores "la evaluación técnica demuestra que PAIGOS es eficiente. Esto se debe a la planificación jerárquica, los cursos, que constan de más de 300 recursos educativos, se generan en menos de un segundo." (Ullrich \& Melis, 2009, p. 9332). Aunque, "el rendimiento real de PAIGOS depende considerablemente de los repositorios y el modelo de aprendiz. En caso de que los componentes residen en servidores diferentes, la latencia de la red por sí sola reduce el rendimiento general." (Ullrich \& Melis, 2009, p. 9331).

Menéndez Domínguez et al. $(2010 ; 2010)$ ofrecen un modelo de ensamblaje de OA centrado en recomendaciones para el etiquetado. La secuenciación la crea el docente apoyado por el sistema para completar los metadatos a través de un modelo de similitud de OA. "El modelo está constituido por dos fases: Desarrollo para la reutilización (Generación) y Desarrollo con reutilización (Composición) [...en la primera] se crean nuevos objetos con un nivel de granularidad fino para un uso inmediato y que cumplan con un objetivo instruccional específico. [...En la segunda] se desarrollan nuevos objetos a partir de otros ya existentes [...]. Se toman OA y se ensamblan en una secuencia que constituye una experiencia de aprendizaje" (Menéndez Domínguez, Prieto Méndez, et al., 2010, p. 57). Los objetos de la primera etapa son considerados asset y los de la segunda SCO (en términos de SCORM) (Menéndez Domínguez, Castellanos Bolaños, et al., 2010). La implementación del modelo propuesto se integró a la plataforma AGORA $^{24}$. Esta aplicación web se constituye en una red de nodos y repositorios distribuidos. Cada nodo cuenta, entre otras funcionalidade ${ }^{25}$, con la posibilidad de gestionar OA utilizando: un generador-etiquetador de objetos, un meta-buscador, un secuenciador-empaquetador de objetos compuestos y un entorno de presentación de contenidos. El sistema emplea los estándares SCORM 2004 para el empaquetado de OA y un etiquetado compatible con LOM. Los usuarios (docentes/estudiantes) deben registrarse para utilizar la herramienta. Se evaluó, por una parte, "la usabilidad de la plataforma para las actividades de gestión de [OA]" a través de una encuesta a 65 usuarios de la plataforma (Menéndez Domínguez, Prieto Méndez, et al., 2010, p. 60) y, por otra, "la utilidad y la facilidad de uso del asistente, y por ende el modelo propuesto, para generar un [OA]" (Menéndez Domínguez, Castellanos Bolaños, et al., 2010, p. 148) a través de un estudio de caso que involucró 10 profesores de educación superior de la Universidad Autónoma de Yucatán, México.

Stănică \& Crișan $(2011,2012)$, presentan un framewok para el ensamblaje y reutilización de OA.

(Ullrich \& Melis, 2009).

23 ActiveMath es un entorno basado en la web, innovador y adaptable, para el aprendizaje de matemática. El sistema guía al estudiantes en el aprendizaje autónomo, y es capaz de adaptarse a los objetivos de aprendizaje, los conocimientos e intereses de cada estudiante (ActiveMath, 2008). Una demo de ActiveMath está disponible en: http://demo.activemath.org/ActiveMath2/main/menu.cmd. Más información sobre ActiveMath puede encontrarse en (Melis et al., 2006).

24 La plataforma AGORA (Asistencia para la Gestión de Objetos Reusables de Aprendizaje) está disponible en: http://smile.esi.uclm.es/agora/.

25 Para más detalles se sugiere consultar el Manual de AGORA disponible en: http://161.67.140.11/agora/manualUsuarioAGORA.pdf. 
Las autoras centran su propuesta en la granularidad de los OA - un tamaño pequeño- y la abstracción -independientes de su uso-, lo que facilita su ensamblaje. La propuesta está centrada en el aprendizaje de matemática. Utilizan cuatro tipos de objetos: simple, elemental, objeto web y lección. Los primeros tres de granularidad baja y la lección compuesta por una combinación de los otros. "El objeto más simple es el componente fundamental, por ejemplo, un texto, una función o una fórmula matemática. Estos apoyarán el desarrollo de objetos de instruccionales y son utilizados para gestionar su interacción. Un componente instruccional adquiere valor cuando se incluye en una lección. Varios componentes se pueden combinar para crear una lección, y al mismo tiempo, un componente se pueden utilizar en diferentes clases." (Stănică \& Crișan, 2012, p. 3). Los componentes más simples son generados a través de plantillas que permiten introducir una guía pedagógica al docente, así como controlar su nivel de interacción con otros OA. Han implementado la propuesta a través de una aplicación de escritorio para la enseñanza de la matemática, donde los docentes ensamblan OA para construir lecciones. El software permite tanto la descomposición de las lecciones en objetos elementales, como el ensamblado de los mismos. La interface de la aplicación permite, al docente, ensamblar los componentes sólo arrastrando con el ratón. Esta acción permite acoplar/desacoplar componentes, que el sistema se encarga de relaciones con los recursos. En ninguno de los trabajos aquí analizados las autoras presentan o mencionan la evaluación de la aplicación/metodología.

Verbert et al. (2012) proponen un framework para el ensamblaje de recursos y materiales educativos basado en patrones. El usuario selecciona una actividad y el sistema sugiere la siguiente, o genera toda la secuencia con una plantilla. Los autores proponen "un enfoque para el ensamblaje semiautomático de contenido para generar secuencias de aprendizaje adaptados a diferentes enfoques pedagógicos. El objetivo general es permitir la selección y ensamblaje de actividades y recursos de aprendizaje pertinentes dentro de secuencias de aprendizaje pedagógicamente sólidas" (Verbert et al., 2012, p. 1257). Los patrones fueron extraídos, utilizando ingeniería inversa, desde diseños de aprendizaje (learning designs) y planificaciones de lecciones (lesson plans) ya existentes. Se identificaron posibles secuencias de aprendizaje que permitieron desarrollar una propuesta de ensamblaje de recursos educativos. La propuesta está centrada en el docente, quien es apoyado para construir la secuencia de aprendizaje. Para comenzar, debe seleccionar uno o más conceptos y cómo desea enseñarlos (método de enseñanza o preferred teaching method). Los autores proponen dos maneras de construir la secuencia: $(i)$ por actividad, donde se toma en cuenta la actividad donde se encontraría el estudiante y el sistema recomienda la siguiente -el docente decide cuál será la actividad-o (ii) por plantillas, el sistema genera toda la secuencia teniendo en cuenta la duración de toda la actividad, el medio (on-line o presencial) y método de enseñanza. En el ensamblaje por actividad, utilizan un algoritmo denominado de ventana deslizable (sliding window algorithm) el cual busca establecer una coincidencia entre la secuencia existente y los patrones, para luego, basado en el patrón elegido definir la siguiente actividad. En la opción de plantillas, los autores utilizan algoritmos de agrupamiento ${ }^{26}$ (clustering algorithm) para agrupar las actividades en función de las preferencias del docente y la duración de la actividad. Una vez definida la secuencia, el sistema recomienda un conjunto de recursos (alojados en repositorios) que serán asociados con la actividad. Los autores desarrollaron un caso de estudio y utilizaron LAMS ${ }^{27}$ para implementar su metodología de ensamblaje. El objetivo del estudio de caso fue evaluar la utilidad y usabilidad de metodología. El estudio se realizó con 20 profesores en octubre de 2011. Los recursos fueron extraídos desde repositorios asociados a $\mathrm{GLOBE}^{28}$, y de sitios como: WikiAnswers ${ }^{29}$, ProProfs $^{30}$ y Wikipedia ${ }^{31}$.

26 Para detalles del algoritmo se sugiere consultar la bibliografía.

27 LAMS (Learning Activity Management System) es un sistema de código abierto, basado en la web, que permite a los autores diseñar, de manera visual, secuencias de aprendizaje (Verbert et al., 2012). Para más detalles sobre esta herramienta puede acceder a http://lamsfoundation.org/.

28 Federación Mundial de Repositorios de Objetos de Aprendizaje (GLOBE): http://www.globe-info.org/.

29 Disponible en: http://www.answers.com/.

30 Disponible en: http://www.proprofs.com/.

31 Disponible en: https://www.wikipedia.org/. 


\subsubsection{Otras metodologías}

Dado que se tomó para el análisis la bibliografía disponible entre 2004 y 2012 inclusive, y que el ensamblaje de OA es una línea de investigación activa, se presentan a continuación un conjunto de investigaciones en la temática publicadas en los años 2013, 2014 y 2015. Las mismas fueron seleccionadas con la misma metodología descrita en el Capítulo $1 \mathrm{y}$ son posteriores al proceso de análisis realizado en esta tesis.

Esta subsección tiene como objetivo, por una parte, dar cuenta de la continuidad y de la evolución de esta línea de investigación y, por otro, complementar el estado de la cuestión con los últimos aportes realizados por los especialistas en la temática.

Se presentan a continuación siete publicaciones, ordenadas de forma cronológica, obtenidas en las bases de IEEE Explorer, ELSEVIER, Springer, iJET y los anales de LACLO. Con investigadores pertenecientes de instituciones de Colombia, Chile, Francia, Italia, España, Túnez, Rusia y Lituania. Sólo una de las publicaciones pertenece a una metodología ya analizada en la sección anterior y da una continuación al trabajo ya revisado.

Becerra et al. (2013) proponen una metodología para la generación automática de itinerarios o rutas de aprendizaje que "recomienda [rutas de aprendizaje] y [OA] basándose en la especificación de lo que el usuario desea aprender y el conocimiento que ya posee" (Becerra et al., 2013, p. 1). Los autores definen los OA "como una entidad, digital o no digital, que puede ser usada para aprendizaje, educación o entrenamiento" (Becerra et al., 2013, p. 1). Esta metodología utiliza ontologías para la representación del dominio y metadatos (LOM) para el etiquetado de los OA. El estudiante, debe declarar lo que quiere aprender y el sistema, automáticamente, organiza los OA dentro del itinerario en función de los aprendizajes previos necesarios para abordar cada OA. En base al itinerario sugerido o recomendado por el sistema, el estudiante "puede seleccionar los [OA] que cumplan con sus expectativas" (Becerra et al., 2013, p. 8). Los autores desarrollaron una aplicación web llamada OASEI sobre la que se realizaron pruebas de laboratorio con 12 usuarios. OASEI "permite a un usuario registrado o administrador seleccionar un conjunto de [OA] pertenecientes a las [rutas de aprendizaje] generada y exportarlo en un formato SCORM. Los [OA] y su metadata en formato IEEE-LOM [...] son almacenados en la plataforma OASEI." (Becerra et al., 2013, p. 6).

Garrido \& Onaindia (2013), proponen una metodología de ensamblaje de OA basada en un planificador inteligente. Esta metodología ya fue descrita en la sección anterior. Este nuevo trabajo se suma al ya analizado, además de la descripción más detallada sobre el uso del perfil del estudiante, se presenta una experiencia de evaluación de la metodología. Dicha experiencia, contó con $(i)$ una prueba de laboratorio que permitió una evaluación cuantitativa de la metodología, en la que se definieron problemas con " $1,2,4,8, \ldots 256$ estudiantes ficticios con diferentes perfiles". Así como también, de una (ii) evaluación cualitativa, en base a un estudio de caso que involucró a 10 estudiantes y 10 docentes de un curso de Inteligencia Artificial. Dentro de las conclusiones de esta nueva experiencia de uso de la metodología, los autores pudieron dar cuenta de que mientras los docentes acuerdan con la secuencia propuesta por los itinerarios de aprendizaje generados por el sistema, observan que "la estructura del curso es demasiado abierta para los estudiantes", y por otra parte, éstos últimos, muestra un alto grado de satisfacción al poder "auto-organizar sus actividades" (Garrido \& Onaindia, 2013, p. 72).

Colace et al. (2014) se enfocan en el problema de la construcción de una Adaptive Educational Hypermedia System, en particular, en la recomendación de itinerarios de aprendizaje (learning path) personalizados con OA. Los autores usan el conceptos de OA, pero no lo definen. "En este trabajo la idea principal es en la generación de una recopilación estandarizada del objeto de aprendizaje con el fin de calificar y cuantificarlos mejor." (Colace et al., 2014, p. 11). La metodología hace uso de ontologías para representar el dominio. Para lograr la personalización, los autores proponen realizar un "análisis de algunas características [...] pedagógicas. [Esta información es obtenida de]: la prueba de desempeño, el tiempo de desempeño, y los tópicos revisados." (Colace et al., 2014, p. 10). Con esta información la metodología propone determinar si el estudiante está listo para pasar a 
la próxima lección del curso. El modelo del estudiante (o user model) también tiene en cuenta los estilos de aprendizaje ${ }^{32}$, los conocimientos previos y el rendimiento del estudiante. Los autores implementaron la metodología como un plug-in para el LMS Moodle y realizaron la evaluación con estudiantes universitarios de tres cursos: Introducción a la las ciencias de la computación (400 estudiantes), redes de computadoras (80 estudiantes) y Tecnología de software para la web (50 estudiantes). Entre los principales resultados se los autores encontraron que, con la aplicación de este enfoque, hubo un incremento en los niveles de conocimiento de los estudiantes en comparación con años anteriores en los mismos cursos.

Torres \& Guzmán-Luna (2015), proponen una metodología para la creación de itinerarios de aprendizaje (learning routes) de OA utilizando un planificador automático y web semántica. Los autores usan el conceptos de OA, pero no lo definen. Los itinerarios deben cubrir los objetivos que el estudiante haya formulado. "La idea principal [...] consiste en la creación de una nueva estructura como una secuencia [...] en el que algunos OA son pre-requisitos de otros. Esta secuencia de OA representa la ruta del curso." (Torres \& Guzmán-Luna, 2015, p. 324). La metodología se basa en la obtención de un problema de planificación (planning problem), el mismo se construye a partir de dos relaciones RequiresBy y IsBasedFor, la primera obliga a que el estudiante complete todas las actividades antes de pasar a la próxima y la segunda alguna de ellas. Los autores desarrollaron una aplicación de escritorio para permitir a los docentes modelar un curso. Realizaron una experiencia de laboratorio para un curso de robótica, convocados a tres expertos en la temática para evaluar las rutas generadas.

Shmelev et al. (2015), proponen una técnica para el desarrollo de itinerarios de aprendizaje (learning path) personalizados a partir de OA. Los autores usan el conceptos de OA, pero no lo definen. La metodología se basa en la utilización de ontologías, algoritmos genéticos y la taxonomía de Bloom (en una versión revisada por Raths \&Wittrock). Shmelev et al. (2015) afirman que "utilizamos algoritmos genéticos para determinar el orden correcto de los OA en una secuencia, [esto involucra]: una ontología para encontrar conexiones entre OA y la versión revisada de la taxonomía de Bloom para evaluar la 'calidad' de las conexiones.” (p. 712). Así pues, la metodología, establece pre-requisitos y resultados esperados para los OA como pares ordenados formados por: (tema/tópico, categoría de Bloom). Estos pares permiten la conexión de los OA. Para la evaluación de la secuencia utilizan algoritmos genéticos. "Un curso (secuencia de LOs) se presenta como un individuo con un cromosoma, y cada gen es un OA. Cada gen tiene una marca especial que es responsable del uso del objeto dentro de una secuencia." (Shmelev et al., 2015, p. 713). Las ontologías representan el conocimiento y lista de áreas de dominio. Para la evaluación de la metodología desarrollaron un prototipo e hicieron pruebas de laboratorio con seis OA creado adhoc.

Štuikys et al. (2015), abordan la secuenciación de OA utilizando un enfoque basado en metaprogramación. Los autores afirman que los OA son "el término más general por el cual expresamos el contenido de aprendizaje [...]. Este término se utiliza en asociación con la reutilización global del contenido..." (Štuikys et al., 2015, p. 151). El ensamblaje de los OA está basados en la definición de un patrón que establece una secuencia en la presentación del contenido: "A $\rightarrow B \rightarrow C$ " (Štuikys et al., 2015, p. 155), donde A son aspectos pedagógicos (como los objetivos), B la teoría o contenidos y $\mathrm{C}$ la práctica. También se establecen un conjunto de operadores que establecen relaciones entre las distintas instancias de los elementos de A, B y C (por ejemplo, $\left\{\mathrm{a}_{1}\right\}$ requires_any_of $\left.\left\{\mathrm{b}_{1}, \mathrm{~b}_{2}\right\}\right)$. Las posibles secuencias de tareas (u OA) son pre-programadas utilizando meta-programación. El estudiante debe elegir entre un conjunto de parámetros y el sistema genera la secuencia con esta información. Para el evaluación de la metodología se implementó un estudio de caso que involucró a 71 estudiantes un curso de Ciencias de la Computación. Los estudiantes contaban con 11 parámetros para elegir, los que ponía a disposición de estos 25920 posibles secuencias para elegir. Dentro de los principales resultados, los autores afirman que "el enfoque permite: (1) generación semi-automático [...] de la secuencia; (2) [...] la

32 Utiliza como modelo para los estilos de aprendizaje, el propuesto por Richard Felder. Utilizados también en las metodologías propuestas por Garrido et. al. (2013; 2009) y Jovanović et al. $(2006,2009)$. 
adaptación de los contenidos a las necesidades de los estudiantes [... y]; (3) un nivel de granularidad más alta de los contenidos." (Štuikys et al., 2015, p. 162). Cabe destacar que esta publicación cuenta con la descripción de importante cantidad (14 trabajos) de metodologías de ensamblaje revisadas en su sección de trabajos relacionados.

Mahmoud et al. (2015), presentan un enfoque para la recomendación de itinerarios de aprendizaje basado en OA. Estos son descritos y publicados como servicios web. Los autores usan el conceptos de OA, pero no lo definen. "Nuestro enfoque define un servicio web de aprendizaje (SW) para cada objeto de aprendizaje (OA) para superar los problemas de interoperabilidad y accesibilidad de los objetos de aprendizaje." (Ben Mahmoud et al., 2015, p. 1). Los itinerarios estarán "adaptados a las necesidades del estudiantes y a los diferentes contextos de aprendizaje" (Ben Mahmoud et al., 2015, p. 3). El estudiante iniciar su proceso de aprendizaje definiendo sus preferencias y el sistema realiza automáticamente la composición de la secuencia de OA (o learning web service) para "un determinado contexto y los objetivos de aprendizaje. Esto se logró sobre la base de la anotación semántica de los servicios web de aprendizaje con ontologías (de dominio y de objetivos)." (Ben Mahmoud et al., 2015, p. 3). Para la descripción de los objetivos se utiliza la taxonomía de Bloom. La publicación no cuenta con información sobre la evaluación de la metodología. Sí se menciona el desarrollo de una aplicación que permite a los diseñadores instruccionales la creación de OA para que el sistema los publique como servicios web educativos.

\subsection{Recapitulación}

Las metodologías de ensamblaje de OA tienen como objetivo principal la definición de un itinerario de aprendizaje en base a un conjunto de objetos alojados en repositorios. La secuencia puede ser generada a través de distintos niveles de automatización (manual, semi-automática o automática) y puede (o no) tener en cuenta el perfil del usuario. Se trata de un proceso de tres etapas: búsqueda de la temática, definición del itinerario de aprendizaje y conexión con los OA (ver Figura 3.5).

Las metodologías de ensamblaje podrían ser agrupadas en tres categorías: aquellas que crean un itinerario de aprendizaje de $\mathrm{OA}$, las que crean un $\mathrm{OA}$ y las que recomiendan un itinerario de aprendizaje o el OA que sigue en la secuena basado en el perfil del usuario. Dependiendo del tipo de metodología pueden contar con distintos modelos o abstracciones de la realidad: un modelo de contenido para representar el $\mathrm{OA}$, un modelo de estudiante que permita reflejar el perfil del estudiante, un modelo profesor para el perfil docente y/o un rol educativo para los contenidos. Todos aportan no solo a la representación de las abstracciones, sino además a concretar secuenciación de contenidos. A esto se le puede agregar la definición de pre-requisito y conocimiento adquirido que también hacen posible el acoplamiento de los OA dentro del itinerario de aprendizaje (ver Figura 3.5).

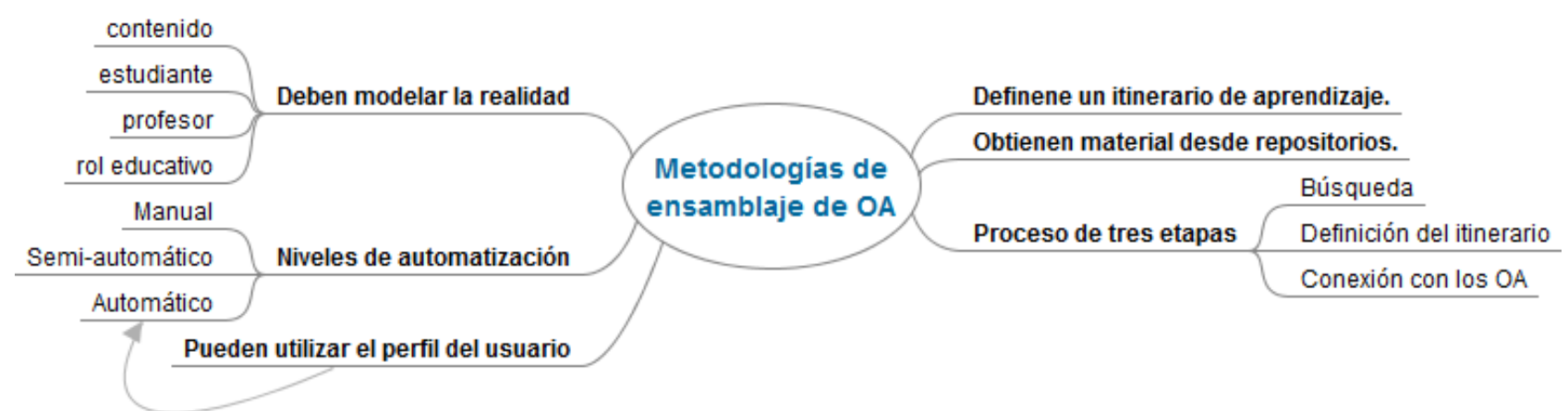

Figura 3.5. Caracterización de las metodologías de ensamblaje de OA.

La representación de las distintas entidades, así como las relaciones que entre ellas se deben establecer para llevar adelante el proceso de ensamblaje -búsqueda, creación de itinerario y selección del material- son representadas utilizando, principalmente, ontologías y metadatos (ver Figura 3.6). 
Las ontologías son utilizadas tanto para la descripción de entidades y sus relaciones (sean éstas contenidos o usuarios), como para hacer comparaciones entre términos sintácticamente distintos y realizar búsquedas semánticas, efectuar mapeos entre distintos modelos de contenido, describir la estructura de los OA, especificar el rol didáctico del contenido o definir trayectorias de aprendizaje a través de los temas. Si bien los usos dados a las ontologías son variados, se debe tener en cuenta que su construcción conlleva un esfuerzo considerable y deben generarse ad-hoc para cada dominio (aunque una vez diseñadas podrían reutilizarse).

El uso de metadatos estandarizados, por su parte, se enfoca tanto en la representación datos técnicos sobre el OA, como en información semántica de estos. Es importante destacar que un correcto funcionamiento del proceso de ensamblaje depende, en parte, de que los metadatos sean completos y tengan descriptores no ambiguos y semánticamente ricos. Como en el caso de las ontologías, los metadatos, presentan una serie de aspectos que pueden afectar al ensamblaje: una falta de semántica de los estándares existentes, la carga de metadatos es una tarea costosa, falta de calidad y completitud de registros de metadatos en el material existente y la complejidad propia del estándar elegido.

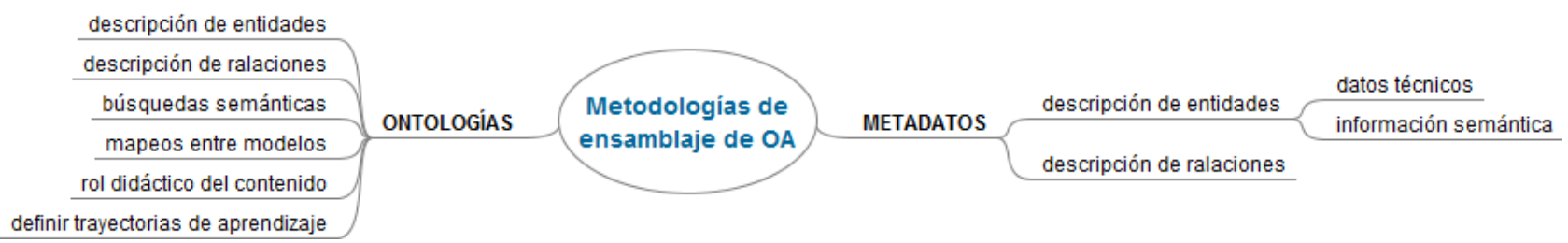

Figura 3.6. Función de metadatos y ontologías en el contexto de las metodología de ensamblaje de OA.

En este capítulo se presentó una breve descripción de cada una de las 27 metodologías de ensamblaje analizadas en esta tesis y una actualización con la revisión de siete metodologías publicadas más recientemente. En el siguiente capítulo se presentan un conjunto de criterios, que también derivan del análisis de las metodologías, y que permitirán una mejor caracterización de las mismas y, por ende, facilitarán la selección de la metodología que mejor se adapta a las necesidades/contexto de los usuarios (sean estos docentes, diseñadores instruccionales o estudiantes). Además, de apoyar la elección de una metodología para el estudio caso desarrollado para este trabajo de tesis. 


\section{CRITERIOS DE EVALUACIÓN}

\section{RESUMEN}

Uno de los sub-objetivos de esta tesis es el de definir un conjunto de criterios que permitan clasificar las metodologías de ensamblaje de OA, seleccionar un SE y elegir un SE para el estudio de caso.

En este cuarto capitulo se definen y aplican los criterios de evaluación a las 27 metodologías estudiadas en esta tesis. En primer lugar se define cada uno de los criterios y se describen los posibles valores para estos. Para luego, mostrar los resultados de la aplicación de los mismos. Con base en esta información, se discute la elección de una metodología de ensamblaje para el estudio de caso. Finalmente, en la recapitulación, se presenta una caracterización de las metodologías a partir de los criterios definidos. 


\subsection{Introducción}

Uno de los sub-objetivos de esta tesis es el de definir criterios que permitan la comparación entre las diferentes metodologías de ensamblaje. Es así, con base en la revisión de las publicaciones seleccionadas, se pudieron identificar distintos focos de análisis de las metodologías de ensamblaje de OA. Desde las motivaciones que llevan a los investigadores a enfocarse en la temática, pasando por cómo representar las distintas entidades involucradas en el proceso de ensamblaje o la forma en que se logra la secuenciación de los OA, hasta la caracterización de los Sistemas Ensambladores que implementan las metodologías y cómo éstas fueron evaluadas. Todos estos son aspectos se deberían tener en cuenta al momento de establecer un conjunto de criterios de evaluación/clasificación de una metodología de ensamblaje.

Así pues, se generó un conjunto de criterios que permiten:

(i) Clasificar las diferentes metodologías de ensamblaje.

(ii) Seleccionar un SE en función de cómo se desea utilizar y del material con el que se cuenta.

(iii) Elegir un SE para el estudio de caso.

Dado que se está abordando una temática en la que la investigación sigue abierta, se hace necesario evaluar varios aspectos de la misma. La forma en la que las metodologías proponen realizar el ensamblaje, los objetivos que persiguen, los destinatarios y la información que requiere de ellos, la granularidad y conceptualización que tienen los OA que se utilizan en la secuencia, el uso de estándares, entre otros.

Se decidió agrupar los criterios en tres categorías:

(a) Estrategia. Los criterios agrupados en esta categoría permiten caracterizar el enfoque de la propuesta subyacente. Esto es, dar una idea general de la metodología estableciendo los lineamientos básicos de la misma. Responder qué tipo metodología es, para qué fue desarrollada, cuál es la problemática que apunta solucionar y con qué tipo de recursos plantea trabajar la metodología. Se incluyen aquí: el enfoque de la metodología, su objetivo y motivación, y cómo se propone realizar el ensamblaje.

(b) Contexto. Se incluyen en esta categoría los criterios vinculados al contexto en el que se aplica la metodología. Al tratarse de propuestas ligadas al proceso de enseñanza o al de aprendizaje, es necesario analizar cómo se abordan aspectos pedagógicos. Indagar sobre quiénes son los destinatarios, qué aspectos pedagógicos son incluidos para realizar el proceso de ensamblaje y cómo fue integrada la metodología en la propuesta áulica al ser evaluada.

(c) Software. Aquellos criterios incluidos en esta categoría están vinculados con la implementación de la metodología en una aplicación informática. Así, se busca caracterizar el software e identificar si está disponible, para qué plataforma, para quienes está diseñado y qué tipo de estándares utiliza.

A continuación, en este capítulo, se presentan y describen el conjunto de criterios (ver Figura 4.1) de evaluación junto con los posibles valores para cada uno de ellos. Para, seguidamente, mostrar y discutir los resultados de la aplicación de los mismos al conjunto de metodologías seleccionadas a partir de la revisión bibliográfica. Luego, se hace una evaluación del conjunto de metodologías candidatas para la implementación del estudio de caso, con base en los resultados obtenidos con la aplicación de todos los criterios. Finalmente, en la recapitulación y síntesis, se analizan los resultados de la aplicación de todos los criterios para cada categoría y se proponen agrupamientos de las metodologías a partir de alguno de los criterios.

Cabe aclarar que el valor "s/c" (sin categorizar), que indica que no se encontró evidencia suficiente sobre ese aspecto en particular, sólo se muestra en los resultados. Se puede utilizar en todos los 
criterios, y no se detallará para evitar redundancia en la explicación; salvo que sea necesaria alguna aclaración sobre la evidencia faltante.

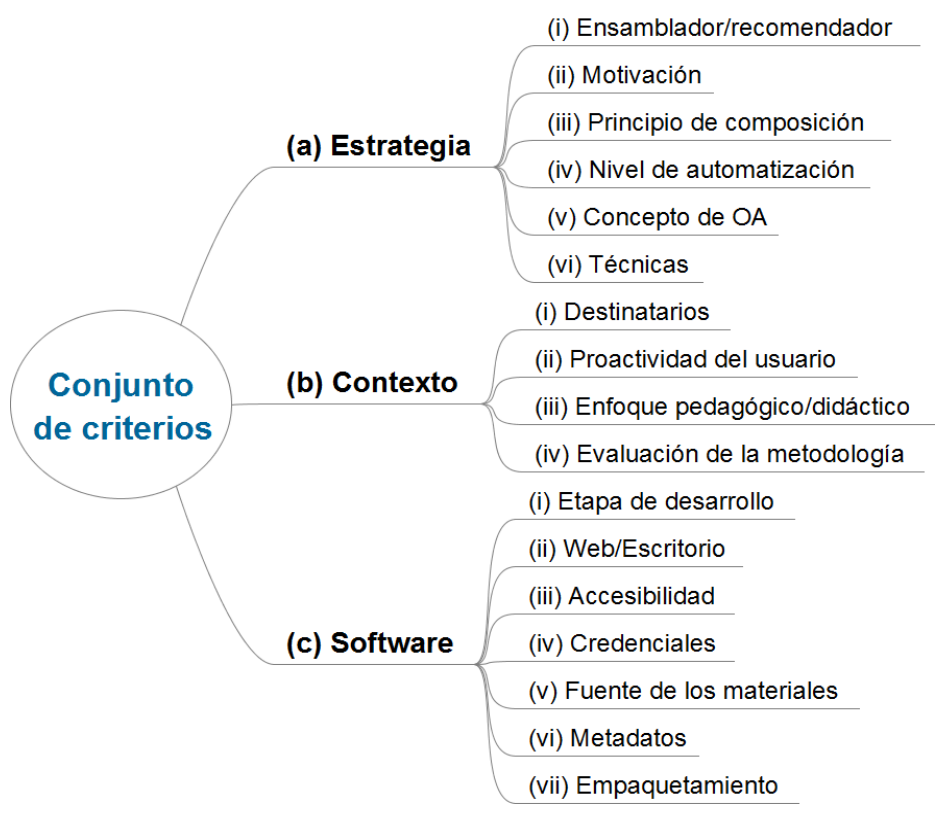

Figura 4.1. Resumen del conjunto de criterios.

\subsection{Definición de criterios de evaluación para SE}

A continuación se definen los criterios que se incluirán en cada una de las categorías descritas anteriormente:

\section{(a) Estrategia}

(i) Ensamblador/recomendador. Este criterio se enfoca en establecer si la propuesta aborda la recomendación además del ensamblaje de OA. Esto permitirá identificar las funciones que debería ofrecer una implementación de la metodología, cuáles serán los requerimientos para los usuarios y cómo la propuesta podría integrarse en una planificación de actividades.

Los valores posibles para este criterio son:

- Ensamblador. Esta etiqueta se utilizará en las metodologías que propongan la construcción de un itinerario de aprendizaje a partir de OA o el contenido de un objeto propiamente dicho. Los OA se ensamblarán en una secuencia que constituye una experiencia de aprendizaje.

- Recomendador. Se etiquetan así aquellas metodologías que tienen como objetivo recomendar un itinerario de aprendizaje creado vía ensamblaje o próximo OA de la secuencia. Se tienen en cuenta, para esto, las preferencias del usuario (docente/estudiante).

- $\mathbf{s} / \mathbf{c}^{1}$. Con este valor se indicará que no se encontró evidencia suficiente, en la/s publicación/es evaluadas, sobre este criterio.

(ii) Motivación. Cada propuesta se lleva adelante motorizada por una motivación. Identificar cuál es la necesidad que llevó a los investigadores a desarrollar la metodología permite, por una parte caracterizar la propuesta, pero también hacer un buen uso de la misma -no generar falsas expectativas sobre los alcances de la metodología-.

1 Este valor se incluye aquí para ejemplificar su utilización y de ahora en más se da por incluido en el resto de los criterios y no se detallará en adelante para evitar redundancia en la explicación. 
Es posible que para caracterizar apropiadamente cada metodología, se deba utilizar más de una de las siguientes opciones.

La lista de valores que puede asumir este criterio son:

- Selección. Esta etiqueta denotará las metodologías que estén motivadas por el problema de seleccionar los OA que mejor se adaptan tanto al contenido o concepto buscado, como al perfil pedagógico del usuario.

- Organización. Con esta etiqueta se identificarán aquellas metodologías que expresen, como problemática motivadora, la organización ineficiente de los los OA por la escasa información estructurada disponible para ellos. Se incluirán aquí también las propuestas que identifiquen que existe una cantidad insuficiente de metadatos semánticos para lograr una secuenciación apropiada de los OA.

- Localización. Esta etiqueta identifica a aquellas propuestas que reconocen que la gran cantidad de OA disponibles en la web es una de las causas que hace más compleja la localización de los mismos. Es decir, la dificultad en la localización es lo que motiva el desarrollo de la metodología.

- Personalización. Las metodologías que sean marcadas con esta etiqueta estarán motivadas por conseguir la personalización del material de acuerdo con el perfil del usuario. Esto debe estar centrado en lo educativo -nivel educativo, conocimientos previos, objetivos de aprendizaje, entre otros-.

- Sistemas e-learning. Refiera a aquellas propuestas que planteen, como motivación, que los sistemas que administran contenido educativos online (LMS, repositorios, LOMS, entre otros) debieran evolucionar y contar con funcionalidades que asistan a los usuarios en la localización, creación y secuenciación de contenidos.

- Secuenciación automática. Con esta etiqueta se identificarán a las metodologías cuyos autores tengan, como parte de la motivación, el conseguir la automatización total o parcial de las diferentes etapas que conlleva el ensamblaje de OA.

- Reutilización. La etiqueta caracterizará a aquellas metodologías, cuyos autores, tienen como motivación generar una genuina reutilización de OA.

(iii) Principio de ensamblaje. Es necesario analizar la forma en la que cada metodología propone realizar el ensamblaje. Esto es, identificar de qué manera relaciona un OA con otro en la secuencia de aprendizaje. Esto ayudará a definir si el conjunto de OA del que dispone o con el que trabaja habitualmente el docente podría ser utilizado directamente a través de una aplicación desarrollada con esta metodología, o si necesitaría de un proceso de adaptación de los mismos.

Es posible que para caracterizar apropiadamente cada metodología se deba utilizar más de una de las siguientes opciones.

La lista de valores que puede asumir este criterio son:

- Metadatos OA. Identifica a aquellas metodologías que hacen uso de los metadatos asociados a los OA en el proceso de ensamblaje.

- Metadatos usuario. Identifica a aquellas metodologías que utilizan los metadatos que describen las preferencias de los usuarios (docentes/estudiantes) en el proceso de ensamblaje.

- Ontologías. Identifica a aquellas metodologías que utilizan ontologías en el proceso de ensamblaje.

- Patrones. Identifica a aquellas metodologías que utilizan algún tipo de red, mapa 
o plantilla diseñado previamente por el docente o experto, el cual guía el proceso de ensamblaje.

(iv) Nivel de automatización. Con este criterio se pretende establecer el nivel de automatización que propone cada metodología. Es importante que los usuarios de la metodología conozcan cuál será su nivel de intervención que se requerirá de su parte en el proceso de ensamblaje.

La lista de valores que puede asumir este criterio son:

- Manual. Esta etiqueta caracteriza a aquellas metodologías de ensamblaje que definen un conjunto de pautas sobre cómo debe crearse el itinerario de aprendizaje y deja en manos de los usuario expertos (docente/diseñador instruccional) el proceso de composición. En este caso, la intervención de un sistema informático se limita a la recuperación de los OA o los recursos que lo componen.

- Semi-automática. Identifica a las metodologías que proponen la automatización de parte del proceso de ensamblaje. Esto contempla a aquellas metodologías donde son los usuarios (en general docentes o expertos) los que definen cómo debe ensamblarse y la automatización está puesta en validar y/o combinar los OA que formarán parte del itinerario de aprendizaje.

- Automática. Esta etiqueta es para las metodologías que proponen una automatización de todo el proceso de ensamblaje. En este caso sólo es necesario indicar un conjunto de requisitos para obtener el itinerario de aprendizaje. Los usuarios (docentes/expertos o estudiantes), en general, pueden hacer uso del itinerario propuesto o proponer modificaciones al mismo.

(v) Concepto de OA. Si bien las publicaciones seleccionados para el análisis trabajan con OA, no han adoptado en todos los casos la misma definición (Sección 2.3.2.1), ni le atribuyen las mismas características. Los usuarios deben conocer qué tipo de material educativo van a poder utilizar y cuáles son sus características. En virtud de lo anterior, se evaluará en este criterio cuáles de las características, que mayor acuerdo tienen en la comunidad especializada (Astudillo, Sanz, \& Willging, 2011), están presentes (o no) en el concepto de OA que usa la metodología. Para ello, se revisa tanto la definición de OA utilizada, como la caracterización que hacen del material con que trabajan.

Se agregan a las características antes mencionadas la etiqueta "Ensamblable" que es un dato de valor para el tipo de análisis propuesto en esta tesis. También se registra cuando "No definen" el concepto de OA. Cabe aclarar que el hecho de que no definan el concepto no invalida la posibilidad de identificar algunas de las demás características.

Dado que todas las metodologías trabajan con material digital $\mathrm{y}$, por ende, esta característica de los OA está presente en todas las publicaciones abordadas, no se incluyó la misma dentro de las etiquetas.

Las etiquetas utilizadas para este criterio serán:

- Intencionalidad pedagógica. Se hace explicita en la caracterización que el OA debe ser diseñado para ser utilizado en un contexto educativo.

- Estructura interna. La metodología, define o explicita el contenido del OA: objetivos educativos, contenidos, actividades y, posiblemente, evaluación.

- Metadatos. En la caracterización se exige que los OA sean descritos utilizando metadatos.

- Reutilizable. El OA se caracteriza como un material reutilizable.

- Autocontenido. El OA se caracteriza como un material que contiene todos los 
recursos necesarios para la consecución de los objetivos de aprendizaje, sin depender de otros recursos o materiales externos al mismo.

- Interoperable. En la caracterización se explicita que el OA podrá ser utilizado sobre diferentes plataformas tecnológicas. Esto incluye a aquellas metodologías, cuya implementación, trabaje con OA en formato estandarizado.

- Accesible. En la caracterización se exige al OA que pueda ser localizado y estar disponible para su utilización desde la web -en general alojado en un repositorio- .

- Granularidad. En la caracterización aborda el tamaño del $\mathrm{OA}^{2}$.

- Ensamblable. En la caracterización del OA se lo considera como bloques de construcción que permiten la creación o composición de unidades de aprendizaje de una granularidad mayor.

- No lo definen. El trabajo utiliza el concepto sin definirlo.

(vi) Técnicas. Un aspecto interesante a identificar se enfoca en qué técnicas subyacentes provenientes de la Matemática, Informática/Computación y/o de las Ciencias de la Educación, son utilizados para definir el proceso de ensamblaje de OA. Si bien este aspecto debiera ser transparente para el usuario final (docente, diseñadores, estudiantes), es de interés para caracterizar las metodologías.

Los valores que se utilizan en este criterio son:

- Matemática. Se incluyen aquí aquellas metodologías que se apoyan en la Matemática (teoría de grafos, teoría de probabilidad, entre otras) para llevar adelante parte del proceso de ensamblaje.

- Informática. Se incluyen aquí aquellas metodologías que se apoyan en la Informática/Computación (servicios, web semántica, ontologías, inteligencia artificial, patrones, entre otros) para llevar adelante parte del proceso de ensamblaje.

- Ciencias de la Educación. Se incluyen aquí aquellas metodologías que se apoyan en diferentes aspectos del área de Ciencias de la Educación (diseño instruccional, estilos de aprendizaje, enfoque constructivista del aprendizaje, aprendizaje basado en competencia, entre otros) para llevar adelante parte del proceso de ensamblaje.

- Otro. Este criterio engloba a aquellas posibles técnicas que se pudieran utilizar en futuras publicaciones de la temática.

\section{(b) Contexto}

(i) Destinatarios. Los SE pueden estar enfocados en las actividades que llevan adelante los estudiantes, los docentes o ambos. Es necesario identificar los destinatarios para saber cómo y en qué momento del proceso de enseñanza y/o de aprendizaje utilizar el SE.

Los valores que se utilizan en este criterio son:

- Docentes. Se identifica con esta etiqueta a las metodologías cuyos destinatarios son únicamente los docentes y/o diseñadores instruccionales. Se enfoca en dar soporte a quien crea el itinerario de aprendizaje.

- Estudiantes. Se identifica con esta etiqueta a las metodologías que están centradas en los estudiantes. Generalmente, la metodología está enfocada en facilitar el auto-aprendizaje. En estas propuestas el docente/experto participa

2 La granularidad se relaciona con el nivel de agregación del OA. Típicamente, el nivel de agregación más pequeño contiene elementos multimedia o fragmentos de estos, y el resto de los niveles refieren a un conjunto de elementos del nivel anterior formando objetos más complejos y de granularidad mayor. (IEEE LTSC, 2002). 
en la definición de las relaciones entre OA (previo al ensamblaje).

- Ambos. Se identifica con esta etiqueta a las metodologías que contemplan como destinatarios tanto a docentes como estudiantes, tomando información de ambos roles.

(ii) Proactividad del usuario. Este criterio está relacionado con el anterior y aporta más información sobre el rol en el que la metodología posiciona al usuario. Los cualificadores "activo" y "pasivo", en las etiquetas, implican una relación inversamente proporcional con la responsabilidad del sistema informático que implementa la metodología. Cuando los usuarios son activos, los SE dependen de ellos para la creación del itinerario y/o la selección de los OA asociados al mismo y viceversa.

Los valores que se utilizan en este criterio son:

- Docente activo. El docente interactúa con el SE publicando OA y creando/editando los itinerarios de aprendizaje. Se incluyen aquí aquellos casos en el que el docente está a cargo de todo el proceso.

- Docente pasivo. La metodología propone que el docente pueda publicar OA y/o definir la temática del itinerario de aprendizaje, y sea el SE quien lo cree. El sistema podría contar con información del docente (esto hace que el nivel de automatización del SE sea aún mayor). Se incluyen aquí aquellas metodologías en las que el docente, finalizado el itinerario, puede editarlo.

- Estudiante activo. El estudiante propone una temática y el sistema le ofrece un conjunto de itinerarios alternativos o bien un conjuntos de OA candidatos a componer el itinerario. El estudiante puede elegir el itinerario o bien participar de su construcción.

- Estudiante pasivo. El estudiante selecciona el tema que desea aprender, el SE le propone itinerario a seguir. Este último puede ser dinámico (basado en su perfil) o estático (definido por un docente/experto).

(iii) Enfoque pedagógico/didáctico. Las metodologías de ensamblaje pueden tener en cuenta un conjunto de elementos derivados de alguna teoría pedagógica. Así registrar preferencias del perfil de usuario y/o características educacionales de los materiales. Esta información permite a los SE llevar adelante la creación del itinerario. Poder clasificar las metodologías de ensamblaje en este aspecto permite conocer el nivel de personalización del itinerario que plantea, así como el costo de la carga de información necesario para utilizar el sistema con los estudiantes o para el diseño de actividades.

Los valores que se utilizan en este criterio son:

- Centrado en el material. Cuando la información está centrada en los OA y se registran, entre otros aspectos, la función educativa, el nivel, objetivos, tiempo estimado de uso, relación con otros OA.

- Centrado en el usuario. Aquellos que representan el perfil del usuario pueden registrar, para cada estudiante: sus objetivos, conocimientos previos, nivel educativo previo y el alcanzado mientras usa el SE, estilos de aprendizaje; y en el caso de docentes su estilo de enseñanza.

- Ambos. Se incluyen aquí las metodologías que registran información sobre el material y el usuario para conseguir una alta personalización del itinerario.

(iv) Evaluación de la metodología. Las diferentes metodologías de ensamblaje revisadas en este tesis, en la mayoría de los casos, han sido probadas con el objetivo de evaluar la propuesta. Desde pruebas controladas de laboratorio, pasando por grupos de usuario pequeños que prueban un prototipo, hasta el uso de una aplicación que implementa la metodología con usuarios reales (docentes/estudiantes). Conocer con qué tipo de 
evaluación cuenta la metodología permite definir si es posible utilizarla o es conveniente esperar hasta que haya sido suficientemente probada antes de incorporarla como una de las herramientas de proceso de enseñanza y/o de aprendizaje.

Los valores que se utilizan en este criterio son:

- EC docentes. Se realizó un Estudio de Caso donde se involucran docentes que diseñan los materiales. En estos casos los estudiantes no utilizan los itinerarios de aprendizaje. La evaluación está centrada en la eficiencia de la metodología para generarlos y/o en la simplificación de la tarea docente.

- EC estudiantes. Se realizó un Estudio de Caso donde se involucra a estudiantes, los cuales deben utilizar los itinerarios de aprendizaje generados a partir de la metodología de ensamblaje. Aquí los docentes/expertos/autores pueden tener una participación que va desde establecer las relaciones entre los $\mathrm{OA}$, hasta tener que generar los itinerarios de aprendizaje utilizando la metodología de ensamblaje como guía.

- Lab. Sólo se hicieron pruebas de laboratorio, con ejemplos totalmente teóricos u OA diseñados ad-hoc para evaluar la metodología/software que la implementa.

- Otros. Este criterio engloba a aquellas posibles técnicas de evaluación que se pudieran utilizar en futuras publicaciones de la temática.

\section{(c) Software}

(i) Etapa de desarrollo. Es importante definir en qué etapa del desarrollo se encuentra el SE que implementa la metodología. Dirimir si se trata de un prototipo que se encuentra en estado de evaluación o una aplicación ya liberada ofrece una perspectiva del tipo de aplicación a la que se accede. Al tratarse de un prototipo debería tenerse en cuenta que algunas de sus funcionalidades pueden no estar disponibles y/o contar con restricciones de uso. El docente debería tener en cuenta esto antes de utilizar la aplicación con sus estudiantes o para el diseño de sus materiales.

Los valores posibles para este criterio son:

- Aplicación. Se denominarán así las implementaciones ya liberadas, estén o no, disponibles para su instalación o uso.

- Prototipo funcional. Se trata de una aplicación que si bien se encuentran en estado de prototipo, es totalmente funcional.

- Prototipo. Esta etiqueta se utilizará para aquellas aplicaciones que estén es su estado de prototipo y que cuenten sólo con parte de sus funciones disponibles.

(ii) Web/Escritorio. Dentro de las soluciones de software que implementan cada metodología de ensamblaje los autores han elegido entre la utilización de tecnología Web o de escritorio para el desarrollo de las mismas. La evolución de la web ha extendido la cantidad de aplicaciones disponible sobre esta plataforma y los SE no son la excepción. Sin embargo, no todas las aplicaciones que implementan las metodologías de ensamblaje se diseñan para la web. Es necesario, entonces, definir si la aplicación es web o es de escritorio. Tanto el acceso como utilización de la aplicación se facilitan si la misma está disponible en la web, sin embargo, es necesario contar una conexión a Internet. Estos aspectos, entre otros, permiten tomar la decisión de utilizar o no el SE.

Los valores posibles para este criterio son:

- Web. Con esta etiqueta se identificarán las aplicaciones que utilicen la web como plataforma. Se incluirán aquí aquellas implementaciones que se incluyan como funcionalidades de repositorios o LCMS y también las que se definan a partir de servicios web. 
- Escritorio. Con esta etiqueta se identificará a las denominadas "aplicaciones de escritorio".

- s/c. Con este valor se indicará que no se encontró evidencia suficiente, en la/s publicación/es evaluadas, que permita afirmar que se desarrolló una aplicación que implemente la metodología, o bien, aunque haya mención al desarrollo de un software, no se pueda identificar para qué plataforma se diseñó.

(iii) Accesibilidad. En este criterio se evaluará si la aplicación está accesible. Esto es, independientemente de su etapa de desarrollo (criterio c.ii), el SE puede estar visible, o no, para los usuarios. Esto determina claramente la posibilidad de uso.

Cabe aclarar que no se aborda en este criterio aspectos de la aplicación de licencias para el uso de software.

Los valores posibles para este criterio son:

- Sí. Aquí se enmarcarán aquellas aplicaciones que pueden ser utilizadas por los usuarios.

- No. Con este valor se etiquetarán las aplicaciones que no estén disponibles para su utilización. Se incluyen aquí, tanto las que no se especifica el enlace para su acceso/descarga, como aquellas en las que el mismo este roto o inaccesible.

(iv) Credenciales ${ }^{3}$. Se identifica, con este criterio, si es necesario ser un usuario registrado para tener acceso a la aplicación informática. Por lo general, esta es una característica de aquellos SE que utilizan información del usuarios para sugerir materiales y/o los itinerarios de aprendizaje. El tener que registrarse para usar el software, en muchos casos es un aspecto que ayuda a determinar su uso en el aula.

Los valores posibles para este criterio son:

- Sí. El SE requiere de la presentación de credenciales para acceder al mismo. Se incluyen aquí aquellas metodologías que, aunque no hacen explícito el requerimiento de credenciales, plantean la adaptación del itinerario de aprendizaje según el perfil del usuario y/o la actualización del mismo.

- No. El SE no requiere de la presentación de credenciales para acceder al mismo.

(v) Fuente de los materiales. El origen desde dónde son extraídos los $\mathrm{OA}$ es de suma importancia ya que define el tipo, cantidad, calidad y la accesibilidad de los mismos. Si el SE puede acceder o trabajar con OA de uno o más repositorios es posible acceder a una variedad de OA y localizar los que mejor se adapten a la actividad y/o estudiantes con los que va a trabajar. Si por el contrario, se trata de un conjunto de OA locales al SE puede ser menos probable hallar los OA apropiados. Cabe aclarar que no se trata del lugar donde se encuentre el repositorio, sino los OA. Es decir, el repositorio podría ser local y contener las referencias a OA que se encuentran accesibles a través de Internet.

Los valores posibles para este criterio son:

- Local. Con este valor se etiquetan aquellos SE que utilizan un conjunto de materiales alojados en un repositorio o base de datos local.

- Externa. Esta etiqueta indicará que el SE utiliza repositorio/s u OA accesibles vía la web. Esto es, cuenta con la posibilidad de conectarse con repositorios externos, o bien, permite la referencia a OA alojados en fuentes externas.

3 Estos valores son independientes de que el criterio “c.iii Accesibilidad” este en No, dado que la información pudo extraerse de la/s publicación/es que abordan la metodología. 
(vi) Metadatos ${ }^{4}$. Los SE, por lo general, utilizan metadatos para representación de los OA. Al conocer qué estándar de metadato utiliza la aplicación es posible identificar, a priori, la cantidad de información que va a requerir al publicar un OA, pero también, sobre la cantidad de información con la que contará la aplicación para el ensamblaje. Dado que, para el etiquetado de material educativo, los estándares de metadatos más utilizados son LOM y DCMI, los mismos son incluidos como posibles valores para este criterio.

Los valores posibles para este criterio son:

- LOM. El SE utiliza el estándar IEEE Learning Object Metadata o IMS Metadata Specification.

- DCMI. El SE utiliza el estándar Dublin Core Metadata Inititive.

- Otros. Este criterio engloba a aquellas posibles estándares de metadatos que se pudieran utilizar en futuras publicaciones de la temática.

- s/c. La/s publicación/es utilizadas para evaluar la metodología no cuenta con información sobre el uso de metadatos o bien son mencionado pero no se indica el estándar utilizado. Se incluyen aquí también aquellas metodologías que no utilizan metadatos.

(vii) Empaquetamiento. Si bien el análisis se centra en aquellas metodologías que utilizan $\mathrm{OA}$, los formatos en los que se pueden presentar estos no es único. Conocer si el SE hace uso de OA que se apegan a un estándar de empaquetamiento permitirá determinar disponibilidad de los mismos en los repositorios y así, estimar la cantidad y variedad de material con el que se contará para crear el itinerario. Así como también el costo y el tipo de aplicación si se deben crear/adaptar los OA.

Dado que, SCORM e IMS-CP son estándares ampliamente utilizados para la creación de OA, los mismos son incluidos como posibles valores para este criterio.

Los valores posibles para este criterio son:

- SCORM. Este valor se utilizará para aquellos SE que hagan uso de OA basados en el estándar SCORM.

- IMS-CP. Este valor se utilizará para aquellos SE que hagan uso de OA basados en el estándar IMS Content Packaging.

- Otro. Este valor es para aquellas metodologías cuyos SE utilizan formatos creados ad-hoc para la implementación de la metodología.

\subsection{Aplicación de los criterios}

A continuación se presenta la aplicación de los criterios al conjunto de metodologías abordadas en este estudio. Previo a la lectura de esta sección se sugiere revisar la Figura 4.1 (p. 67) que muestra un esquema que resume el conjunto de criterios descritos, en extenso, en la sección anterior.

Con el fin de favorecer la lectura de los resultados se codificaron las 27 publicaciones ${ }^{5}$ utilizando, como base, los códigos asignados a las mismas en el proceso de selección (ver Tabla 2.3, Capítulo 2). Cabe aclarar que se agruparon las publicaciones que abordan las mismas metodologías. La recodificación se presenta en la siguiente Tabla 4.1.

4 En algunas de las publicaciones se menciona el uso del estándar IMS Metadata Specification, sin embargo, aquí se registrarán como IEEE LOM. Esto es en virtud de que una vez liberado el estándar IEEE LOM (junio de 2002), IMS hizo los ajustes para adaptar su especificación a la de IEEE se puede llamar LOM a cualquiera de las dos especificaciones (Barker, 2005).

5 Cabe aclarar que las 27 publicaciones analizadas son las correspodientes al período 2004-2012. 
Tabla 4.1: Asignación de una codificación a cada metodología.

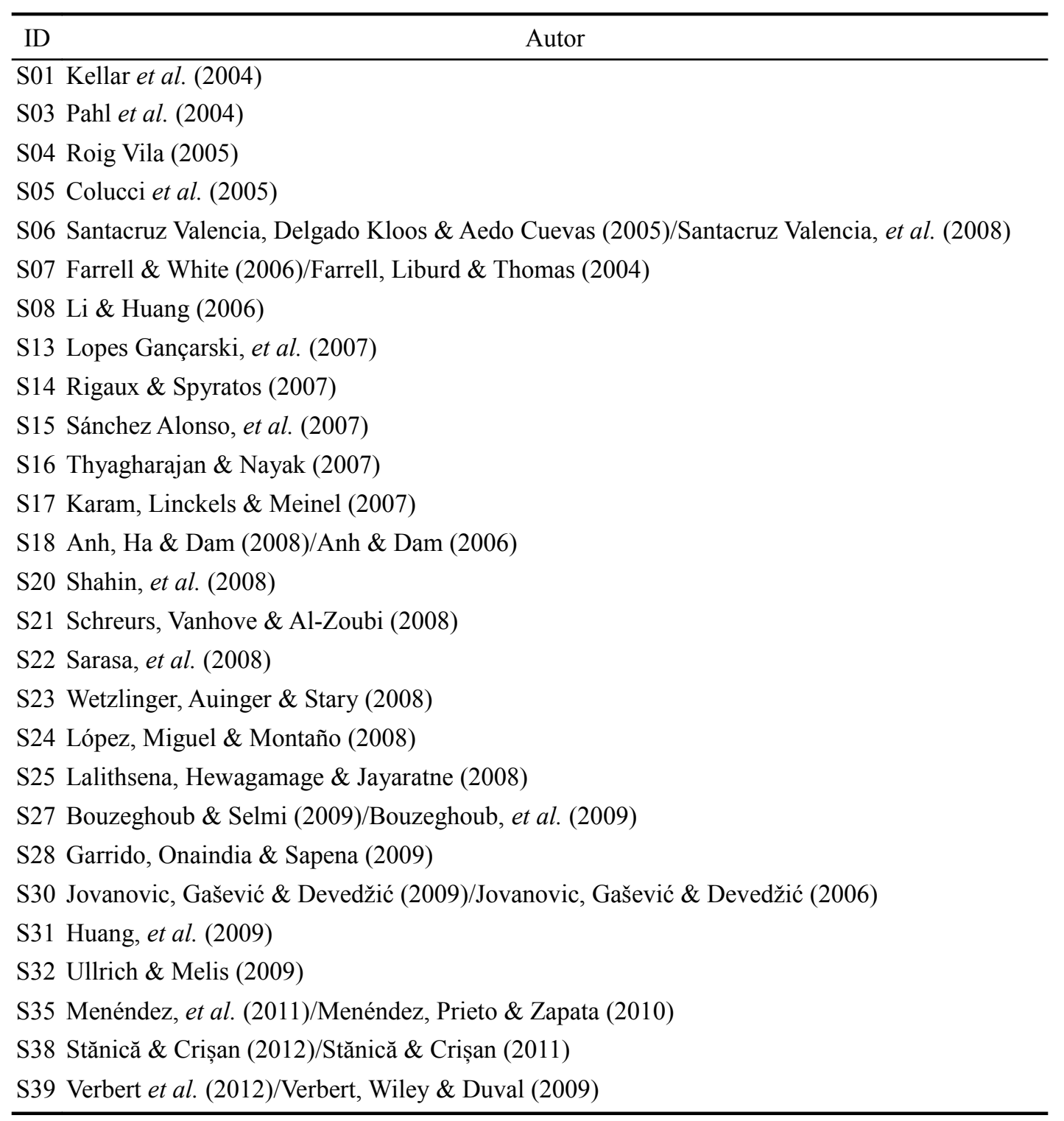

Para una mejor lectura, en las sub-secciones que se encuentran a continuación, se analizará cada categoría y, dentro ella, cada uno de los criterios aplicados a las metodologías revisadas en esta tesis.

\subsubsection{La estrategia de ensamblaje}

Al realizar la revisión de las 27 metodologías, analizadas en este estudio, puede observarse (Figura 4.2) que no hay una tendencia clase en el diseño de metodologías, respecto a aquellas que se enfocan sólo en el ensamblaje, frente a aquellas que toman características de los Sistemas Recomendadores. Cabe destacar que la mayoría (15:27) se enfocan únicamente en el proceso de ensamblaje, mientras que en una menor proporción (12:27) generan los itinerarios de aprendizaje teniendo en cuenta, además, las preferencias de los usuarios que le permiten la recomendación de itinerarios en función de esta información (ver Tabla 1, Anexo II). 


\section{Ensambladores vs Recomendadores}

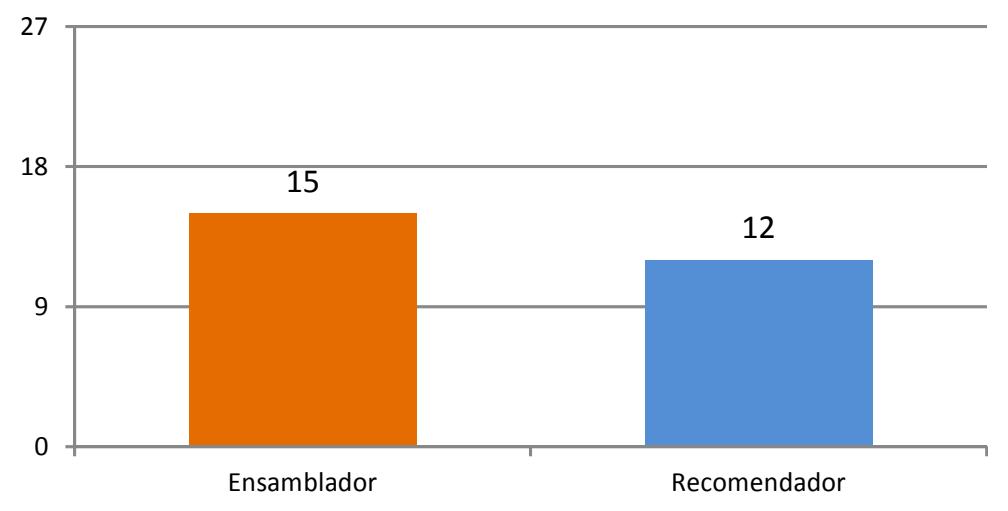

Figura 4.2. Criterio a.i: ensambladores vs recomendadores

Respecto a la motivación que llevó a los investigadores a desarrollar la metodología, no hay unanimidad entre ellos (ver Tabla 4.2 y Figura 4.3), ni tampoco un alto consenso en este criterio. Sin embargo, podrían tomarse como las principales motivaciones la personalización del material educativo y el mejoramiento de los sistemas de e-learning, y en un segundo grupo, podrían ubicarse la selección del material educativo más apropiado para los estudiantes y la reutilización.

Tabla 4.2. Criterio a.ii: motivación que llevó a los investigadores a desarrollar la metodología.

\begin{tabular}{lc}
\hline \multicolumn{1}{c}{ Motivación $(*)$} & Cant. \\
\hline Localización de MED & 2 \\
Organización del conocimiento & 4 \\
Secuenciación automática de contenidos & 5 \\
Selección de los MED apropiados & 6 \\
Reutilización del contenido & 7 \\
Mejoramiento de los sistemas e-learning & 10 \\
Personalización del contenido & 10 \\
\hline
\end{tabular}

(*) Ver Tabla 2 (Anexo II) para más detalles.

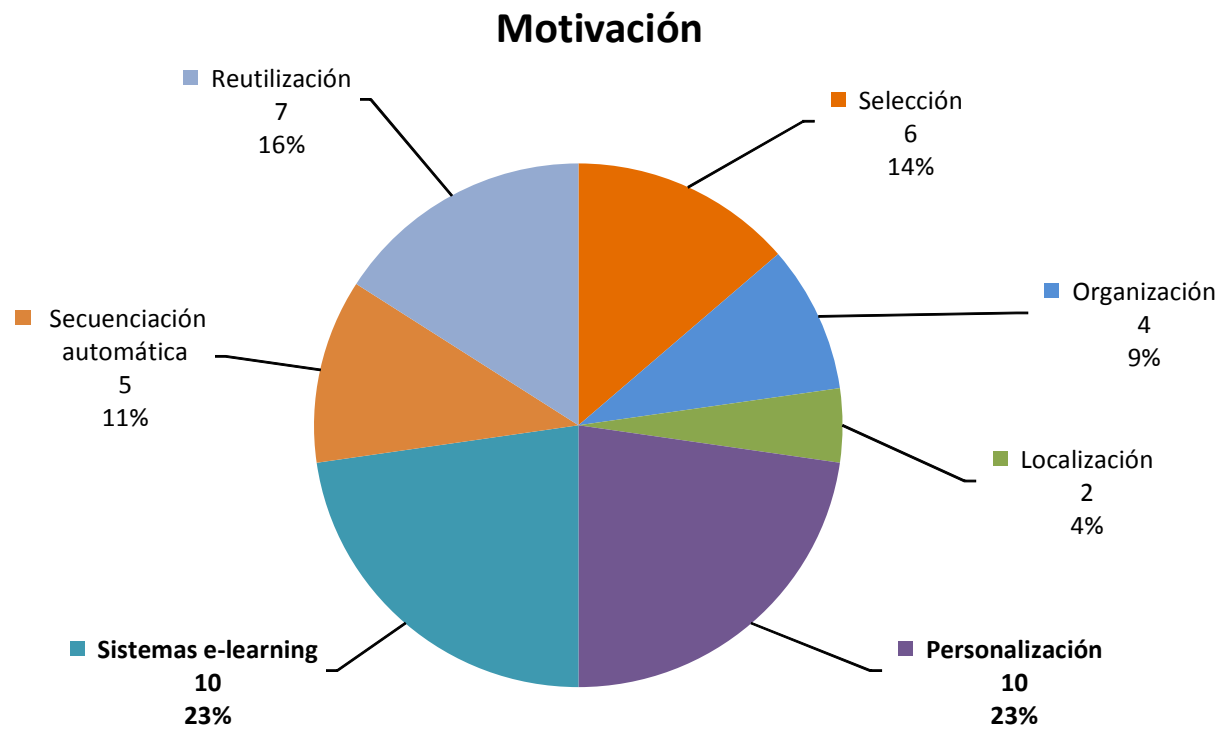

Figura 4.3. Criterio a.ii: motivación que llevó a los investigadores a desarrollar la metodología.

¿De qué forma las metodologías llevan adelante el proceso de composición? Esto es, cómo 
relacionan un $\mathrm{OA}$ con el siguiente. Al aplicar el criterio a.iii:Principio de ensamblaje (ver Tabla 3, Anexo II y Figura 4.4) se pudo determinar que, del conjunto de metodologías analizadas, se utilizan en igual proporción (16:27) metadatos asociados a los objetos y algún tipo de patrón, como guía del proceso de ensamblaje. Estos últimos (patrones), en general definidos por expertos, permiten establecer la secuencia en la que deben ser ensamblados los OA y son, luego, los metadatos asociados a estos últimos los que permiten determinar a qué material accederá el estudiante en cada estación del itinerario.

Cabe destacar, respecto de los patrones, que la mayoría de las propuestas presentan un enfoque topdown, donde los expertos generan los patrones, sin embargo S39 toma el camino contrario y generan los patrones desde la práctica.

\section{Principio de ensamblaje}

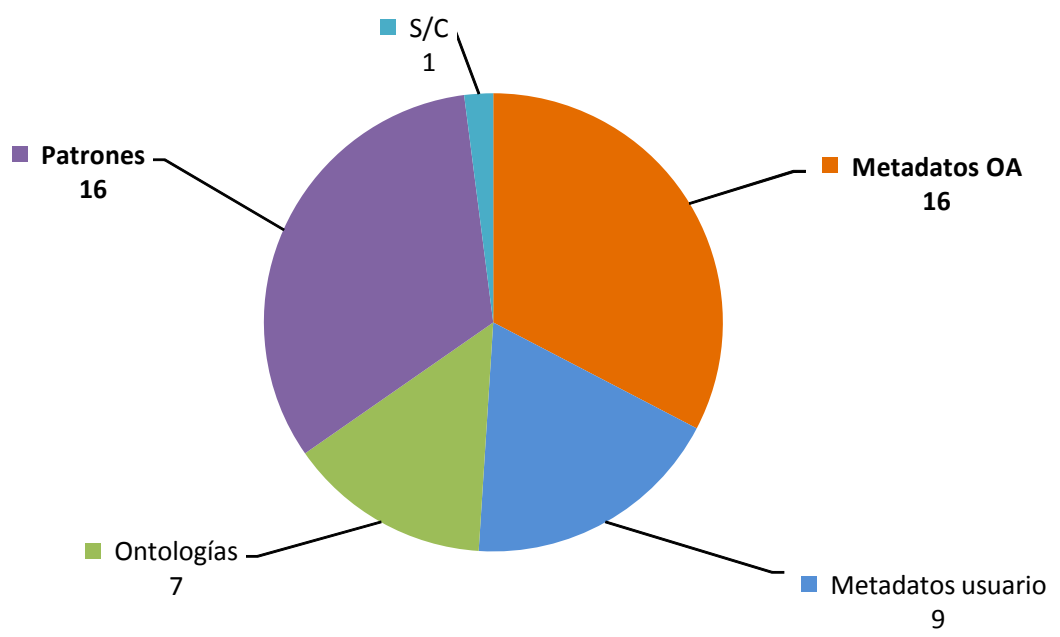

Figura 4.4. Criterio a.iii: cómo relaciona la metodología un OA con el siguiente.

Al aplicar el criterio a.iv:Nivel de automatización pudo observarse que las metodologías se inclinan por un enfoque de alta automatización en el proceso de ensamblaje (ver Tabla 4, Anexo II). Dentro de éstas, predominan las que deciden automatizar todo el proceso de ensamblaje (13:27) y, en una proporción algo menor están los enfoques semi-automáticos (11:27), que automatizan parte del proceso de ensamblaje. Sin embargo, también existen, aunque en menor proporción, las que proponen un proceso manual (3:27), dejando en manos de los expertos todo el proceso de selección y ensamblaje de los OA (Figura 4.5).

Dentro del grupo de enfoque manual, podría destacarse a S04 que retoma el concepto de WebQuest para llevar adelante la creación del itinerario. En el grupo que opta por propuestas semiautomáticas, tres de las propuestas coinciden en ofrecer al docente ensamblar de a pares (S06, S22 y S27), mientras que otros tres (S18, S28 y S20) proponen la definición de un plan o mapa de contenidos con todos los OA. S39, por su parte, propone tanto ofrecer la secuencia completa, como la recomendación de la siguiente actividad en función de la que está realizando el estudiante. Las del grupo mayoritario, que eligen el ensamblaje automático, se podrían subdividir en dos grandes sub-grupos: las que recomiendan el itinerario con base en una evaluación aplicada al usuario con el fin de conocer su perfil ( $\mathrm{S} 01, \mathrm{~S} 08$ y S25) y aquellas que en las que basan la recomendación en una consulta inicial que parte del usuario (S05, S07, S14, S16 y S17). 


\section{Nivel de automatización}

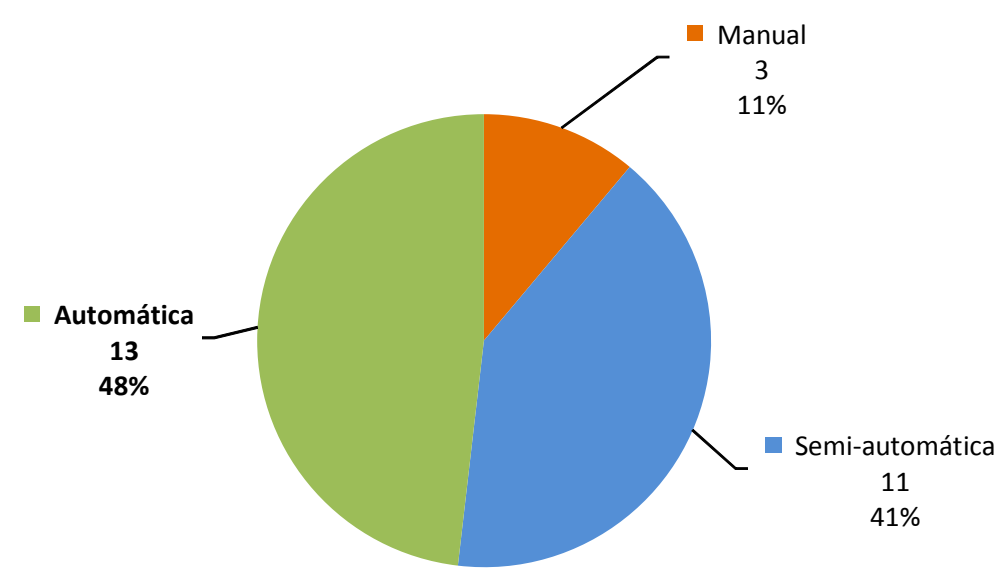

Figura 4.5. Criterio a.iv: niveles de automatización del proceso de ensamblaje.

Al aplicar este criterios a.v (ver Tabla 5 en Anexo II, gráficamente en Figura 4.6) puede afirmarse que no hay unanimidad en la conceptualización de OA. A pesar de que los OA carecen, actualmente, de una definición aceptada mayoritariamente por los expertos en la temática, 9:27 publicaciones usan el concepto de OA, pero no lo definen. Sin embargo, cabe aclarar, que en todas las publicaciones puede observarse algún nivel de caracterización de los mismos. Es posible identificar, entonces, tres características que son utilizadas mayoritariamente: intencionalidad pedagógica (20:27), metadatos (20:27) y reutilizable (17:27). En un segundo grupo se adoptan las características interoperable (12:27), ensamblable (11:27), accesible (11:27), también, definen la estructura del OA (12:27) y abordan la granularidad (12:27). Sobre ésta último, la mayoría (S01, S06, S08, S16, S21, S22, S30, S32 y S38) utiliza el concepto de granularidad en términos de niveles de agregación y el resto (S14, S23 y S35) se enfoca en el tipo de recurso que compondrá el OA. Por otra parte, sólo 4:27 exigen que se cumpla la característica de auto-contenido. Cabe aclarar que la idea de OA como material digital, subyace en todas las publicaciones.

La definición de OA, adoptada en esta tesis ${ }^{6}$, atiende a las principales características en las que acuerdan los autores de referencia en el tema (Sanz, 2015), por lo que podría constituirse en un buen punto de comparación sobre cuánto se ajustan, en general, la caracterización de OA por la que opta cada metodología. En la Tabla 4.3 se propone dicha comparativa y se resaltan las metodologías con mayor coincidencia. Puede observarse que S06, al contar con una estructura interna de los OA, cuenta con el nivel más alto de concordancia. Al agregar los datos tomados del criterio c.vii: empaquetamiento, se agregan a la lista S05, S20, S23 y S35. Cabe destacar que si bien S06 no ofrece un modelo de empaquetamiento estándar, su modelo de contenidos se basa en SCORM (ver 3.1.1).

6 "Un tipo de MED, que se caracteriza, desde el punto de vista pedagógico, por orientarse a un objetivo específico de aprendizaje, y por presentar: una serie de contenidos con el fin de abordar la temática relacionada con el objetivo, actividades que permitan al alumno poner en práctica o problematizar el contenido presentado, y una autoevaluación que posibilite conocer al alumno, si ha podido comprender esos contenidos vinculados al objetivo. Desde el punto de vista tecnológico, se caracteriza por contener un conjunto de metadatos estandarizados para su búsqueda, y recuperación, y estar integrado, utilizando un modelo de empaquetamiento que respete estándares, y de esta manera, permita su diálogo con diferentes entornos tecnológicos.” (Sanz, 2015, p. 34). 
Tabla 4.3. Caracterización de OA. Comparativa con la definición de Sanz (2015).

\begin{tabular}{|c|c|c|c|c|c|c|}
\hline Sanz & $\begin{array}{c}\text { Material } \\
\text { educativo } \\
\text { digital }\end{array}$ & $\begin{array}{l}\text { Punto de } \\
\text { vista } \\
\text { pedagógico }\end{array}$ & $\begin{array}{c}\text { Objetivo } \\
\text { específico }{ }^{+} \\
\text {actividades }^{+} \\
\text {contenido }+ \\
\text { autoevaluación }\end{array}$ & $\begin{array}{c}\text { Metadatos } \\
\text { estandarizados }\end{array}$ & $\begin{array}{c}\text { Diálogo con } \\
\text { diferentes } \\
\text { entornos } \\
\text { tecnológicos }\end{array}$ & $\begin{array}{l}\text { Modelo de } \\
\text { empaquetamiento } \\
\text { estándar }\end{array}$ \\
\hline Criterio & Digital & $\begin{array}{c}\text { Intensio- } \\
\text { nalidad } \\
\text { pedagógica }\end{array}$ & Estructura interna & Metadatos & Interoperable & SCORM/IMSCP (*) \\
\hline S01 & $\checkmark$ & $\checkmark$ & & $\checkmark$ & & $\bar{\checkmark}$ \\
\hline S03 & $\checkmark$ & $\checkmark$ & & $\checkmark$ & & \\
\hline S04 & $\checkmark$ & $\checkmark$ & & & & \\
\hline S05 & $\checkmark$ & $\checkmark$ & & $\checkmark$ & $\checkmark$ & $\checkmark$ \\
\hline S06 & $\checkmark$ & $\checkmark$ & $\checkmark$ & $\checkmark$ & $\checkmark$ & \\
\hline S07 & $\checkmark$ & $\checkmark$ & & $\checkmark$ & & $\checkmark$ \\
\hline S08 & $\checkmark$ & $\checkmark$ & & $\checkmark$ & $\checkmark$ & \\
\hline $\mathrm{S} 13$ & $\checkmark$ & $\checkmark$ & & $\checkmark$ & $\checkmark$ & \\
\hline S14 & $\checkmark$ & & & $\checkmark$ & & \\
\hline $\mathrm{S} 15$ & $\checkmark$ & $\checkmark$ & & $\checkmark$ & & \\
\hline S16 & $\checkmark$ & $\checkmark$ & & & & \\
\hline S17 & $\checkmark$ & $\checkmark$ & & & & \\
\hline S18 & $\checkmark$ & $\checkmark$ & & 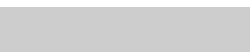 & $\checkmark$ & $\checkmark$ \\
\hline S20 & $\checkmark$ & $\checkmark$ & & $\checkmark$ & $\checkmark$ & $\checkmark$ \\
\hline $\mathrm{S} 21$ & $\checkmark$ & $\checkmark$ & & $\checkmark$ & $\checkmark$ & \\
\hline S22 & $\checkmark$ & & & $\checkmark$ & $\checkmark$ & $\checkmark$ \\
\hline $\mathrm{S} 23$ & $\checkmark$ & $\checkmark$ & & $\checkmark$ & $\checkmark$ & $\checkmark$ \\
\hline S24 & $\checkmark$ & $\checkmark$ & & & $\checkmark$ & $\checkmark$ \\
\hline S25 & $\checkmark$ & & & & & \\
\hline S27 & $\checkmark$ & $\checkmark$ & & $\checkmark$ & & \\
\hline S28 & $\checkmark$ & & & $\checkmark$ & & $\checkmark$ \\
\hline $\mathrm{S} 30$ & $\checkmark$ & & & $\checkmark$ & & \\
\hline S31 & $\checkmark$ & & & $\checkmark$ & $\checkmark$ & $\checkmark$ \\
\hline S32 & $\checkmark$ & $\checkmark$ & & $\checkmark$ & & \\
\hline S35 & $\checkmark$ & $\checkmark$ & & $\checkmark$ & $\checkmark$ & $\checkmark$ \\
\hline S38 & $\checkmark$ & $\checkmark$ & & $\checkmark$ & & \\
\hline S39 & $\checkmark$ & & & & & \\
\hline
\end{tabular}

(*) Datos tomados del criterio c.vii.Empaquetamiento.

Otro aspecto que puede resultar de interés con la aplicación de este criterio $(a . v)$ es observar qué metodologías exigen que el material con el que trabajan tenga la capacidad de ensamblarse. Como puede verse en la Tabla 5 (Anexo II), menos de la mitad (11:27) de las metodologías exigen esta característica en los OA. Tres de ellas (S03, S07 y S14) incluyen en la definición la idea de bloque de construcción, mientras que el resto (S05, S06, S08, S13, S17, S21, S23 y S38) le asocian la capacidad de poder formar parte de una composición.

El último criterio de esta categoría, a.vi, indaga sobre la disciplina a la que pertenece la técnica utilizada por las metodologías. Así pues, al aplicar este criterio (ver Tabla 7 en Anexo II, gráficamente en Figura 4.7) puede observarse que todas hacen uso de la Informática; mientras que 10:27 toman recursos de la Matemática y sólo 5:27 dicen acudir a las Ciencias de la Educación. Otro análisis que posibilita este criterio es preguntar sobre cuáles metodologías hacen uso de la interdisciplinaridad y entre qué disciplinas (ver Figura 4.8). La mayoría (15:27) se valen sólo de la informática para el desarrollo de la metodología, mientras de 7:27 le suman a ésta la matemática. Sólo 2:27 combinan informática y pedagogía, y hay 3:27 que combinan las tres disciplinas.

La aplicación de este criterio también permitió observar que, dentro del campo de la informática, las metodologías se concentraron en: la web semántica, la teoría de grafos y la inteligencia artificial; 
mientras que para la matemática lo hicieron principalmente, con la teoría de probabilidad. La pedagogía, por su parte, aportó desde el diseño instruccional, la teoría constructivista, los estilos de aprendizaje y el aprendizaje basado en competencias.

\section{Concepto de OA}

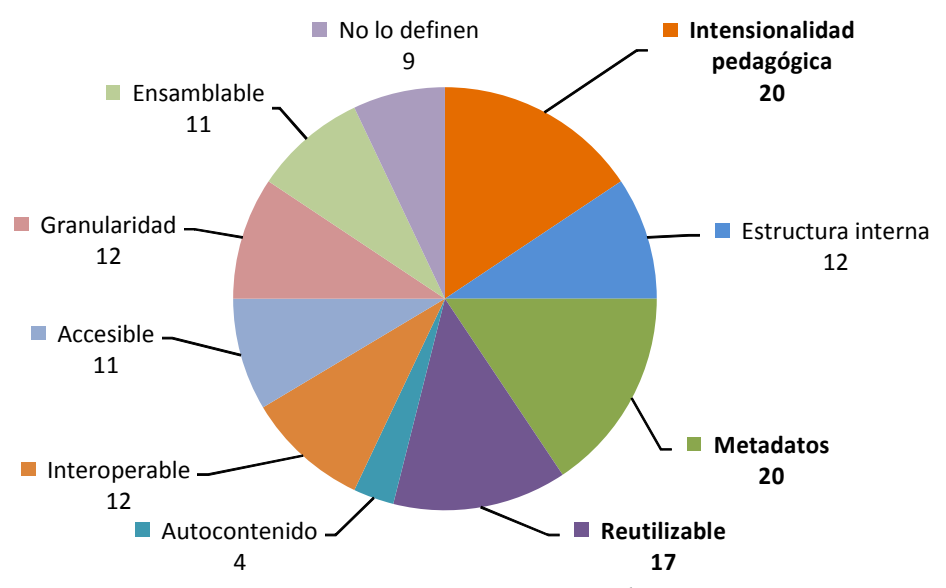

Figura 4.6. Criterio a.v: concepto de OA.

Técnicas

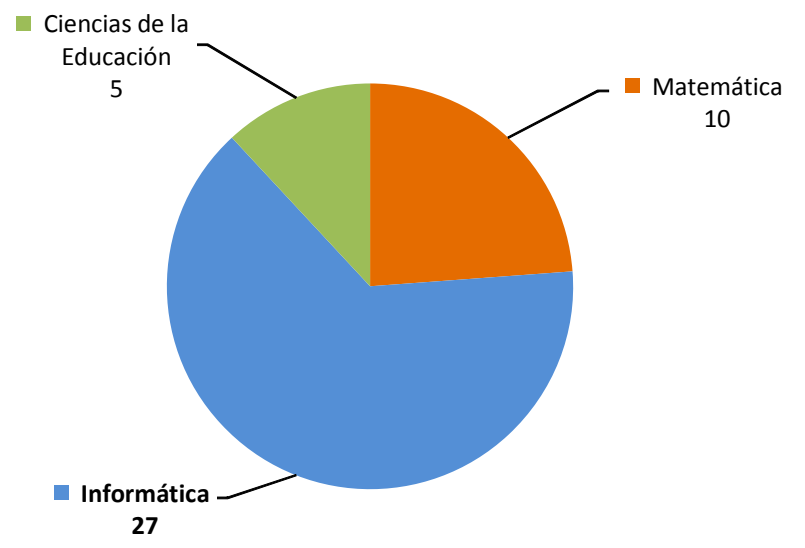

Figura 4.7. Las metodologías recurren a distintas disciplinas para su diseño.

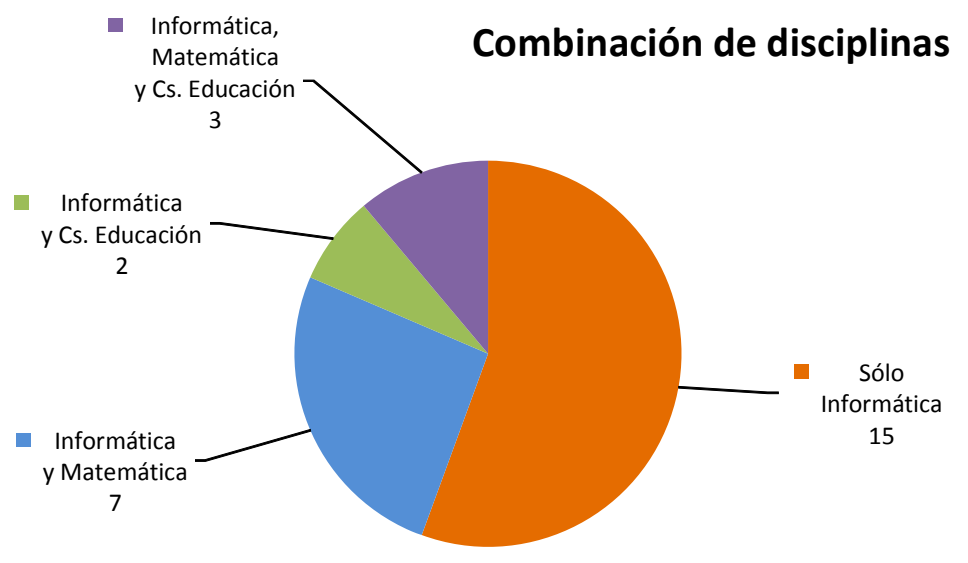

Figura 4.8. Presencia de las tres disciplinas analizadas en la construcción de la metodología. 


\subsubsection{El contexto de aplicación de la metodología}

Esta categoría, contiene criterios vinculados al contexto en el que se aplica la metodología. El primero de ellos, b.i, se enfoca en los destinatarios de la misma (ver Tabla 7 en Anexo II, gráficamente en Figura 4.9). Casi la mitad (14:27) de las metodologías, analizadas en este estudio, fueron diseñadas para ser utilizadas tanto por docentes como por estudiantes. Es decir, permiten tanto la creación del itinerario, como su utilización por parte del estudiante. De las 13 restantes, 7:27 se enfocan únicamente en los docentes (como herramienta de autor) y 6:27 sólo en los estudiantes.

\section{Los destinatarios}

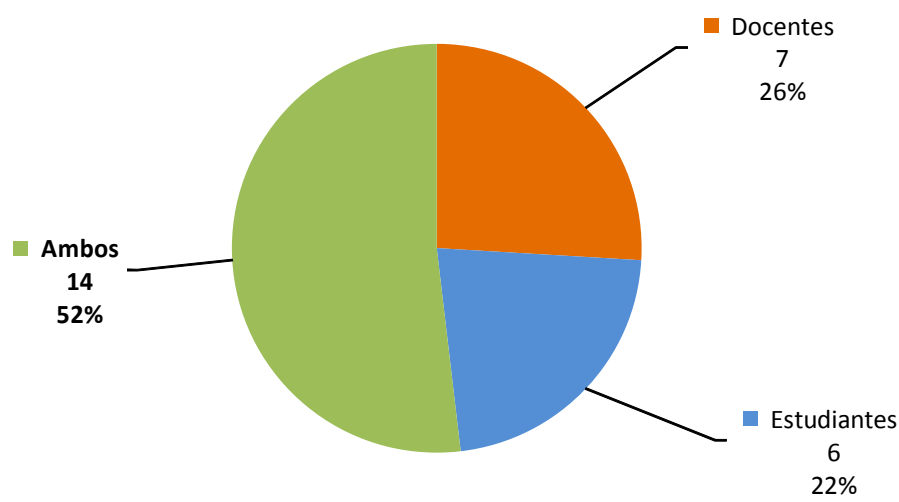

Figura 4.9. Destinatarios de la metodología.

El segundo criterio, b.ii, complementa al anterior y avanza sobre el nivel de proactividad que le permite, la metodología, a los destinatarios. En la Tabla 8 (Anexo II) se puede observar que se le da, mayoritariamente, al docente un rol activo (17:27), mientras que el estudiante tiene un rol pasivo (16:27). Esto implica que existe un mayor nivel de automatización de cara a los estudiantes y, viceversa, una mayor dependencia de la proactividad de los docentes.

Al hacer foco sobre las 14 metodologías que tienen como destinatarios a ambos usuarios, y combinarlas con los resultados obtenidos de la aplicación de este criterio, puede observarse (Figura 4.10) que los docentes tienen un rol activo (7:13 y 3:13). Sólo en 3:13 de los casos, los estudiantes están activos, en el resto, los estudiantes son pasivos (7:13 y 4:13). Esto vuelve a mostrar la tendencia de que las metodologías tienen un alto nivel de dependencia de los docentes y ofrecen un alto nivel de automatización a los estudiantes. Cabe destacar que S03, S14, S30, S31 son las únicas cuatro metodologías que ofrecen un alto nivel de automatización al docente y como se aprecia en la gráfica, hacen lo propio con los estudiantes (DPEP).

Las 8 metodologías que se enfocan únicamente en los docentes (ver Tabla 8, Anexo II) el rol del docente es activo. Mientras que en las centradas en el estudiante (6:27) dos proponen un estudiante activo y las cuatro restantes pasivo.

El tercer criterio, b.iii, indaga sobre dónde recarga cada metodología el aspecto pedagógico/didáctico: en el material, en los usuarios o en ambos. La aplicación de este criterio (ver Tabla 9, Anexo II y Figura 4.11), muestra que la mayor proporción la tienen los usuarios (9:27 y 12:27). Los aspectos tenidos en cuenta por las metodologías que se enfocan en aspecto pedagógicos asociados a los usuarios (etiquetados como "Usuario" y "Ambos"), podrían resumirse en: su estilo de aprendizaje (S01 y S30), su conocimiento previo (S13, S14, S17 y S27) y sus objetivos de aprendizaje (S28 y S32). Otros lo manifiestan de forma más genérica a través de sus preferencias (S16, S20, S21, S25 y S28) o necesidades (S08 y S23). Algunos incluyen más de uno de estos aspectos: S25 tiene en cuenta el conocimiento previo y las competencias, S16 y S20 el conocimiento previo y los objetivos de aprendizaje.

Respecto de los que se enfocan en los materiales (teniendo en cuenta o no al usuario) requieren de competencias a abordar, duración, nivel dificultad, tipo de material, requisitos previos. Cabe 
destacar que el uso de pre-requisitos, asociados a los OA, es utilizado por varias de las metodologías (S06, S07, S08, S18, S25, S28, S30 y S32).

\section{Proactividad de los usuarios}

(Ambos destinatarios)

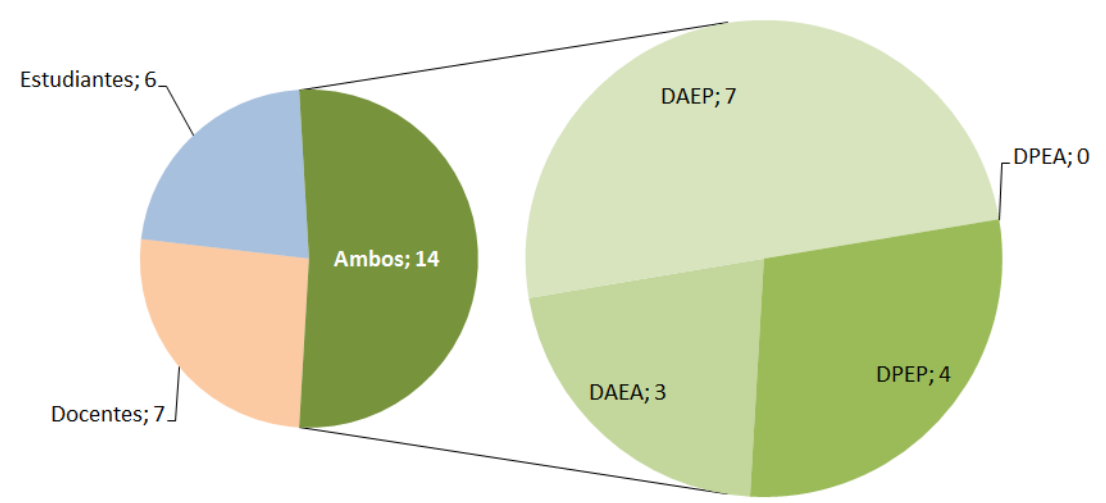

Figura 4.10. Nivel de actividad para metodologías destinadas a estudiantes y docentes. DA:Docente Activo - DP:Docente Pasivo - EA:Estudiante Activo - EP:Estudiante Pasivo

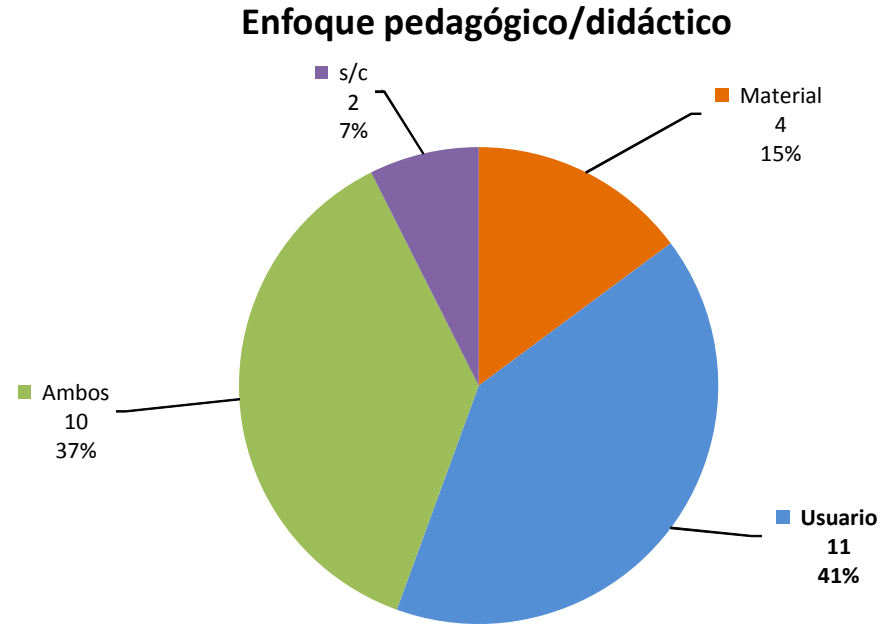

Figura 4.11. De qué forma las metodologías abordan el aspecto pedagógico.

El cuarto criterio, $b . i v$, pone el foco sobre la evaluación de las metodologías. En la Figura 4.12 (ver también Tabla 10, Anexo II) se observa que, según las publicaciones analizadas en esta tesis, en 6:27 de las metodologías no se encontró evidencia de que le hayan realizado pruebas para su evaluación. Además, de las 21 restantes, a la mayoría de ellas le hicieron pruebas de laboratorio (13:21); mientras que en 8:27 manifiestan haber realizado estudios de caso para evaluarlas. De las ocho, tres lo hicieron sobre docentes y cinco estudiantes.

De tres estudios de caso realizados sobre docentes: S39 lo hizo sobre 20 docentes de nivel universitario que generaron tres lecciones cada uno, utilizando LAMS; S35, por su parte, llevó adelante el estudio con 10 docentes, también de educación superior universitaria, los que generaron, cada uno, dos OA utilizando la herramienta $\mathrm{AGORA}^{7}$; S01, en tanto, desarrolló materiales para estudiantes de matemática de una escuela secundaria y seis módulos para informática de la salud para CANARIE Network, pero no se especifica la cantidad de docentes que participaron, ni sus características. Habría que agregar aquí a S31 (representado en el gráfico de la Figura 4.12 con su estudio a estudiantes) que realizó el estudio de caso incluyendo ambos actores, para los docentes que involucró a 23 docentes universitarios (no ofrece detalles sobre el material).

Para los cinco que realizaron estudios de casos con estudiantes se muestra un resumen en la Tabla 4.4 .

7 AGORA implementa la metodología propuesta por S35. Disponible en: http://smile.esi.uclm.es/agora/. 
Tabla 4.4. Estudio de caso realizados con estudiantes.

\begin{tabular}{cl}
\hline Autores & \multicolumn{1}{c}{ Estudio de Caso } \\
\hline S07 & Realizado sobre 114 empleados de IBM. \\
S25 & Las pruebas fueron realizadas sobre 10 estudiantes universitarios (para aprender JAVA). \\
S28 & Un curso complementario sobre Inteligencia Artificial para ocho estudiantes de doctorado. \\
S30 & El estudio se hizo sobre 27 estudiantes universitarios de la asignatura Sistemas de información \\
& inteligentes. \\
S31 & El estudio se hizo sobre 57 estudiantes de segundo año de la Universidad de Taiwan. \\
\hline
\end{tabular}

\section{Evaluación de la metodología}

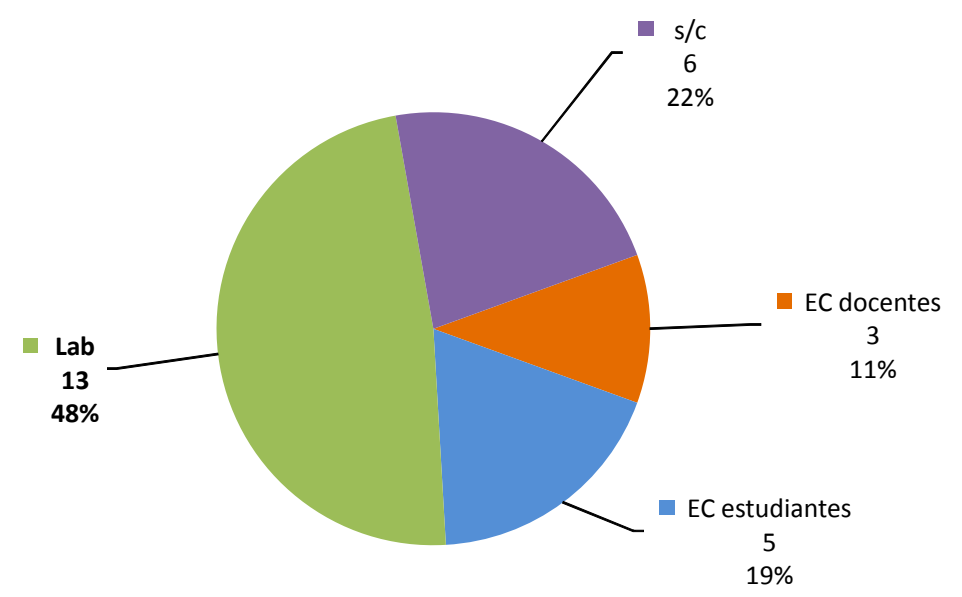

Figura 4.12. Las distintas estrategias de evaluación de las metodologías.

\subsubsection{El software que implementa la metodología}

En esta sub-sección se muestra la aplicación de los criterios pertenecientes a la categoría Software. Se busca caracterizar las aplicaciones informáticas (SE) que se desarrollaron basadas en las distintas metodologías.

El primer criterio, c.i, permite distinguir las metodologías que realizaron una aplicación informática, de las que no. Cabe aclarar que éstas últimas son identificadas como la etiqueta "s/c", dado que podría haberse desarrollado un software y no haber encontrado evidencia publicada de ello.

Como se puede observar en la Tabla 11 (Anexo II) y, gráficamente, en la Figura 4.13, casi la totalidad (25:27) de las metodologías cuentan con su correlato en un software que las implementa. De éstas, la mayoría son prototipos (16:25) y las nueve restantes manifiestan haber desarrollado aplicaciones que superaron esta etapa.

El segundo criterio, c.ii, que refina el anterior, se propone la disquisición entre aquellas aplicaciones informáticas desarrolladas para ser utilizadas desde la web y las de escritorio. Se puede notar (Tabla 12, Anexo II y Figura 4.14) que la mayoría de las aplicaciones informáticas se desarrollaron para la web (17:25), mientras que otras cinco deben utilizarse en un entorno de escritorio y, los tres restantes (S03, S14 y S20) no ofrecen datos a éste respecto. 


\section{Etapa de desarrollo}

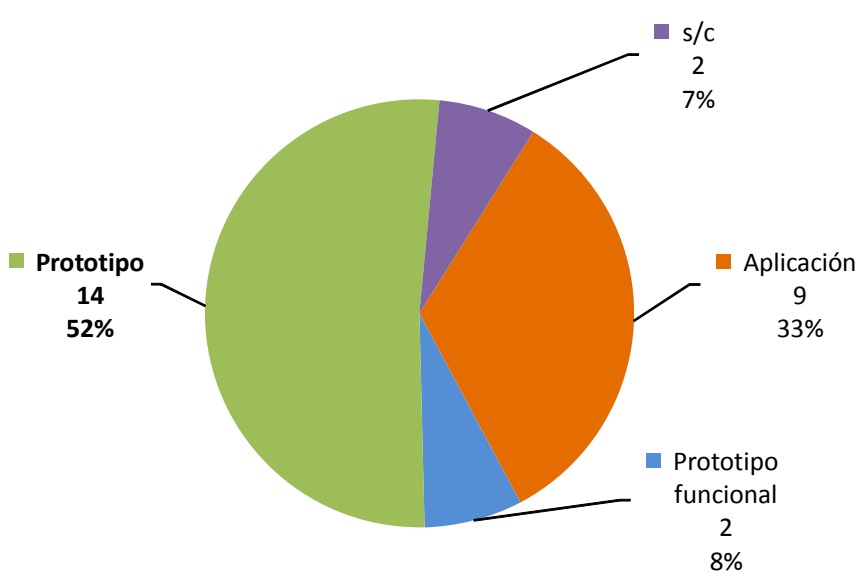

Figura 4.13. Implementación de las metodologías vía un software.

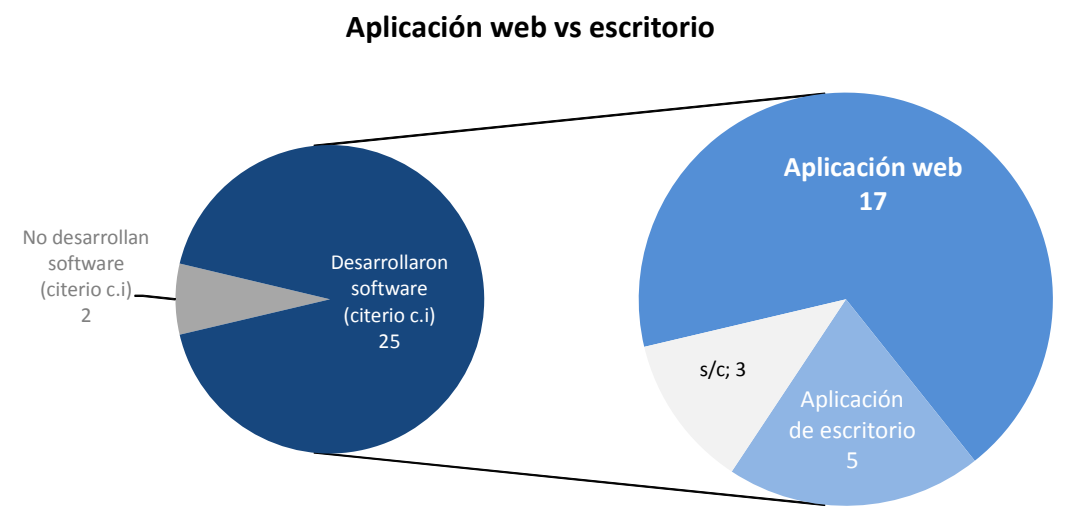

Figura 4.14. Disquisición entre aplicaciones web y de escritorio

El siguiente criterio (c.iii), establece si las aplicaciones informáticas desarrolladas están accesibles o no para ser utilizadas. De los $25 \mathrm{SE}$ desarrollados, sólo cinco (ver Tabla 4.5) están disponibles para ser utilizados (ver Tabla 13, Anexo II). Se trata, en todos los casos, de aplicaciones web. Cabe aclarar que sólo el prototipo funcional creado por S06 ofrece una experiencia en la que el usuario (docente en este caso) trabaja de forma directa con la metodología, mientras que para el resto, éste debe hacer uso de un sistema de e-learning al que se le incorporó la metodología. Esto es, en el primer caso el contacto del usuario con la metodología es directo, cuando en el resto debe interactuar, además, con otras funcionalidades del sistema.

De las 20 aplicaciones que no están disponibles (ver Figura 4.14), la mayoría (18:20) no ofrece información al respecto, es decir no se encontraron datos sobre cómo acceder al software. Las 12 aplicaciones web, o bien no ofrecen una URL o la que presentan en el artículo está inaccesible ${ }^{8}$ (ver Tabla 4.5).

El cuarto criterio, c.iv, avanza sobre la necesidad de credenciales (ver Tabla 14, Anexo II). De las 25 aplicaciones informáticas desarrolladas se desprende, del análisis de los textos, que la mayoría (13:25) no requieren de autenticación para acceder a al software. Las 12 restantes, indican que harán un seguimiento del usuario y por tanto solicitan autenticación (ver Figura 4.15).

Respecto a las cinco aplicaciones accesibles, dos de ellas no necesitan credenciales (S04 y S06), mientras que las otras tres (S17, S32 y S35) requieren contar con un usuario registrado. Cabe aclarar que S32, que requiere de credenciales, permite ver el material sin ellas y el usuario es requerido para editar y para el seguimiento.

$8 \mathrm{Al}$ intentar acceder el navegador muestra un error 404. 
Tabla 4.5. URL de las aplicaciones accesibles ${ }^{9}$

\begin{tabular}{cl}
\hline \multicolumn{1}{c}{ ID } & \multicolumn{1}{c}{ URL } \\
\hline S04 & http://www.edutic.ua.es \\
S06 & http://solaris.fdi.ucm.es:40000/elo-toolv1/index.html \\
S17 & http://www.tele-task.de/ \\
S32 & http://demo.activemath.org \\
S35 & http://smile.esi.uclm.es/agora/ \\
\hline
\end{tabular}

Tabla 4.6. Aplicaciones no accesibles

\begin{tabular}{cl}
\hline \multicolumn{1}{c}{ ID } & \multicolumn{1}{c}{ Tipo de error } \\
\hline S01 & NO URL \\
S05 & Inaccesible (error 404) \\
S07 & NO URL \\
S15 & Servicio $\left({ }^{*}\right)$ \\
S16 & NO URL \\
S18 & NO URL \\
S23 & NO URL \\
S24 & Inaccesible (error 404) \\
S25 & NO URL \\
S30 & NO URL \\
S31 & NO URL \\
S39 & NO URL \\
(*) Crean un servicio, pero no $^{\text {indican dónde está publicado. }}$
\end{tabular}

\section{Necesidad de credenciales}

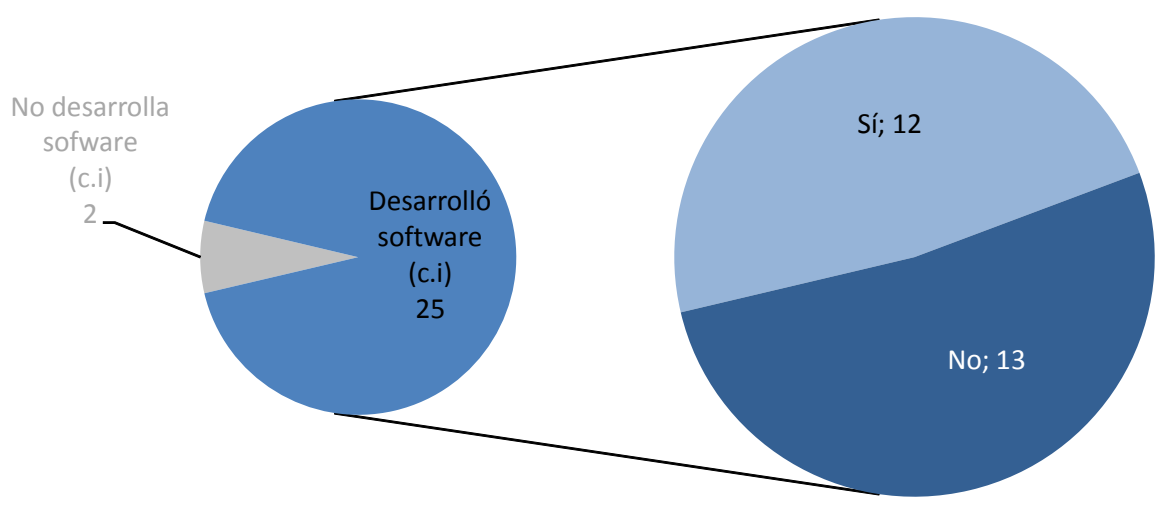

Figura 4.15. Necesidad de credenciales, criterio aplicado sobre las metodologías que desarrollaron software.

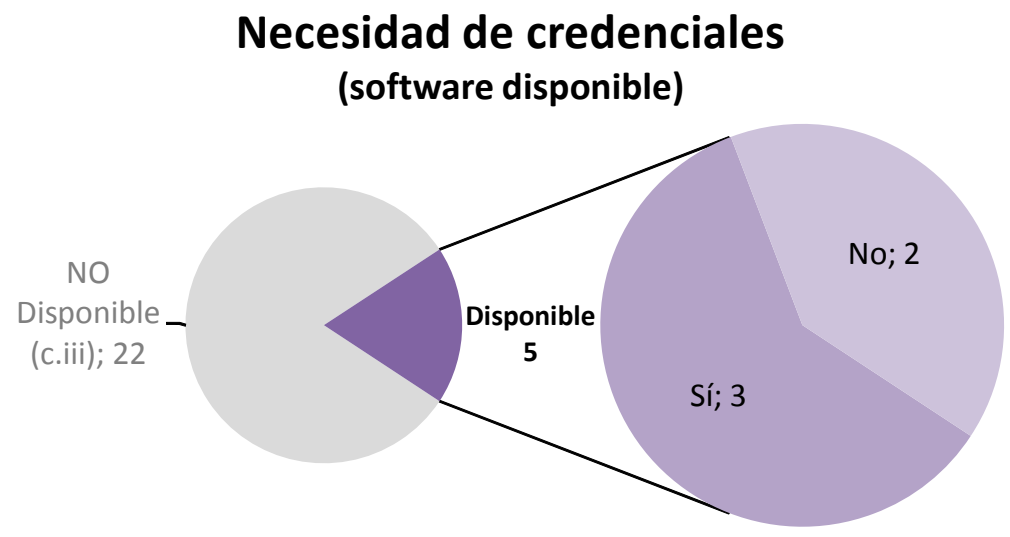

Figura 4.16. Necesidad de credenciales, criterio aplicado sobre el software accesible.

En el quinto criterio, c.v, se intenta identificar la fuente de los materiales (ver Tabla 15, Anexo II). Es decir, desde dónde se obtienen los OA que formarán parte de los itinerarios. Se indaga, si se trata

9 Recuperadas por última vez el 3 de febrero de 2016. 
de un repositorio local que aloja los OA o si los mismos están localizados en otros repositorios vía Internet. Si se observa la Figura 4.17, no hay una tendencia, se reparten prácticamente en iguales proporciones el trabajo con OA locales (13:25) y externos (12:25). De los 12, que manifiestan el uso de OA de fuentes externas, siete (S03, S05, S13, S15, S28, S32 y S35) lo expresan de forma genérica con frases como "en repositorios" o "en la web", mientras que cinco (S06, S07, S08, S14 y S23) expresan que utilizan XML para describir los OA y, sólo dos dan nombres concretos sobre la fuente de datos con la que trabajan: S04 afirma obtener el material desde diez repositorios externos, entre ellos: CAREO ${ }^{10}$, IDEAS ${ }^{11}$ o Applet de Java para Matemática ${ }^{12}$; mientras que S39 de GLOBE ${ }^{13}$, WikiAnswers $^{14}$, ProProfs ${ }^{15}$ y Wikipedia ${ }^{16}$.

\section{Fuente de los materiales}

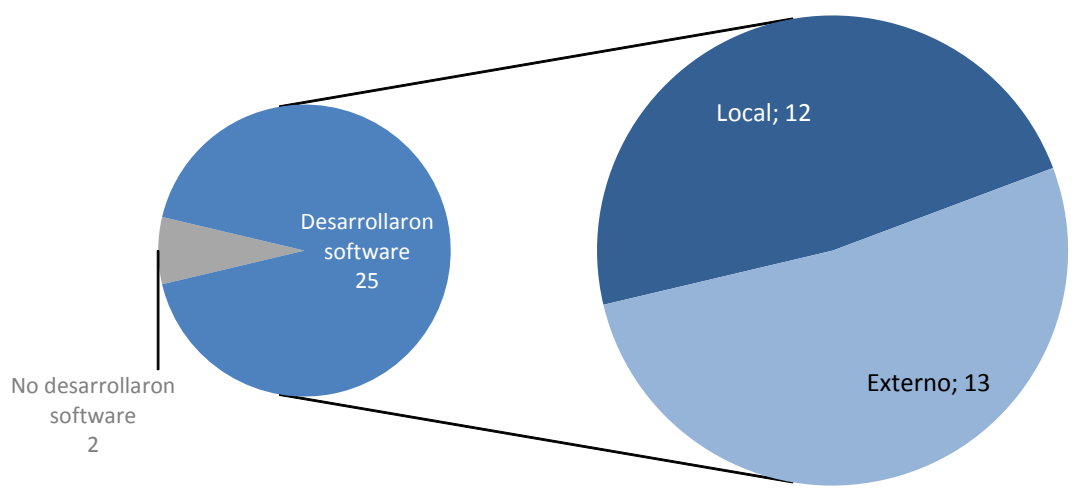

Figura 4.17. Fuente de datos de las 25 metodologías que desarrollaron software.

En el ante-último criterio (c.vi), se revisa qué estándar de metadatos utiliza cada metodología (ver Tabla 16, Anexo II). Al detenerse en la Figura 4.18, la gráfica muestra que más de la mitad de las metodologías utilizan el estándar LOM (16:27) y de éstas, hay seis que lo extienden (S06, S07, S15, S20, S27 y S28). Mientras que sólo una (S30) manifiesta usar DCMI. Se muestra, también, una importante cantidad (10:27) de metodologías que no definen con qué estándar trabajan, de éstas, seis (S08, S14, S18, S25, S32 y S38) manifiestan utilizar metadatos y las cuatro restantes no mencionan el tema.

\section{Estándar de metadatos utilizados}

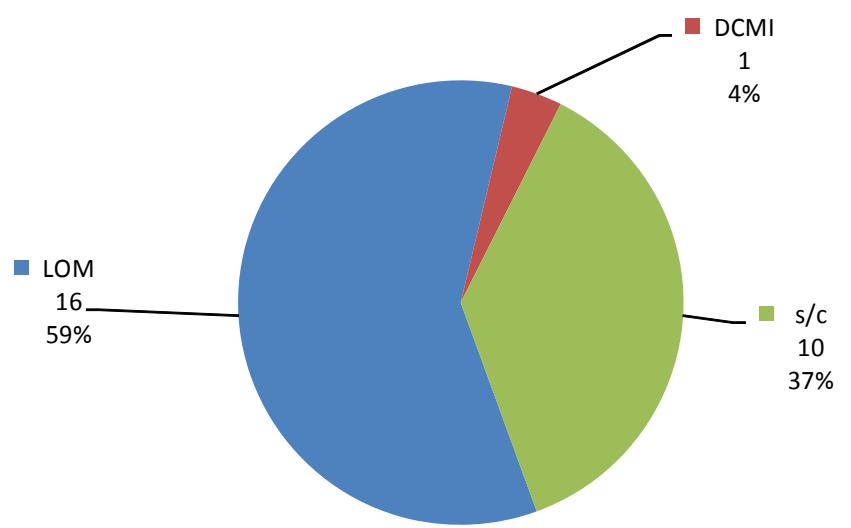

Figura 4.18. Estándar de metadatos utilizados por las metodologías en sus implementaciones.

10 Campus Alberta Repository of Educational Objects. Disponible en: http://www.careo.org/.

11 Interactive Dialogue with Educators from Across of State. Disponible en: http://ideas.wisconsin.edu/.

12 Disponible en: http://www.walter-fendt.de/m14s/index.html.

13 Federación Mundial de Repositorios de Objetos de Aprendizaje (GLOBE): http://www.globe-info.org/.

14 Disponible en: http://www.answers.com/.

15 Disponible en: http://www.proprofs.com/.

16 Disponible en: https://www.wikipedia.org/. 
Finalmente, se presenta la aplicación del criterio c.vii, en el cual se identifica para cada metodología qué modelo de empaquetamiento utiliza (ver Tabla 17, Anexo II). En la Figura 4.19, se puede observar que una importante cantidad (11:27) utilizan un estándar para OA (8 SCORM y 3 IMS$\mathrm{CP}$ ), en la misma proporción aparecen las que utilizan otros modelos $\mathrm{y}$, para los cinco restantes, no se pudo encontrar información a este respecto.

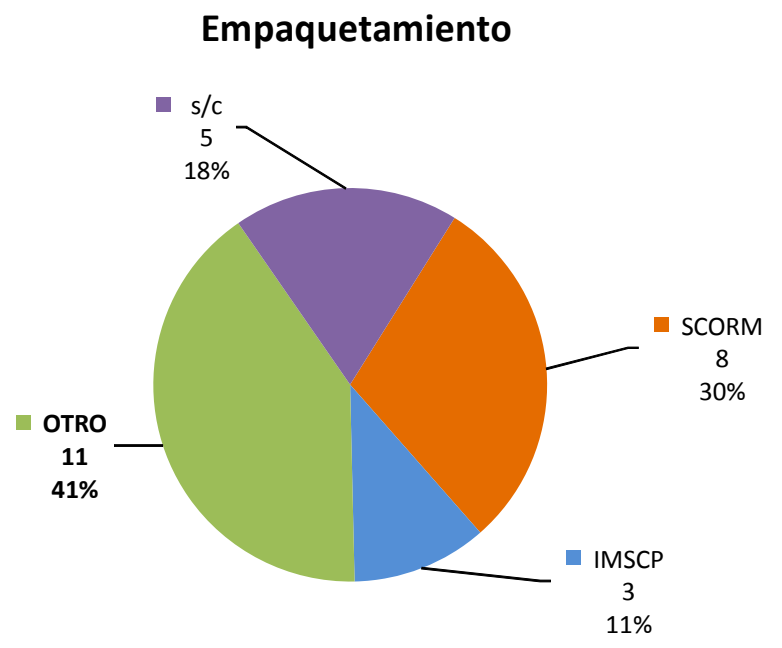

Figura 4.19. Tipo de material utilizado por las metodologías

De las cinco metodologías que tienen disponibles el software para utilizar, sólo una (S35) utiliza un estándar (SCORM), las restantes: S04 utiliza webQuest; S06 define ELO pudiendo referenciarse todo tipo de material digital disponible en la web, desde contenido crudo (imágenes, documentos, sonidos, etc.) hasta la creación de unidades didácticas; S17, por su parte, utiliza lo que denomina "lecture subparts", que de la lectura se desprende que se trata de diapositivas correspondientes a exposiciones de docentes; mientras que S32 utiliza un tipo de material que denomina libro, sobre el que no se brindan mayores detalles ${ }^{17}$.

\subsection{Selección de una metodología para el estudio de caso}

Uno de los objetivos del análisis realizado de las metodologías, a partir de los criterios, es elegir la aplicación para el estudio de caso. Para llevar adelante esta selección, se busca una metodología enfocada principalmente en el ensamblaje y, preferentemente, que incluya a los docentes, dado que es de interés para esta investigación conocer la opinión de los docentes sobre el uso de una metodología de ensamblaje para el diseño de materiales educativos. Asimismo, sería deseable que cuente con una aplicación web y haga uso de estándares tanto para el etiquetado (si lo implementa), como para el empaquetado de los OA.

A continuación, se hace una caracterización de las metodologías candidatas a la aplicación del estudio con base al conjunto de criterios definidos en este capítulo.

Cabe aclarar que:

- Dado que es necesario, para llevar adelante el estudio de caso, contar con un Sistema Ensamblador que implemente la metodología, se realizó un primer filtrado por accesibilidad (criterio c.iii). De forma tal que se caracterizan a continuación, únicamente las cinco metodologías que cumplen con este criterio (ver Tabla 4.5).

- Para la caracterización no se tuvieron en cuenta los criterios a.ii y a.vi por considerar que la motivación y la técnica en la que se basa la metodología tendrían poco valor en la selección de la metodología para este estudio de caso.

17 Para más información sobre sobre el formato y cómo construir un libro, pude acceder a la ayuda del software: http://demo.activemath.org/ActiveMath2/main/help.cmd. 


\section{S04. Roig Vila}

Esta metodología propone un SE que utiliza patrones basados en webQuest, donde el docente, con un rol activo, crea manualmente del itinerario de aprendizaje con base en la información educativa asociada los OA. Esta metodología, asume un enfoque genérico para los OA, entendiéndolo como un material educativo digital reutilizable. El software que implementa la metodología es una aplicación web que no requiere de credenciales y utiliza una fuente externa para recuperar los OA que formarán parte del itinerario. No se obtuvieron datos del uso de estándares para el etiquetado de los OA y no utiliza estándares de empaquetamiento. No hay registro sobre la evaluación de la metodología en las publicaciones revisadas.

\section{S06. Santacruz Valencia, Delgado Kloos \& Aedo Cuevas}

Esta metodología propone, también, un SE basado en el uso de los metadatos asociados a los OA y ontologías de dominio para la creación, semi-automática, del itinerario de aprendizaje. Está centrada en el docente, quien tiene un rol activo. El material es quien reviste las características educacionales. La metodología tiene una apropiada caracterización de los OA, entendiéndolos como material educativo digital, reutilizable e interoperable, que cuenta con una estructura interna y metadatos que permiten su etiquetado y su ensamblaje. El software que implementa la metodología es un prototipo totalmente funcional diseñado para la web que no requiere de credenciales para su uso. Los OA se encuentran en almacenes externos y utiliza una extensión del estándar LOM para el etiquetado. No utiliza estándares de empaquetamiento. La metodología sólo fue evaluada en pruebas de laboratorio.

\section{S17. Karam, Linckels \& Meinel}

Esta metodología propone un SR de itinerarios de aprendizaje. Esta centrada en los estudiantes, que utiliza los metadatos de estos usuarios y ontologías de dominio para la recomendación, automática, del itinerario de aprendizaje. Propone un rol de usuario es pasivo. El software que implementa la metodología es un prototipo funcional diseñado para la web que requiere de la creación de un usuario para contar con un perfil pedagógico del estudiante. No hay registro del uso de estándares de metadatos y no utiliza empaquetamiento. La aplicación hace uso de un repositorio local donde almacena los OA que utiliza en los itinerarios. Los OA son caracterizados, genéricamente, como un material educativo digital reutilizable y ensamblable. La metodología sólo fue evaluada en pruebas de laboratorio.

\section{S32. Ullrich \& Melis}

Esta metodología propone un SR de itinerarios de aprendizaje, semi-automático, con funcionalidades de autor para docentes (usuario activo) y de uso para los estudiantes (usuario pasivo). Utiliza los metadatos asociados a los OA, así como los datos de los estudiantes para recomendar el material e ir formando, así, el itinerario de aprendizaje. La metodología se implementa a través de un prototipo web que necesita del registro del docente para crear los itinerarios y del estudiante para poder realizar las sugerencias en función del perfil de éste (aunque pueden usarse los itinerarios sin loguearse al sistema). La aplicación utiliza un repositorio externo donde almacenan los OA que utiliza en los itinerarios. No hay registro del uso de estándares de metadatos y no utiliza empaquetamiento. Los OA son caracterizados, en el contexto de esta metodología, como un material educativo digital reutilizable, accesible, auto-contenido y etiquetado con metadatos. La metodología sólo fue evaluada en pruebas de laboratorio. Cabe aclarar que la temática del material está centrada en la matemática.

\section{S35. Menéndez et al.}

Esta metodología propone un SR de itinerarios de aprendizaje, semi-automático, centrado en los docentes, a los que otorga un rol activo para crear el itinerario de aprendizaje. No se encontraron datos sobre el principio de composición y tampoco, respecto a qué asocia las preferencias pedagógico/didácticas. Los OA son caracterizados por esta metodología como un material educativo digital reutilizable, interoperable, accesible y etiquetado con metadatos. Desarrollaron una aplicación web, que utiliza una fuente externa para obtener los OA y los estándares LOM (para los 
metadatos) y SCORM (para el empaquetamiento). Requiere credenciales para su uso. La metodología fue evaluada a través de un estudio de casos con docentes.

Para finalizar en esta sección, se presenta una tabla (ver Tabla 4.7) comparativa de las cinco metodologías, donde se resumen pros y contras de la elección de cada metodología para llevar adelante el estudio de caso. En la misma, puede observarse que la metodología S06 cuenta con la mayor cantidad de características que favorecen su elección para implementar el estudio de caso. Por lo tanto, esta metodología es la que se utilizará en este trabajo para llevar adelante dicho estudio.

Tabla 4.7. Comparativa para la selección de la metodología para el estudio de caso.

\begin{tabular}{ll}
\hline \multicolumn{1}{c}{ Metodología } & \multicolumn{1}{c}{ Pros } \\
\hline S04. Roig Vila & Esta metodología propone un SE \\
& El software no requiere de credenciales y \\
utiliza una fuente externa para recuperar & los OA \\
& \\
S06. Santacruz Valencia, Delgado & Esta metodología propone SE. \\
& Está centrada en el docente. La \\
& metodología tiene una apropiada \\
& caracterización de los OA. El software es \\
& un prototipo totalmente funcional \\
diseñado para la web que no requiere de & credenciales para su uso. \\
& Los OA se encuentran en almacenes \\
& externos. Utiliza una extensión del \\
& estándar LOM para el etiquetado. \\
& La metodología fue evaluada en pruebas \\
de laboratorio.
\end{tabular}

S17. Karam, Linckels \& Meinel El software es un prototipo funcional diseñado para la web.

La metodología fue evaluada en pruebas de laboratorio.

\section{S32. Ullrich \& Melis}

S35. Menéndez et al.
Funcionalidades de autor para docentes (usuario activo) y de uso para los estudiantes (usuario pasivo).

El software es un prototipo web.

La aplicación utiliza un repositorio externo.

Los OA están caracterizados apropiadamente.

La metodología fue evaluada en pruebas de laboratorio.

Los OA son caracterizados apropiadamente.

Desarrollaron una aplicación web, que utiliza una fuente externa para obtener los OA y los estándares LOM (para los metadatos) y SCORM (para el empaquetamiento).

La metodología fue evaluada a través de un estudio de casos con docentes.
Propone un SR centrado en los estudiantes. Requiere de la creación de un usuario. No hay registro del uso de estándares de metadatos y no utiliza empaquetamiento. La aplicación utiliza un repositorio local. Los OA son caracterizados genéricamente.

Necesita del registro del docente para crear materia y del estudiante.

No hay registro del uso de estándares de metadatos y no utiliza empaquetamiento.

La temática del material está centrada en la matemática.

Esta metodología propone un SR

No se encontraron datos sobre el principio de composición y tampoco, respecto a qué asocia las preferencias pedagógico/didácticas.

Requiere credenciales para su uso. 


\subsection{Evolución y tendencias}

El ensamblaje de OA continúa siendo una línea de investigación activa, esto puede observarse en las publicaciones analizadas en la Sección 3.6.1.

Con base en dicho análisis se puede observar que los autores adoptan como línea de investigación las metodologías de tipo 3 (recomendadores de itinerarios de aprendizaje). Las mismas, son diseñadas, principalmente, utilizando ontologías y metadatos, y cuentan con el registro del perfil del usuario, esto continúa siendo una tendencia en los nuevos trabajos analizados.

Desde el punto de vista pedagógico, cabe destacar el uso de taxonomía de propuesta por Benjamin Bloom (o sus revisiones) para describir los objetivos de aprendizaje (3:7) y de la clasificación de estilos de aprendizaje hecha por Richard Felder (3:5).

Por otra parte, continúa sin establecerse qué es un OA, en el contexto de la metodología, para la mayoría de las publicaciones. Sólo 2:7 presentan una definición de OA.

Si bien la mayoría de las nuevas publicaciones analizadas (6:7) presentan la evaluación de las mismas, las aplicaciones que implementan las metodologías no están accesibles. Aquí se puede observar una tendencia a incorporar las metodologías de ensamblaje a los LMS. Esto se condice con una de las motivaciones que se observó impulsan la investigación en la temática: mejorar los sistemas de e-learning.

\subsection{Recapitulación y Síntesis}

En este capítulo se enunciaron y se aplicaron a cada una de las metodologías de ensamblaje de OA analizadas en esta tesis 17 criterios de análisis definidos en la marco de este trabajo. Los mismos, divididos en categorías, permiten la caracterización de las metodologías desde tres enfoques diferentes: estrategia de ensamblaje, contexto de aplicación y el software que la implementa (SE).

La aplicación de cada criterio, al interior de cada categoría, proporciona en sí mismo la posibilidad de realizar un análisis puntual de ese aspecto de metodología. Sin embargo, es posible, realizar un análisis tomando los resultados obtenidos en cada categoría.

Tomando toda la información de la categoría (a) Estrategia, podría ser de interés focalizar sobre la disquisición ensamblador-recomendador. Tomando, entonces, a.i como criterio inicial, se identifican dos grandes grupos: los ensambladores y los recomendadores (ver Figura 4.20). Ahora es posible, utilizando el resto de los criterios caracterizar estos dos sub-grupos.

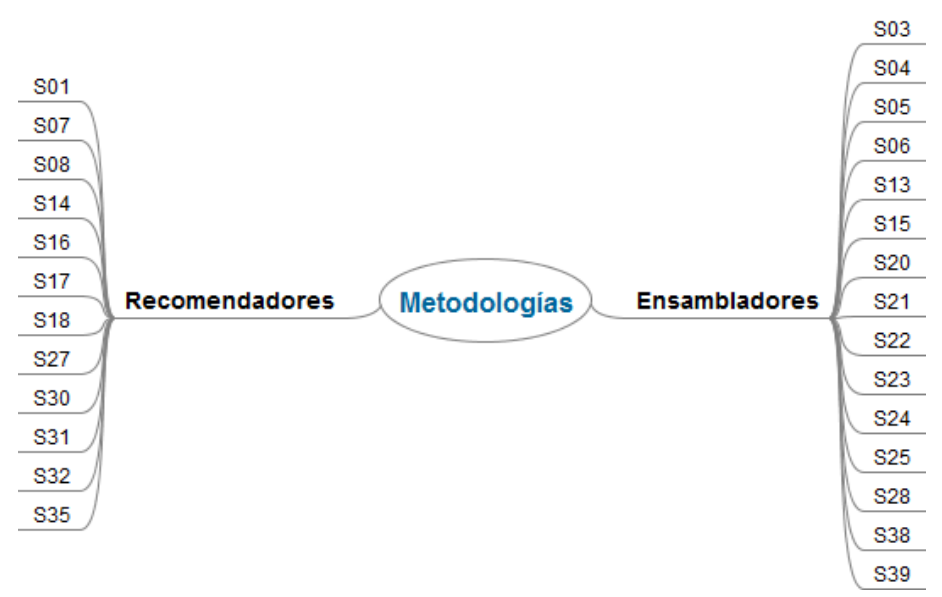

Figura 4.20. Aplicación del criterio a.i

Los SE analizados se crearon mayormente motivados por lograr la personalización en la distribución de contenidos educativos y el mejoramiento de las herramientas de e-learning a partir de funcionalidades que permitan crear itinerarios de aprendizaje con OA. Se sustentan, en general, en metodologías automáticas o semi-automáticas que utilizan técnicas informáticas que, basadas en metadatos y patrones, generan los itinerarios de aprendizaje con OA. Estos últimos son entendidos, 
por la mayoría de los autores revisados, como una material digital reutilizable e interoperable etiquetado con metadatos.

El segundo grupo, los recomendadores, sin una motivación que los aglutine, muchos hacen foco en una mejor organización del conocimiento y la personalización del material educativo acorde a, por ejemplo, el perfil de los estudiantes. Se apoyan en la Informática y la Matemática (principalmente la probabilidad) para proponer metodologías automáticas que recomienden el itinerario de aprendizaje con base en los metadatos de usuarios y OA. Estos trabajos consideran a estos últimos como un material digital accesible y reutilizable etiquetado con metadatos.

Haciendo lo propio con la categoría (b) Contexto, es posible también hacer una clasificación de las metodologías en este aspecto. Si se toma como primer variable de análisis el criterio b.i Destinatarios, podría dividirse al conjunto de metodologías en tres grupos: las metodologías enfocadas en los estudiantes, las que lo hacen en los docentes y en ambos.

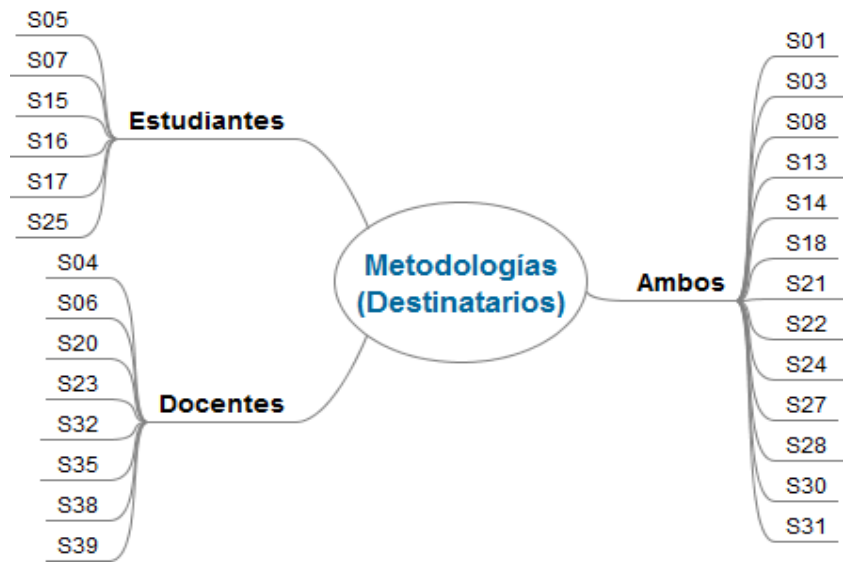

Figura 4.21: Agrupación de las metodologías por Destinatarios (criterio b.i)

Las primeras (centradas en el estudiante) muestran que, en general, proponen un estudiante pasivo. Cuentan con información de éste (conocimientos previos, objetivos de aprendizaje, estilo de aprendizaje, entre otros) y/o registran el seguimiento de las actividades realizadas. Esto requiere de un importante nivel de automatización del SE. Se han hecho, principalmente, pruebas de laboratorio, aunque hay algunos registros de estudios con estudiantes reales.

Cuando las metodologías se enfocan en los docentes, en general, proponen usuarios activos que participar en el diseño de los itinerarios de aprendizaje, con un software con función de apoyo. Para esto, las metodologías disponen de información sobre los aspectos pedagógico/didáctico del material. Se han hecho, principalmente, pruebas de laboratorio.

Las metodologías que plantean tener en cuenta ambos tipos de usuarios, proponen mayoritariamente un docente activo y un estudiante pasivo. Cuentan con información del perfil del estudiante y, en algunos casos, lo combinan con información del material. El software va a cumplir funciones de apoyo para que el docente publique los $\mathrm{OA}$ e indique las relaciones entre ellos, para que luego, el estudiante, haga uso de un itinerario generado automáticamente y basado en su perfil. Se han hecho, principalmente, pruebas de laboratorio, aunque hay registro de estudios con usuarios reales.

Finalmente, analizando los criterios agrupados en la categoría (c) Software, es posible definir distintos agrupamientos de las metodologías. En busca de analizar la accesibilidad de las aplicaciones desarrolladas podría hacerse un primer análisis con el criterio c.ii Web/Escritorio, y en una segunda instancia por c.iv Credenciales. Se formarían así cuatro grupos con distintos niveles de accesibilidad (ver Figura 4.22).

Las aplicaciones informáticas de escritorio, en general, no necesitan de credenciales para ser operadas y utilizan fuente de datos local. Respecto a los estándares: mayoritariamente trabajan con LOM y, si bien no con unanimidad, algunas usan OA en formatos estandarizados.

Las aplicaciones web, que requieren de credenciales para su uso, están principalmente en estado de 
prototipo, pero existen algunas ya publicadas. Utilizan, en general, fuentes locales para recuperar los materiales. Si bien algunas utilizan el estándar LOM, la mayoría no proporciona información a este respecto. Con relación al uso de empaquetamiento, no hay unanimidad, pero varios optan por SCORM. Sólo un número reducido de aplicaciones están accesibles, actualmente.

Las aplicaciones web que no hacen uso de credenciales, por su parte, están mayoritariamente en estado de prototipo. Utilizan fuentes externas para la recuperación de los OA que se incluirán en los itinerarios. La mayoría hace uso del estándar LOM y, si bien no es unánime, varias adoptan SCORM como base para la definición de su modelo de contenido. Aunque la mayoría de las aplicaciones no están disponibles para su uso, actualmente, hay algunas accesibles.

En el siguiente capítulo se describe, en detalle, el estudio de caso. Se presentan los objetivos del mismo, así como la metodología de investigación, las herramientas de recolección de datos y las preguntas de investigación.

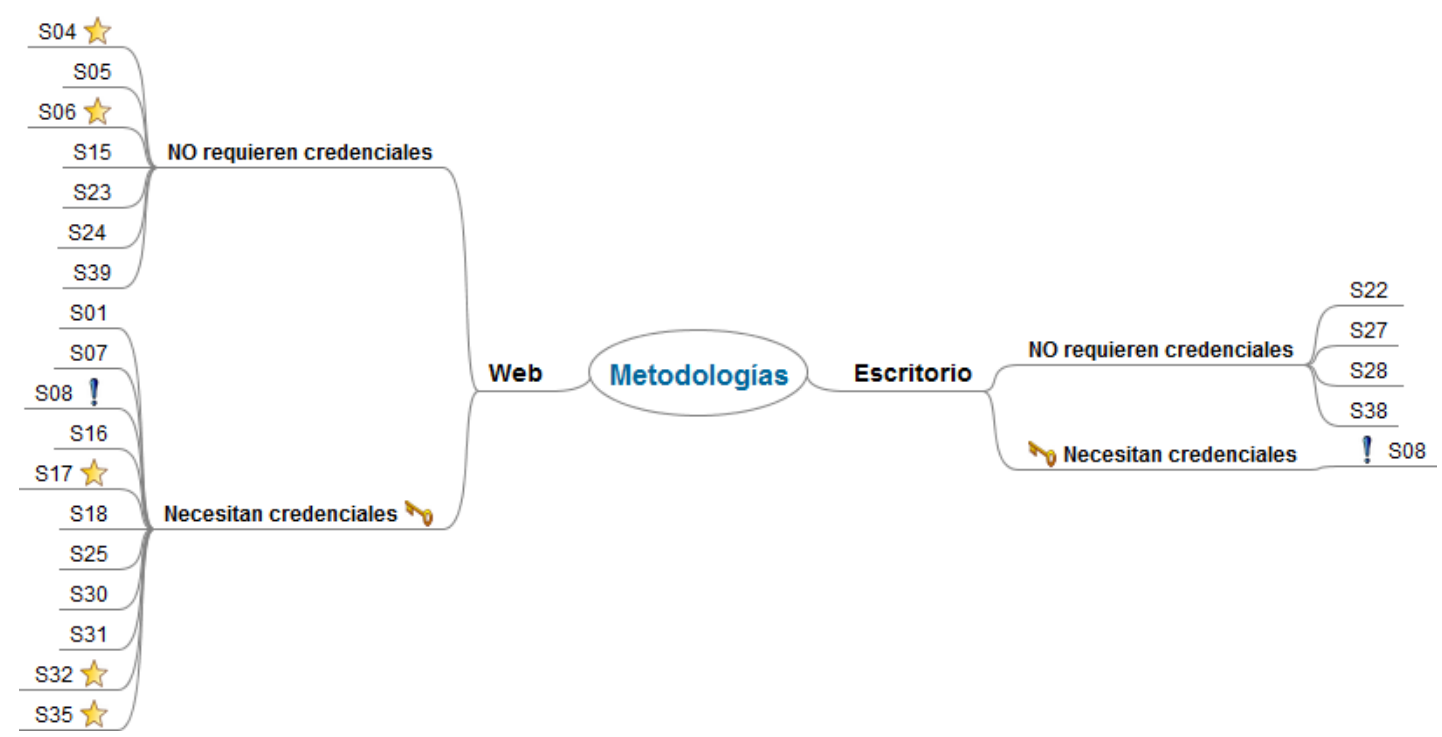

Figura 4.22. Accesibilidad del software desarrollado.

La estrella destaca el software que implementa la metodología pueden ser utilizado.

?

El signo de admiración indica que S08 está en dos categorías.

$\checkmark$ La llave indica uso de credenciales. 


\section{Estudio de Caso}

\section{RESUMEN}

Otro de los sub-objetivos de esta tesis es elegir un SE para llevar adelante un estudio de caso. Hecho esto, se implementó un taller de ensamblaje de OA para docentes universitarios que utilizaron la metodología y el SE ELO-Tool.

En este quinto capitulo se describe en detalle el estudio de caso y el taller sobre el que se bizo la implementación del mismo. Primeramente, se definen los objetivos, el contexto y la muestra con la que se trabajó. Luego, se describe la metodología de Investigación. Seguidamente, se presenta la selección del SE, la adaptación realizada para la implementación del taller y una descripción de éste. Se cierra con una recapitulación. 


\subsection{Introducción}

Como se puede observar en el estado del arte (Capítulos 2 y 3) existe una importante cantidad de metodologías de ensamblaje de OA. Las mismas cuentan, por lo general (ver Capítulo 4), con una aplicación que la implementa (de forma total o parcial). Si bien la implementación de un SE queda fuera del alcance de este trabajo, se llevó adelante un estudio de caso para la evaluación de una metodología y el software que la implementa.

Existen varias tipologías de estudios de caso, en este trabajo se eligió la evaluativa. Este tipo de estudio describe y explica, pero además se orienta a la formulación de juicios de valor que constituyan la base para tomar decisiones (Merriam, 1998). La elección está basada en la intencionalidad del presente estudio de evaluar tanto la metodología, como el software que la implementa, pero también realizar aportes que permitan el mejoramiento o evolución de ambos.

Para el estudio de caso se diseñó una secuencia de actividades que, se estima, debería llevar adelante un docente que desea generar un itinerario de aprendizaje a partir de OA, utilizando la metodología seleccionada y el software que la implementa. Adicionalmente, se diseñó un software que funciona como soporte de la herramienta seleccionada, posibilitando a los docentes visualizar los recursos OA e itinerarios de aprendizaje de forma más amigable y hacer su trabajo más palpable.

Para llevar adelante el estudio de caso se realizó un taller en el que se utilizó la herramienta de ensamblaje ELO-Tool ${ }^{1}$ para generar itinerarios de aprendizaje con base en el ensamblaje de diferentes OA. La temática utilizada para la generación de dichas secuencias de aprendizaje estuvo centrada en la programación de computadoras, contenidos que abordan las asignaturas Introducción a la Computación y Programación I².

A continuación, en este capítulo, se presentan los objetivos del estudio de caso, se define el contexto en el que se desarrolla y la muestra seleccionada. Seguidamente, se presenta la metodología de investigación, donde se describen: los procedimientos, los instrumentos de recolección de datos y variables de estudio. Luego, se justifica la elección de la herramienta utilizada para el estudio de caso y se explica el conjunto de adaptaciones realizadas para la implementación del mismo. Finalmente, se ofrecen los detalles del diseño del taller con base en la metodología seleccionada y a las adaptaciones realizadas a la misma. El capítulo cierra con una recapitulación.

\subsection{Objetivos}

Este estudio de caso permite evaluar la metodología de ensamblaje propuesta por SantacruzValencia et al. (2005) a través de la utilización de la herramienta ELO-Tool que la implementa. Los objetivos planteados para este estudio de caso son: $(i)$ conocer la opinión de algunos docentes sobre el posible impacto del ensamblaje de $\mathrm{OA}$ en la generación de itinerarios de aprendizaje, (ii) identificar fortalezas y debilidades de la metodología de ensamblaje seleccionada, (iii) evaluar el nivel de usabilidad de las herramientas de software utilizadas, tendientes a realizar recomendaciones para su mejora y (iv) generar un proceso metodológico de aplicación de la herramienta elegida, en este caso ELO-Tool, para ser utilizado por docentes del área de ciencias de la computación.

Particularmente se indagó si a los docentes participantes:

(i) la metodología les facilita el ensamblaje de OA.

(ii) la metodología les facilita la creación de itinerarios de aprendizaje con OA.

(iii) la adaptación propuesta les permite utilizar OA para la creación de itinerarios de aprendizaje.

(iv) el nivel de automatización propuesto por la metodología -e implementado en la

1 Disponible en: http://solaris.fdi.ucm.es:40000/elo-toolv1/index.html

2 Facultad de Ciencias Exactas y Naturales - UNLPam (La Pampa, Argentina) 
herramienta- les resulta suficiente para crear itinerarios de aprendizaje.

(v) la metodología les resulta adecuada para la reutilización de recursos educativos de distinta granularidad.

(vi) la metodología les resulta adecuada para la reutilización de los OA alojados en el repositorio de ELO-Tool.

(vii) ELO-Tool les ofrece un nivel aceptable de usabilidad.

(viii) ELOs-Viewer les ofrece un nivel aceptable de usabilidad.

\subsection{Definición del contexto y la muestra}

El diseño de MED, y el de OA en particular, continúa siendo tema de investigación para la comunidad científica internacional. Se busca, entre otros objetivos, facilitar el trabajo de docentes y diseñadores instruccionales en la compleja tarea de crear este tipo de materiales digitales.

Las investigaciones derivan, en muchos casos, en la creación de herramientas de software que implementan metodologías de ensamblaje. Así pues, las diferentes metodologías -descritas en la sección 3.6- tienen, en su mayoría, su correlato en un software que las implementa (ver 4.4.3).

Los SE, por lo general, han sido evaluados principalmente sobre casos de laboratorio (ver Tabla 10, Anexo II). Se hace necesario entonces, dado el avance de las investigaciones, llevar adelante estudios de caso que involucren actividades y materiales educativos concretos que permita la opinión de usuarios reales.

Para desarrollar las actividades que este tipo de investigación conlleva, se hace necesario contar con una aplicación que sea completamente funcional. En particular, para este trabajo de investigación, que permita el trabajo con OA como insumo para el ensamblaje. Ambos requerimientos favorecen la implementación de actividades concretas y con usuarios reales.

Para la definición de las temáticas específicas, abordadas en el taller, se contó con la participación de docentes y auxiliares de las asignaturas Introducción a la Computación y Programación I. Se tomó como eje la "programación estructurada", contenido común para ambas asignaturas.

El repositorio de ELO-Tool no contaba, al momento de la implementación de este estudio de caso, con un conjunto de OA que permitiera la correcta implementación del mismo. Se hizo necesario pues, la localización de recursos educativos, afines a la temática seleccionada, que permitieran la creación vía ensamblaje de los OA destinado al uso en el Taller.

Por tanto, se llevó adelante la búsqueda (en la web) de los recursos educativos ${ }^{3}$ relacionados con la temática "Programación Estructurada", así como el posterior almacenamiento de sus metadatos en el repositorio de ELO-Tool.

Como resultado de la búsqueda, se localizaron más de 120 recursos y se crearon ad hoc otros 27 . Del total, 49 fueron alojados en el repositorio de la herramienta. Los mismos permitieron, vía las opciones de ensamblaje, la creación de OA y, posteriormente durante el taller, la generación de itinerarios de aprendizaje ajustados al currículo de las asignaturas involucradas.

Con base en el conjunto de recursos educativos alojados en el repositorio de ELO-Tool, se llevó adelante, en un proceso previo al Taller, la creación de OA -vía el proceso de ensamblaje-. En el desarrollo del Taller, los docentes, pudieron crear itinerarios de aprendizaje utilizando dichos OA. Esta decisión permitió centrar el trabajo de los participantes en el ensamblaje de OA. Así se evitó que perdieran el foco del estudio con las tareas previas, que fueron realizadas por el autor de este trabajo.

Fijar una temática, por su parte, permitió que el material localizado tuviera entre sí una vinculación.

3 Cabe aclarar que se trata de "cualquier material [digital] que, en un contexto educativo determinado, sea utilizado con una finalidad didáctica o para facilitar el desarrollo de las actividades formativas" (Marquès, 2011) y no constituyen en si mismos un OA. 
Se procuró que los recursos obtenidos -además de focalizarse en la temática- al ser ensamblados pudieran ser abordados por los estudiantes de las asignaturas involucradas. Esto es, se tuvieron en cuenta las concepciones previas, pero además, que los requerimientos para abordar los OA pudieran ser cubiertos por las competencias alcanzadas a partir del abordaje de otro objeto (o una combinación de éstos). Por tanto, las competencias alcanzadas al utilizar un OA ofrecerían los conocimientos necesarios para el abordaje del próximo en una secuencia de aprendizaje, permitiendo así su ensamblaje de acuerdo a la metodología.

Para la obtención de la muestra se realizó un muestreo por conveniencia ${ }^{4}$. Esto permitió definir los participantes del taller sobre el que se realizaron las observaciones para este estudio de caso. Los individuos seleccionados pertenecen a las categorías: (i) "docente de Introducción a la Computación" y (ii) "docente de Programación I". La selección de la muestra abarcó todos los docentes de ambas categorías.

Participaron del taller cinco docentes, de entre 40-50 años de edad, tres mujeres y dos varones. El grupo se conformó de dos profesores y tres auxiliares. La muestra incluyó individuos con formación en matemática y en informática, dos con formación docente, un Profesor de Matemática y un Profesor en Matemática y Computación; y tres con formación informática pura, un Licenciado en Informática, un Ingeniero en Sistemas y un Analista de Sistemas. Todos con más de 15 años de experiencia en docencia universitaria. Si bien se trata de una muestra pequeña, permitió conocer la opinión de un grupo de docentes representativo.

Las asignaturas mencionadas abordan temas relacionados -además de ser correlativas-, lo que permitió la implementación de actividades comunes y dio lugar a la creación de itinerarios de aprendizaje tanto intra-cátedra, como inter-cátedra.

El taller se realizó durante el mes de agosto de 2015 en aulas de la Facultad de Ciencias Exactas y Naturales (UNLPam), sita en la ciudad de Santa Rosa, La Pampa, Argentina.

\subsection{Metodología de Investigación}

De la considerable variedad de métodos que pueden ser empleados en una investigación de estudio de caso para la recogida de datos, en esta ocasión, se focalizó sobre: la entrevista, la encuesta y la observación participante.

La entrevista y la observación participante son métodos que se complementan. A través de la primera es posible recabar la opinión de aquellos involucrados en la experiencia, "conocer y captar lo que un informante piensa y cree, cómo interpreta su mundo y qué significados utiliza y maneja; pero no [...] asegura que lo expresado verbalmente sea el contenido de su acción" (LACE, 1999, p. 16).

La observación participante, por su parte, permite registrar las acciones en su propio contexto. Este método de observación necesita que el investigador se involucre en "el ambiente cotidiano de los sujetos e informantes, recogiendo datos de un modo sistemático y no intrusivo." (Taylor \& Bogdan, 1986, p. 31). En este estudio, este aspecto estuvo cubierto en virtud de que los sujetos informantes fueron -y son- colegas con los que se tiene en común más de diez años ininterrumpidos de trabajo docente. Esto permitió, como afirman Cohen \& Lawrence (2002, p. 168) "desarrollar una relación íntima e informal" con aquellos a quienes se estaba observando.

La relación establecida con los sujetos observados permite una mayor relajación y contribuye a construir un ambiente natural, de forma que las observaciones se correspondan con el trabajo cotidiano de los informantes. Es así que la función como docente del taller derivó en lo que se

4 Muestreo por conveniencia o intencional. "Consiste en elegir los individuos más próximos para servir como informantes" (Cohen \& Lawrence, 2002, p. 138). Este tipo de muestreo se caracteriza por un esfuerzo deliberado de obtener muestras representativas mediante la inclusión en la muestra de grupos típicos. También puede ser que el investigador seleccione directa e intencionadamente los individuos de la población. El caso más frecuente de este procedimiento es utilizar como muestra a los individuos a los que se tiene fácil acceso (los profesores de universidad emplean con mucha frecuencia a sus propios alumnos) (Larios Rodríguez \& Figueroa Preciado, 2008). 
denomina una participación moderada, es decir "aquella en la que se mantiene un equilibrio entre estar dentro y fuera de la situación, entre participar y observar." (LACE, 1999, p. 19).

\subsubsection{Procedimientos. Momentos del Taller}

El taller se desarrolló en dos sesiones o momentos:

1. Metodología de ensamblaje (45')

En una primera sesión, se presentó la metodología de ensamblaje con la que trabaja ELOTool. El tipo de material con el que se construyeron los OA con los que se trabajaría en la sesión de ensamblaje. Así como la función de los metadatos y las ontologías en el contexto de la metodología.

Esta sesión también se utilizó para explicar las adaptaciones que se realizaron para la implementación del estudio de caso en función de la definición de Objeto de Aprendizaje adoptada en esta tesis.

\subsection{Familiarización con las herramientas y el contenido (60')}

En la segunda sesión, se realizó un proceso exploratorio tanto de ELO-Tool, como de ELOsViewer donde los participantes se familiarizaron con las mismas y con el material del repositorio de ELO-Tool.

Si bien las temáticas, sobre la que tratan los OA con los que se trabajó en el taller, fueron provistas por los docentes participantes, no fueron ellos quienes los publicaron. Es por esto que fue importante dejar tiempo para este proceso.

\subsection{Ensamblaje (60')}

Luego de familiarizarse con las herramientas y el material, también durante la segunda sesión, se asignó a cada docente un itinerario de aprendizaje. Cada participante contó con una imagen del itinerario (indicando qué OA lo componen) y una ayuda (documento PDF) para la carga de los metadatos. Con esta información debían crear el itinerario de aprendizaje utilizando, libremente, tanto ELO-Tool como ELOs-Viewer.

\subsubsection{Diseño de instrumentos}

Para la recogida de datos se utilizaron:

- Registro fílmico de la actividad del taller.

- Planilla estructurada de observación.

- Encuestas para evaluar cada momento del taller.

- Encuesta para caracterizar las usabilidad de ELO-Tool/ELOs-Viewer.

- Entrevistas a los participantes, indagando sobre fortalezas y debilidades de: $(i)$ la metodología de ensamblaje y (ii) del proceso de ensamblaje utilizando ELO-Tool.

La información obtenida en la observación participante, provino del registro audiovisual de las sesiones y una planilla estructurada de observación. Se observaron las dificultades que los docentes tuvieron al utilizar la metodología y la herramienta que la implementa, centrándose en la gestión y el ensamblaje de OA. Asimismo, se observó si los docentes pudieron crear itinerarios de aprendizaje a partir de los OA disponibles en el repositorio de la herramienta.

La planilla estructurada de observación (utilizada en la segunda sesión), contiene la descripción de la actividades que se le plantearon a los participantes en el taller, el código del participante y una escala de 0 a 5 (0: sin dificultad, 5: les resulto muy compleja) para registrar el nivel de dificultad que tuvieron los docentes al realizar la tarea. También cuenta con una columna de observaciones 
donde se registró la percepción del observador sobre cómo cada participante llevó adelante el proceso de ensamblaje.

Luego de cada sesión del taller se realizó una encuesta en la que los participantes pudieron exponer sus opiniones respecto de la actividad realizada (las encuestas están disponibles en el Anexo III). En las encuestas se consultó, a los participantes, lo siguiente:

- Respecto a la metodología de ensamblaje seleccionada

- El concepto de ensamblaje

- El conocimiento asociado

- El itinerario de aprendizaje

- La función de las ontologías

- Las modificaciones realizadas a la metodología a partir de la adaptación propuesta

- Respecto a la experiencia de ensamblaje

- La complejidad de la experiencia

- El nivel de automatización ofrecido por la metodología y reflejado por la herramienta

- El uso de metadatos, ontologías y conocimiento asociado

- La experiencia en el contexto de la creación de itinerarios de aprendizaje

Al finalizar el taller se les realizó una encuesta de satisfacción, de carácter anónimo, a cada uno de los docentes participantes. En ella se indagó sobre aspectos de usabilidad de las aplicaciones.

Respecto a la usabilidad de ELO-Tool se consultó, a los participantes, sus percepciones acerca de:

- La claridad de las opciones versus las tareas que realizan

- La navegabilidad

- $\quad$ El aspecto

- La carga de información

- El uso de la herramienta por parte de otros docentes

Respecto a la usabilidad de ELOs-Viewer se consultó, a los participantes, sus percepciones acerca de:

- La forma en la que se visualizan los ELOs

- La forma en la que se visualizan los metadatos

- El uso de la herramienta por parte de otros docentes

Las entrevistas, a los participantes del taller, consistieron en encuentros dirigidos hacia la comprensión de las perspectivas que tiene cada informante en cuanto al uso de la metodología de ensamblaje, y la herramienta que la implementa, para la creación de itinerarios de aprendizaje a partir de OA. Recabando así información puntual tanto sobre la metodología, como sobre el uso de la herramienta de software que la implementa, su aprovechamiento y la utilidad para él como docente.

Se utilizó un tipo de entrevista semi-estructurada. Por lo que se llevó adelante un trabajo de planificación de las preguntas a partir del análisis de las observaciones realizadas, del registro fílmico y del análisis de los metadatos que completaron al crear los itinerarios de aprendizaje. Esto permitió, en base preguntas de respuesta abierta, profundizar sobre la experiencia de los 
informantes en su proceso de creación del itinerario de aprendizaje. Las preguntas realizadas a cada participante están disponibles en el Anexo III.

\subsubsection{Variables de estudio}

En función de las herramientas diseñadas para la recolección de datos, en el presente estudio de caso se analizaron las siguientes variables:

- Planilla estructura de observación, Encuesta 2 y entrevista

- Nivel de dificultad que tuvo, cada participante, al completar la tarea.

- Encuesta 1 (metodología de ensamblaje) y entrevista

- Nivel de comprensión de la metodología de ensamblaje utilizada.

- Nivel de comprensión de las adaptaciones realizadas a la metodología.

- Encuesta 2 (experiencia de ensamblaje) y entrevista

- Porcentaje de la actividad completada exitosamente por cada parcialmente.

- Fortalezas y debilidades de la metodología de ensamblaje.

- Problemas detectados en la carga de metadatos.

- Utilidad de la metodología para crear itinerarios de aprendizaje (a través del ensamblaje de OA).

- Encuesta de satisfacción y entrevista

- Nivel de satisfacción de los usuarios en el uso del software.

- Fortalezas de las aplicaciones.

- Debilidades de las aplicaciones.

\subsection{Selección del SE}

El SE seleccionado para llevar adelante este estudio de caso es ELO-Tool (ver Anexo IV). Esta aplicación web fue desarrollada por la Dra. Liliana Patricia Santacruz-Valencia, co-directora de esta tesis, como parte del trabajo para su tesis doctoral (Santacruz-Valencia et al., 2005). Esta herramienta utiliza conocimiento asociado (ver 5.3.2), representado en los metadatos de cada OA, y ontologías de dominio para llevar adelante el proceso de ensamblaje.

Los criterios definidos en el Capítulo 4 fueron la base para la selección de la herramienta. ELOTool se eligió por $(i)$ tratarse de un prototipo totalmente funcional, (ii) que se encuentra accesible en la web, (iii) sin necesidad de uso de credenciales. Asimismo, (iv) la definición de OA propuesta por la autora tiene coincidencias suficientes con la propuesta en esta tesis y $(v)$ permitió realizar las adaptaciones necesarias, de aquellos aspectos de la misma, no contemplados por la metodología. También, (vi) cuenta con una definición precisa de la estructura interna del OA, lo que facilitó la localización y creación de los mismos. En virtud de que el repositorio de ELO-Tool sólo almacena los metadatos de los recursos y OA, ( $v i$ ) pueden referenciarse los mismos a partir de su URL en la web, lo que propició la reutilización y minimizó la creación de material ad hoc para el estudio de caso. Por otra parte, al tratarse de una herramienta enfocada en el docente, (vii) hizo posible centrar el estudio de caso en el proceso de creación del itinerario de aprendizaje. Lo cual, permitió excluir del estudio el uso de los OA por parte de los estudiantes, aspecto que excede el análisis propuesto en el presente trabajo de investigación.

Cabe destacar que:

- El uso de ontologías de dominio y del repositorio local en el proceso de ensamblaje 
exigieron, por una parte, la creación de ontologías (ver Anexo VI) que permitieran representar el dominio de las asignaturas y del lenguaje de programación utilizado y, por otra, el almacenamiento en repositorio de ELO-Tool de los recursos y la generación, vía ensamblaje, de los OA necesarios para la creación de los itinerarios de aprendizaje (ver Anexo V).

- La denominación de ELO (ver 3.2.1 para más detalle sobre el modelo de contenido) incluye tanto a Unidades de Información (UI), como a Unidades de Contenido (UC) y Unidades Didácticas (UD); en esta tesis, se considerarán las UI y UC como recursos educativos y las UD como OA. Por tanto, al mencionar los ELOs se hará referencia tanto al conjunto de recursos educativos, como a los OA.

\subsection{Adaptación de la metodología}

Para la implementación del Taller de Ensamblaje de Objetos de Aprendizaje se hizo necesario realizar un conjunto de adaptaciones a la metodología de ensamblaje propuesta la Dra. SantacruzValencia. Asimismo, se estimó pertinente desarrollar una aplicación web que permitiera una visualización de los ELOs más amigable para el usuario que la propuesta por ELO-Tool.

La principal motivación, de las adaptaciones realizadas, está dada por las diferencias entre definición de OA adoptada en esta tesis y la utilizada por la metodología seleccionada.

Para Santacruz-Valencia et al. (2005):

"Un ELO se define como "aquel recurso educativo descrito por meta-datos y organizado en una estructura multicapa, de tal forma que los elementos más avanzados de esta estructura incluyen conocimiento asociado (competencias y requisitos), con vistas al ensamblaje y la reutilización." (p. 74).

En tanto, en esta tesis, un Objeto de Aprendizaje se define como (Sanz, 2015, p. 34):

"Un tipo de material educativo digital, que se caracteriza, desde el punto de vista pedagógico, por orientarse a un objetivo específico de aprendizaje, y presentar mínimamente: una serie de contenidos con el fin de abordar la temática relacionada con el objetivo, una actividad que permita al alumno poner en práctica o problematizar el contenido presentado, y una autoevaluación que posibilite conocer al alumno, si ha podido comprender esos contenidos vinculados al objetivo. Desde el punto de vista tecnológico, se caracteriza por contener un conjunto de metadatos estandarizados para su búsqueda, y recuperación, y estar integrado, utilizando un modelo de empaquetamiento que respete estándares, y de esta manera, permita su diálogo con diferentes entornos tecnológicos.”.

Si bien existen entre ambas definiciones un conjunto de coincidencias, como el etiquetado con metadatos, la idea de reutilización y la especificación de una estructura para el material, se presentan también algunas diferencias.

En la definición de la Dra. Sanz, está la exigencia de "un objetivo específico de aprendizaje", esto aparece en los metadatos de los ELOs de nivel superior (Uds), pero no se especifica que deba ser único. También "una serie de contenidos" y "una autoevaluación", esto también aparece asociados a los metadatos a las UDs, pero no se reflejan en actividades concretas. Dado que, como ya se mencionó, se asume en esta tesis que las UDs son OA, es necesario garantizar que tengan un objetivo único, contenidos y una autoevaluación. Por otra parte, en la Tabla 5.1 puede observarse que la combinación, vía ensamblaje, de dos UDs da como resultado otra UD. Como cada UD cuenta con, al menos, un objetivo de aprendizaje, la combinación de dos (o más) UDs generaría OA con dos o más objetivos, lo cual iría en contra la definición aquí adoptada. 
Tabla 5.1. Posibles resultados de ensamblar dos ELOs (Santacruz-Valencia et al., 2005, p. 81 Tabla 3.5).

\begin{tabular}{cccc}
\hline & UI & UC & UD \\
\hline UI & UC & UC & $\times$ \\
UC & UC & UD & UD \\
UD & $\times$ & UD & UD \\
\hline
\end{tabular}

En busca de subsanar estas diferencia, y haciendo uso de la flexibilidad de la metodología elegida para llevar adelante el taller, se implementaron las siguientes adaptaciones:

- Se re-definió la estructura básica para cada UD (ver Figura 5.1), en base en la definición de $\mathrm{OA}$, con vistas a la generación de las misma vía el proceso de ensamblaje. Así pues, para poder crear una UD es necesario contar con UIs apropiados. Debe haber, entonces, UIs que referencien objetivos, otras a contenidos, a ejemplos y otras a evaluación.

Basado en las UIs, descritas arriba, se forman las UCs. Se propone, por tanto, la existencia de dos tipos de UC: aquellas que contienen el objetivo de aprendizaje y las que contienen ejemplo/s y auto-evaluación/ejercitación ${ }^{5}$. Con la combinación de ambas UCs se conforma un OA que refleje la estructura expuesta en la definición adoptada: "el objetivo, una actividad que permita al alumno poner en práctica o problematizar el contenido presentado, y una autoevaluación".

Cabe aclarar que la metodología propone el ensamblaje de a pares y que cada UC debe, obligatoriamente, referenciar al menos dos UIs.

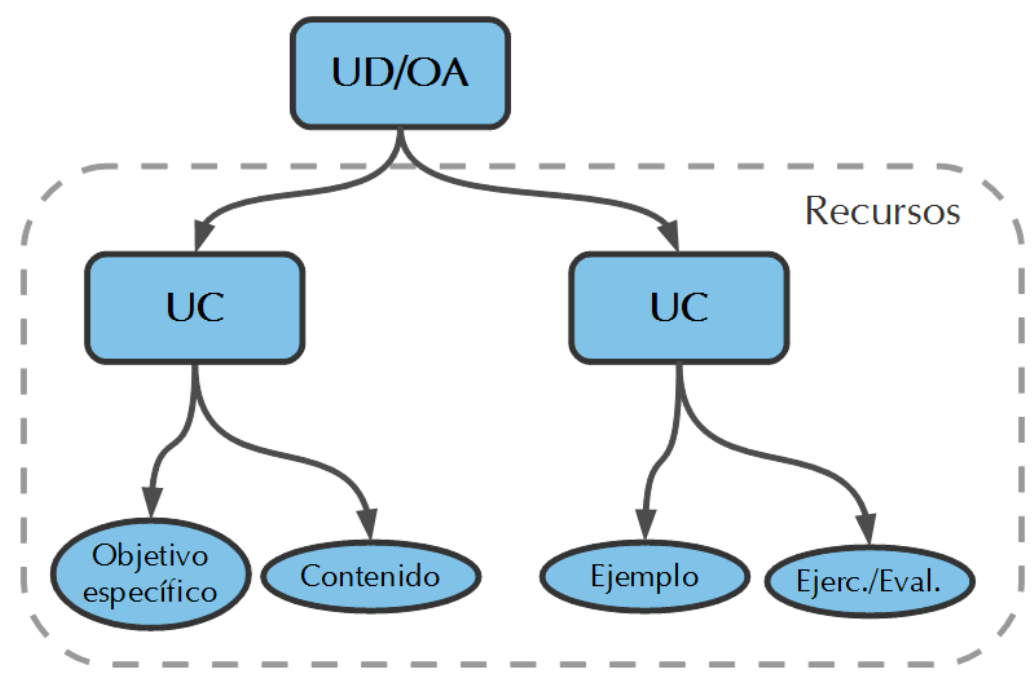

Figura 5.1. Estructura básica un $O A$.

- Para poder garantizar la correcta composición de los OA, vía el ensamblaje, se hizo necesaria otra adaptación. Dado que el proceso de ensamblaje se basa en el CA y que el mismo se refleja a través de una ontología, se incorporó una nueva ontología que pudiera reflejar los componentes del material con los se pensaba trabajar: objetivo, contenido, ejemplo, ejercitación y auto-evaluación.

A continuación se presenta un ejemplo. Cabe aclarar que la definición de los requerimientos y competencias de cada UC/UD son determinados exclusivamente por el docente que lleva adelante el proceso de ensamblaje -con base en la ontología-.

Para crear el OA ESTRUCTURA REPETITIVA FOR ${ }^{6}$ (ver Figura 5.3) cuyo objetivo es "Utilizar apropiadamente la sentencia repetitiva FOR del lenguaje Pascal para resolver

5 Se asume que el planteo de ejercicios (preferentemente con solución) en este tipo de materiales educativos funcionaría de forma similar a una auto-evaluación.

6 Disponible, vía ELOs-Viewer, en: http://goo.gl/2nb9Nv 
ejercicios/problemas", se utilizaron dos $\mathrm{UCs}^{7}$ :

- UC01. DESCRIPCIÓN CICLO FOR. Donde se presenta el objetivo, se describe la estructura repetitiva FOR y se muestra el DFD correspondiente. El CA para esta UC es la noción de Secuencia (requerimiento), y la noción de Repeticion y uso de la sentencia ForToDo (competencias).

- UC02. EJERCICIOS CICLO FOR. Donde se presentan ejemplos y ejercicios para la estructura repetitiva FOR en Pascal. El CA para esta UC es el concepto de Repeticion y el uso de la sentencia ForToDo (requerimientos), y se consolida el concepto de Repetición (competencia).

Como se puede observar es posible ensamblarlos ya que las competencias de UC01 cubre a UC02 (competencias Repeticion y ForToDo).

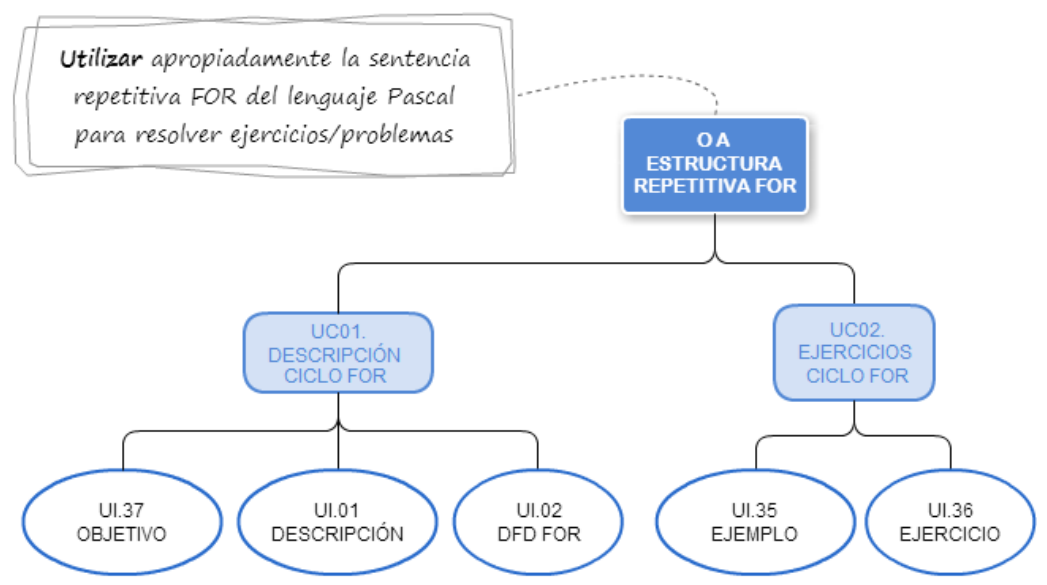

Figura 5.2. Esquema del OA: ESTRUCTURA REPETITIVA FOR

- Otra adaptación que se implementó es la extensión en la granularidad de los materiales. La propuesta original utiliza UIs, UCs y UDs, y como se aprecia en la Tabla 5.1, la combinación, vía ensamblaje, de dos UDs da como resultado otra UD. Al asumir que las UDs son OA, la combinación de dos OA no puede dar como resultado otro OA (tendrían más de un objetivo). Por tanto, se asumirá que el ensamblaje de dos (o más) OA da como resultado un itinerario de aprendizaje.

De forma similar que para las UCs, se agregó como requerimiento de cada OA: Objetivo y Ejercicio. Es decir que todos los OA además de los requerimientos de contenidos van a tener, obligatoriamente, estos dos requerimientos. Se busca garantizar con este agregado que el ensamblaje se produzca con otro OA, o bien, con un itinerario al que se le agrega un OA (y sigue siendo un itinerario de aprendizaje).

A continuación se presenta un ejemplo:

Para crear el itinerario de aprendizaje INTRODUCCIÓN A ARREGLOS PASCAL ${ }^{8}$ (ver Figura 5.3), cuyos objetivos son: "Utilizar apropiadamente la sentencia repetitiva FOR del lenguaje Pascal para resolver ejercicios/problemas" y "Utilizar arreglos unidimenciones en Pascal", se utilizaron dos OA:

- UD01. ESTRUCTURAS REPETITIVAS PASCAL. EL FOR. Descrita en el ejemplo anterior. Cuyo CA es: se debe conocer el concepto de Secuencia (requerimiento), y se

7 Las palabras en cursiva son los nombres con el que aparecen las ontologías y las clases y sub-clases de las mismas en el sistema, es por eso que no se les colocó acento y, algunas, se conforman de la unión de dos o más palabras sin espacio.

8 Disponible, vía ELOs-Viewer, en: http://goo.gl/sFPDNf 
aborda el concepto de Repeticion y la práctica con la sentencia ForToDo (competencias).

- UD02. ARREGLOS EN PASCAL. Donde se presenta el objetivo, se describe el concepto de arreglo (genérico) y se muestra un ejemplo. Luego, se ofrecen ejemplos y ejercitación sobre arreglos en Pascal. El CA para este OA es: se debe conocer el concepto de Repetición (requerimiento) y se aborda la noción de Arreglos y se practica con el tipo de datos Array de Pascal (competencias).

Cabe aclarar que las requerimientos y competencias (CA) del nuevo itinerario, fueron calculadas por ELO-Tool de forma automática. Las primeras pertenecen al primer OA de la secuencia y, las segundas, surgen de la unión de las competencias de los OA ensamblados.

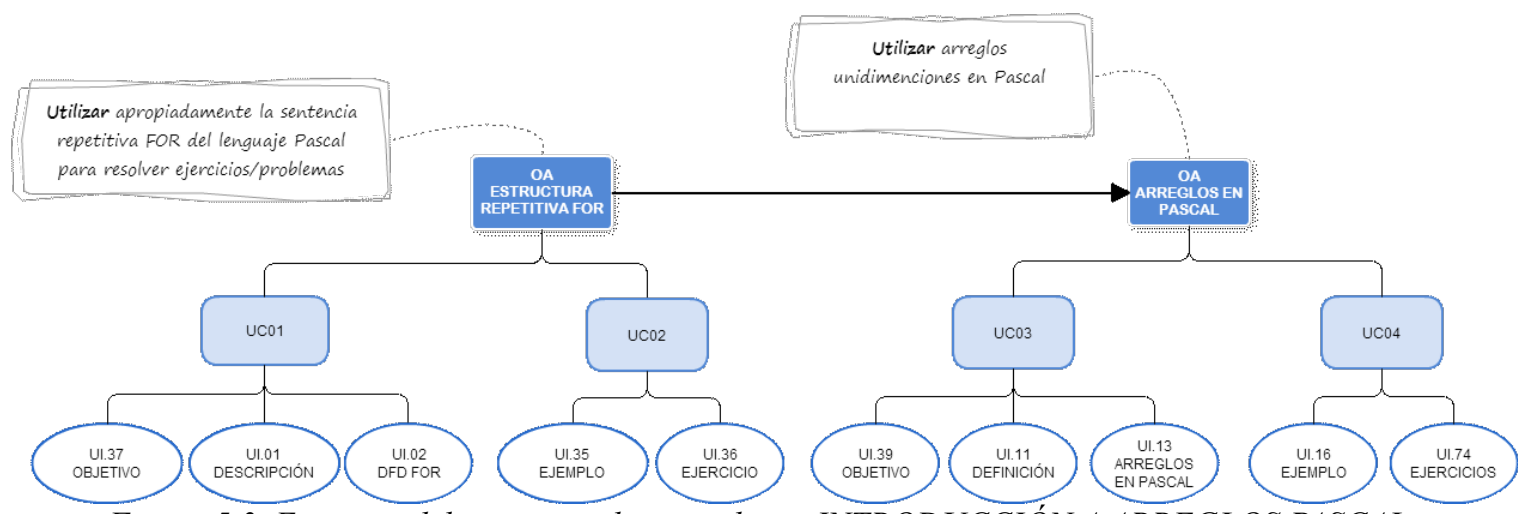

Figura 5.3. Esquema del itinerario de aprendizaje INTRODUCCIÓN A ARREGLOS PASCAL.

- En busca de visualizar los ELOs de forma más amigable para el usuario se diseñó una aplicación web a tal efecto. Si bien ELO-Tool cuenta con la opción para mostrar los ELOs, en ella se muestran los datos en crudo de los metadatos de los ELOs (ver Figura 5.4). Se estimó importante que los participantes del taller tuvieran acceso a la información asociada con cada ELO de forma directa y visualmente agradable.

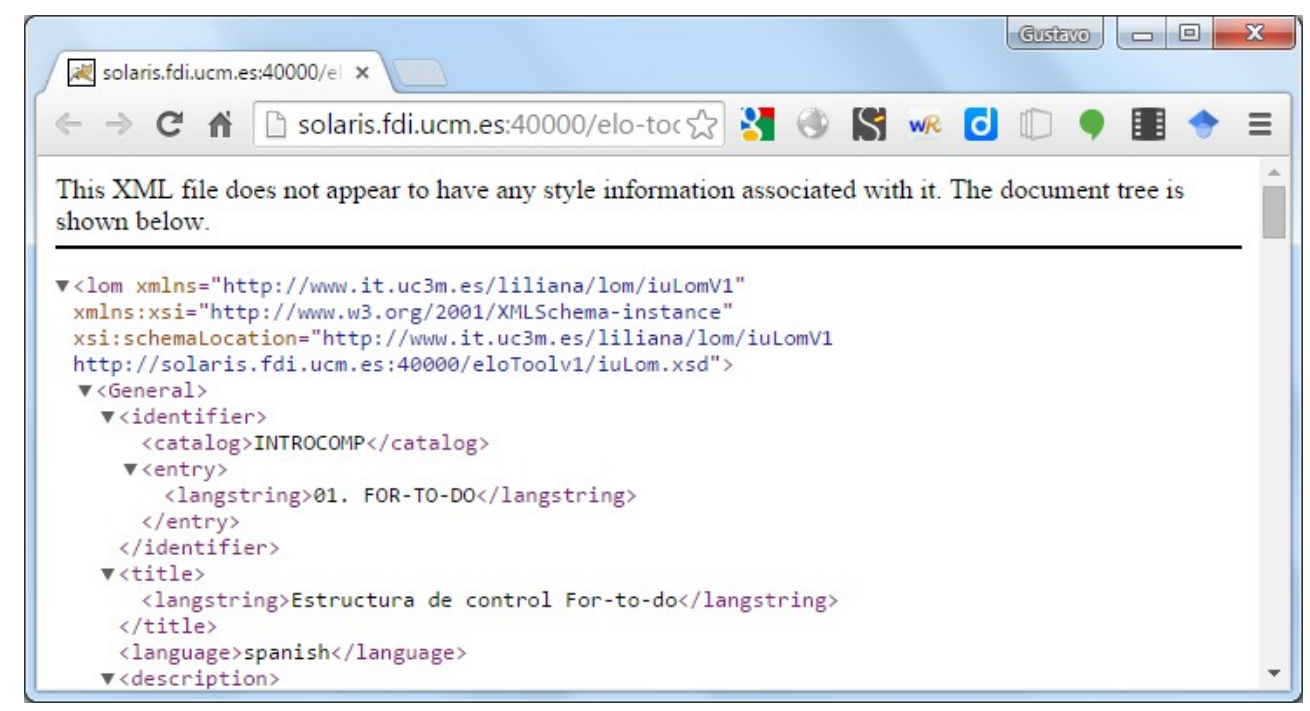

Figura 5.4. Visualizando un ELO en ELO-Tool.

La aplicación diseñada se denomina ELOs-Viewer ${ }^{9}$ y cuenta con dos funciones:

- Búsqueda de ELOs. Esta opción permite localizar ELOs alojados en el repositorio de ELO-Tool a través de la búsqueda por palabra clave sobre los nombres con el que los ELOs se representan en la base de datos de ELO-Tool (no sobre los metadatos de los mismos, función con la que ya cuenta ELO-Tool). Esta búsqueda se puede combinar con

9 Disponible en: http://elosviewer.exactas.unlpam.edu.ar/ 
el filtrado por tipo de ELO (UI, UC o UD). Dado que la base de datos de ELO-Tool registra sólo estos tres tipos de ELOs, en esta primera versión de la aplicación, tanto OA, como itinerarios de aprendizaje se localizan bajo el filtro de "UD" (ver Figura 5.5).

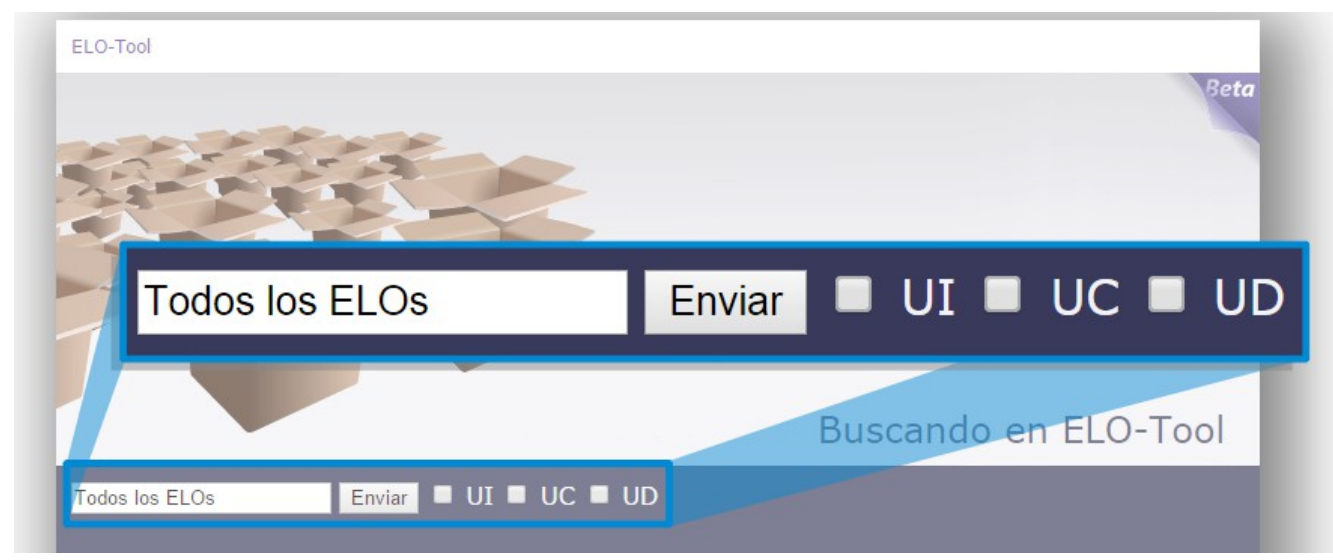

Figura 5.5. Espacio de búsqueda en ELOs-Viewer.

- Visualización de ELOs. Para la visualización de los ELOs se seleccionaron parte de los metadatos que los describen. El recorte se hizo en base a los destinatarios (docentes) y a la información más relevante para al momento de llevar adelante la actividad de ensamblaje con ELO-Tool dentro del taller.

Así pues, los metadatos seleccionados fueron:

- UI/UC/UD: Título, Autor, Descripción, Palabras clave, Tipo de interactividad, Nivel de interactividad, Densidad semántica y Nivel de dificultad.

- UI: Además, la Localización (URL del recurso).

- UC/UD: Se agregan a los del ítem anterior Requerimientos y Competencias;

- UD: Se sumó Objetivos, Resumen y Evaluación.

Respecto de la información relevante para ensamblaje:

- "Denominación para ensamblaje", permite seleccionar y copiar al portapapeles el nombre con que se identifica al ELO, para luego sólo tener que pegarlo, dentro del campo, al momento del ensamblaje.

- "URL en el repositorio" tiene, para los OA, la dirección de los mismos en el repositorio en el formato que utiliza ELO-Tool para referenciarlo al momento del ensamblaje. Esto es útil cuando se desea ensamblar más de dos OA (para dos lo completa el propio ELO-Tool).

- Tanto requerimientos, como competencias, se listan con el formato: Clase (ontología\#). Esta información se hace necesaria cuando se desean agregar requerimientos/competencias al momento del ensamblaje (ELO-Tool calcula automáticamente el CA).

Respecto a la visualización de ELOs e itinerarios de aprendizaje:

- UI. La aplicación muestra un ícono con el enlace directo al recurso.

- UC y UD/OA. La aplicación utiliza un esquema de árbol (ver Figura 5.6). Donde la raíz representa el ELO que se está accediendo, las hojas contiene los enlaces directos a los recursos, y los nodos intermedios, permiten visualizar los ELOs que componen el material, dentro del propio ELOs-Viewer. También hay una referencia icónica que representa la granularidad del ELO (UI:una pieza de rompecabezas, UC: dos piezas unidas y UD: tres piezas unidas). 


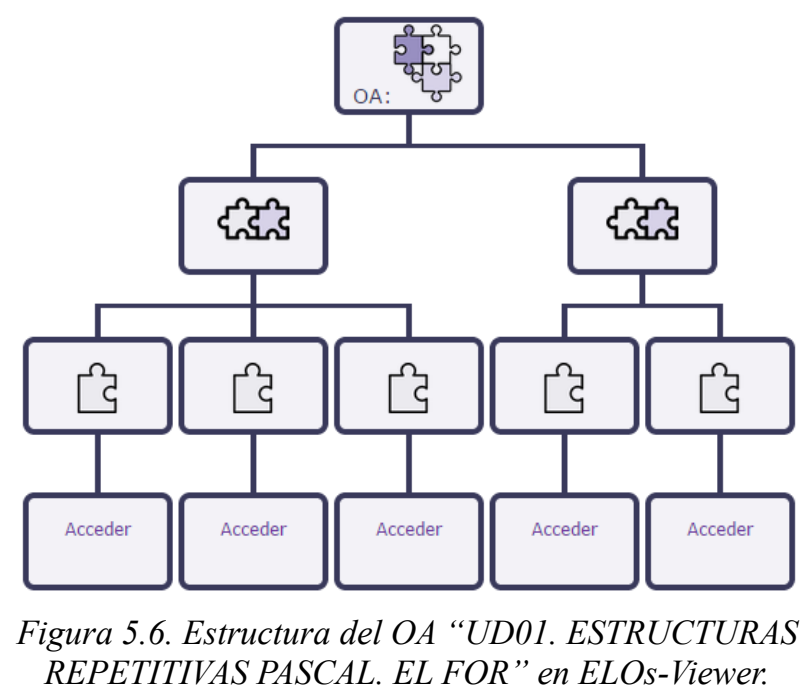

- Itinerario de aprendizaje. La aplicación presenta este tipo de material como una secuencia a nodos unidos por una flecha (ver Figura 5.7). Cada nodo es un enlace al OA dentro de ELOs-Viewer. Este formato visual intenta reforzar la idea planteada antes de que dos OA combinados vía ensamblaje dan como resultado un itinerario de aprendizaje.

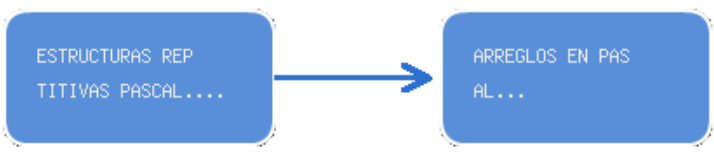

Figura 5.7. Estructura de Itinerario "IA01.

INTRODUCCIÓN A ARREGLOS PASCAL” en ELOs-Viewer.

- Lista completa de metadatos. Al final de cada vista se ofrece un enlace que muestra la lista completa de metadatos a través de un enlace directo al archivo XML en el repositorio de ELO-Tool.

\subsection{Diseño del taller}

\subsubsection{Diseño de itinerarios de aprendizaje}

Dado que se trata de material educativo, se hace necesario poder realizar, previo al ensamblaje, el diseño del itinerario de aprendizaje. De esta manera definir: los objetivos, qué materiales se desea incluir en el itinerario, en qué orden se le presentará a los estudiantes en función del objetivo de aprendizaje y qué ejercitación y/o qué tipo de evaluación se va a proponer.

La herramienta ELO-Tool, no cuenta con una funcionalidad que permita editar el material (OA/itinerarios). Es por esto que se hizo necesaria la utilización de una herramienta externa que permitiera llevar adelante dicha actividad. En este caso se utilizó $\operatorname{Cacoo}^{10}$, una aplicación web para el diseño de diagramas, que es colaborativa y que se puede incluir como complemento de Google Drive. Espacio, éste último, que se utilizó para compartir el material con los participantes del taller.

Se llevó adelante entonces - previo al inicio del taller-, el diseño de los itinerarios de aprendizaje que iban a ser presentados a los participantes del taller para que llevaran adelante el proceso de ensamblaje.

Se diseñaron, con base en los temas de interés planteados por los docentes participantes, cinco itinerarios de aprendizaje (los diagramas pueden apreciarse con más detalle en el Anexo V) ${ }^{11}$ : 
- IA01. BURBUJA. Este itinerario propone un recorrido por los distintos conceptos involucrados en el proceso de ordenación de arreglos con el método Burbuja. Integra contenidos de Introducción a la Computación (estructuras de control repetitivas y arreglos) con Programación I (métodos de ordenación). Se compone de tres OA, seis UCs y 14 UIs (ver Figura 5.8).

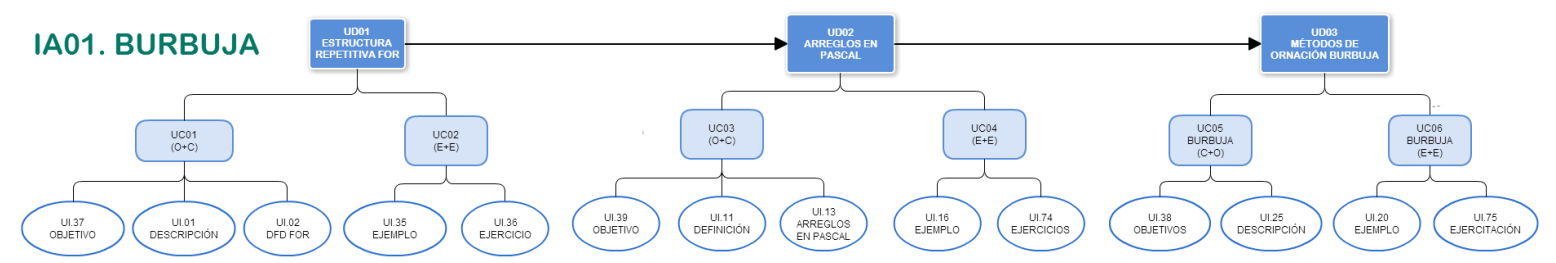

Figura 5.8. Diagrama que muestra la estructura del Itinerario de Aprendizaje "IA01. BURBUJA".

- IA02. SELECCION. Este itinerario propone un recorrido por los distintos conceptos involucrados en el proceso de ordenación de arreglos con el método Selección Directa. Integra contenidos de Introducción a la Computación (estructuras de control repetitivas y arreglos) con Programación I (métodos de ordenación). Se compone de tres OA, dos de los cuales comparte con IA01 (reutilización), seis UCs y 14 UIs (ver Figura 5.9).

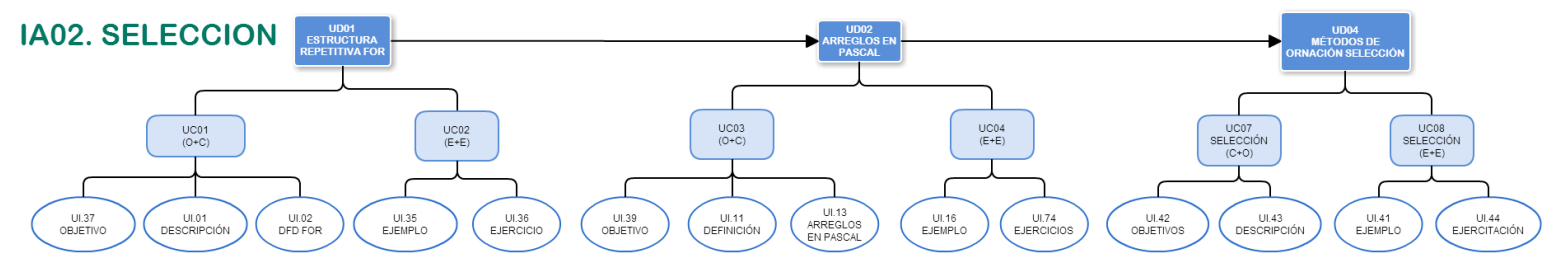

Figura 5.9. Diagrama que muestra la estructura del Itinerario de Aprendizaje "IA02. SELECCION".

- IA03. COMPARAR REPETITIVAS. Este itinerario propone repasar de las estructuras de control repetitivas en Pascal con el fin de comparar el funcionamiento y contexto de uso. Aborda contenidos de Introducción a la Computación (estructuras de control repetitivas). Se compone de cuatro OA, el inicial se comparte con IA01 y IA02 (reutilización), ocho UCs y 20 UIs (ver Figura 5.10). Se presenta dividido en dos partes (IA03A e IA03B) ya que este itinerario se pensó para ser construido por dos participantes. Cada uno ensamblara su parte y una vez que ambos itinerarios estuvieran disponibles en el repositorio de ELO-Tool se ensamblaron para crear IA03.

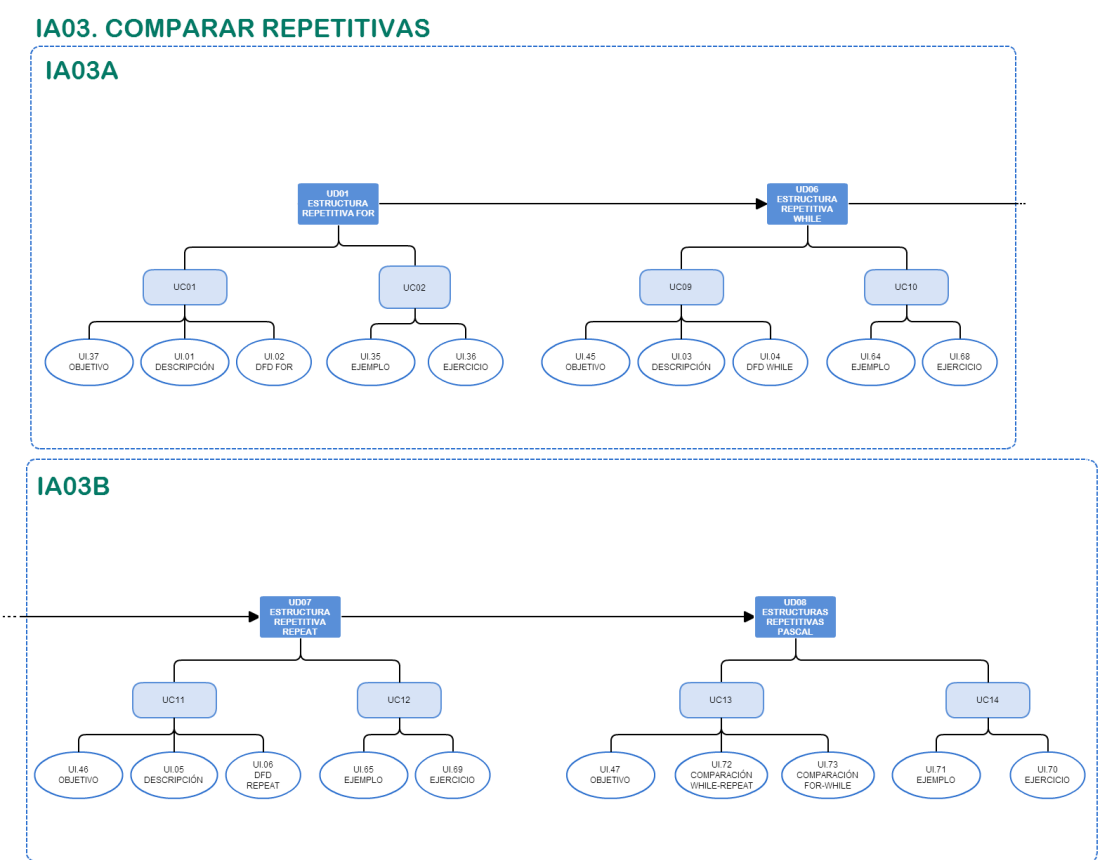

Figura 5.10. Diagrama del Itinerario de Aprendizaje "IA03. COMPARAR REPETITIVAS". 
- IA04. ARCHIVO SECUENCIAL. Este itinerario propone, inicialmente, repasar el concepto de archivo o fichero, para luego implementar en Pascal programas que manejen ficheros de texto o acceso secuencial. Integra contenidos de Programación I. Se compone de dos OA, cuatro UCs y ocho UIs (ver Figura 5.11).

\section{IA04. ARCHIVO SECUENCIAL}

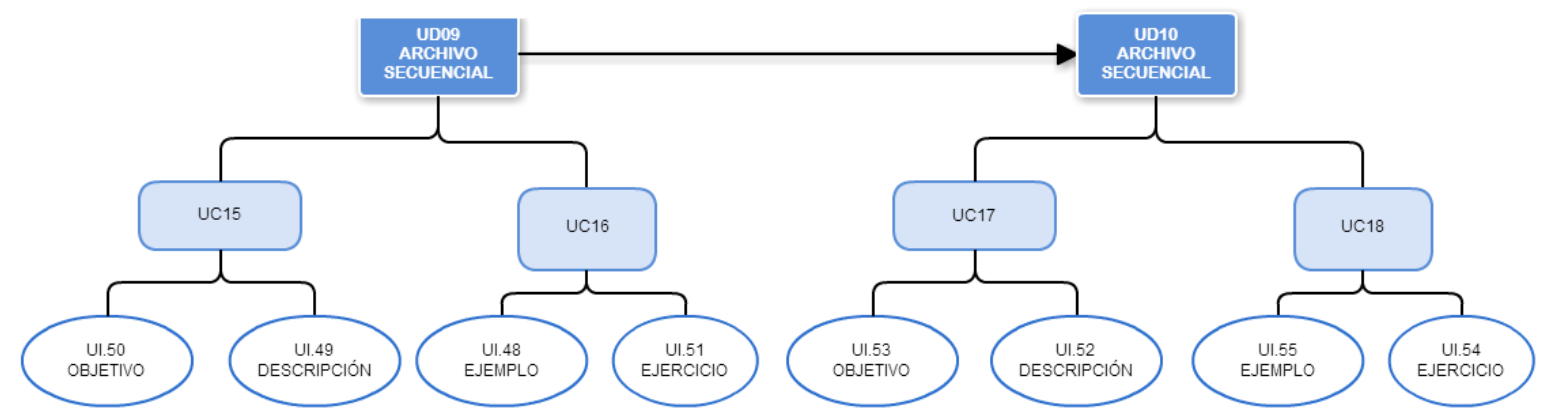

Figura 5.11. Estructura del Itinerario de Aprendizaje "IA04. ARCHIVO SECUENCIAL".

- IA05. POLYA VS DESARROLlO. Este itinerario propone comparar la metodología propuesta por George Polya para la resolución de problemas con uno de los métodos utilizados en el desarrollo de software. Aborda contenidos de vistos en el Taller de Introducción a la Programación ${ }^{12}$ y retomados en Introducción a la Computación. Se compone de dos OA, cuatro UCs y ocho UIs (ver Figura 5.12).

\section{IA05. POLYA VS DESARROLLO}

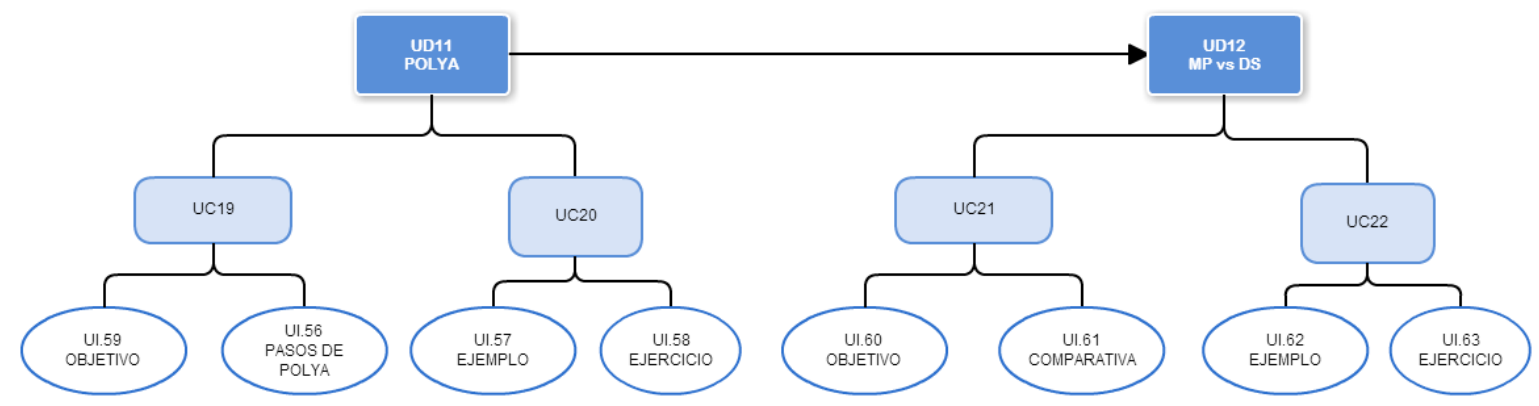

Figura 5.12. Estructura del Itinerario de Aprendizaje "IA05. POLYA VS DESARROLLO".

\subsubsection{Búsqueda de Unidades de Información}

Luego del proceso de diseño de los itinerarios de aprendizaje, comenzó la tarea de búsqueda de las UIs que permitirían la creación de las UCs y, finalmente, los OA para el estudio de caso. Cabe aclarar que en todos los casos los recursos que referencian las UIs debían estar disponibles en la web -dado que el repositorio de ELO-Tool sólo puede alojar los metadatos de los ELOs y no el recurso en sí mismo-.

Si bien existen, en la web, una importante cantidad de material sobre la temática del taller, localizar recursos con la granularidad apropiada para constituirse en una UI fue una tarea compleja. Tal es así que, en algunos casos, se optó por el diseño de UIs ad hoc.

A los 121 recursos seleccionados de la web se le sumaron 27 de creación propia (como por ejemplo, imágenes que describen los objetivos y presentaciones con ejercitación y su solución). De estas 148 UIs se publicaron 49 en el repositorio de ELO-Tool. Las mismas se consideraron como las más apropiadas para el abordaje de las distintas temáticas y conforman el conjunto de UIs necesarias para la creación de los OA que se utilizaron en el taller.

Resumiendo, con las 49 UIs se crearon 22 UCs y con éstas 11 OA. Con ellos los cinco itinerarios de aprendizaje descritos antes.

12 Taller dictado para ingresantes por el equipo de cátedra de Introducción a la Computación. 
La actividad de diseño, búsqueda y creación de los OA fue realizada por el autor de esta tesis y quitó carga de trabajo a los docentes participantes del taller, permitiendo que los mismos se focalizaran en las actividades de ensamblaje de OA.

\subsubsection{Creación de ontologías}

Otra actividad que llevó adelante, previa a la implementación del taller, es la creación y publicación de las ontologías. Las mismas representan los distintos dominios de las temáticas que se usaron en el taller: el lenguaje Pascal y los temas abordados en las asignaturas Introducción a la Computación y Programación I. Asimismo, se creó la ontología que representa los componentes del material que debía incluir cada OA en función de la adaptación propuesta (ver Sección 5.5).

En una primera instancia se accedió a algunas ontologías propuestas por otros autores referenciadas en la bibliografía consultada para este tesis-, sin embargo no eran compatible con ELO-Tool y/o no representaban apropiadamente el dominio. Se realizó, además, una búsqueda en la web sobre ontologías que permitieran representar el dominio del lenguaje Pascal, pero esta búsqueda fue infructuosa. Ante la necesidad de representar el dominio de asignaturas concretas, se crearon ad hoc las ontologías para las mismas.

Esto llevó a la creación, con base en ontologías existentes en ELO-Tool, de las tres ontologías de dominio y, la que representa elementos presentes en un material educativo (las ontologías pueden revisarse en detalle en el Anexo VI):

- IntroCompb\#. Describe el dominio de la asignatura Introducción a la Computación, representando, a través de sus clases, todos los temas que se abordan en la asignatura.

- ProgI\#. Describe el dominio de la asignatura Programación I, representando, a través de sus clases, todos los temas que se abordan en la asignatura.

- Pascalb\#. Describe el dominio del lenguaje de programación Pascal representando, a través de sus clases, las distintas conceptos asociados al lenguaje y que se abordan en las asignaturas.

- ComponentesDelMaterial\#. Esta ontología representa los componentes que debe contener un material educativo (por ejemplo un UCs o un OA). Como ya se mencionó, permitió la adecuada composición de la estructura interna de los $\mathrm{OA}$ al crearlos vía el proceso de ensamblaje.

Cabe aclarar que las ontologías fueron trabajadas con los docentes participantes del taller en encuentros informales, previos al taller, que permitieron la evolución y validación de las mismas.

\subsection{Recapitulación}

En este capítulo se describió el estudio de caso (ver Figura 5.14) que se realizó a partir de un taller a un grupo de docentes de la Facultad de Ciencias Exactas y Naturales de la UNLPam. Para las actividades de dicho taller se utilizó el SE ELO-Tool. El mismo implementa una metodología de ensamblaje desarrollada por la Dra. Santacruz-Valencia que permite generar itinerarios de aprendizaje, vía ensamblaje, con base en el conocimiento asociado de los OA.

Se seleccionó esta metodología, y el software que la implementa, en virtud de que cuenta con un prototipo totalmente funcional, disponible en la web. Asimismo, la definición de OA propuesta por la Dra. Santacruz-Valencia tiene coincidencias suficientes con la propuesta en esta tesis y cuenta con una descripción precisa de la estructura interna del OA. Tanto la metodología, como la herramienta, propician la reutilización y facilitan la realización de las adaptaciones -necesarias en para el contexto de implementación del taller de ensamblaje-. Todo esto hizo posible llevar adelante el estudio de caso y centrarlo en el proceso de creación de itinerarios de aprendizaje, vía ensamblaje, a partir de la reutilización de OA.

Este estudio de caso permitió: (i) conocer la opinión de los docentes en relación a la creación de 
itinerarios de aprendizaje con OA, (ii) identificar fortalezas y debilidades de la metodología de ensamblaje seleccionada, (iii) evaluar el nivel de usabilidad de las herramientas de software utilizadas y $(i v)$ generar un proceso metodológico de aplicación de ELO-Tool para ser utilizado por docentes del área de ciencias de la computación.

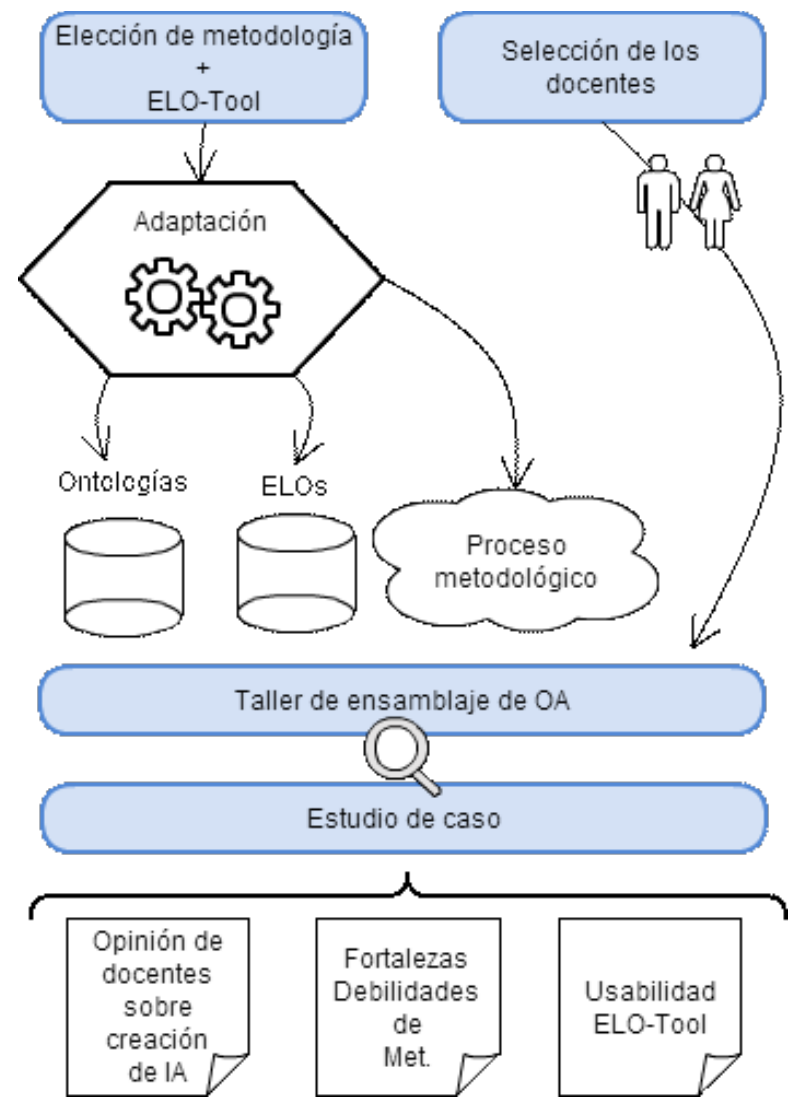

Figura 5.13. Resumen del proceso realizado para el estudio de caso.

Para la obtención de la muestra se realizó un muestreo por conveniencia sobre las asignaturas Introducción a la Computación y Programación I. Esto permitió definir una muestra de cinco docentes participantes del taller. Sobre los cuales se realizaron las observaciones para este estudio de caso.

Para la recogida de datos se utilizaron: la entrevista, la encuesta y la observación participante. Además se realizó un registro fílmico de la actividad del taller y se tomaron registros utilizando una planilla estructura de observación. Esto permitió medir el nivel de dificultad que tuvo cada participante al completar la tarea y el nivel de comprensión de la metodología de ensamblaje utilizada. También, el porcentaje de la actividad completada exitosamente por cada participante, las fortalezas y debilidades de la metodología de ensamblaje y los potenciales problemas en la carga de metadatos. Asimismo, se evaluó la utilidad de la metodología para crear itinerarios de aprendizaje (a través del ensamblaje de OA). Con respecto al software utilizado se midió el nivel de satisfacción de los usuarios tras el uso del mismo y, las fortalezas y debilidades de las aplicaciones.

El taller se desarrolló en dos sesiones: en la primera se explicó la metodología de ensamblaje y las adaptaciones que se realizaron para la implementación del estudio; y en la otra los participantes se familiarizaron con las herramientas de software y el contenido del repositorio, para luego crear, cada uno, un itinerario de aprendizaje. La temática utilizada para la generación dichos itinerarios estuvo centrada en contenidos de las asignaturas Introducción a la Computación y Programación I.

Para la implementación del estudio de caso fue necesario realizar una serie de adaptaciones. Las mismas se centraron la adaptación del modelo de contenidos propuesto por la metodología de ensamblaje en términos de la definición de OA utilizada en esta tesis. Por una parte se re-definió la estructura básica para cada UD de forma de que la misma contara con UIs que representen los distintos componentes de un OA: objetivos, contenidos, actividades y auto-evaluación. También se 
adaptó el CA para que permitiera representar esta nueva estructura y se creó una ontología que reflejara el tipo de material y las exigencias que se definieron para la conformación de un OA. Sumado a lo anterior, se extendió en la granularidad de los materiales propuestos por la metodología y se estableció que la combinación de dos o más UDs, vía ensamblaje, darán como resultado un itinerario de aprendizaje. Finalmente, se diseñó una aplicación web, denominada ELOs-Viewer, para facilitar a la visualización del material alojado en el repositorio de ELO-Tool y apoyar a los docentes en las actividades de ensamblaje del taller.

Para la implementación del taller se trabajó con más 120 UIs, de las cuales se publicaron 49 en el repositorio de ELO-Tool. Con ellas se crearon 22 UCs y con éstas 11 OA. También se diseñaron y publicaron en el repositorio de ELO-Tool tres ontologías de dominio y una para representar los componentes de los OA. Estos y las ontologías fueron utilizados por los docentes, participantes del taller, para crear los itinerarios de aprendizaje.

La actividad de diseño de los itinerarios y búsqueda del material, así como la creación de OA y ontologías, fue realizada por el autor de esta tesis y quitó carga de trabajo a los docentes participantes del taller, permitiendo que los mismos se focalizaran en las actividades de ensamblaje de OA, tema central de esta tesis.

En el siguiente capítulo se presentan y discuten los resultados obtenidos de la implementación del estudio de caso. 


\section{RESULTADOS Y DisCuSIÓN}

\section{RESUMEN}

A partir de la observación participante y del conjunto de berramientas de registro, se recolectaron los datos que resultaron de las experiencia de los informantes al realizar el taller de ensamblaje de OA.

En este sexto capitulo, se presentan y discuten los resultados obtenidos del estudio de caso. En primer lugar, se bace una descripción del material utilizado en el taller. Seguidamente, se presentan los resultados organizados a partir de las variables de estudio. Para finalizar, en la recapitulación, se presentan, además de una revisión del capítulo, un esquema que resume los resultados obtenidos. 


\subsection{Introducción}

En esta tesis se llevó adelante un estudio de caso (ver Capítulo 5) para el cual se diseñó un taller de ensamblaje de OA. Dicho diseño implicó, no sólo la diagramación e implementación de las actividades del mismo, sino también, la selección y adaptación de una metodología de ensamblaje de Objetos de Aprendizaje y la localización y publicación del material para el taller a través de la herramienta ELO-Tool.

Dado que se desarrolló una experiencia real, donde docentes de la FCEyN-UNLPam tuvieron que generar itinerarios de aprendizaje con vistas a la utilización de los mismos en sus propias cátedras (Introducción a la Computación y Programación I), el material seleccionado para el taller debió cumplir con las siguientes características:

- Ser apropiado para estudiantes del primer año de la carrera Profesorado en Computación.

- Estar centrado en las temáticas de las asignaturas en cuestión.

- Contar con la granularidad apropiada que permitiera su utilización en el proceso de ensamblaje con base en la metodología seleccionada.

- Permitir la creación, vía ensamblaje, de Objetos de Aprendizaje.

- Estar accesible vía la web.

- Ser de dominio público o permitir su reutilización en el contexto educativo.

Luego de la búsqueda, localización, selección y almacenamiento de los recursos (UIs) en el repositorio de ELO-Tool, se concretó la creación, vía ensamblaje, del conjunto de OA que se utilizarían en el taller.

Cumplidas las etapas descritas en los párrafos anteriores, se implementó el taller. La metodología de investigación que se utilizó fue observación participante. Para la recolección de datos, se utilizó:

- Planilla estructurada. Registro del observador.

- Encuesta 1. Encuesta de opinión administrada luego de la primer sesión del taller.

- Encuesta 2. Encuesta de opinión administrada luego de la segunda sesión del taller (en la cual se realizó la creación de los itinerarios vía el ensamblaje de OA).

- Encuesta de satisfacción sobre ELO-Tool. Encuesta de satisfacción administrada luego de la segunda sesión del taller.

- Encuesta de satisfacción sobre ELOs-Viewer. Encuesta de satisfacción administrada luego de la segunda sesión del taller.

- Registro audiovisual. Videos registrados durante ambas sesiones del taller.

- Entrevista. Registros sonoros de las entrevistas realizadas a cada uno de los participantes del taller.

Se cuenta para el análisis con cinco filmaciones, una de la $(i)$ primera sesión del taller, cuatro de la segunda sesión del taller: (ii) primera parte de la explicación, (iii) segunda parte de la explicación, (iv) inicio del proceso de ensamblaje (actividad) y (v) proceso de ensamblaje del itinerario IA3a. En adelante se utilizará la denominación "registro audiovisual" para todas las filmaciones obtenidas durante el taller. Se hará lo propio denominando "Entrevista" a todos los registros de audio obtenidos durante las mismas - en la Tabla 6.1, pueden observarse detalles de las mismas-. 
Tabla 6.1. Codificación e información sobre el registro de audio de las entrevistas.

\begin{tabular}{ccccc}
\hline Registro & Fecha & Hora & Duración & Descripción del registro \\
\hline Nota de voz 001 & $09 / 07 / 15$ & $08: 55$ & $10: 16$ & Registro de la entrevista con el informante P2 \\
Nota de voz 002 & $09 / 08 / 15$ & $08: 05$ & $13: 12$ & Registro de la entrevista con el informante P4 \\
Nota de voz 003 & $09 / 08 / 15$ & $11: 12$ & $07: 54$ & Registro de la entrevista con el informante P1 \\
Nota de voz 004 & $09 / 09 / 15$ & $09: 45$ & $15: 28$ & Registro de la entrevista con el informante P5 \\
Nota de voz 005 & $09 / 10 / 15$ & $10: 26$ & $08: 33$ & Registro de la entrevista con el informante P3 \\
\hline
\end{tabular}

A continuación se resume el proceso de búsqueda de los recursos educativos utilizados en el estudio. Luego se presentan los resultados obtenidos a partir de las herramientas de recolección de datos y se plantea la discusión de los mismos.

\subsection{Recursos educativos utilizados en el estudio}

\subsubsection{Unidades de Información}

La web es una enorme fuente de recursos, sin embargo, se debían localizar aquellos con la granularidad apropiada para constituirse en una UI y con la temática de los itinerarios de aprendizaje definidos para el taller. Esta fue una tarea compleja y, en algunos casos, se consideró pertinente crear material ad hoc para la implementación del taller.

Se seleccionaron, de la web, 121 recursos como candidatos a ser las UIs que formaron parte del material del taller (ver Figura 6.1). A los anteriores se agregaron 27 de creación propia, principalmente para representar los objetivos y la ejercitación (ver Tabla 6.2). Lo que totalizó 148 UIs.

\section{Cantidad de Uls por tema}

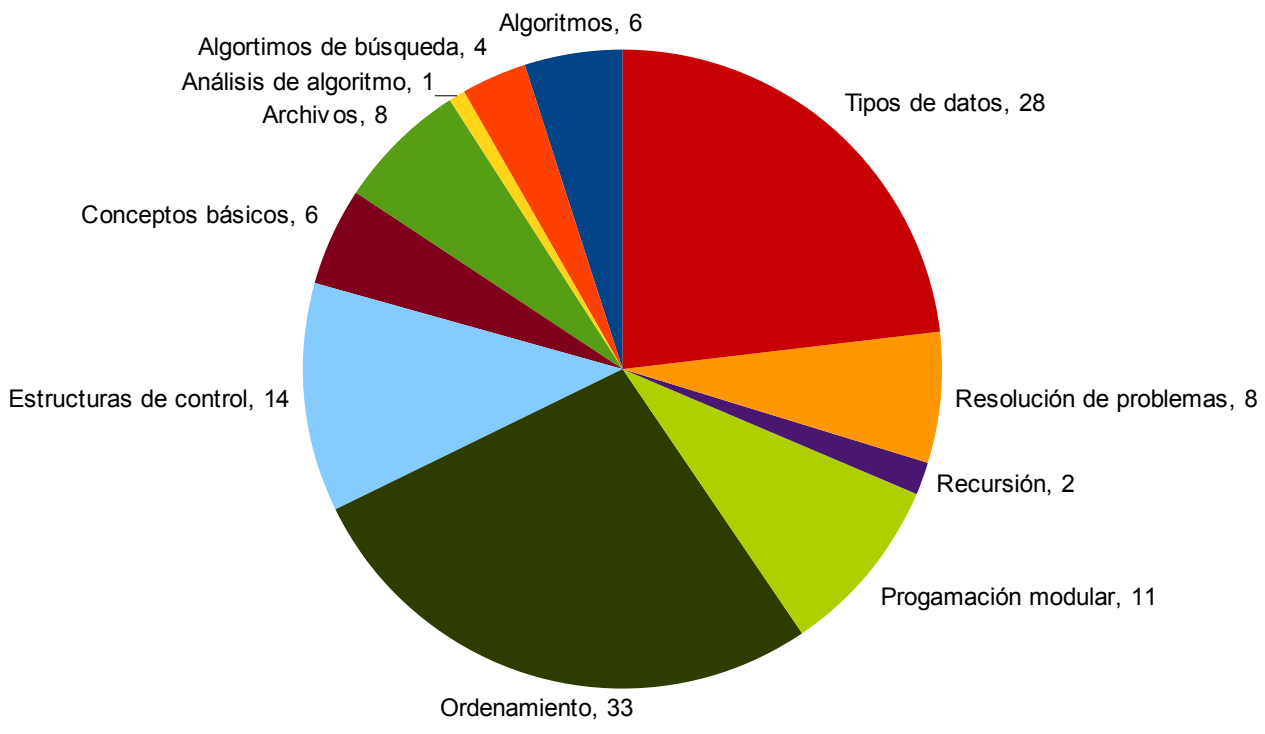

Figura 6.1. Distribución de UIs localizadas en la web por tema.

Del total, se publicaron en el repositorio de ELO-Tool 49 UIs. Las mismas se consideraron las más apropiadas para el abordaje de las distintas temáticas y conforman el conjunto de UIs necesarias 
para la creación de los OA que se utilizaron en el taller. De las 49, el 55\% fueron de producción propia. Estos corresponden a la incorporación de objetivos y ejercicios a los OA. La reutilización de recursos estuvo centrada en los conceptos y ejemplos (ver Tabla 6.2).

Tabla 6.2. Cantidad de UIs en función de la fuente de obtención de las mismas.

\begin{tabular}{lccc} 
& $\begin{array}{c}\text { Producción } \\
\text { Propia }\end{array}$ & $\begin{array}{c}\text { Localizado } \\
\text { en la Web }\end{array}$ & Total \\
\hline Objetivos & 11 & 0 & 11 \\
Contenidos & 3 & 13 & 16 \\
Ejemplos & 3 & 8 & 11 \\
Ejercitación & 10 & 1 & 11 \\
\hline Total & 27 & 22 & $\mathbf{4 9}$ \\
\hline
\end{tabular}

Con base en las 49 UIs se crearon 22 UCs y con éstas 11 OA. Los mismos fueron utilizados $-\mathrm{y}$ reutilizados- por los docentes participantes del taller para crear cinco itinerarios de aprendizaje. Además se realizó la creación del itinerario de ejemplo que se utilizó en la explicación del proceso de ensamblaje durante la segunda sesión del taller (ver Tabla 6.3).

Tabla 6.3: Lista de itinerarios de aprendizaje (finalizado el taller).

\begin{tabular}{|c|c|c|}
\hline Denominación & Creador & URL \\
\hline $\begin{array}{l}\text { IA01. INTRODUCCIÓN A ARREGLOS } \\
\text { PASCAL }\end{array}$ & Observador* & http://solaris.fdi.ucm.es:40000/elo-toolv1/elos/elo788.xml \\
\hline IA02. SELECCIÓN & $\mathrm{P} 3$ & http://solaris.fdi.ucm.es:40000/elo-toolv1/elos/elo801.xml \\
\hline $\begin{array}{l}\text { IA03.ESTRUCTURAS REPETITIVAS EN } \\
\text { PASCAL (REPEAT, FOR y WHILE) }\end{array}$ & $\mathrm{P} 4 / \mathrm{P} 5$ & http://solaris.fdi.ucm.es:40000/elo-toolv1/elos/elo800.xml \\
\hline $\begin{array}{l}\text { IA03a.ESTRUCTURAS REPETITIVAS EN } \\
\text { PASCAL (FOR Y WHILE) }\end{array}$ & P4 & http://solaris.fdi.ucm.es:40000/elo-toolv1/elos/elo795.xml \\
\hline $\begin{array}{l}\text { IA03b.ESTRUCTURAS REPETITIVAS EN } \\
\text { PASCAL (REPEAT). COMPARACIONES }\end{array}$ & P5 & http://solaris.fdi.ucm.es:40000/elo-toolv1/elos/elo799.xml \\
\hline $\begin{array}{l}\text { IA04. ARCHIVOS SECUENCIALES EN } \\
\text { PASCAL }\end{array}$ & $\mathrm{P} 2$ & http://solaris.fdi.ucm.es:40000/elo-toolv1/elos/elo796.xml \\
\hline IA05 - POLYA VS DESARROLLO & $\mathrm{P} 1$ & http://solaris.fdi.ucm.es:40000/elo-toolv1/elos/elo793.xml \\
\hline
\end{tabular}

* Este itinerario lo crea el autor de la tesis como ejemplo para el trabajo en el taller.

Luego de haber llevado adelante una extensa búsqueda, en base a los resultados y al propio proceso de búsqueda puede observarse que la reutilización es una opción viable para la creación de materiales educativos, sin embargo, se hace necesaria la participación del docente para la adecuación del materiales.

Como se mencionó arriba, la reutilización de recursos se pudo llevar adelante, principalmente, para la representación del contenido. Es decir, la explicación y ejemplos del tema abordado en el OA. Sin embargo, la localización de recursos en la web que se constituyeran en UIs que representaran los objetivos de aprendizaje del OA fue inviable. Asimismo, la localización de recursos que permitieran la consolidación de los aprendizajes, dados los objetivos y los contenidos del OA, también se tornó una tarea compleja. Si bien, se pudo hallar material que ofreciera ejercitación sobre la temática, el mismo estaba acompañado de material complementario, era muy extenso y/o se enfocaba en otros lenguajes de programación. Es decir, dependía del contexto para el cual se había diseñado. 
Otro aspecto importante es que los recursos localizados no cuentan con metadatos que pudieran ser utilizados directamente por la aplicación ELO-Tool. Esto es, si bien la mayoría de los recursos cuentan con algún tipo de metadatos estandarizado, no cuentan con la posibilidad de tomar la información en el estándar deseado. Esto sumó al trabajo de curación, el de etiquetado para poder publicarlo en el repositorio de la herramienta.

Una vez publicados los UIs y generados los OA vía ensamblaje, se hizo posible la reutilización de los mismos (siempre que el CA lo permitiera). Cabe destacar que el proceso de ensamblaje conserva -y extiende- el conjunto de metadatos de los OA lo que favorece la reutilización dentro de la herramienta y potencialmente fuera de ella, dado que el repositorio de la misma es abierto.

\subsection{Resultados posteriores al taller}

Los resultados se organizaron con base en las variables de estudio (ver 5.4.3). Cada sub-sección aborda una de las variables analizadas (ver Figura 6.2) y se consignan aquellos aportes, de los informantes, obtenidos con los distintos instrumentos de recolección de datos, que resultan de importancia o interés para dicha variable.

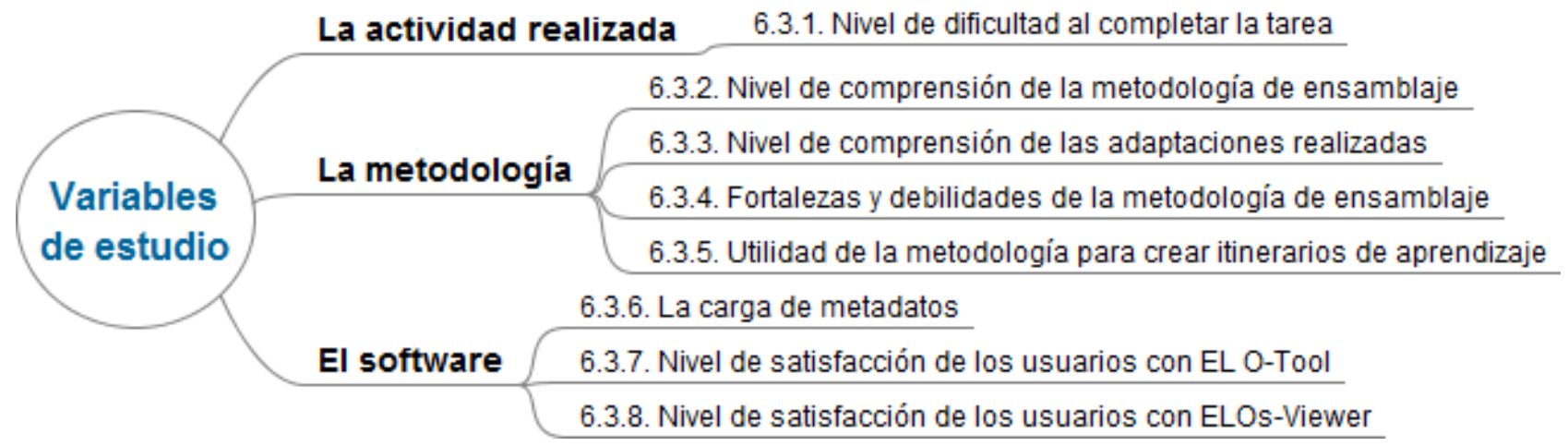

Figura 6.2. Esquema visual que muestra las variables de estudio

\subsubsection{Nivel de dificultad al completar la tarea}

Como se puede observar en la Tabla 6.3 los cinco participantes del taller (P1, P2, P3, P4 y P5) pudieron crear su itinerario de aprendizaje. Esto también se refleja en la Encuesta 2, donde todos los informantes responden afirmativamente a las preguntas ¿Pudo completar la actividad? y ¿Pudo crear el Itinerario de Aprendizaje?

Al ser consultados sobre ¿Qué tan complejo le resultó el proceso de ensamblaje? (Encuesta 2) la mayoría (3:5) respondió que le resultó un proceso fácil o relativamente fácil, mientras que uno expresó que es un proceso de dificultad media y para el otro participante el proceso fue complejo (ver Figura 6.3). En este sentido, en la Encuesta de satisfacción sobre ELO-Tool se les consultó sobre el nivel de dificultad del ensamblaje (al usar de la herramienta) y los informantes coinciden en que se trata una actividad fácil o muy fácil (ver Figura 6.4).

\section{Complejidad del proceso}

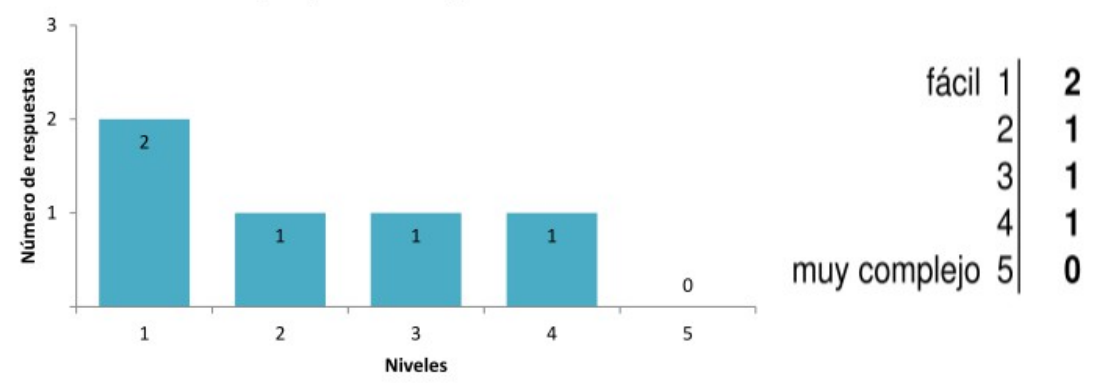

Figura 6.3. Encuesta 2. ¿Qué tan complejo le resultó el proceso de ensamblaje? 


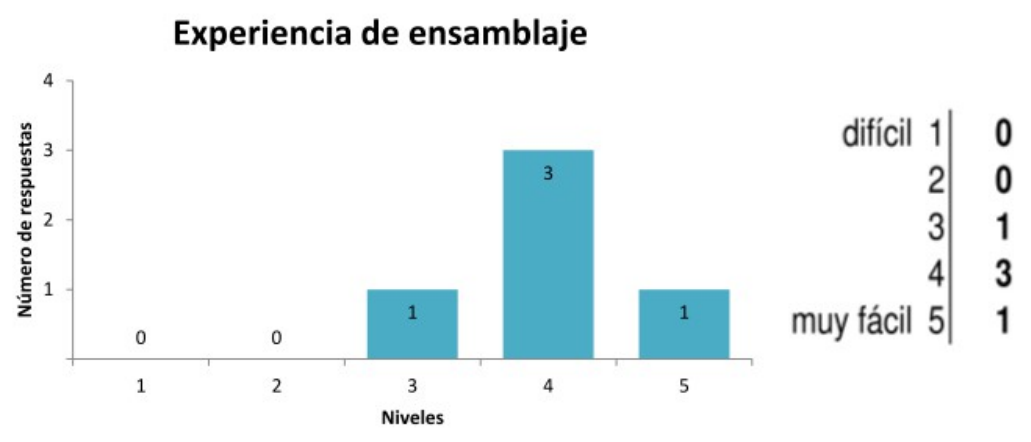

Figura 6.4. Encuesta de satisfacción sobre ELO-Tool. "El ensamblaje de los OA le resultó:"

En este mismo sentido, durante la Entrevista, se les consultó sobre la experiencia al llevar adelante el proceso de ensamblaje. A lo que los informantes P1, P2 y P3 respondieron (ver Tabla 1, en Anexo VII) que la experiencia fue buena. A P4 y P5, por su parte, se le consultó cómo fue la experiencia de trabajar en grupo y centraron su respuesta en la experiencia grupal y no en el ensamblaje.

Otro aspecto sobre los que se los consultó en la Entrevista fue si recordaban cuánto tiempo les había llevado el proceso de ensamblaje. Todos coincidieron en que la percepción fue poco tiempo, dos estiman unos diez minutos, otros dos una media hora y el restante no precisa una duración (ver Tabla 2, en Anexo VII). En el registro audiovisual, puede observarse que a P4 y P5 (que trabajaron en grupo) le llevó casi 19 minutos realizar su primer itinerario, a P1 poco más de 14 minutos y a P2 le llevó casi 25 minutos (ver Tabla 6.4).

Tabla 6.4. Tiempo de creación del itinerario. Comparativa entre la percepción del informante y el registro audiovisual

\begin{tabular}{ccc}
\hline Participante & Respuestas & $\begin{array}{c}\text { Dato desde el registro } \\
\text { audiovisual }\end{array}$ \\
\hline $\mathbf{P 1}$ & "no más de 10 minutos" & $\sim 14$ minutos \\
$\mathbf{P 2}$ & "unos 20/25 minutos." & $\sim 25$ minutos \\
$\mathbf{P 4}$ & "Media hora me imagino." & - \\
$\mathbf{P 5}$ & "no me pareció mucho" & $\sim 19$ minutos \\
\hline
\end{tabular}

* Hizo el itinerario luego de la segunda sesión del taller, no aparece en el registro audiovisual.

También en la Entrevista, al ser consultados sobre si les resultó complicado encontrar los OA que debían utilizar en el ensamblaje, primer paso del proceso, la mayoría afirmó que no (ver Tabla 3, en Anexo VII).

Como puede observarse, todos los registros estarían indicando que el proceso final del ensamblaje no reviste dificultad para los participantes, ni desde el punto de vista de la metodología, ni desde la implementación propuesta desde la herramienta. Más aún, se registra la misma percepción de dificultad durante el proceso (registro audiovisual), días después (Encuesta 2) y un mes después (Entrevista). Esta característica es uno de los aspectos centrales para la adopción de la misma como estrategia para la creación de OA.

Cabe aclarar, sin embargo, que parte del proceso que lleva a la consecución de los itinerarios fue absorbido por el autor de esta tesis. Esto es, el proceso completo, al hacerlo desde cero, consta de la búsqueda, localización, etiquetado y creación de los OA, para finalmente, crear los itinerarios de aprendizaje a partir de ellos. Además de la creación de las ontologías.

Retomando el registro, a la pregunta (Encuesta 2): Si Ud. tuvo problemas al ensamblar o no pudo 
terminar el proceso de ensamblaje... ¿Podría indicar si los problemas que tuvo fueron por alguno/s de los siguientes motivos? De los seis potenciales problemas ofrecidos como opciones, sólo uno fue elegido por un único informante: "Tuve problemas al cargar los metadatos"; y la opción abierta, de esta misma pregunta, tuvo dos respuestas: "Editar IA"1 y "no heredaba los requisitos del primer OA".

Se registraron, entonces, sólo tres problemas durante el proceso de ensamblaje llevado adelante en el taller, uno con metadatos, otro con el CA y otro relacionado con las funcionalidades de ELOTool. Al solicitarle más detalles del problema (¿Podría describir con algo de detalle el/los problema/s que seleccionó arriba?):

Para el primer caso el informante manifiesta haber encontrado la solución:

"Los problemas o dificultades seleccionados arriba se allanaron rápidamente por la modalidad de trabajo en el taller, que aportó mucho trabajo previo del docente a cargo del mismo, pero cuando no se está familiarizado con la herramienta, la dificultad mayor es saber qué se está requiriendo en cada caso (en la carga de metadatos)"

El segundo y tercer caso, los informantes coinciden en un problema con el $\mathrm{CA}^{2}$ :

\section{“[...]2) algunos campos con metadatos de los OA que ensamblamos no se heredaban" \\ "ELO-Tools no heredaba los requisitos del primer OA al momento de ensamblar y generar el IA (en algunos casos. Nuestro grupo armó 3 IA).[...]"}

En este sentido, al reproducir el proceso de ensamblaje de los participantes que armaron tres itinerarios ("Nuestro grupo armó 3 IA"), puede observarse el problema en concreto en el itinerario "IA03b. ESTRUCTURAS REPETITIVAS EN PASCAL (REPEAT). COMPARACIONES". Esto se puede observar en la Figura 6.5 donde se reproduce el ensamblaje de dicho itinerario y los requisitos aparecen vacíos.

Cabe aclarar que, tal como se observa en el registro audiovisual (ver Tabla 4 en Anexo VII), uno de los integrantes del grupo (P4), al momento de completar los requerimientos del itinerario "IA03a.ESTRUCTURAS REPETITIVAS EN PASCAL (FOR Y WHILE)", manifiesta que cuenta con los requerimientos del primer OA. Por lo que el error sólo ocurre en uno de los itinerarios.

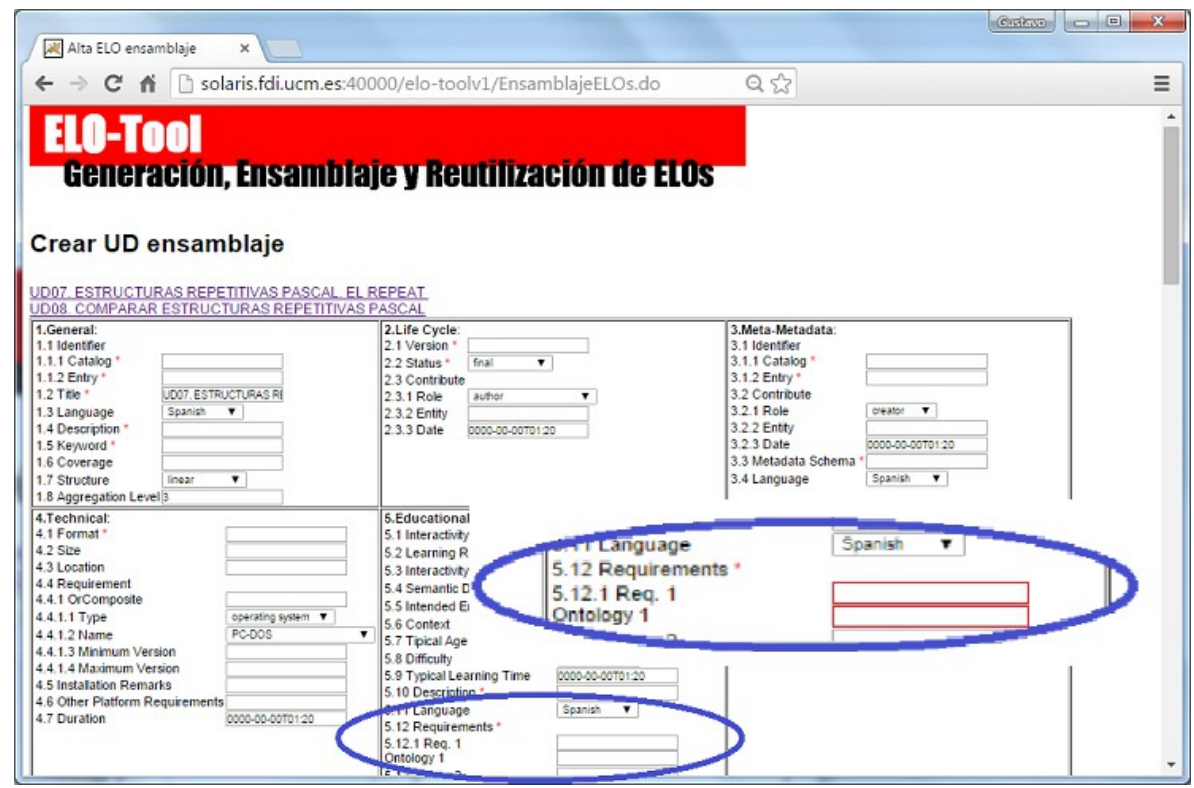

Figura 6.5. La imagen muestra una captura de pantalla donde al ensamblar los OA correspondientes al IAO3b los requerimientos aparecen vacios.

1 "IA": El informante hace referencia a Itinerario de Aprendizaje.

2 Se destaca en negrita los problemas con el CA. Las observaciones sobre los problemas con el software serán abordadas en la sub-Sección que aborda el uso de los mismos. 
De acuerdo al registro realizado por el observador en la Planilla estructura de observación (ver Tabla 6.5), si bien, algunos de los participantes tuvieron algunas dificultades las pudieron superar.

Tabla 6.5. Contenido de la Planilla estructura de observación.

\begin{tabular}{ccl} 
Participante & $\begin{array}{c}\text { Dificultad } \\
\text { (valores entre 0 y 5)* }\end{array}$ & \multicolumn{1}{c}{ Observaciones } \\
\hline P1 & 0 & $\begin{array}{l}\text { No hizo preguntas sobre cómo utilizar o ensamblar (se valió del material) } \\
\text { No pudo acceder al material en la web, se lo pase en un pendrive y pudo } \\
\text { ensamblar sin problemas }\end{array}$ \\
P3 & 2 & $\begin{array}{l}\text { Hizo fuera de la clase } \\
\text { P4 y P5 trabajaron en conjunto, crearon IA3A, IA3B y luego los } \\
\text { ensamblaron para crear IA03. Crearon pero además eliminaron itinerarios } \\
\text { que le habían quedado mal los nombres } \\
\text { P4 }\end{array}$ \\
Idem P4 porque trabajaron juntos.
\end{tabular}

\begin{abstract}
* Donde 0 no tuvo dificultad, 1 tuvo problemas mínimos (fueron solucionados), 2 tuvo algunos problemas que obstaculizaron el proceso (fueron solucionados), 3 tuvo varios problemas que obstaculizaron el proceso (fueron solucionados), 4 tuvo varios problemas que obstaculizaron el proceso (solo algunos fueron solucionados) y 5 tuvo varios problemas que obstaculizaron el proceso (la mayoría no fueron solucionados).
\end{abstract}

Los datos obtenidos del relevamiento realizado a partir del taller muestran que, además de completar exitosamente el ensamblaje de OA, lo hicieron prácticamente sin dificultades. Asimismo, los obstáculos, en todos los casos, pudieron ser superados a partir de una simple consulta sobre la semántica de los metadatos a cargar y con el relleno manual de los valores faltantes.

Se puede concluir, entonces, que el nivel de dificultad al completar la tarea fue bajo, ya que el proceso ensamblaje de OA se pudo llevar adelante de forma fácil y con mínimas dificultades.

\title{
6.3.2 Nivel de comprensión de la metodología de ensamblaje
}

Para explicar la metodología a utilizar se ocupó la primer sesión del taller (45'). Terminada la sesión se les envió por correo electrónico, a los participantes, el enlace a la Encuesta 1 que indaga sobre distintos aspectos de la metodología. Las respuestas fueron emitidas dentro de la primera semana posterior al envío del correo.

Al ser consultados sobre qué entendían por ensamblaje (Encuesta 1), la mayoría (3:5) optó por dos de las opciones: "Agrupar los OA de acuerdo con el CA" y "Crear un itinerario de aprendizaje". Mientras que en menor medida (2:5) lo hicieron por "Agrupar recursos educativos en una secuencia de aprendizaje". Descartando de plano la opción de "Crear un OA" (ver Figura 6.6). Así, los informantes tomaron las opciones que mejor describen al ensamblaje de la metodología presentada.

Un aspecto central en la metodología elegida es el CA. Al consultarlos (Encuesta 1) por este concepto mayoritariamente (5:5) se inclinaron por la opción "Metadatos que describen al OA". Sin embargo, no descartaron las otras opciones, en igual proporción (2:5) seleccionaron: "Las competencias necesarias para abordar el OA", "Los requisitos necesarios para abordar el OA" y "Las competencias alcanzadas tras abordar el OA" (ver Figura 6.7).

Respecto, también, al CA y su función dentro del ensamblaje, pudo observarse en el registro audiovisual algunos diálogos entre los informantes que dan cuenta de la comprensión sobre este aspecto. P2 explica a P4 la relación entre competencias y requerimientos: "los requerimientos están contenidos en las competencias. Pueden ser menos requerimientos que las competencias, pero no más..." (ver Tabla 5 Anexo VII). Luego P4 hace lo propio con P5: "las competencias de éste... éstas tienen que estar incluidas allá... Si éstos no te dan no lo podés ensamblar con el otro..." (Tabla 6 Anexo VII).

Se pude apreciar entonces, en ambos registros, que no todos los informantes lograron una comprensión acabada del concepto. Si bien, efectivamente, el CA se representa a través de 
metadatos, su función principal es constituirse como los requisitos para abordar el OA y las competencias adquiridas una vez utilizado el mismo.

\section{Qué es el ensamblaje de OA}

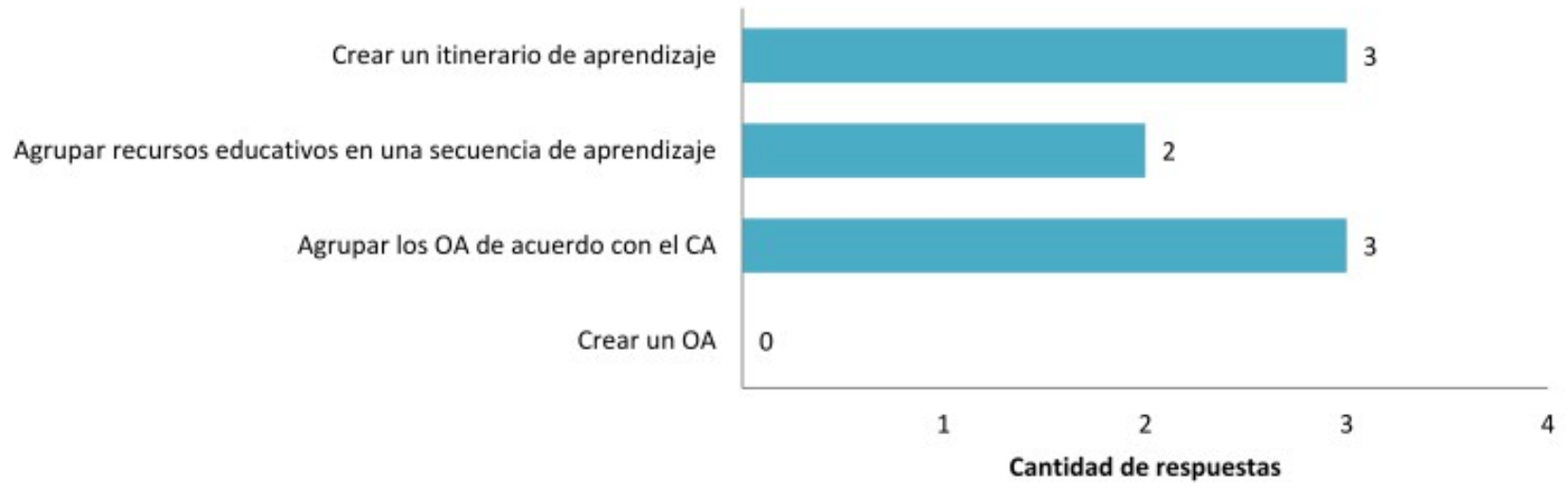

Figura 6.6. Encuesta 1. "Según lo expuesto hasta aquí en el taller, considera Ud. que el ensamblaje de OA es"

\section{Qué es el Conocimiento Asociado}

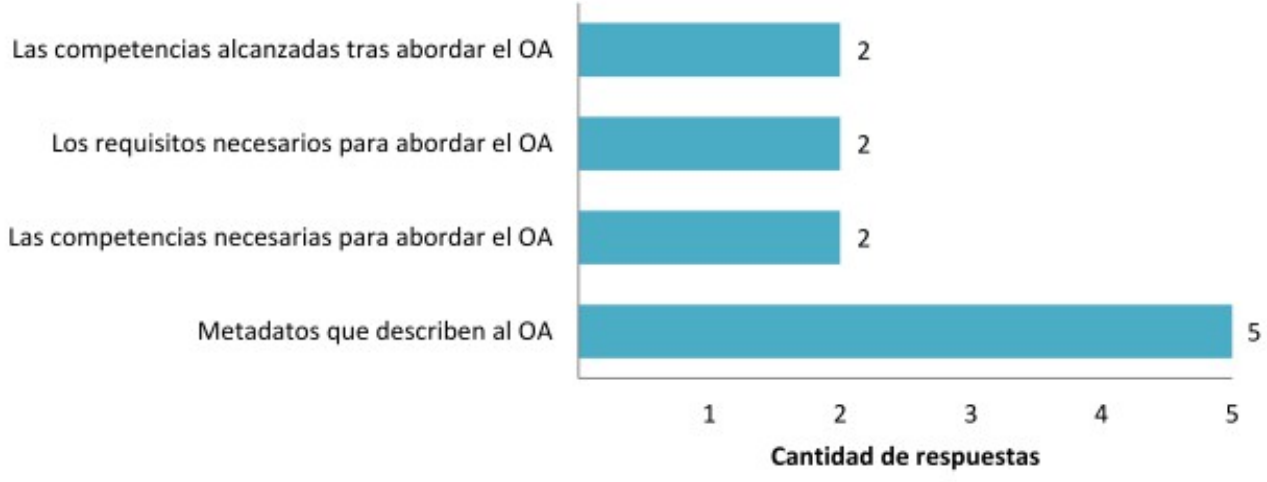

Figura 6.7. Encuesta 1. "En la metodología de ensamblaje, el CA es"

Respecto de cómo se genera el CA, en la Encuesta 1, tres de los cinco creen que los requerimientos los obtiene del primer OA de la secuencia, y en la misma proporción acuerdan con que las competencias son tomadas del último OA de la secuencia.

Durante la actividad de ensamblaje, según los registros audiovisuales, hubo dudas sobre requerimientos, no así sobre las competencias. El registro audiovisual muestra a dos participantes que abordan el tema y, en charla con el Observador, desean confirmar si las competencias se generan por suma o unión de todas las competencias de la secuencia (ver Tabla 7 en Anexo VII).

En este caso, si bien la comprensión de la generación del CA durante el proceso de ensamblaje es inicialmente parcial, en la sesión de ensamblaje esto no parece reflejado en la actividad. Es decir, es correcto afirmar que los requerimientos son obtenidos del primer OA de secuencia, sin embargo las competencias son generadas a partir de la unión de las competencias de todos los OA del itinerario y no sólo de último. Pero, al crear los itinerarios no hay cuestionamientos sobre cómo se crean las competencias, sino más bien, una confirmación de lo que muestra el software al cargar los metadatos.

El CA está representado en forma de metadatos dentro de los OA, los cuales hacen referencia a ontologías del dominio. Al consultar a los informantes por éstas últimas, varios (3:5) las asociaron más a los metadatos, al acordar en que "permiten describir los OA", pero al mismo tiempo una mayoría (4:5) acuerdan en que "permiten describir el dominio" (ver Tablas 6.6). Si bien, está mayoría contesta correctamente, asociarlos con la descripción de los $\mathrm{OA}$, en esta metodología, no es correcto. 
Aunque mayoritariamente (4:5) acuerdan con que las ontologías "permiten el ensamblaje de OA heterogéneos", no acuerdan (3:5) con que "permiten la comparación del CA de cada OA" (ver Tablas 6.7). Estas respuestas son un tanto contradictorias ya que para poder ensamblar los OA deben poder compararse.

Tabla 6.6. Encuesta 1. Comparativa de respuestas de los participantes acerca de qué describen las ontologías.

En la metodología de ensamblaje, las ontologías:

Permiten describir los OA

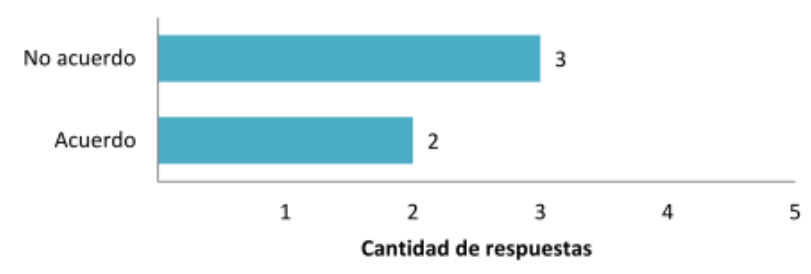

\section{Permiten describir el dominio}

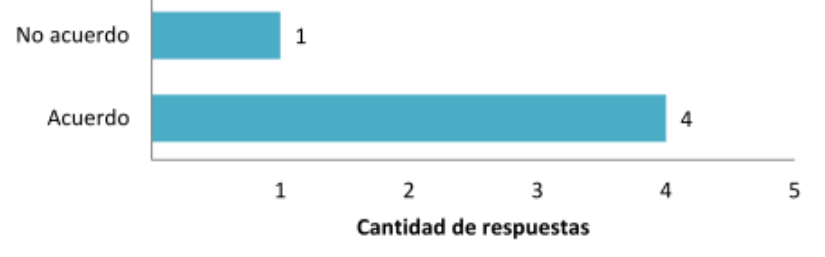

Tabla 6.7. Encuesta 1. Comparativa de respuestas de los participantes acerca la función de las ontologías.

En la metodología de ensamblaje, las ontologías:

\section{Permiten el ensamblaje de OA heterogéneos}

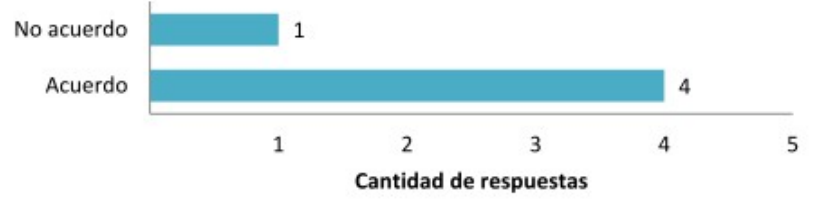

\section{Permiten la comparación del CA de cada OA}

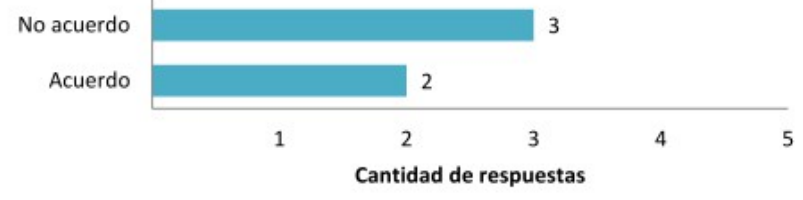

Por su parte, en la Entrevista, al ser consultados sobre si recordaban cómo es que la metodología utiliza las ontologías para ensamblar, tres respondieron afirmativamente, pero cuando se explayaron, lo hicieron con respuestas poco precisas. Los otros dos participantes manifiestan no acordarse del tema (ver Tabla 9 en Anexo VII).

Se puede observar entonces, que el concepto de ontología no fue comprendido en su totalidad por los participantes del taller.

Se podría concluir, con base en los registros, que el nivel de comprensión de la metodología de ensamblaje es medio o bajo, ya que si bien $(i)$ los informantes tomaron las opciones que mejor describen al ensamblaje de la metodología presentada, (ii) no todos lograron una comprensión acabada del concepto CA y de la forma en que el mismo se genera. A esto se le suma (iii) una ambigua noción de la función de las ontologías en el proceso de ensamblaje.

\subsubsection{Nivel de comprensión de las adaptaciones realizadas}

Para llevar adelante el estudio de caso se realizaron un conjunto de adaptaciones a la metodología (ver Sección 5.5), las mismas fueron explicadas y luego se le consultó a los informantes sobre distintos aspectos de las mismas para evaluar el nivel de comprensión de los cambios realizados.

Uno de los cambios fue la determinación de denominar recursos educativos a las UIs y UCs, y OA a las UDs. Consultados sobre este respecto, en la Encuesta 1, los informantes en su mayoría (3:5) consideran recursos a las UCs y UDs, y sólo un 1:5 a las UIs. Por otra parte la mayoría (3:5) cree que un OA es una UD (ver Figuras 6.8 y 6.9).

Como puede observarse en las Figuras 6.8 y 6.9, los informantes no pueden reconocer, con base en la clasificación propuesta, los distintos tipos de recursos. Es decir, si bien es correcto afirmar que las $\mathrm{UC}$ son recursos, las UD (que equivalen a OA) no son considerados recursos. 
Qué ELO son recursos educativos

(ADAPTACIÓN)

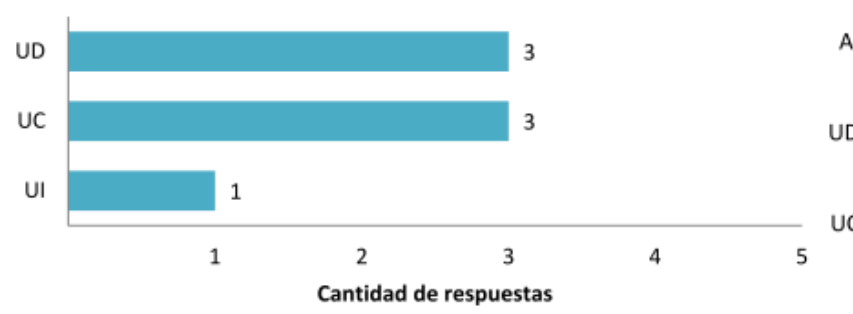

Figura 6.8. Encuesta 1. "Según la ADAPTACIÓN propuesta, qué tipo de ELO son considerados recursos educativos"

\section{Un OA equivale a (ADAPTACIÓN)}

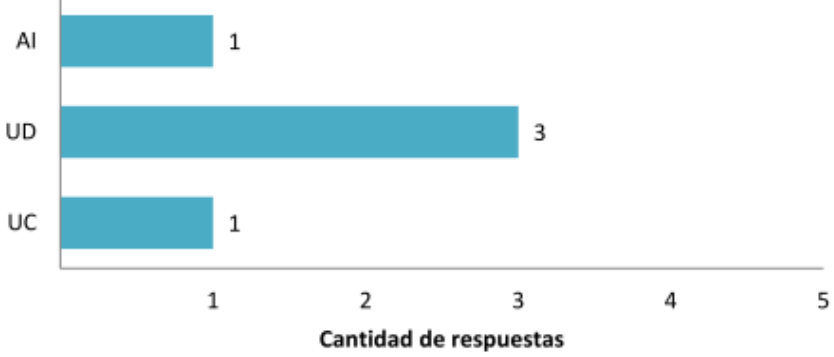

Figura 6.9. Encuesta 1. "Según la ADAPTACIÓN propuesta, un Objeto de Aprendizaje equivale a"

Otra de las adaptaciones propuestas se basa en la definición de OA con la que se trabaja en esta tesis donde un OA debe contener: objetivo específico, contenido, actividad, evaluación y metadatos. También se los consultó a los informantes sobre este punto.

La Encuesta 1 muestra que todos coinciden en que los OA deben contar con actividades y contenidos. Una importante mayoría (4:5) metadatos estandarizados y evaluación. En menor número (3:5) seleccionaron objetivo y sólo uno exige la presencia de ontologías. Cabe destacar que ninguno de los informantes considera que los OA debe tener "Tantos objetivos como UC contenga" (ver Figura 6.10). Para el caso de la definición de OA, la mayoría de los informantes caracterizan adecuadamente la estructura interna de un objeto de aprendizaje.

\section{Un OA debe contener (ADAPTACIÓN)}

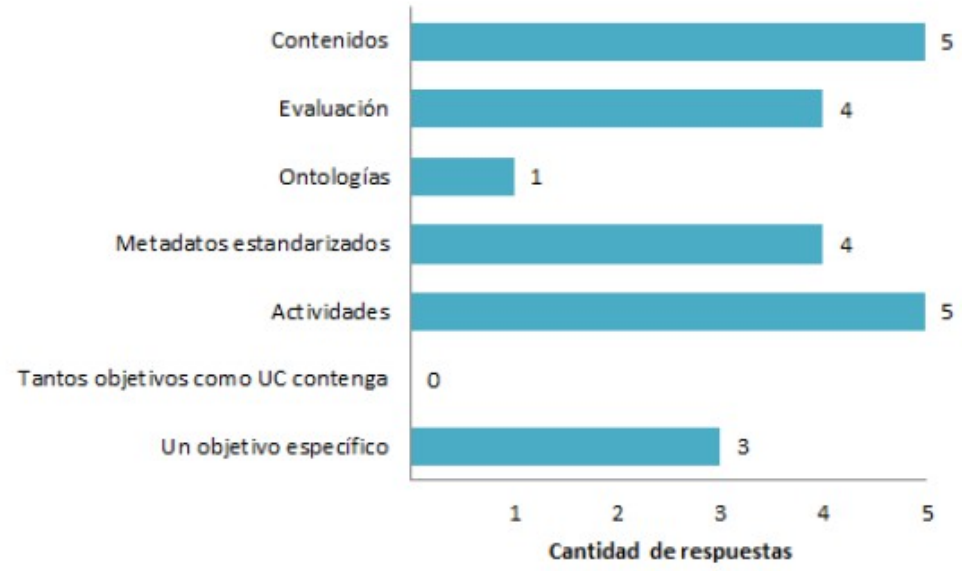

Figura 6.10: Encuesta 1. "Según la ADAPTACIÓN propuesta, un OA debe contener"

Otra de las adaptaciones propuestas está basada en el hecho de que un OA, según la definición adoptada, debe tener un objetivo específico de aprendizaje. Así pues, toda combinación de OA se considera un itinerario de aprendizaje -más de un objetivo-.

A este respecto se los consultó (Encuesta 1) sobre qué se obtiene al ensamblar, según la metodología elegida, para luego, hacer lo propio con la adaptación propuesta.

Para el caso de la metodología original, todos coincidieron en que UI+UC es una UC y que una UC+UD es una UD. Un alto porcentaje (4:5) coincidieron en que UC+UC es una UD. En menor cantidad (3:5) estiman que dos UI dan como resultado una UC y que dos UD generan otra UD. Ninguno de los informantes cree que al combinar una UC con una UD se obtenga un itinerario de aprendizaje (IA). Cabe destacar que cuatro de los cinco participantes afirman que una UI+UD es UD, y en un porcentaje menor $(2: 5)$ que dos UD, al ensamblar crean un IA. Lo primero está prohibido en la metodología original y en lo segundo, no está contemplado en la misma. La granularidad mayor la tiene la UD (ver Figura 6.11). Salvo por estos errores podría considerarse que en su mayoría los participantes pudieron identificar qué tipo de ELO es obtenido al combinar otros dos. 
Luego de consultar por los resultados al ensamblar dos ELOs, se indagó sobre los itinerarios de aprendizaje (Encuesta 1). La mayoría (3:5) acuerda con que está conformado por una secuencia de dos UDs, y la misma cantidad acuerda con que es un conjunto de dos o más OA -podían elegir más de una opción-. Sólo dos de los cinco acuerdan con que no es posible crear un itinerario con UIs.

En la Entrevista, se los consultó sobre qué tipo de material se utiliza para crear los itinerarios de aprendizaje. Cabe aclarar que en todos los casos se debió listar el conjunto de materiales: UI, UC, UD y OA. Las respuestas fueron dispares, dos contestaron correctamente (objetos de aprendizaje), otros dos optaron por las unidades de contenido y el restante asume que un itinerario está dentro de una unidad didáctica (ver Tabla 10 en Anexo VII).

Para el caso de esta adaptación, inicialmente, la mayoría de los informantes tenían una concepción correcta sobre cómo se conformaba un itinerario de aprendizaje. Sin embargo, al consultarlos tiempo después, esta idea fue perdiendo precisión.

\section{Qué se obtiene al ensamblar}

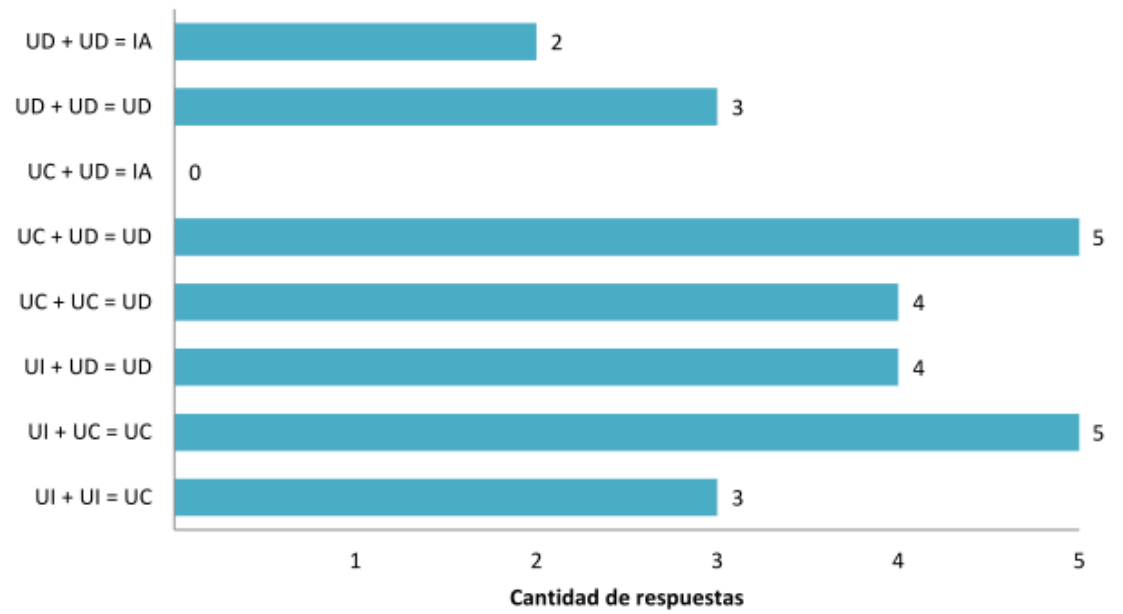

Figura 6.11. Encuesta 1. "Según la metodología de ensamblaje, qué se obtiene al ensamblar"

Durante la actividad de ensamblaje según se puede observar en el registro audiovisual, los participantes, si bien recuerdan que deben incluirse metadatos extras, no recuerdan cuáles son (ver Tabla 11 y 12 en Anexo VII). También en el registro audiovisual, se puede apreciar como P1 y P4 intercambian opiniones con el Observador respecto de cuáles son los requerimientos que autocompleta la aplicación y cuáles deben ser agregados (ver Tabla 8 en Anexo VII).

Cabe destacar, en éste punto, que los participantes se acercaban por primera vez a estos conceptos y sólo fueron expuestos brevemente a los mismos en una sesión de 45'. Asimismo, no se les proporcionó, desde el taller, material bibliográfico o de consulta sobre la temática.

Esto último podría explicar el hecho de que los informantes no hayan podido reconocer adecuadamente los distintos tipos de recursos y algunas de las adaptaciones propuestas. Sin embargo, y pese a la reducida experiencia en la temática, la mayoría de los informantes caracterizan adecuadamente la estructura interna de un objeto de aprendizaje, qué se obtiene al combinar dos ELOs y cómo está conformado un itinerario de aprendizaje. Se podría concluir, con base en los registros, que el nivel de comprensión de las adaptaciones realizadas a la metodología es medio.

\subsubsection{Fortalezas y debilidades de la metodología de ensamblaje}

Uno de los objetivos de este estudio de caso es identificar fortalezas y debilidades de la metodología. Por tanto, se indagó a los informantes sobre los distintos aspectos que están involucrados en el proceso de ensamblaje en el que ellos participaron.

El nivel de automatización ofrecido por la metodología fue observado a través del uso de la aplicación ELO-Tool. Consultados en este sentido (Encuesta 2), mayoritariamente respondieron en favor de la suficiencia ofrecida por la metodología en este aspecto (ver Figura 6.12). 


\section{El nivel de automatización}

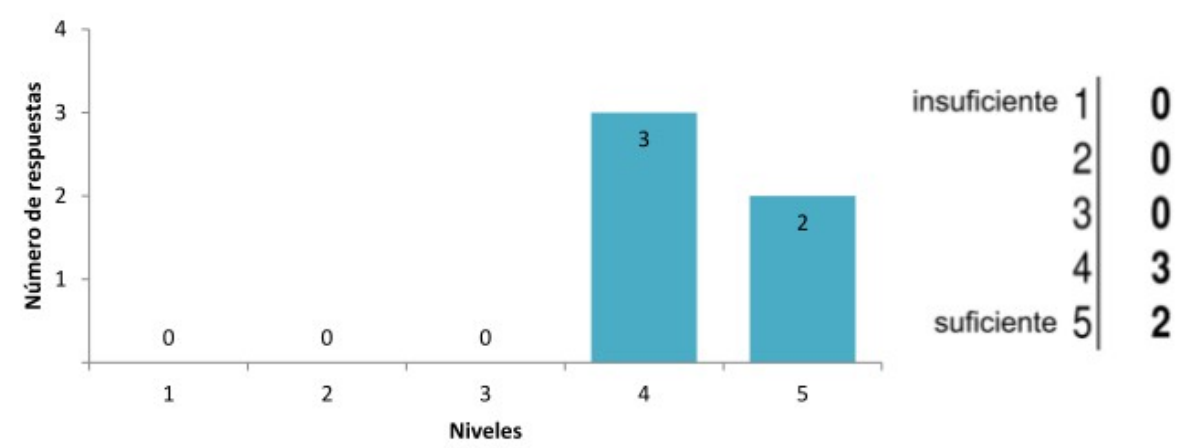

Figura 6.12. Encuesta 2. "El nivel de automatización del proceso de ensamblaje que le propone la metodología le pareció"

Con respecto a la cantidad de metadatos que tuvieron que cargar -la metodología utiliza LOM, un estándar extenso-, al ser consultados (Encuesta 2), los informantes respondieron de forma variada. La mayoría (3:5) afirma que es aceptable, mientras que a uno le parece apropiada y, en el otro extremo, a otro le parece caso excesiva (ver Figura 6.13).

La cantidad de metadatos que tuvo que

cargar

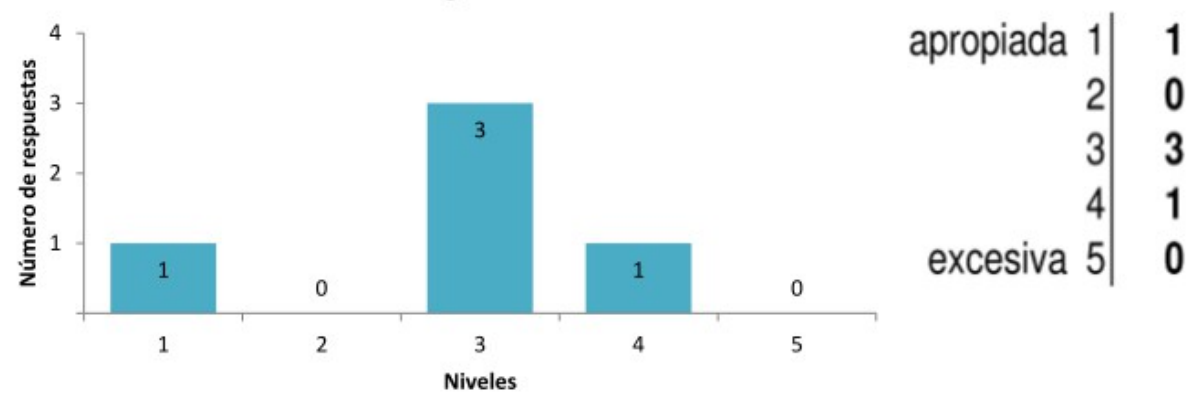

Figura 6.13. Encuesta 2. "La cantidad de metadatos que tuvo que cargar para concretar el ensamblaje, le pareció"

Respecto del CA, los informantes opinan que la cantidad de información que aporta el mismo no llega a ser suficiente para el proceso de ensamblaje. Esto es, eligieron los niveles 3 (2:5) y 4 (3:5) para una escala de 1 a 5, donde 1 es insuficiente y 5 suficiente (ver Figura 6.14).

La cantidad de información que aporta

el CA

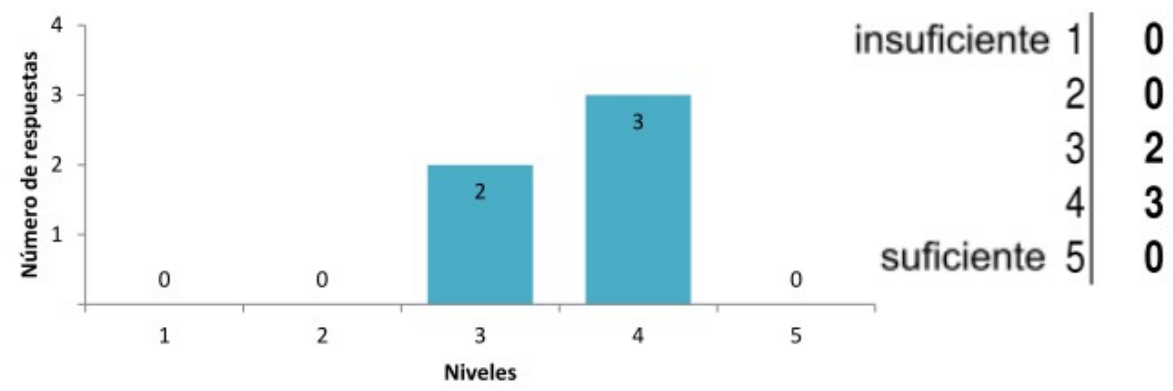

Figura 6.14: Encuesta 2. "La cantidad de información que aporta el Conocimiento Asociado (CA) para el ensamblaje, en todos los casos, le parece"

También relacionado al CA, está la elección de la clase de la ontología que mejor represente los requerimientos y las competencias, esta tarea le resultó a la mayoría de una complejidad media (3:5) y al resto sencilla (Encuesta 2, ver Figura 6.15). 
Elección del Conocimiento Asociado

(CA) más apropiado

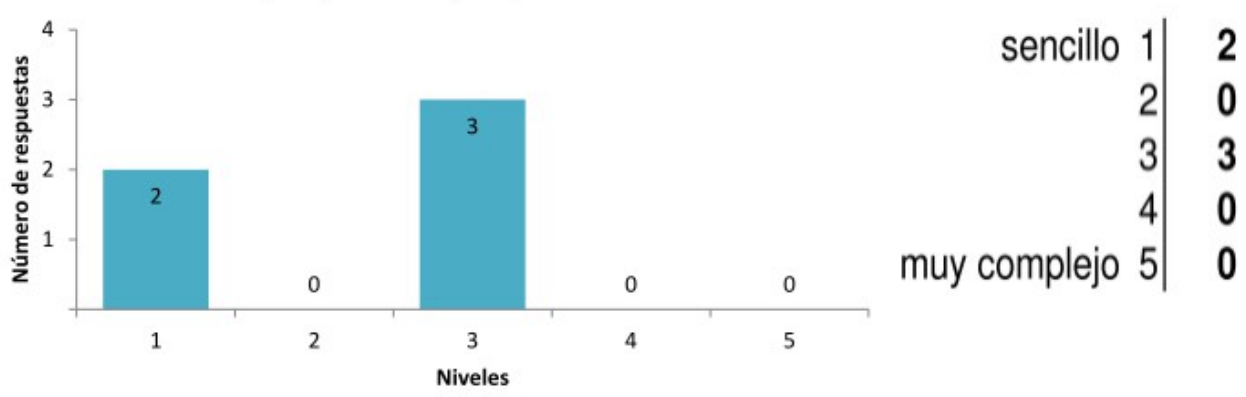

Figura 6.15: Encuesta 2. "Cuando tuvo que utilizar las ontologías para completar los requerimientos/competencias, la elección del Conocimiento Asociado (CA) más apropiado le resultó"

La metodología es dependiente de las ontologías para el ensamblaje. Se les consultó a los participantes del taller (Encuesta 2) qué tan flexible era la metodología al depender de ontologías y, en sus respuestas, uno afirma que es flexible, mientras que la mayoría se inclinan por una flexibilidad moderada o media (ver Figura 6.16).

\section{Dependencia de ontologías}

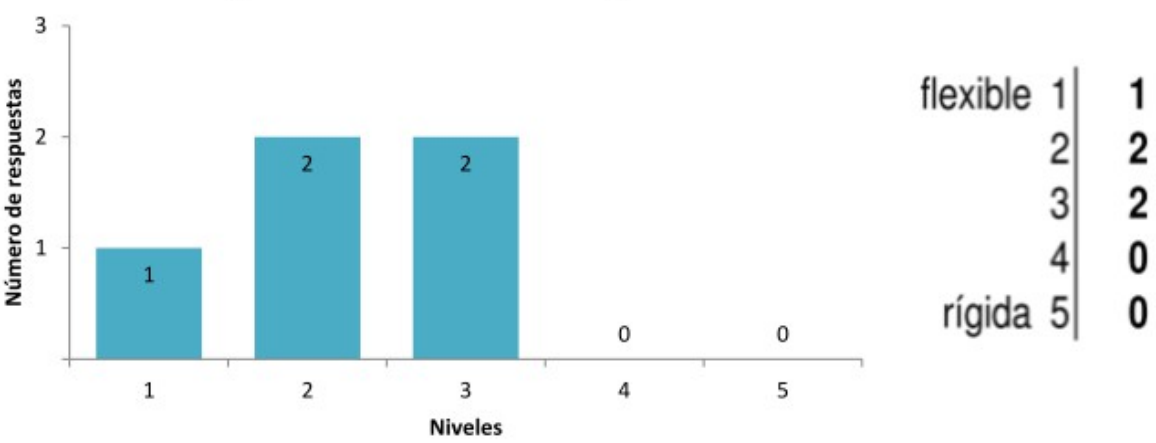

Figura 6.16. Encuesta 2. "La metodología depende de las ontologías para el ensamblaje, esto la vuelve una metodología"

Como se puede apreciar en las Figuras 6.12 a 6.16, las respuestas de los informantes se encuentran en los niveles medio o centrales. Sin embargo, podría distinguirse como aspectos positivos o fortalezas que: se trata de una metodología con un buen nivel de automatización, solicita al usuario una cantidad aceptable de metadatos (incluyendo aquí la elección del CA) y, el hecho de que dependa de ontologías, la hace una metodología medianamente flexible. Por otra parte, la principal debilidad estaría en la cantidad de conocimiento asociado que utiliza para el ensamblaje.

\subsubsection{Utilidad de la metodología para crear itinerarios de aprendizaje}

Uno de los objetivos de la metodología, y de la herramienta que la implementa, está enfocado en el apoyo a los docentes para crear itinerarios de aprendizaje. Es por esto que se consultó a los informantes a éste respecto.

La mayoría (3:5) afirma que seguramente volvería a utilizar una metodología de ensamblaje para la creación de su material didáctico, mientras que el 2:5 restante está bastante seguros de hacerlo (ver Figura 6.17). 


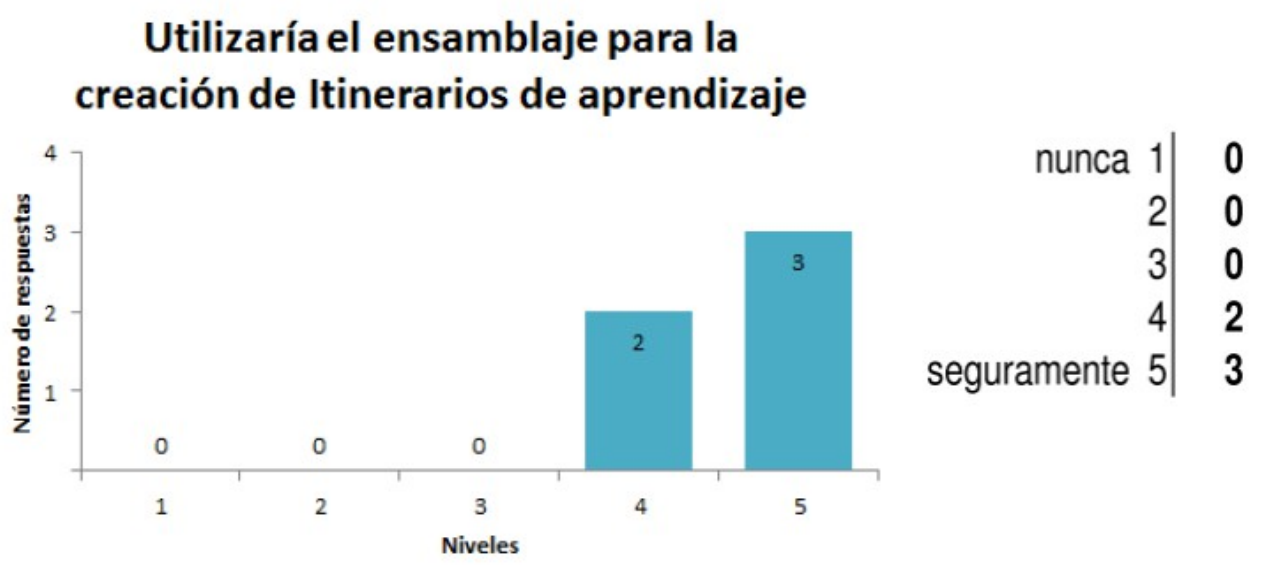

Figura 6.17: Encuesta 2. "Utilizaría, en el futuro, el ensamblaje (con esta herramienta u otra) como una técnica para la creación de itinerarios de aprendizaje"

Por otra parte, la totalidad de los informantes están de acuerdo en que el ensamblaje de OA le facilitaría su tarea como docente. Al solicitarles que justificaran su respuesta, tres coincidieron en que favorece la reutilización del material educativo y los otros dos se enfocan en que facilita parte de la creación del material al hacerlo de forma incremental (ver Tabla 14 en Anexo VII).

La reutilización también se abordó en una pregunta específica, ya que es otro de los objetivos de la metodología. Consultados sobre este aspecto, todos los informantes coinciden en que propicia la reutilización de OA (Tabla 15 Anexo VII).

Se hace evidente, en las respuestas de los informantes, que éstos ven en la metodología el potencial para la creación de material educativo a través de la reutilización de recursos existentes. Este aspecto está dentro de las motivaciones de la creación de la metodología y esta intensionalidad se ve reflejada en las opiniones de los participantes del taller.

\subsubsection{La carga de metadatos}

La metodología utiliza una extensión del estándar de metadatos LOM, un estándar con una importante cantidad de campos por completar. En el caso de las UDs el formulario supera los 74 campos a cargar (dependiendo a cuántos requisitos, cuántas competencias y cuántos objetivos tenga). Si bien sólo 24 de los campos son obligatorios y algunos de ellos son completados por ELOTool, esta importante cantidad de datos solicitados, suele ser un aspecto negativo o, al menos, controversial en el proceso de creación de material educativo. Es por esto que tanto en la Encuesta 2, como en la de satisfacción sobre ELO-Tool y en la Entrevista, se les consultó a los participantes del taller sobre la carga de metadatos.

En la Encuesta 2, con respecto a la cantidad de metadatos que tuvieron que cargar, hubo distintos pareceres a este respecto: mientras para la mayoría es aceptable (3:5), a uno le pareció apropiada y al otro casi excesiva (ver Sección 6.2.4, Figura 6.13).

Cuando en la Encuesta de satisfacción de ELO-Tool se les consultó sobre cómo les resultó la carga de los metadatos usando esta herramienta, a la mayoría le resultó una actividad fácil (3:5), a otro medianamente fácil y al último un tanto difícil (ver Figura 6.18). Un resultado similar se obtuvo para los metadatos que representan el CA: a la mayoría (3:5) le resultó fácil y al resto medianamente fácil (ver Figura 6.19).

En ambos registros puede observarse que, pese a tratarse de una número importante de metadatos a los informantes les resultó, en general, una cantidad apropiada. También una actividad sencilla, pese a algunas dificultades que manifiestan, durante la Entrevista, acerca de la complejidad semántica de los metadatos. 


\section{La carga de metadatos}

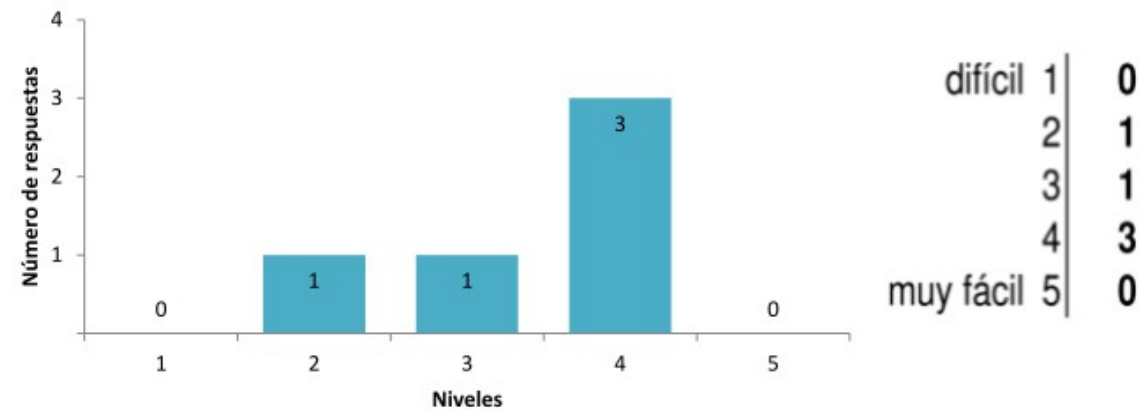

Figura 6.18. Encuesta de satisfacción de ELO-Tool . "La carga de metadatos le resultó"

La carga de CA

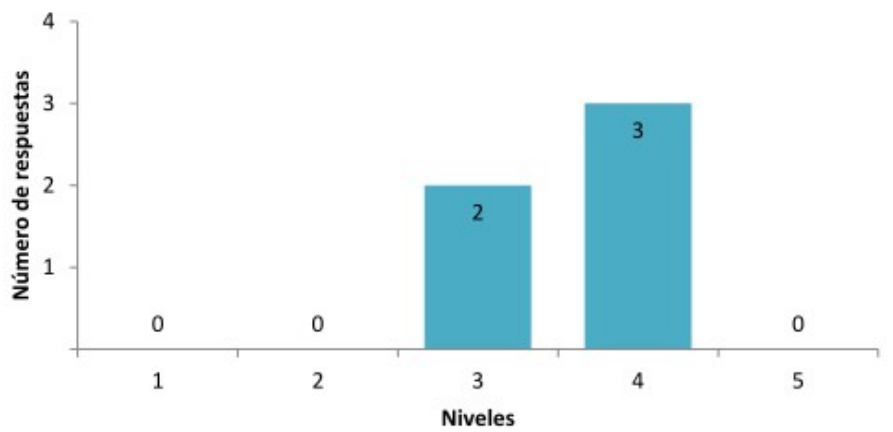

Figura 6.19. Encuesta de satisfacción de ELO-Tool. "La carga de CA de los OA le resultó"

Durante la Entrevista se le pidió que relatara su experiencia con la carga de metadatos y se le preguntó si le había resultado un "trabajo 'pesado". Analizando las respuestas (ver Tabla 16 en Anexo VII), se puede observar que, a cuatro de los cinco participantes no les resultó un trabajo pesado. Sin embargo, dos de los participantes (P1 y P2) manifestaron dudas sobre la semántica de algunos metadatos. Por otra parte, P2, P3 y P4, observan que inicialmente tuvieron la sensación de que se trataba de muchos metadatos. También se puede destacar que la metodología y la herramienta colaboran para que no haya que cargar tantos datos (P3 y P4). Finalmente, P4 y P5, destacan que una actividad en la que hay que "estar atento" y "prestar atención".

Con el objetivo de facilitarle a los participantes la carga de los metadatos, durante el proceso de ensamblaje, se les proporcionó una ayuda ${ }^{3}$. Se les consultó sobre el nivel de uso de este material para llevar adelante la carga de metadatos. A este respecto, cuatro de los cinco participantes manifiestan haber utilizado el material y que les fue de utilidad. P4, por su parte, afirma no haberlo utilizado, sin embargo, puede observarse en el registro audiovisual que sí utiliza el material (ver Tabla 17 en Anexo VII).

Al consultarlos por los campos que debieron completar solos ( $\sin$ la ayuda), por tratarse de contenido que debe cargar el docente, todos concuerdan en que pudieron hacerlo sin problema (ver Tabla 18 en Anexo VII).

Puede observarse, que los dos aspectos más complejos de la carga de metadatos, cantidad y semántica de los mismos, aparecen reflejados como preocupaciones en parte de los registros obtenidos. Asimismo, es notable la facilidad con la que fue llevado adelante el proceso de carga de metadatos, dado que se encontraban por primera vez con este tipo de actividad en el contexto de un proceso de ensamblaje. Estas respuestas podrían encontrar su justificación en que la herramienta que les facilitó el trabajo, sin embargo, la opinión de los informantes a este respecto no es unánime. Durante la Entrevista, se consultó si creían que ELO-Tool les facilitó la carga de los metadatos. Tres manifestaron que sí y los otros dos afirman que, al no poder compararla con otra aplicación, no pueden definir si les facilitó el trabajo (ver Tabla 19 en Anexo VII). También debe considerase que el hecho de contar con material de apoyo para completar los metadatos facilitó la carga.

3 El documento estaba organizado en diapositivas y se distribuyó en formato PDF. 


\subsubsection{Nivel de satisfacción de los usuarios con ELO-Tool}

Luego de la Sesión de ensamblaje, se consultó a los participantes sobre el nivel de usabilidad de ELO-Tool.

Consultados respecto a la claridad ELO-Tool permite realizar las tareas, a la mayoría le parece clara (3:5), uno bastante clara y a otro medianamente clara (ver Figura 6.20). Los mismos resultados se obtuvieron al consultarlos por la navegación a través del sistema (ver Figura 6.21).

\section{Claridad del sistema para las tareas solicitadas}

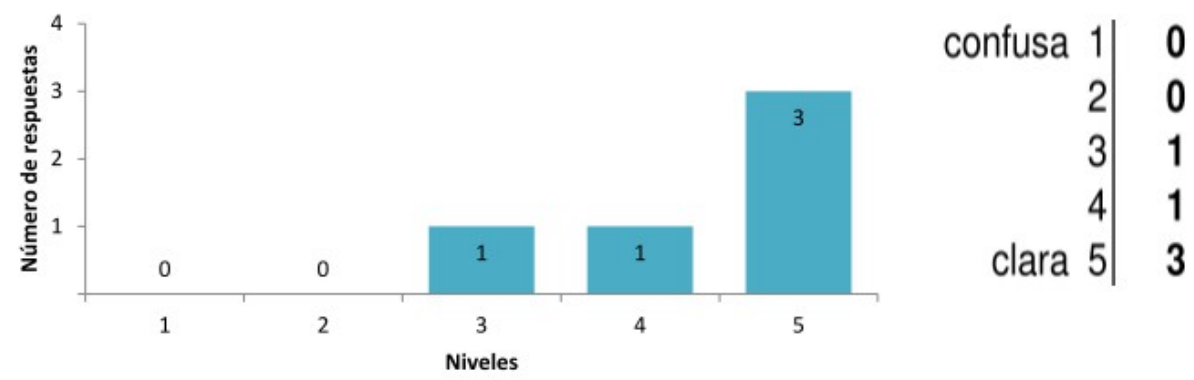

Figura 6.20. Encuesta de satisfacción de ELO-Tool. "Su percepción en relación a la claridad de cómo el sistema le permite realizar las tareas solicitadas es"

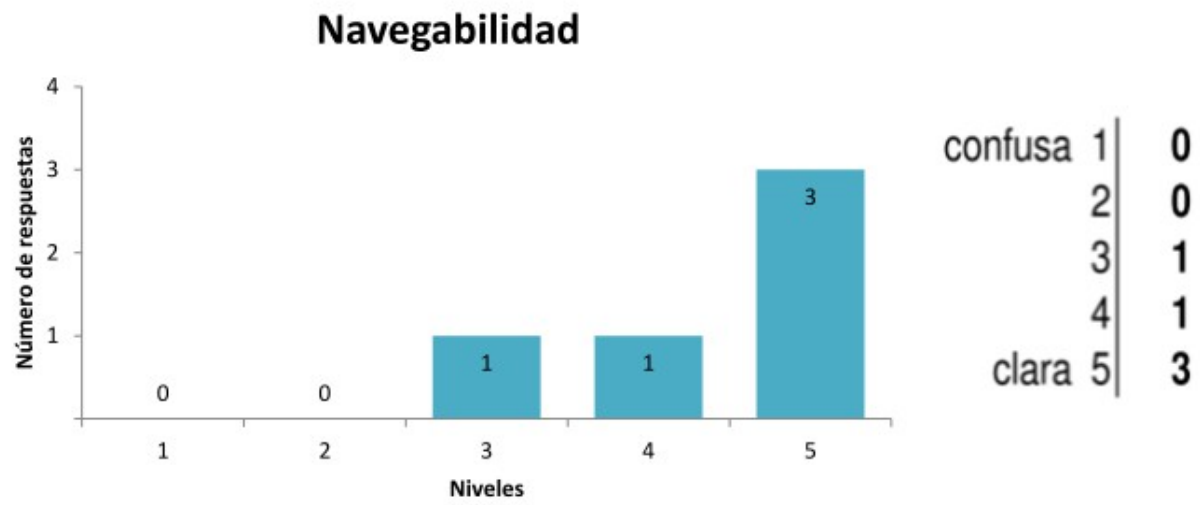

Figura 6.21. Encuesta de satisfacción de ELO-Tool. "La navegación a través del sistema le resulta"

Respecto de la apariencia de ELO-Tool, 2:5 afirman que es medianamente agradable, uno que tiene una apariencia bastante agradable y otros dos que es agradable (ver Figura 6.22).

\section{Apariencia general del sistema}

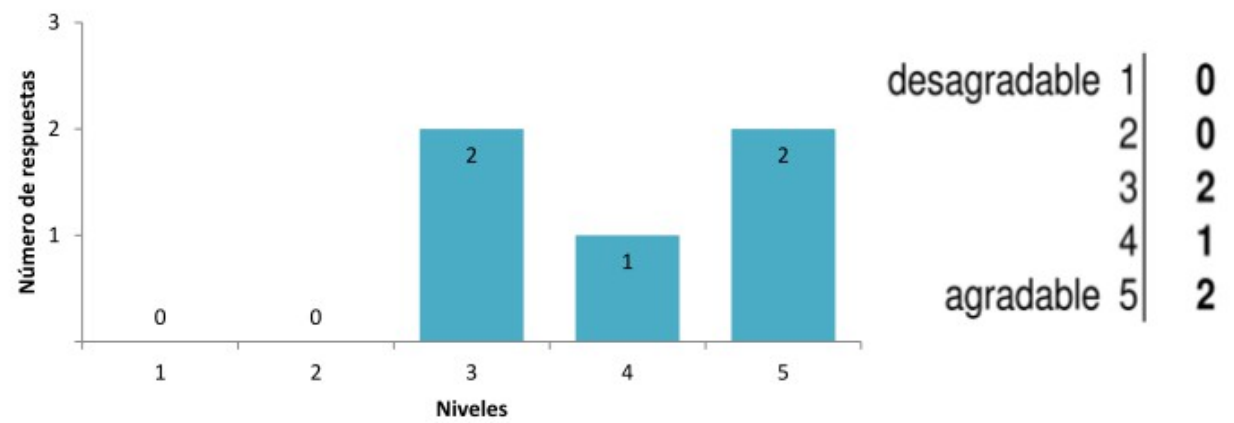

Figura 6.22. Encuesta de satisfacción de ELO-Tool. "La apariencia general del sistema le resulta"

En este sentido, durante la Entrevista, P1 dice "por ahí desde el punto de vista de la usabilidad y de la belleza y demás... no es lo más, pero en cuanto a la funcionalidad que ofrece creo que está bien, que es adecuada.", por su parte, P4 afirma "la interfaz gráfica es básica digamos, no tiene mucho".

La interfaz de ELO-Tool se opera, principalmente, a través de enlaces textuales. Al consultar a los informantes acerca de la representatividad del texto de las opciones respecto a la función de las 
mismas, le parecen claras (2:5) o bastante claras (3:5) (ver Figura 6.23). Un resultado similar se obtuvo al consultarlos por la estructura y organización del sistema -una organización jerárquica de dos o tres niveles- es clara (3:4) o bastante clara (1:4). Uno de los informantes no respondió esta pregunta (ver Figuras 6.24).

Texto de las opciones y su función

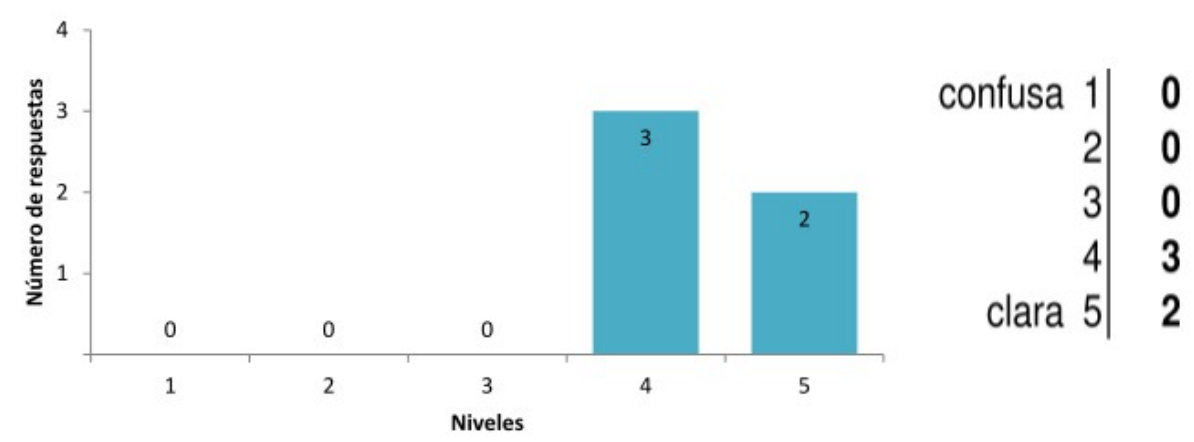

Figura 6.23. Encuesta de satisfacción de ELO-Tool. "La representatividad del texto de las opciones respecto a la función de las mismas le resultaron"

\section{Estructura y organización del sistema}

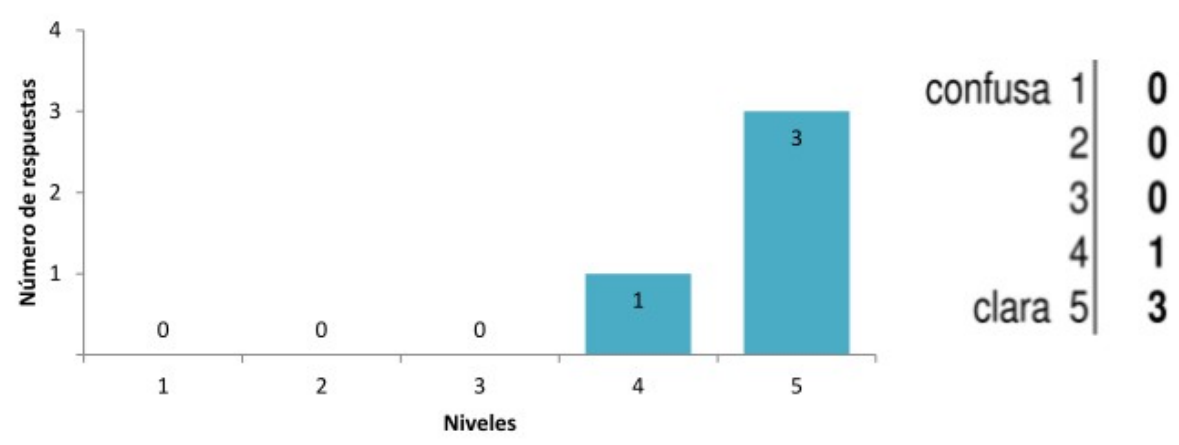

Figura 6.24. Encuesta de satisfacción de ELO-Tool. "La estructura y organización del sistema le resultó"

Al consultarlos por su opinión acerca de qué tan fácil le resultaría ELO-Tool a otros usuarios de la Facultad, la mayoría (3:5) cree que medianamente fácil, del resto, para uno fácil y el otro muy fácil (ver Figura 6.25).

\section{El sistema para usuarios de la Facultad}

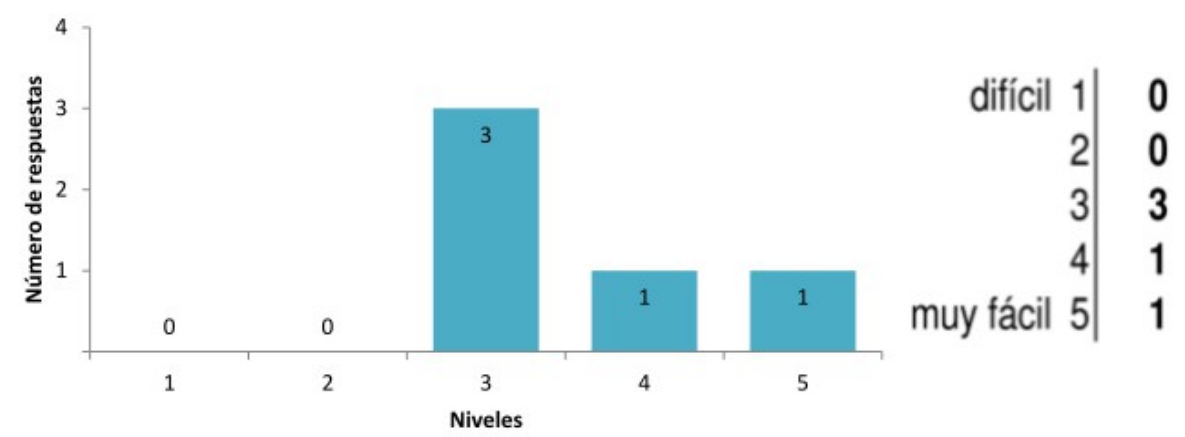

Figura 6.25. Encuesta de satisfacción de ELO-Tool. "El sistema para los distintos tipos de usuario que hay en la Facultad resulta"

Puede observarse que aunque se trata de una herramienta sencilla en su diseño, en general, los participantes tienen una opinión positiva de la misma. Lo mismo ocurre con las funcionalidades que ofrece. Sin embargo, sobre este último aspecto, se observan algunos comentarios negativos.

En la encuesta sobre Encuesta 2, manifiestan algunos problemas surgidos en uso de la herramienta:

- Problemas con la herencia de los requerimientos:

- “2) algunos campos con metadatos de los OA que ensamblamos no se heredaban" 
- "ELO-Tools no heredaba los requisitos del primer OA al momento de ensamblar y generar el IA"

- También se hicieron observaciones acerca de la falta de una opción que permita editar:

- "1)no pudimos editar un IA para corregirlo"

- "me hubiera gustado poder editar un campo de los metadatos que quedó con un error, y no fue posible la edición del mismo"

- Asimismo, uno de los informantes, manifiesta sus reparos con la función Dar de baja ELO:

- "Muy fácil eliminar (dar de baja) un OA, debería mostrar una advertencia antes"

Respecto a este último punto, puede observarse la preocupación de P4 por este aspecto de ELOTool en el registro audiovisual - previo a la actividad de ensamblaje-. Dice el informante "Y vos tenés instalado en tu máquina... Porque vos podés eliminar de tu máquina". Dado que presentaba esta duda y que durante la actividad de ensamblaje tuvo que usar (junto con P5) la funcionalidad de Dar de baja ELO, en la entrevista se le hizo la pregunta: ¿Les resultó fácil borrar un ELO? Te preocupó el hecho de que borraras directamente del repositorio ¿Por qué?:

"Fue muy fácil, y esto de que no te diga el cartel 'está seguro que...' mmmm.

O sea lo que te preguntamos también ese día... ¿qué pasa? ¿Yo puedo volver a una versión anterior? Al estilo Dropbox o Google Drive, ¿se puede? Ese es el tema, vos borraste ¿y?"

Por su parte P5, respondió:

"No, borrar, borramos re-fácil. La parte de borrado era re-fácil, lo que pasa es que tenés que estar super atento para no hacer macanas. Porque te da la posibilidad de hacer un montón de macanas."

Otro de los aspectos señalados, durante el proceso de ensamblaje, fue la posibilidad de guardar mientras se están cargando los datos. Dice P5 “¿Esto se puede ir grabando?”, P2 responde "No, hasta que no envias...".

Se puede observar, también, la preocupación de P1, luego de haber creado su itinerario de aprendizaje y pese a haber completado la descripción de la evaluación, la misma no aparece al ver el itinerario. Dice P1, mirando su itinerario de aprendizaje en ELOs-Viewer, “¿Cómo no se especifica evaluación? Yo le puse....”. Esto se ilustra en la Figura 6.26 que muestra el itinerario de aprendizaje creado por P1.

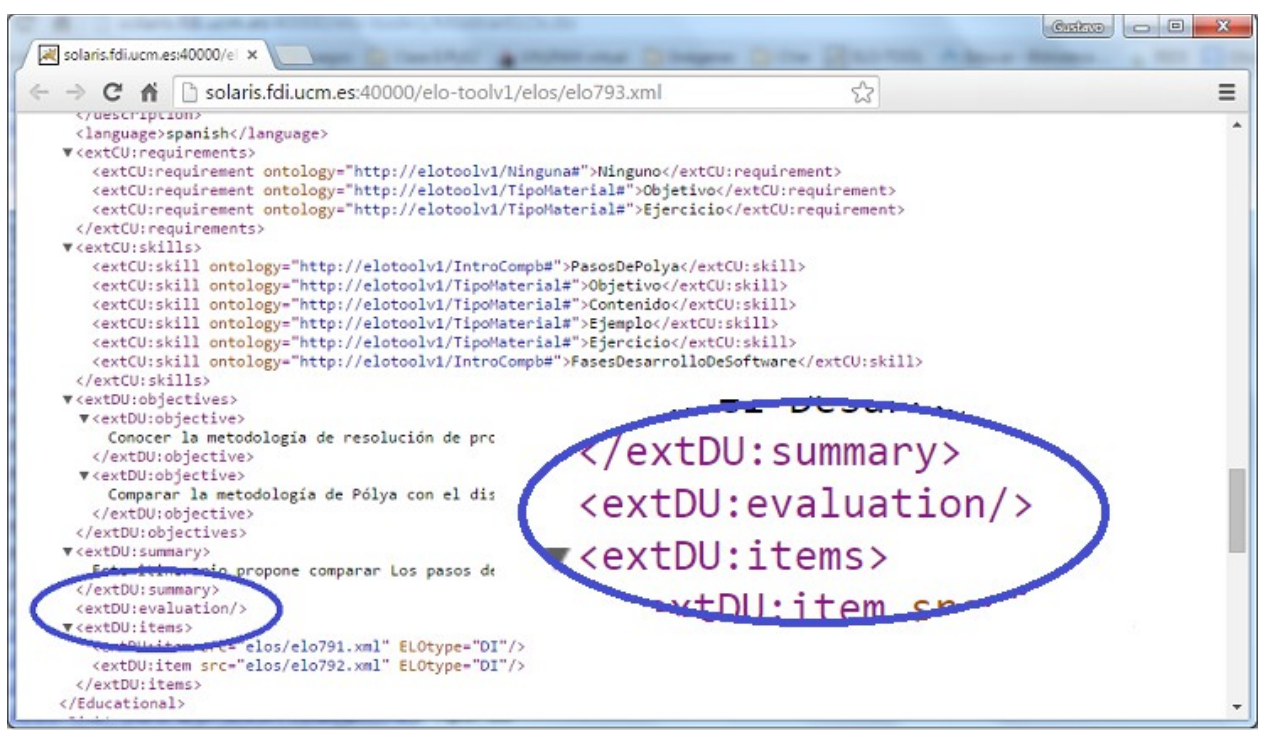

Figura 6.26. Parte de los metadatos del itinerario "IA05 - POLYA VS DESARROLLO". El círculo muestra la evaluación vacía. Disponible en: http://solaris.fdi.ucm.es:40000/elo-toolv1/elos/elo793.xml 


\subsubsection{Nivel de satisfacción de los usuarios con ELOs-Viewer}

En busca de ofrecer a los participantes del taller una vista amigable de los ELOs, se desarrolló la aplicación denominada ELOs-Viewer. La misma fue pensada como complemento de ELO-Tool, para ser utilizada en el taller para visualizar los ELOs. Sin embargo, al momento de crear los itinerarios los participantes podían utilizarla para acceder a una vista de los metadatos necesarios para el ensamblaje. Terminado el taller, también se hizo una encuesta de satisfacción de esta herramienta. Asimismo, en la entrevista se los consultó acerca de cómo usaron dicha aplicación.

Al responder la encuesta de satisfacción, y consultados por la percepción que tenían en relación a la claridad de cómo el sistema le permite realizar las tareas solicitadas 2:5 afirman que es clara, otros 2:5 optan por bastante clara y uno de los participantes considera que es medianamente clara (ver Figura 6.27).

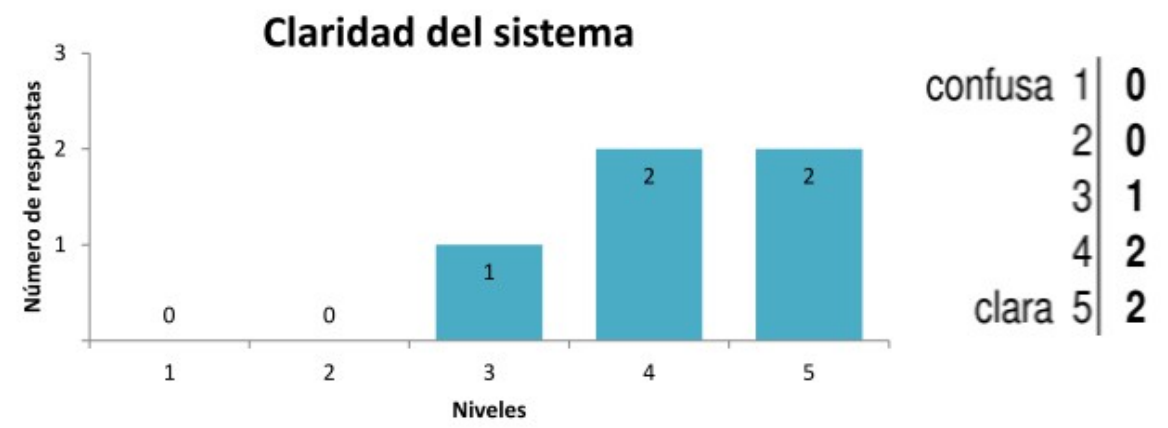

Figura 6.27: Encuesta de satisfacción de ELO-Viewer. "Su percepción en relación a la claridad de cómo el sistema le permite realizar las tareas solicitadas es"

Para la mayoría de los informantes (3:5), la navegación del sistema es clara, mientras que uno de ellos la considera bastante clara y el otro medianamente clara (ver Figura 6.28).

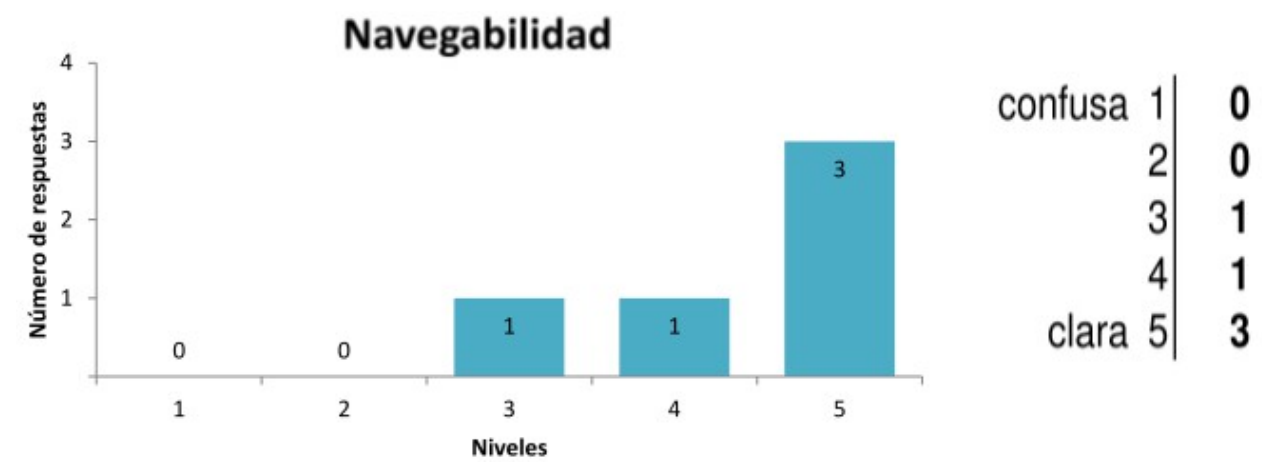

Figura 6.28: Encuesta de satisfacción de ELO-Viewer. "La navegación a través del sistema le resulta"

Respecto a la apariencia de ELOs-Viewer, les parece agradable (3:5) o bastante agradable (2:5) (ver Figura 6.29).

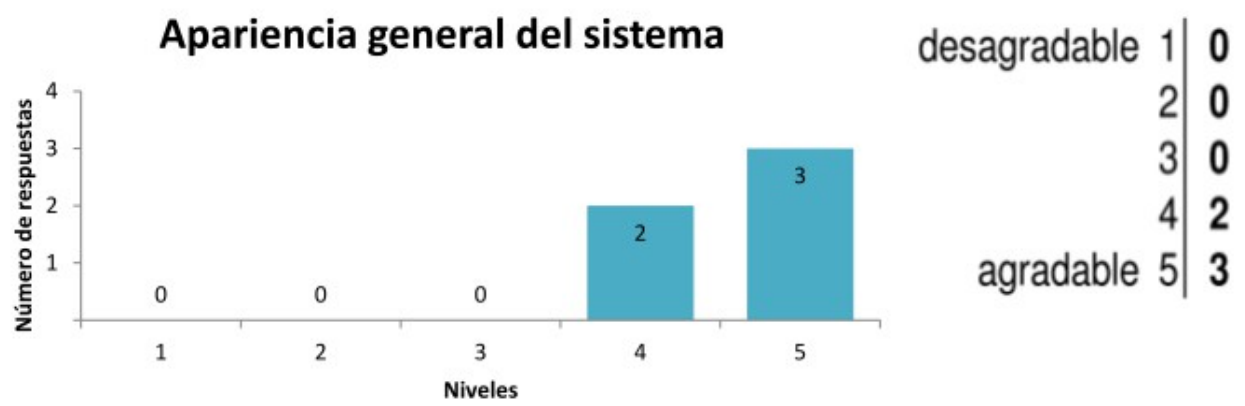

Figura 6.29: Encuesta de satisfacción de ELO-Viewer. "La apariencia general del sistema le resulta"

De forma similar a la anterior, dos de los informantes coinciden en que la representatividad del texto de las opciones respecto a la función de las mismas le resultaron claras y los otros tres bastante claras (ver Figura 6.30). 


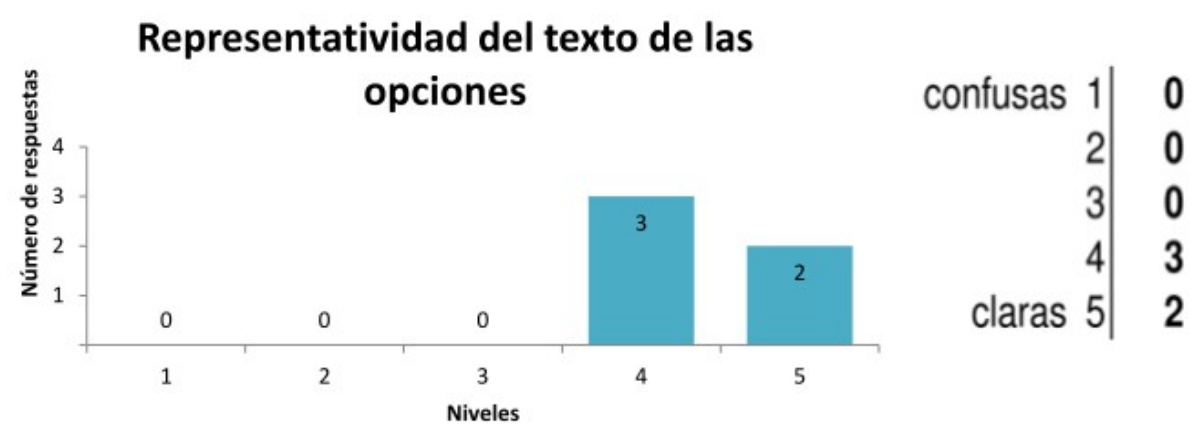

Figura 6.30: Encuesta de satisfacción de ELO-Viewer. "La representatividad del texto de las opciones respecto a la función de las mismas le resultaron"

Para tres de los informantes la estructura y organización de ELOs-Viewer le resultó clara, mientras que para uno fue bastante clara y para el restante medianamente clara (ver Figura 6.31).

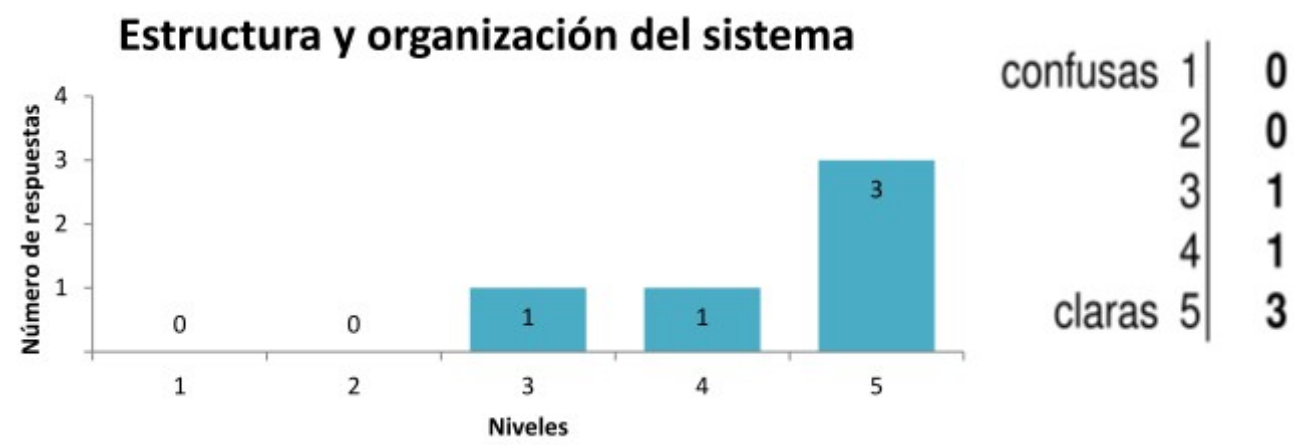

Figura 6.31: Encuesta de satisfacción de ELO-Viewer. "La estructura y organización del sistema le resultó"

Respecto a la presentación de los ELOs, para la mayoría (3:5) la misma es clara y para el resto es bastante clara (1:5) y medianamente clara (1:5) (ver Figura 6.32).

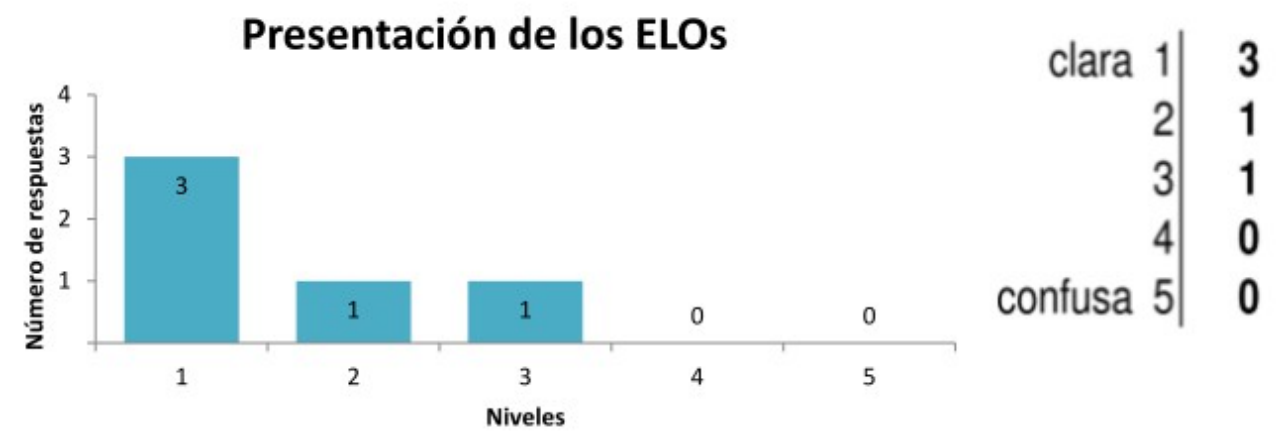

Figura 6.32: Encuesta de satisfacción de ELO-Viewer. "La forma en la que se presentan los ELOs le resultó"

Al ser consultados por la presentación de los metadatos en ELOs-Viewer, hubo respuestas disímiles. Para dos de los informantes se hizo de forma clara, para otro medianamente clara y para el restante algo confusa (ver Figura 6.33). De forma similar respondieron para el conocimiento asociado: dos optaron por clara, otro por bastante clara y, en este caso, los dos restantes por algo confusa (ver Figura 6.34).

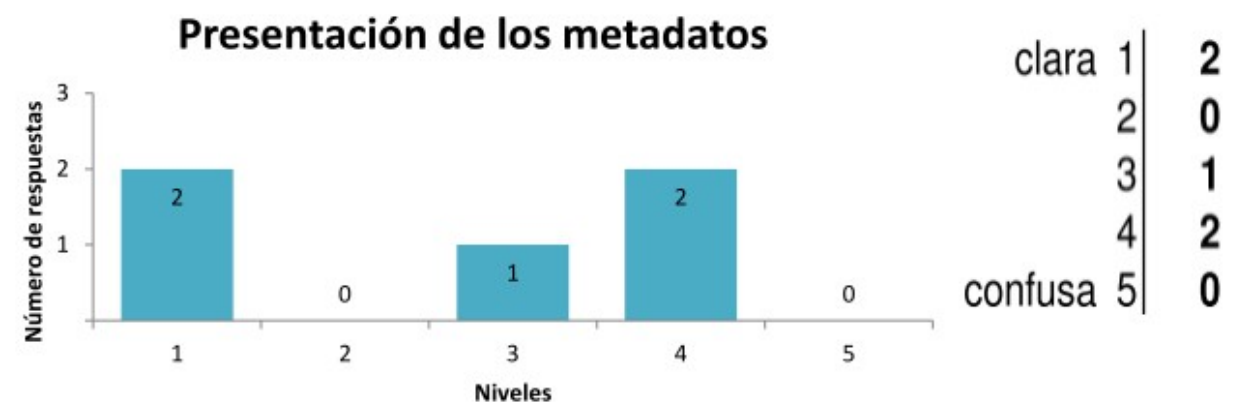

Figura 6.33: Encuesta de satisfacción de ELO-Viewer. "La forma en la que se presentan los metadatos le resultó" 


\section{Presentación del CA}

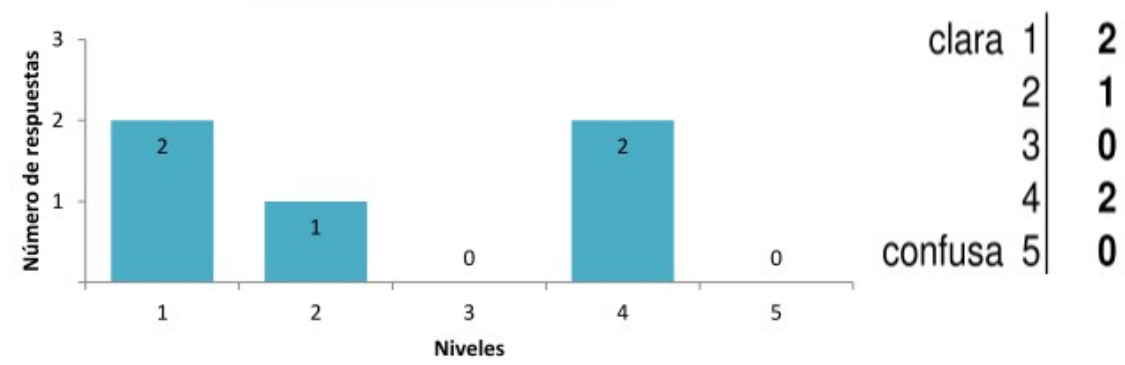

Figura 6.34: Encuesta de satisfacción de ELO-Viewer. "La forma en la que se presenta el CA de los OA le resultó"

Pese a las respuestas dispares del punto anterior, a la gran mayoría (4:5) le fue muy útil la información que muestra la ELOs-Viewer en el momento de realizar el de ensamblaje y al restante útil (ver Figura 6.35).

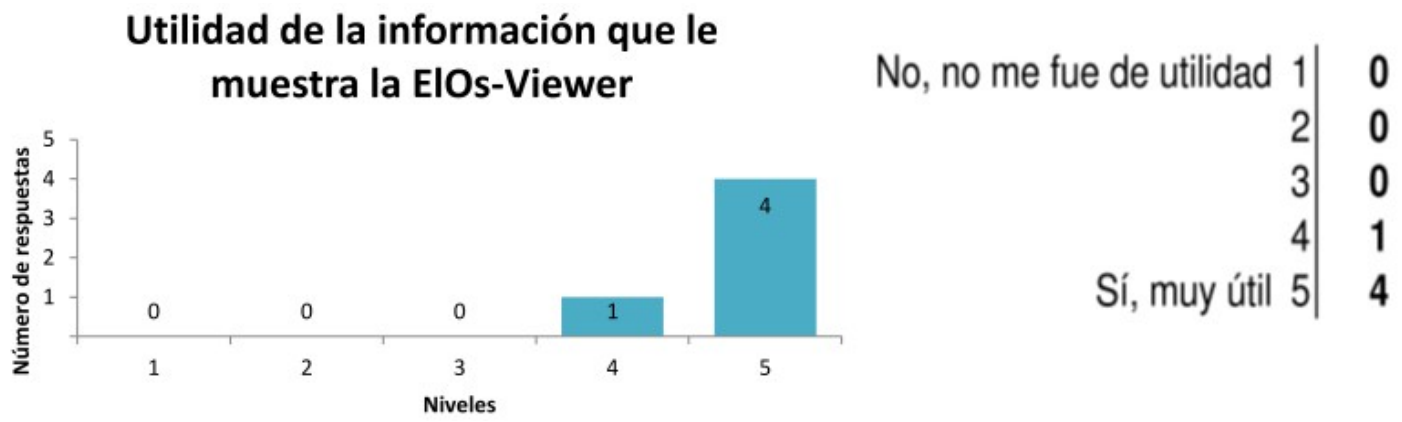

Figura 6.35: Encuesta de satisfacción de ELO-Viewer. "La información que le muestra la herramienta le fue de utilidad en el proceso de ensamblaje"

Respecto al uso de la herramienta durante el proceso de ensamblaje, dos de los participantes (P2 y P4) manifiestan que les fue de utilidad, mientras que otros dos (P1 y P3) recuerdan haber usado sólo la ayuda en PDF y el restante no recuerda si lo utilizó (ver Tabla 20 en Anexo VII). Sin embargo, en la siguiente pregunta (ver Tabla 21 en Anexo VII) P1 afirma haber tomado de ELOsViewer "los objetivos".

Luego, también en la Entrevista, se les consultó si recordaban para qué habían utilizado ELOsViewer. De las respuestas (ver Tabla 21 en Anexo VII) puede observarse que se destacan las búsqueda y visualización de los OA.

Finalmente, al consultarlos sobre qué tan difícil les sería usar ELOs-Viewer a los distintos usuarios de la Facultad, dos de los informantes afirman que fácil y los tres restantes medianamente fácil (ver Figura 6.36).

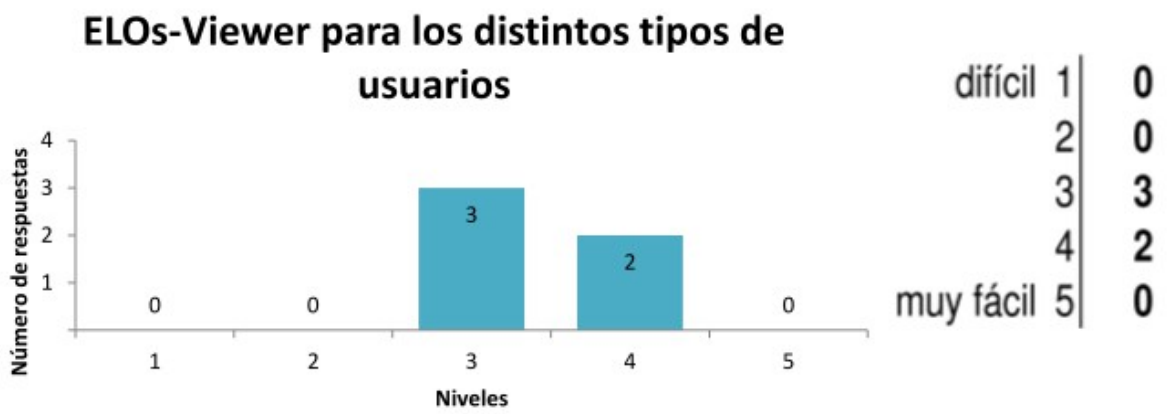

Figura 6.36: Encuesta de satisfacción de ELO-Viewer. "El sistema para los distintos tipos de usuario que hay en la Facultad resulta"

No se registraron problemas con el uso de esta aplicación en ninguna de las herramientas que se utilizaron para la toma de datos.

Como puede observarse la aplicación cumplió de forma aceptable su cometido de actuar como una herramienta sencilla y de soporte de ELO-Tool durante el proceso de ensamblaje. Así como también para la visualización de los OA y los itinerarios de aprendizaje. 


\subsection{Recapitulación}

En esta tesis se llevó adelante un estudio de caso sobre un taller de ensamblaje de OA, con el objetivo de evaluar la metodología de ensamblaje propuesta por Santacruz-Valencia et al. (2005). El diseño de este estudio implicó, por una parte, la adaptación de una metodología de ensamblaje con vista a la implementación del taller y, por otra, la localización y publicación de las UIs, la creación de los OA vía ensamblaje y la implementación del taller donde se crearon los itinerarios de aprendizaje (ver Figura 6.37).

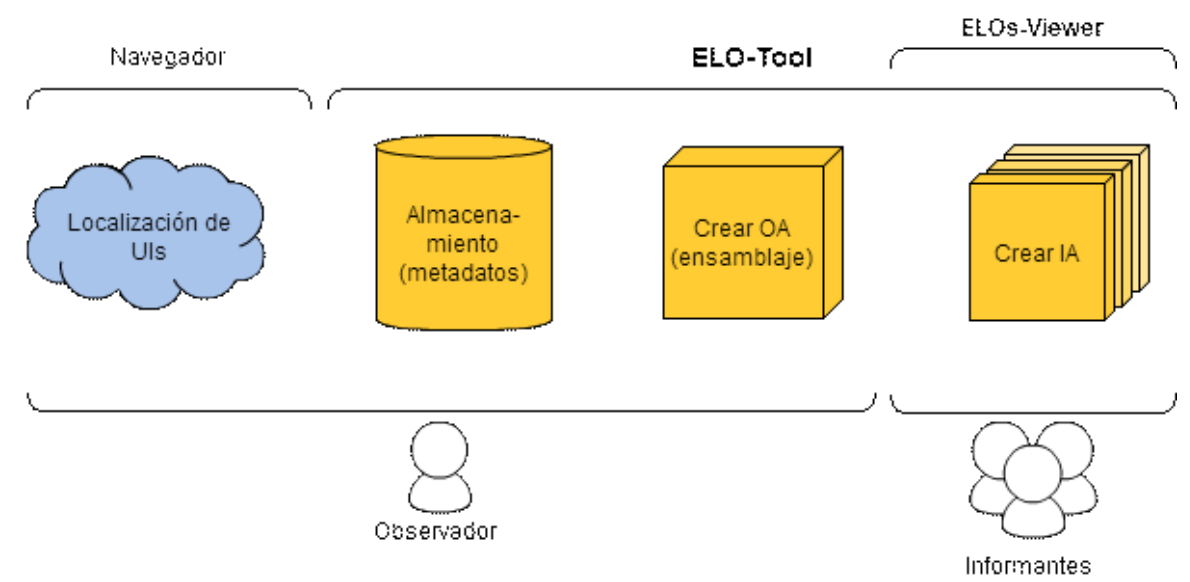

Figura 6.37. El diagrama ilustra el proceso de creación de IA en este estudio de caso.

Del proceso de selección de UIs se publicaron 49 UIs (ver Figura 6.38) con las que crearon los 11 OA que se utilizaron en el taller. Los mismos fueron utilizados $-\mathrm{y}$ reutilizados- por los docentes participantes para crear seis itinerarios de aprendizaje.

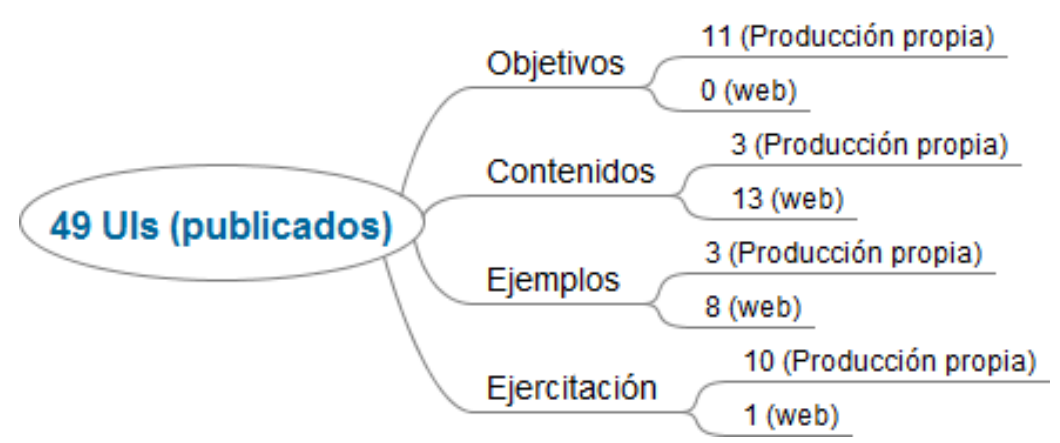

Figura 6.38. Distribución de las UIs alojadas en ELO-Tool de acuerdo a la fuente de obtención.

A partir de las distintas herramientas de registros se pudieron dar respuesta a las ocho preguntas de investigación (ver Figura 6.39).

Los participantes del taller llevaron adelante, exitosamente, la creación de sus itinerarios de aprendizaje, y consideraron que se trató de una tarea sencilla o fácil de llevar adelante. En este proceso les resultó apropiada la cantidad de metadatos que tuvieron que cargar, actividad central en el mismo, y el nivel de automatización que propone la metodología. También considerando que volverían a utilizar una técnica de ensamblaje para la creación de itinerarios de aprendizaje ya que propicia la reutilización y facilita la tarea docente. También se mostraron, en general, satisfechos con las aplicaciones de software utilizadas tanto en su aspecto estético como en su funcionalidad.

Si la muestra con la que se trabajó es pequeña y no permite la generalización de los resultados de esta experiencia, desde los aspectos a trabajar se puede considerar que:

- Las restricciones impuestas al material (ver Figura 6.40) dificultó la localización de las UIs y se impuso como un factor externo, al propio recurso, que limita el nivel de reutilización de los mismos.

- El tiempo dedicado a la explicación de la metodología de ensamblaje, y las adaptaciones, no fue el suficiente para que los participantes se apropiaran de la misma. Es posible que de 
haber incluido a los participantes en todo el proceso (ver Figura 6.37) hubiese favorecido la consolidación de la metodología.

- En la misma línea del párrafo anterior podríamos ubicar a las dificultades con la semántica de los metadatos y las dudas respecto a las ontologías y al CA. Conceptos, estos últimos, con los que los docentes no estaban familiarizados.

- Finalmente, para el caso de ELO-Tool, sería deseable que permitiera la edición de los metadatos de un ELO ya creado, así como también que diera advertencia previa al borrado de ELOs.

También es importante destacar que se trabajó con docentes con formación en las área de matemática y ciencias de la computación, lo que facilitó el abordaje de los aspectos más técnicos de la metodología durante el desarrollo del taller.

En el siguiente capítulo se presentan las conclusiones obtenidas a partir de la elaboración del presente trabajo de tesis, así como posibles trabajos futuros.

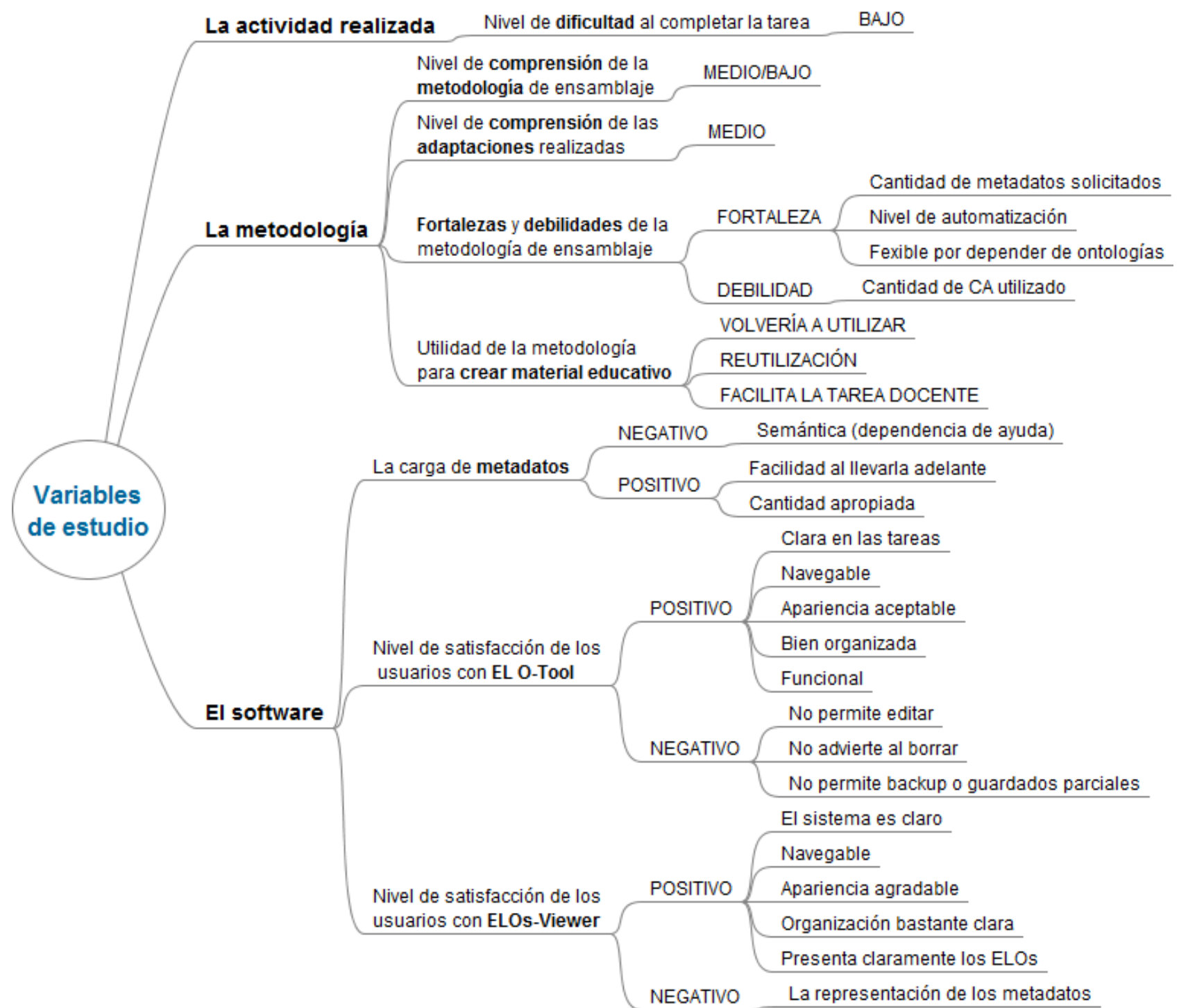

Figura 6.39. El diagrama muestra las variables de estudio y los valores obtenidos en base a los registros.

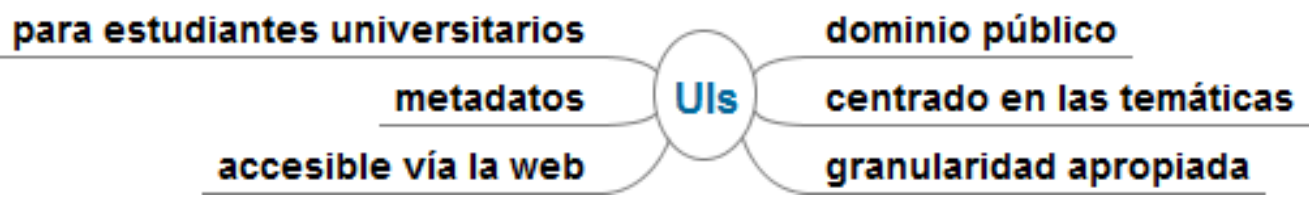

Figura 6.40. Restricciones impuestas a las UIs para ser publicadas con vistas al taller. 


\section{CONClusiones y TRabajos Futuros}

\section{RESUMEN}

El presente trabajo de investigación ba tenido como objetivo principal realizar un estudio y análisis de las metodologías de ensamblaje de OA que favorezcan la reutilización.

En este séptimo, y último capítulo, se presentan las conclusiones y trabajos futuros. Primeramente, se exponen los aportes realizados como producto del desarrollo de este trabajo de tesis. Luego, se presentan las conclusiones divididas en: 1) generales (resultado de todo el proceso de investigación), 2) específicas, que a su vez se subdividen en (a) las que devienen de la aplicación de los criterios de evaluación y (b) las obtenidas del estudio de caso. Para finalizar, se bace una breve descripción de los trabajos futuros derivados del proceso de investigación. 


\subsection{Introducción}

El presente trabajo de investigación ha tenido como objetivo principal realizar un estudio y análisis de las metodologías de ensamblaje de OA que favorezcan la reutilización, debido a que, la creciente demanda de formación virtual -tanto en modalidades híbridas o a distancia- ha puesto el foco en este tipo de estrategias para la creación de MED, así como el diseño y re-utilización de OA, y en la evolución de los sistemas que los administran.

Se ha descompuesto el objetivo principal en sub-objetivos, los que se han alcanzado dado que:

- Se han estudiado y analizado 27 metodologías de ensamblaje.

- Se definieron 17 criterios que permiten la comparación entre diferentes metodologías de ensamblaje y el análisis de las mismas.

- Se han estudiado y analizado herramientas disponibles actualmente que permiten el ensamblaje y se ha realizado una comparativa de las mismas.

- Se ha seleccionado una metodología de ensamblaje y el sistema ensamblador que la implementa, y se utilizaron en un estudio de caso.

- Se ha realizado el análisis de los resultados obtenidos que permiten vislumbrar algunas dificultades percibidas por los docentes intervinientes y algunos aspectos que se consideran favorables en el uso de la metodología de ensamblaje.

A partir del cumplimiento de los objetivos, se realizan los siguientes aportes en la temática: se compone el estado del arte, se propone un conjunto de criterios de evaluación/análisis, una tipología para metodologías de ensamblaje de OA y una estrategia metodológica para utilizar una metodología de ensamblaje de OA en un contexto real. Además, se pone a disposición de la comunidad interesada: un conjunto de MED de distinta granularidad, una lista de herramientas de software que implementan metodologías de ensamblaje, un visualizador de ELOs y un conjunto de ontologías de dominio.

Para llegar a la consecución del objetivo y los sub-objetivos, en esta tesis, se llevó adelante la selección de un conjunto de publicaciones que abordan la temática y el posterior análisis de las metodologías de ensamblaje de OA descritas en las mismas. Esto permitió identificar un conjunto de focos de análisis y definir un grupo de criterios de evaluación de metodologías de ensamblaje de OA. Dichos criterios, permitieron llevar adelante el análisis en profundidad de las metodologías y la selección de una de ellas, y la aplicación informática que la implementa, para realizar un estudio de caso. Los objetivos de éste último fueron: $(i)$ conocer la opinión de los docentes sobre el ensamblaje de OA en la generación de itinerarios de aprendizaje, (ii) identificar fortalezas y debilidades de la metodología de ensamblaje seleccionada, (iii) evaluar el nivel de usabilidad de las herramientas de software utilizadas, tendientes a realizar recomendaciones para su mejora y $(i v)$ generar un proceso metodológico de aplicación de ELO-Tool para ser utilizado por docentes del área de ciencias de la computación. El estudio, se hizo con docentes universitarios en el contexto de un taller de ensamblaje de OA.

El desarrollo de cada uno de los capítulos anteriores ha dado lugar a un conjunto de aportes y conclusiones que se exponen en las secciones subsiguientes.

En la sección 7.2 de este capítulo, se presentan los aportes realizados por esta tesis y, seguidamente, en la sección 7.3, las conclusiones. Para finalizar en la sección 7.4, se enuncian los trabajos futuros. 


\subsection{Aportes de esta tesis}

\subsubsection{Aportes generales}

El desarrollo de este trabajo de tesis ha permitido:

- La composición de un estado del arte para los Sistemas Ensambladores de OA. La revisión de las 35 publicaciones dio lugar al análisis y consecución de un estado de la cuestión. El mismo, se ha constituyó en los cimientos de esta tesis y ha permitido concretar del resto del trabajo de investigación realizado. Asimismo, se trata de un estudio sobre el que no se han hallado precedentes en la temática.

- La definición de un conjunto de criterios de evaluación para metodologías y sistemas de ensamblaje de OA. No se encontró, previo a este trabajo de tesis, un conjunto de criterios que permitieran el análisis de metodologías de ensamblaje de OA y/o las aplicaciones de ellas derivadas. Se definieron pues, un conjunto de criterios para el análisis y comparación de metodologías de ensamblaje de OA. Los mismos posibilitan: (i) clasificar y caracterizar las diferentes metodologías de ensamblaje; (ii) seleccionar un SE en función de cómo se desea utilizar y/o en función del material con el que se cuenta y, (iii) en el caso puntual de esta tesis, permitió elegir un SE para el estudio de caso.

- La publicación de un conjunto de MED de distinta granularidad. Dado que la metodología elegida para el estudio de caso fue evaluada solamente a través de una experiencia de laboratorio, fue necesario poblar el repositorio de ELO-Tool con ELOs centrado en las temáticas abordadas en las asignaturas Introducción a la Computación y Programación I. Esto pone a disposición de la comunidad educativa 49 UIs, 22 UCs, 11 OA y cinco itinerarios de aprendizaje, en distintas temáticas de programación estructurada, diseñados por docentes universitarios.

- La definición de una estrategia metodológica (marco de trabajo procedimental) para el diseño de actividades de ensamblaje con OA. La necesidad de realizar un taller de ensamblaje dio lugar a la creación de una estrategia metodológica para llevar adelante un proceso de ensamblaje con OA. La misma permite, a quienes deseen utilizar la metodología de ensamblaje elegida para el estudio de caso y el software ELO-Tool, contar con un marco de trabajo para la creación de itinerarios de aprendizaje basados OA. Los mismos pueden estar generados a partir de ELOs o bien referenciar OA de otros repositorios.

- La definición de una tipología para SE. A partir del análisis realizado, sobre el conjunto de las publicaciones seleccionadas, se pudo obtener la definición de una tipología para SE. La misma los clasifica en sistemas ensambladores de OA, sistemas creadores de OA vía ensamblaje y sistemas recomendadores de itinerarios de aprendizaje. Esta disquisición puede utilizarse para realizar una clasificación inicial y tener una primera definición de las funcionalidades del SE.

\subsubsection{Aportes específicos}

Desde el punto de vista tecnología digital esta tesis aporta:

- Una lista de herramientas de software que permiten el ensamblaje de OA. Del análisis de las publicaciones y la posterior aplicación de los criterios de evaluación, se deja a disposición para docentes/investigadores interesados en estas temáticas, información sobre cinco herramientas. Las mismas fueron desarrollas por distintos especialistas en la temática, implementan SE de OA y que están accesibles vía la web.

- Un visualizador de ELOs. Dado que ELO-Tool ofrece una visualización de los ELOs basada en el acceso a los metadatos en crudo, se desarrolló una aplicación web, en el marco de esta tesis, para visualizar ELOs e itinerarios de aprendizaje, denominada ELOs-Viewer. La 
misma está disponible en: http://elosviewer.exactas.unlpam.edu.ar/.

- Un conjunto de ontologías de dominio. Para la implementación de estudio de caso, como parte de la contextualizacion del taller, se desarrollaron ontologías de dominio -utilizando OWL- que representan a las asignaturas Introducción a la Computación y Programación I; y el lenguaje Pascal. Las mismas están disponibles en el Anexo VI o vía el repositorio de ontologías de ELO-Tool.

\subsubsection{Otros aportes}

Para la difusión de resultados preliminares, obtenidos con la realización de este trabajo de tesis, se publicaron artículos en congresos. Los mismos se listan a continuación:

- Astudillo, G. J., Sanz, C. V., \& Santacruz-Valencia, L. P. (2013). Análisis de metodologías de recomendación y ensamblado de Objetos de Aprendizaje, a partir de la definición de criterios. En VIII Congreso de Tecnología en Educación y Educación en Tecnología (p. 10 p.). Santiago del Estero, Argentina: RedUNCI. Recuperado a partir de http://sedici.unlp.edu.ar/handle/10915/27530.

- Astudillo, G. J., Sanz, C. V., \& Santacruz-Valencia, L. P. (2014). Sistemas ensambladores de objetos de aprendizaje. En IX Congreso sobre Tecnología en Educación \& Educación en Tecnología (pp. 373-382). La Rioja: RedUNCI. http://doi.org/10.13140/2.1.1947.8404.

- Maldonado, J., \& Astudillo, G. (2014). Los Objetos de Aprendizaje: Un estado del arte en Iberoamérica. Presentado en CCITA, Miami: EEUU.

\subsection{Conclusiones}

El desarrollo del presente trabajo de tesis permitió arribar a las siguientes conclusiones:

- La falta de una definición acordada y de consenso generalizado sobre el concepto de OA, dificulta la comparación de las metodologías de ensamblaje. Máxime cuando éste no se define de forma explícita en el contexto de la metodología de ensamblaje.

- La mayoría de las definiciones utilizadas por los autores de metodologías de ensamblaje de OA no tienen en cuenta las principales características, que cuentan con el acuerdo de la comunidad científica especializada en la temática.

- Si bien se utilizan estándares para el etiquetado de los OA (principalmente LOM), no ocurre lo mismo en el empaquetamiento de los mismos. Sólo unas pocas hacen uso de SCORM/IMS-CP, en general, utilizan materiales de diversos formatos y diseñados ad hoc para la implementación de la metodología. No obstante esto, varias de las metodologías incluso algunas de las que no empaquetan- derivan su modelo de contenidos en el estándar SCORM.

- Algunas metodologías de ensamblaje hacen uso de ontologías para representar las distintas abstracciones que deben manejar (usuario y/o OA). El uso de ontologías, si bien ofrece semántica a las entidades, puede actuar como una limitante debido al esfuerzo y el requerimiento de expertos que implica la construcción de las mismas.

- La mayoría de las metodologías de ensamblaje utilizan metadatos para la descripción del perfil del usuario y/o el contenido del OA. Esto genera un esfuerzo inicial en la representación de las entidades, aunque algunos SE automatizan parte del proceso. La dependencia de esta información vuelve, a estos sistemas, un tanto vulnerables a los errores de carga o a la falta de datos. Esto ocurre, principalmente, en los OA ya que para el caso de información de perfil de los usuarios, los SE suelen solicitar esta información al inicio y mantenerla actualizada. Otro problema asociado a los metadatos derivan de las extensiones del estándar LOM propuesto por varias metodologías, ya que los OA alojados en 
repositorios, no cuentan con la información que se requiere desde la extensión propuesto, la cual es necesaria para el ensamblaje.

De los resultados obtenidos de la aplicación de los criterios de evaluación:

- Se realizó la aplicación del conjunto de criterios para evaluar la estrategia utilizadas por las metodologías de ensamblaje. Esto permitió distinguir entre aquellas que llevan adelante el proceso basadas únicamente en el ensamblaje y las que utilizan algoritmos de recomendación para personalizar los itinerarios. Así como también, identificar la utilización de patrones y metadatos (y combinación de éstos) como principal principio de ensamblaje. En general con un alto nivel de automatización.

- En cuanto al contexto de aplicación de las metodologías, se encontró evidencia que indicaría una falta de evaluación de las mismas con usuarios y materiales reales. También se pudo observar que, aunque una parte importante de las metodologías orientadas a docentes presentan un nivel alto de automatización, aún requieren de docentes y/o expertos proactivos que diseñen patrones y etiqueten los OA. El otro tipo de destinatario, el estudiante, principalmente hace uso de los itinerarios y, en muchos casos, los mismos están ajustados a su perfil como estudiantes y/o su rendimiento académico.

- Si bien la mayoría de las metodologías han desarrollado un software que las implementa o bien han incorporado las mismas en algún sistema de gestión de contenidos, la mayoría, al momento de desarrollar esta tesis, no se encuentran disponibles para su utilización o prueba.

En relación a los objetivos planteados para el estudio de caso y los resultados obtenidos a partir del diseño e implementación del mismo:

- El proceso de creación de materiales educativos con OA debe hacerse con base en una definición que permita identificar qué es y qué no es un OA. Con la definición adoptada en esta tesis, localizar OA que se ajusten a la misma fue dificultoso. En su lugar, sí fue posible hallar recursos con los que componer o crear OA.

- Es posible la creación de OA vía ensamblaje a partir de la reutilización de recursos alojados en repositorios disponibles en la web. Sin embargo, en varios casos la personalización/contextualización de los recursos, así como su granularidad, limita las posibilidades de reutilización y requiere de la creación de algunos recursos ad hoc.

- Es posible llevar adelante el diseño de itinerario de aprendizaje a partir del ensamblaje de OA. Sin embargo, debe tenerse en cuenta el trabajo previo requerido para la capacitación de los docentes por tratarse de un enfoque, en la creación de materiales, que no está ampliamente difundido.

- La metodología utilizada en el estudio de caso requiere de ontologías, esto permitió la adaptación de ELO-Tool al contexto de aplicación en el estudio de caso. Sin embargo, la creación de ontologías de dominio es un proceso que requiere de un esfuerzo considerable y que debe ser previo a la creación de los itinerarios y por ende al proceso de ensamblaje. Se cree, por tanto, que esta tarea debe ser acompañada de la participación de expertos (grupo interdisciplinario) y previa a que los docentes se involucren con el proceso de ensamblaje.

- Si bien, todos los participantes pudieron completar el ensamblaje de los itinerarios asignados, y la tarea les resultó inicialmente sencilla de concretar, no se apropiaron completamente de la metodología.

- La aplicación ELO-Tool facilitó el proceso y permitió completar con éxito la actividad. Aunque se trata de una herramienta sencilla en su diseño y se marcaron aspectos a mejorar, en general, los participantes tienen una opinión positiva de la misma. 


\subsection{Líneas de trabajo futuros}

A partir de las conclusiones de esta tesis surge la siguiente línea de trabajo:

- Generar una herramienta web libre, basada en estándares reconocidos de etiquetado y empaquetado, que contribuya de manera integrada en la recomendación de OA sujetos a ser ensamblados, y que permita componer itinerarios de aprendizaje personalizados.

Esta línea será retomada como parte de mi trabajo de tesis doctoral.

Con base en el estudio de caso realizado en esta tesis se platean nuevas líneas de trabajo, a saber:

- Llevar adelante una experiencia donde una mayor cantidad de docentes intervinientes hagan todo el proceso de ensamblaje. Desde la localización de los recursos, pasando por la definición o evaluación de las ontologías, la creación de los OA y el ensamblaje de los mismos en itinerarios. Esto permitiría una evaluación más detallada de la metodología y se estima que esto favorecería, también, la apropiación de la misma.

Cabe aclarar que el grupo de docentes debiera contar con una formación tal que le permitiera abordar los conceptos más técnicos (como ontologías), o bien, contar con un grupo interdisciplinario que lleve adelante distintas tareas según su experticia.

- Extender ELO-Tool para contemplar los aspectos que han surgido como puntos de mejora en el proceso del estudio de caso.

Otras líneas de trabajo:

- Vincular las posibilidades de ensamblaje de OA a las funcionalidades actuales de los EVEA, y entornos MOOC.

- Evaluar el aporte de las metodologías de ensamblaje en la personalización de los aprendizaje de los estudiantes. Dado que muchas de las metodologías se crearon motivadas por el mejoramiento de los sistemas de e-learning y/o la generación de materiales ajustados al perfil educativo de usuario, sería importante indagar en qué medida se puedan alcanzar este objetivo. 


\section{RESUMEN}

En este octavo capítulo, se presenta la bibliografía utilizada a lo largo de todo el trabajos de tesis. 


\section{Bibliografía}

ActiveMath. (2008). Overview ActiveMath [Página Web]. Recuperado 8 de febrero de 2014, a partir de http://www.activemath.org/Home/Teachers.

ADL. (2009a). SCORM® 2004 4th Edition Content Aggregation Model (CAM). Advanced Distributed Learning. Recuperado a partir de http://www.adlnet.gov/Technologies/scorm/SCORMSDocuments/SCORM\%202004\%204th \%20Ed\%20V1.1/Documentation\%20Suite/SCORM_2004_4ED_v1_1_Doc Suite.zip.

ADL. (2009b). SCORM® 2004 4th Edition Overview Version 1.0. Advanced Distributed Learning. Recuperado a partir de http://www.adlnet.gov/wpcontent/uploads/2011/07/SCORM_2004_4ED_v1_1_Doc_Suite.zip.

Anh, N. V., \& Dam, H. S. (2006). ACGs: Adaptive Course Generation System - An Efficient Approach to Build E-Learning Course. En Computer and Information Technology, 2006. CIT '06. The Sixth IEEE International Conference on (pp. 259-259). IEEE. http://doi.org/10.1109/CIT.2006.32.

Anh, N. V., Ha, N. V., \& Dam, H. S. (2008). Constructing a Bayesian belief network to generate learning path in adaptive hypermedia system. Journal of Computer Science and Cybermetics, 24(1), 12-19.

APROA. (2004). Qué es un OA [Página Web]. Recuperado 11 junio 2010, a partir de http://www.aproa.cl/.

Astudillo, G. J., Sanz, C. V., \& Santacruz Valencia, L. P. (2013). Análisis de metodologías de recomendación y ensamblado de Objetos de Aprendizaje, a partir de la definición de criterios. En VIII Congreso de Tecnología en Educación y Educación en Tecnología. Santiago del Estero, Argentina: RedUNCI. Recuperado a partir de http://sedici.unlp.edu.ar/handle/10915/27530.

Astudillo, G. J., Sanz, C. V., \& Willging, P. A. (2011). Análisis del estado del arte de los objetos de aprendizaje. Revisión de su definición y sus posibilidades (Trabajo final). Universidad Nacional de La Plata, La Plata, Buenos Aires. Recuperado a partir de http://sedici.unlp.edu.ar/ARG-UNLP-TPG-0000002954/12061.pdf.

Astudillo, G. J., Willging, P. A., \& García, P. M. (2011). Estado del arte de los repositorios de materiales educativos en Latinoamérica. Presentado en VI Congreso de Tecnología en Educación y Educación en Tecnología, Salta, Argentina: RedUNCI. Recuperado a partir de http://sedici.unlp.edu.ar/handle/10915/18431.

Barker, P. (2005). What is IEEE Learning Object Metadata (the LOM) / IMS Learning Resource Meta-data. CETIS (Centre for Educational Technology Interoperability Standards). Recuperado a partir de http://metadata.cetis.ac.uk/guides/WhatIsLOM.pdf.

Barritt, C., Lewis, D., \& Wieseler, W. (1999, junio 25). Cisco Systems Reusable Information Object Strategy. Definition, Creation Overview, and Guidelines. Cisco Systems, Inc. Recuperado a partir http://www.cisco.com/warp/public/779/ibs/solutions/learning/whitepapers/el_cisco_rio.pdf.

Becerra, C., Calvo, J., Muñoz, R., \& Noël, R. (2013). Generador de Rutas de Aprendizaje para el Dominio Informático. Conferencias LACLO, 4(1). Recuperado a partir de http://www.laclo.org/papers/index.php/laclo/article/view/77. 
Ben Mahmoud, C., Azaiez, I., Bettahar, F., Abel, M.-H., \& Gargouri, F. (2015). A learning semantic Web service for generating learning paths. En Computer and Information Science (ICIS), 2015 IEEE/ACIS 14th International Conference on (pp. 627-631). http://doi.org/10.1109/ICIS.2015.7166668.

Betrián, D. P., Hilera, J. R., \& Pagés-Arévalo, C. (2011). ISO/IEC 19788 MLR: Un Nuevo Estándar de Metadatos para Recursos Educativos. IEEE-RITA, 6(3), 140-145.

Bhargava, R. (2009). Manifesto For The Content Curator: The Next Big Social Media Job Of The Future? [Blog]. Recuperado 12 de marzo de 2013, a partir de http://www.rohitbhargava.com/2009/09/manifesto-for-the-content-curator-the-next-bigsocial-media-job-of-the-future-.html.

Bouzeghoub, A., \& Selmi, M. (2009). Authoring Tool for Structural and Semantic Coherence Validation of Composed Learning Objects. En Advanced Learning Technologies, 2009. ICALT 2009 (pp. 175-177). Riga, Letonia. http://doi.org/10.1109/ICALT.2009.197.

Bouzeghoub, A., Buffat, M., Lopes Gançarski, A., Lecocq, C., Benjemaa, A., Selmi, M., \& Maillet, K. (2009). Search and Composition of Learning Objects in a Visual Environment. En U. Cress, V. Dimitrova, \& M. Specht (Eds.), Learning in the Synergy of Multiple Disciplines. Vol. 5794. (pp. 763-768). Springer Berlin Heidelberg. Recuperado a partir de http://dx.doi.org/10.1007/978-3-642-04636-0_81.

Bouzeghoub, A., Defude, B., Ammour, S., Duitama, J.-F., \& Lecocq, C. (2004). A RDF description model for manipulating learning objects. En Advanced Learning Technologies, 2004. Proceedings. IEEE International Conference on (pp. 81-85). http://doi.org/10.1109/ICALT.2004.1357379.

Casali, A., Cechinel, C., \& Ochoa, X. (2015). Edición Especial: Estrategias para Mejorar la Usabilidad de Repositorios Educativos. IEEE-RITA, 3(2), 76-77.

Chan Núñez, M. E. (2003). Objetos de aprendizaje y planetarización del conocimiento (pp. 1-5). Presentado en Educa-Online Barcelona, Barcelona, España.

Chan Núñez, M. E. (2004). Tendencias en el diseño educativo para entornos de aprendizaje digitales. Revista Digital Universitaria [en línea], 5(10). Recuperado a partir de http://www.revista.unam.mx/vol.5/num10/art68/int68.htm.

Chellatamilan, T., \& Suresh, R. M. (2012). Automatic classification of learning objects through dimensionality reduction and feature subset selections in an e-learning system. En Technology Enhanced Education (ICTEE), 2012 IEEE International Conference on (pp. 1 -6). http://doi.org/10.1109/ICTEE.2012.6208621.

Chiappe, A., Segovia, Y., \& Rincon, Y. (2007). Toward an instructional design model based on learning objects (pp. 671-681). Presentado en Educational Technology Research and Development, Association for Educational Communications and Technology. http://doi.org/10.1007/s11423-007-9059-0.

Cobo Romaní, C., \& Pardo Kuklinski, H. (2007). Planeta Web 2.0. Inteligencia colectiva o medios fast food. Barcelona/ México DF: Grup de Recerca d'Interaccions Digitals, Universitat de Vic. Flacso México. Recuperado a partir de http://www.planetaweb2.net/.

Cohen, L., \& Lawrence, M. (2002). Métodos de investigación educativa (2da Edición). Madrid, España: La Muralla.

Colace, F., Santo, M., \& Greco, L. (2014). E-Learning and Personalized Learning Path: A Proposal Based on the Adaptive Educational Hypermedia System. International Journal of Emerging Technologies in Learning (iJET), 9(2), 9-16. 
Collis, B., \& Strijker, A. (2004). Technology and Human Issues in Reusing Learning Objects. Journal of Interactive Media in Education, 2004(1). Recuperado a partir de http://jime.open.ac.uk/article/2004-4-collis/187.

Colucci, S., Di Noia, T., Di Sciascio, E., Donini, F. M., \& Ragone, A. (2005). Semantic-based automated composition of distributed learning objects for personalized e-learning. En The Semantic Web: Research and Applications (pp. 633-648). Springer.

de Benito, B., Darder, A., \& Salinas, J. (2012). Los itinerarios de aprendizaje mediante mapas conceptuales como recurso para la representación del conocimiento. Revista Electrónica de Tecnología Educativa, (39), 1-14.

Doan, B.-L., Bourda, Y., \& Dumitrascu, V. (2006). A Semi-Automatic Tool using Ontology to Extract Learning Objects. En Advanced Learning Technologies, 2006. Sixth International Conference on (pp. 92-93). http://doi.org/10.1109/ICALT.2006.1652374.

Dodge, B. (1997). Some Thoughts About WebQuests [Página Web]. Recuperado 15 de julio de 2014, a partir de http://webquest.sdsu.edu/about webquests.html.

Duitama, F., Defude, B., Bouzeghoub, A., \& Lecocq, C. (2005). A Framework for the Generation of Adaptive Courses Based on Semantic Metadata. Multimedia Tools and Applications, 25(3), 377-390. http://doi.org/10.1007/s11042-005-6541-8.

Farrell, R. (2006). Dynamic Assembly of Learning Materials in a Corporate Context. Educational Technology-Saddle Brook Then Englewood Cliffs NJ, 46(1), 70 - 73.

Farrell, R. G., Liburd, S. D., \& Thomas, J. C. (2004). Dynamic Assembly of Learning Objects. En Proceedings of the 13th International World Wide Web Conference on Alternate Track Papers \&Amp; Posters (pp. 162-169). New York, NY, USA: ACM. http://doi.org/10.1145/1013367.1013394.

Felder, R. M., \& Silverman, L. K. (1988). Learning and teaching styles in engineering education. Engineering education, 78(7), 674-681.

Friesen, N. (2001). What are Educational Objects? Recuperado 20 de abril de 2010, a partir de http://www.ucalgary.ca/commons/careo/objectpaper.htm.

Friesen, N., Caws, C., \& Beaudoin, M. (2006). A New Learning Object Repository for Language Learning: Methods and Possible Outcomes. Interdisciplinary Journal of Knowledge and Learning Objects, 2, 111-124.

Gançarski, A. L., Bouzeghoub, A., Defude, B., \& Lecocq, C. (2007). Iterative search of composite learning objects. En IADIS Internationnal Conference WWW/Internet (pp. 8-12). Vila Real, Portugal.

García Aretio, L. (2005). Objetos de aprendizaje. Características y repositorios. Boletín Electrónico de Noticias de Educación a Distancia (BENED). Recuperado 5 de septiembre de 2014, a partir de http://e-spacio.uned.es/fez/eserv/bibliuned:327/editabril2005.pdf.

García Aretio, L. (2013). MOOC: objetos de aprendizaje. Contextos Universitarios Mediados, 19(13), 1-6.

Garrido, A., Onaindia, E., \& Sapena, O. (2009). Automated Planning for Personalised Course Composition. En Advanced Learning Technologies (pp. 178 -182). Riga, Latvia. http://doi.org/10.1109/ICALT.2009.39.

Garrido, A., \& Onaindia, E. (2013). Assembling Learning Objects for Personalized Learning: An AI Planning Perspective. Intelligent Systems, IEEE, 28(2), 64-73. http://doi.org/10.1109/MIS.2011.36. 
Gerard, R. (1969). Shaping the Mind: Computers in Education (Training \& Development Journal, Vol. 23). New York: Academic Press.

Gruber, T. (2009). Ontology. En L. Liu \& M. T. Özsu (Eds.), Encyclopedia of Database Systems (pp. 1963-1965). Springer US. Recuperado a partir de http://dx.doi.org/10.1007/978-0-38739940-9 1318.

Haas, H., \& Brown, A. (2004). Web Services Glossary. W3C Working Group. Recuperado 15 de septiembre de 2014, a partir de http://www.w3.org/TR/ws-gloss/.

Hilera González, J. R., \& Hoya Marón, R. (2010). Estándares de e-learning: guía de consulta. España: Universidad de Alcalá.

Huang, Y.-M., Huang, T.-C., Wang, K.-T., \& Hwang, W.-Y. (2009). A Markov-Based Recommendation Model for Exploring the Transfer of Learning on the Web. Educational Technology \& Society, 12(2), 144-162.

ICE. (2007). Los objetos de aprendizaje como recurso para la docencia universitaria: criterios para su elaboración. Editorial de la UPV. Recuperado 5 de abril de 2013, a partir de http://www.aqu.cat/doc/doc 22391979 1.pdf.

IEEE LTSC. (2002). Draft Standard for Learning Object Metadata. Recuperado 11 de marzo de 2013, a partir de http://ltsc.ieee.org/wg12/files/LOM_1484_12_1_v1_Final_Draft.pdf.

IMS Global Learning Consortium. (2003). Learning Design Specification [Página Web]. Recuperado 4 de febrero de 2014, a partir de http://www.imsglobal.org/learningdesign/.

IMS Global Learning Consortium. (2007). IMS Content Packaging Information Model. IMS GLC. Recuperado 15 de marzo de 2014, a partir de http://www.imsglobal.org/content/packaging/.

Johnson, L., Adams Becker, S., Cummins, M., Estrada, V., Freeman, A., \& Ludgate, H. (2013). NMC Horizon Report: 2013 Higher Education Edition. Austin, Texas: The New Media Consortium.

Jovanović, J., Gašević, D., \& Devedžić, V. (2006). Dynamic Assembly of Personalized Learning Content on the Semantic Web. En Y. Sure \& J. Domingue (Eds.), The Semantic Web: Research and Applications (Vol. 4011, pp. 545-559). Springer Berlin Heidelberg. Recuperado a partir de http://dx.doi.org/10.1007/11762256 40.

Jovanović, J., Gašević, D., \& Devedžić, V. (2009). TANGRAM for personalized learning using the semantic web technologies. Journal of emerging technologies in web intelligence, 1(1), 621. http://doi.org/doi:10.4304/jetwi.1.1.6-21.

Jovanovic, J., Gasevic, D., Verbert, K., \& Duval, E. (2005). Ontology of Learning Object Content Structure. En AIED (pp. 322-329).

Jovanović, J., Gasevic, D., Verbert, K., \& Duval, E. (2010). ALOCoM Content Type Ontology. Working Draft [Página Web]. Recuperado 6 de marzo de 2014, a partir de http://jelenajovanovic.net/ontologies/loco/alocom-content-type/spec.

Karam, N., Linckels, S., \& Meinel, C. (2007). Semantic Composition of Lecture Subparts for a Personalized e-Learning. En E. Franconi, M. Kifer, \& W. May (Eds.), The Semantic Web: Research and Applications (Vol. 4519, pp. 716-728). Springer Berlin Heidelberg. Recuperado a partir de http://dx.doi.org/10.1007/978-3-540-72667-8 50.

Kellar, M., Stern, H., Watters, C., \& Shepherd, M. (2004). Information architecture to support dynamic composition of interactive lessons and reuse of learning objects. En System Sciences, 2004. Proceedings of the 37th Annual Hawaii International Conference on System Sciences (pp. 1-10). Hawaii: IEEE. http://doi.org/10.1109/HICSS.2004.1265048. 
Kitchenham, B. (2004). Procedures for performing systematic reviews (Technical Report No. TR/SE-0401) (p. 27). Reino Unido: Keele University. Recuperado a partir de http://testszingarelli.googlecode.com/svn-history/r336/trunk/2-

Disciplinas/MetodPesquisa/kitchenham_2004.pdf.

L'Allier, J. (1998). NETg's Precision Skilling: The linking of occupational skills descriptors to training interventions. Recuperado 15 de diciembre de 2009, a partir de http://www.netg.com/research/pskillpaper.htm.

LACE. (1999). Introducción al estudio de casos en educación. Laboratorio para el Análisis del Cambio Educativo. Universidad de Cádiz. Recuperado desde http://www2.uca.es/lace/documentos/EC.pdf.

Lalithsena, R. S. S., Hewagamage, K. P., \& Jayaratne, K. L. (2008). A Semantic Web Model for the Personalized e-Learning (pp. 30-39). Presentado en 9th International Information Technology Conference, Colombo, Sri Lanka. Recuperado a partir de http://www.icter.org/conference/sites/default/files//icter/IITC-2008p30.pdf.

Larios Rodríguez, I. N., \& Figueroa Preciado, G. (2008). Muestro. Proyecto de seguimiento a los cursos de Estadística. Universidad de Sonora. Recuperado desde http://www.estadistica.mat.uson.mx/Material/elmuestreo.pdf.

Leal Fonseca, D. E. (2008). Iniciativa colombiana de objetos de aprendizaje: situación actual y potencial para el futuro. Apertura, 8(8), 76-85.

Ledda, R. (2013, junio 18). Tin Can API, porque aprendemos en cualquier lugar [Tin Can API, porque aprendemos en cualquier lugar | PUNTOMOV]. Recuperado a partir de http://puntomov.com/2013/06/18/que-es-tin-can-api/.

Li, Y., \& Huang, R. (2006). Dynamic composition of curriculum for personalized e-learning. IOS Press, 151(Learning by Effective Utilization of Technologies: Facilitating Intercultural Understanding), 569 - 576.

Llamas Nistal, M., Vaz de Carvalho, C., \& Rueda Artunduaga, C. (Eds.). (2007). TICs para el aprendizaje de la ingeniería. IEEE, Sociedad de Educación. Recuperado a partir de http://romulo.det.uvigo.es/ticai/index.php/ticai-2007/ticai2007.

Longmire, W. (2000). A primer on learning objects [Página Web]. Recuperado 20 de mayo de 2008, a partir de http://www.learningcircuits.org/mar2000/primer.html.

Lopes Gançarski, A., Bouzeghoub, A., Defude, B., \& Lecocq, C. (2007). Iterative search of composite learning objects. En IADIS Internationnal Conference WWW/Internet (pp. 8-12). Vila Real, Portugal. Recuperado a partir de http://www.iadis.net/dl/final_uploads/200712C050.pdf.

Lopez Cobo, J.-M., Arroyo, S., Sicilia, M.-A., \& Sánchez, S. (2007). A Semantic Web Service Architecture for Learning Object Repositories. En Advances in Electronic Business (Vol. 2, pp. 141-158). Hershey, Pennsylvania: Cybertech publishing. Recuperado a partir de http://www.cc.uah.es/ssalonso/papers/AEB_SWS_CoboEtAl.pdf.

López, M. G., Miguel, V., \& Montaño, N. E. (2008). Sistema Generador de AMBientes de Enseñanza-ApRendizaje Constructivistas basados en Objetos de Aprendizaje (AMBAR): la Interdisciplinariedad en los ambientes de aprendizaje en línea. Revista de Educación a Distancia, (19), 1-14.

Maldonado, J., \& Astudillo, G. (2014). Los Objetos de Aprendizaje: Un estado del arte en Iberoamérica. Presentado en CCITA, Miami: EEUU.

Manouselis, N., Drachsler, H., Verbert, K., \& Duval, E. (2012). Recommender systems for learning. New York: Springer. 
Marquès, P. (2011). Los medios didácticos [Página Web]. Recuperado en Junio 21, 2014, desde http://peremarques.pangea.org/medios.htm.

McGreal, R. (2004). Online Education using Learning Objects. New York: RoutledgeFalmer.

Melis, E., Goguadze, G., Homik, M., Libbrecht, P., Ullrich, C., \& Winterstein, S. (2006). Semanticaware components and services of ActiveMath. British Journal of Educational Technology, $37(3), 405-423$.

Menéndez Domínguez, V. H., Castellanos Bolaños, M. E., Zapata González, A., \& Prieto Méndez, M. E. (2010). Generación de objetos de aprendizaje empleando un enfoque asistido. PixelBit: Revista de medios y educación, (38), 141-153.

Menéndez Domínguez, V. H., Prieto Méndez, M. E., \& Zapata González, A. (2010). Sistemas de Gestión Integral de Objetos de Aprendizaje. IEEE-RITA, 5(2), 56-62.

Menéndez, V., Prieto, M., \& Zapata, A. (2010). Sistemas de Gestión Integral de Objetos de Aprendizaje. Latin-American Learning Technologies Journal, 5(2), 56-62.

Merriam, S. B. (1998). Qualitative Research and Case Study Applications in Education. Revised and Expanded from «Case Study Research in Education.». San Francisco, CA: ERIC.

Merrill, M. D. (1983). Component display theory. Instructional-design theories and models: An overview of their current status, 1, 282-333.

Merrill, M. D., Li, Z., \& Jones, M. K. (1990). Second generation instructional design (ID2). Educational Technology, 30, 7-14.

Pahl, C., \& Barrett, R. (2004). A web services architecture for learning object discovery and assembly. En Proceedings of the 13th international World Wide Web conference on Alternate track papers \& posters (pp. 446-447). New York, NY, USA: ACM. http://doi.org/10.1145/1013367.1013518.

Parrish, P. E. (2004). The trouble with learning objects. Educational Technology Research and Development, 52(1), 49-67. http://doi.org/10.1007/BF02504772.

Polsani, P. R. (2003). Use and Abuse of Reusable Learning Objects. Journal of Digital Information, 3(4). Recuperado a partir de https://journals.tdl.org/jodi/index.php/jodi/article/view/89.

Rigaux, P., \& Spyratos, N. (2007). Selene report: Metadata management and learning object composition in a self elearning network (Research Report). Londres: School of Computer Science and Information Systems, Birkbeck College, University of London. Recuperado a partir de http://www.dcs.bbk.ac.uk/selene/reports.

Roig Vila, R. (2005). Diseño de materiales curriculares electrónicos a través de Objetos de Aprendizaje. RED. Revista de Educación a Distancia, (IV), 1-9.

Rustici Software. (2016). What is the Tin Can API? [Página Web]. Recuperado 2 de abril de 2016, a partir de https://tincanapi.com/overview/.

Sánchez-Alonso, S., Sicilia, M., López-Cobo, J., \& Arroyo, S. (2007). Design by Contract-Based Selection and Composition of Learning Objects. En B. Fernández-Manjón, J. SánchezPérez, J. Gómez-Pulido, M. Vega-Rodríguez, \& J. Bravo-Rodríguez (Eds.), Computers and Education (pp. 179-191). Springer Netherlands. Recuperado a partir de http://dx.doi.org/10.1007/978-1-4020-4914-9 16.

Santacruz-Valencia, L. P., Delgado Kloos, C., \& Cuevas Aedo, I. (2005). Automatización de los procesos para la generación ensamblaje y reutilización de Objetos de Aprendizaje (Tesis doctoral). Universidad Carlos III de Madrid, Madrid. Recuperado desde www.lite.etsii.urjc.es/liliana/Defensa_Tesis_LPSV.pdf. 
Santacruz-Valencia, L. P., Navarro, A., Delgado Kloos, C., \& Aedo, I. (2008). ELO-Tool: Taking Action in the Challenge of Assembling Learning Objects. Journal of Educational Technology \& Society, 11(1), 102-117.

Sanz, C. V. (2015). Los objetos de aprendizaje, un debate abierto y necesario. Bit \& Byte, 1(1), 3335. Recuperado a partir de http://sedici.unlp.edu.ar/handle/10915/46722.

Sarasa, A., Piquer, J., Arriola, R., \& Iglesia, S. (2008). LOMEditor: Composition and Classification of Learning Objects. En A. Mendes, I. Pereira, \& R. Costa (Eds.), Computers and Education (pp. 241-249). Springer London. Recuperado a partir de http://dx.doi.org/10.1007/978-184628-929-3 24.

Schreurs, J., Vanhove, B., \& Al-Zoubi, A. (2008). Assembling content into dynamic learning objects versus authoring of e-learning courses. International Journal of Emerging Technologies in Learning, 3(3), 15-20.

Shahin, R., Barakat, L., Mahmoud, S., \& Alkassar, M. (2008). Dynamic Generation of Adaptive Courses. En Information and Communication Technologies: From Theory to Applications, 2008 (pp. 1-4).

Shmelev, V., Karpova, M., \& Dukhanov, A. (2015). An Approach of Learning Path Sequencing Based on Revised Bloom's Taxonomy and Domain Ontologies with the Use of Genetic $\begin{array}{lllllll}\text { Algorithms. } & \text { Procedia } & \text { Computer } & \text { Science, } & 66, & 711\end{array}$ http://doi.org/http://dx.doi.org/10.1016/j.procs.2015.11.081.

Sicilia Urbán, M. A. (2005). Reusabilidad y reutilización de objetos didácticos: mitos, realidades y posibilidades. Revista de Educación a Distancia, IV(Número monográfico II), 1-12. Recuperado a partir de http://www.um.es/ead/red/M2/sicilia46.pdf.

Sicilia Urbán, M.-A., \& Sánchez Alonso, S. (2009a). Introducción a los estándares de learning objects. Presentado en Diseño y Evaluación de contenidos y actividades educativas reutilizables, Information Engineering Research Unit. Universidad de Alcalá.

Stănică, J. L., \& Crișan, D. A. (2011). Framework For Flexible Reuse And Assembly Of Learning Objects-A Pilot Project. Journal of Information Systems \& Operations Management, 5(2.1), $478-484$.

Stănică, J. L., \& Crișan, D. A. (2012). Dynamic Development And Assembly Of Learning Objects In A Math Learning Environment. Journal of Information Systems \& Operations Management, 6(1), 29-40.

Štuikys, V., Burbaite, R., \& Bespalova, K. (2015). The LO Sequencing Problem and Its Solution Using Meta-Programming-Based Approach. En G. Dregvaite \& R. Damasevicius (Eds.), Information and Software Technologies: 21st International Conference (pp. 151-164). Druskininkai, Lituania: Springer International Publishing. Recuperado a partir de http://dx.doi.org/10.1007/978-3-319-24770-0 14.

Taylor, S., \& Bogdan, R. (1986). Introducción a los métodos de investigación cualitativa. Buenos Aires: Paidós.

Thyagharajan, K., \& Nayak, R. (2007). Adaptive content creation for personalized e-learning using web services. Journal of Applied Sciences Research, 3(9), 828-836.

Torres, I.-D., \& Guzmán-Luna, J. A. (2015). Composition of Learning Routes Using Automatic Planning and Web Semantics. En T. Sobh \& K. Elleithy (Eds.), Innovations and Advances in Computing, Informatics, Systems Sciences, Networking and Engineering (Vol. 313, pp. 321328). Online: Springer International Publishing. Recuperado a partir de http://dx.doi.org/10.1007/978-3-319-06773-5 43. 
Ullrich, C. (2008). Course Generation as a Hierarchical Task Network Planning Problem. KI, 3, $72-$ 74. Recuperado a partir de http://www.carstenullrich.net/pubs/Ullrich-CG-KI08.pdf.

Ullrich, C., \& Melis, E. (2009). Pedagogically founded courseware generation based on HTNplanning. Expert Systems with Applications, 36(5), 9319 - 9332. Recuperado a partir de http://doi.org/10.1016/j.eswa.2008.12.043.

Verbert, K., \& Duval, E. (2004). Towards a Global Architecture for Learning Objects: A Comparative Analysis of Learning Object Content Models. En Proceedings of World Conference on Educational Multimedia, Hypermedia and Telecommunications 2004 (pp. 202-208). Lugano, Switzerland: AACE. Recuperado a partir de http://www.editlib.org/p/12933.

Verbert, K., \& Duval, E. (2008). ALOCOM: a generic content model for learning objects. International Journal on Digital Libraries, 9(1), 41-63. http://doi.org/10.1007/s00799-0080039-8.

Verbert, K., Ochoa, X., Derntl, M., Wolpers, M., Pardo, A., \& Duval, E. (2012). Semi-automatic assembly of learning resources. Computers \& Education, 59(4), 1257 - 1272. http://doi.org/10.1016/j.compedu.2012.06.005.

Verbert, K., Wiley, D., \& Duval, E. (2009). A Methodology and Framework for the Semi-automatic Assembly of Learning Objects. En Learning in the Synergy of Multiple Disciplines (Vol. 5794, pp. 757-762). Springer Berlin / Heidelberg. Recuperado a partir de http://dx.doi.org/10.1007/978-3-642-04636-0 80.

Wetzlinger, W., Auinger, A., \& Stary, C. (2008). Ad-hoc Composition of Distributed Learning Objects using Active XML. International Journal of Emerging Technologies in Learning (iJET), 3(3), pp. 33-39. http://doi.org/10.3991/ijet.v3i3.279.

Wiley, D. (1999a). Learning objects and the new CAI: So what do I do with a learning object. opencontent.org. Recuperado a partir de http://opencontent.org/docs/instruct-arch.pdf.

Wiley, D. (1999b). The post-LEGO learning object. Recuperado a partir de http://opencontent.org/docs/post-lego.pdf.

Wiley, D. (2000). Connecting learning objects to instructional design theory: A definition, a metaphor, and a taxonomy. En The Instructional Use of Learning Objects (online version). Recuperado a partir de http://reusability.org/read/chapters/wiley.doc.

Wiley, D. (2007). The learning objects literature. En Handbook of research on educational communications and technology (David Jonassen, Michael J. Spector, Marcy Driscoll, M. David Merrill, Jeroen van Merrienboer, Marcy P. Driscoll, pp. 345-353). New York/London: Taylor \& Francis. Recuperado a partir de http://www.opencontent.org/docs/wiley-lo-reviewfinal.pdf.

Wiley, D., Gibbons, A., \& Recker, M. (2000). A reformulation of the issue of learning object granularity and its implications for the design of learning objects. En The instructional use of learning objects. Bloomington, Indiana: Agency for Instructional Technology and Association for Educational Communications of Technology.

Wiley, D., Waters, S., Dawson, D., Lambert, B., Barclay, M., Wade, D., \& Nelson, L. (2004). Overcoming the Limitations of Learning Objects. Journal of Educational Multimedia and Hypermedia, 13(4), 507-521.

Zapata Ros, M. (2005). Secuenciación de contenidos y objetos de aprendizaje. Revista de Educación a Distancia, IV(Número monográfico II), 1-39. Recuperado a partir de http://www.um.es/ead/red/M2/zapata47.pdf. 
Zapata Ros, M. (2010). Secuenciación de contenidos. Especificaciones para la secuenciación instruccional de objetos de aprendizaje (Tesis doctoral). Universidad de Alcalá, España.

Zapata, D., \& Zapata, M. (2009). ¿Qué es un Objeto de Aprendizaje? [Objeto de aprendizaje]. Recuperado a partir de http://aprendeenlinea.udea.edu.co/lms/men/oac1.html.

Zouaq, A., Nkambou, R., \& Frasson, C. (2007). An integrated approach for automatic aggregation of learning knowledge objects. Interdisciplinary Journal of Knowledge and Learning Objects, 3, 135-162. 


\section{ACRÓNIMOS}

A

ACG Adaptive Course Generation System

ACM Association for Computing Machinery

ADL Advanced Distributed Learning

AGORA Plataforma de Ayuda para la Gestión de Objetos Reusables de Aprendizaje

AICC Aviation Industry Computer-Based Training Committee

ALO Atamic Learning Object

ALOCoM Abstract Learning Object Content Model

AMBAR Sistema Generador de AMBientes de Enseñanza-ApRendizaje Constructivistas basados en Objetos de Aprendizaje

C

CA Conocimiento Asociado

CO Content Object

D

DCMES Dublin Core Metadata Element Set

DCMI Dublin Core Metadata Inititive

DFD Diagrama de Flujo de Datos

E

ELO Electronic Learning Object

EVEA Entono Virtual de Enseñanza y Aprendizaje

$\mathbf{F}$

FCEyN Facultad de Ciencias Exactas y Naturales - UNLPam 
G

GLOBE Global Learning Objects Brokered Exchange

HTN Hierarchical Task Network

HTML HyperText Markup Language

ID Identifier

IEEE Institute of Electrical and Electronics Engineers

ILO-QL Iterative Learning Object Query Language

IMS Instructional Management System Global Learning Consortium

IMS-LD IMS Learning Design

IMS-CP IMS Content Packaging

IMS-LRMS IMS Learning Resource Meta-data Specification

ISO/IEC International Organization for Standardization/International Electrotechnical Commission

$\mathbf{L}$

LACLO Latin American Community of Learning Objects

LAMS Learning Activity Management System

LCMS Learning Content Management System

LMS Learning Management System

LO Learning Object

LOM Learning Object Metadata

LOMS Learning Object Management System

M

MC Modelo de Contenido

MED Material Educativo Digital

MENC Ministerio de Educación Colombiano

MLR Metadata Learning Resource

MOOC Massive Open Online Course

$\mathbf{0}$

OA Objetos de Aprendizaje 
OpenDOAR The Directory of Open Access Repositories

OWL Ontology Web Language

$\mathbf{R}$

RIO Reusable Information Object

RLO Reusable Learning Objects

$\mathbf{S}$

SCO Shareable Content Objects

SCORM Sharable Content Object Referente Model

SE Sistema Ensamblador

SR Sistema Recomendador

$\mathbf{T}$

TIC Tecnologías de la Información y la Comunicación

$\mathbf{U}$

UC Unidad de Contenido

UD Unidad Didáctica

UI Unidad de Información

UNLPam Universidad Nacional de La Pampa - La Pampa - Argentina

URL Uniform Resource Locator

$\mathbf{X}$

XML Extensible Markup Language 


\section{LISTA DE PUBLICACIONES SELECCIONADAS}

Este anexo presenta las tablas con los resultados de la aplicación de los criterios de inclusión y exclusión sobre todo el conjunto de publicaciones. Se presentan dos tablas, una con datos sobre las publicaciones y otra conteniendo los resultados de las preguntas de evaluación. 
Tabla 1. Lista de publicaciones seleccionadas. Datos generales.

\begin{tabular}{|c|c|c|c|c|}
\hline ID & Autor & Fecha & Fuente & País \\
\hline S01 & Kellar et al. & 2004 & IEEE & Canada \\
\hline S02 & Farrell, Liburd \& Thomas & 2004 & otro & EEUU \\
\hline S03 & Pahl et al. & 2004 & otro & Irlanda \\
\hline S04 & Roig Vila & 2005 & otro & España \\
\hline S05 & Colucci et al. & 2005 & Springer & Italia \\
\hline S06 & Santacruz Valencia, Delgado Kloos \& Aedo Cuevas & 2005 & otro & España \\
\hline S07 & Farrell \& White & 2006 & otro & EEUU \\
\hline S08 & Li \& Huang & 2006 & otro & China \\
\hline S09 & Anh \& Dam & 2006 & IEEE & Korea \\
\hline $\mathrm{S} 10$ & Jovanovic, Gašević \& Devedžić & 2006 & Springer & Rusia/Candá \\
\hline S11 & Lopes Gançarski, et al. & 2007 & otro & Francia \\
\hline $\mathrm{S} 12$ & Rigaux \& Spyratos & 2007 & otro & Francia \\
\hline S13 & Sánchez Alonso, et al. & 2007 & Springer & España \\
\hline S14 & Thyagharajan \& Nayak & 2007 & otro & India \\
\hline $\mathrm{S} 15$ & Karam, Linckels \& Meinel & 2007 & Springer & Alemania \\
\hline S16 & Anh, Ha \& Dam & 2008 & otro & Korea \\
\hline S17 & Santacruz Valencia, et al. & 2008 & otro & España \\
\hline S18 & Shahin, et al. & 2008 & IEEE & Siria \\
\hline S19 & Schreurs, Vanhove \& Al-Zoubi & 2008 & otro & Belgica y Jordania \\
\hline S20 & Sarasa, et al. & 2008 & otro & España \\
\hline $\mathrm{S} 21$ & Wetzlinger, Auinger \& Stary & 2008 & otro & Australia \\
\hline $\mathrm{S} 22$ & López, Miguel \& Montaño & 2008 & otro & Venezuela \\
\hline $\mathrm{S} 23$ & Lalithsena, Hewagamage \& Jayaratne & 2008 & otro & Sri Lanka \\
\hline S24 & Bouzeghoub, et al. & 2009 & Springer & Francia \\
\hline S25 & Bouzeghoub \& Selmi & 2009 & IEEE & Francia \\
\hline S26 & Garrido, Onaindia \& Sapena & 2009 & IEEE & España \\
\hline S27 & Verbert, Wiley \& Duval & 2009 & Springer & Belgica y EEUU \\
\hline S28 & Jovanovic, Gašević \& Devedžić & 2009 & Springer & Rusia y Canadá \\
\hline S29 & Huang, et al. & 2009 & otro & Taiwan \\
\hline S30 & Ullrich \& Melis & 2009 & otro & China y Alemania \\
\hline S31 & Menéndez, Prieto \& Zapata & 2010 & IEEE & México \\
\hline S32 & Menéndez, et al. & 2011 & otro & México \\
\hline S33 & Stănică \& Crișan & 2011 & otro & Rumania \\
\hline S34 & Stănică \& Crișan & 2012 & otro & $\begin{array}{c}\text { Rumania } \\
\text { Belgica, Ecuador, }\end{array}$ \\
\hline S35 & Verbert et al. & 2012 & otro & Alemania y España \\
\hline S36 & Tsai, Chiu, Lee \& Wang & 2006 & IEEE & Taiwan \\
\hline S37 & Doan, Bourda \& Dumitrascu & 2006 & IEEE & Francia \\
\hline S38 & Ruiz-Iniesta, Jiménez-Díaz \& Gómez-Albarrán & 2010 & IEEE & España \\
\hline S39 & Caro Piñares, Hernández \& Jiménez Builes & 2011 & otro & Colombia \\
\hline $\mathrm{S} 40$ & Chellatamilan \& Suresh & 2012 & IEEE & India \\
\hline
\end{tabular}


Tabla 2. Lista de publicaciones seleccionadas. Resultados de las preguntas de evalaución.

\begin{tabular}{|c|c|c|c|c|c|c|c|c|}
\hline ID & Autor & PE1 & PE2 & PE3 & PE4 & PE5 & PE6 & PE7 \\
\hline S01 & Kellar et al. & $\mathrm{S}$ & $\mathrm{S}$ & $\mathrm{N}$ & $\mathrm{S}$ & $\mathrm{S}$ & $\mathrm{S}$ & $\mathrm{S}$ \\
\hline S02 & Farrell, Liburd \& Thomas & $\mathrm{S}$ & S & $\mathrm{S}$ & $\mathrm{S}$ & $\mathrm{S}$ & $\mathrm{S}$ & $\mathrm{S}$ \\
\hline $\mathrm{S} 03$ & Pahl et al. & $\mathrm{S}$ & S & $\mathrm{S}$ & $\mathrm{S}$ & $\mathrm{S}$ & $\mathrm{S}$ & $\mathrm{N}$ \\
\hline S04 & Roig Vila & $\mathrm{S}$ & S & $\mathrm{N}$ & $\mathrm{S}$ & $\mathrm{S}$ & $\mathrm{S}$ & $\mathrm{S}$ \\
\hline S05 & Colucci et al. & $\mathrm{S}$ & $\mathrm{S}$ & $\mathrm{S}$ & $\mathrm{N}$ & $\mathrm{S}$ & $\mathrm{S}$ & $\mathrm{N}$ \\
\hline S06 & Santacruz Valencia, Delgado Kloos \& Aedo Cuevas & $\mathrm{S}$ & S & $\mathrm{S}$ & $\mathrm{S}$ & $\mathrm{S}$ & $\mathrm{S}$ & $\mathrm{S}$ \\
\hline S07 & Farrell \& White & $\mathrm{S}$ & $\mathrm{S}$ & $\mathrm{S}$ & $\mathrm{S}$ & $\mathrm{S}$ & $\mathrm{S}$ & $\mathrm{S}$ \\
\hline S08 & Li \& Huang & $\mathrm{S}$ & $\mathrm{S}$ & $\mathrm{S}$ & $\mathrm{S}$ & $\mathrm{S}$ & $\mathrm{S}$ & $\mathrm{S}$ \\
\hline S09 & Anh \& Dam & $\mathrm{S}$ & $\mathrm{S}$ & $\mathrm{S}$ & $\mathrm{S}$ & $\mathrm{S}$ & $\mathrm{N}$ & $\mathrm{S}$ \\
\hline S10 & Jovanovic, Gašević \& Devedžić & $\mathrm{S}$ & $\mathrm{S}$ & $\mathrm{S}$ & $\mathrm{S}$ & $\mathrm{S}$ & $\mathrm{N}$ & $\mathrm{S}$ \\
\hline S11 & Lopes Gançarski, et al. & $\mathrm{S}$ & $\mathrm{S}$ & $\mathrm{N}$ & $\mathrm{S}$ & $\mathrm{S}$ & $\mathrm{N}$ & $\mathrm{S}$ \\
\hline $\mathrm{S} 12$ & Rigaux \& Spyratos & $\mathrm{S}$ & $\mathrm{S}$ & $\mathrm{S}$ & S & $\mathrm{S}$ & $\mathrm{S}$ & $\mathrm{S}$ \\
\hline S13 & Sánchez Alonso, et al. & $\mathrm{S}$ & $\mathrm{S}$ & $\mathrm{S}$ & $\mathrm{S}$ & $\mathrm{S}$ & $\mathrm{N}$ & $\mathrm{S}$ \\
\hline S14 & Thyagharajan \& Nayak & $\mathrm{S}$ & $\mathrm{S}$ & $\mathrm{N}$ & $\mathrm{S}$ & $\mathrm{S}$ & $\mathrm{N}$ & $\mathrm{N}$ \\
\hline S15 & Karam, Linckels \& Meinel & $\mathrm{S}$ & $\mathrm{S}$ & $\mathrm{S}$ & $\mathrm{S}$ & $\mathrm{S}$ & $\mathrm{S}$ & $\mathrm{N}$ \\
\hline S16 & Anh, Ha \& Dam & $\mathrm{S}$ & $\mathrm{S}$ & $\mathrm{S}$ & $\mathrm{S}$ & $\mathrm{S}$ & $\mathrm{N}$ & $\mathrm{S}$ \\
\hline S17 & Santacruz Valencia, et al. & $\mathrm{S}$ & S & $\mathrm{S}$ & $\mathrm{S}$ & $\mathrm{S}$ & $\mathrm{S}$ & $\mathrm{S}$ \\
\hline S18 & Shahin, et al. & $\mathrm{S}$ & $\mathrm{S}$ & $\mathrm{S}$ & $\mathrm{S}$ & $\mathrm{S}$ & $\mathrm{N}$ & $\mathrm{N}$ \\
\hline S19 & Schreurs, Vanhove \& Al-Zoubi & $\mathrm{S}$ & $\mathrm{S}$ & $\mathrm{N}$ & $\mathrm{S}$ & $\mathrm{S}$ & $\mathrm{S}$ & $\mathrm{N}$ \\
\hline S20 & Sarasa, et al. & $\mathrm{S}$ & S & $\mathrm{S}$ & $\mathrm{S}$ & $\mathrm{S}$ & $\mathrm{S}$ & $\mathrm{S}$ \\
\hline $\mathrm{S} 21$ & Wetzlinger, Auinger \& Stary & $\mathrm{S}$ & $\mathrm{S}$ & $\mathrm{N}$ & $\mathrm{N}$ & $\mathrm{S}$ & $\mathrm{S}$ & $\mathrm{N}$ \\
\hline S22 & López, Miguel \& Montaño & $\mathrm{S}$ & S & $\mathrm{N}$ & $\mathrm{S}$ & $\mathrm{S}$ & $\mathrm{S}$ & $\mathrm{N}$ \\
\hline $\mathrm{S} 23$ & Lalithsena, Hewagamage \& Jayaratne & $\mathrm{S}$ & $\mathrm{S}$ & $\mathrm{S}$ & $\mathrm{S}$ & $\mathrm{S}$ & $\mathrm{N}$ & $\mathrm{S}$ \\
\hline S24 & Bouzeghoub, et al. & $\mathrm{S}$ & $\mathrm{S}$ & $\mathrm{S}$ & $\mathrm{S}$ & $\mathrm{S}$ & $\mathrm{S}$ & $\mathrm{S}$ \\
\hline S25 & Bouzeghoub \& Selmi & $\mathrm{S}$ & $\mathrm{S}$ & $\mathrm{S}$ & $\mathrm{S}$ & $\mathrm{S}$ & $\mathrm{S}$ & $\mathrm{S}$ \\
\hline S26 & Garrido, Onaindia \& Sapena & $\mathrm{S}$ & $\mathrm{S}$ & $\mathrm{S}$ & $\mathrm{S}$ & $\mathrm{S}$ & $\mathrm{N}$ & $\mathrm{S}$ \\
\hline S27 & Verbert, Wiley \& Duval & $\mathrm{S}$ & $\mathrm{S}$ & $\mathrm{S}$ & $\mathrm{S}$ & $\mathrm{S}$ & $\mathrm{N}$ & $\mathrm{N}$ \\
\hline S28 & Jovanovic, Gašević \& Devedžić & $\mathrm{S}$ & $\mathrm{S}$ & $\mathrm{S}$ & $\mathrm{S}$ & $\mathrm{S}$ & $\mathrm{N}$ & $\mathrm{S}$ \\
\hline S29 & Huang, et al. & $\mathrm{S}$ & $\mathrm{S}$ & $\mathrm{S}$ & $\mathrm{S}$ & $\mathrm{S}$ & $\mathrm{N}$ & $\mathrm{S}$ \\
\hline $\mathrm{S} 30$ & Ullrich \& Melis & $\mathrm{S}$ & S & $\mathrm{S}$ & $\mathrm{S}$ & $\mathrm{S}$ & $\mathrm{S}$ & $\mathrm{S}$ \\
\hline S31 & Menéndez, Prieto \& Zapata & $\mathrm{S}$ & $\mathrm{S}$ & $\mathrm{N}$ & $\mathrm{S}$ & $\mathrm{S}$ & $\mathrm{S}$ & $\mathrm{N}$ \\
\hline S32 & Menéndez, et al. & $\mathrm{S}$ & $\mathrm{S}$ & $\mathrm{N}$ & $\mathrm{S}$ & $\mathrm{S}$ & $\mathrm{S}$ & $\mathrm{N}$ \\
\hline S33 & Stănică \& Crișan & $\mathrm{S}$ & $\mathrm{S}$ & $\mathrm{N}$ & $\mathrm{S}$ & $\mathrm{S}$ & $\mathrm{S}$ & $\mathrm{S}$ \\
\hline S34 & Stănică \& Crișan & $\mathrm{S}$ & $\mathrm{S}$ & $\mathrm{N}$ & $\mathrm{S}$ & $\mathrm{S}$ & $\mathrm{S}$ & $\mathrm{S}$ \\
\hline S35 & Verbert et al. & $\mathrm{S}$ & $\mathrm{S}$ & $\mathrm{S}$ & $\mathrm{S}$ & $\mathrm{S}$ & $\mathrm{N}$ & $\mathrm{N}$ \\
\hline S36 & Tsai, Chiu, Lee \& Wang & $\mathrm{S}$ & $\mathrm{N}$ & $\mathrm{N}$ & $\mathrm{N}$ & $\mathrm{S}$ & $\mathrm{S}$ & $\mathrm{N}$ \\
\hline S37 & Doan, Bourda \& Dumitrascu & $\mathrm{S}$ & $\mathrm{N}$ & $\mathrm{N}$ & $\mathrm{S}$ & $\mathrm{S}$ & $\mathrm{S}$ & $\mathrm{S}$ \\
\hline S38 & Ruiz-Iniesta, Jiménez-Díaz \& Gómez-Albarrán & $\mathrm{S}$ & $\mathrm{N}$ & $\mathrm{N}$ & $\mathrm{N}$ & $\mathrm{S}$ & $\mathrm{N}$ & $\mathrm{N}$ \\
\hline S39 & Caro Piñares, Hernández \& Jiménez Builes & $\mathrm{S}$ & $\mathrm{N}$ & $\mathrm{N}$ & $\mathrm{N}$ & $\mathrm{S}$ & $\mathrm{N}$ & $\mathrm{S}$ \\
\hline $\mathrm{S} 40$ & Chellatamilan \& Suresh & $\mathrm{S}$ & $\mathrm{N}$ & $\mathrm{N}$ & $\mathrm{N}$ & $\mathrm{S}$ & $\mathrm{S}$ & $\mathrm{N}$ \\
\hline
\end{tabular}


Este anexo presenta las tablas con los registros de la aplicación de los criterios de evaluación. Los mismos se agrupan en tres categorías (ver Capitulo 4) por lo que se conserva la misma agrupación.

\section{Referencia}

La siguiente tabla muestra la lista con los códigos asignados a cada metodología y los autores de las mismas. Por cuestiones de espacio, cuando se necesario se utilizará sólo esta codificación sin los autores.

\begin{tabular}{ll}
\hline ID & \\
\hline S01 & Kellar et al. \\
S03 & Pahl et al. \\
S04 & Roig Vila \\
S05 & Colucci et al. \\
S06 & Santacruz Valencia, Delgado Kloos \& Aedo Cuevas \\
S07 & Farrell et al. \\
S08 & Li \& Huang \\
S13 & Lopes Gançarski, et al. \\
S14 & Rigaux \& Spyratos \\
S15 & Sánchez Alonso, et al. \\
S16 & Thyagharajan \& Nayak \\
S17 & Karam, Linckels \& Meinel \\
S18 & Anh et al. \\
S20 & Shahin et al. \\
S21 & Schreurs, Vanhove \& Al-Zoubi \\
S22 & Sarasa et al. \\
S23 & Wetzlinger, Auinger \& Stary \\
S24 & López, Miguel \& Montaño \\
S25 & Lalithsena, Hewagamage \& Jayaratne \\
S27 & Bouzeghoub et al. \\
S28 & Garrido, Onaindia \& Sapena \\
S30 & Jovanovic et al. \\
S31 & Huang et al. \\
S32 & Ullrich \& Melis \\
S35 & Menéndez et al. \\
S38 & Stănică \& Crișan \\
S39 & Verbert et al. \\
& \\
\hline
\end{tabular}




\section{(a) Estrategia}

\section{(i) Ensamblador/recomendador}

Tabla 1. Aplicación del criterio a.i

\begin{tabular}{|c|c|c|c|}
\hline ID & Autores & Ensamblador & Recomendador \\
\hline $\mathrm{S} 01$ & Kellar et al. & & $\bar{\checkmark}$ \\
\hline S03 & Pahl et al. & $\checkmark$ & \\
\hline S04 & Roig Vila & $\checkmark$ & \\
\hline S05 & Colucci et al. & $\checkmark$ & \\
\hline S06 & Santacruz Valencia, Delgado Kloos \& Aedo Cuevas & $\checkmark$ & \\
\hline S07 & Farrell et al. & & $\checkmark$ \\
\hline S08 & Li \& Huang & & $\checkmark$ \\
\hline S13 & Lopes Gançarski, et al. & $\checkmark$ & \\
\hline S14 & Rigaux \& Spyratos & & $\checkmark$ \\
\hline S15 & Sánchez Alonso, et al. & $\checkmark$ & \\
\hline S16 & Thyagharajan \& Nayak & & $\checkmark$ \\
\hline S17 & Karam, Linckels \& Meinel & & $\checkmark$ \\
\hline S18 & Anh et al. & & $\checkmark$ \\
\hline S20 & Shahin et al. & $\checkmark$ & \\
\hline S21 & Schreurs, Vanhove \& Al-Zoubi & $\checkmark$ & \\
\hline S22 & Sarasa et al. & $\checkmark$ & \\
\hline S23 & Wetzlinger, Auinger \& Stary & $\checkmark$ & \\
\hline S24 & López, Miguel \& Montaño & $\checkmark$ & \\
\hline S25 & Lalithsena, Hewagamage \& Jayaratne & $\checkmark$ & \\
\hline S27 & Bouzeghoub et al. & & $\checkmark$ \\
\hline S28 & Garrido, Onaindia \& Sapena & $\checkmark$ & \\
\hline S30 & Jovanovic et al. & & $\checkmark$ \\
\hline S31 & Huang et al. & & $\checkmark$ \\
\hline S32 & Ullrich \& Melis & & $\checkmark$ \\
\hline S35 & Menéndez et al. & & $\checkmark$ \\
\hline S38 & Stănică \& Crișan & $\checkmark$ & \\
\hline S39 & Verbert et al. & $\checkmark$ & \\
\hline
\end{tabular}




\section{(ii) Motivación}

\section{Referencias:}
A. Selección de los MED apropiados
B. Organización del conocimiento
C. Localización de MED
D. Personalización del contenido
E. Mejoramiento de los sistemas e-learning
F. Secuenciación automática de contenidos
G. Reutilización del contenido

Tabla 2. Aplicación del criterio a.ii

\begin{tabular}{|c|c|c|c|c|c|c|c|}
\hline ID & A. & B. & C. & D. & $E$. & F. & G. \\
\hline S01 & & & & $\checkmark$ & $\bar{\checkmark}$ & & \\
\hline $\mathrm{S} 03$ & & & & & $\checkmark$ & & \\
\hline S04 & & & & $\checkmark$ & & & \\
\hline S05 & & & & $\checkmark$ & & & \\
\hline S06 & & & & & $\checkmark$ & $\checkmark$ & $\checkmark$ \\
\hline S07 & & $\checkmark$ & $\checkmark$ & & & & \\
\hline S08 & $\checkmark$ & $\checkmark$ & & $\checkmark$ & & $\checkmark$ & $\checkmark$ \\
\hline $\mathrm{S} 13$ & $\checkmark$ & & & & & & $\checkmark$ \\
\hline S14 & & & & & & $\checkmark$ & \\
\hline $\mathrm{S} 15$ & & & & & $\checkmark$ & & \\
\hline S16 & & $\checkmark$ & & $\checkmark$ & & & \\
\hline S17 & $\checkmark$ & & $\checkmark$ & & & & \\
\hline $\mathrm{S} 18$ & $\checkmark$ & & & & & & \\
\hline $\mathrm{S} 20$ & $\checkmark$ & & & & & $\checkmark$ & \\
\hline $\mathrm{S} 21$ & & & & $\checkmark$ & & & \\
\hline $\mathrm{S} 22$ & & & & & $\checkmark$ & & \\
\hline S23 & $\checkmark$ & & & & & & \\
\hline $\mathrm{S} 24$ & & & & & $\checkmark$ & & \\
\hline $\mathrm{S} 25$ & & & & $\checkmark$ & $\checkmark$ & & \\
\hline S27 & & & & & $\checkmark$ & & $\checkmark$ \\
\hline S28 & & & & $\checkmark$ & $\checkmark$ & & $\checkmark$ \\
\hline $\mathrm{S} 30$ & & & & $\checkmark$ & & & $\checkmark$ \\
\hline S31 & & $\checkmark$ & & & & & \\
\hline $\mathrm{S} 32$ & & & & & $\checkmark$ & & \\
\hline S35 & & & & & & & \\
\hline S38 & & & & & & & $\checkmark$ \\
\hline S39 & & & & $\checkmark$ & & $\checkmark$ & \\
\hline
\end{tabular}


(iii) Principio de composición

Tabla 3. Aplicación del criterio a.iii

\begin{tabular}{|c|c|c|c|c|c|}
\hline ID & Metadatos OA & Metadatos usuario & Ontologías & Patrones & $\mathrm{S} / \mathrm{C}$ \\
\hline S01 & $\checkmark$ & $\checkmark$ & & & \\
\hline S03 & $\checkmark$ & $\checkmark$ & & $\checkmark$ & \\
\hline S04 & & & & $\checkmark$ & \\
\hline S05 & & & $\checkmark$ & & \\
\hline S06 & $\checkmark$ & & $\checkmark$ & & \\
\hline S07 & $\checkmark$ & & $\checkmark$ & & \\
\hline S08 & $\checkmark$ & $\checkmark$ & & $\checkmark$ & \\
\hline $\mathrm{S} 13$ & $\checkmark$ & & & $\checkmark$ & \\
\hline S14 & $\checkmark$ & $\checkmark$ & & $\checkmark$ & \\
\hline S15 & $\checkmark$ & & & $\checkmark$ & \\
\hline S16 & $\checkmark$ & $\checkmark$ & & & \\
\hline S17 & & $\checkmark$ & $\checkmark$ & & \\
\hline S18 & & $\checkmark$ & & $\checkmark$ & \\
\hline $\mathrm{S} 20$ & $\checkmark$ & $\checkmark$ & & $\checkmark$ & \\
\hline $\mathrm{S} 21$ & & & & $\checkmark$ & \\
\hline $\mathrm{S} 22$ & $\checkmark$ & & & $\checkmark$ & \\
\hline S23 & $\checkmark$ & & & & \\
\hline S24 & $\checkmark$ & & $\checkmark$ & $\checkmark$ & \\
\hline S25 & & & $\checkmark$ & & \\
\hline S27 & $\checkmark$ & & & $\checkmark$ & \\
\hline S28 & $\checkmark$ & & & $\checkmark$ & \\
\hline S30 & & & $\checkmark$ & & \\
\hline $\mathrm{S} 31$ & & & & $\checkmark$ & \\
\hline S32 & $\checkmark$ & $\checkmark$ & & & \\
\hline S35 & & & & & $\checkmark$ \\
\hline S38 & & & & $\checkmark$ & \\
\hline S39 & & & & $\checkmark$ & \\
\hline
\end{tabular}


(iv) Nivel de automatización

Tabla 4. Aplicación del criterio a.vi

\begin{tabular}{|c|c|c|c|c|}
\hline ID & Autores & Manual & Semi-automática & Automática \\
\hline S01 & Kellar et al. & & & $\checkmark$ \\
\hline S03 & Pahl et al. & & & $\checkmark$ \\
\hline S04 & Roig Vila & $\checkmark$ & & \\
\hline S05 & Colucci et al. & & & $\checkmark$ \\
\hline S06 & Santacruz Valencia, Delgado Kloos \& Aedo Cuevas & & $\checkmark$ & \\
\hline S07 & Farrell et al. & & & $\checkmark$ \\
\hline S08 & Li \& Huang & & & $\checkmark$ \\
\hline S13 & Lopes Gançarski, et al. & & $\checkmark$ & \\
\hline S14 & Rigaux \& Spyratos & & & $\checkmark$ \\
\hline S15 & Sánchez Alonso, et al. & & & $\checkmark$ \\
\hline S16 & Thyagharajan \& Nayak & & & $\checkmark$ \\
\hline S17 & Karam, Linckels \& Meinel & & & $\checkmark$ \\
\hline S18 & Anh et al. & & $\checkmark$ & \\
\hline $\mathrm{S} 20$ & Shahin et al. & & $\checkmark$ & \\
\hline $\mathrm{S} 21$ & Schreurs, Vanhove \& Al-Zoubi & $\checkmark$ & & \\
\hline $\mathrm{S} 22$ & Sarasa et al. & & $\checkmark$ & \\
\hline $\mathrm{S} 23$ & Wetzlinger, Auinger \& Stary & & & $\checkmark$ \\
\hline $\mathrm{S} 24$ & López, Miguel \& Montaño & & $\checkmark$ & \\
\hline $\mathrm{S} 25$ & Lalithsena, Hewagamage \& Jayaratne & & & $\checkmark$ \\
\hline $\mathrm{S} 27$ & Bouzeghoub et al. & & $\checkmark$ & \\
\hline S28 & Garrido, Onaindia \& Sapena & & $\checkmark$ & \\
\hline $\mathrm{S} 30$ & Jovanovic et al. & & & $\checkmark$ \\
\hline $\mathrm{S} 31$ & Huang et al. & & & $\checkmark$ \\
\hline $\mathrm{S} 32$ & Ullrich \& Melis & & $\checkmark$ & \\
\hline $\mathrm{S} 35$ & Menéndez et al. & & $\checkmark$ & \\
\hline $\mathrm{S} 38$ & Stănică \& Crișan & $\checkmark$ & & \\
\hline S39 & Verbert et al. & & $\checkmark$ & \\
\hline
\end{tabular}




\section{(v) Concepto de Objeto de Aprendizaje}
A. Intensionalidad pedagógica
B. Estructura interna
C. Metadatos
D. Reutilizable
E. Auto-contenido
F. Interoperable
G. Accesible
H. Ensamblable
I. No lo definen (*)

Tabla 5. Aplicación del criterio a.v

\begin{tabular}{|c|c|c|c|c|c|c|c|c|c|}
\hline ID & A. & B. & C. & D. & E. & F. & G. & H. & I. \\
\hline S01 & $\checkmark$ & & $\checkmark$ & $\checkmark$ & & & & & \\
\hline S03 & $\checkmark$ & & $\checkmark$ & $\checkmark$ & & & & $\checkmark$ & \\
\hline S04 & $\checkmark$ & & & $\checkmark$ & & & & & \\
\hline S05 & $\checkmark$ & & $\checkmark$ & $\checkmark$ & & $\checkmark$ & & $\checkmark$ & \\
\hline S06 & $\checkmark$ & $\checkmark$ & $\checkmark$ & $\checkmark$ & & $\checkmark$ & & $\checkmark$ & \\
\hline S07 & $\checkmark$ & & $\checkmark$ & & & & $\checkmark$ & $\checkmark$ & \\
\hline S08 & $\checkmark$ & & $\checkmark$ & $\checkmark$ & $\checkmark$ & $\checkmark$ & & $\checkmark$ & \\
\hline S13 & $\checkmark$ & & $\checkmark$ & & & $\checkmark$ & & $\checkmark$ & \\
\hline S14 & & & $\checkmark$ & & & & & $\checkmark$ & \\
\hline S15 & $\checkmark$ & & $\checkmark$ & $\checkmark$ & & & $\checkmark$ & & $\checkmark$ \\
\hline S16 & $\checkmark$ & & & $\checkmark$ & & & & & $\checkmark$ \\
\hline S17 & $\checkmark$ & & & $\checkmark$ & & & & $\checkmark$ & \\
\hline S18 & $\checkmark$ & & & & & $\checkmark$ & & & $\checkmark$ \\
\hline S20 & $\checkmark$ & & $\checkmark$ & $\checkmark$ & & $\checkmark$ & & & $\checkmark$ \\
\hline S21 & $\checkmark$ & & $\checkmark$ & & & $\checkmark$ & & $\checkmark$ & \\
\hline S22 & & & $\checkmark$ & $\checkmark$ & & $\checkmark$ & $\checkmark$ & & \\
\hline S23 & $\checkmark$ & & $\checkmark$ & $\checkmark$ & $\checkmark$ & $\checkmark$ & $\checkmark$ & $\checkmark$ & \\
\hline S24 & $\checkmark$ & & & $\checkmark$ & & $\checkmark$ & $\checkmark$ & & \\
\hline S25 & & & & & & & $\checkmark$ & & $\checkmark$ \\
\hline S27 & $\checkmark$ & & $\checkmark$ & & & & $\checkmark$ & & \\
\hline S28 & & & $\checkmark$ & $\checkmark$ & & & & & $\checkmark$ \\
\hline S30 & & & $\checkmark$ & & & & $\checkmark$ & & $\checkmark$ \\
\hline S31 & & & $\checkmark$ & & & $\checkmark$ & $\checkmark$ & & $\checkmark$ \\
\hline S32 & $\checkmark$ & & $\checkmark$ & $\checkmark$ & $\checkmark$ & & $\checkmark$ & & \\
\hline S35 & $\checkmark$ & & $\checkmark$ & $\checkmark$ & & $\checkmark$ & $\checkmark$ & & \\
\hline S38 & $\checkmark$ & & $\checkmark$ & $\checkmark$ & $\checkmark$ & & & $\checkmark$ & \\
\hline S39 & & & & & & & & & $\checkmark$ \\
\hline
\end{tabular}


(vi) Técnicas

Tabla 6. Aplicación del criterio a.vi

\begin{tabular}{|c|c|c|c|}
\hline ID & Matemática & Informática & $\begin{array}{c}\text { Ciencias de la } \\
\text { Educación }\end{array}$ \\
\hline S01 & & $\checkmark$ & \\
\hline S03 & & $\checkmark$ & \\
\hline S04 & & $\checkmark$ & \\
\hline S05 & & $\checkmark$ & \\
\hline S06 & & $\checkmark$ & \\
\hline S07 & $\checkmark$ & $\checkmark$ & \\
\hline S08 & $\checkmark$ & $\checkmark$ & \\
\hline S13 & $\checkmark$ & $\checkmark$ & \\
\hline S14 & $\checkmark$ & $\checkmark$ & \\
\hline S15 & & $\checkmark$ & \\
\hline S16 & & $\checkmark$ & \\
\hline S17 & & $\checkmark$ & \\
\hline S18 & $\checkmark$ & $\checkmark$ & \\
\hline S20 & $\checkmark$ & $\checkmark$ & $\checkmark$ \\
\hline S21 & & $\checkmark$ & \\
\hline S22 & & $\checkmark$ & \\
\hline S23 & & $\checkmark$ & \\
\hline S24 & & $\checkmark$ & $\checkmark$ \\
\hline S25 & & $\checkmark$ & \\
\hline S27 & $\checkmark$ & $\checkmark$ & \\
\hline S28 & $\checkmark$ & $\checkmark$ & \\
\hline S30 & & $\checkmark$ & $\checkmark$ \\
\hline S31 & $\checkmark$ & $\checkmark$ & $\checkmark$ \\
\hline S32 & $\checkmark$ & $\checkmark$ & $\checkmark$ \\
\hline S35 & & $\checkmark$ & \\
\hline S38 & & $\checkmark$ & \\
\hline S39 & & $\checkmark$ & \\
\hline
\end{tabular}




\section{(b) Contexto}

\section{(i) Destinatarios}

Tabla 7. Aplicación del criterio b.i

\begin{tabular}{|c|c|c|c|c|}
\hline ID & Autores & Docentes & Estudiantes & Ambos \\
\hline S01 & Kellar et al. & & & $\checkmark$ \\
\hline S03 & Pahl et al. & & & $\checkmark$ \\
\hline S04 & Roig Vila & $\checkmark$ & & \\
\hline S05 & Colucci et al. & & $\checkmark$ & \\
\hline S06 & Santacruz Valencia, Delgado Kloos \& Aedo Cuevas & $\checkmark$ & & \\
\hline S07 & Farrell et al. & & $\checkmark$ & \\
\hline S08 & Li \& Huang & & & $\checkmark$ \\
\hline S13 & Lopes Gançarski, et al. & & & $\checkmark$ \\
\hline S14 & Rigaux \& Spyratos & & & $\checkmark$ \\
\hline S15 & Sánchez Alonso, et al. & & $\checkmark$ & \\
\hline S16 & Thyagharajan \& Nayak & & $\checkmark$ & \\
\hline S17 & Karam, Linckels \& Meinel & & $\checkmark$ & \\
\hline S 18 & Anh et al. & & & $\checkmark$ \\
\hline S20 & Shahin et al. & $\checkmark$ & & \\
\hline $\mathrm{S} 21$ & Schreurs, Vanhove \& Al-Zoubi & & & $\checkmark$ \\
\hline $\mathrm{S} 22$ & Sarasa et al. & & & $\checkmark$ \\
\hline $\mathrm{S} 23$ & Wetzlinger, Auinger \& Stary & $\checkmark$ & & \\
\hline $\mathrm{S} 24$ & López, Miguel \& Montaño & & & $\checkmark$ \\
\hline $\mathrm{S} 25$ & Lalithsena, Hewagamage \& Jayaratne & & $\checkmark$ & \\
\hline S27 & Bouzeghoub et al. & & & $\checkmark$ \\
\hline $\mathrm{S} 28$ & Garrido, Onaindia \& Sapena & & & $\checkmark$ \\
\hline $\mathrm{S} 30$ & Jovanovic et al. & & & $\checkmark$ \\
\hline S31 & Huang et al. & & & $\checkmark$ \\
\hline $\mathrm{S} 32$ & Ullrich \& Melis & & & $\checkmark$ \\
\hline $\mathrm{S} 35$ & Menéndez et al. & $\checkmark$ & & \\
\hline $\mathrm{S} 38$ & Stănică \& Crișan & $\checkmark$ & & \\
\hline S39 & Verbert et al. & $\checkmark$ & & \\
\hline
\end{tabular}


(ii) Proactividad del usuario

Tabla 8. Aplicación del criterio b.ii

\begin{tabular}{|c|c|c|c|c|c|}
\hline ID & $\begin{array}{c}\text { Docente } \\
\text { activo }\end{array}$ & $\begin{array}{c}\text { Docente } \\
\text { pasivo }\end{array}$ & $\begin{array}{c}\text { Estudiante } \\
\text { activo }\end{array}$ & $\begin{array}{l}\text { Estudiante } \\
\text { pasivo }\end{array}$ & Criterio b. $i$ \\
\hline S01 & $\checkmark$ & & $\checkmark$ & & $\mathrm{A}$ \\
\hline S03 & & $\checkmark$ & & $\checkmark$ & A \\
\hline S04 & $\checkmark$ & & & & D \\
\hline S05 & & & & $\checkmark$ & E \\
\hline S06 & $\checkmark$ & & & & D \\
\hline S07 & & & $\checkmark$ & & E \\
\hline S08 & $\checkmark$ & & $\checkmark$ & & A \\
\hline S13 & $\checkmark$ & & & $\checkmark$ & A \\
\hline S14 & & $\checkmark$ & & $\checkmark$ & A \\
\hline S15 & & & & $\checkmark$ & E \\
\hline S16 & & & & $\checkmark$ & $\mathrm{E}$ \\
\hline S17 & & & & $\checkmark$ & E \\
\hline $\mathrm{S} 18$ & $\checkmark$ & & & $\checkmark$ & A \\
\hline S20 & $\checkmark$ & & & & D \\
\hline $\mathrm{S} 21$ & $\checkmark$ & & & $\checkmark$ & A \\
\hline $\mathrm{S} 22$ & $\checkmark$ & & $\checkmark$ & & A \\
\hline $\mathrm{S} 23$ & $\checkmark$ & & & & D \\
\hline S24 & $\checkmark$ & & & $\checkmark$ & A \\
\hline S25 & & & & $\checkmark$ & E \\
\hline S27 & $\checkmark$ & & & $\checkmark$ & A \\
\hline S28 & $\checkmark$ & & & $\checkmark$ & A \\
\hline $\mathrm{S} 30$ & & $\checkmark$ & & $\checkmark$ & A \\
\hline S31 & & $\checkmark$ & & $\checkmark$ & A \\
\hline S32 & $\checkmark$ & & & $\checkmark$ & A \\
\hline S35 & $\checkmark$ & & & & D \\
\hline S38 & $\checkmark$ & & & & D \\
\hline S39 & $\checkmark$ & & & & $\mathrm{D}$ \\
\hline
\end{tabular}

A: Ambos, E:Estudiantes, D:Docentes 
(iii) Enfoque pedagógico/didáctico

Tabla 9. Aplicación del criterio b.iii

\begin{tabular}{|c|c|c|c|c|}
\hline ID & Material & Usuario & Ambos & $\mathrm{S} / \mathrm{C}$ \\
\hline S01 & & $\checkmark$ & & \\
\hline $\mathrm{S} 03$ & & $\checkmark$ & & \\
\hline S04 & $\checkmark$ & & & \\
\hline S05 & & $\checkmark$ & & \\
\hline S06 & $\checkmark$ & & & \\
\hline S07 & & & $\checkmark$ & \\
\hline S08 & & $\checkmark$ & & \\
\hline $\mathrm{S} 13$ & & $\checkmark$ & & \\
\hline S14 & & $\checkmark$ & & \\
\hline S15 & & $\checkmark$ & & \\
\hline S16 & & $\checkmark$ & & \\
\hline S17 & & $\checkmark$ & & \\
\hline S18 & & & $\checkmark$ & \\
\hline S20 & & & $\checkmark$ & \\
\hline S21 & & $\checkmark$ & & \\
\hline $\mathrm{S} 22$ & & & & $\checkmark$ \\
\hline $\mathrm{S} 23$ & & & $\checkmark$ & \\
\hline S24 & $\checkmark$ & & & \\
\hline $\mathrm{S} 25$ & & & $\checkmark$ & \\
\hline S27 & & & $\checkmark$ & \\
\hline $\mathrm{S} 28$ & & & $\checkmark$ & \\
\hline $\mathrm{S} 30$ & & & $\checkmark$ & \\
\hline S31 & & $\checkmark$ & & \\
\hline S32 & & & $\checkmark$ & \\
\hline S35 & & & & $\checkmark$ \\
\hline S38 & & $\checkmark$ & & \\
\hline S39 & $\checkmark$ & & & \\
\hline
\end{tabular}




\section{(iv) Evaluación de la metodología}

Tabla 10. Aplicación del criterio b.iv

\begin{tabular}{|c|c|c|c|c|}
\hline ID & $\begin{array}{c}\text { EC } \\
\text { docentes }\end{array}$ & $\begin{array}{c}\text { EC } \\
\text { estudiantes }\end{array}$ & Lab & $\mathbf{S} / \mathbf{C}$ \\
\hline S01 & $\checkmark$ & & & \\
\hline $\mathrm{S} 03$ & & & & $\checkmark$ \\
\hline S04 & & & & $\checkmark$ \\
\hline S05 & & & & $\checkmark$ \\
\hline S06 & & & $\checkmark$ & \\
\hline S07 & & $\checkmark$ & & \\
\hline S08 & & & $\checkmark$ & \\
\hline $\mathrm{S} 13$ & & & & $\checkmark$ \\
\hline $\mathrm{S} 14$ & & & $\checkmark$ & \\
\hline S15 & & & $\checkmark$ & \\
\hline S16 & & & $\checkmark$ & \\
\hline S17 & & & $\checkmark$ & \\
\hline $\mathrm{S} 18$ & & & $\checkmark$ & \\
\hline $\mathrm{S} 20$ & & & $\checkmark$ & \\
\hline $\mathrm{S} 21$ & & & $\checkmark$ & \\
\hline $\mathrm{S} 22$ & & & & $\checkmark$ \\
\hline $\mathrm{S} 23$ & & & $\checkmark$ & \\
\hline S24 & & & $\checkmark$ & \\
\hline $\mathrm{S} 25$ & & $\checkmark$ & & \\
\hline $\mathrm{S} 27$ & & & $\checkmark$ & \\
\hline $\mathrm{S} 28$ & & $\checkmark$ & & \\
\hline $\mathrm{S} 30$ & & $\checkmark$ & & \\
\hline S31 & & $\checkmark$ & & \\
\hline S32 & & & $\checkmark$ & \\
\hline S35 & $\checkmark$ & & & \\
\hline S38 & & & & $\checkmark$ \\
\hline S39 & $\checkmark$ & & & \\
\hline
\end{tabular}

(*) Incluyó en el estudio de caso a docentes. 


\section{(c) Software}

(i) Etapa de desarrollo

Tabla 11. Aplicación del criterio c.i

\begin{tabular}{|c|c|c|c|c|}
\hline ID & Aplicación & $\begin{array}{l}\text { Prototipo } \\
\text { funcional }\end{array}$ & Prototipo & $\mathbf{S} / \mathbf{C}$ \\
\hline S01 & & & $\checkmark$ & \\
\hline $\mathrm{S} 03$ & & & $\checkmark$ & \\
\hline S04 & $\checkmark$ & & & \\
\hline S05 & & & $\checkmark$ & \\
\hline S06 & & $\checkmark$ & & \\
\hline S07 & $\checkmark$ & & & \\
\hline S08 & $\checkmark$ & & & \\
\hline $\mathrm{S} 13$ & & & & $\checkmark$ \\
\hline S14 & & & $\checkmark$ & \\
\hline $\mathrm{S} 15$ & & & $\checkmark$ & \\
\hline S16 & $\checkmark$ & & & \\
\hline S17 & & $\checkmark$ & & \\
\hline $\mathrm{S} 18$ & & & $\checkmark$ & \\
\hline $\mathrm{S} 20$ & & & $\checkmark$ & \\
\hline $\mathrm{S} 21$ & & & & $\checkmark$ \\
\hline $\mathrm{S} 22$ & $\checkmark$ & & & \\
\hline $\mathrm{S} 23$ & & & $\checkmark$ & \\
\hline $\mathrm{S} 24$ & & & $\checkmark$ & \\
\hline $\mathrm{S} 25$ & & & $\checkmark$ & \\
\hline S27 & & & $\checkmark$ & \\
\hline $\mathrm{S} 28$ & $\checkmark$ & & & \\
\hline $\mathrm{S} 30$ & & & $\checkmark$ & \\
\hline $\mathrm{S} 31$ & $\checkmark$ & & & \\
\hline $\mathrm{S} 32$ & & & $\checkmark$ & \\
\hline $\mathrm{S} 35$ & $\checkmark$ & & & \\
\hline $\mathrm{S} 38$ & $\checkmark$ & & & \\
\hline S39 & & & $\checkmark$ & \\
\hline
\end{tabular}




\section{(ii) Web/Escritorio}

Tabla 12. Aplicación del criterio c.ii

\begin{tabular}{|c|c|c|c|}
\hline ID & $\begin{array}{c}\text { Aplicación } \\
\text { web }\end{array}$ & $\begin{array}{l}\text { Aplicación de } \\
\text { escritorio }\end{array}$ & $\mathbf{S} / \mathbf{C}$ \\
\hline S01 & $\checkmark$ & & \\
\hline S03 & & & $\checkmark$ \\
\hline S04 & $\checkmark$ & & \\
\hline S05 & $\checkmark$ & & \\
\hline S06 & $\checkmark$ & & \\
\hline S07 & $\checkmark$ & & \\
\hline S08 & $\checkmark$ & $\checkmark$ & \\
\hline $\mathrm{S} 13(*)$ & & & $\checkmark$ \\
\hline S14 & & & $\checkmark$ \\
\hline S15 & $\checkmark$ & & \\
\hline S16 & $\checkmark$ & & \\
\hline S17 & $\checkmark$ & & \\
\hline S18 & $\checkmark$ & & \\
\hline S20 & & & $\checkmark$ \\
\hline $\mathrm{S} 21\left(^{*}\right)$ & & & $\checkmark$ \\
\hline S22 & & $\checkmark$ & \\
\hline S23 & $\checkmark$ & & \\
\hline S24 & $\checkmark$ & & \\
\hline S25 & $\checkmark$ & & \\
\hline S27 & & $\checkmark$ & \\
\hline $\mathrm{S} 28$ & & $\checkmark$ & \\
\hline S30 & $\checkmark$ & & \\
\hline S31 & $\checkmark$ & & \\
\hline S32 & $\checkmark$ & & \\
\hline S35 & $\checkmark$ & & \\
\hline S38 & & $\checkmark$ & \\
\hline S39 & $\checkmark$ & & \\
\hline
\end{tabular}




\section{(iii) Accesibilidad}

Tabla 13. Aplicación del criterio c.iii

\begin{tabular}{|c|c|c|c|}
\hline ID & Autor & Sí & No \\
\hline S01 & Kellar et al. & & $\checkmark$ \\
\hline S03 & Pahl et al. & & $\checkmark$ \\
\hline S04 & Roig Vila & $\checkmark$ & \\
\hline S05 & Colucci et al. & & $\checkmark$ \\
\hline S06 & Santacruz Valencia, Delgado Kloos \& Aedo Cuevas & $\checkmark$ & \\
\hline S07 & Farrell et al. & & $\checkmark$ \\
\hline S08 & Li \& Huang & & $\checkmark$ \\
\hline S13 & Lopes Gançarski, et al. $(*)$ & & $\checkmark$ \\
\hline S14 & Rigaux \& Spyratos & & $\checkmark$ \\
\hline S15 & Sánchez Alonso, et al. & & $\checkmark$ \\
\hline S16 & Thyagharajan \& Nayak & & $\checkmark$ \\
\hline S17 & Karam, Linckels \& Meinel & $\checkmark$ & \\
\hline S18 & Anh et al. & & $\checkmark$ \\
\hline S20 & Shahin et al. & & $\checkmark$ \\
\hline S21 & Schreurs, Vanhove \& Al-Zoubi (*) & & $\checkmark$ \\
\hline S22 & Sarasa et al. & & $\checkmark$ \\
\hline S23 & Wetzlinger, Auinger \& Stary & & $\checkmark$ \\
\hline S24 & López, Miguel \& Montaño & & $\checkmark$ \\
\hline S25 & Lalithsena, Hewagamage \& Jayaratne & & $\checkmark$ \\
\hline S27 & Bouzeghoub et al. & & $\checkmark$ \\
\hline S28 & Garrido, Onaindia \& Sapena & & $\checkmark$ \\
\hline S30 & Jovanovic et al. & & $\checkmark$ \\
\hline S31 & Huang et al. & & $\checkmark$ \\
\hline S32 & Ullrich \& Melis & $\checkmark$ & \\
\hline S35 & Menéndez et al. & $\checkmark$ & \\
\hline S38 & Stănică \& Crișan & & $\checkmark$ \\
\hline S39 & Verbert et al. & & $\checkmark$ \\
\hline
\end{tabular}

(*) No desarrollaron software. 


\section{(iv) Credenciales}

Tabla 14. Aplicación del criterio c.iii

\begin{tabular}{|c|c|c|c|}
\hline ID & Autores & Sí & No \\
\hline S01 & Kellar et al. & $\checkmark$ & \\
\hline S03 & Pahl et al. & $\checkmark$ & \\
\hline S04 & Roig Vila & & $\checkmark$ \\
\hline S05 & Colucci et al. & & $\checkmark$ \\
\hline S06 & Santacruz Valencia, Delgado Kloos \& Aedo Cuevas & & $\checkmark$ \\
\hline S07 & Farrell et al. & $\checkmark$ & \\
\hline S08 & Li \& Huang & $\checkmark$ & \\
\hline S13 & Lopes Gançarski, et al. (*) & & $\checkmark$ \\
\hline S14 & Rigaux \& Spyratos & & $\checkmark$ \\
\hline $\mathrm{S} 15$ & Sánchez Alonso, et al. & & $\checkmark$ \\
\hline S16 & Thyagharajan \& Nayak & $\checkmark$ & \\
\hline S17 & Karam, Linckels \& Meinel & $\checkmark$ & \\
\hline S18 & Anh et al. & $\checkmark$ & \\
\hline S20 & Shahin et al. & & $\checkmark$ \\
\hline S21 & Schreurs, Vanhove \& Al-Zoubi $(*)$ & & $\checkmark$ \\
\hline S22 & Sarasa et al. & & $\checkmark$ \\
\hline $\mathrm{S} 23$ & Wetzlinger, Auinger \& Stary & & $\checkmark$ \\
\hline $\mathrm{S} 24$ & López, Miguel \& Montaño & & $\checkmark$ \\
\hline $\mathrm{S} 25$ & Lalithsena, Hewagamage \& Jayaratne & $\checkmark$ & \\
\hline S27 & Bouzeghoub et al. & & $\checkmark$ \\
\hline S28 & Garrido, Onaindia \& Sapena & & $\checkmark$ \\
\hline $\mathrm{S} 30$ & Jovanovic et al. & $\checkmark$ & \\
\hline $\mathrm{S} 31$ & Huang et al. & $\checkmark$ & \\
\hline S32 & Ullrich \& Melis & $\checkmark$ & \\
\hline S35 & Menéndez et al. & $\checkmark$ & \\
\hline S38 & Stănică \& Crișan & & $\checkmark$ \\
\hline S39 & Verbert et al. & & $\checkmark$ \\
\hline
\end{tabular}

(*) No desarrollaron software. 


\section{(v) Fuente de los materiales}

Tabla 15. Aplicación del criterio c.v

\begin{tabular}{llcc}
\hline \multicolumn{1}{c}{ ID } & \multicolumn{1}{c}{ Autor } & Local & Externo \\
\hline S01 & Kellar et al. & $\checkmark$ & \\
S03 & Pahl et al. & & $\checkmark$ \\
S04 & Roig Vila & & $\checkmark$ \\
S05 & Colucci et al. & & $\checkmark$ \\
S06 & Santacruz Valencia, Delgado Kloos \& Aedo Cuevas & & $\checkmark$ \\
S07 & Farrell et al. & & $\checkmark$ \\
S08 & Li \& Huang & & $\checkmark$ \\
S13 & Lopes Gançarski, et al. & & $\checkmark$ \\
S14 & Rigaux \& Spyratos & & $\checkmark$ \\
S15 & Sánchez Alonso, et al. & & \\
S16 & Thyagharajan \& Nayak & $\checkmark$ & \\
S17 & Karam, Linckels \& Meinel & $\checkmark$ & \\
S18 & Anh et al. & $\checkmark$ & \\
S20 & Shahin et al. & $\checkmark$ & \\
S21 & Schreurs, Vanhove \& Al-Zoubi & $\checkmark$ & \\
S22 & Sarasa et al. & $\checkmark$ & \\
S23 & Wetzlinger, Auinger \& Stary & & \\
S24 & López, Miguel \& Montaño & $\checkmark$ & \\
S25 & Lalithsena, Hewagamage \& Jayaratne & $\checkmark$ & \\
S27 & Bouzeghoub et al. & $\checkmark$ & \\
S28 & Garrido, Onaindia \& Sapena & & \\
S30 & Jovanovic et al. & $\checkmark$ & $\checkmark$ \\
S31 & Huang et al. & $\checkmark$ & \\
S32 & Ullrich \& Melis & & \\
S35 & Menéndez et al. & & \\
S38 & Stănică \& Crișan & & \\
S39 & Verbert et al. & & \\
\hline
\end{tabular}

(*) No desarrollan aron software. 
(vi) Metadatos

Tabla 16. Aplicación del criterio c.vi

\begin{tabular}{|c|c|c|c|c|}
\hline ID & Autor & LOM & DCMI & $\mathbf{S} / \mathbf{C}$ \\
\hline S01 & Kellar et al. & $\bar{\checkmark}$ & & \\
\hline S03 & Pahl et al. & $\checkmark$ & & \\
\hline S04 & Roig Vila & & & $\checkmark$ \\
\hline S05 & Colucci et al. & $\checkmark$ & & \\
\hline S06 & Santacruz Valencia, Delgado Kloos \& Aedo Cuevas & $\checkmark$ & & \\
\hline S07 & Farrell et al. & $\checkmark$ & & \\
\hline S08 & Li \& Huang & & & $\checkmark$ \\
\hline $\mathrm{S} 13$ & Lopes Gançarski, et al. & $\checkmark$ & & \\
\hline $\mathrm{S} 14$ & Rigaux \& Spyratos & & & $\checkmark$ \\
\hline $\mathrm{S} 15$ & Sánchez Alonso, et al. & $\checkmark$ & & \\
\hline S16 & Thyagharajan \& Nayak & & & $\checkmark$ \\
\hline S17 & Karam, Linckels \& Meinel & & & $\checkmark$ \\
\hline $\mathrm{S} 18$ & Anh et al. & & & $\checkmark$ \\
\hline $\mathrm{S} 20$ & Shahin et al. & $\checkmark$ & & \\
\hline $\mathrm{S} 21$ & Schreurs, Vanhove \& Al-Zoubi & $\checkmark$ & & \\
\hline $\mathrm{S} 22$ & Sarasa et al. & $\checkmark$ & & \\
\hline $\mathrm{S} 23$ & Wetzlinger, Auinger \& Stary & $\checkmark$ & & \\
\hline $\mathrm{S} 24$ & López, Miguel \& Montaño & $\checkmark$ & & \\
\hline $\mathrm{S} 25$ & Lalithsena, Hewagamage \& Jayaratne & & & $\checkmark$ \\
\hline S27 & Bouzeghoub et al. & $\checkmark$ & & \\
\hline $\mathrm{S} 28$ & Garrido, Onaindia \& Sapena & $\checkmark$ & & \\
\hline $\mathrm{S} 30$ & Jovanovic et al. & & $\checkmark$ & \\
\hline S31 & Huang et al. & $\checkmark$ & & \\
\hline $\mathrm{S} 32$ & Ullrich \& Melis & & & $\checkmark$ \\
\hline $\mathrm{S} 35$ & Menéndez et al. & $\checkmark$ & & \\
\hline $\mathrm{S} 38$ & Stănică \& Crișan & & & $\checkmark$ \\
\hline S39 & Verbert et al. & & & $\checkmark$ \\
\hline
\end{tabular}




\section{(vii) Empaquetamiento}

Tabla 17. Aplicación de criterio c.vii

\begin{tabular}{|c|c|c|c|c|c|}
\hline ID & Autores & SCORM & IMSCP & OTRO & $\mathbf{S} / \mathbf{C}$ \\
\hline S01 & Kellar et al. & & $\checkmark$ & & \\
\hline S03 & Pahl et al. & & & & $\checkmark$ \\
\hline S04 & Roig Vila & & & $\checkmark$ & \\
\hline S05 & Colucci et al. & $\checkmark$ & & & \\
\hline S06 & Santacruz Valencia, Delgado Kloos \& Aedo Cuevas & & & $\checkmark$ & \\
\hline S07 & Farrell et al. & & $\checkmark$ & & \\
\hline S08 & Li \& Huang & & & $\checkmark$ & \\
\hline S13 & Lopes Gançarski, et al. & & & & $\checkmark$ \\
\hline S14 & Rigaux \& Spyratos & & & $\checkmark$ & \\
\hline $\mathrm{S} 15$ & Sánchez Alonso, et al. & & & & $\checkmark$ \\
\hline S16 & Thyagharajan \& Nayak & & & $\checkmark$ & \\
\hline S17 & Karam, Linckels \& Meinel & & & $\checkmark$ & \\
\hline S18 & Anh et al. & $\checkmark$ & & & \\
\hline S20 & Shahin et al. & $\checkmark$ & & & \\
\hline S21 & Schreurs, Vanhove \& Al-Zoubi & & & $\checkmark$ & \\
\hline S22 & Sarasa et al. & $\checkmark$ & & & \\
\hline S23 & Wetzlinger, Auinger \& Stary & $\checkmark$ & & & \\
\hline S24 & López, Miguel \& Montaño & $\checkmark$ & & & \\
\hline $\mathrm{S} 25$ & Lalithsena, Hewagamage \& Jayaratne & & & & $\checkmark$ \\
\hline S27 & Bouzeghoub et al. & & & $\checkmark$ & \\
\hline S28 & Garrido, Onaindia \& Sapena & & $\checkmark$ & & \\
\hline S30 & Jovanovic et al. & & & $\checkmark$ & \\
\hline S31 & Huang et al. & $\checkmark$ & & & \\
\hline S32 & Ullrich \& Melis & & & $\checkmark$ & \\
\hline S35 & Menéndez et al. & $\checkmark$ & & & \\
\hline S38 & Stănică \& Crișan & & & & $\checkmark$ \\
\hline S39 & Verbert et al. & & & $\checkmark$ & \\
\hline
\end{tabular}




\section{Anexo}

Se presentan en este Anexo las cuatro encuestas administradas a los informantes del taller de ensamblaje de OA, asi como las preguntas que se le realizaron en la entrevista a la cada participante. Las preguntas para la entrevista se diseñaron en base a los videos, la planilla de observación, las encuestas y los itinerarios de aprendizaje generados. Las encuestas fueron diseñadas, publicadas y administradas a través de formularios de Google Drive.

\section{Encuestas}

\section{Encuesta 1: "Metodología de ensamblaje"}

Esta encuesta forma parte de los instrumentos de recolección de datos para la Tesis "Estrategias de diseño y ensamblaje de Objetos de Aprendizaje"

Referencias:

OA: Objetos de Aprendizaje

CA: Conocimiento Asociado

*Obligatorio

- Según lo expuesto hasta aquí en el taller, considera Ud. que el ensamblaje de OA es: *

(elija tantas opciones como desee)

- Crear un OA

- Agrupar los OA de acuerdo con el CA

- Agrupar recursos educativos en una secuencia de aprendizaje

- Crear un itinerario de aprendizaje

- En la metodología de ensamblaje, el CA es: *

(elija tantas opciones como desee)

- Metadatos que describen al OA

- Las competencias necesarias para abordar el OA

- Los requisitos necesarios para abordar el OA

- Las competencias alcanzadas tras abordar el OA 
- Según la metodología de ensamblaje, qué se obtiene al ensamblar: (Marque todas las que desee)
- $\mathrm{UI}+\mathrm{UI}=\mathrm{UC}$
○ $\quad \mathrm{UI}+\mathrm{UC}=\mathrm{UC}$
- $U I+U D=U D$
- UC + UC = UD
- UC + UD = UD
- $\quad U C+U D=I A$
- UD + UD = UD
- $\quad U D+U D=I A$
○ $\quad U I+U D=U D$

- En la metodología de ensamblaje, las ontologías: * (indique si acuerda o no con las siguientes afirmaciones)

$\begin{array}{lcc} & \text { Acuerdo } & \text { No acuerdo } \\ \text { permiten describir el dominio } & \text { [ ] } & \text { [ ] } \\ \text { permiten describir los OA } & \text { [ ] } & \text { [ ] } \\ \text { permiten el ensamblaje de OA heterogéneos } & \text { [ ] } & \text { [ ] } \\ \text { permiten la comparación del CA de cada OA } & \text { [ ] } & \text { [ ] }\end{array}$

- Según la ADAPTACIÓN propuesta, qué tipo de ELO son considerados recursos educativos (Puede elegir varias opciones)

- Una Unidad de Información (UI)

- Una Unidad de Contenido (UC)

- Una Unidad de Didáctica (UD)

- Según la ADAPTACIÓN propuesta, un Objeto de Aprendizaje equivale a:

- Una Unidad de Didáctica (UD)

- Un Itinerario de Aprendizaje (IA)

- Una Unidad de Contenido (UC)

- Una Unidad de Información (UI)

- Según la ADAPTACIÓN propuesta, un OA debe contener: (Marque todas las opciones que desee)

- Actividades

- Tantos objetivos como UC contenga

- Contenidos

- Ontologías

- Metadatos estandarizados

- Evaluación

- Un objetivo simple

- Actividades 
- Según la ADAPTACIÓN propuesta, un Itinerario de Aprendizaje (IA): (Indique si acuerda o no con las siguientes afirmaciones)

$\begin{array}{lcc} & \text { Acuerdo } & \text { No acuerdo } \\ \text { no puede crearse a partir de Uls } & {[} & \text { [ ] } \\ \text { es un conjunto de dos o más OAs } & {[} & \text { [ ] } \\ \text { esta conformado por una secuencia de dos UDs } & {[\text { ] }} & \text { [ ] } \\ \text { las competencias los obtiene del último OA de las secuencia } & {[\text { ] }} & \text { [ ] } \\ \text { los requisitos los obtiene de la primer OA de las secuencia } & \text { [ ] } & \text { [ ] }\end{array}$

- Alguna observación que desee realizar sobre lo expuesto en el taller:

\section{Encuesta 2: "Ensamblaje"}

Esta encuesta forma pare de los instrumentos de recolección de datos para la Tesis "Estrategias de diseño y ensamblaje de Objetos de Aprendizaje"

Referencias:

OA: Objetos de Aprendizaje

- ¿Pudo completar la actividad?

$\begin{array}{lllllll}\text { No } & 1 & 2 & 3 & 4 & 5 & \text { Sí }\end{array}$

- ¿Pudo crear el Itinerario de Aprendizaje?

Sí/No

- Por favor, indique el nombre del Itinerario de Aprendizaje generado en ELO-Tool:

- Por favor, indique el nombre de los OA que componen el Itinerario de Aprendizaje: (Los nombres deben estar separado por coma)

- ¿Qué tan complejo le resultó el proceso de ensamblaje?
fácil 1
2
34
5 muy complejo

- El nivel de automatización del proceso de ensamblaje que le propone la metodología le pareció:

$\begin{array}{lllllll}\text { insuficiente } & 1 & 2 & 3 & 4 & 5 & \text { suficiente }\end{array}$

- La cantidad de metadatos que tuvo que cargar para concretar el ensamblaje, le pareció:

$\begin{array}{lllllll}\text { apropiada } & 1 & 2 & 3 & 4 & 5 & \text { excesiva }\end{array}$

- La cantidad de información que aporta el Conocimiento Asociado (CA) para el ensamblaje, en todos los casos, le parece:

$\begin{array}{lllllll}\text { insuficiente } & 1 & 2 & 3 & 4 & 5 & \text { suficiente }\end{array}$

- Cuando tuvo que utilizar las ontologías para completar los requerimientos/competencias, la elección del Conocimiento Asociado (CA) más apropiado le resultó:
sencillo
$\begin{array}{lll}1 & 2 & 3\end{array}$
4
5 muy complejo

- La metodología depende de las ontologías para el ensamblaje, esto la vuelve una metodología:(flexible: que puede ser adaptada a varios contextos y evolucionar)
flexible 1
2
3
4
5 rígida 
- Utilizaría, en el futuro, el ensamblaje (con esta herramienta u otra) como una técnica para la creación de itinerarios de apren

$\begin{array}{lllllll}\text { nunca } & 1 & 2 & 3 & 4 & 5 & \text { seguramente }\end{array}$

- ¿Cree Ud. que el ensamblaje de OA le facilitaría su tarea como docente?

Sí/No

- ¿Por qué? [

- ¿Cree Ud. que el ensamblaje propicia la reutilización del material educativo? Sí/No

- ¿Por qué? [

- ¿Podría indicar si los problemas que tuvo fueron por alguno/s de los siguientes motivos?(puede elegir una 0 varias opciones)

- No supe cómo llevar adelante el ensamblaje

- Los OA que necesitaba no estaban disponibles

- Tuve problemas al cargar los metadatos

- Tuve problemas al elegir requerimientos/competencias

- Tuve un error porque ingresé mal el nombre de los $0 \mathrm{~A}$

- Cuando solicité que se ensamblaran los OA, ELO-Tool mostró un error

- Otro/s problema/s

- ¿Podría describir cuál o cuáles otro/s?

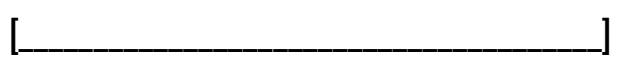

- ¿Podría describir con algo de detalle el/los problema/s que seleccionó arriba?

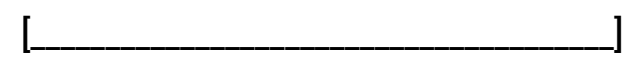

- En caso de tener alguna sugerencia u observación adicional, por favor, refiérala a continuación:

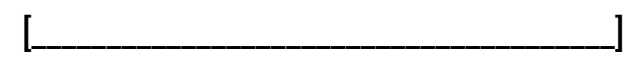

\section{Encuesta de satisfacción (usabilidad) para ELO-Tool}

Esta encuesta forma pare de los instrumentos de recolección de datos para la Tesis "Estrategias de diseño y ensamblaje de Objetos de Aprendizaje"

Referencias:

OA: Objetos de Aprendizaje

CA: Conocimiento Asociado

- Su percepción en relación a la claridad de cómo el sistema le permite realizar las tareas solicitadas es: $\begin{array}{lllllll}\text { confusa } & 1 & 2 & 3 & 4 & 5 & \text { clara }\end{array}$

- La navegación a través del sistema le resulta:

$\begin{array}{lllllll}\text { confusa } & 1 & 2 & 3 & 4 & 5 & \text { clara }\end{array}$

- La apariencia general del sistema le resulta: $\begin{array}{lllllll}\text { desagradable } & 1 & 2 & 3 & 4 & 5 & \text { agradable }\end{array}$

- La representatividad del texto de las opciones respecto a la función de las mismas le resultaron: $\begin{array}{lllllll}\text { confusa } & 1 & 2 & 3 & 4 & 5 & \text { clara }\end{array}$ 
- La estructura y organización del sistema le resultó:

$\begin{array}{lllllll}\text { confusa } & 1 & 2 & 3 & 4 & 5 & \text { clara }\end{array}$

- El ensamblaje de los OA le resultó:

$\begin{array}{lllllll}\text { difícil } & 1 & 2 & 3 & 4 & 5 & \text { muy fácil }\end{array}$

- La carga de metadatos le resultó:

$\begin{array}{lllllll}\text { difícil } & 1 & 2 & 3 & 4 & 5 & \text { muy fácil }\end{array}$

- La carga de CA de los OA le resultó:

$\begin{array}{lllllll}\text { difícil } & 1 & 2 & 3 & 4 & 5 & \text { muy fácil }\end{array}$

- El sistema para los distintos tipos de usuario que hay en la Facultad resulta: (Considere que ELO Tools debería ser utilizado por docentes).
difícil
23
45 muy fácil

\section{Encuesta de satisfacción (usabilidad) para ELOs-Viewer}

Esta encuesta forma pare de los instrumentos de recolección de datos para la Tesis "Estrategias de diseño y ensamblaje de Objetos de Aprendizaje"

Referencias:

OA: Objetos de Aprendizaje

CA: Conocimiento Asociado

- Su percepción en relación a la claridad de cómo el sistema le permite realizar las tareas solicitadas es:

$\begin{array}{lllllll}\text { confusa } & 1 & 2 & 3 & 4 & 5 & \text { clara }\end{array}$

- La navegación a través del sistema le resulta:

$\begin{array}{lllllll}\text { confusa } & 1 & 2 & 3 & 4 & 5 & \text { clara }\end{array}$

- La apariencia general del sistema le resulta:

$\begin{array}{lllllll}\text { desagradable } & 1 & 2 & 3 & 4 & 5 & \text { agradable }\end{array}$

- La representatividad del texto de las opciones respecto a la función de las mismas le resultaron: $\begin{array}{lllllll}\text { confusa } & 1 & 2 & 3 & 4 & 5 & \text { clara }\end{array}$

- La estructura y organización del sistema le resultó:

$\begin{array}{lllllll}\text { confusa } & 1 & 2 & 3 & 4 & 5 & \text { clara }\end{array}$

- La forma en la que se presentan los ELOs le resultó:

$\begin{array}{llllllll}\text { clara } & 1 & 2 & 3 & 4 & 5 & \text { confusa }\end{array}$

- La forma en la que se presentan los metadatos le resultó:

$\begin{array}{llllllll}\text { clara } & 1 & 2 & 3 & 4 & 5 & \text { confusa }\end{array}$

- La forma en la que se presenta el CA de los OA le resultó:

$\begin{array}{lllllll}\text { clara } & 1 & 2 & 3 & 4 & 5 & \text { confusa }\end{array}$

- La información que le muestra la herramienta le fue de utilidad en el proceso de ensamblaje
No, no me fue de utilidad
1
2
34
5 Sí, muy útil

- El sistema para los distintos tipos de usuario que hay en la Facultad resulta:

(Considere que ELO Tools debería ser utilizado por docentes).

$\begin{array}{lllllll}\text { difícil } & 1 & 2 & 3 & 4 & 5 & \text { muy fácil }\end{array}$ 


\section{Entrevistas}

\section{Preguntas para P1}

- ¿Cómo fue la experiencia de trabajar sola para llevar adelante el proceso de ensamblaje?

- ¿Tenés una idea aproximada de cuántos te llevó crear el itinerario?

\section{Metadatos}

- ¿Cuál fue tu experiencia con la carga de metadatos? ¿Te resultó un trabajo "pesado"?

- Los que tuviste que definir vos ¿te resultó complejo decidir qué escribir en cada caso? ¿Cómo decidiste el nombre de la IA?

- ¿Tuviste que usar la ayuda que les dejé sobre metadatos en el ensamblaje? ¿Crees que sin la ayuda no lo hubieses podido lograr?

- Tuviste dudas con algunos metadatos (como la evaluación y con la densidad semántica) ¿te quedaron más claros luego del taller?

\section{Ensamblaje}

- ¿Te resultó complicado encontrar los OA que tenías que usar para generar el itinerario de aprendizaje que te tocó?

- Asociaste los requerimientos con los conocimientos previos y las competencias con logras después de pasar por el material. Después de haber pasado por la experiencia del taller ¿seguís de acuerdo con esa afirmación?

- Se entendió cómo es que la metodología utiliza las ontologías para ensamblar...

\section{Adaptación}

- ¿Te quedó claro porqué se agregaron "objetivo" y "ejercicio" como parte de los requerimientos?

- ¿Con qué tipo de material se deben crear los itinerarios de aprendizaje según mi propuesta? (x encuesta)

\section{Herramientas}

- Crees que ELO-Tool ¿Te facilitó la carga de los metadatos?

- ¿Usaste ELOs-viewer en el proceso o te alcanzó con la ayuda en PDF para ensamblar en ELO-Tool?

- ¿Te acordás para qué usaste ELOs-Viewer?

\section{Preguntas para P2}

- ¿Cómo fue la experiencia de trabajar sola para llevar adelante el proceso de ensamblaje?

- ¿Crees que el hecho de no haber accedido a la imagen que mostraba el itinerario te complicó en el proceso de ensamblaje? ¿0 sólo con la indicación sobré qué debías ensamblar te alcanzó?

- ¿Tenés una idea aproximada de cuántos te llevó crear el itinerario?

\section{Metadatos}

- ¿Cuál fue tu experiencia con la carga de metadatos? ¿Te resultó un trabajo "pesado"?

- Los que tuviste que definir vos ¿te resultó complejo decidir qué escribir en cada caso? ¿Cómo decidiste el nombre de la IA?

- ¿Tuviste que usar la ayuda que les dejé sobre metadatos en el ensamblaje? ¿Crees que sin la ayuda no lo hubieses podido lograr?

\section{Ensamblaje}


- ¿Te resultó complicado encontrar los OA que tenías que usar para generar el itinerario de aprendizaje que te tocó?

- Se entendió cómo es que la metodología utiliza las ontologías para ensamblar...

\section{Adaptación}

- ¿Te quedó claro porqué se agregaron "objetivo" y "ejercicio" como parte de los requerimientos?

- ¿Con qué tipo de material se deben crear los itinerarios de aprendizaje según mi propuesta? ( $\mathrm{x}$ encuesta)

\section{Herramientas}

- Crees que ELO-Tool ¿Te facilitó la carga de los metadatos?

- ¿Usaste ELOs-viewer en el proceso o te alcanzó con la ayuda en PDF para ensamblar en ELO-Tool?

- ¿Te acordás para qué usaste ELOs-Viewer?

\section{Preguntas para P3}

- ¿Cómo fue la experiencia de trabajar sólo (fuera de la clase)?

- ¿Tenés una idea aproximada de cuántos te llevó crear el itinerario?

- ¿Crees que el haber trabajado en clase hubieses demorado lo mismo?

\section{Metadatos}

- ¿Cuál fue tu experiencia con la carga de metadatos? ¿Te resultó un trabajo "pesado"?

- Los que tuviste que definir vos ¿te resultó complejo decidir qué escribir en cada caso? ¿Cómo decidiste el nombre de la IA?

- ¿Tuviste que usar la ayuda que te pasé sobre la carga de metadatos en el ensamblaje? ¿Crees que sin la ayuda no lo hubieses podido lograr?

\section{Ensamblaje}

- ¿Te resultó complicado encontrar los OA que tenías que usar para generar el itinerario de aprendizaje que te tocó?

- Se entendió cómo es que la metodología utiliza las ontologías para ensamblar...

\section{Adaptación}

- ¿Te quedó claro porqué se agregaron "objetivo" y "ejercicio" como parte de los requerimientos?

- ¿Con qué tipo de material se deben crear los itinerarios de aprendizaje según mi propuesta? (x encuesta)

\section{Herramientas}

- Crees que ELO-Tool ¿Te facilitó la carga de los metadatos?

- ¿Usaste ELOs-viewer en el proceso o te alcanzó con la ayuda en PDF para ensamblar en ELO-Tool?

- ¿Te acordás para qué usaste ELOs-Viewer?

\section{Preguntas para P4}

- Vos trabajaste en grupo con Claudio... ¿No?

- ¿Tenés una idea aproximada de cuántos les llevó crear cada itinerario?

- Crees que el haber trabajado en grupo con Claudio te facilitó el trabajo o si hubieses trabajado sola hubieses demorado lo mismo (para hacer un itinerario). 


\section{Metadatos}

- ¿Cuál fue tu experiencia con la carga de metadatos? ¿Te resultó un trabajo "pesado"?

- Los que tenían que definir uds, con Claudio, ¿les resultó complejo definir qué escribir en cada caso? ¿Cómo decidieron qué nombre ponerle al IA?

- ¿Tuvieron que usar la ayuda que les dejé sobre metadatos en los tres ensamblaje o la fueron dejando de lado en el 2do y el 3ro?

\section{Ensamblaje}

- ¿Te resultó complicado encontrar los $0 \mathrm{~A}$ que tenías que usar para generar el itinerario de aprendizaje que te tocó?

- Con Claudio discutieron sobre si las competencias cubren los requerimientos o viceversa ¿te quedó claro cómo funciona el CA al ensamblar?

- ¿Qué opinás de que la cobertura para ensamblar sea del $100 \%$ ?

- Se entendió cómo es que la metodología utiliza las ontologías para ensamblar...

\section{Adaptación}

- ¿Te quedó claro porqué se agregaron "objetivo" y "ejercicio" como parte de los requerimientos?

- ¿Te parece que al tener que combinar UCs con objetivos + contendos con otras que tengan ejemplos + ejercicios se reflejan bien todos los componentes de un material educativo?

- ¿Con qué tipo de material se deben crear los itinerarios de aprendizaje según mi propuesta? (x encuesta)

\section{Herramientas}

- Crees que ELO-Tool ¿Te facilitó la carga de los metadatos?

- ¿Usaste ELOs-viewer en el proceso o te alcanzó con la ayuda en PDF para ensamblar en ELO-Tool?

- ¿Te acordás para qué usaron ELOs-Viewer?

- ¿Les resultó fácil borrar un ELO? Te preocupó el hecho de que borraras directamente del repositorio ¿Por qué?

\section{Preguntas para P5}

- Vos trabajaste en grupo con Lorena... ¿No?

- ¿Tenés una idea aproximada de cuántos les llevó crear cada itinerario?

- Crees que el haber trabajado en grupo con Lorena te facilitó el trabajo o si hubieses trabajado solo hubieses demorado lo mismo (para hacer un itinerario).

\section{Metadatos}

- ¿Cuál fue tu experiencia con la carga de metadatos? ¿Te resultó un trabajo "pesado"?

- Los que tenían que definir uds, con Lorena, ¿les resultó complejo definir qué escribir en cada caso? ¿Cómo decidieron qué nombre ponerle al IA?

- ¿Tuvieron que usar la ayuda que les dejé sobre metadatos en los tres ensamblaje o la fueron dejando de lado en el 2 do y el 3 ro?

\section{Ensamblaje}

- ¿Les resultó complicado encontrar los OA que tenían que usar para generar los itinerarios de aprendizaje que les tocaron?

- ¿Te quedó claro cómo es que se realiza en ensamblaje? Discutieron un poco con Lorena sobre requerimientos y competencias... 
- Cuando unís dos $\mathrm{OA}$ ¿Te acordás de dónde se obtienen los requerimientos y las competencias? (x encuesta)

- Se entendió cómo es que la metodología utiliza las ontologías para ensamblar...

\section{Adaptación}

- ¿Te quedó claro porqué se agregaron "objetivo" y "ejercicio" como parte de los requerimientos?

- ¿Con qué tipo de material se deben crear los itinerarios de aprendizaje según mi propuesta? ( $\mathrm{x}$ encuesta)

\section{Herramientas}

- Crees que ELO-Tool ¿Te facilitó la carga de los metadatos?

- Te noté preocupado porque no podías grabar mientras cargaban los metadatos en el ensamblaje...

- ¿Les resultó fácil borrar un ELO?

- Usaron ELOs-viewer en el proceso o les alcanzó con la ayuda en PDF para ensamblar en ELO-Tool

- ¿Te acordás para qué usaron ELOs-Viewer? 


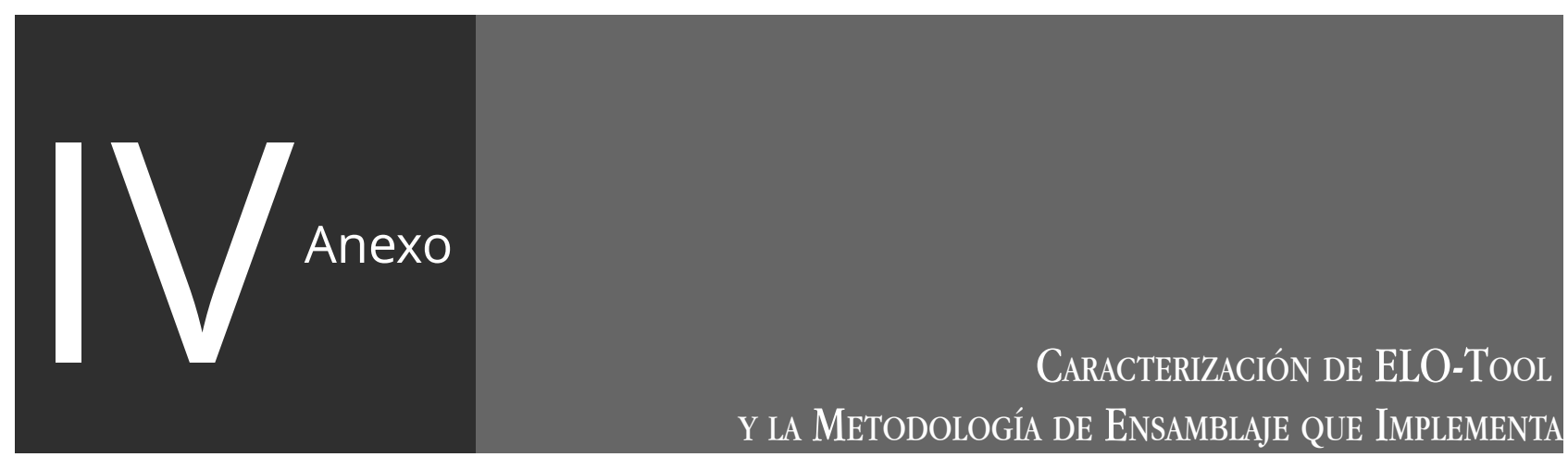

En este anexo se describe la aplicación web ELO-Tool y la metodología de ensamblaje que implementa, propuestos por la Dra. Santacruz-Valencia.

ELO-Tool es una aplicación web que permite la búsqueda, generación, ensamblaje y reutilización de ELOs. La herramienta pone a disposición de los usuarios cinco módulos totalmente funcionales disponibles a través de opciones (Figura 1):

- Gestión de ontologías: través de esta opción se cargan las ontologías de dominio para su uso en los procesos de ensamblaje.

- Gestión y Búsqueda de ELOs: la opción permite la creación y visualización de los ELOs. Así como también, se encarga de la localización, en el repositorio de la herramienta, de los ELOs a través de la búsqueda sobre los metadatos de los mismos.

- Traductor: tiene como función describir aquellos ELOs generados con herramientas externas a ELO-Tool con los metadatos apropiados para ser procesados por la esta aplicación.

- Ensamblaje de ELOs: permite ensamblar dos ELOs teniendo en cuenta sus requisitos y competencias.

ELO-Tool interactúa con dos repositorios locales: $(i)$ repositorio de ELOs (generados por la herramienta o traducidos) y (ii) repositorio de ontologías. El primero permite almacenar los metadatos de los ELOs. Esto puede hacerse vía la opción de Gestión de ELOs, en la cual es posible crear o dar de alta los mismos. El segundo repositorio, aloja las ontologías de dominio que definen el vocabulario y hacen posible la comparación de los requerimientos y competencias entre ELOs con vistas al ensamblaje.

Para crear ELOs la herramienta ofrece formularios que adaptan sus campos a los distintos niveles de granularidad. Permitiendo cargar los metadatos, asociados de los recursos u OA, con base en un perfil de metadatos basado en el estándar LOM $^{1}$. Para dar de alta tanto recursos, como OA es necesario completar los metadatos en un archivo en formato XML cuya estructura interna se corresponde con el estándar de metadatos LOM.

La opción de Gestión de ELOs también cuenta con las opciones Mostrar ELOs y Búsqueda de ELOs. La primera ofrece un listado de los ELOs alojados en el repositorio de la herramienta. Dicha nómina muestra los nombres de los ELOs, los cuales actúan como un enlace a sus metadatos. Al hacer clic en cualquiera de los hipervínculos se tiene acceso a un listado completo de los metadatos (en formato XML, Figura 2).

Para la búsqueda ofrece un subconjunto de los campos del estándar LOM correspondientes a las categorías: General, Técnica y Educativa (ver Figura 3).

1 Para más detalles sobre el estándar Proyecto de Final 1484.12.1-2002 esta disponible en: http://grouper.ieee.org/groups/ltsc/wg12/files/LOM $1484 \quad 12$ 1 v1 Final Draft.pdf. 


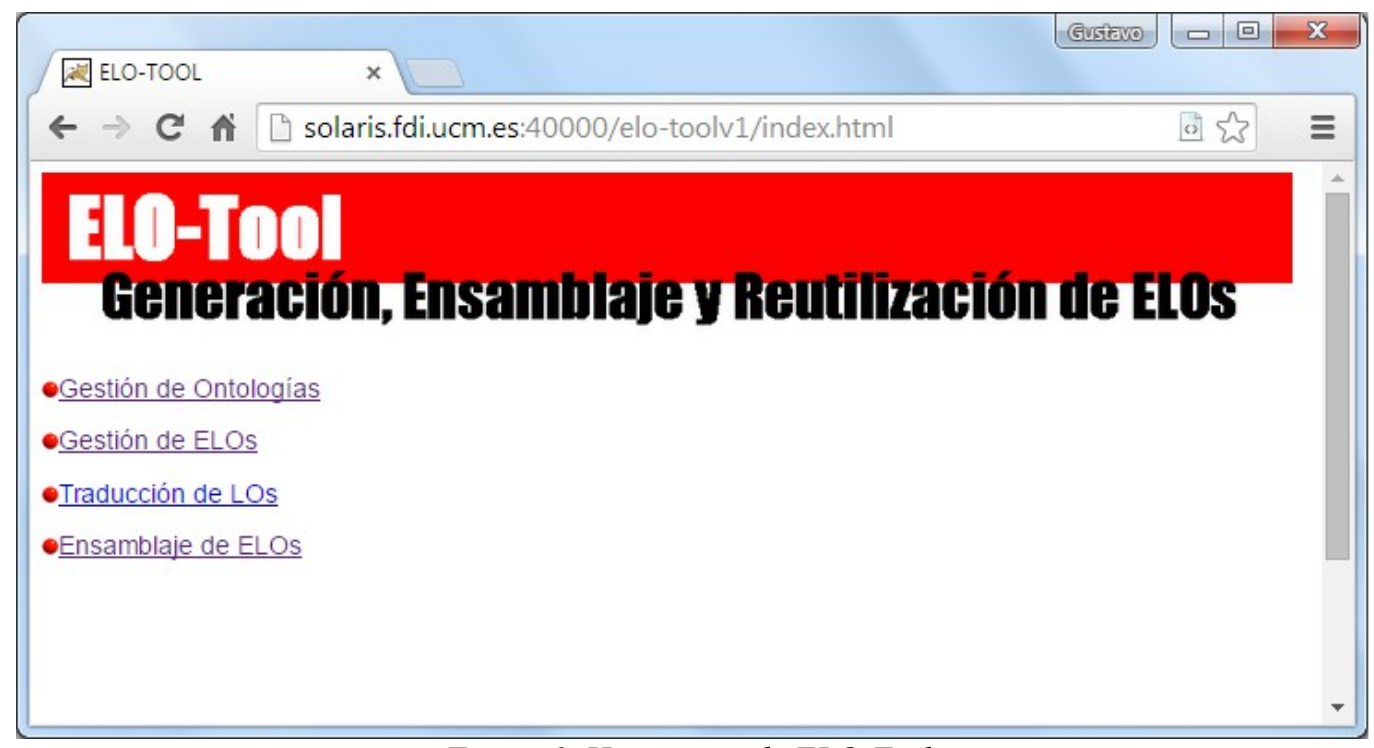

Figura 1. Home page de ELO-Tool.

solaris.fdi.ucm.es:40000/el $\times$

\begin{tabular}{|l|l|l|l|}
\hline Gustavo \\
\hline
\end{tabular}

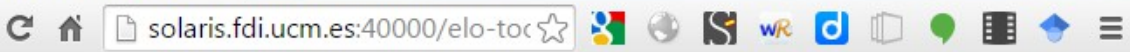

This XML file does not appear to have any style information associated with it. The document tree is shown below.

$\mathbf{v}<$ lom xmlns="http://www. it.uc3m.es/liliana/lom/iuLomv1"

xmlns: xsi="http://www.w3.org/2001/XMLSchema-instance"

xsi:schemaLocation="http://www.it.uc3m.es/liliana/lom/iuLomV1

http://solaris.fdi.ucm.es:40000/eloToolv1/iuLom.xsd">

$\mathbf{v}$ General>

$\checkmark<$ identifier>

<catalog>INTROCOMP</catalog>

$\checkmark<$ entry>

$<$ langstring $>01$. FOR-TO-DO</langstring $>$

$</$ entry $\rangle$

/identifier $>$

$\mathbf{v}\langle$ title $>$

<langstring>Estructura de control for-to-do</langstring>

$\langle/$ title $>$

$<$ language $>$ spanish $</$ language $>$

$\mathbf{v}<$ description $>$

Figura 2. Visualizando un ELO en ELO-Tool.

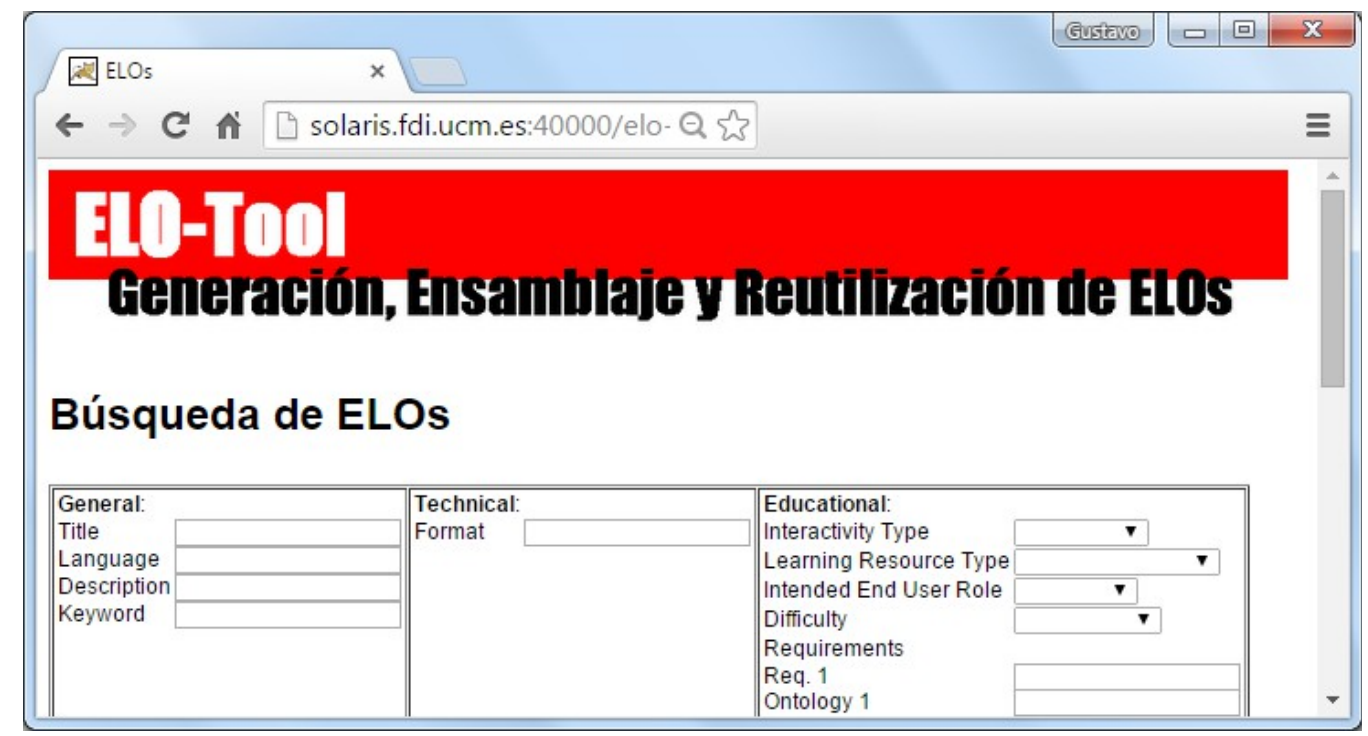

Figura 3. Parte del formulario de búsqueda de ELO-Tool. 
Para la gestión de ontologías la herramienta cuenta con tres opciones: (i) Dar de alta ontología, (ii) Dar de baja ontología y (iii) Mostrar ontologías.

Para Dar de alta una ontología se debe generar, por fuera de la herramienta, un archivo en formato XML en el que se define la jerarquía de clases correspondiente al dominio que se desea representar, así como también las relaciones o mapping con otras ontologías.

La ontología se describe mediante el lenguaje $\mathrm{OWL}^{2}$ (Ontology Web Language). Para definir la estructura de clases se utilizan los descriptores de clase ${ }^{3}$ : owl:Class, rdfs:subclassof y owl:equivalentclass. Los dos primeros permiten definir clases y sub-clases, y el tercero establece la equivalencia de una clase con otra contenida en una ontología diferente (relación de mapping con otra ontología). En la Figura 4 se muestra un ejemplo con parte de una de las ontologías generadas para el estudio de caso del presente trabajo de investigación.

Al igual que para los ELOs al elegir la opción Mostrar ontologías, aparece un listado de las mismas cuyo nombre funciona como un enlace a los archivos XML. Al hacer clic en cualquiera de los hipervínculos se muestra, en el navegador, el archivo XML correspondiente a la ontología (ver Figura 4a).

Para Dar de baja una ontología sólo es necesario indicar el nombre con el que figura y la misma se elimina del repositorio. Las ontologías son centrales en el proceso de ensamblaje y el sistema no confirma la operación elegida, hay que realizar esta acción con extrema precaución.

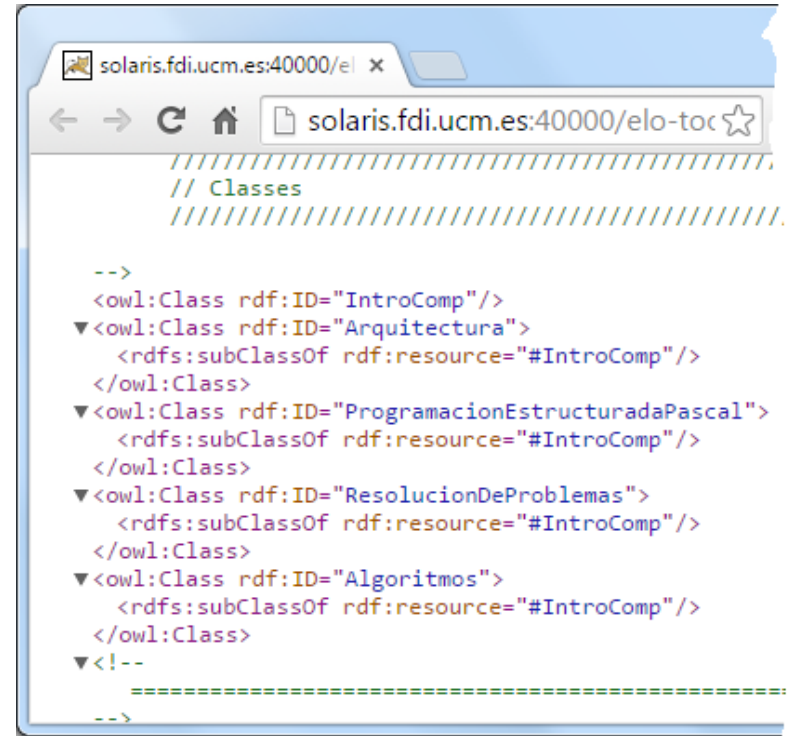

(a) Código fuente

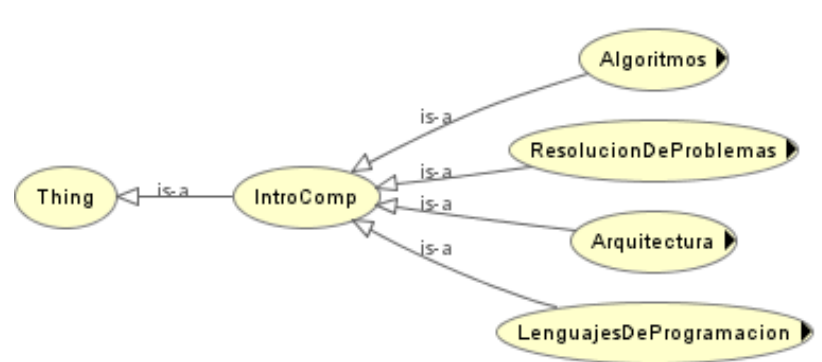

(b) Representación gráfica en Protégé

Figura 4. Parte de la ontología que representa el dominio de Introducción a la Computación. Disponible en: http://solaris.fdi.ucm.es:40000/elo-toolv1/ontologias/ont160.xml

Para el Ensamblaje de ELOs, sólo es necesario completar los nombres (con el que aparecen al Mostrar ELOs) de los dos recursos u OA que se desean ensamblar y el sistema se encarga de hacer la validación sobre el conocimiento asociado (ver Sección 1, Capítulo 3) y, si es posible el ensamblaje, ofrece un formulario para completar metadatos (Figura 5). La herramienta completa parte de los metadatos del ELO resultante del ensamblaje.

El repositorio de ELO-Tool aloja ELOs con distinta granularidad (ver Sección 1, Capítulo 3), los cuales están disponibles para su ensamblaje. Como muestra la Tabla 1 la combinación de éstos da como resultado diferentes tipos de ELOs. A modo de ejemplo, en la Figura 5 puede observarse que de dos UCs (cuyos nombres en el repositorio son UC14 y UC15) se obtiene una UD (tal como lo muestra el título).

2 La referencia del lenguaje esta disponible en: http://www.w3.org/TR/owl-ref/

3 La definición para cada uno de los descriptores puede acceder en http://www.w3.org/TR/owl-ref/\#ClassAxioms 


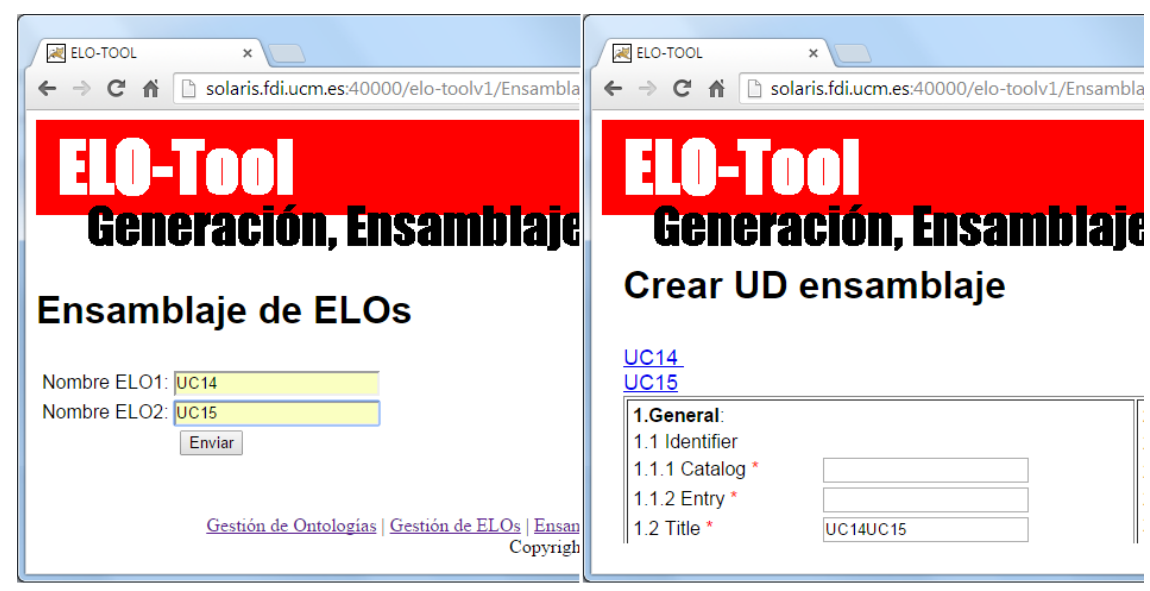

Figura 1. Ejemplo de ensamblaje de dos UCs en ELO-Tool.

Tabla 1. Posibles resultados de ensamblar dos ELOs (Santacruz-Valencia et al., 2005, p. 81 Tabla 3.5).

\begin{tabular}{cccc}
\hline & UI & UC & UD \\
\hline UI & UC & UC & $\times$ \\
UC & UC & UD & UD \\
UD & $\times$ & UD & UD \\
\hline
\end{tabular}

\section{Modelo de Contenido de ELO-Tool}

El modelo de contenidos propuesto para los ELOs cuentan con una estructura multicapa y metadatos que permiten su recuperación y ensamblaje.

Los objetos más complejos (UC y UD) cuentan con Conocimiento Asociado (CA), "se refiere a los requisitos necesarios para la comprensión del ELO y las competencias adquiridas después de su comprensión.” (Santacruz-Valencia et al., 2005, p. 74).

La estructura de los ELOs está formada por tres tipos, de acuerdo a su nivel de granularidad -de simple a compleja- se definen como (Santacruz-Valencia et al., 2005, pp. 76-80):

- UI. Son elementos atómicos representados por un único archivo multimedia. Si bien cuentan con metadatos, carecen de CA. Estas unidades, no representan en sí mismas una experiencia educativa.

- UC. Se forma con un conjunto de UIs, o UIs combinadas con UCs. Representan una experiencia educativa y por tanto, necesitan CA: requisitos para su comprensión y proporcionan unas competencias tras su abordaje.

- UD. Se construyen combinando UCs, UCs con UDs, o varias UDs. Representan, también, una experiencia educativa en la que abordan varios contenidos. Las UDs cuentan con CA. Pero además deben tener, dentro de sus metadatos, un resumen, los objetivos educativos y una descripción del proceso de evaluación. El resumen, los objetivos y la evaluación, si bien son obligatorios, no inciden en el proceso de ensamblaje.

El CA es representado a través de los metadatos que describen a los ELOs en el SE. La autora ha elegido el estándar de metadatos LOM para representar el CA. Dicho estándar no cuenta, por defecto, con la información necesaria para representar dicho conocimiento. Dado que el mismo es el que permite el ensamblaje de UCs y las UDs, propuso una extensión del estándar. Así pues, en la categoría 5.Educational del estándar (ver Figura 7) añadió las sub-categorías que permiten representar los requisitos y competencias de los ELOs mencionados. Las categorías agregadas son (Santacruz-Valencia et al., 2005, pp. 77-80):

- Para UCs: (i) 5.12 requirements, conjunto de requerimientos; (ii) 5.12.1 requirement, define 
cada uno de los requerimientos en base a las clases definidas en la ontología de dominio. (iii) 5.13 competencies conjunto de competencias; 5.13.1 competency define cada una de las competencias adquiridas al abordar la UC en base a las clases definidas en la ontología de dominio. (iv) 5.14 files, conjunto archivos asociados a la UC; y (v) 5.14.1 file, indica la dirección (URL) en el que se encuentran los ELOs utilizados en la UC.

- Para UDs: además de los metadatos requirements y competencies -que se describieron para las UCs- se agregan (i) 5.15 objective, que enuncia los objetivos de aprendizaje de la UD; (ii) 5.16 summary, que describe la UD y (iii) 5.17 evaluation, donde se explicitan las estrategias de evaluación. Además, se agregan (iv) 5.18 items y (v) 5.18 .1 item, donde se colocan las URLs de cada ELO utilizando en el ensamblaje, así como el tipo del mismo.

Cabe aclarar que en la caso de las UIs la URL referencia un recurso que se encuentra fuera del repositorio. Mientras que UCs y UDs puede apuntar a ELOs que se encuentre tanto dentro como fuera del repositorio de ELO-Tool. En el caso de utilizar la opción de ensamblaje, ésta información, hace referencia a los ELOs del repositorio y es completada de forma automática por el SE.

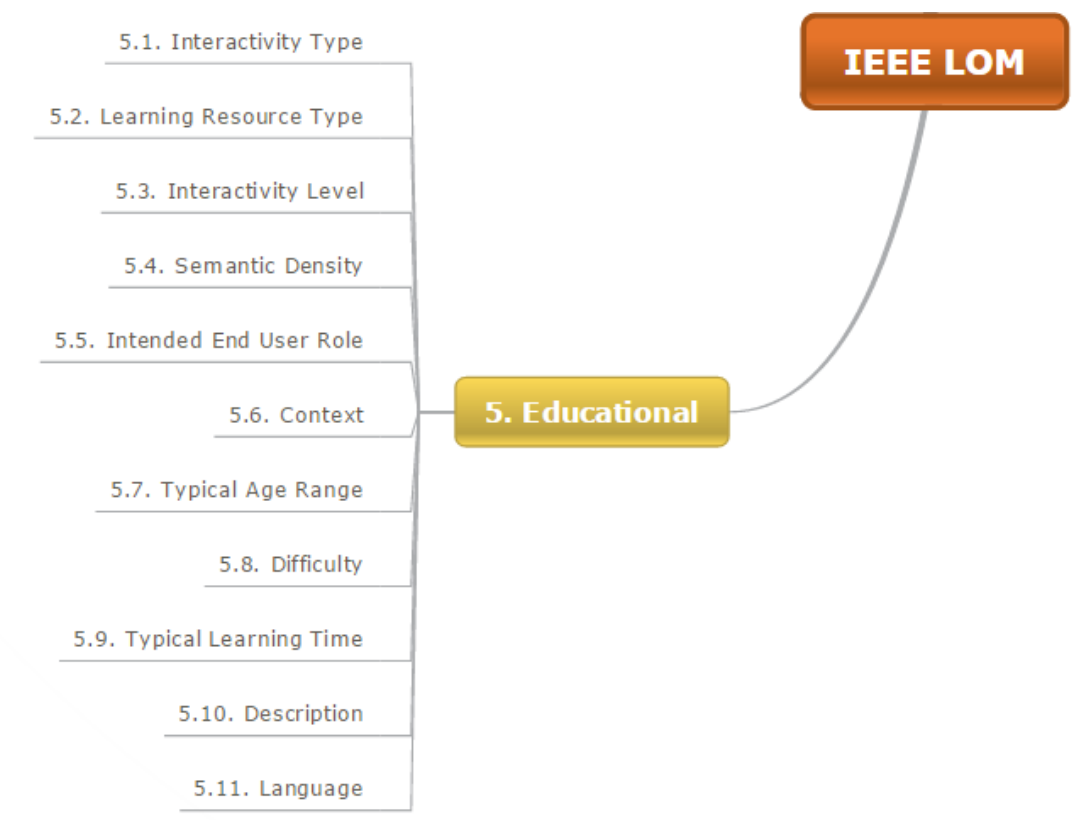

Figura 2. Parte del esquema de metadatos IEEE LOM. La figura muestra la Categoría 5. Educational

\section{Metodología de Ensamblaje}

El proceso de ensamblaje propuesto, en esta metodología, se basa en el CA. Así pues, dos ELOs pueden ser ensamblados si las competencias de uno cubren los requerimientos del otro (SantacruzValencia et al., 2005).

Tanto los requerimientos como las competencias, son representados en la metodología a través de los metadatos asociados a UC y UD. Pero además, este CA se refleja en las ontologías de dominio. Utilizando de manera combinada los metadatos y las ontologías se hace posible definir en ensamblaje entre dos ELOs.

El mecanismo que permite el ensamblaje de ELOs heterogéneos se denomina OntoGlue. El mismo "es un mecanismo [...], basado en antologías que permite comparar semánticamente los requisitos y las competencias pertenecientes a ELOs heterogéneos. Así pues, los requisitos y las competencias representan clases que pertenecen a ontologías particulares. El problema fundamental al que se da solución con la aplicación de OntoGlue es a la diferencia sintáctica entre conceptos, puesto que se establecen mappings entre ontologías" (Santacruz-Valencia et al., 2005, p. 94).

Cuando el CA se encuentra representado en el mismo dominio, la comparación de los metadatos 
para evaluar si es posible su ensamblaje es sintáctica. Es decir, dado los objetos $O A_{1}$ y $O A_{2}$ cuyo CA está representado en el mismo dominio, si un objeto $O A_{2}$ requiere de "Tipo de datos simples" y el objeto $O A_{1}$ aborda esta temática y dentro de las competencias del mismo aparece "Tipo de datos simples" (Figura 7), entonces, las competencias de ambos ELOs son sintáctica y semánticamente iguales. Por tanto, es posible su ensamblaje. Se dice que la competencia de $O A_{1}$ cubre al requisito de $\mathrm{OA}_{2}$ o simplemente que $\mathrm{OA}_{1}$ cubre a $\mathrm{OA}_{2}$.

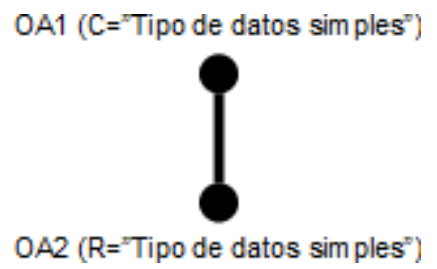

Figura 3. CA de dos $O A$ que pertenecen al mismo dominio

Al comparar las competencias de un ELO con los requisitos de otro, cuya semántica permitiría su ensamblaje, puede haber diferencias en la sintaxis que impidan el proceso. Esto es, supongamos que los objetos $O A_{1}$ y $O A_{2}^{\prime}$ son representados sobre distintos dominios, el $O A_{2}^{\prime}$ requiere para su abordaje "Sentencias repetitivas", mientras que entre las competencias del objeto $O A_{1}$ está "Sentencias iterativas". Claramente, en el ejemplo, las competencias del $O A_{1}$ son semánticamente coincidentes con los requerimientos del $O A_{2}^{\prime}$, sin embargo, una comparación estrictamente sintáctica impediría el ensamblaje. Para este caso, la metodología propone un mapping o mapeo entre ontologías. Es decir, definir una relación de equivalencia entre clases de ontología diferentes. De esta manera, se hace posible realizar la comparación y también el ensamblaje de los ELOs ya que $O A_{1}$ cubre a $O A_{2}^{\prime}$, a través del mapping. La Figura 8 representa esta situación de forma esquemática. Como se puede apreciar en ella, el CA a $O A_{1}$ y el CA a $O A_{1}^{\prime}$ son equivalentes, además, $O A_{1}^{\prime}$ cubre a $O A_{2}^{\prime}$, por tanto $O A_{1}$ cubre a $O A_{2}^{\prime}$ y, por tanto, podrían ensamblase.

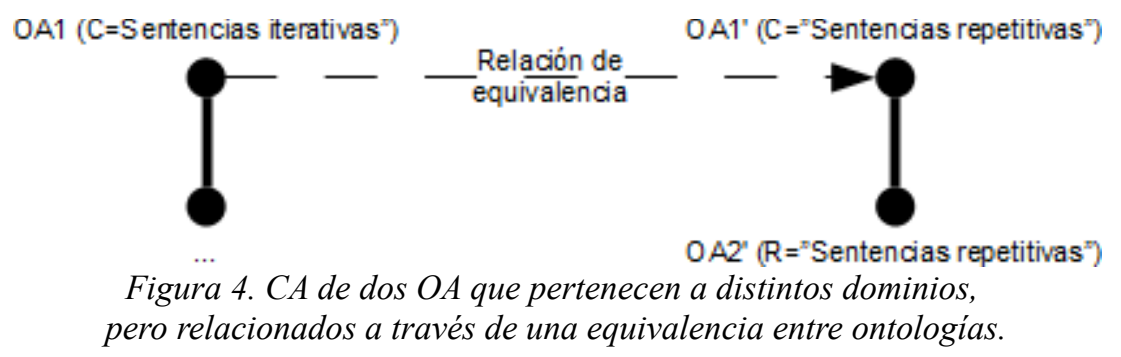

También puede ocurrir que dos ELOs que tienen su CA representado en dos ontologías de dominio diferentes pudieran ser relacionados a través de una tercera ontología. Estableciendo lo que se denomina una cadena de mapping. Esto es, si existe un mapping entre ontologías que relaciona los objetos $O A_{l}$ y $O A_{l}{ }^{\prime}$ (como en el caso anterior), y además, otro mapping que hace lo propio con $O A_{l}{ }^{\prime}$ y $O A_{1}^{\prime \prime}$, y su vez $O A_{1}{ }^{\prime \prime}$ cubre a $O A_{2} "$, entonces es posible la comparación (y ensamblaje) entre $O A_{1}$ y $\mathrm{OA}_{2} "$ (ver Figura 9).

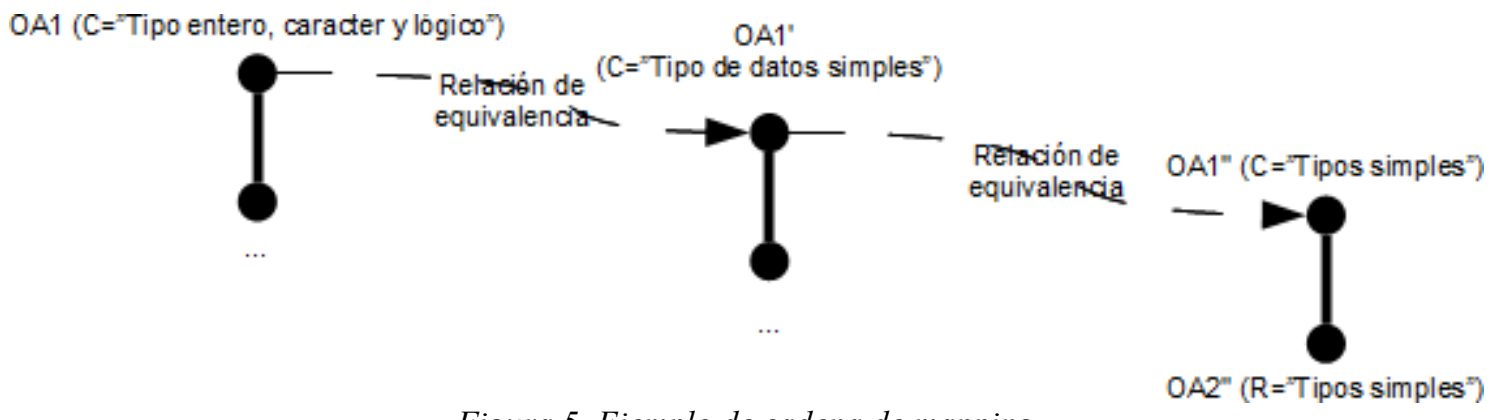

Figura 5. Ejemplo de cadena de mapping.

Otro caso contemplado por la metodología es cuando existe una ontología intermedia que permite relacionar dos ELOs para su ensamblaje. Si se observa la Figura 10, $O A_{l}$ cubre a $O A_{2}$ y el CA de éste es equivalente al de $O A_{l}{ }^{\prime}$, pero también el CA de $O A_{l}$ " es equivalente a $O A_{l}{ }^{\prime}$, además, $O A_{l}{ }^{\prime \prime}$ 
cubre a $O A_{2} "$. La metodología implementa los cálculos necesarios para determinar que $O A_{1}$ cubre a $O A_{2}$ ", y por tanto ensamblarlos.

OA1 (C="Tipo entero, caracter y lógicon)

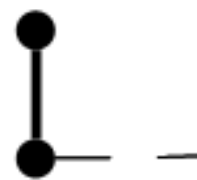

OA2 (R="Tipo entero, caracter y lógic $\left.\infty^{n}\right)$
OA1"

(C ="Tipo de datos simples")

Relación de

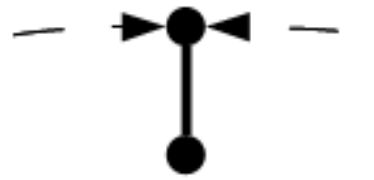

Relación de equivalercja

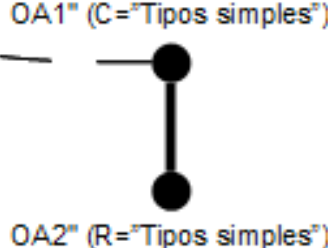

Figura 6. Ensamblaje a partir de ontologías intermedias. 
Este anexo contiene los diagramas que describen los itinerarios de aprendizaje diseñados para el taller de ensamblaje de OA. Este material fue provisto a los participantes del taller para que crearan los itinerarios.

Referencias

Unidades de información. La abreviatura "UI" y el número
forman parte del nombre que la unidad tiene en el repositorio de
ELO-Tool.

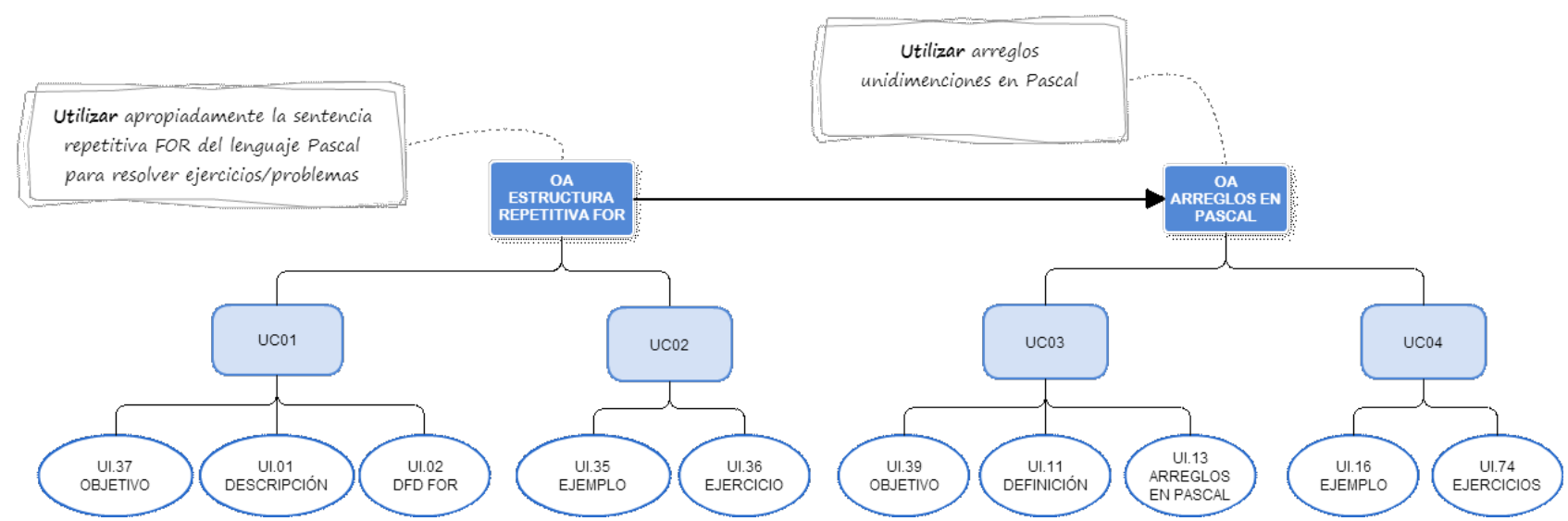

Figura 1. Diagrama del itinerario de aprendizaje que se utilizó como ejemplo durante el taller. 

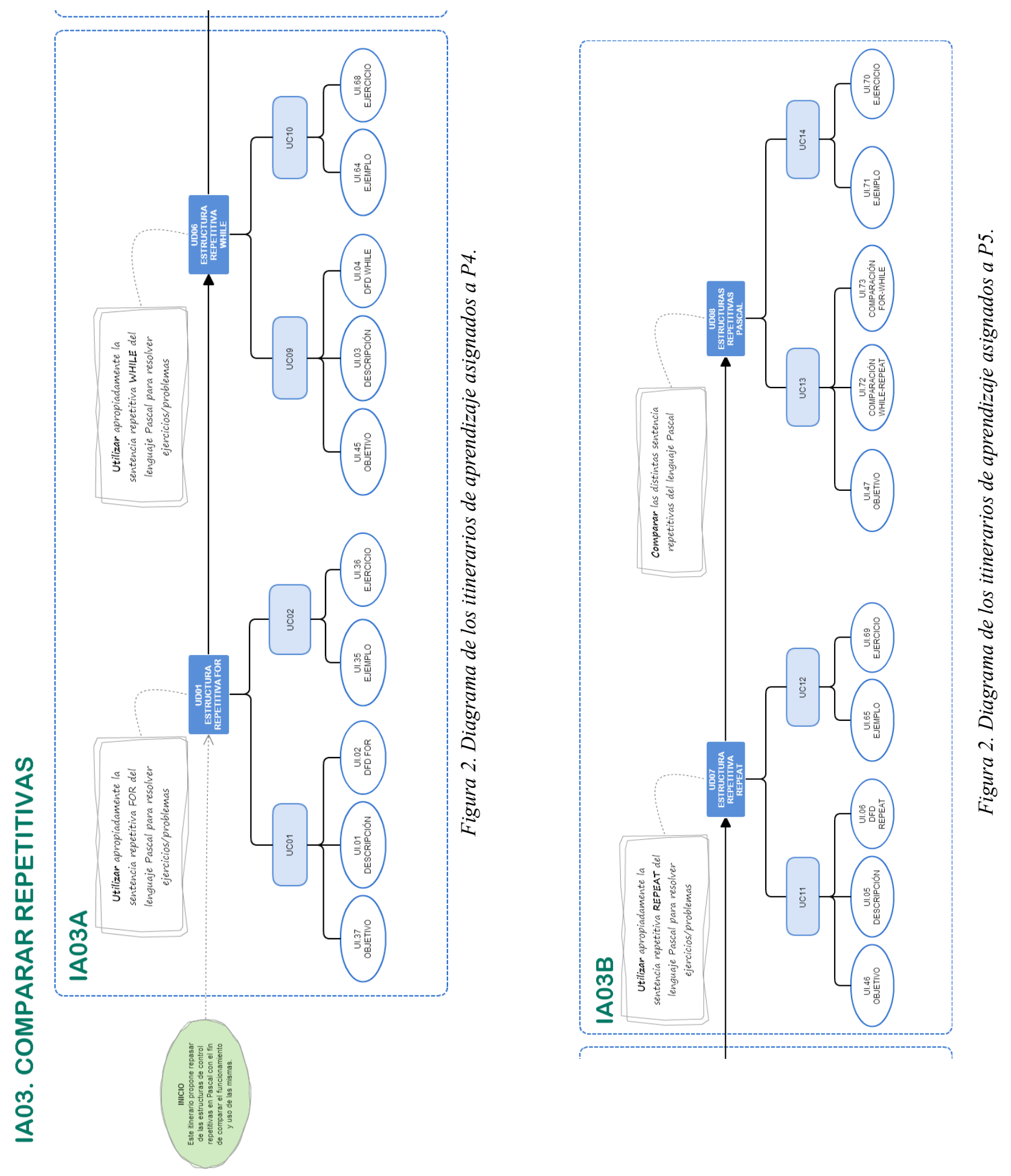


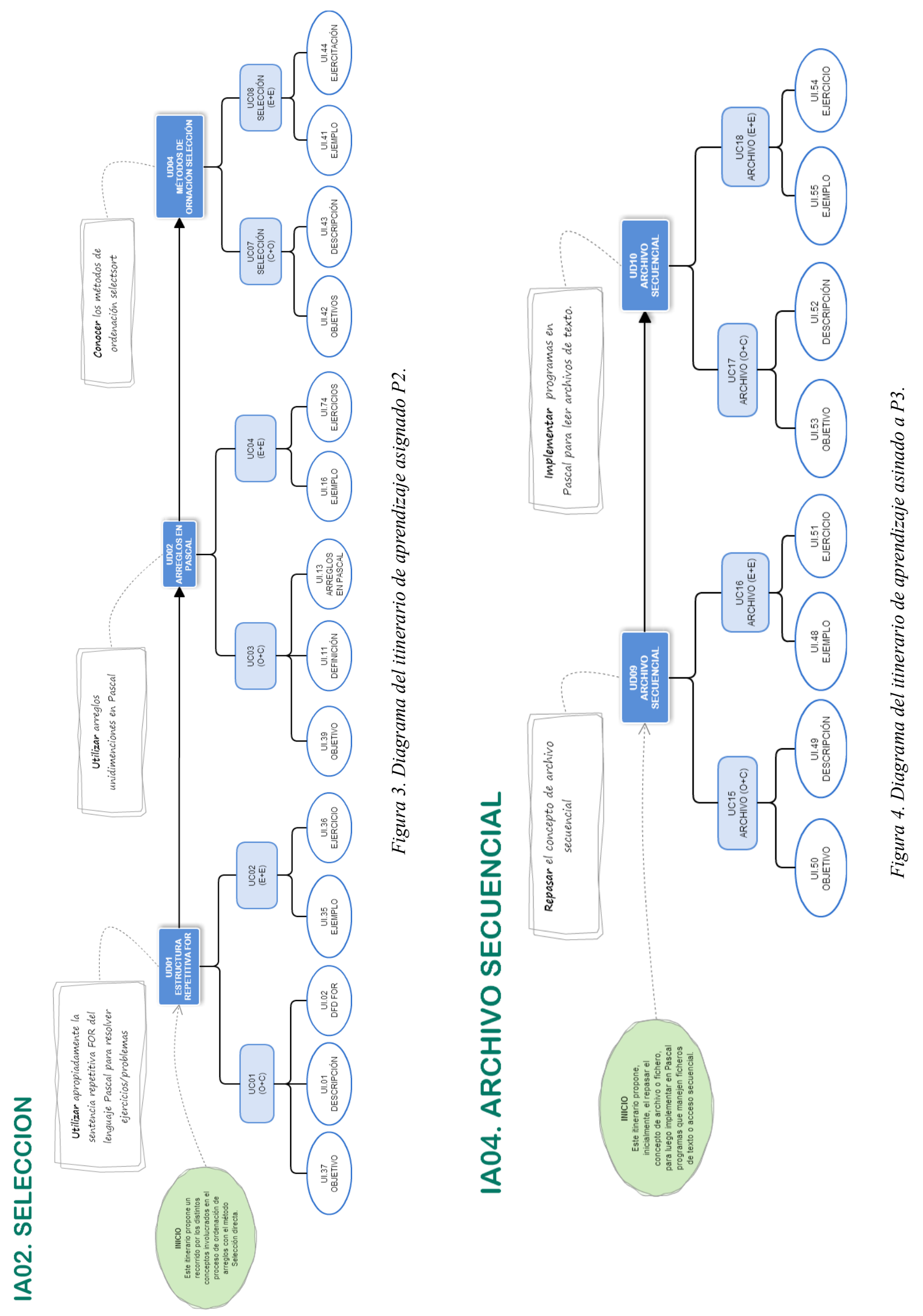




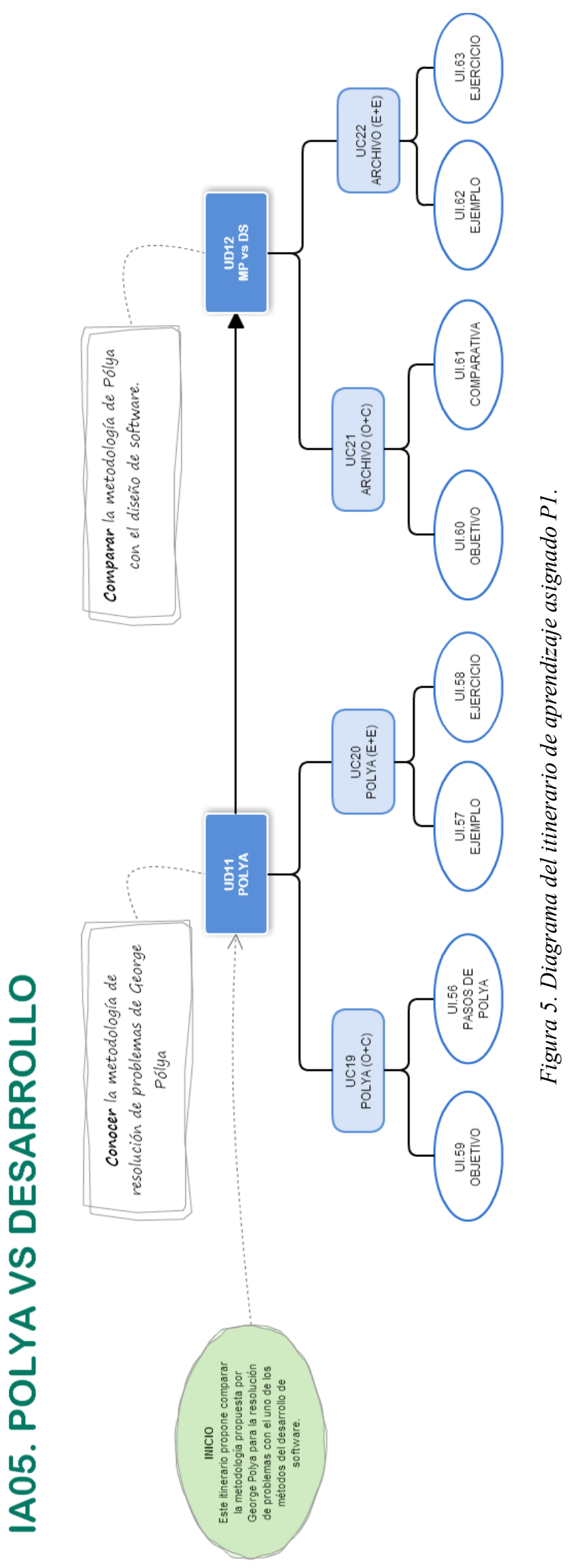




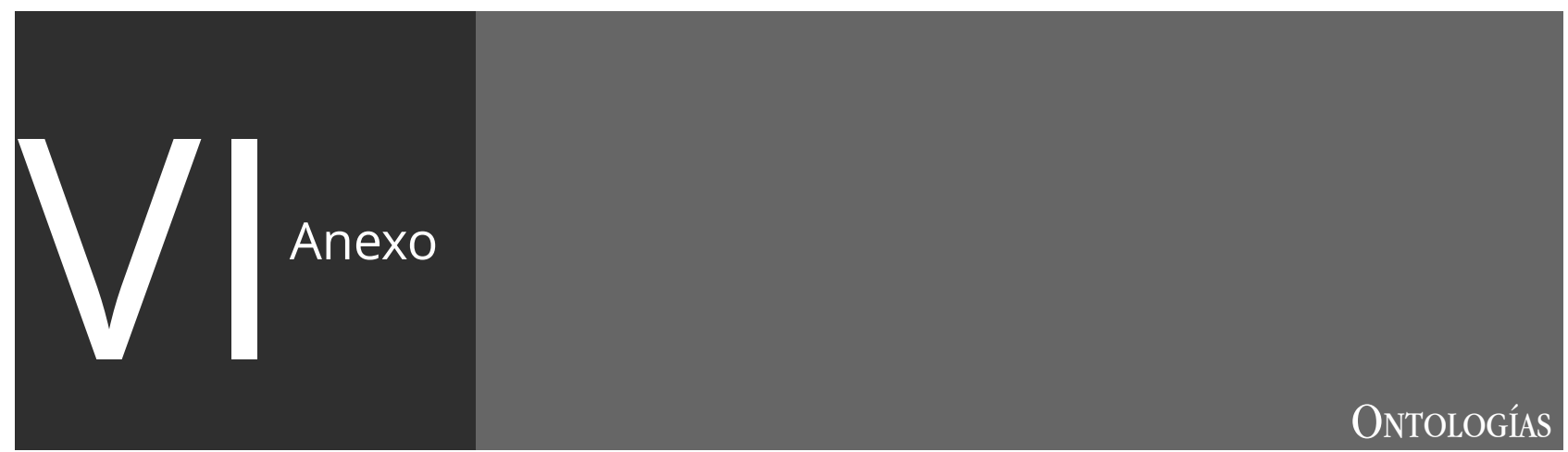

Este anexo contiene las tres ontologías de dominio y la de tipo de material creadas ad-hoc para la utilización de ELO-Tool en el contexto del taller de ensamblaje de OA que se diseñó para el estudio de caso.

\section{Ontologías de dominio}

\section{Introducción a la computación}

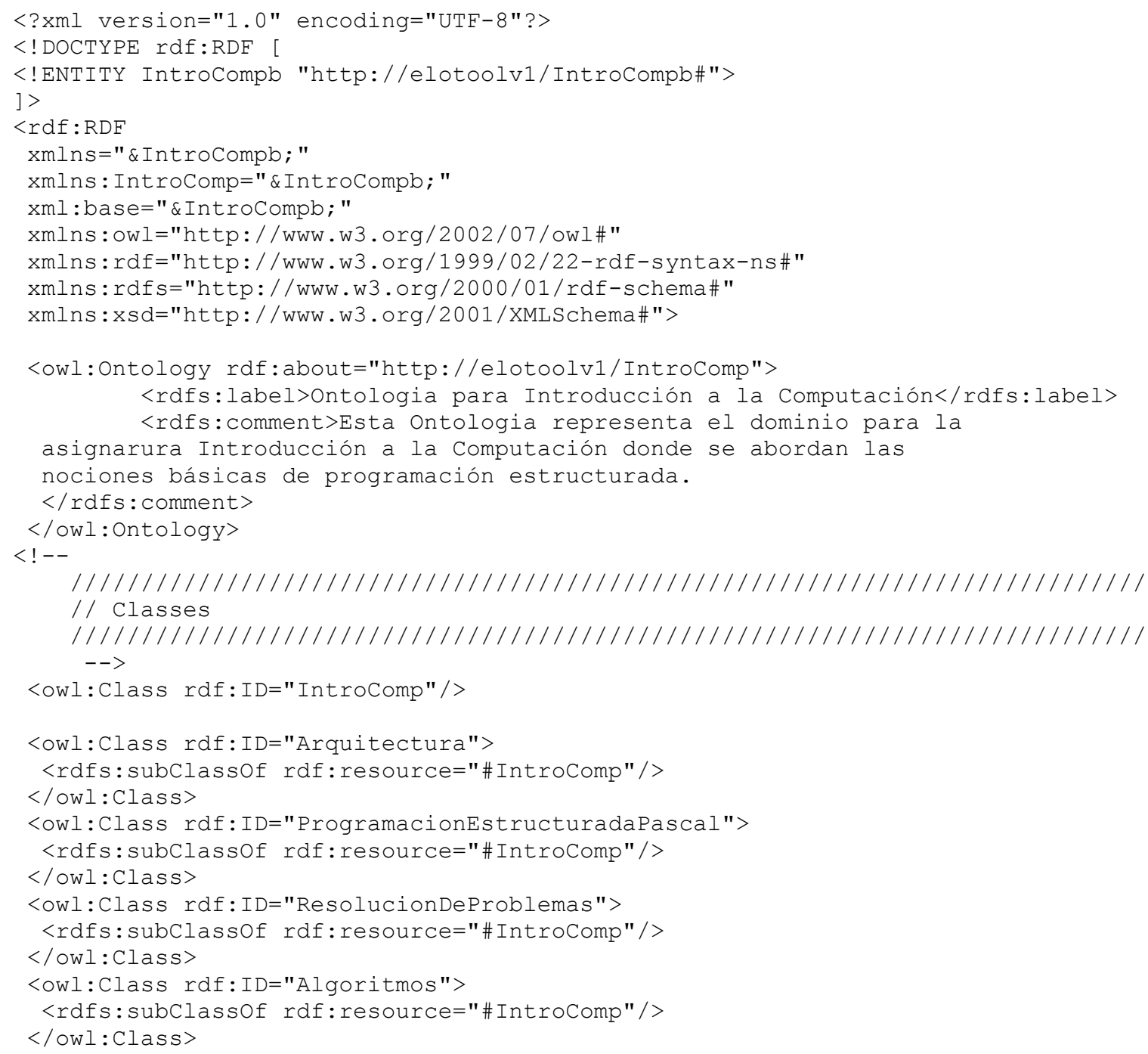




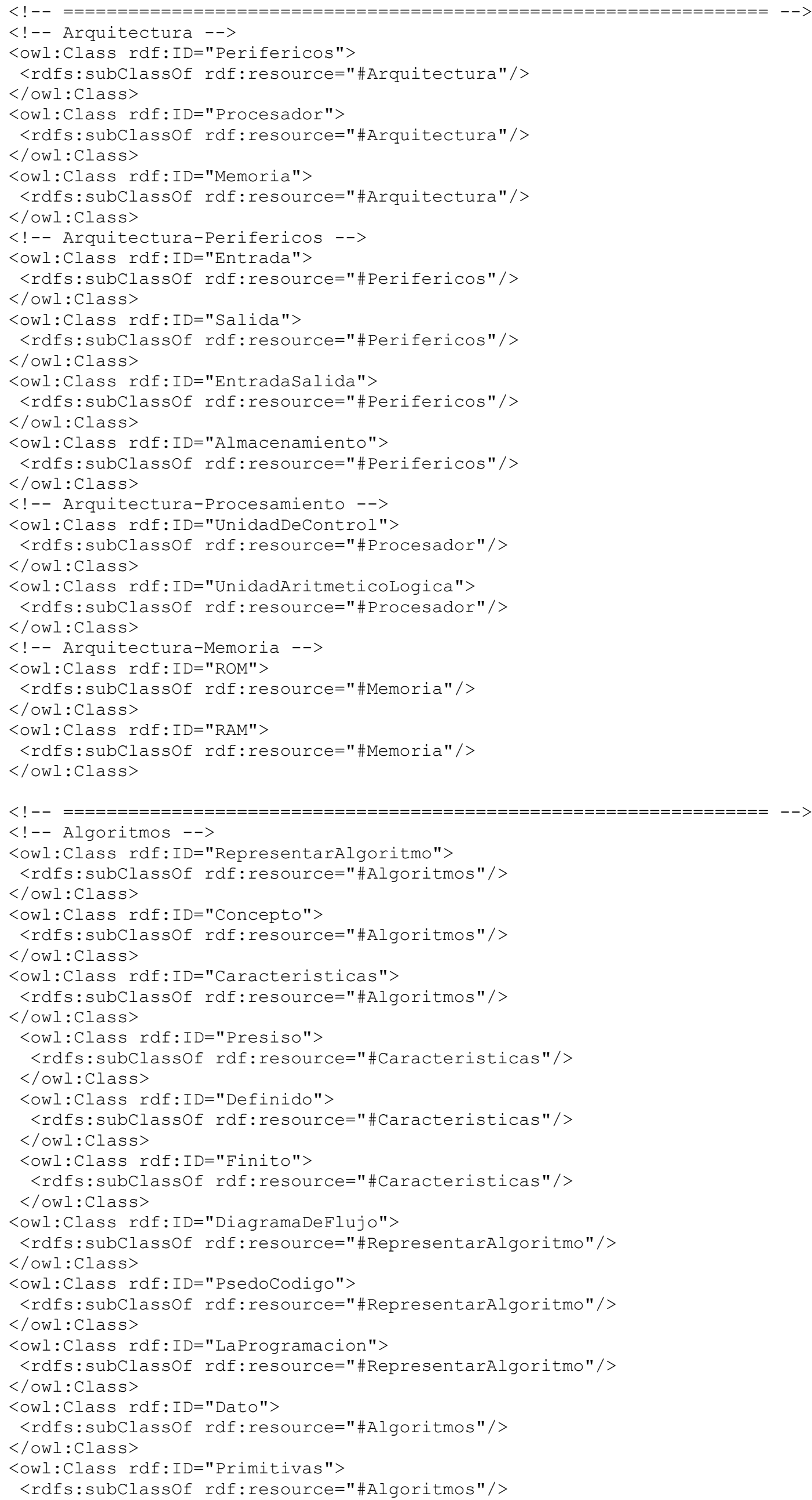


$</$ owl:Class $>$

<owl:Class rdf:ID="PrimitivaSecuencia">

<rdfs:subclassof rdf:resource="\#Primitivas"/>

$</$ owl:Class $>$

<owl:Class rdf:ID="PrimitivaSeleccion">

<rdfs:subclassof rdf:resource="\#Primitivas"/>

$<$ owl:Class $>$

<owl:Class rdf:ID="PrimitivaRepeticion">

<rdfs:subclassof rdf:resource="\#Primitivas"/>

$</$ owl:Class $>$

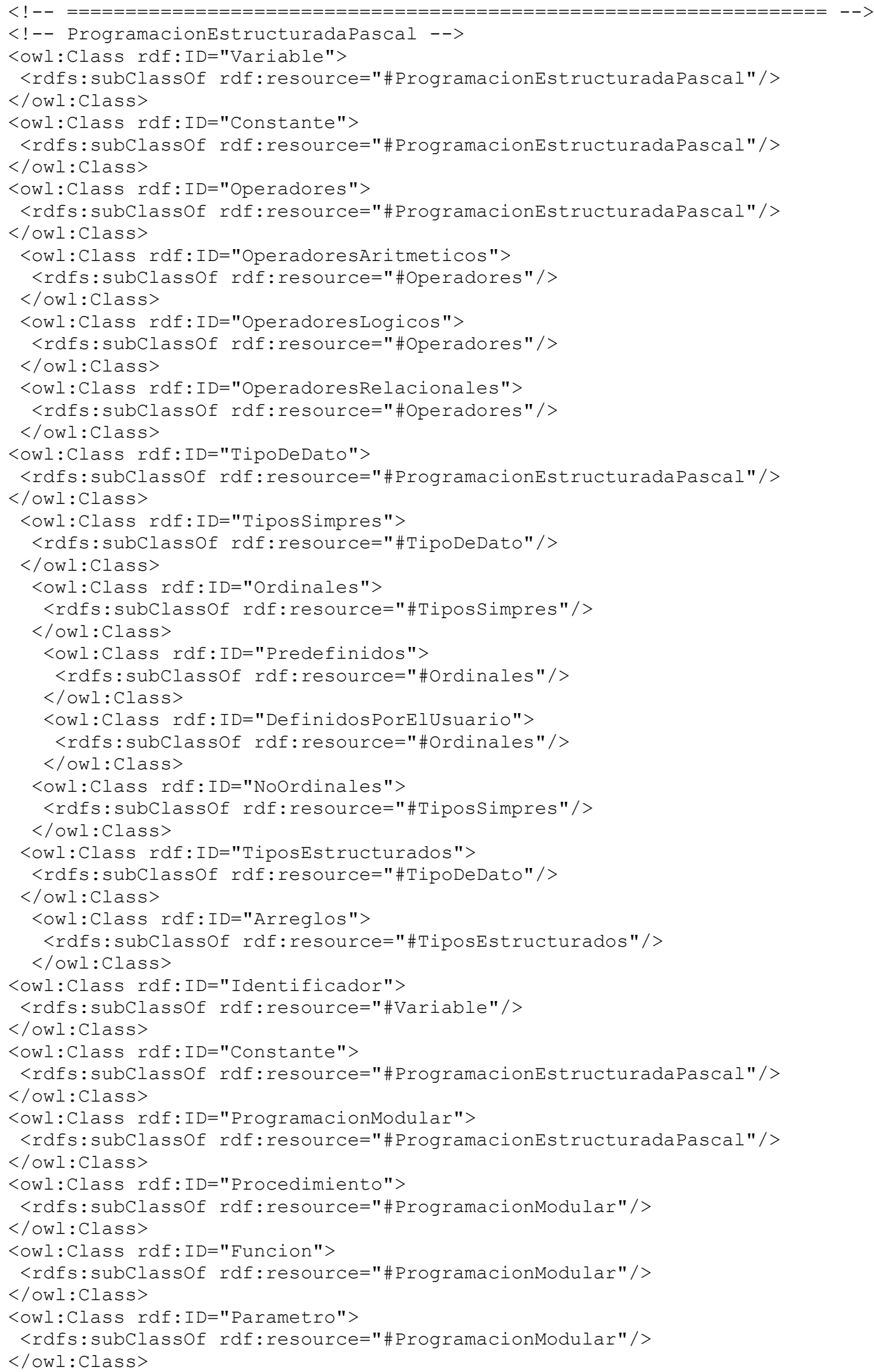


<owl:Class rdf:ID="ParametroValor">

<rdfs:subclassof rdf:resource="\#Parametro"/>

$</$ owl:Class $>$

<owl:Class rdf:ID="ParametroVariable">

$<$ rdfs:subclassof rdf:resource="\#Parametro"/>

$</$ owl:Class $>$

<owl:Class rdf:ID="EstructuraDePrograma">

<rdfs:subclassof rdf:resource="\#ProgramacionEstructuradaPascal"/>

$<$ owl:Class $>$

<owl:Class rdf:ID="BloqueEncabezamiento">

<rdfs:subclassof rdf:resource="\#EstructuraDePrograma"/>

$<$ owl:Class $>$

<owl:Class rdf:ID="BloqueDeclaracion">

<rdfs:subClassOf rdf:resource="\#EstructuraDePrograma"/>

$</$ owl:Class $>$

<owl:Class rdf:ID="BloqueEjecutable">

<rdfs:subClassof rdf:resource="\#EstructuraDePrograma"/>

$<$ owl:Class $>$

<owl:Class rdf:ID="Secuencia">

<rdfs:subclassof rdf:resource="\#BloqueEjecutable"/>

$</$ owl:Class $>$

<owl:Class rdf:ID="EstructurasDeControl">

<rdfs:subClassof rdf:resource="\#BloqueEjecutable"/>

$</$ owl:Class $>$

<owl:Class rdf:ID="Seleccion">

<rdfs:subClassof rdf:resource="\#EstructurasDeControl"/>

$<$ owl:Class $>$

<owl:Class rdf:ID="Repeticion">

<rdfs:subClassof rdf:resource="\#EstructurasDeControl"/>

$</$ owl : Class $>$

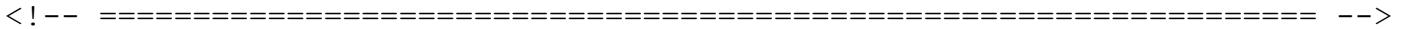

$<$ !-- Resolucion De Problemas -->

<owl:Class rdf:ID="PasosDePolya">

<rdfs:subClassof rdf:resource="\#ResolucionDeProblemas"/>

$</$ owl:Class $>$

<owl:Class rdf:ID="FasesDesarrolloDeSoftware">

<rdfs:subclassof rdf:resource="\#ResolucionDeProblemas"/>

$</$ owl:Class $>$

<owl:Class rdf:ID="CoprenderElProblema">

<rdfs:subClassof rdf:resource="\#PasosDePolya"/>

$</$ owl:Class $>$

<owl:Class rdf:ID="TrazarUnPlan">

<rdfs:subclassof rdf:resource="\#PasosDePolya"/>

$</$ owl:Class $>$

<owl:Class rdf:ID="ImplementarElPlan">

<rdfs:subclassof rdf:resource="\#PasosDePolya"/>

$</$ owl:Class $>$

<owl:Class rdf:ID="ComprobarLosResultados">

<rdfs:subClassof rdf:resource="\#PasosDePolya"/>

$<$ owl:Class $>$

<owl:Class rdf:ID="AnalisisDeRequisitos">

<rdfs:subClassof rdf:resource="\#FasesDesarrolloDeSoftware"/>

$</$ owl:Class $>$

<owl:Class rdf:ID="Disenio">

<rdfs:subClassOf rdf:resource="\#FasesDesarrolloDeSoftware"/>

$</$ owl:Class $>$

<owl:Class rdf:ID="Programacion">

<rdfs:subClassof rdf:resource="\#FasesDesarrolloDeSoftware"/>

$<$ lowl:Class $>$

<owl:Class rdf:ID="Pruebas">

<rdfs:subClassof rdf:resource="\#FasesDesarrolloDeSoftware"/>

$</$ owl:Class $>$

<owl:Class rdf:ID="Documentacion">

<rdfs:subclassof rdf:resource="\#FasesDesarrolloDeSoftware"/>

$</$ owl:Class $>$

<owl:Class rdf:ID="Mantenimiento">

<rdfs:subClassof rdf:resource="\#FasesDesarrolloDeSoftware"/>

$</$ owl:Class $>$

$</$ rdf $:$ RDF $>$ 


\section{Programación I}

<?xml version="1.0" encoding="UTF-8"?>

$<$ ! DOCTYPE rdf:RDF [

<!ENTITY ProgI "http://elotoolv1/ProgI\#"> ] $>$

$<\operatorname{rdf}: \operatorname{RDF}$

xmlns="\&ProgI;"

xmlns:ProgI="\&Prog $I ; "$

$\mathrm{xml}:$ base $=" \& \operatorname{Prog} I ; "$

xmlns:owl="http://www.w3.org/2002/07/owl\#"

xmlns:rdf="http://www.w3.org/1999/02/22-rdf-syntax-ns\#"

xmlns:rdfs="http://www.w3.org/2000/01/rdf-schema\#"

xmlns:xsd="http://www.w3.org/2001/XMLSchema\#">

<owl:Ontology rdf:about="http://elotoolv1/ProgI">

<rdfs:label>ontologia para Programación I</rdfs:label>

<rdfs:comment>Esta ontologia representa el dominio para la

asignarura Programación I donde se abordan los

conceptos de búsqueda, ordenación, recursividad, tipos dinámicos

(punteros y archivos), listas, análisis de algoritmos (Bigo).

$</$ rdfs:comment $>$

$</$ owl: Ontology $>$

$<!--$

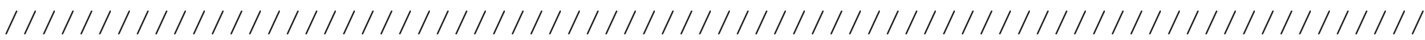

// Classes

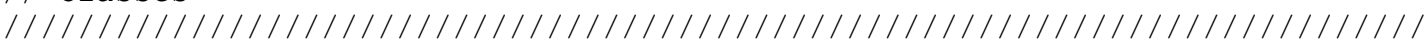
$-->$

<Owl:Class rdf:ID="ProgI"/>

<owl:Class rdf:ID="Ordenacion">

<rdfs:subClassof rdf:resource="\#ProgI"/>

$</$ owl:Class $>$

<owl:Class rdf:ID="Busqueda">

<rdfs:subClassof rdf:resource="\#ProgI"/>

$<$ /owl:Class $>$

<owl:Class rdf:ID="Recursividad">

<rdfs:subClassof rdf:resource="\#QuickSort"/>

<rdfs:subclassof rdf:resource="\#Binaria"/>

$<$ rdfs:subclassof rdf:resource="\#ProgI"/>

$<$ owl:Class $>$

<owl:Class rdf:ID="TiposDeDatos">

<rdfs:subclassof rdf:resource="\#ProgI"/>

$</$ owl:Class $>$

<owl:Class rdf:ID="AnalisisDeAlgortimo">

<rdfs:subClassof rdf:resource="\#ProgI"/>

$</$ owl:Class $>$

$<$ !-- Ordenacion $-->$

<owl:Class rdf:ID="MetodosExternos">

<rdfs:subclassof rdf:resource="\#Ordenacion"/>

$<$ owl:Class $>$

<owl:Class rdf:ID="Merge">

<rdfs:subclassof rdf:resource="\#MetodosExternos"/>

$</$ owl:Class $>$

<owl:Class rdf:ID="MetodosInternos">

<rdfs:subClassof rdf:resource="\#Ordenacion"/>

$</$ owl:Class $>$

<owl:Class rdf:ID="Directos">

<rdfs:subClassof rdf:resource="\#MetodosInternos"/>

$<$ /owl:Class $>$

<owl:Class rdf:ID="MetodoBurbuja">

<rdfs:subclassof rdf:resource="\#Directos"/>

$</$ owl : Class $>$

<owl:Class rdf:ID="MetodoSeleccion">

<rdfs:subClassof rdf:resource="\#Directos"/>

$<$ owl:Class $>$

<owl:Class rdf:ID="MetodoInsercion">

<rdfs:subClassof rdf:resource="\#Directos"/>

$<$ owl:Class $>$

<owl:Class rdf:ID="Indirectos">

<rdfs:subClassof rdf:resource="\#MetodosInternos"/>

$</$ owl:Class $>$ 
<owl:Class rdf:ID="QuickSort">

<rdfs:subclassof rdf:resource="\#Indirectos"/> $<$ owl:Class $>$

<owl:Class rdf:ID="Shell">

<rdfs:subclassof rdf:resource="\#Indirectos"/>

$</$ owl:Class $>$

$<!--$ Busqueda -->

<owl:Class rdf:ID="Secuencial">

<rdfs:subclassof rdf:resource="\#Busqueda"/>

$<$ /owl:Class $>$

<owl:Class rdf:ID="Binaria">

<rdfs:subclassof rdf:resource="\#Busqueda"/>

$<$ owl:Class>

$<!--$ TiposDeDatos -->

<owl:Class rdf:ID="Punteros">

<rdfs:subClassof rdf:resource="\#TiposDeDatos"/>

$</$ owl:Class $>$

<owl:Class rdf:ID="Listas">

<rdfs:subClassof rdf:resource="\#TiposDeDatos"/>

$<$ /owl:Class $>$

<owl:Class rdf:ID="Pilas">

<rdfs:subClassof rdf:resource="\#Listas"/>

$</$ owl:Class $>$

<owl:Class rdf:ID="Colas">

<rdfs:subClassof rdf:resource="\#Listas"/>

$<$ owl:Class $>$

<owl:Class rdf:ID="Registros">

<rdfs:subClassof rdf:resource="\#TiposDeDatos"/>

$<$ owl:Class $>$

<owl:Class rdf:ID="Archivos">

<rdfs:subClassof rdf:resource="\#iposDeDatos"/>

$</$ owl:Class $>$

<owl:Class rdf:ID="ArchivosSecuenciales">

<rdfs:subclassof rdf:resource="\#Archivos"/>

$<$ owl:Class $>$

<owl:Class rdf:ID="ArchivosAccesoDirecto">

<rdfs:subClassof rdf:resource="\#Archivos"/>

$</$ owl:Class $>$

$<$ !-- AnalisisDeAlgortimo -->

<owl:Class rdf:ID="Complejidad">

<rdfs:subClassof rdf:resource="\#AnalisisDeAlgortimo"/>

$<$ owl:Class $>$

<owl:Class rdf:ID="Eficiencia">

<rdfs:subClassof rdf:resource="\#AnalisisDeAlgortimo"/>

$</$ owl:Class $>$

$</$ rdf :RDF $>$

\section{Lenguaje de programación Pascal}

$<$ ?xml version="1.0" encoding="UTF-8"?>

$<$ ! DOCTYPE rdf:RDF [

<!ENTITY pascalb "http://elotoolvi/pascalb\#">

] $>$

$<r d f: R D F$

xmlns $=" \&$ pascalb; $"$

xmlns:pascalb="\&pascalb;"

xml: base="\&pascalb;"

xmlns: owl="http://www.w3.org/2002/07/owl\#"

xmlns:rdf="http://www.w3.org/1999/02/22-rdf-syntax-ns\#"

xmlns:rdfs="http://www.w3.org/2000/01/rdf-schema\#"

xmlns:xsd="http://www.w3.org/2001/XMLSchema\#">

<owl:Ontology rdf:about="http://elotoolv1/pascalb\#">

<rdfs:comment>

Esta Ontologia representa el dominio del lenguaje de programacion Pascal

$</$ rdfs: comment $>$

$<$ rdfs:label>Ontologia para Pascal</rdfs:label>

$<$ /owl: Ontology>

<owl:Class rdf:ID="ProgramaPascal"/> 
$<!--$ DECLARACIONES -->

<owl:Class rdf:ID="Declaraciones">

<rdfs:subClassof rdf:resource="\#ProgramaPascal"/>

$</$ owl:Class $>$

<owl:Class rdf:ID="Uses">

<rdfs:subclassof rdf:resource="\#Declaraciones"/>

$<$ owl:Class $>$

<owl:Class rdf:ID="Const">

<rdfs:subclassof rdf:resource="\#Declaraciones"/>

$</$ owl:Class $>$

<owl:Class rdf:ID="Var">

<rdfs:subClassof rdf:resource="\#Declaraciones"/>

$<$ /owl:Class $>$

<owl:Class rdf:ID="Type">

<rdfs:subclassof rdf:resource="\#Declaraciones"/>

$<$ /owl:Class $>$

$<$ !-- DECLARACIONES - TIPOS DE DATOS -->

<owl:Class rdf:ID="TiposDeDatos">

<rdfs:subclassof rdf:resource="\#ProgramaPascal"/>

$<$ owl:Class $>$

<owl:Class rdf:ID="DatosEstaticos">

<rdfs:subClassof rdf:resource="\#tiposDeDatos"/>

$</$ owl:Class $>$

<owl:Class rdf:ID="TiposSimples">

<rdfs:subClassof rdf:resource="\#DatosEstaticos"/>

$<$ owl:Class $>$

<owl:Class rdf:ID="Tipoordinal">

<rdfs:subClassof rdf:resource="\#Tipossimples"/>

$</$ owl:Class $>$

<owl:Class rdf:ID="TipoPreDefinido">

<rdfs:subclassof rdf:resource="\#Tipoordinal"/>

$</$ owl:Class $>$

<owl:Class rdf:ID="Integer">

<rdfs:subClassof rdf:resource="\#ipoPreDefinido"/>

$</$ owl:Class $>$

<owl:Class rdf:ID="Char">

<rdfs:subclassof rdf:resource="\#TipoPreDefinido"/>

$</$ owl:Class $>$

<owl:Class rdf:ID="Boolean">

<rdfs:subClassof rdf:resource="\#TipoPreDefinido"/>

$</$ owl:Class $>$

<owl:Class rdf:ID="TipoDefinidoPorUsuario">

<rdfs:subclassof rdf:resource="\#TipoOrdinal"/>

$<$ owl:Class $>$

<owl:Class rdf:ID="Enumerado">

<rdfs:subClassof rdf:resource="\#TipoDefinidoPorUsuario"/>

$</$ owl:Class $>$

<owl:Class rdf:ID="Subrango">

<rdfs:subClassof rdf:resource="\#TipoDefinidoPorUsuario"/>

$</$ owl:Class $>$

<owl:Class rdf:ID="TipoNoOrdinal">

<rdfs:subclassof rdf:resource="\#Tipossimples"/>

$<$ owl:Class $>$

<owl:Class rdf:ID="Real">

<rdfs:subClassof rdf:resource="\#TipoNoOrdinal"/>

$<$ owl:Class $>$

<owl:Class rdf:ID="TiposEstructurados">

<rdfs:subClassof rdf:resource="\#DatosEstaticos"/>

$<$ owl:Class $>$

<owl:Class rdf:ID="Array">

<rdfs:subclassof rdf:resource="\#TiposEstructurados"/>

$</$ owl:Class $>$

<owl:Class rdf:ID="Set">

<rdfs:subClassof rdf:resource="\#iposEstructurados"/>

$</$ owl:Class $>$

<owl:Class rdf:ID="Record">

<rdfs:subClassof rdf:resource="\#TiposEstructurados"/>

$<$ owl:Class $>$

<owl:Class rdf:ID="File">

<rdfs:subclassof rdf:resource="\#TiposEstructurados"/>

$</$ owl:Class $>$

<owl:Class rdf:ID="TipoCadena"> 
<rdfs:subClassof rdf:resource="\#DatosEstaticos"/>

$<$ owl:Class $>$

<owl:Class rdf:ID="String">

<rdfs:subclassof rdf:resource="\#TipoCadena"/>

$</$ owl:Class $>$

<owl:Class rdf:ID="DatosDinamicos">

<rdfs:subClassof rdf:resource="\#tiposDeDatos"/>

$</$ owl:Class $>$

<owl:Class rdf:ID="Pointer">

<rdfs:subClassof rdf:resource="\#DatosDinamicos"/>

$</$ owl:Class $>$

$<!--$ MODULOS -->

<owl:Class rdf:ID="ModulosPascal">

<rdfs:subclassof rdf:resource="\#ProgramaPascal"/>

$<$ owl:Class $>$

<owl:Class rdf:ID="Procedimientos">

<rdfs:subClassof rdf:resource="\#ModulosPascal"/>

$</$ owl:Class $>$

<owl:Class rdf:ID="ProcedimientosPreDefinidos">

<rdfs:subClassof rdf:resource="\#Procedimientos"/>

$</$ owl: Class $>$

<owl:Class rdf:ID="ProcedimientosString">

<rdfs:subclassof rdf:resource="\#ProcedimientosPreDefinidos"/>

$</$ owl:Class $>$

<owl:Class rdf:ID="Insert">

<rdfs:subClassof rdf:resource="\#ProcedimientosString"/>

$</$ owl:Class $>$

<owl:Class rdf:ID="Delete">

<rdfs:subClassof rdf:resource="\#ProcedimientosString"/>

$</$ owl:Class $>$

<owl:Class rdf:ID="Procedure">

<rdfs:subclassof rdf:resource="\#Procedimientos"/>

$</$ owl:Class $>$

<owl:Class rdf:ID="Funciones">

<rdfs:subclassof rdf:resource="\#ModulosPascal"/>

$<$ owl:Class $>$

<owl:Class rdf:ID="FuncionesPreDefinidas">

<rdfs:subClassof rdf:resource="\#Funciones"/>

$<$ owl:Class $>$

<owl:Class rdf:ID="FuncionesString">

<rdfs:subclassof rdf:resource="\#FuncionesPreDefinidas"/>

$</$ owl: Class $>$

<owl:Class rdf:ID="Concat">

<rdfs:subClassOf rdf:resource="\#FuncionesString"/>

$</$ owl:Class $>$

<owl:Class rdf:ID="Copy">

<rdfs:subClassOf rdf:resource="\#FuncionesString"/>

$<$ owl:Class $>$

<Owl:Class rdf:ID="Pos">

<rdfs:subclassof rdf:resource="\#FuncionesString"/>

$</$ owl:Class $>$

<owl:Class rdf:ID="Length">

<rdfs:subClassof rdf:resource="\#FuncionesString"/>

$</$ owl:Class $>$

<owl:Class rdf:ID="Function">

<rdfs:subclassof rdf:resource="\#Funciones"/>

$</$ owl:Class $>$

<owl:Class rdf:ID="Parametros">

<rdfs:subclassof rdf:resource="\#ModulosPascal"/>

$<$ owl:Class $>$

<owl:Class rdf:ID="Parametrovalor">

<rdfs:subClassof rdf:resource="\#Parametros"/>

$</$ owl:Class $>$

<owl:Class rdf:ID="ParametroVariable">

<rdfs:subclassof rdf:resource="\#Parametros"/>

$<$ /owl:Class $>$

$<!--$ SENTENCIAS -->

<owl:Class rdf:ID="Sentencias">

<rdfs:subclassof rdf:resource="\#ProgramaPascal"/>

$</$ owl:Class $>$

<owl:Class rdf:ID="Operadores"> 
<rdfs:subclassof rdf:resource="\#Sentencias"/>

$<$ /owl:Class $>$

<owl:Class rdf:ID="OperadoresAritmeticos">

<rdfs:subClassof rdf:resource="\#Operadores"/>

$</$ owl:Class $>$

<owl:Class rdf:ID="OperadoresLogicos">

<rdfs:subClassof rdf:resource="\#Operadores"/>

$</$ owl:Class $>$

<owl:Class rdf:ID="OperadoresRelacionales">

<rdfs:subClassof rdf:resource="\#Operadores"/>

$<$ owl:Class $>$

<owl:Class rdf:ID="Expresiones">

<rdfs:subclassof rdf:resource="\#Sentencias"/>

$</$ owl:Class $>$

<owl:Class rdf:ID="Condiciones">

<rdfs:subclassof rdf:resource="\#texpresiones"/>

$</$ owl:Class $>$

<owl:Class rdf:ID="Aritmeticas">

<rdfs:subClassof rdf:resource="\#Expresiones"/>

$</$ owl:Class $>$

<owl:Class rdf:ID="Secuencia">

<rdfs:subclassof rdf:resource="\#Sentencias"/>

$<$ owl:Class $>$

<owl:Class rdf:ID="Asignacion">

<rdfs:subclassof rdf:resource="\#Secuencia"/>

$</$ owl: Class $>$

<owl:Class rdf:ID="EntradaSalida">

<rdfs:subclassof rdf:resource="\#Secuencia"/>

$</$ owl:Class $>$

<owl:Class rdf:ID="Writeln">

<rdfs:subclassof rdf:resource="\#EntradaSalida"/>

$</$ owl:Class $>$

<owl:Class rdf:ID="Readln">

<rdfs:subClassof rdf:resource="\#EntradaSalida"/>

$<$ owl:Class $>$

<Owl:Class rdf:ID="Operaciones">

<rdfs:subclassof rdf:resource="\#Secuencia"/>

$</$ owl:Class $>$

<owl:Class rdf:ID="EstructurasDeControlEnPascal">

<rdfs:subclassof rdf:resource="\#Sentencias"/>

$<$ owl:Class $>$

<owl:Class rdf:ID="SeleccionEnPascal">

<rdfs:subClassof rdf:resource="\#EstructurasDeControlEnPascal"/>

$</$ owl: Class $>$

<owl:Class rdf:ID="IfThen">

<rdfs:subclassof rdf:resource="\#SeleccionEnPascal"/>

$</$ owl:Class $>$

<owl:Class rdf:ID="IfThenElse">

<rdfs:subclassof rdf:resource="\#SeleccionEnPascal"/>

$</$ owl:Class $>$

<owl:Class rdf:ID="Caseof">

<rdfs:subclassof rdf:resource="\#SeleccionEnPascal"/>

$</$ owl:Class $>$

<owl:Class rdf:ID="RepeticionEnPascal">

<rdfs:subclassof rdf:resource="\#EstructurasDeControlEnPascal"/>

$</$ owl:Class $>$

<owl:Class rdf:ID="RepeatUntil">

<rdfs:subclassof rdf:resource="\#RepeticionEnPascal"/>

$</$ owl:Class $>$

<owl:Class rdf:ID="WhileDo">

<rdfs:subClassof rdf:resource="\#RepeticionEnPascal"/>

$</$ owl:Class $>$

<owl:Class rdf:ID="ForToDo">

<rdfs:subclassof rdf:resource="\#RepeticionEnPascal"/>

$</$ owl:Class $>$

$</$ rdf :RDF $>$

\section{Ontología de Componentes de un material educativo}

$<$ ?xml version="1.0" encoding="UTF-8"?>

$<$ !DOCTYPE rdf:RDF [

<!ENTITY ComponentesDelMaterial "http://elotoolvl/ComponentesDelMaterial\#"> 


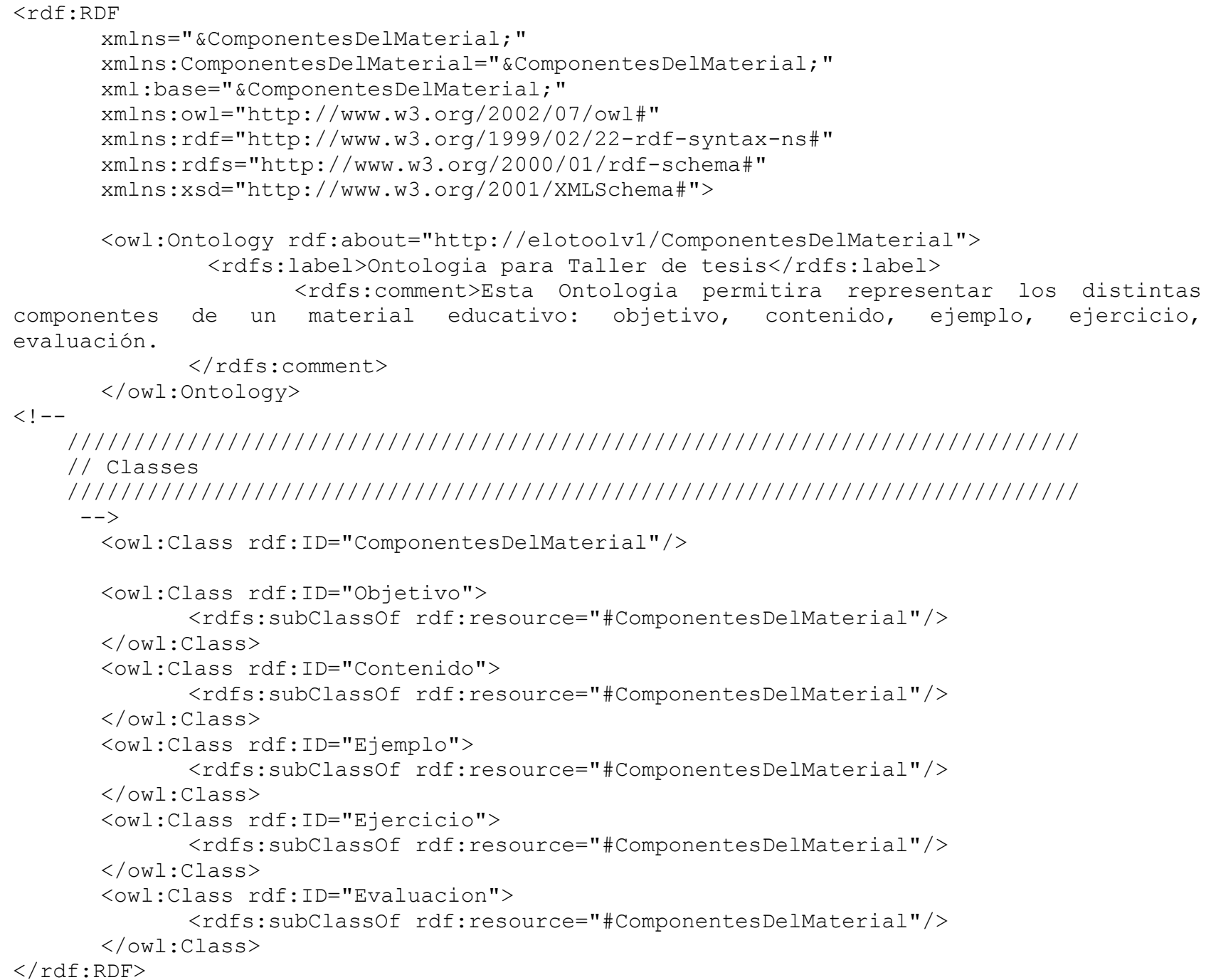




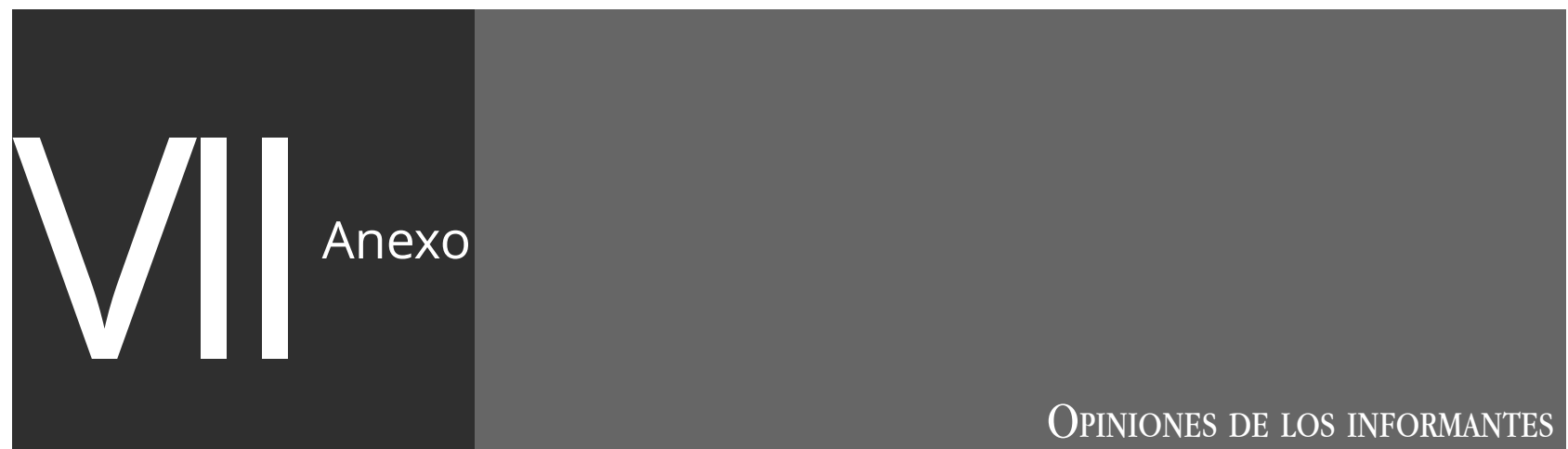

Este anexo contiene parte de las opiniones de los informantes recogidas durante y en el post taller de ensamblaje de OA a través de las distintas herramientas de recolección de datos ${ }^{1}$. Dichas opiniones se presentan aqui organizadas en tablas y fueron seleccionadas de las transcripciones completas por contener información relevante sobre las variables de estudio de esta Tesis.

Los cinco participantes de taller se identifican con: P1, P2, P3, P4 y P5.

Tabla 1. Entrevista. Respuestas de los participantes a:

¿Cómo fue la experiencia de trabajar solo/a para llevar adelante el proceso de ensamblaje?

\begin{tabular}{|c|c|}
\hline Participante & Respuestas \\
\hline P1 & $\begin{array}{l}\text { "A mí me pareció amigable, para mí el único punto fue tratar de } \\
\text { completar la mayor cantidad de metadatos posible, si bien trae } \\
\text { bastante cuando vas copiando del objeto de aprendizaje o las } \\
\text { unidades que ya tenés, trae algunas cosas cuando las copias, igual } \\
\text { hay que completar algunos datos más que es la parte más tediosa." }\end{array}$ \\
\hline P2 & $\begin{array}{l}\text { "La experiencia fue buena, era la primera vez que trabajaba con } \\
\text { material y herramientas de éste tipo, pero la experiencia fue } \\
\text { buena." }\end{array}$ \\
\hline P3 & $\begin{array}{l}\text { "Mirá, primero uno poco-bastante descolgado de algunos temas } \\
\text { que no sabía ni de qué se trataba prácticamente al principio. Pero } \\
\text { después, nada, me pareció sencillo porque empecé, digamos, con } \\
\text { bastante desconocimiento ni de qué se trataba, ni cómo funcionaba } \\
\text { el taller, ni como era todo esto de los objetos de ensamblaje, pero } \\
\text { después me pareció sencillo hasta el momento de llegar a la prueba } \\
\text { o la práctica que es lo que más te queda, aparte de lo que se charla, } \\
\text { es cuando lo ponés en práctica, entonces llevarlo a la práctica fue } \\
\text { bastante sencillo, porque estaba todo bastante definido. } \\
\text { Sinceramente, no sé cómo hubiese sido si tuviera que haber } \\
\text { definido cada uno de los objetos que vos ibas a ensamblar en la } \\
\text { práctica. Pero después sencillo, bastante sencillo, muy simple" }\end{array}$ \\
\hline
\end{tabular}

1 Cabe aclarar que al mostrar las respuestas de los informantes se resaltarán en negrita parte de las respuestas, que a juicio del autor, tengan más relevancia para el tema abordado. 
Tabla 2. Entrevista. Respuestas de los participantes a:

¿Tenés una idea aproximada de cuántos les llevó crear cada itinerario?

\begin{tabular}{|c|c|}
\hline Participante & Respuestas \\
\hline P1 & $\begin{array}{l}\text { "Yo creo que poquito, no más de } \mathbf{1 0} \text { minutos debe haber sido, una } \\
\text { cosa así. Pero ya teníamos muchas cosas listas." }\end{array}$ \\
\hline $\mathbf{P 2}$ & "Creo que aproximadamente unos $20 / 25$ minutos." \\
\hline P3 & $\begin{array}{l}\text { "Media hora me imagino. Sí porque estaba con la imagen que } \\
\text { mandaste de los elementos que estaban... yo pensé que iba a llevar } \\
\text { mucho tiempo pero nada, tres objetos, y sí me costó el primero, el } \\
\text { segundo y el tercero salieron rápidos." }\end{array}$ \\
\hline $\mathbf{P 4}$ & $\begin{array}{l}\text { "Nosotros hicimos tres itinerarios, porque pese a que teníamos } \\
\text { designado dos, decidimos con hacer... hicimos juntos los dos, pero } \\
\text { después decidimos unirlos y que quede uno solo, así que armamos } \\
\text { tres. Sí recuerdo que nos quedamos más tiempo que el resto, pero } \\
\text { no fue muy muy... después de que le agarramos la mano, le } \\
\text { encontrás la técnica, no fue mucho tiempo, no me pareció mucho. } \\
\text { Ni tampoco difícil." }\end{array}$ \\
\hline P5 & “¿Diez minutos? Menos, menos de diez minutos.” \\
\hline $\begin{array}{r}\text { Tabla 3. Entre } \\
\text { OA que te }\end{array}$ & $\begin{array}{l}\text { a. Respuestas de los participantes a: ¿Les resultó complicado encontrar los } \\
\text { nque usar para generar los itinerarios de aprendizaje que les tocaron? }\end{array}$ \\
\hline Participante & Respuestas \\
\hline P1 & "No, super fácil.” \\
\hline $\mathbf{P 2}$ & "No, no me resultó complicado..." \\
\hline P3 & $\begin{array}{l}\text { "No, no porque estaban definidos, estaban bien con el nombre y } \\
\text { aparte con la identificación..." }\end{array}$ \\
\hline $\mathbf{P 4}$ & "No porque usamos el ELOs-Viewer." \\
\hline P5 & $\begin{array}{l}\text { "No sé si complicado es la palabra... pero sí, tuvimos que pensarlo } \\
\text { sí" }\end{array}$ \\
\hline
\end{tabular}

Tabla 4. Registro audiovisual: dialogo inicia 19:43. Analizan el CA del itinerario “IA03a.ESTRUCTURAS REPETITIVAS EN PASCAL (FOR Y WHILE)”.

\begin{tabular}{cl}
\hline Participante & Respuestas \\
\hline P1 & $\begin{array}{l}{[\text { P1 debe comenzar con la carga de los requerimientos }]} \\
\text { "Requerimientos..." }\end{array}$ \\
P1 & "Los requerimientos del primero..." [en un intercambio con P5] \\
P4 & "Trae el primero, pero si querés más..." \\
\hline
\end{tabular}


Tabla 5. Registro audiovisual. P4 explica a P2 cómo funciona el CA en el ensamblaje.

\begin{tabular}{|c|c|}
\hline Participante & Dialogo \\
\hline Observador & “QQué tengo que mirar para poder ensamblar?” \\
\hline $\mathbf{P 2}$ & "Si el CA..." \\
\hline P4 & "Si esas competencias son las mismas o están incluídas..." \\
\hline $\mathbf{P 2}$ & $\begin{array}{l}\text { "No los requerimientos... Si los requerimientos están contenidos } \\
\text { en las competencias. Pueden ser menos requerimientos que las } \\
\text { competencias, pero no más..." }\end{array}$ \\
\hline P4 & “¡Está bien!” \\
\hline
\end{tabular}

Tabla 6. Registro audiovisual. Analizan el CA de los OA que deben ensamblar UD06 y UD01 utilizando ELOs-Viewer.

\begin{tabular}{cl}
\hline Participante & \multicolumn{1}{c}{ Dialogo } \\
\hline P4 & $\begin{array}{l}\text { "Al revés... lo que tengo que mirar qué requerimientos tiene es el } \\
\text { seis." }\end{array}$ \\
P5 & "Si estos están incluidos..." \\
P4 & "Los requerimientos del seis son éstos: repetición, objetivo y \\
& ejercicio" \\
P5 & "Está bien! Pero las competencias" \\
P4 & "Y... tengo que mirar en el uno" \\
P5 & "iNo! Al revés..." \\
P4 & $\begin{array}{l}\text { "No... las competencias de éste... éstas tienen que estar } \\
\text { incluidas allá... Si éstos no te dan no lo podés ensamblar con el } \\
\text { otro, el otro necesita como contenido mínimo, algo de lo que te de } \\
\text { esto. Estos saberes..." }\end{array}$ \\
P5 & "Lo había interpretado al revés."
\end{tabular}

Tabla 7. Registro audiovisual. La generación de las competencias. Consultas de P1 y P2 al Observador.

\begin{tabular}{ccc}
\hline Participante & Mm:ss & \multicolumn{1}{c}{ Respuestas } \\
\hline P1 & $21: 55: 00$ & $\begin{array}{l}\text { “iAh! Las competencias eran uniones de las } \\
\text { otras... Pero esas aparecen, ¿no?” }\end{array}$ \\
P2 & $29: 37: 00$ & $\begin{array}{l}\text { “iLas competencias pone por defecto la suma de } \\
\text { las dos competencias?” }\end{array}$ \\
\hline
\end{tabular}


Tabla 8. Registro audiovisual. Se intercambian opiniones sobre cómo se genera el CA (requerimientos), al momento de tener que completarlos.

\begin{tabular}{cl}
\hline Participante & Diálogo \\
\hline $\mathbf{P 1}$ & "Requerimientos..." \\
$\mathbf{P 4}$ & "Los requerimientos del primero..." \\
$\mathbf{P 1}$ & "Pero si eso lo trae ¿o no?" \\
\hline
\end{tabular}

Tabla 9. Entrevista. Respuestas de los participantes a:

"Se entendió cómo es que la metodología utiliza las ontologías para ensamblar..."

\begin{tabular}{|c|c|}
\hline Participante & Respuestas \\
\hline P1 & "Sí” \\
\hline P2 & $\begin{array}{l}\text { "Sí ... eso más o menos... eso quedó bastante claro, con el tema de } \\
\text { palabras clave o conceptos comunes o por lo menos algo que } \\
\text { vincule los objetos que uno quiere ensamblar." }\end{array}$ \\
\hline P3 & $\begin{array}{l}\text { "Sí, sí bastante. Al principio me costó más entender algunos } \\
\text { términos de fundamentación de la educación, digamos, de los } \\
\text { objetos de aprendizaje, pero después sí" }\end{array}$ \\
\hline P4 & $\begin{array}{l}\text { "No, más o menos... Quizás... no sé, conocer bien la definición de } \\
\text { lo que es ontología, ahí me quedé como que me faltó algún otro } \\
\text { ejemplo, alguna otra cosa para" }\end{array}$ \\
\hline P5 & "No, no me acuerdo eso..." \\
\hline
\end{tabular}

Tabla 10. Entrevista. Parte de las respuestas de los participantes a: ¿Con qué tipo de material se deben crear los itinerarios de aprendizaje según mi propuesta?

\begin{tabular}{|c|c|}
\hline Participante & Respuestas \\
\hline P1 & $\begin{array}{l}\text { "Yo creo que lo habíamos armado objetos de aprendizaje o unidad } \\
\text { didáctica" }\end{array}$ \\
\hline P2 & $\begin{array}{l}\text { "... Y un itinerario tiene, tal vez, un... el desarrollo de un tema } \\
\text { dentro de una unidad didáctica ..." }\end{array}$ \\
\hline P3 & $\begin{array}{l}\text { "Unidades de contenido. Eso es lo que más me costó, digamos, al } \\
\text { principio ubicarme en esos conceptos" }\end{array}$ \\
\hline P4 & $\begin{array}{l}\text { "Pasa que todos van armándose... unidades de contenido formas un } \\
\text { objeto de aprendizaje, podés juntar dos objetos de aprendizaje } \\
\text { para armar un itinerario de aprendizaje." }\end{array}$ \\
\hline P5 & $\begin{array}{l}\text { "Unidades de contenido trabajamos... . Yo no me acuerdo los } \\
\text { nombres tengo que refrescarlos." }\end{array}$ \\
\hline
\end{tabular}


Tabla 11. Registro audiovisual. Intercambio entre P1, P4 y el Observador sobre la incorporación de Objetivo y Ejercicio, al momento de tener que completar requerimientos.

\begin{tabular}{cl}
\hline Participante & \multicolumn{1}{c}{ Diálogo } \\
\hline P4 & "Trae el primero" \\
Observador "Claro... después habría que incluirles..." \\
P1 "Los del segundo" \\
Observador "No" \\
P4 "Todos los del primero" \\
Observador "Claro y habría que incluirle ejercitación y objetivo."
\end{tabular}

Tabla 12. Registro audiovisual. Consulta de P2 al Observador sobre la incorporación de requerimientos, al momento de tener que completarlos.

\begin{tabular}{cl}
\hline Participante & \multicolumn{1}{c}{ Diálogo } \\
\hline P2 & "¿Los requerimientos algunos había que agregarlos, no?" \\
Observador & "Mirá son éstos..." [Se resaltan en pantalla, sobre el IA de ejemplo, \\
& con ayuda de ELOs-Viewer] \\
$\mathbf{P 2}$ & "Sí objetivo y ejercicio \\
& $\begin{array}{l}\text { Ah! Sí la dirección es siempre la misma..." [en referencia a la URL } \\
\text { de las ontología a la que corresponden los requerimientos] }\end{array}$ \\
\hline
\end{tabular}

Tabla 13. Entrevista. Respuestas de los participantes a: ¿Te quedó claro por qué se agregaron "objetivo" y "ejercicio" como parte de los requerimientos?

\begin{tabular}{|c|c|}
\hline Participante & Respuestas \\
\hline P1 & $\begin{array}{l}\text { "En el momento me suena todo lo que me decís y lo habían } \\
\text { entendido, de por qué era, ahora no sé. Porque ensamblábamos } \\
\text { unidades... habíamos dicho que un objeto de aprendizaje constaba } \\
\text { de objetivos, ejercicios, digamos todo el objeto estaba constituido } \\
\text { de esas cosas, incluía también una evaluación... para ser un } \\
\text { objeto de aprendizaje tenía que constar de esas partes y la } \\
\text { podíamos ir armando". }\end{array}$ \\
\hline $\mathbf{P 2}$ & $\begin{array}{l}\text { "Creo que eso de alguna manera completa un poco los objetos de } \\
\text { aprendizaje que uno tiene para ensamblar y de alguna manera te } \\
\text { permite un ensamblaje más rico y permite crear unidades más } \\
\text { completas." }\end{array}$ \\
\hline P3 & $\begin{array}{l}\text { "No. Ah! Dijiste sí, pero que eran los mismos objetivos que estaban } \\
\text { definidos en cada uno de los objetos ¿o no?¿Algo así era?" }\end{array}$ \\
\hline P4 & $\begin{array}{l}\text { "No. Sí sé que estaban que estaban las actividades y la evaluación, } \\
\text { eso sí. Se me ocurre con que tiene que ver netamente con } \\
\text { cuestiones docente, digamos, pedagógicas: tener cuáles son los } \\
\text { objetivos para poder trabajar y trazar después el itinerario. Pero, } \\
\text { no" }\end{array}$ \\
\hline P5 & $\begin{array}{l}\text { “...¿cómo era eso? objetivos y ejercicios, eso para poder usarlos } \\
\text { para otro ensamblaje entonces teníamos objetivos en común, } \\
\text { entonces después buscábamos esos objetivos para... porque uno } \\
\text { estaba incluido dentro del otro, entonces buscábamos esos en } \\
\text { comunes para poderlos ensamblar ¿era así, no?" }\end{array}$ \\
\hline
\end{tabular}


Tabla 14. Apreciaciones de los informantes sobre por qué la metodología facilitaría su tarea como docentes.

"lo facilitaría por la reutilización del material educativo"

"Si tenemos como docentes la intencionalidad de reutilizar una unidad didáctica creada por otros, no siempre se adapta o amolda a nuestras necesidades, conocimiento asociado, intenciones pedagógicas. Al ensamblar OAs podemos conseguir el "paquete" que uno desea."

"Porque me permitiría crear material de mayor complejidad y abarcar así varios procesos relativos al aprendizaje ( objetivos, introducción del tema, aplicaciones, ejercicios y problemas, auto-evaluación, entre otras)"

"Porque me permitiría armar itinerarios de aprendizaje con material ya existente, o con parte e material existente y liberaría al docente (en caso de que el ensamblaje de efectúe automáticamente) de la tarea de buscar al cobertura de requisitos y competencias entre OAs"

"Sería más práctica para una planificación de temas, y asociativa para los mismos."

Tabla 15. Apreciaciones de los informantes sobre por qué la metodología facilitaría la reutilización de OA.

"se ahorra tiempo en cuanto a la creación del material a usar"

"Creo que si la granularidad del material es la adecuada será posible la reutilización de los materiales educativos. Será posible entonces usar el material en distintos itinerarios"

"Porque el ensamblaje puede ser gradual: a medida que creamos OA, podemos ir ensamblándolos con itinerarios ya creados y obtener así otros IA más complejos"

"Por lo que expliqué en la pregunta anterior"

"Una vez definidos, puede reutilizarse ya que hay muchos temas asociados.-"

Tabla 16. Entrevista. Respuestas de los participantes a:

¿Cuál fue tu experiencia con la carga de metadatos? ¿Te resultó un trabajo "pesado”?

\begin{tabular}{|c|c|}
\hline Participante & Respuestas \\
\hline P1 & $\begin{array}{l}\text { "Había algunas cosas que no me quedaban claras, lo de } \\
\text { densidad semántica que después seguimos investigando un poquito } \\
\text { y qué quería decir... Es un trabajo que si queremos que se } \\
\text { encuentren las cosas más rápidamente y demás, hay que tomarse el } \\
\text { tiempo para hacerlo. Sí, pero fue lo más tedioso de hacer." }\end{array}$ \\
\hline P2 & $\begin{array}{l}\text { "Y en principio uno tiene la sensación de que es excesiva la } \\
\text { cantidad datos que te piden, pero pesado en realidad... un poco } \\
\text { difícil en algunos casos de determinar qué es lo que me están } \\
\text { pidiendo." }\end{array}$ \\
\hline P3 & $\begin{array}{l}\text { "No para nada. Vah! cuando lo ves que ves que son tantos datos } \\
\text { que se solicitan, tantas variables, tantos... decís ¿todos estos? Pero } \\
\text { después por el power point que vos nos diste que estuve leyendo } \\
\text { eran pocos. Digamos al principio parecía como que iban a ser } \\
\text { muchos y complicado, pero terminaron siendo pocos datos de } \\
\text { todos esos los que se iban a utilizar y sencillos" }\end{array}$ \\
\hline
\end{tabular}

Continúa... 
Tabla 16 (continuación). Entrevista. Respuestas de los participantes a: ¿Cuál fue tu experiencia con la carga de metadatos? ¿Te resultó un trabajo "pesado”?

\begin{tabular}{|c|c|}
\hline Participante & Respuestas \\
\hline P4 & $\begin{array}{l}\text { "Resultó largo, pero... era prestar atención, focalizada en eso } \\
\text { para no errarle, pero no, no me fue difícil. Sabiendo que eso era } \\
\text { un montón de campos y que muchos venían solos porque ya } \\
\text { venían heredados o no se completaban. Si hubieses pedido los 'no } \\
\text { se cuántos campos', sí..." }\end{array}$ \\
\hline P5 & $\begin{array}{l}\text { "[cuando tuvieron que completar el formulario...] } \\
\text { Emmh, no no. Lo que pasa que tenés que estar ahí al toque } \\
\text { atento, bien concentrado con el tema de los metadatos sino después } \\
\text { se te empiezan a, no sé si olvidar, pero tenés que empezar a tomarte } \\
\text { más tiempo para empezar a recordarlos" }\end{array}$ \\
\hline
\end{tabular}

Tabla 17. Entrevista. Respuestas de los participantes a:

¿Tuviste que usar la ayuda que les dejé sobre metadatos en el ensamblaje?

¿Crees que sin la ayuda no lo hubieses podido lograr?

\begin{tabular}{|c|c|}
\hline Participante & Respuestas \\
\hline \multirow[t]{3}{*}{ P1 } & "Para completar los metadatos? Sí la usé \\
\hline & {$[\ldots]$} \\
\hline & me fue de mucha ayuda, "la ayuda"" \\
\hline $\mathbf{P 2}$ & $\begin{array}{l}\text { "Sí use la ayuda porque eso me ahorró tiempo, porque el hecho de } \\
\text { tener que pensar y tener que deducir que es lo que me pide en cada } \\
\text { categoría del ingreso de metadatos y cada uno de los sectores, eso } \\
\text { insume tiempo de aprender qué es lo que pide y sí me fue de } \\
\text { utilidad la ayuda y me ahorró tiempo en el ensamblaje." }\end{array}$ \\
\hline P3 & $\begin{array}{l}\text { "Ya te digo, la primera vez sí, iba siguiendo los pasos... es como } \\
\text { cuando haces con el asistente que te indicaba paso a paso cómo } \\
\text { completar, ya la segunda vez no, porque ya era prácticamente lo } \\
\text { mismo y cosas de las que había escrito, la reutilizás viste con el } \\
\text { navegador las vas reutilizando en la medida que completás." }\end{array}$ \\
\hline P4 & $\begin{array}{l}\text { "No, creo que ni lo miramos... porque íbamos leyendo los } \\
\text { campos y... no. Además, te acordás que con vos estuvimos } \\
\text { practicando con un ejemplo. No, me parece que no lo } \\
\text { miramos..." }\end{array}$ \\
\hline P5 & "Sí lo usamos..." \\
\hline
\end{tabular}


Tabla 18. Entrevista. Respuestas de los participantes a: Los que tenían que definir uds ¿les resultó complejo definir qué escribir en cada caso?

\begin{tabular}{cl}
\hline Participante & \multicolumn{1}{c}{ Respuestas } \\
\hline P1 & "No, no me resultó para nada complejo" \\
P2 & $\begin{array}{l}\text { "No los datos que yo tuve que definir eran bastante específicos de } \\
\text { acuerdo al tema y al tipo de material }[. . .] \text { no fue difícil porque el } \\
\text { tema era bastante acotado, conocido por mí }\end{array}$ \\
& "No para nada" \\
P3 & "No" \\
P4 & "No, en eso nos pusimos de acuerdo enseguida" \\
\hline
\end{tabular}

Tabla 19. Entrevista. Respuestas de los participantes a: Crees que ELO-Tool ¿Te facilitó la carga de los metadatos?

\begin{tabular}{|c|c|}
\hline Participante & Respuestas \\
\hline P1 & $\begin{array}{l}\text { "Sí, sí }[\ldots] \text { en cuanto a la funcionalidad que ofrece creo que está } \\
\text { bien, que adecuada." }\end{array}$ \\
\hline P2 & $\begin{array}{l}\text { "Sí, yo creo que facilita la carga porque queda claro cuáles con los } \\
\text { requerimientos de los metadatos... sí creo que facilitó la carga." }\end{array}$ \\
\hline P3 & $\begin{array}{l}\text { "Sí porque estaba bien detallada, es como un diccionario de datos. } \\
{[\ldots . .] "}\end{array}$ \\
\hline P4 & $\begin{array}{l}\text { "No sé si facilitar porque tendría que poder compararlo con algún } \\
\text { otro [...]. No me resultó difícil." }\end{array}$ \\
\hline P5 & $\begin{array}{l}\text { "Sí, va no sé si facilitó... es lo que vimos. Como es lo único que vi } \\
\text { no sé si me facilitó o no... es lo que vi en el momento. Lo que pasa } \\
\text { es que no tenía otra opción para saber si me facilitada o no." }\end{array}$ \\
\hline
\end{tabular}

Tabla 20. Entrevista. Respuestas de los participantes a: ¿Usaste ELOs-Viewer en el proceso o te alcanzó con la ayuda en PDF para ensamblar en ELO-Tool?

\begin{tabular}{|c|c|}
\hline Participante & Respuestas \\
\hline P1 & "No, creo que me arreglé con tu PDF." \\
\hline $\mathbf{P 2}$ & $\begin{array}{l}\text { "No, me fue de utilidad ELOs-Viewer, porque cuando uno } \\
\text { visualiza los objetos que va a ensamblar aparece información } \\
\text { importante que es muy útil para la carga de metadatos y aparece } \\
\text { de forma clara y, digamos, facilita el encontrar la información que } \\
\text { uno necesita para la carga de metadatos." }\end{array}$ \\
\hline P3 & "No, el power point $[\ldots] "$ \\
\hline $\mathbf{P 4}$ & $\begin{array}{l}\text { "ELOs-Viewer sí. De ese íbamos mirando qué tenía, digamos, lo } \\
\text { que te decía, a uno le entra por la vista, la interfaz gráfica los vos } \\
\text { veías en ELOs-Viewer nos fue ayudando para ensamblaje." }\end{array}$ \\
\hline P5 & $\begin{array}{l}\text { "[...] Eso no me acuerdo, el PDF me acuerdo que lo usamos, el } \\
\text { ELOs-Viewer no me acuerdo si lo habíamos usado..." }\end{array}$ \\
\hline
\end{tabular}


Tabla 21. Entrevista. Respuestas de los participantes a: ¿Te acordás para qué usaste ELOs-Viewer?

\begin{tabular}{ll}
\hline Participante & Respuestas \\
\hline
\end{tabular}

P1 "ELOs-Viewer, sí para buscar los objetos de aprendizaje con los que iba a hacer el itinerario y mirar adentro alguna de los... bueno los requisitos y copiar los objetivos, sí"

P2 "Sí, porque la visulización que da la herramienta da en forma fácil y práctica datos necesarios para la carga de metadatos."

P3 “Al principio para ver cada uno... inclusive miré algunos cómo estaban definidos. Sí creo que sí, pero muy poquito al principio, antes de comenzar a completar uno."

P4 "Fue buscar de ahí los objetos de aprendizaje[...]"

P5 "Sí, pero no me acuerdo si lo habíamos usado..." 